

\section{DISCLAIMER}

This report was prepared as an account of work sponsored by an agency of the United States Government. Neither the United States Government nor any agency Thereof, nor any of their employees, makes any warranty, express or implied, or assumes any legal liability or responsibility for the accuracy, completeness, or usefulness of any information, apparatus, product, or process disclosed, or represents that its use would not infringe privately owned rights. Reference herein to any specific commercial product, process, or service by trade name, trademark, manufacturer, or otherwise does not necessarily constitute or imply its endorsement, recommendation, or favoring by the United States Government or any agency thereof. The views and opinions of authors expressed herein do not necessarily state or reflect those of the United States Government or any agency thereof. 


\section{DISCLAIMER}

Portions of this document may be illegible in electronic image products. Images are produced from the best available original document. 
WAPD-TM-1275

Special External Distribution

AIRWAY_A FORTRAN COMPUTER PROGRAM TO ESTIMATE RADIATION DOSE COMMITMENTS TO MAN FROM THE ATMOSPHERIC RELEASE OF RADIONUCLIDES

(LWBR Development Program)

\section{J. L. Rider}

Contract EY-76-C-11-0014

Printed in the United States of America

Available from the

National Technical Information Service

U. S. Department of Commerce

5285 Port Royal Road

Springfield, Virginia 22151

This document is an interim memorandum prepared primarily for internal reference and does not repre-
sent a final expression of the opinion of Westinghouse. When this memorandum is distributed externally,
it is with the express understanding that Westinghouse makes no representation as to completeness,
accuracy, or usability of information contained therein.

\section{BETTIS ATOMIC POWER LABORATORY WEST MIFFLIN, PENNSYLVANIA}

Operated for the U. S. Department of Energy by. WESTINGHOUSE ELECTRIC CORPORATION 
This report was prepared as an account of work sponsored by the United States Government. Neither the United States, nor the United States Department of Energy, nor any of their employees, nor any of their contractors, subcontractors, or their employees, makes any warranty, express or implied, or assumes any legal liability or responsibility for the accuracy, completeness or usefulness of any information, apparatus, product or process disclosed, or represents that its use would not infringe privately owned rights. 


\section{FOREWORD}

The Shippingport Atomic Power Station located in Shippingport, Pennsylvania was the first large-scale, central-station nuclear power plant in the United States and the first plant of such size in the world operated solely to produce electric power. This program was started in 1953 to confirm the practical application of nuclear power for large-scale electric power generation. It has provided much of the technology being used for design and operation of the commercial, central-station nuclear power plants now in use.

Subsequent to development and successful operation of the Pressurized Water Reactor in the DOE-owned reactor plant at the Shippingport Atomic Power Station, the Atomic Energy Commission in 1965 undertook a research and development program to design and build a Light Water Breeder Reactor core for operation in the Shippingport Station.

The objective of the Light Water Breeder Reactor (LWBR) program has been to develop a technology that would significantly improve the utilization of the nation's nuclear fuel resources employing the well-established water reactor technology. To achieve this objective, work has been directed toward analysis, design, component tests, and fabrication of a water-cooled, thorium oxide fuel cycle breeder reactor for installation and operation at the Shippingport Station. The LWBR core started operation in the Shippingport Station in the Fall of 1977 and is expected to be operated for about 3 to 4 years. At the end of this period, the core will be removed and the spent fuel shipped to the Naval Reactors Expended Core Facility for a detailed examination to verify core performance including an evaluation of breeding characteristics.

In 1976, with fabrication of the Shippingport LWBR core nearing completion. the Energy Research and Development Administration established the Advanced Water Breeder Applications (AWBA) program to develop and disseminate technical information which would assist U.S. industry in evaluating the LWBR concept for commercial-scale applications. The program will explore some of the problems that would be faced by industry in adapting technology confirmed in the LWBR program. Information to be developed includes concepts for commercial-scale prebreeder cores which would produce uranium-233 for light water breeder cores while producing electric power, improvements for breeder cores based on the technology developed to fabricate and operate the Shippingport LWBR core, and other information and technology to aid in evaluating commercial-scale application of the LWBR concept.

All three development programs (Pressurized Water Reactor, Light Water Breeder Reactor, and Advanced Water Breeder Applications) have been administered by the Division of Naval Reactors with the goal of developing practical improvements in the utilization of nuclear fuel resources for generation of electrical energy using water-cooled nuclear reactors.

Technical information developed under the Shippingport, LWBR, and AWBA programs has been and will continue to be published in technical memoranda, one of which is this present report. 


\section{TABLE OF CONTENTS}

I. INTRODUCTION 1

II. MODEL DESCRIPTION

III. MODEL EQUATIONS

A. Air and Ground Activity Concentration

1. Air Concentrations-Vicinity of Releases

a. During Releases. 17

b. Subsequent to Releases 17

2. Air Concentration-Beyond Vicinity of Releases 18

a. During Release 18

b. Subsequent to Releases 18

3. Ground Concentration-Vicinity of Releases 18

a. During Releases 18

b. Subsequent to Releases 19

4. Ground Concentrations-Beyond Vicinity of Releases

B. Dose Commitments 19

1. Immersion Dose Commitments 20

a. Vicinity of Releases 20

b. Beyond Vicinity of Releases 21

2. Surface Deposit Dose Commitments

a. Vicinity of Releases $\quad . \quad 22$

b. Beyond Vicinity of Releases

3. Ingestion Dose Commitments 25

a. Food Supply Subjected to Direct Deposition 25

b. Food Supply Produced in Previously Contaminated Ground 29

4. Inhalation Dose Commitments 33

a. Radionuclides Not Previously Deposited

b. Resuspended Radionuclides

C. Parameters 39

1. Precipitation Scavenging Constant 39

2. Root Uptake Factor 39

3. Standard Deviation of Vertical Dispersion 40

4. Dry Deposition Integral Term 40

5. Modified Release Rates 41

6. Number Concentrations

IV. PROGRAM DESCRIPTION

V. ACKNOWLEDGEMENTS 46

$\begin{array}{ll}\text { VI. REFERENCES } & 47\end{array}$

APPENDIX A-AIRWAY PROGRAM ABSTRACT

APPENDIX B—AIRWAY PROGRAM EQUATIONS

APPENDIX C-AIRWAY INP̈ÜT DESCRIPTION

APPENDIX D-SAMPLE PROBLEM 
TABLE OF CONTENTS (Cont)

APPENDIX E-PROGRAM VERIFICATION AND QUALIFICATION

APPENDIX F-GENERAL INPUT DATA

\section{LIST OF ILLUSTRATIONS}

Figure

Title

Page

1 Typical Air Concentration of Gaseous Radionuclides in the Vicinity of the Release

Point as a Function of Time

'l'ypical Air C'oncentration of Particulate Kadionuclides in the Vicinity of the Release Point as a Function of Time

Typical Ground Concentration of Released Radionuclides in the Vicinity of the Release Point as a Function of Time

Typical Ground Concentration of Daughter Radionuclides in the Vicinity of the Release Point as a Function of Time

Typical Air Concentration of Radionuclides in Affected Regions Beyond 80 to $100 \mathrm{~km}$ as a Function of Time

6 Typical Ground Concentration of Radionuclides in Affected Regions Beyond 80 to $100 \mathrm{~km}$ as a Function of Time 


\section{NOMENCLATURE}

Symbol

$\AA$

$A$

$A C_{d}$

$\mathrm{AC}_{\mathrm{b}}$

$\mathrm{AC}_{\mathrm{c}}$

$\mathrm{AC}_{\mathrm{d}}$

$\bar{A} \bar{Z}_{\mathrm{a}}$

$\mathbf{A}_{\chi_{\text {a }}}$

$\mathbf{A} \chi_{\mathrm{b}}$

$\mathrm{A}_{\chi_{\mathrm{c}}}$

B

$\mathrm{B}_{\mathrm{a}}$

$\mathrm{C}_{\mathrm{a}}$

$\mathrm{C}_{\mathrm{b}}$

Subscripted term used in the equation defining the ground concentrations during releases in the region affected by the releases which is beyond the immediate neighborhood of the release point. Defined by Equation $(12)-\mathrm{Ci} / \mathrm{m}^{3}$

Surface area of the regions beyond the immediate vicinity of the release point which are affected by the releases $-m^{2}$

The number concentrations on the ground in the region immediately surrounding the release point during the time that releases are occurring-\# atoms $/ \mathrm{m}^{2}$

The number concentrations on the ground in the region immediately surrounding the release point after the releases have stopped-\# atoms $/ \mathrm{m}^{2}$

The number concentrations on the ground in the region beyond the immediate neighborhood of the release point which is affected by the releases during the period that releases are occurring-\# atoms $/ \mathrm{m}^{2}$

The number concentration on the ground in the region beyond the immediate neighborhood of the release point which is affected by the releases after the releases have stopped-\# atoms $/ \mathrm{m}^{2}$

The total number of atoms of a radionuclide available for precipitation scavenging in a column of air over a unit area of the region immediately surrounding the release point during the period that releases are occurring-\# atoms $/ \mathrm{m}^{2}$

The number concentration in the air at ground level in the region immediately surrounding the release point during the period that releases are occurring-\# atoms $/ \mathrm{m}^{3}$

The number concentration in the atmosphere of the region beyond the immediate vicinity of the release point which is affected by the releases during the period that releases are occurring-\# atoms $/ \mathrm{m}^{3}$

The number concentration in the atmosphere of the region beyond the immediate vicinity of the release point which is affected by the releases after they have stopped-\# atoms $/ \mathrm{m}^{3}$

Subscripted term used in the equation defining the ground concentration during release in the region affected by the release which is beyond the immediate neighborhood of the release point. Defined by Equation (13).

Breathing rate of people subject to the inhalation pathways $-\mathrm{m}^{3} / \mathrm{hr}$

The activity concentration on the ground in the region immediately surrounding the release point during the period that releases are occurring $-\mathrm{Ci} / \mathrm{m}^{2}$

The activity concentration on the ground in the region immediately surrounding the release point after the releases have stopped $-\mathrm{Ci} / \mathrm{m}^{2}$ 
$\mathrm{C}_{c}$

$\mathrm{C}_{\mathrm{d}}$

$\mathrm{C}_{\mathrm{p}}$

$\mathrm{C}_{\mathrm{E}}$

$\bar{C}_{a}$

$\overline{\mathrm{C}}$

$\mathrm{D}_{\mathrm{I}}$

$\mathrm{D}_{\mathrm{II}}$

$\mathrm{D}_{\text {III }}$

$\mathrm{D}_{\mathrm{IV}}$

d

ds

E

$\mathbf{F}$

FMAX

$f$

$\mathbf{G}$
The activity concentration on the ground in the region affected by the releases which is beyond the immediate neighborhood of the release point during the period that releases are occurring- $\mathrm{Ci} / \mathrm{m}^{2}$

The activity concentration on the ground in the region affected by the releases which is beyond the immediate neighborhood of the release point after the releases have stopped- $\mathrm{Ci} / \mathrm{m}^{2}$

Concentration of a nuclide in food and forage plants grown in soil with a concentration $\mathrm{C}_{\mathrm{s}} \rightarrow$ grams nuclide/gram plant

Concentration of a nuclide in the soil-grams nuclide/gram soil

I'he ground concentration in the region immediately surrounding the release point averaged over the area in this region in which food is produced while releases are occurring- $\mathrm{C} i / \mathrm{m}^{2}$

The ground concentration in the region immediately surrounding the release point averaged over the area in this region in which food is produced after the releases have stopped- $\mathrm{C} i / \mathrm{m}^{2}$

Dose commitment due to immersion in a cloud of radionuclides-rem

Dose commitment due to exposure to a surface deposit of radioactive nuclides-rem

Dose commitment due to the ingestion of radionuclides-rem

Dose commitment due to the inhalation of radionuclides-rem

The number of increments used to approximate the integration over the intervals between the radial distances at which the standard deviation of vertical dispersion values are provided in calculating the effect of dry deposition

Depth of the root-bearing layer of soil in which food is produced-cm

Subseripted term ueed in tho oquation which defincs the ground concentration during releases in the region affected by the releases which is beyond the immediate neighborhood of the release point. Defined by Equation (14).

Multiplication factor applied to the pathway dose commitments

Maximum number of food types considered

Fraction of the time that the wind is blowing into a given sector with a specific wind speed while a given meteorological stability category exists

Subscripted term used in the equation which defines the dose commitment due to ingestion of food produced in the immediate vicinity of the release point which is contaminated via deposition. Defined by Equation (36)- $\mathrm{Ci} / \mathrm{m}^{2}$ 
$\mathrm{H}$

$\mathrm{h}$

I

J

JMAX

$\mathrm{K}$

KMAX

$\mathrm{K}_{\mathrm{I}}$

$\mathrm{K}_{\mathrm{II}}$

$\dot{K}_{\text {III }}$

$\mathrm{K}_{\mathrm{IV}}$

$\mathrm{K}_{0}$

$\mathrm{K}_{1}$

L

Subscripted term used in the equation which defines the dose.commitment due to the ingestion of food prosiuced in ground in the vicinity of the release point which had beer contaminated by the deposition of radionuclides prior to its use to produce food. Defined by Equation ( 50$)-\mathrm{Ci} / \mathrm{m}^{2}$

Height above the surface of the ground of the point at which the radionuclides are released-m

Subscripted term used in the equation which defines the dose commitment due to the ingestion of food produced in soil in the vicinity of the release point which was conta minated by radionuclides prior to its use to produce food. Defined by Equation $(51)-\mathrm{Ci} / \mathrm{m}^{2}$

Subscripted term used in the equation which defines the ground concentration in the region immediately surrounding the release point after the releases have stopped. Defined by Equation ( 7$)-\mathrm{Ci} / \mathrm{m}^{2}$

The total number of angular sectors into which the region surrounding the release point is subdivided

Amount of activity taken up per unit mass of food due to a unit activity concentration in the food producing area per unit thickness of the root-bearing layer- $\mathrm{m}^{2} / \mathrm{gm}$

The total number of annuli into which the region surrounding the release point is subdivided

Dose rate conversion factor defining the dose rate resulting from immersion in a cloud containing a unit activity concentration-rem $/ \mathrm{hr}$ per $\mathrm{Ci} / \mathrm{m}^{3}$

Dose rate conversion factor defining the dose rate resulting from exposure to a surface deposit containing a unit surface activity concentration-rem $/ \mathrm{hr}$ per $\mathrm{Ci} / \mathrm{m}^{2}$

Dose commitment conversion factor defining the dose accumulated during a given time interval due to the ingestion of a unit quantity of activity at the beginning of the time interval-rem/Ci

Dose commitment conversion factor defining the dose accumulated during a given time interval due to the inhalation of a unit quantity of activity at the beginning of the time interval-rem/Ci

The coefficient of the exponential term of the expression used to define the relationship between resuspended material and the material concentration on the ground- $m^{-1}$

The constant term of the expression used to define the relationship between the resuspended material and the material concentration on the ground $-\mathrm{m}^{-1}$

Subscripted term used in the equation which defines the dose commitment due to the ingestion of food produced in soil in the vicinity of the release point which was contaminated by the deposition of activity prior to its use to produce food. Defined by Equation $(52)-\mathrm{Ci} / \mathrm{m}^{2}$ 
M

MMAX

$\mathbf{N}$

NMAX

$\ddot{\mathbf{P}}$

$\mathbf{P}_{\mathrm{T}}$

Q

$Q^{\prime}$

$q$

$q_{0}$

k

RMAX

$\mathbf{R}_{\mathrm{S}}$

$\mathbf{r}$

$\mathrm{S}$

$\mathrm{S}_{\mathrm{s}}$

$\mathrm{T}_{\mathrm{fa}}$

Subscripted term used in the equation which defines the dose commitment due to the ingestion of food produced in soil in the vicinity of the release point which was con- taminated by radionuclides prior to its use to produce food. Defined by Equation (53) $-\mathrm{Ci} / \mathrm{m}^{2}$

The total number of stability categories used to define the meteorological conditions

Subscripted term used in the equation which defines the dose commitment due to the ingestion of food produced in soil in the vicinity of the release point which was contaminated by the deposition of activity prior to its use to produce food. Defined by Equation (54) $\cdots-\mathrm{Ci} / \mathrm{m}^{2}$

The total number of wind velocity increments used to define the meteorological condítions

The population in the angular seyments into which the region immediately surrounding the release point is subdivided at the time releases start

The population in the regions beyond the immediate vicinity of the release point which are affected by the releases at the time releases start

Constant rate of continuous release of activity- $\mathrm{Ci} / \mathrm{hr}$.

The rate at which activity is being introduced in the region beyond the boundary of the immediate neighborhood of the release point- $\mathrm{Ci} / \mathrm{hr}$

The amount of food consumed per person per unit time-gm/day (solids) or liters/day (liquid)

The normal intake of food per person per unit time- $\mathrm{gm} /$ day (solids) or liters/day (liquids)

Subscripted term used in the equation which defines the ground concentration in the region beyond the immediate neighborhood of the release point after releases have. stopped. Defined by Equation $(16)-\mathrm{Ci} / \mathrm{m}^{2}$

The radial distance to the boundary of the region immediately surrounding the release point $-m$

Precipitation rale- $\mathrm{m} / \mathrm{hr}$

Radial distance-m

Subscripted term used in the equation which defines the ground concentration in the region beyond the immediate neighborhood of the release point after releases have stopped. Defined by Equation (17) ..... $\mathrm{Ci} / \mathrm{m}^{2}$

The precipitation scavenging ratio (by volume)

The time at which the deposition of activity upon the food crops begins measured from the time that releases start-hr 
$\mathrm{T}_{\mathrm{fb}}$

$\mathrm{T}_{\mathrm{I}}$

$T_{L}$

$\mathrm{T}_{\mathrm{S}}$

$\mathrm{T}_{\mathrm{SI}}$

$\mathrm{T}_{\mathrm{SL}}$

t

U

VMAX

$\mathrm{V}_{\mathrm{a}}$

v

$\mathbf{w}$

$\mathrm{Y}$

$\bar{Y}$

y

Z

$\mathrm{Z}_{\mathrm{s}}$

$\overline{\mathrm{z}_{\mathrm{a}}}$

$\alpha$
The time at which the deposition of activity upon the food crops ends measured from the time that releases start-hr

$\infty$

The beginning of the time interval over which the dose commitments are to be obtained measured from the time at which releases start-hr

The end of the time interval over which the dose commitments are to be obtained measured from the time at which releases start-hr

The time that releases stop measured from the time at which the releases begin- $\mathrm{hr}$

The upper limit of the time interval over which the dose commitments during releases are calculated-hr

The lower limit of the time interval over which the dose commitments after releases stop are calculated-hr

Time measured from the start of releases-hr

Subscripted term used in the equations for R and S. Defined by Equation (18).

The total number of radial positions at which the standard deviation of vertical dispersion is available for each of the meteorological stability conditions considered

Volume of the atmosphere in the region beyond the immediate vicinity of the release point which is affected by the releases- $\mathrm{m}^{3}$

Dry deposition velocity-m/sec

The activity intake rate associated with a $q_{0}$ food intake rate per unit deposition rate per unit area- $\mathrm{m}^{2}$

Rate at which activity is depositing per unit area due to dry deposition mechanisms and precipitation scavenging- $\mathrm{Ci} / \mathrm{m}^{2} \mathrm{hr}$

Spatially averaged rate at which activity is depositing per unit area due to dry deposition mechanisms and precipitatin scavenging- $\mathrm{Ci} / \mathrm{m}^{2} \mathrm{hr}$

Parameter used to define the radial distances in the intervals between the distances at which the standard deviation of vertical dispersion date is available

Height of the atmosphere-m

Height of the rain-bearing layer in the atmosphere-m

The activity available for precipitation scavenging in a column of air over a unit area of the region immediately surrounding the release point during the period that releases are occurring- $\mathrm{Ci} / \mathrm{m}^{2}$

Loss coefficient term representing all (sum of) ground concentrations removal mechanisms $-\mathrm{hr}^{-1}$ 
$\beta$

$\gamma$

$\Delta \mathbf{r}$

$\boldsymbol{\theta}$

$\wedge$

$\lambda_{\mathrm{d}}$

$\lambda_{\mathrm{pl}}$

$\lambda_{\mathrm{p} 2}$

$\lambda_{r}{ }^{\circ}$

$\lambda_{\mathrm{s}}$

$\lambda_{\text {w }}$

$\bar{\lambda}$

$\bar{\mu}$

$\rho_{\mathrm{s}}$

$\sigma_{z}$

$x_{\mathrm{a}}$

$x_{b}$

$x_{c}$

$\overline{\chi_{b}}$

Resuspension factor relating the amount of activity which is resuspended in the air to the activity concentrations on the surface of the ground $-\mathrm{m}^{-1}$

Loss coefficient term representing all (sum of) the deposition mechanisms from the activity concentrations in the atmosphere of the region beyond the immediate neighborhood of the release point which is affecled by the releases-m/hr

Width of the increments into which the intervals between the distances at which the standard deviations of the vertical dispersion are subdivided-m

Width of an azimuthal sector-radians

Radioactive decay constant-hr 1

Loss rate constant of the exponential term of the expression used to define the relationship between the resuspended activity in the air and the surface activity concentration on the ground $-\mathrm{hr}^{-1}$

Population growth rate constant in the region immediately surrounding the release point- $\mathrm{hr}^{-1}$

Population growth rate constant in the region beyond the immediate neighborhood of the release point which is affected by the releases- $\mathrm{hr}^{-1}$

Resuspension loss rate constant- $\mathrm{hr}^{-1}$

Precipitation scavenging rate constant $-\mathrm{hr}^{-1}$

Erosion and ground migration (weathering) constant-hr 1

Loss rate constant term representing all (sum of) the removal mechanisms from the activity concentrations in the air $-\mathrm{hr}^{-1}$

Average wind speed $-\mathrm{m} / \mathrm{sec}$

Density of the soil in which food is produced- $-\mathrm{gm} / \mathrm{cm}^{3}$

Standard deviation of the vertical dispersion-m

Activity concentration in the air at ground level in the region immediately surroundiny the suluast puint dusing the period that release are occurring- $\mathrm{Cl} / \mathrm{m}^{3}$

The activity concentration in the atmosphere of the region beyond the immediate vicinity of the release point during the period that releases are occurring- $\mathrm{Ci} / \mathrm{m}^{3}$

The activity concentration in the atmusphere of the region beyond the immediate vicinity of the release point after the releases have stopped $-\mathrm{Ci} / \mathrm{m}^{3}$

The average activity concentration in the atmosphere of the regions beyond the immediate vicinity of the release point during the period that food is being produced while releases are occurring- $\mathrm{Ci} / \mathrm{m}^{3}$ 
$\overline{\chi_{c}}$

Subscripts f

$g$

i

j

k

$\ell$

m

$\mathbf{v}$

The average activity concentration in the atmosphere of the regions beyond the immediate vicinity of the release point during the period that food is being produced after the releases have stopped- $-\mathrm{Ci} / \mathrm{m}^{3}$

Exponent of term used in defining the standard deviation of vertical dispersion

Refers to a specific food type (i.e., plants, beef, milk)

Refers to the organ which is being subjected to radiation doses

Refers to the released radionuclides

Refers to the angular sectors into which the region immediately surrounding the release point is subdivided

Refers to the annuli into which the region immediately surrounding the release point is subdivided

Refers to the daughters of a released radionuclide i $\left(\ell=0\right.$ refers to the $o^{\text {th }}$ daughter (i.e., the released radionuclide i))

Refers to the stability categories of the meteorological conditions

Refers to the wind speeds

Refers to the distances at which standard deviations of vertical dispersions are available 
The AIRWAY computer program was developed to estimate the radiation dose commitments accrued by all the people affected by the atmospheric release of radionuclides from a nuclear facility. This computer program provides dose commitment estimates for people on the boundary of the facility, in the immediate vicinity (i.e., within $80-100 \mathrm{~km}$ ) and in the portions of the world beyond the immediate vicinity which are affected by the release. The AIRWAY program considers dose commitments resulting from immersion in the atmosphere containing the released radionuclides, ingestion of contaminated food, inhalation of gaseous and suspended radioactivity, and exposure to ground deposits. The dose commitments for each of these pathways is explicitly calculated for each radionuclide released into the atmosphere and for each daughter of each released radionuclide. This is accomplished by calculating the air and ground concentrations of each daughter in each of the regions of interest using the release rate of the parent radionuclide. The AIRWAY computer program is considered to be a significant improvement over other programs in which the effect of daughter radionuclides must be approximated by separate releases.

\title{
AIRWAY-A FORTRAN COMPUTER PROGRAM TO ESTIMATE RADIATION DOSE COMMITMENTS TO MAN FROM THE ATMOSPHERIC RELEASE OF RADIONUCLIDES (LWBR Development Program)
}

\author{
J. L. Rider
}

\section{INTRODUCTION}

The Light Water Breeder Reactor (LWBR) program was undertaken to develop the technology necessary to use a light water breeder reactor based on the thorium-uranium fuel cycle as a means to provide an alternate energy source and to improve the utilization of the available nuclear fuel resources. To fully assess the benefits and the impacts which may result from the use of LWBR's, an environmental impact statement (Reference 1) was prepared for an LWBR and its supporting fuel cycle industry ranging from the mining and milling facilities through the reprocessing and refabrication facilities.

As part of the overall program to develop the LWBR environmental statement, it was necessary to estimate the radiation dose commitments accrued by man from the release of radionuclides from the various facilities which comprise a hypothetical LWBR industry. Estimates of these radiation dose commitments were made for the maximum individual, the population in the vicinity of the radionuclide releases and the world population. These estimates were made for liquid and airborne releases of radioactive material from each facility of an LWBR industry. This report presents a description of the AIRWAY computer program which was developed during the preparation of the LWBR environmental statement to estimate the radiation dose commitment resulting from the release of radionuclides to the atmosphere. The AIRWAY computer program provides the means of implementing the models presented in References 2 and 3 to estimate the dose commitments arising from the atmospheric release of radioactive material. The primary purpose of this report is to present a detailed description of the AIRWAY computer program and thus enable others who are involved in similar analyses to utilize this computer program in their analyses subsequent to judging the validity of using this program for their specific applicatons.

\section{MODEL DESCRIPTION}

The AIRWAY computer program calculates the dose commitments arising from the atmospheric release of radionuclides including those resulting from the daughters of the released isotopes formed subsequent to the release for six specific pathways by which the released radionuclides and their daughters may reach man. The six specific pathways presently considered in the AIRWAY computer program are:

1. Immersion in the cloud of released radionuclides

2. Exposure to surfaces on which the released radioactive material has been deposited.

3. Ingestion of foods on which the released radionuclides have been deposited. This pathway includes the consumption of products such as meat, milk, and eggs which have been obtained from animals which have eaten foods on which radioactive material was directly deposited. 
4. Ingestion of foods produced on lands which have been subjected to prior deposition of radionuclides.

5. Inhalation of the air in the cloud of released radionuclides.

6. Inhalation of radioactive material which has been resuspended in the air subsequent to its deposition from the original cloud of released radionuclides.

The AIRWAY program calculates the dose commitments arising from each of these pathways for the population in the immediate vicinity of the releases (i.e., within approximately a 100 -kilometer radius) and for the population in the remainder of the world beyond the immediate vicinity which is affected by the releases. The dose commitments in the immediate neighborhood of the release point are based upon a constant release rate and upon a constant set of meteorological conditions throughout the period that releases are occurring. Thus, when the AIRWAY program is used to estimate doses resulting from releases which occur over a time period in which the meteorological conditions change, the average weather conditions during the release period must be used. Similarly, if the rate at which the radionuclides are released fluctuates during the release period, an average release rate should be used. Conversely, if average release rates and weather data are not considered applicable over the entire time of release, the release period must be subdivided into segments which can be adequately represented by constant release rates and meteorological data and the dose commitment from each of these time segments summed to obtain the total doses associated with the given relcuoc.

With constant release rates and weather conditions, the concentration of the released radionuclides and their daughters in the air at a given location in the vicinity of the release point will remain constant throughout the duration of the release. Thus, the rates at which the released radionuclides and their daughters in particulate form are being deposited on the ground surfaces at a given location are also constant during the release period. Deposition occurs as the result of precipitation scavenging and by dry deposition mechanisms. However, the concentrations of the radionuclides on the ground at a given location will be increasing with time (approaching equilibrium) during the period that releases are occurring.

Once the releases have stopped, the concentrations in air of the released isotopes and their daughters at a given location in the vicinity of the release point are significantly reduced. If the released radionuclides and/or their daughters are gaseous, then the air concentrations of these nuclides are reduced to the concentrations resulting from the spread of these nuclides throughout the atmosphere of the world from the earlier portions of the release. These air concentrations will then decrease further with time as world wide distribution of these gaseous nuclides occurs and as they decay. If the released radionuclides and/or their daughters are in the form of particulates, then the air concentrations of these nuclides in the immediate vicinity of the release point will be reduced essentially to zero, since these particulate nuclides will be deposited upon the earth's surface prior to encircling the earth (this is based upon the release of these materials occurring within a few hundred meters of the earth's surface). Actually, there will be a low concentration of the particulate nuclides in the air at a given location in the vicinity of the release point after the releases have stopped due to the resuspension of deposited radionuclides. The concentration of the released radionuclides on the ground after the releases have stopped will immediately begin to decrease due to decay, and in the case of long-lived radioisotopes, due to erosion and ground migration effects. The concentrations on the ground of the daughter radionuclides may continue to increase after the releases (and thus, deposition) have stopped due to their formation via the decay of their parents. However, the rate at which the concentrations of the daughter radionuclides on the ground is increasing will be slowed subsequent to the release stopping and at some time after the releases stop, the ground concentrations of the daughter nuclides will peak and then decay away. The typical behavior of the air and ground concentrations of the released radionuclides and their daughters in the immediate vicinity of the release point is shown on Figures 1 through 4.

The dose commitment estimates to the people in the region beyond that affected directly by the releases (i.e., beyond approximately a 100-kilometer radius) are based upon the uniform dispersion of the released radionuclides and their daughters in the atmosphere of the region affected by the release. The extent of the region actually affected by the release is dependent upon the material released. Thus, the release of a long-lived gaseous isotope (e.g., ${ }^{85} \mathrm{Kr}$ with a 10.7 -yr half life) would affect the entire world, while the release of a short-lived gaseous isotope (e.g. ${ }^{135} \mathrm{Xe}$ with a $9.2 \mathrm{hr}$ half life) may affect only a region a few thousand kilometers in radius. The largest region actually affected by radionuclides in particulate form would be limited to approximately the size of the smallest region affected by gaseous radionuclides since 


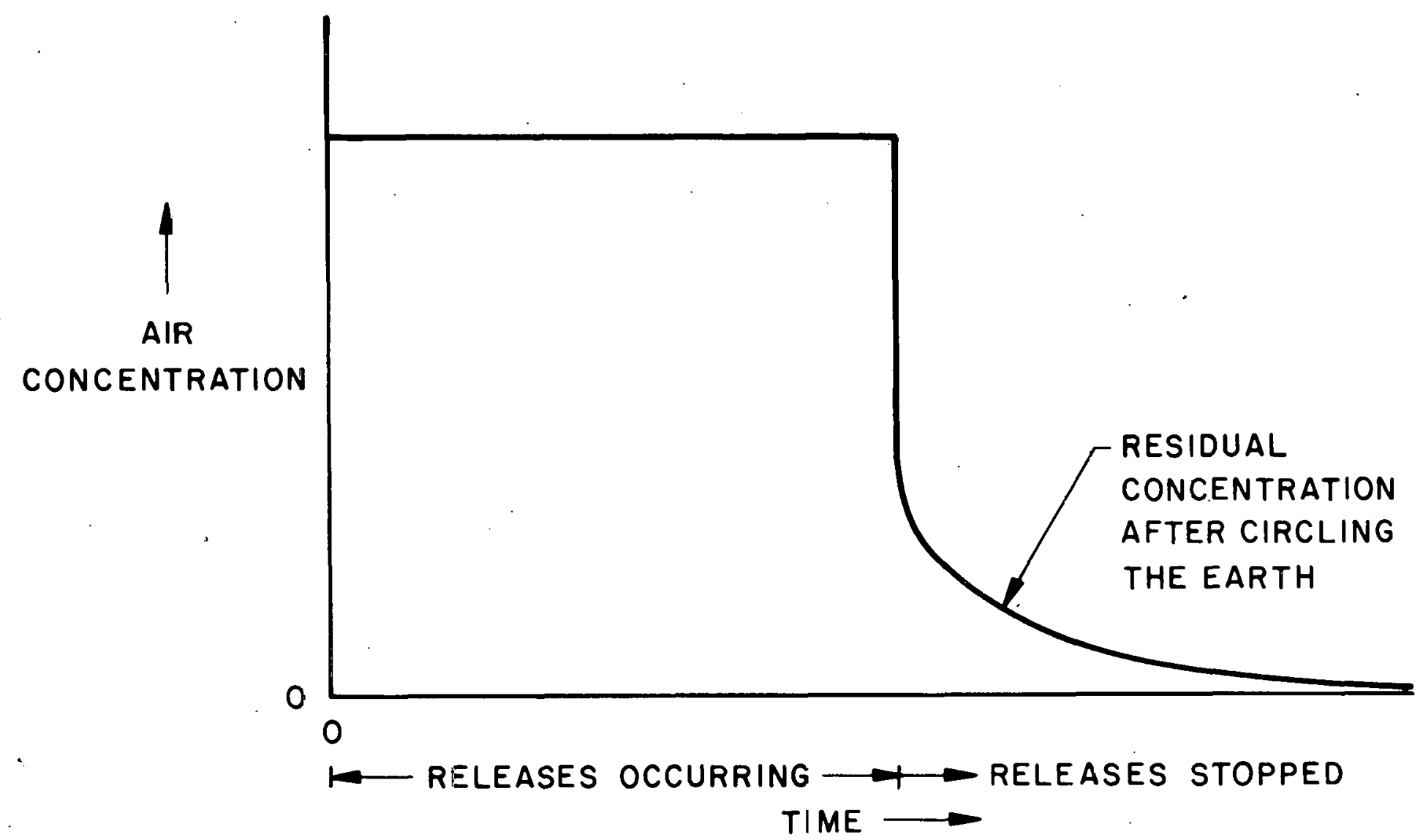

Figure 1. Typical Air Concentration of Gaseous Radionuclides in the Vicinity of the Release Point as a Function of Time 


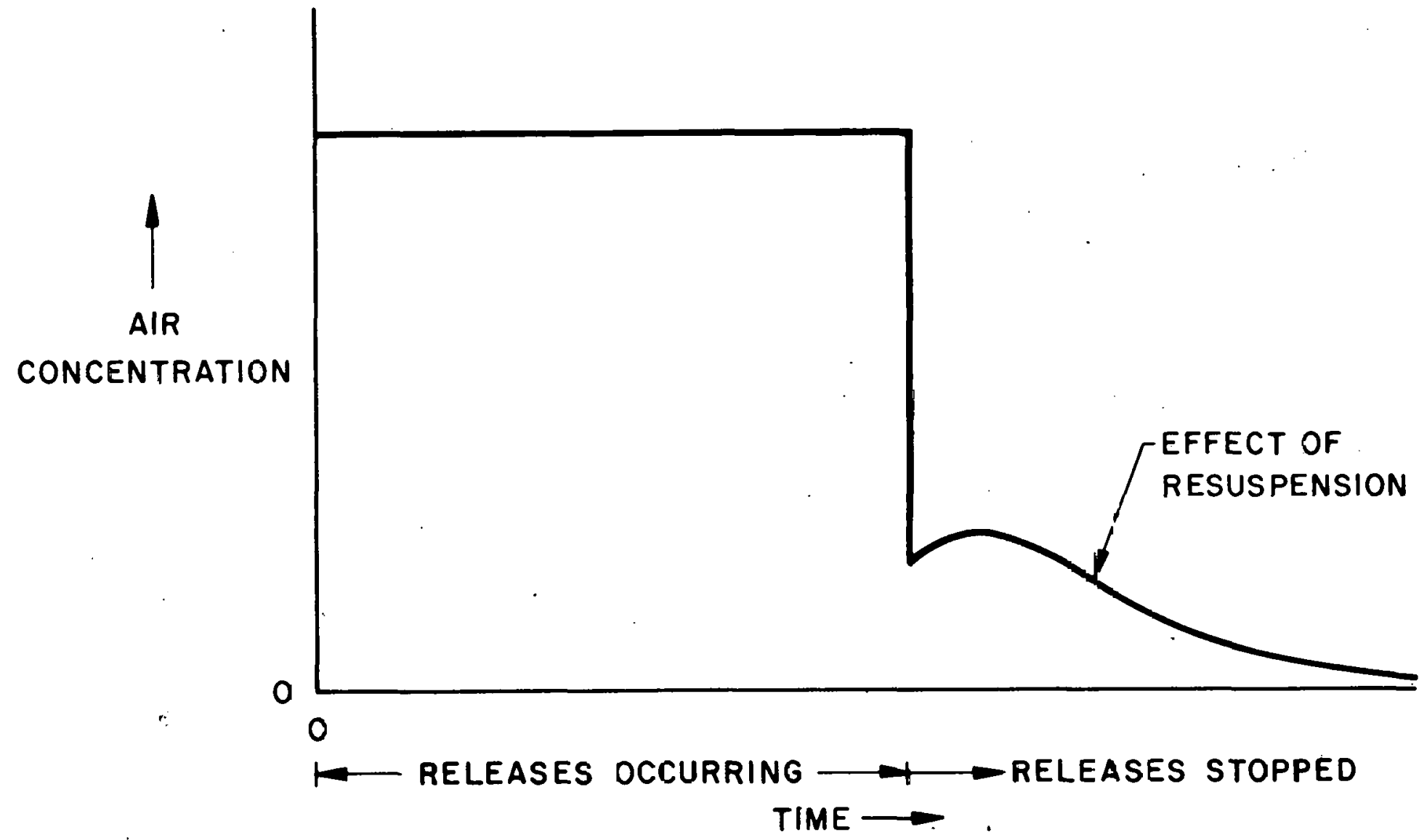

Figure 2. Typical $A_{i r}$ Concentration of Particulate Radionuclides in the 'Jicinity of the Release Point as a Function of Time 


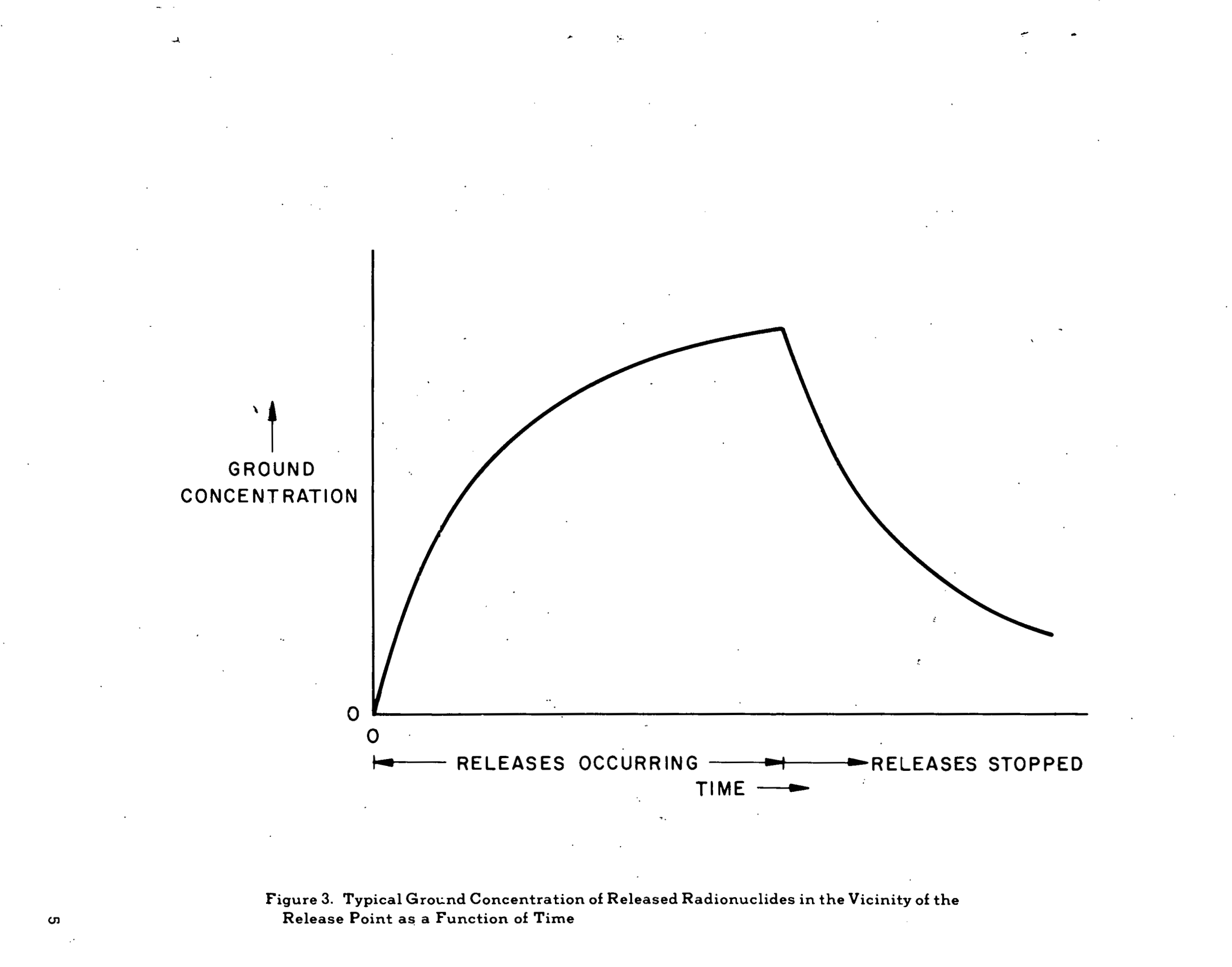


a

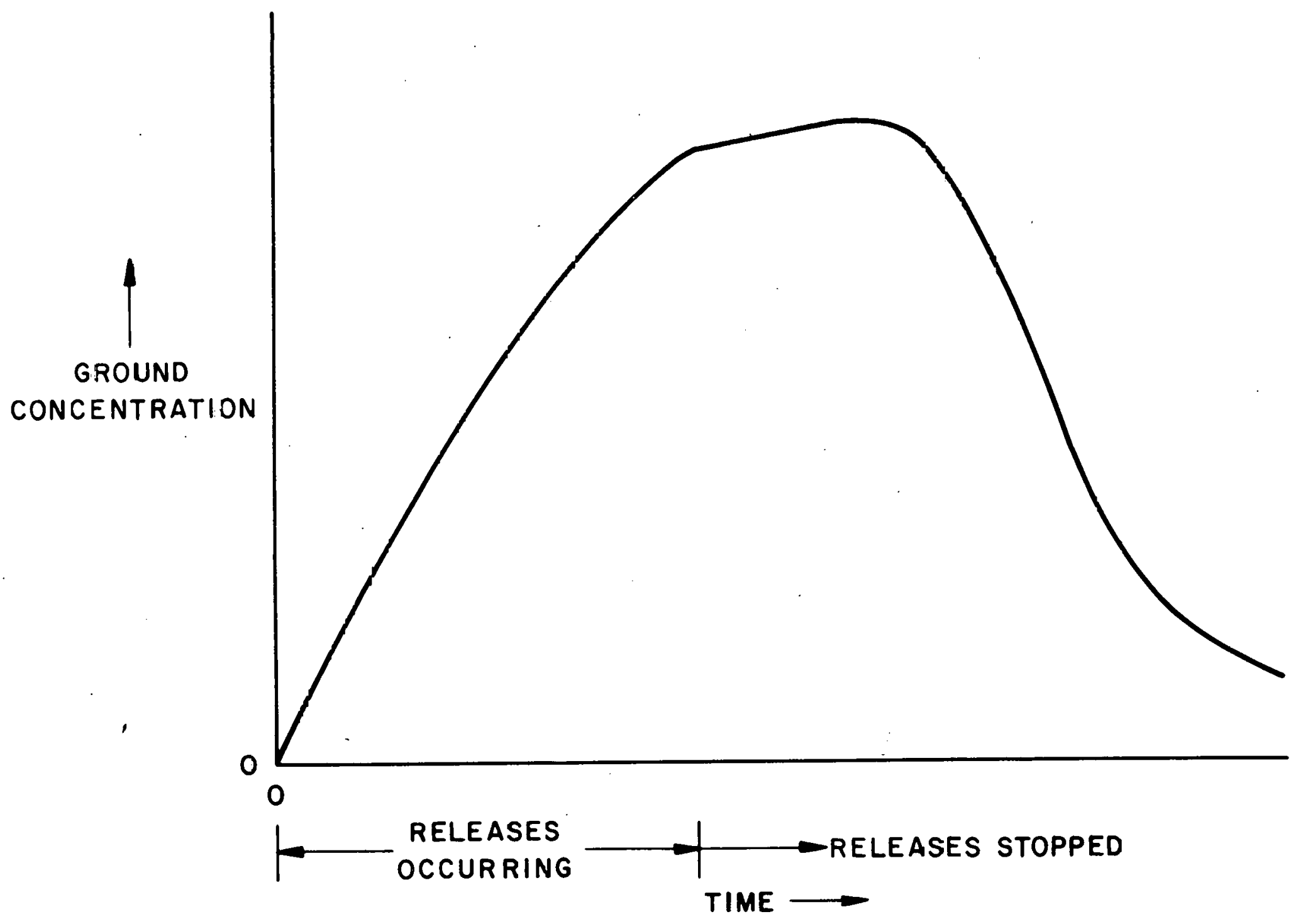

Figure 4. Typical Ground Concentration oE Daughter Radionuclide in the Vicinity of the Release Point as a Function of Time 
all particulates will have deposited within this distance due to the effect of precipitation scavenging aid dry deposition mechanisms. The amount of material which enters the regions beyond the immediate vicinity of the release point (i.e., beyond 100 kilometers) per unit time and thus is available for dispersion, is adjusted by any deposition which occurred during the traverse of the first 100 kilometers. The radionuclides which reach the regions beyond the immediate neighborhood of the release point and then disperse uniformly throughout the overall region affected by the release are then subjected to dry deposition, precipitation scavenging and decay effects. Those radionuclides which have been deposited in the region affected by the release beyond the immediate vicinity of the release point are subject to resuspension, ground migration, and decay.

The concentrations in the air of the released radionuclides and their daughters in the region affected by the releases are increasing towards an equilibrium concentration throughout the period that releases are occurring. However, once the releases have stopped and thus, are no longer feeding the regions beyond the immediate vicinity of the release point, the air concentrations of the released radionuclides in the overall region affected by the releases will begin to decrease via decay and deposition. The air concentrations of the daughter radionuclides in the affected regions may continue to increase for a time after the releases stop, but eventually they will peak and then begin to decrease when their decay rate exceeds the formation rate from their parents. The concentrations on the ground of the released particulates and their daughters in the regions affected by the releases increase during the period that releases are occurring. These ground concentrations continue to increase after the releases have stopped until the rate of formation of these nuclides from deposition and from decay of their precursors becomes less than the rate at which they are decaying. The typical time-dependent behavior of the air and ground concentrations of the released radionuclides and their daughters in the regions beyond 100 kilometers which are affected by the releases is depicted in Figures 5 and 6.

The AIRWAY computer program uses the concept of dose commitments to define the effect of radiation upon man. A dose commitment is defined to be the total dose received during a specific period of time as the direct result of exposure to radioactive material. This exposure may occur via any of many pathways, such as immersion in air containing radioactive material and ingestion of food upon which radioactive material had deposited. Further, the effect of an exposure may last well beyond the duration of the exposure. For example, essentially the entire dose accrued by eating food which contains radioactive material is caused by the decay of the ingested material and its daughters at times subsequent to the actual act of ingestion. Thus, a 70 -year dose commitment is the total dose that would be accrued by an individual or by a group of people in a 70-year period as the direct result of exposure to radioactive material. The total dose commitment is the sum of the dose commitments from all of the pathways by which man may be exposed to radiation. The AIRWAY program calculates these dose commitments for a total of nine organs, including the total body dose.

Provisions are made in the AIRWAY program to permit the population to change exponentially as a function of time, to define the overall extent of the region affected by the releases and to permit the local food sources to be produced on farms and gardens within a few hundred meters of the population being exposed or to be produced throughout the entire 100-kilometer radial region surrounding the release point. The program also includes the ability to vary the times at which the initial exposure occurs relative to the start of the release and the time intervals over which the exposure to the radiation environment continues.

\section{MODEL EQUATIONS}

This section of the report presents the equations utilized in the AIRWAY computer program to define the activity concentrations in the air and on the ground arising from the atmospheric release of radionuclides and the dose commitments resulting from these releases. The general equations defining these activity concentrations and the resulting dose commitments were developed in References 2 and 3 . $\AA$ complete summary of all the detailed equations used in the AIRWAY program is presented in Appendix B.

\section{A. Air and Ground Activity Concentration}

The AIRWAY computer program is designed to estimate the dose commitments which will be received from the continuous release of radionuclides into the atmosphere over a relatively long period of time (i.e., 


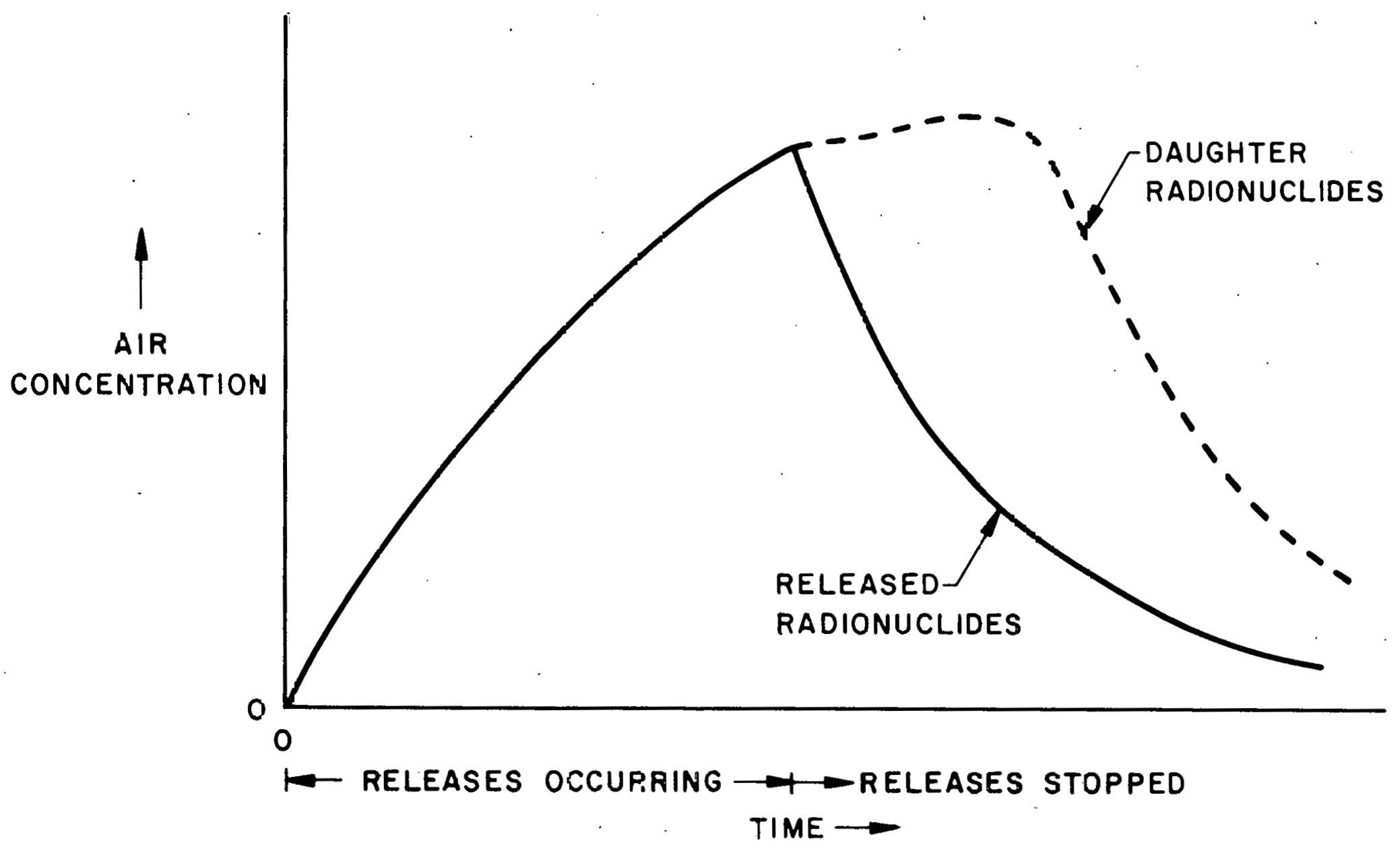

Figure 5. Typical Air Concentration of Radionuclides in Affected Regions Beyond 30-100 km as a Function of Time 


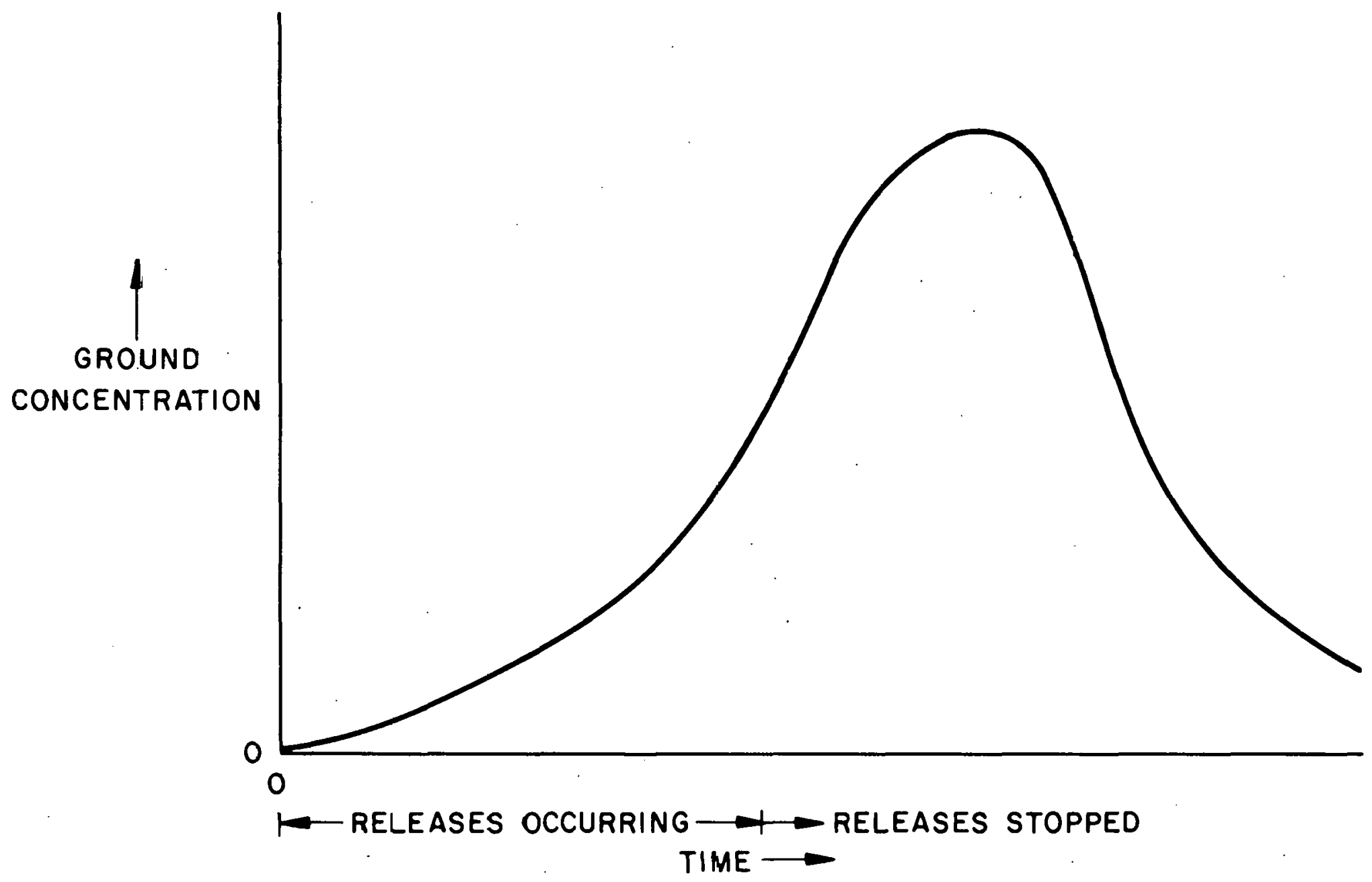

6

Figure 6. Typical Ground Concentration of Radionuclide in Affected Regions Beyond $80-100 \mathrm{~km}$ as a Function of Time 
days, weeks, months, years). Further, these dose commitment estimates are to be'made for people in the immediate vicinity of the point of release (i.e., within 100 kilometers) and for the people beyond this immediate region who are affected by the releases. To obtain these dose commitment estimates it is necessary to define the radionuclide activity concentration in the air and on the ground both during the period that releases are occurring and after the releases have stopped. These air and ground concentrations must also be provided within the immediate vicinity of the release and in the regions beyond the 100 -kilometer radial boundary of the immediate neighborhood of the release point.

These concentrations of the radioactivity in the air and on the qround are obtained from various combinations of the following specific seven quantities.

1. The ground level air concentrations of the $l^{\text {th }}$ daughter of the released radionuclide $i$ at a given radial position $r_{k}$ in a given azimuthal sector of width $\theta_{j}$ during the initial passage of the released nuclide and its daughters through the neighborhood immediately surrounding the release point. (The radial and azimuthal subdivisions of the region in the immediate vicinity of the release point are depicted on Figure 7).

2. The ground concentrations at a given time $t$ measured from the time that releases start of the $\ell^{\text {th }}$ daughter of the released radionuclide $i$ at the distance $r_{k}$ in the angular sector of width $\theta_{i}$ due to the deposition of the released nuclide and its daughters during their initial passage through the neighborhood immediately surrounding the release point.

3. The residual concentration at a time $t$ measured from the time releases start of the $\ell^{\text {th }}$ daughter of the released radionuclide $i$ remaining on the ground after the releases stop at a radial position $r_{k}$ in the $\mathrm{j}^{\text {th }}$ angular sector due to the deposition of the released radionuclide $i$ and its daughters at this location during their initial passage through the immediate neighborhood surrounding the release point.

4. The air concentration of the $\ell^{\text {th }}$ daughter of the radionuclide $i$ in the overall region affected by the releases at a given time $t$ during the period that releases are occurring (i.e., $0 \leq \mathrm{t} \leq \mathrm{T}_{\mathrm{s}}$ ).

5. The residual concentration of the $\ell^{\text {th }}$ daughter of the radionuclide i remaining in the atmosphere of the overall region affected by the releases at a given time $t\left(t>T_{s}\right)$ after the releases stop.

6 . The ground concentrations of the $l^{\text {th }}$ daughter of the radionuclide $i$ on the surface of the overall region affected by the releases at a given time during the period that releases are occurring due to the deposition of the released radionuclide and its daughters earlier during the release period.

7. The concentration of the $l^{\text {th }}$ daughter of the radionuclide $i$ on the surfares $n$ the nverall reging aff= fected by the releases at a qiven time after the releases stop due to the artivity remaining on the ground from deposition of the released nuclide and its daughters at earlier times and due to the ongoing deposition of the nuclide of interest present in the atmosphere of the region affected by the release at the current time.

The equations defining these seven quantities are presented below along with a brief summary of their basis. These equations are developed in detail in References 2 and 3.

The average ground level air concentrations $\chi_{\text {ailijh }}$ of the. radioactivity of the $\ell^{\text {th }}$ daughter of the re= leased radionuclide $i$ at the distance $r_{k}$ in the $j^{\text {th }}$ angular sector of regions in the vicinity of the release point during the initial passage of the radioactivity through the immediate neighborhood of the release point is given by

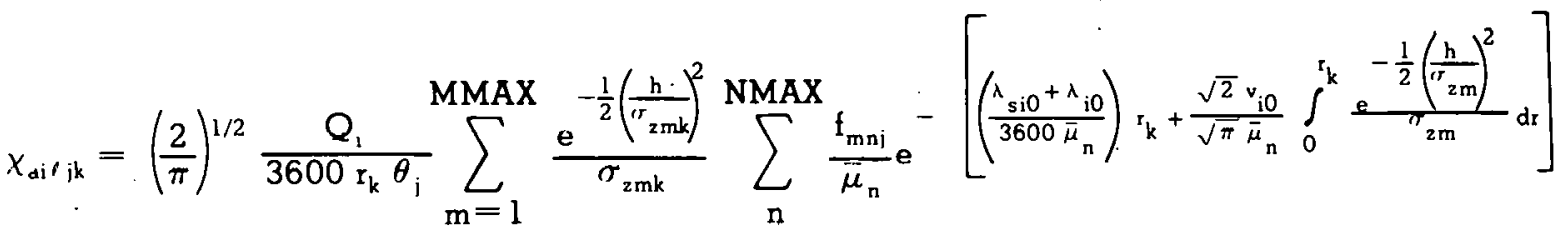

$$
\begin{aligned}
& \left\{\frac { \lambda _ { i 1 } \lambda _ { i 2 } \ldots \lambda _ { i \ell } } { ( 1 - e ^ { - \overline { \lambda } _ { i 0 } { } ^ { \prime } / 3 6 0 0 \overline { \mu } _ { n } } ) } \left[\frac{1}{\bar{\lambda}_{i 1} \bar{\lambda}_{i 2} \ldots \bar{\lambda}_{i \ell}}\right.\right.
\end{aligned}
$$




$$
\left.\left.-\bar{\lambda}_{i 0} \sum_{q=0}^{q=l} \frac{(-1)^{q} e^{-\frac{\bar{\lambda}_{i q}{ }^{r}}{3600 \bar{\mu}_{n}}}}{\left(\bar{\lambda}_{i q}-\bar{\lambda}_{i 0}\right)\left(\bar{\lambda}_{i q}-\bar{\lambda}_{i 1}\right) \ldots\left(\bar{\lambda}_{i q}-\bar{\lambda}_{i q-1}\right) \bar{\lambda}_{i q}\left(\bar{\lambda}_{i q+1}-\bar{\lambda}_{i q}\right)\left(\bar{\lambda}_{i q}+2-\bar{\lambda}_{i q}\right) \ldots\left(\bar{\lambda}_{i \ell}-\bar{\lambda}_{i q}\right)}\right]\right\}
$$

where

$$
\bar{\lambda}_{\text {iq }}=\lambda_{\text {iq }}+\lambda_{\text {siq }}+3600 v_{\text {iq }} A_{a} / V_{a}
$$

The preceding expression for $\chi_{\text {ailik }}$ is developed in detail in Reference 2 . The equation shows that the average ground level activity concentration in the air is dependent upon the rate of release of the $\mathrm{i}^{\text {th }}$ radionuclide $Q_{i}$, the meteorological conditions which exist throughout the release period, the losses due to decay, scavenging (rain, etc.), and dry deposition mechanisms, and the production of the radionuclide of interest via the decay of its precursors. The meteorological dispersion effects are dependent upon the radial distance from the release point, $\mathrm{r}_{\mathrm{k}}$, the width of the azimuthal sector being considered, $\theta_{\mathrm{i}}$, the height of the release, $h$, the mean wind velocity, $\bar{\mu}_{n}$ and the standard deviation of the vertical dispersion, $\sigma_{2 m}$. The effect of the changing meteorological conditions throughout the period that releases are occurring is handled by modifying the dispersion and loss effects associated with each wind speed-stability category encountered by the fraction of the time that the wind is blowing into the sector of interest with a given wind speed while a given stability category exists, $\mathrm{f}_{\mathrm{jmn}}$ and then summing over the $\mathrm{n}$ wind speeds and m stability categories. The loss and production effects are dependent upon the decay constant, $\lambda_{i}$, the scavenging coefficient, $\lambda_{\text {si }}$, the dry deposition velocity, $v_{i}$, the distance from the release point, $r_{k}$, the time for the released radionuclide to reach the $r_{k}$ radial distance (defined by $r_{k} / \bar{\mu}_{n}$ ), the surface area, $A_{a}$, of the regions affected by the release, and the overall volume of the atmosphere, $V_{a}$ in which the releases are dispersed.

The ground concentration $C_{a i \ell j k}$ at a given time $t$ measured from the time that releases start of the $l^{\text {th }}$ daughter of the released radionuclide $i$ at the radial distance $r_{k}$ in the $j^{\text {th }}$ angular sector of the region immediately surrounding the release point due to the deposition of particulates during their initial passage through the immediate neighborhood surrounding the release point is given by

$$
\begin{aligned}
& \mathrm{C}_{\mathrm{ai} \ell, \mathrm{jk}}=\frac{\sum_{\mathrm{q}=0}^{\mathrm{q}=\ell} \alpha_{\mathrm{i} 0} \alpha_{\mathrm{i} 1} \ldots \alpha_{\mathrm{iq}-1} \mathrm{Y}_{\mathrm{iqjk}} \lambda_{\mathrm{iq}+1} \lambda_{\mathrm{iq}+2 \ldots \lambda_{\mathrm{i} \ell}}}{\alpha_{\mathrm{i} 0} \alpha_{\mathrm{i} 1} \ldots \alpha_{\mathrm{i} \ell}}-\sum_{\mathrm{q}=0}^{\mathrm{q}=\ell}(-1)^{\mathrm{q}} \mathrm{e}^{-a_{\mathrm{iq}} \mathrm{\prime}} \\
& \sum_{\mathrm{h}=0}^{\mathrm{h}=\mathrm{q}} \frac{(-1)^{\mathrm{h}} \mathrm{Y}_{\mathrm{ihjk}} \lambda_{\mathrm{ih}+1} \lambda_{\mathrm{ih}+2} \ldots \lambda_{\mathrm{i} \ell}}{\left(\alpha_{\mathrm{iq}}-\alpha_{\mathrm{ih}}\right)\left(\alpha_{\mathrm{iq}}-\alpha_{\mathrm{ih}+1}\right) \ldots\left(\alpha_{\mathrm{iq}}-\alpha_{\mathrm{iq}-1}\right) \alpha_{\mathrm{iq}}\left(\alpha_{\mathrm{iq}+1}-\alpha_{\mathrm{iq}}\right)\left(\alpha_{\mathrm{iq}+2}-\alpha_{\mathrm{iq}}\right) \ldots\left(\alpha_{\mathrm{i},}-\alpha_{\mathrm{iq}}\right)}
\end{aligned}
$$

where

$$
\alpha_{\text {iq }}=\lambda_{\text {iq }}+\lambda_{\text {wiq }}
$$

$\mathrm{Y}_{\mathrm{iqjk}}$ is the rate per unit area at which deposition of the $\mathrm{q}^{\text {th }}$ daughter of the $i$ released radionuclide is occurring due to dry deposition mechanisms and precipitation scavenging. $Y_{\text {iqji }}$ is given by the following expression 


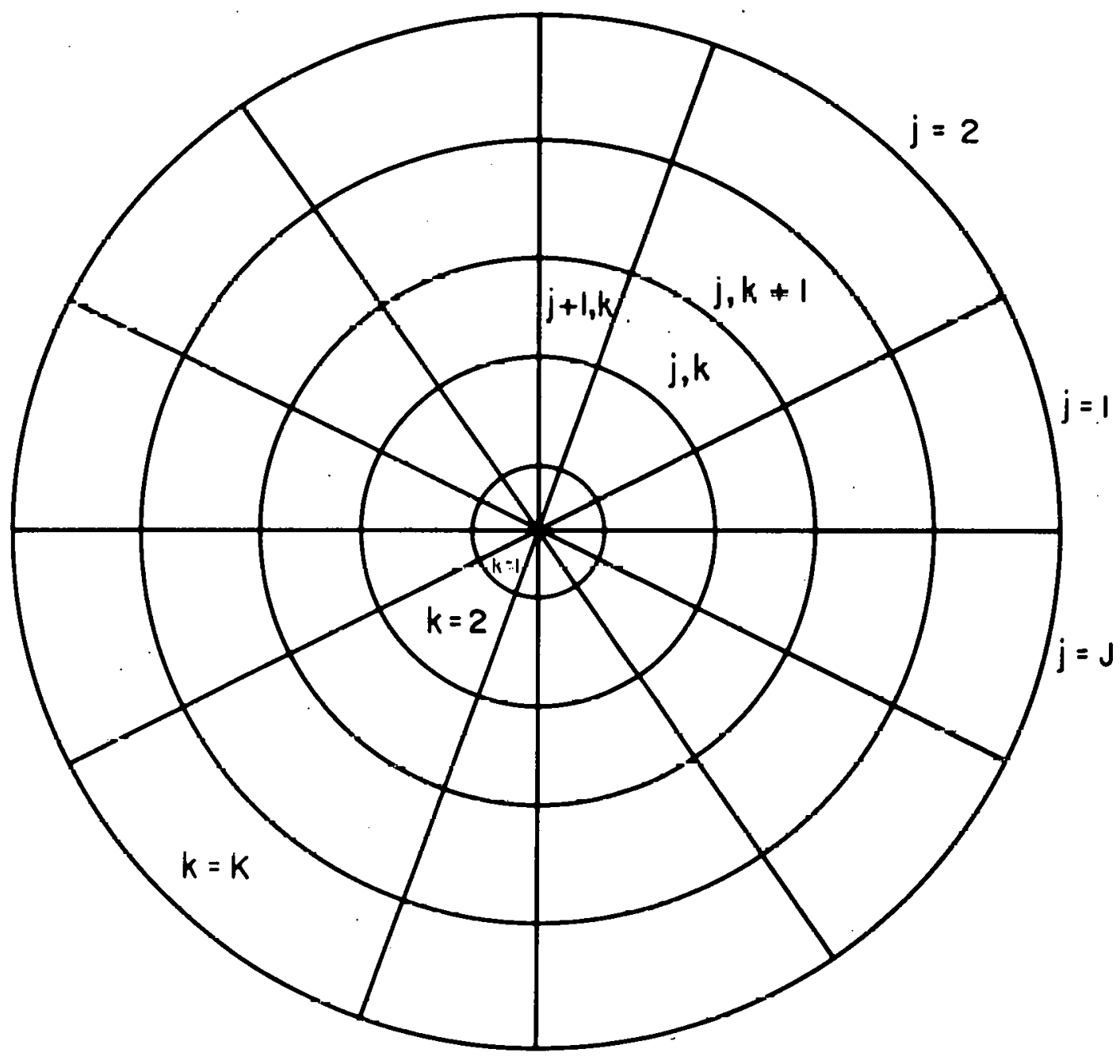

Figurc 7. Subdivision of Reyiun in Vicinity of Release Point 


$$
\begin{aligned}
& \left.Y_{i q j k}=3600 v_{i q} \chi_{a i q j k}+\frac{\lambda_{s} \theta_{i}}{3600 r_{k} \theta_{i}} \sum_{m=1}^{\text {MMAX }} \sum_{n=1}^{\operatorname{NMAX}} \frac{f_{m n i}}{\bar{\mu}_{n}} e^{-\left[\left(\frac{\lambda_{s}+\lambda_{i 0}}{3600 \bar{\mu}_{n}}\right) r_{k}\right.}+\frac{\sqrt{2} v_{i 0}}{\sqrt{\pi} \bar{\mu}_{n}} \int_{0}^{r_{k}} \frac{e^{-\frac{1}{2}\left(\frac{h}{r_{z m}}\right)^{2}} d r}{r_{z m}}\right] \\
& \left\{\frac { \lambda _ { \mathrm { i } 1 } \lambda _ { \mathrm { i } 2 } \cdots \lambda _ { \mathrm { iq } } } { 1 - \mathrm { e } ^ { - ( \overline { \lambda } _ { \mathrm { i } 0 } { } _ { \mathrm { r } } ^ { \mathrm { r } } / 3 6 0 0 \overline { \mu } _ { \mathrm { n } } ) } } \left[\frac{1}{\bar{\lambda}_{\mathrm{i} 1} \bar{\lambda}_{\mathrm{i} 2} \ldots \bar{\lambda}_{\mathrm{iq}}}\right.\right. \\
& \left.\left.-\bar{\lambda}_{\mathrm{i} 0} \sum_{\mathrm{h}=0}^{\mathrm{h}=\mathrm{q}} \frac{(-1)^{\mathrm{h}} \mathrm{e}^{-\frac{\bar{\lambda}_{\mathrm{ih}}^{\mathrm{r}} \mathrm{k}}{3600 \bar{\mu}_{\mathrm{n}}}}}{\left(\bar{\lambda}_{\mathrm{ih}}-\bar{\lambda}_{\mathrm{i} 0}\right)\left(\bar{\lambda}_{\mathrm{ih}}-\bar{\lambda}_{\mathrm{il}}\right) \ldots\left(\bar{\lambda}_{\mathrm{ih}}-\bar{\lambda}_{\mathrm{ih}-1}\right) \bar{\lambda}_{\mathrm{ih}}\left(\bar{\lambda}_{\mathrm{ih}+1}-\bar{\lambda}_{\mathrm{ih}}\right)\left(\bar{\lambda}_{\mathrm{ih}+2}-\bar{\lambda}_{\mathrm{ih}}\right) \ldots\left(\bar{\lambda}_{\mathrm{iq}}-\bar{\lambda}_{\mathrm{ih}}\right)}\right)\right\}
\end{aligned}
$$

and $\chi_{\text {diujk }}$ is defined by Equation (1).

The preceding set of equations for the ground concentration of the $l^{\text {th }}$ daughter of the released radionuclide $i$ in the immediate vicinity of the release point is developed in detail in Reference 2. In general the ground concentration of a radioactive nuclide is dependent upon the deposition of that nuclide or one of its precursors due to precipitation scavenging and dry deposition mechanisms. Deposition due to dry deposition mechanisms is dependent upon the air concentration of the depositing nuclide at ground level and the deposition associated with precipitation scavenging is dependent upon the concentration distribution of the depositing nuclide throughout the rain-bearing layer of the atmosphere. The ground concentration of a given radionuclide is also dependent upon the time since deposition began, the decay constants and the weathering or migration coefficients $\lambda_{\text {wiq }}$. The derivation in Reference 2 of this set of equations to define the ground concentrations in the vicinity of the release point during releases considered the effect of resuspension upon these ground concentrations, via a resuspension coefficient $\lambda_{\text {riq }}$ which is added into the $\alpha_{\text {iq }}$ term. Thus, its effect is to reduce the ground concentrations of the released radionuclide and its daughters. However, the effect of resuspension of the deposited material was neglected in the AIRWAY program in defining all ground concentrations of the released radionuclide and its daughters. As a result the ground concentrations are overestimated in the AIRWAY program.

The residual concentration $\mathrm{C}_{\mathrm{bi} \ell \mathrm{jk}}$ at a given time t of the $\ell^{\text {th }}$ daughter of the released radionuclide i remaining on the ground after the releases stop at the radial position $\mathrm{r}_{\mathrm{k}}$ in the angular sector of width $\theta_{\mathrm{j}}$ in the immediate vicinity of the release point due to the deposition of the released nuclide and its daughters during their initial passage through the immediate neighborhood of the release point is given by

$$
C_{b i \ell j k}=\sum_{q=0}^{q=\ell} J_{i \ell q i k} e^{-\alpha_{i q}\left(t-T_{s}\right)}
$$

where

$$
\begin{aligned}
\mathrm{J}_{\mathrm{i} \ell \mathrm{qik}}= & \frac{\lambda_{\mathrm{iq}+1} \lambda_{\mathrm{iq}+2} \ldots \lambda_{\mathrm{i} \ell}}{\left(\alpha_{\mathrm{iq}+1}-\alpha_{\mathrm{iq}}\right)\left(\alpha_{\mathrm{iq}+2}-\alpha_{\mathrm{iq}}\right) \ldots\left(\alpha_{\mathrm{i} \ell}-\alpha_{\mathrm{iq}}\right)} \\
& \left.\sum_{\mathrm{h}=0}^{\mathrm{h}=\mathrm{q}} \frac{(-1)^{\mathrm{q}+\mathrm{h}} \lambda_{\mathrm{ih}+1} \lambda_{\mathrm{ih}+2} \ldots \lambda_{\mathrm{iq}}}{\left(\alpha_{\mathrm{iq}}-\alpha_{\mathrm{ih}}\right)\left(\alpha_{\mathrm{iq}}-\alpha_{\mathrm{ih}+1}\right) \ldots\left(\alpha_{\mathrm{iq}}-\alpha_{\mathrm{iq}-1}\right)} \mathrm{C}_{\mathrm{aihjk}}\right|_{1-\mathrm{T}_{\mathrm{s}}}
\end{aligned}
$$


The $\mathrm{C}_{\mathrm{aihj}}$ term in the above equation represents the ground concentration of the $\mathrm{h}^{\text {th }}$ daughter of the radionuclide $i$ at the time releases stop and is obtained by solving Equation (3) with $t=T_{s}$ the time at which releases stop.

The preceding equations are derived in detail in Reference 2. The equations are obtained by considering the effect of decay and weathering upon the activity concentrations at the time releases stop of the released nuclide and its daughters which have been deposited on the ground during their initial passage through the immediate neighborhood of the release point. As noted before, the effect of resuspension upon the ground concentrations of the deposited material was neglected in the AIRWAY program.

During the period that releases are occurring the air concentration $\chi_{b i l}$ of the $l^{\text {th }}$ daughter of the radionuclide $\mathrm{i}$ in the region beyond the boundaries of the immediate neighborhood of the releases (usually considered to be approximately $100 \mathrm{~km}$ from the point of release) is given by the following expression.

$$
\begin{aligned}
& x_{\mathrm{bi} \ell}=\frac{\lambda_{\mathrm{i} 1} \lambda_{\mathrm{i} 2} \ldots \lambda_{\mathrm{i} \ell} \mathrm{Q}_{\mathrm{i} \ell}^{\prime}}{\mathrm{V}_{\mathrm{l}}}\left\lceil\frac{1}{\bar{\lambda}_{0} \bar{\lambda}_{11} \ldots \bar{\lambda}_{\mathrm{i} \ell}}\right. \\
& \left.-\sum_{q=0}^{q=\ell} \frac{(-1)^{q} e^{-\bar{\lambda}_{i q}}}{\left(\bar{\lambda}_{i q}-\bar{\lambda}_{i 0}\right)\left(\bar{\lambda}_{i q}-\bar{\lambda}_{i l}\right) \ldots\left(\bar{\lambda}_{i q}-\bar{\lambda}_{i q-1}\right) \bar{\lambda}_{i q}\left(\bar{\lambda}_{i q+1}-\bar{\lambda}_{i q}\right)\left(\bar{\lambda}_{i q+2}-\bar{\lambda}_{i q}\right) \ldots\left(\bar{\lambda}_{i l}-\bar{\lambda}_{i q}\right)}\right]
\end{aligned}
$$

The preceding equation for $\chi_{\text {hi }}$, is developed in detail in Reference 3. It is based upon the assumption that the released material is dispersed uniformly throughout the volume of the atmosphere associated with the region affected by the release and that this dispersion occurs immediately upon release. The ground level air concentration of the $l^{\text {th }}$ daughter of the $i^{\text {th }}$ released radionuclide in the overall region affected by the releases is dependent upon the time since the releases started $t$, the rate at which the $\mathrm{i}^{\text {th }}$ radionuclide is being released into the regions beyond the boundary of the immediate neighborhood of the release point $\mathrm{Q}_{\mathrm{i}}^{\prime}$ and the volume of the atmosphere of the overall region affected by the release. It is further dependent upon the decay constants, scavenging coefficients and dry deposition velocities of the released radionuclide $i$ and its daughters and upon the surface area of the region affected by the release.

The residual concentration $X_{\text {oil }}$ at a given time after the releases stop of the $\ell^{\text {th }}$ danghter of the radio= nuclide $i$ remaining in the atmosphere of the overall region affected by the release is given by

$$
\begin{aligned}
& x_{c i l}=\sum_{q=0}^{q=\ell}\left[\frac{\lambda_{i q+1} \lambda_{i q+2} \cdots \lambda_{i \ell}}{\left(\bar{\lambda}_{i q+1}-\bar{\lambda}_{i q}\right)\left(\bar{\lambda}_{i q+2}-\bar{\lambda}_{i q}\right) \ldots\left(\bar{\lambda}_{i \ell}-\bar{\lambda}_{i q}\right)}\right. \\
& \left.\left.\sum_{h=0}^{h=q} \frac{(-1)^{q+h} \lambda_{i h+1} \lambda_{i h+2} \ldots \lambda_{i q}}{\left(\bar{\lambda}_{i q}-\bar{\lambda}_{i h}\right)\left(\bar{\lambda}_{i q}-\bar{\lambda}_{i h+1}\right) \ldots\left(\bar{\lambda}_{i q}-\bar{\lambda}_{i q-1}\right)} x_{\text {iih }}\right|_{t=T_{s}}\right] e^{-\bar{\lambda}_{i q}^{\left(t-T_{s}\right)}}
\end{aligned}
$$

The $\left.X_{b i h}\right|_{1}=T_{s}$ term in the above equation represents the air concentration of the $h^{\text {th }}$ daughter of the radionuclide $i$ in the overall region affected by the releases at the time releases stop and is obtained by solving Equation (8) with $\mathrm{t}=\mathrm{T}_{\mathrm{s}}$.

The preceding equation is developed in detail in Reference 3 . It is obtained by considering the effect of decay, precipitation scavenging and dry deposition mechanisms upon the air concentration of the released radionuclide $i$ and its daughters in the overall region affected by the releases at the time releases stop. 
During the period that releases are occurring, the ground concentration of the $\ell^{\text {th }}$ daughter of the radionuclide $i$ on the surface of the overall region affected by the releases due to the deposition of the released radionuclide $i$ and its daughters earlier in the release period is given by

$$
\begin{aligned}
& \mathrm{C}_{\mathrm{ci} \ell}=\sum_{\mathrm{q}=0}^{\mathrm{q}=\ell}\left[\frac{\gamma_{\mathrm{iq}} \AA_{\mathrm{iq}} \lambda_{\mathrm{iq}+1} \lambda_{\mathrm{iq}+2} \ldots \lambda_{\mathrm{i} \ell}}{\alpha_{\mathrm{iq}} \alpha_{\mathrm{iq}+1} \ldots \alpha_{\mathrm{i} \ell}}\right. \\
& +e^{-\bar{\lambda}_{i q}{ }^{h=l}} \sum_{h=q}^{h} \frac{\gamma_{i h} B_{i h} E_{i h q} \lambda_{i h+1} \lambda_{i h+2} \ldots \lambda_{i \ell}}{\left(\alpha_{i h}-\bar{\lambda}_{i q}\right)\left(\alpha_{i h+1}-\bar{\lambda}_{i q}\right) \ldots\left(\alpha_{i \ell}-\bar{\lambda}_{i q}\right)} \\
& +\frac{(-1)^{q+1} \lambda_{i q+1} \lambda_{i q+2} \ldots \lambda_{i \ell}}{\left(\alpha_{i q+1}-\alpha_{i q}\right)\left(\alpha_{i q+2}-\alpha_{i q}\right) \ldots\left(\alpha_{i \ell}-\alpha_{i q}\right)} e^{-\alpha_{i q}} \\
& \sum_{h=0}^{h=q} \frac{(-1)^{h} \gamma_{i h} \lambda_{i h+1} \lambda_{i h+2} \ldots \lambda_{i q}}{\left(\alpha_{i q}-\alpha_{i h}\right)\left(\alpha_{i q}-\alpha_{i h+1}\right) \ldots\left(\alpha_{i q}-\alpha_{i q-1}\right)} \\
& \left.\left\{\frac{A_{i h}}{\alpha_{i q}}-B_{i h} \sum_{p=0}^{p=h} \frac{E_{i h p}}{\alpha_{i q}-\bar{\lambda}_{i p}}\right\}\right]
\end{aligned}
$$

where

$$
\begin{aligned}
& \gamma_{\mathrm{iq}}=3600 \mathrm{v}_{\mathrm{iq}}+\lambda_{\mathrm{siq}} \mathrm{Z}_{\mathrm{s}} \\
& \mathrm{A}_{\mathrm{iq}}=\frac{\lambda_{\mathrm{i} 1} \lambda_{\mathrm{i} 2} \ldots \lambda_{\mathrm{iq}} \mathrm{Q}_{\mathrm{iq}}^{\prime}}{\bar{\lambda}_{\mathrm{i} 1} \bar{\lambda}_{\mathrm{i} 2} \ldots \bar{\lambda}_{\mathrm{iq}}} \frac{\mathrm{V}_{\mathrm{q}}}{\mathrm{B}_{\mathrm{iq}}}=\lambda_{\mathrm{i} 1} \lambda_{\mathrm{i} 2} \ldots \lambda_{\mathrm{iq}} \mathrm{Q}_{\mathrm{iq}}^{\prime} \mathrm{V}_{\mathrm{a}} \\
& \mathrm{E}_{\mathrm{ihq}}=\frac{(-1)^{\mathrm{q}}}{\left(\bar{\lambda}_{\mathrm{iq}}-\bar{\lambda}_{\mathrm{iq}}\right)\left(\bar{\lambda}_{\mathrm{iq}}-\bar{\lambda}_{\mathrm{i} 1}\right) \ldots\left(\bar{\lambda}_{\mathrm{iq}}-\bar{\lambda}_{\mathrm{iq}-1}\right) \bar{\lambda}_{\mathrm{iq}}\left(\bar{\lambda}_{\mathrm{iq}+1}-\bar{\lambda}_{\mathrm{iq}}\right)\left(\bar{\lambda}_{\mathrm{iq}+2}-\bar{\lambda}_{\mathrm{iq}}\right) \ldots\left(\bar{\lambda}_{\mathrm{ih}}-\bar{\lambda}_{\mathrm{iq}}\right)}
\end{aligned}
$$

The preceding set of equations for the ground concentration of the $\ell^{\text {th }}$ daughter of the released radionuclide $\mathrm{i}$ in the overall region affected by the releases is derived in detail in Reference 3 . As noted previously the ground concentration of a radionuclide is dependent upon its deposition or the deposition of one of its precursors due to precipitation scavenging or dry deposition mechanisms. These deposition effects are in turn dependent upon the air concentrations of the nuclides being deposited. The air concentration of the depositing nuclides in the overall region affected by the release is primarily a function of the time since. releases begin, the volume of the atmosphere of the overall region affected by the release and the rate of introduction of the radionuclide $i$ into that volume. The rate at which deposition occurs is dependent upon the dry deposition velocity, the precipitation scavenging coefficient and the height $Z_{3}$ of the rain-bearing layer. 
After the releases stop the ground concentration $C_{d i l}$ of the $\ell^{\text {th }}$ daughter of the radionuclide $i$ on the surfaces of the overall region affected by the release is given by

$$
C_{d i \ell}=\sum_{q=0}^{q=\ell}\left[R_{i \ell q} e^{-\alpha_{i q}\left(t-T_{s}\right)}+S_{i \ell q} e^{-\dot{\lambda}_{i q}^{\left(t-\tau_{s}\right)}}\right]
$$

where

$$
\begin{aligned}
& R_{i \ell q}=\frac{\lambda_{i q+1} \lambda_{i q+2} \ldots \lambda_{i \ell}}{\left(\alpha_{i q+1}-\alpha_{i q}\right)\left(\alpha_{i q+2}-\alpha_{i q}\right) \ldots\left(\alpha_{i \ell}-\alpha_{i q}\right)} . \\
& \sum_{h=U}^{h=q}(-1)^{q+h}\left[\left.\frac{\lambda_{i h+1} \lambda_{i h+2} \ldots \lambda_{i q}}{\left(\alpha_{i q}-\alpha_{i h}\right)\left(\alpha_{i q}-\alpha_{i h+1}\right) \ldots\left(\alpha_{i q q}-\alpha_{i q-1}\right)} C_{c i h}\right|_{t=T_{s}}\right. \\
& -\frac{1}{\alpha_{i q}-\bar{\lambda}_{i h}}\left\{\left.\sum_{p=0}^{p=h} \frac{(-1)^{h+p} \lambda_{i p+1} \lambda_{i p+2} \ldots \lambda_{i h}}{\left(\bar{\lambda}_{i h}-\bar{\lambda}_{i p}\right)\left(\bar{\lambda}_{i h}-\bar{\lambda}_{i p+1}\right) \ldots\left(\bar{\lambda}_{i h}-\bar{\lambda}_{\text {ih }-1}\right)} \chi_{b i p}\right|_{t=T_{s}}\right\}
\end{aligned}
$$

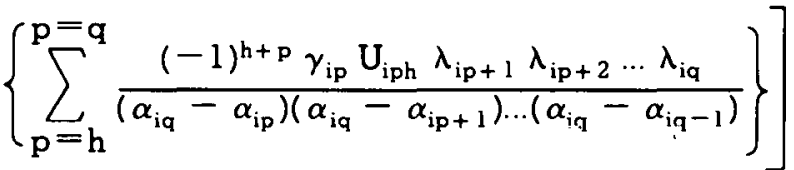

$$
\begin{aligned}
& S_{i, q}=\left[\sum_{h=q}^{h=l} \frac{\gamma_{i h} U_{i h q} \lambda_{i h+1} \lambda_{i h+2} \ldots \lambda_{i l}}{\left(\alpha_{i h}-\bar{\lambda}_{i q}\right)\left(\alpha_{i h+1}-\bar{\lambda}_{i q}\right) \ldots\left(\alpha_{i \ell}-\bar{\lambda}_{i q}\right)}\right] \\
& {\left[\left.\sum_{h=0}^{h=q} \frac{(-1)^{q+h} \lambda_{i h+1} \lambda_{i h+2} \ldots \lambda_{i q}}{\left(\bar{\lambda}_{i q}-\bar{\lambda}_{i h}\right)\left(\bar{\lambda}_{i q}-\bar{\lambda}_{i h+1}\right) \ldots\left(\bar{\lambda}_{i q}-\bar{\lambda}_{i q-1}\right)} x_{b i h}\right|_{t=T_{s}}\right]} \\
& U_{i h q}=\frac{\lambda_{i q+1} \lambda_{i q+2} \ldots \lambda_{i h}}{\left(\bar{\lambda}_{i q+1}-\bar{\lambda}_{i q}\right)\left(\bar{\lambda}_{i q+2}-\bar{\lambda}_{i q}\right) \ldots\left(\bar{\lambda}_{i h}-\bar{\lambda}_{i q}\right)}
\end{aligned}
$$

The term $\mathrm{C}_{\text {cihl }}=\mathrm{TS}_{\mathrm{S}}$ in Equation (16) represents the ground concentration at the time releases stop of the $\mathrm{h}^{\text {th }}$ daughter of the nuclide $i$ on the surface of the overall region affected by the releases. The term $\left.\chi_{\mathrm{bix}}\right|_{t=\mathrm{TS}}$ in Equations (16) and (17) represents the air concentration at the time releases stop of the $x^{\text {th }}$ daughter of the radionuclide $\mathrm{i}$ in the atmosphere of the overall region affected by the releases.

The preceding set of equations for $\mathrm{C}_{\mathrm{di} \ell}$ was developed in detail in Reference 3 . The ground concentrations after releases stop of the $\ell^{\text {th }}$ daughter of the radionuclide $i$ on the surfaces of the overall region affected by the releases is dependent upon the effect of decay and weathering and upon the activity on the ground due to the deposition of the released radionuclide and its daughters at earlier times. It also is dependent upon the deposition via precipitation scavenging and dry deposition mechanisms of the released radionuclide and its daughters remaining in the atmosphere of the overall region affected by the release after the releases stop. 
The total air and ground concentrations in the vicinity of the release point and in the regions beyond the immediate neighborhood of the nuclear point both during releases and after releases have stopped are obtained from specific combinations of the preceding quantities. These specific combinations for the air and ground concentrations of interest are defined in the remainder of this section.

\section{Air Concentrations-Vicinity of Releases}

a. During Releases-In general there are three contributions to the total ground level concentration of the activity in the air of the $\ell^{\text {th }}$ daughter of the released radionuclide $i$ in the immediate vicinity of the release point during the period that releases are occurring. The first of these contributors is $\chi_{\mathrm{ai} / \mathrm{ik}}$, the concentration of the $\ell^{\text {th }}$ daughter of the $i^{\text {th }}$ released radionuclide during its initial passage through the region within $100 \mathrm{~km}$ of the release point (i.e., within 30 hours of the time of release).

The second contributor is the activity in the air of the $\ell^{\text {th }}$ daughter of the radionuclide $i$ which had been released earlier during the release and has since encircled the earth one or more times. Thus, this activity has been dispersed throughout a significant portion of the earth's atmosphere. The concentration in air of the released radionuclide and its daughters which have encircled the earth one or more times is a special case of $\chi_{\text {bi }}$, the concentration in air of the $\ell^{\text {th }}$ daughter of the released radionuclide $i$ in the overall region affected by the release after its initial traverse through the immediate vicinity of the release point. In this case the overall region affected by the release should be chosen to include at least the portion of the atmosphere which is traversed by the released radionuclide and its daughters on their initial passage around the earth. Further, sufficient time should have elapsed subsequent to the release to permit the released nuclide and its daughters to have encircled the earth. Reference 4 indicates that it takes approximately 30 days for released material to encircle the earth and that the material has dispersed in a 40 to $50^{\circ}$ latitude band when it has returned to its release point the first time.

The final contributor to the total ground level air concentration in the immediate vicinity of the release point is due to the resuspension of the ground concentrations of the radioactive material deposited earlier during the release period. This contribution is given by the product of the resuspension factor $\beta$ and the ground concentration of the $l^{\text {th }}$ daughter of the $i^{\text {th }}$ released radionuclide in the vicinity of the release point. There are two components to the ground concentrations in the vicinity of the release point. The first of these is $C_{a i l j k}$ the ground concentration of the $\ell^{\text {th }}$ daughter of the $i^{\text {th }}$ released radionuclide resulting from the deposition of the radioactive nuclides during their initial passage through the region immediately adjacent to the release point. The second of these components is the ground concentration of the $\ell^{\text {th }}$ daughter of the released radionuclide i resulting from the deposition of nuclides which had encircled the earth one or more times prior to deposition. This ground concentration is a special case of $\mathrm{C}_{\mathrm{cil}}$, the ground concentration of the released nuclide and its daughter in the overall region affected by the releases during the period that releases are occurring. As before, the overall region should consist of at least the portion of the earth's surface traversed by the released material and its daughters during their initial passage around the earth.

Thus the ground level concentration of the radioactivity in the air of the $\ell^{\text {th }}$ daughter of the released radionuclide $i$ in the immediate vicinity of the release point during the period that releases are occurring is given by

$$
\chi_{\mathrm{a} i \ell \mathrm{jk}}+\chi_{\mathrm{bi} \ell}+\beta\left(\mathrm{C}_{\mathrm{ai} \ell \mathrm{jk}}+\mathrm{C}_{\mathrm{ci} \ell}\right)
$$

b. Subsequent to Releases-After the releases stop there are two contributors to the total ground level concentration of the activity in the air of the $l^{\text {th }}$ daughter of the released radionuclide $i$ in the immediate vicinity of the release point. The first of these contributors is the air concentration in the immediate vicinity of the release point of the $l^{\text {th }}$ daughter of the released radionuclide $i$ which has encircled the earth one or more times. This contribution is a special case of $\chi_{\mathrm{c} i l}$, the concentration in the air of $\ell^{\text {th }}$ daughter of the radionuclide $i$ remaining in the atmosphere of the overall region affected by the release subsequent to the initial passage of the released nuclide and its daughters through the regions immediately surrounding the release point. In this case the overall region affected by the release should include at least the portion of the earth's atmosphere traversed by the released material and its daughters during their initial passage around the earth.

The second contribution to the total ground level air concentrations in the immediate vicinity of the release point after releases stop is due to the resuspension of the radioactive material deposited earlier. This 
contribution is given by the product of the resuspension factor $\beta$ and the concentration of the $\ell^{\text {th }}$ daughter of the radionuclide $i$ on the ground in the immediate vicinity of the release point after the releases stop. There are two components to the ground concentrations in the vicinity of the release point after the releases stop. The first of these components is $\mathrm{C}_{\mathrm{bi} / \mathrm{jk}}$ the residual concentration on the ground after the releases have stopped resulting from the deposition of the released radionuclide and its daughters during their initial traverse of the region immediately surrounding the release point. The second component of the ground concentration in the vicinity of the release point after releases have stopped is the ground concentration which resulted from the deposition of nuclides which have encircled the earth one or more times. This ground concentration is a special case of the ground concentrations of the released radionuclide $i$ and its daughters in the overall region affected by the release after the releases stop. In particular the overall region affected by the releases must encompass at least the portion of the earth's surface traversed by the released material and its daughters during their initial passage around the globe. Further, sufficient time must elapse subsequent to the release to permit the nuclides to circle the earth.

Thus, the ground level concentration of the radioactivity in the air of the $\ell^{\text {th }}$ daughter of the relcascd radionuclide $\mathrm{i}$ in the immediate vicinity of the release point after the releases have stopped is given by

$$
x_{\mathrm{ci} \ell}+\beta\left(\mathrm{C}_{\mathrm{bi} \ell \mathrm{jk}}+\mathrm{C}_{\mathrm{di} \ell}\right)
$$

\section{Air Concentration-Beyond Vicinity of Releases}

a. During Release-There are two contributors to the total ground level air concentration of the activity in the region beyond the immediate vicinity of the release point during the period that releases are occurring. The first of these contributors is the air concentration $\chi_{\mathrm{bi} l}$ of the $l^{\text {th }}$ daughter of the released radionuclide i during the period that releases are occurring in the overall region affected by the release after the initial traverse through the immediate vicinity of the release point. The second of these contributors is the result of resuspension of material which had been deposited earlier in the release period in the overall region affected by the releases. This contribution is given by the product of the resuspension factor $\beta$ and the ground concentration $C_{\mathrm{ci} l}$ of the $\ell^{\text {th }}$ daughter of the radionuclide $\mathrm{i}$ in the overall region affected by the releases during the period that releases are occurring. Thus, the total ground level air concentration of the $\ell^{\text {th }}$ daughter of the released radionuclide $i$ in the overall region affected by the release during the period that releases are occurring is given by

$$
\chi_{\mathrm{bi} \ell}+\beta \mathrm{C}_{\mathrm{ci} \ell} .
$$

b. Subsequent to Releases-There are two contributors to the total ground level air concentration of the activity in the regions beyond the immediate vicinity of the release point after the releases stop. The first of these contributors is $\chi_{\mathrm{ci} l}$, the residual concentration of the $l^{\text {th }}$ daughter of the radionuclide i remaining in the atmosphere of the overall region affected by the releases after the releases stop. The second contributor is the result of the resuspension of material whish had been deposited previously in the overall rc... gion affected by the releases. This contribution is given by the product of the resuspension factor $\beta$ and the ground concentrations $C_{d i l}$ of the $l^{\text {th }}$ daughter of the radionuclide $i$ in the nverall reginn affected by the releases after the releases stop. Thus, the total ground level air concentration of the $l^{\text {th }}$ dauqhter of the released radionuclide i in the overall region affected by the releases after the releases stop is given by

$$
\chi_{\mathrm{ci} \ell}+\beta \mathrm{C}_{\mathrm{di} \ell} \text {. }
$$

\section{Ground Concentration-Vicinity of Releases}

a. During Releases-As identified earlier there are two contributors to the ground concentrations of radioactivity in the vicinity of the release point during the period that releases are occurring. The first of these contributors is $\mathrm{C}_{\mathrm{ai} \ell \mathrm{j} \mathrm{k}^{\prime}}$ the ground concentration of the $l^{\text {th }}$ daughter of the $\mathrm{i}^{\text {th }}$ released radionuclide resulting from their deposition during their initial passage through the regions immediately adjacent to the release point. The second contributor is a special case of $\mathrm{C}_{\mathrm{cil}}$, the ground concentration of the released radionuclide and its daughters in the overall region affected by the release during the period that releases 
are occurring where the overall region affected by the releases must include at least the portion of the earth's surface traversed during the initial passage of the released material and its daughters around the world. Thus, the total ground concentration of the.$^{\text {th }}$ daughter of the released radionuclide $i$ in the vicinity of the release point while the releases are going on is given by

$$
\mathrm{C}_{\mathrm{ai} \ell \mathrm{jk}}+\mathrm{C}_{\mathrm{cil}}
$$

b. Subsequent to Releases-As noted previously there are two components to the ground concentration of radioactivity in the neighborhood of the release point after the releases have stopped. The first of these is $\mathrm{C}_{\mathrm{bi} \ell_{\mathrm{j}} \text {, }}$ the residual concentration remaining after the releases stop on the ground in the vicinity of the release point due to the deposition of the released radionuclide and its daughters during their initial passage through the region immediately surrounding the release point. The second component is the ground concentrations resulting from the deposition of radionuclides which have encircled the earth one or more times. This latter component is a special case of the ground concentration $C_{d i l}$ of the $l^{\text {th }}$ daughter of the released radionuclide $i$ in the overall region affected by the releases after the releases stop. In this special case the overall region affectd by the releases must contain at least the part of the earth's surface traversed by the released radionuclide and its daughters during their initial passage around the earth. Thus, the total ground concentration of the $\ell^{\text {th }}$ daughter of the released radionuclide $i$ in the vicinity of the release point after releases stop is given by

$$
\mathrm{C}_{\mathrm{bilik}}+\mathrm{C}_{\mathrm{dil}}
$$

\section{Ground Concentrations-Beyond Vicinity of Releases}

The ground concentration of the $l^{\text {th }}$ daughter of the released radionuclide $i$ in the overall region affected by the releases during the period that releases are occurring is given by $\mathrm{C}_{\mathrm{cil}}$. After the releases have stopped the ground concentrations of the $\ell^{\text {th }}$ daughter of the released radionuclide $i$ in the overall region affected by the releases is given by $\mathrm{C}_{\mathrm{d} i \ell}$.

\section{B. Dose Commitments}

The AIRWAY computer program estimates the dose commitments from six specific pathways by which radionuclides released into the atmosphere may reach man. These dose commitments are made for the people in the immediate vicinity of the release point and for the people beyond this immediate region but within the overall region affected by the releases. Since the dose commitments may be accrued over a relatively large time interval (i.e., 70 years), the number of people in the immediate neighborhood of the releases and in the overall region affected by the releases may change significantly during such an interval. Thus, provisions are made in the AIRWAY program to permit an exponential growth or decay of the number of people in these regions. Further these dose commitments are made for a specific period of time which in general encompasses all or a part of the time that releases are occurring and some time after the releases stop. The equations presented in this section are written to reflect part of the overall dose commitment being accrued while releases are occurring and the remainder of the dose commitment obtained after releases stop. The AIRWAY program uses the actual time interval over which the dose commitments are to be obtained to define which part (if any) of the period during which releases are occurring is used to estimate the dose commitments. The dose commitment equations presented in this section are developed in detail in References 2 and 3 and are modified here to reflect the quantities incorporated into the AIRWAY program. 


\section{Immersion Dose Commitments}

a. Vicinity of Releases-The dose commitment $\mathrm{D}_{\mathrm{Ii} / \mathrm{gjk}}$ to the $\mathrm{g}^{\text {th }}$ organ of the people in the $\mathrm{k}^{\text {th }}$ annulus of the $j^{\text {th }}$ sector of the region immediately surrounding the release point due to immersion in a plume containing the $\ell^{\text {th }}$ daughter of the $\mathrm{i}^{\text {th }}$ released radionuclide is given by

$$
\begin{aligned}
& D_{\mathrm{Ii} \ell \mathrm{jj}}=\int_{\mathrm{T}_{\mathrm{I}}}^{\mathrm{T}_{\mathrm{S}}} \mathrm{K}_{\mathrm{Ii} \ell \mathrm{g}} \boldsymbol{H}_{\mathrm{ik}} \mathrm{e}^{\mathrm{A} \mathrm{PI}^{\prime}}\left\lfloor\chi_{\mathrm{ai} \ell \mathrm{jk}}+\chi_{\mathrm{bi} \ell}+\beta\left(\mathrm{C}_{\mathrm{ai} \ell \mathrm{jk}}+\mathrm{C}_{\mathrm{ci} \ell}\right)\right] \mathrm{dt} \\
& +\int_{\bar{T}_{\mathcal{G}}}^{\mathrm{T}_{\mathrm{L}}} \mathrm{K}_{\mathrm{Ii \ell g}} \mathrm{F}_{\mathrm{jk}} \mathrm{e}^{\lambda \mathrm{F})^{t}}\left(\dot{\chi}_{\mathrm{cil}}+\beta\left(\mathcal{C}_{\mathrm{bi} \ell \mathrm{jk}}+\mathcal{C}_{\mathrm{di} \ell}\right)\right] \mathrm{dt}
\end{aligned}
$$

Thus, the dose commitment is given by the time integral of the product of the immersion dose commitment conversion tactor $\mathrm{K}_{\mathrm{I} \ell \mathrm{l} g}$, the number of people at a given location in the vicinity of the release point as a function of time $P_{j k} e^{\lambda P 1^{T}}$ and the total ground level air concentration of the radionuclide of interest in the vicinity of the release point. The overall dose commitment time interval between $T_{1}$, the beginning of the interval and $T_{L}$, the end of the interval is divided into two segments. The first of these extends from $T_{I}$ to the time at which releases stop $T_{S}$ and thus defines the dose commitments which are accrued while the releases are occurring. The second segment extends from $T_{S}$ to $T_{L}$ and defines the dose commitments received after the releases stop.

The AIRWAY program considers that the population growth in the vicinity of the release point is represented by an exponential term which uses the number of people at a given radial position in a specific sector at the time releases begin $P_{j k}$ as an initial base and a growth constant $\lambda_{P 1}$ which is spatially independent. It also considers that the immersion dose commitment conversion factor $\mathrm{K}_{\mathrm{ILl} q}$ is independent of time. Further the AIKWAY computer program neglects the contribution to the immersion dose commitment due to the resuspension of previously deposited radionuclides on the ground in the immediate vicinity of the releases. Thus, the immersion dose commitment to the people in the immediate vicinity of the release point is given by

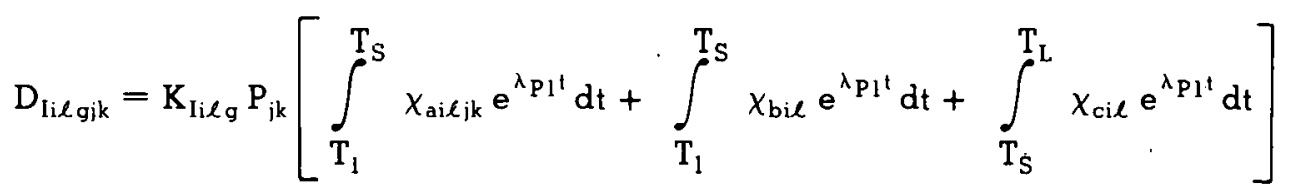

Noting that $\chi_{\text {a } i, j k}$ is independent of time, using the expresssions for $\chi_{\text {bil }}$ and $\chi_{\text {eir }}$ given in Equations (8) and (9) respectively and performing the indicated integration as done in References 2 and 3 yields the following equation for the dose commitment to the people in the $\mathrm{k}^{\text {th }}$ annulus of the $j^{\text {th }}$ sector of the region immediately surrounding the release point due to immersion in air containing the $\ell^{\text {th }}$ daughter of the released radionuclide $i$.

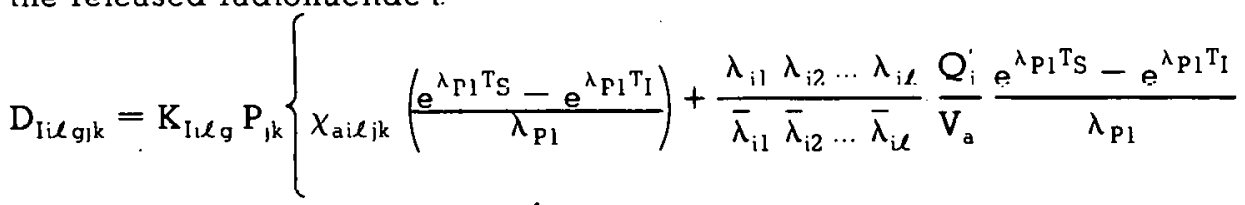

$$
\begin{aligned}
& -\lambda_{i 1} \lambda_{i 2} \ldots \lambda_{i \ell} \frac{Q_{i}^{\prime}}{V_{a}} \sum_{q=0}^{q=\ell} \frac{e^{-\left(\bar{\lambda}_{i q}-\lambda_{P 1}\right) T_{I}}-e^{-\left(\bar{\lambda}_{i q}-\lambda_{P 1}\right) T_{S}}}{\bar{\lambda}_{i q}-\lambda_{P 1}}
\end{aligned}
$$




$$
\begin{aligned}
& {\left[\frac{(-1)^{q}}{\left(\bar{\lambda}_{i q}-\bar{\lambda}_{i q}\right)\left(\bar{\lambda}_{i q}-\bar{\lambda}_{i 1}\right) \ldots\left(\bar{\lambda}_{i q}-\bar{\lambda}_{i q-1}\right) \bar{\lambda}_{i q}\left(\bar{\lambda}_{i q+1}-\bar{\lambda}_{i q}\right)\left(\bar{\lambda}_{i q+2}-\bar{\lambda}_{i q}\right) \ldots\left(\bar{\lambda}_{i \ell}-\bar{\lambda}_{i q}\right)}\right]} \\
& +\sum_{q=0}^{q=\ell} \frac{\lambda_{i q+1} \lambda_{i q+2} \ldots \lambda_{i \ell} e^{\bar{\lambda}_{i q} T_{S}}}{\left(\bar{\lambda}_{i q+1}-\bar{\lambda}_{i q}\right)\left(\bar{\lambda}_{i q+2}-\bar{\lambda}_{i q}\right) \ldots\left(\bar{\lambda}_{i \ell}-\bar{\lambda}_{i q}\right)}\left(\frac{e^{-\left(\bar{\lambda}_{i q}-\lambda_{p 1}\right) T_{S}}-e^{-\left(\bar{\lambda}_{i q}-\lambda_{p 1}\right) T_{L}}}{\bar{\lambda}_{i q}-\lambda_{p l}}\right) \\
& \left.\left.\sum_{h=0}^{h=q} \frac{(-1)^{q+h} \lambda_{i h+1} \lambda_{i h+2} \ldots \lambda_{i q}}{\left(\bar{\lambda}_{i q}-\bar{\lambda}_{i h}\right)\left(\bar{\lambda}_{i q}-\bar{\lambda}_{i h+1}\right) \ldots\left(\bar{\lambda}_{i q}-\bar{\lambda}_{i q \cdot 1}\right)} x_{b i h}\right|_{t=T_{S}}\right\}
\end{aligned}
$$

If the overall region affected by the release of the radionuclide $i$ is not large enough to include at least one traverse of the earth, the only contributions to the ground level air concentration of the $l^{\text {th }}$ daughter of the released radionuclide $i$ would occur during the initial passage through the region surrounding the release point. Thus, the ground level air concentration would reduce to $\chi_{\mathrm{ai} / \mathrm{jk}}$ and the immersion dose commitment to

$$
D_{l i \ell g j k}=K_{I i \ell g} P_{j k} \chi_{a i l j k}\left(\frac{e^{\lambda P 1} T_{S}-e^{\lambda} P_{1} T_{I}}{\lambda_{P 1}}\right)
$$

b. Beyond Vicinity of Releases-The dose commitment of the $\mathrm{g}^{\text {th }}$ organ of the people in the overall region affected by the release beyond the immediately neighborhood of the release point due to immersion in air containing the $\ell^{\text {th }}$ daughter of the released radionuclide $i$ is given by

$$
D_{\text {Ii } \ell g}=\int_{T_{I}}^{T_{S}} K_{I i \ell g} P_{T} e^{\lambda P 2^{t}}\left(\chi_{b i \ell}+\beta C_{c i l}\right) d t+\int_{T_{S}}^{T_{L}} K_{I i \ell g} P_{T} e^{\lambda P 2^{t}}\left(\chi_{c i l}+\beta C_{d i \ell}\right) d t
$$

Thus, llie duse commitmont is given by the integral over time of the product of the immersion dose commitment conversion factor, the number of people in the overall region affected by the release beyond the immediate neighborhood of the release point $\mathrm{P}_{\mathrm{T}} \mathrm{e}^{{ }_{\mathrm{P} 2 \mathrm{t}}}$ and the concentration of the radionuclide of interest in the atmosphere of the overall region affected by the release.

The AIRWAY program considers that the population growth in the overall region affected by the releases is represented by an exponential term which uses the total number of people in the region affected by the releases at the time releases start $P_{T}$ as an initial base and a growth constant $\lambda_{P 2}$. The contribution to the inmersion dose commitmont from the resuspension of radionuclide previously deposited on the ground in the overall region affected by the releases is again neglected. Noting that the immersion dose commitment convereion factor is independent of time, the immersion dose commitment to the people beyond the immediate neighborhood of the release point but within the overall region affected by the releases is given by

$$
D_{\mathrm{I} i \ell g}=K_{\mathrm{I} i \ell g} P_{T}\left[\int_{T_{I}}^{T_{S}} \chi_{b i \ell} e^{\lambda_{P} t} d t+\int_{T_{S}}^{T_{L}} x_{c i l} e^{\lambda_{P 2} t} d t\right]
$$


Using the expressions for $\chi_{\mathrm{bil}}$ and $\chi_{\mathrm{cil}}$ given in Equations (8) and (9) respectively and performing the indicated integration provides the following equation for the dose commitment to the people in the overall region affected by the releases due to immersion in air containing the $\ell^{\text {th }}$ daughter of the released radionuclide $\mathrm{i}$.

$$
\begin{aligned}
& \mathrm{D}_{\mathrm{Ii} \ell g}=\mathrm{K}_{\mathrm{I} \ell \mathrm{g}} \mathrm{P}_{\mathrm{T}}\left\{\frac{\lambda_{\mathrm{i} 1} \lambda_{\mathrm{i} 2} \ldots \lambda_{\mathrm{i} \ell}}{\bar{\lambda}_{\mathrm{i} 0} \bar{\lambda}_{\mathrm{i} 1} \ldots \bar{\lambda}_{\mathrm{i} \ell}} \frac{\mathrm{Q}_{\mathrm{i}}^{\prime}}{\mathrm{V}_{\mathrm{a}}} \frac{\mathrm{e}^{\lambda_{\mathrm{P} 2 \mathrm{~T}}}-\mathrm{e}^{\lambda_{\mathrm{P} 2} \mathrm{~T}_{\mathrm{I}}}}{\lambda_{\mathrm{p} 2}}\right. \\
& -\lambda_{\mathrm{i} 1} \lambda_{\mathrm{i} 2} \ldots \lambda_{\mathrm{i} \ell} \frac{\mathrm{Q}_{\mathrm{i}}^{\mathrm{q}}}{\mathrm{V}_{\mathrm{a}}} \sum_{\mathrm{q}=0} \frac{\mathrm{e}^{-\left(\bar{\lambda}_{\mathrm{iq}}-\lambda_{\mathrm{P} 2}\right) \mathrm{T} \mathrm{I}}-\mathrm{e}^{-\left(\bar{\lambda}_{\mathrm{iq}}-\lambda_{\mathrm{P} 2}\right) \mathrm{T}} \mathrm{S}}{\bar{\lambda}_{\mathrm{iq}}-\lambda_{\mathrm{P} 2}}
\end{aligned}
$$

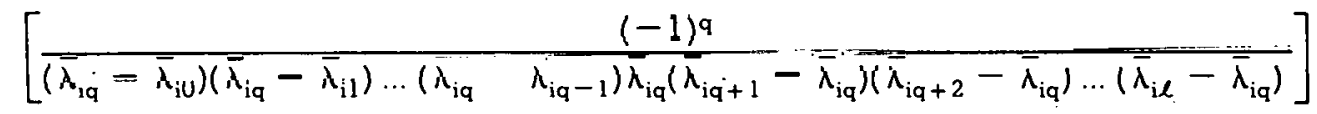

$$
\begin{aligned}
& +\sum_{q=0}^{q=\ell} \frac{\lambda_{i q+1} \lambda_{i q+2} \cdots \lambda_{i \ell} e^{\bar{\lambda}_{i q} T_{S}}}{\left(\bar{\lambda}_{i q+1}-\bar{\lambda}_{i q}\right)\left(\bar{\lambda}_{i q+2}-\bar{\lambda}_{i q}\right) \ldots\left(\bar{\lambda}_{i \ell}-\bar{\lambda}_{i q}\right)}\left(\frac{e^{-\left(\bar{\lambda}_{i q}-\lambda_{p 2}\right) T_{S}}-e^{-\left(\bar{\lambda}_{i q}-\lambda_{p 2}\right) T_{L}}}{\bar{\lambda}_{i q}-\lambda_{p 2}}\right) \\
& \left.\left.\sum_{h=0}^{h=q} \frac{(-1)^{q+h} \lambda_{i h+1} \lambda_{i h+2} \ldots \lambda_{i q}}{\left(\bar{\lambda}_{i q}-\bar{\lambda}_{i h}\right)\left(\bar{\lambda}_{i q}-\bar{\lambda}_{i h+1}\right) \ldots\left(\bar{\lambda}_{i q}-\bar{\lambda}_{i q-1}\right)} x_{b i l}\right|_{t=T_{S}}\right\}
\end{aligned}
$$

\section{Surface Deposit Dose Commitments}

a. Vicinity of Releases-The dose commitment $D_{I T i / j^{i}}$ in the $q^{\text {th }}$ organ of the people in the $k^{\text {th }}$ annulus of the $j^{\text {th }}$ sector of the region immediately surrounding the release point due to a surface deposit of the $\ell^{\text {th }}$ daughter of the released radionuclide $i$ is given by

$$
\begin{aligned}
D_{I I L \ell g k}= & \int_{T_{I}}^{T_{S}} K_{I I i \ell g} P_{i k} e^{\lambda P I^{\prime}}\left(C_{a i \ell j k}+C_{c i \ell}\right) d l \\
& +\int_{T_{S}}^{T_{L}} K_{I I i \ell g} P_{i k} e^{\lambda_{P} I^{t}}\left(C_{b i \ell j k}+C_{d i \ell}\right) d t
\end{aligned}
$$

Thus, the surface deposit dose commitment is given by the time integral of the product of the surface deposit dose commitment conversion factor $\mathrm{K}_{\mathrm{II} i \ell, g^{\prime}}$ the number of people at a given position in the immediate neighborhood of the release point and the ground concentration of the radionuclide of interest at the same position in the vicinity of the release point. Using the expressions for $C_{a i l j k}, C_{b i l j k^{\prime}} C_{c i l}$, and $C_{d i l}$ given in Equations (3), (6), (10), and (15), respectively, and performing the indicated integration results in the following equation for the dose commitment to the $\mathrm{g}^{\text {th }}$ organ of the people in the $\mathrm{k}^{\text {th }}$ annulus of the $\mathrm{j}^{\text {th }}$ sector of the region surnounding the release point due to exposure of surface deposits of the $l^{\text {th }}$ daughter of the released radionuclide $i$. 


$$
\begin{aligned}
& D_{I I l \ell g j k}=K_{I I l q g} P_{i k}\left[\frac{e^{\lambda_{P 1} T_{S}}-e^{\lambda_{P 1} T_{I}}}{\lambda_{P 1}} \frac{\sum_{q=0}^{q=\ell} \alpha_{i 0} \alpha_{i 1} \ldots \alpha_{i q-1} Y_{i q i k} \lambda_{i q+1} \lambda_{i q+2} \ldots \lambda_{i \ell}}{\alpha_{i 0} \alpha_{i 1} \ldots \alpha_{i \ell}}\right. \\
& -\sum_{q=0}^{q=l}(-1)^{q} \frac{e^{-\left(\alpha_{i q}-\lambda_{p 1}\right) T_{I}}-e^{-\left(\alpha_{i q}-\lambda_{p 1}\right) T_{S}}}{\alpha_{i q}-\lambda_{P 1}} \\
& \sum_{h=0}^{h=q} \frac{(-1)^{h} Y_{i h j k} \lambda_{i h+1} \lambda_{i h+2} \ldots \lambda_{i \ell}}{\left(\alpha_{i q}-\alpha_{i h}\right)\left(\alpha_{i q}-\alpha_{i h+1}\right) \ldots\left(\alpha_{i q}-\alpha_{i q-1}\right) \alpha_{i q}\left(\alpha_{i q+1}-\alpha_{i q}\right)\left(\alpha_{i q+2}-\alpha_{i q}\right) \ldots\left(\alpha_{i \ell}-\alpha_{i q}\right)} \\
& +\sum_{q=0}^{q=\ell}\left\{\frac{\gamma_{i q} A_{i q} \lambda_{i q+1} \lambda_{i q+2} \cdots \lambda_{i \ell}}{\alpha_{i q} \alpha_{i q+1} \cdots \alpha_{i \ell}} \frac{e^{\lambda_{P 1} T_{S}}-e^{\lambda_{P 1} T_{I}}}{\lambda_{P 1}}\right. \\
& +\frac{e^{-\left(\bar{\lambda}_{i q}-\lambda_{P 1}\right) T_{I}}-e^{-\left(\bar{\lambda}_{i q}-\lambda_{p 1}\right) T_{S}}}{\bar{\lambda}_{i q}-\lambda_{p 1}} \sum_{h=q}^{h=\ell} \frac{\gamma_{i h} B_{i h} E_{i h} \lambda_{i h+1} \lambda_{i h+2} \ldots \lambda_{i l}}{\left(\alpha_{i h}-\bar{\lambda}_{i q}\right)\left(\alpha_{i h+1}-\bar{\lambda}_{i q}\right) \ldots\left(\alpha_{i \ell}-\bar{\lambda}_{i q}\right)} \\
& +\frac{(-1)^{q+1} \lambda_{i q+1} \lambda_{i q+2} \ldots \lambda_{i \ell}}{\left(\alpha_{i q+1}-\alpha_{i q}\right)\left(\alpha_{i q+2}-\alpha_{i q}\right) \ldots\left(\alpha_{i \ell}-\alpha_{i q}\right)} \frac{e^{-\left(\alpha_{i q}-\lambda_{p 1}\right) T_{I}}-e^{-\left(\alpha_{i q}-\lambda_{p 1}\right) T_{S}}}{\alpha_{i q}-\lambda_{\mathrm{Pl}}} \\
& \left.\sum_{h=0}^{h=q} \frac{(-1)^{h} \gamma_{i h} \lambda_{i h+1} \lambda_{i h+2} \ldots \lambda_{i q}}{\left(\alpha_{i q}-\alpha_{i h}\right)\left(\alpha_{i q}-\alpha_{i h+1}\right) \ldots\left(\alpha_{i q}-\alpha_{i q}-1\right)}\left[\frac{A_{i h}}{\alpha_{i q}}-B_{i h} \sum_{p=0}^{p=h} \frac{E_{i h p}}{\alpha_{i q}-\bar{\lambda}_{i p}}\right]\right\} \\
& +\sum_{q=0}^{q=\ell} J_{i \ell q i k} e^{\alpha_{i q} T_{S}} \frac{e^{-\left(\alpha_{i q}-\lambda_{p 1}\right) T_{S}}-e^{-\left(\alpha_{i q}-\lambda_{P 1}\right) T_{L}}}{\alpha_{1 q}-\lambda_{p 1}} \\
& +\sum_{q=0}^{q=\ell}\left\{R_{i \ell q} e^{\alpha_{i q} T_{S}} \frac{e^{-\left(\alpha_{i q}-\lambda_{P 1}\right) T_{S}}-e^{-\left(\alpha_{i q}-\lambda_{P 1} T_{L}\right.}}{\alpha_{i q}-\lambda_{P 1}}\right. \\
& \left.+S_{i \ell q} e^{\bar{\lambda}_{i q} T_{S}} \frac{e^{-\left(\bar{\lambda}_{i q}-\lambda_{p 1}\right) T_{S}}-e^{\left(\bar{\lambda}_{i q}{ }^{\left.\lambda_{p 1}\right) T_{L}}\right.}}{\bar{\lambda}_{i q}-\lambda_{p l}}\right\}
\end{aligned}
$$

where $Y_{i q j k^{\prime}} A_{i q^{\prime}} B_{i h^{\prime}}, E_{i h q}, J_{i \ell q j k^{\prime}} R_{i \ell q^{\prime}}$ and $S_{i \ell q}$ are given by Equations (5), (12), (13), (14), (7), (16), and (17), respectively.

If the overall region affected by the release of the radionuclide $i$ is not sufficiently large to permit at least one traverse of the earth, the only contributions to the ground concentration in the vicinity of the release point are the result of the deposition of the released radionuclide and its daughters during their initial passage through the region immediately surrounding the release point. Thus, the ground concentration at a 
given position in the vicinity of the release point would reduce to $C_{a i / j k}$ during releases and to $C_{b i / j k}$ after the releases stop. In this case the dose commitment to the $\mathrm{g}^{\text {th }}$ organ of the people at a specific location in the immediate vicinity of the release point due to surface deposits of the $\ell^{\text {th }}$ daughter of the released radionuclide $\mathrm{i}$ is given by

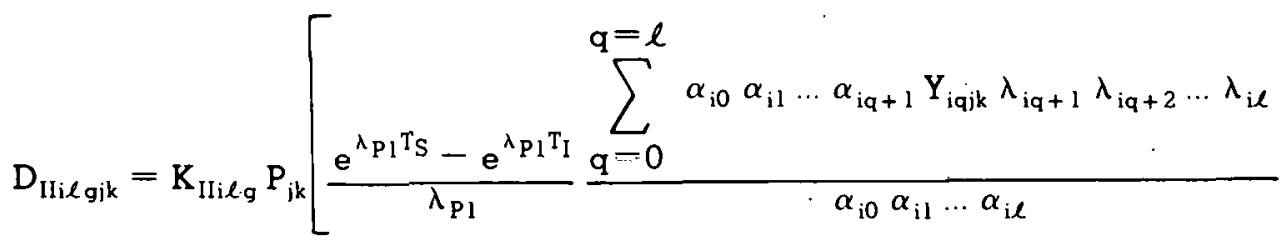

$$
-\sum_{q=0}^{q=\ell}(-1)^{q} \frac{e^{-\left(\alpha_{i q}-\lambda_{P 1}\right) T_{1}}-e^{-\left(\alpha_{i q}-\lambda_{P 1}\right) T_{S}}}{\alpha_{i q}-\lambda_{P 1}}
$$

$$
\begin{aligned}
& \sum_{\mathrm{h}=0}^{\mathrm{h}=\mathrm{q}} \frac{(-1)^{\mathrm{h}} \mathrm{Y}_{\mathrm{ihjk}} \lambda_{\mathrm{ih}+1} \lambda_{\mathrm{ih}+2} \ldots \lambda_{\mathrm{il}}}{\left(\alpha_{\mathrm{iq}}-\alpha_{\mathrm{ih}}\right)\left(\alpha_{\mathrm{iq}}-\alpha_{\mathrm{ih}+1}\right) \ldots\left(\alpha_{\mathrm{iq}}-\alpha_{\mathrm{iq}-1}\right) \alpha_{\mathrm{iq}}\left(\alpha_{\mathrm{iq}+1}-\alpha_{\mathrm{iq}}\right)\left(\alpha_{\mathrm{iq}+2}-\alpha_{\mathrm{iq}}\right) \ldots\left(\alpha_{\mathrm{i} \ell}-\alpha_{\mathrm{iq}}\right)} \\
& \left.+\sum_{q=0}^{q=\ell} J_{i \ell q j k} e^{\alpha_{i q} T_{S}} \frac{e^{-\left(\alpha_{i q}-\lambda_{P 1}\right) T_{S}}-e^{-\left(\alpha_{i q}-\lambda_{P 1}\right) T_{L}}}{\alpha_{i q}-\lambda_{P 1}}\right] .
\end{aligned}
$$

where $\mathrm{Y}_{\mathrm{iqik}}$ and $\mathrm{J}_{\mathrm{i} \ell \mathrm{qik}}$ are given by Equations (5) and (7), respectively.

b. Beyond Vicinity of Releases-The dose commitment to the $\mathrm{g}^{\text {th }}$ organ of the people in the overall region affected by the release beyond the immediate neighborhood of the release point resulting from ex $\bar{x}-$ posure to a surface deposit of the $l^{\text {th }}$ daughter of the released radionuclide $i$ is given by

$$
D_{I I i \ell g}=\int_{T_{I}}^{T_{S}} K_{I I i \ell g} P_{T} e^{\lambda P 2 t} C_{c i \ell} d t+\int_{T_{S}}^{T_{L}} K_{I I i \ell g} P_{T} e^{\lambda P 2 t} C_{d i \ell} d t
$$

This dose commitment is given by the time integral of the product of the surface deposit dose commitment conversion factor, the number of people in the overall region affected by the release beyond the immediate vicinity of the release point and the ground concentration of the $\ell^{\text {th }}$ daughter of the released radionuclide i. Using the expressions for $C_{o i l}$ and $C_{d i \ell}$ given by Equations (10) and (15), respectively, and performing the indicated integration results in the following equation for the dose commitment to the $\mathrm{g}^{\text {th }}$ organ of the people in the overall region aftected by the release due to exposure to a ground surface deposit containing the $\ell^{\text {th }}$ daughter of the $\mathrm{i}^{\text {th }}$ released radionuclide.

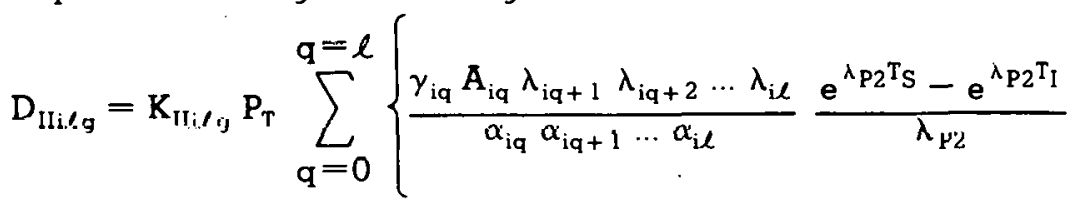

$$
\begin{aligned}
& +\frac{e^{-\left(\bar{\lambda}_{i q}-\lambda_{p 2}\right) T_{1}}-e^{-\left(\bar{\lambda}_{i q}-\lambda_{p 2}\right) T_{S}}}{\bar{\lambda}_{i q}-\lambda_{P 2}} \sum_{h=q}^{h=\ell} \frac{\gamma_{i h} B_{i h} E_{i h q} \lambda_{i h+1} \lambda_{i h+2} \ldots \lambda_{i \ell}}{\left(\alpha_{i h}-\bar{\lambda}_{i q}\right)\left(\alpha_{i h+1}-\bar{\lambda}_{i q}\right) \ldots\left(\alpha_{i \ell}-\bar{\lambda}_{i q}\right)}
\end{aligned}
$$




$$
\begin{aligned}
& +\frac{(-1)^{\mathrm{q}+1} \lambda_{\mathrm{iq}+1} \lambda_{\mathrm{iq}+2} \ldots \lambda_{\mathrm{i} \ell}}{\left(\alpha_{\mathrm{iq}+1}-\alpha_{\mathrm{iq}}\right)\left(\alpha_{\mathrm{iq}+2}-\alpha_{\mathrm{iq}}\right) \ldots\left(\alpha_{\mathrm{i} \ell}-\alpha_{\mathrm{iq}}\right)} \frac{\mathrm{e}^{-\left(\alpha_{\mathrm{iq}}-\lambda_{\mathrm{P} 2}\right) \mathrm{T}_{\mathrm{I}}-\mathrm{e}^{-\left(\alpha_{\mathrm{iq}}-\lambda_{\mathrm{P} 2}\right) \mathrm{T} \mathrm{S}}}}{\alpha_{\mathrm{iq}}-\lambda_{\mathrm{P} 2}} \\
& \sum_{\mathrm{h}=0}^{\mathrm{h}=\mathrm{q}} \frac{(-1)^{\mathrm{h}} \gamma_{\mathrm{ih}} \lambda_{\mathrm{ih}+1} \lambda_{\mathrm{ih}+2} \ldots \lambda_{\mathrm{iq}}}{\left(\alpha_{\mathrm{iq}}-\alpha_{\mathrm{ih}}\right)\left(\alpha_{\mathrm{iq}}-\alpha_{\mathrm{ih}+1}\right) \ldots\left(\alpha_{\mathrm{iq}}-\alpha_{\mathrm{iq}-1}\right)}\left[\frac{\mathrm{A}_{\mathrm{ih}}}{\alpha_{\mathrm{iq}}}-\mathrm{B}_{\mathrm{ih}} \sum_{\mathrm{p}=0}^{\mathrm{p}=\mathrm{h}} \frac{\mathrm{E}_{\mathrm{ihP}}}{\alpha_{\mathrm{iq}}-\bar{\lambda}_{\mathrm{iP}}}\right] \\
& +R_{i \ell q} e^{\alpha_{i q} T_{S}} \frac{e^{-\left(\alpha_{i q}-\lambda_{P 2}\right) T}-e^{-\left(\alpha_{i q}-\lambda_{P 2}\right) T_{L}}}{\alpha_{i q}-\lambda_{P 2}} \\
& \left.+S_{i \ell q} e^{\bar{\lambda}_{i q} T_{S}} \frac{e^{-\left(\bar{\lambda}_{i q}-\lambda_{P 2}\right) T_{S}}-e^{-\left(\bar{\lambda}_{i q}-\lambda_{P 2}\right) T_{L}}}{\bar{\lambda}_{i q}-\lambda_{P 2}}\right\}
\end{aligned}
$$

\section{Ingestion Dose Commitments}

The ingestion dose commitments are divided into two separate pathways in the AIRWAY program. The first of these pathways is concerned with the dose commitments which may be obtained from the particulate radionuclides which are deposited directly upon existing above surface crops. These crops may be those which are eaten directly by man such as fruits, lettuce, spinach and other above ground vegetables or they may be ingested indirectly through the consumption of milk and meat which are produced from animals which were pastured on ground subjected to the deposition of radionuclides. Thus, the ingestion dose commitments associated with this pathway are dependent upon the rate at which the particulate radionuclides are being deposited on the ground due to dry deposition mechanisms and to precipitation scavenging. The second of these ingestion pathways considered by the AIRWAY program defines the dose commitments which may be obtained by the ingestion of food which is produced in ground which has been previously contaminated by prior deposition. Thus, this second pathway includes all pasture and food grown on land subsequent to the deposition of radionuclides on that land. The dose commitments associated with this second ingestion pathway are dependent upon the ground concentrations of the various radionuclides during the period of the crop growth.

\section{a. Food Supply Subjected to Direcl Deposition}

(1) Vicinity of Releases-The dose commitment from the ingestion of food subjected to the direct deposition of radinnuclides is given by the time inteqral of the product of the ingestion dose commitment conversion factor $\mathrm{K}_{\text {IIIl } / g}$ the number of people at the position of interest, the intake rate of the radionuclide of interest per unit deposition rate per unit area of the nuclide of interest $W_{f i}$ the fraction of the normal daily intake of the $f^{\text {th }}$ food type which is obtained from the region subjected to deposition $\mathrm{q}_{\mathrm{f}} / \mathrm{q}_{\mathrm{Of}}$, and the average rate at which deposition of the radionuclide of interest occurs. Thus, the dose commitment $D_{\text {IIIle gik }}$ to the $\mathrm{g}^{\text {th }}$ organ of the people in the $\mathrm{k}^{\text {th }}$ annulus of the $\mathrm{j}^{\text {th }}$ sector of the region immediately surrounding the release point due to the ingestion of food subjected to the deposition of the $l^{\text {th }}$ daughter of the released radionuclide $i$ is given by

$$
\begin{aligned}
& D_{I I I \ell \ell j k}=\int_{T_{I}}^{T_{S}} 1.0 \times 10^{6} K_{I I I i \ell g} P_{j k} e^{\lambda P^{t}} \sum_{f} \frac{q_{f}}{q_{0 f}} W_{i l}\left(\bar{Y}_{i \ell l}+\gamma_{i \ell} \bar{\chi}_{b i \ell l}\right) d t \\
& +\int_{T_{S}}^{T_{L}} 1.0 \times 10^{6} K_{\text {IIlilg }} P_{i k} e^{\lambda P^{t}} \sum_{f} \frac{q_{1}}{q_{u f}} w_{U \ell} \gamma_{i l} \bar{\gamma}_{\text {Lill }} d t
\end{aligned}
$$


where $\bar{\chi}_{\mathrm{bi} \ell \mathrm{f}}$ and $\bar{\chi}_{\mathrm{ci} \ell \mathrm{f}}$ are the time averaged concentrations of the $\ell^{\text {th }}$ daughter of the released radionuclide $i$ in the overall region affected by the release during the period that releases are occurring and after releases have stopped, respectively. These averages are obtained over the period that deposition on the food is occurring and are defined by the following expressions.

$$
\begin{aligned}
& \bar{\chi}_{\mathrm{bi} \ell \mathrm{t}}=\int_{\mathrm{T}_{\mathrm{fa}}}^{\mathrm{T}_{\mathrm{S}}} x_{\mathrm{bi} \ell} \mathrm{dt} / \int_{\mathrm{T}_{\mathrm{fa}}}^{\mathrm{T}_{\mathrm{S}}} \mathrm{dt} \\
& \bar{\chi}_{\mathrm{ci \ell t}}=\int_{\mathrm{T}_{\mathrm{S}}}^{\mathrm{T}_{\mathrm{fb}}} x_{\mathrm{ci} \ell} \mathrm{dt} / \int_{\mathrm{T}_{\mathrm{S}}}^{\mathrm{T}_{\mathrm{fb}}} \mathrm{dt}
\end{aligned}
$$

where $\mathrm{T}_{\mathrm{fa}}$ and $\mathrm{l} \mathrm{l}_{\mathrm{fb}}$ are the times at which deposition on the food supply beqins and ends, respertively

The preceding definitions of the average concentrations of the radionuclides in the atmosphere reflect the situation when deposition is occurring within the period that dose commitments are being accrued and extend over a time interval which includes the time at which releases stop. This presentation is consistent with the manner in which the dose commitment equations are presented. The AIRWAY program, however, uses the times defining the period during which deposition on the food supply is occurring relative to the time at which releases stop and to the time interval over which the dose commitments are obtained to define the actual intervals over which the averages are made.

Using the preceding definitions for $\bar{\chi}_{\text {bilf }}$ and $\bar{\chi}_{c i l f}$ in Equation (3l) and then rearranging yields the following expression for the ingestion dose commitments arising from the direct deposition of radionuclides on the food supply.

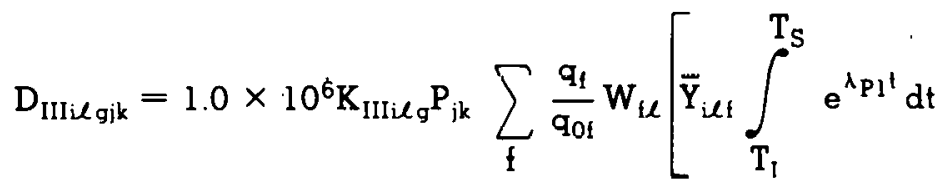

$$
\begin{aligned}
& \left.+\frac{\gamma_{i \ell}}{T_{S}-T_{i a}} \int_{T_{l a}}^{T_{S}} \chi_{b i \ell} d t \int_{T_{I}}^{T_{S}} e^{\lambda}{ }^{\lambda} d t+\frac{\gamma_{i \ell}}{T_{f b}-T_{S}} \int_{T_{S}}^{T_{t b}} \chi_{c i l} d t \int_{T_{G}}^{T_{L}} e^{\lambda_{P} 1^{t}} d t\right]
\end{aligned}
$$

Substituting the expressions for $\chi_{\mathrm{bi} \ell}$ and $\chi_{\mathrm{ci} \ell}$ given by Equations (8) and (9), respectively, into the preceding equation and then performing the indicated integration yields the following

$$
\begin{aligned}
& D_{111 \ell g i k}=1.0 \times 10^{6} K_{11 ! \ell g} P_{j k} \sum_{f}-\frac{q_{1}}{q_{0 l}} W_{L \ell}\left[\bar{Y}_{i \ell t} \frac{e^{\lambda_{P 1} T_{S}}-e^{\lambda_{P 1} T_{I}}}{\lambda_{P 1}}\right. \\
& +\gamma_{i \ell} A_{i \ell}-\frac{\gamma_{i \ell} B_{i \ell}}{T_{S}-T_{i a}} \sum_{q=0}^{q=\ell} E_{i \ell q} \frac{e^{-\bar{\lambda}_{i q} T_{i n}}-e^{-\bar{\lambda}_{i{ }_{1}} T_{S}}}{\bar{\lambda}_{i q}}-\frac{e^{{ }^{A} P 1} T_{S}-e^{\lambda_{P 1} T_{I}}}{\lambda_{P 1}} \\
& \left.+\frac{\lambda_{i \ell}}{T_{f b}-T_{S}} \sum_{q=0}^{q=\ell} G_{i \ell q} \frac{1-e^{-\bar{\lambda}_{i q}\left(T_{i b}-T_{S}\right)}}{\bar{\lambda}_{i q}} \frac{e^{\lambda_{P 1} T_{L}}-e^{\lambda_{P 1} T_{S}}}{\lambda_{P 1}}\right]
\end{aligned}
$$


where $A_{i \ell}, B_{i \ell}$ and $E_{i \ell q}$ are given by Equations (12), (13), and (14), respectively, and $G_{i \ell q}$ is defined as

$$
\begin{aligned}
G_{i \ell q}= & \frac{\lambda_{i q+1} \lambda_{i q+2} \ldots \lambda_{i \ell}}{\left(\bar{\lambda}_{i q+1}-\bar{\lambda}_{i q}\right)\left(\bar{\lambda}_{i q+2}-\bar{\lambda}_{i q}\right) \ldots\left(\bar{\lambda}_{i \ell}-\bar{\lambda}_{i q}\right)} \\
& \left.\sum_{h=0}^{h=q} \frac{(-1)^{q+h} \lambda_{i h+1} \lambda_{i h+2} \ldots \lambda_{i q}}{\left(\bar{\lambda}_{i q}-\bar{\lambda}_{i h}\right)\left(\bar{\lambda}_{i q}-\bar{\lambda}_{i h+1}\right) \ldots\left(\bar{\lambda}_{i q}-\bar{\lambda}_{i q-1}\right)} x_{b i h}\right|_{t=T}
\end{aligned}
$$

If the overall region affected by the release of the radionuclide i does not extend to include one passage around the earth, the deposition on the food crops in the vicinity of the release point can only result from the deposition of the released radionuclides and their daughters during their initial passage through the region surrounding the release point. Thus, the expression for this ingestion dose commitment would reduce to

$$
D_{\text {III } i \ell \text { gik }}=1.0 \times 10^{6} K_{I I I ~ j \ell g} P_{j k} \frac{e^{\lambda_{P 1} T_{S}}-e^{\lambda_{P 1} T_{I}}}{\lambda_{P 1}} \sum_{f} \frac{q_{f}}{q_{0 I}} W_{L l} \bar{Y}_{i l t}
$$

The $\bar{Y}_{i l 1}$ term in the preceding equations is the spatially averaged rate at which deposition of the $\ell^{\text {th }}$ daughter of the released radionuclide $i$ is occurring per unit area due to precipitation scavenging and dry deposition mechanisms. This spatial average is defined over the area on which the food ingested by the people at a given location is produced and is given by the following expression.

$$
\bar{Y}_{i \ell l}=\int_{r_{i}} \int_{\theta_{1}} Y_{i \ell j k} r_{i} d r d \theta / \iint_{r_{i}} \int_{\theta_{i}} r_{f} d r d \theta
$$

If the integrals in the preceding expression are approximated by sums over the $k$ annuli and the $\mathrm{j}$ sectors into which the region immediately surrounding the release point is subdivided, then the spatially averaged deposition rate becomes

$$
\bar{Y}_{i \ell f}=\frac{\sum_{k_{f}} \sum_{j_{i}} \theta_{i}\left[\left(\frac{r_{k+1}+r_{k}}{2}\right)^{2}-\left(\frac{r_{k}+r_{k}-1}{2}\right)^{2}\right] Y_{i \ell j k}}{\sum_{k_{f}} \sum_{j_{f}} \theta_{j}\left[\left(\frac{r_{k+1}+r_{k}}{2}\right)^{2}-\left(\frac{r_{k}+r_{k}-1}{2}\right)^{2}\right]} .
$$

where the summations are performed over the annular segments where food is being produced for consumption by the people located in the $\mathrm{k}^{\text {th }}$ annulus of the $\mathrm{j}^{\text {th }}$ sector of the region immediately surrounding the release point.

In particular, if the food consumed by the people residing in a given annular segment of the region immediately surrounding the release point is produced in the same annular segment, then the average deposition rate per unit area of the $\ell^{\text {th }}$ daughter of the released radionuclide i on the food consumed by these people in the $k^{\text {th }}$ annulus of the $j^{\text {th }}$ sector of the region in the immediate vicinity of the release point is given by

$$
\bar{Y}_{i \ell f}=Y_{i \ell j k}
$$


However, if the food consumed by the people in a given location is produced throughout the entire region surrounding the release point, then the average deposition rate per unit area of the $\ell^{\text {th }}$ daughter of the released radionuclide $i$ on the food consumed by the people in any location within the immediate vicinity of the release point is given by

$$
\bar{Y}_{i \ell l}=\frac{\sum_{k=1}^{\text {KMAX JMAX }} \sum_{j=1}^{\text {KMAX JMAX }} \theta_{i}\left[\left(\frac{r_{k+1}+r_{k}}{2}\right)^{2}-\left(\frac{r_{k}+r_{k-1}}{2}\right)^{2}\right] Y_{i \ell j k}}{\sum_{k=1}^{\text {JMAX }} \sum_{j=1} \theta_{j}\left[\left(\frac{r_{k+1}+r_{k}}{2}\right)^{2}-\left(\frac{r_{k}+r_{k-1}}{2}\right)^{2}\right]} .
$$

where KMAX and JMAX are the total number of annuli and sectors, respectively, into which the rcgion immediately surrounding the release point is subdivided. It should be noted that when $k=1$ then the $\left(r_{k}+r_{k-1}\right) / 2$ term is set to zero and when $k=\operatorname{KMAX}$ the $\left(r_{k+1}+r_{k}\right) / 2$ term is set to RMAX, the outer radius of the region immediately surrounding the release point.

(2) Beyond Vicinity of Releases-The dose commitment to the $\mathrm{g}^{\text {th }}$ organ of the people in the overall region affected by the release beyond the immediate neighborhood of the release point due to the ingestion of food which has been subjected to the deposition of the $\ell^{\text {th }}$ daughter of the released radionuclide $i$ is given by

$$
\begin{aligned}
& D_{\text {III } l g}=\int_{T_{T}}^{T_{S}} 1.0 \times 10^{6} K_{\text {IIIL }} P_{T} e^{\lambda P 2^{t}} \sum_{f} \frac{q_{f}}{q_{0 f}} W_{L \ell} \gamma_{i l} \bar{X}_{b i l f} d t
\end{aligned}
$$

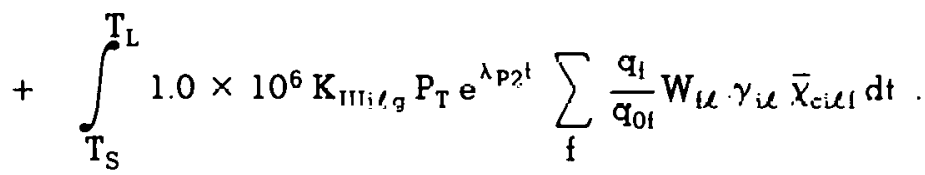

Substituting the expressions for $\bar{\chi}_{\text {bilf }}$ and $\bar{\chi}_{c i l f}$ defined by Equations (32) and (33), respectively, into the preceding equation, rearranging and performing the indicated integration yields the following expression for the ingestion dose commitment associated with the deposition of radionuclides upon the food consumed by the people in the overall region affected by the releases beyond the immediate vicinity of the release point

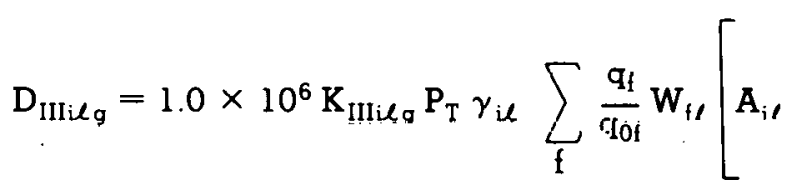

$$
\begin{aligned}
& -\frac{B_{i \ell}\left(e^{\lambda_{P 2} T_{S}}-e^{\lambda_{P 2} T_{1}}\right)}{\left(T_{S}-T_{1 a}\right) \lambda_{12}} \sum_{q=0}^{q=\ell} \frac{E_{i \ell q}\left(e^{-\bar{\lambda}_{i q} T_{f a}}-e^{-\bar{\lambda}_{i q} T_{S}}\right)}{\bar{\lambda}_{i q}}
\end{aligned}
$$

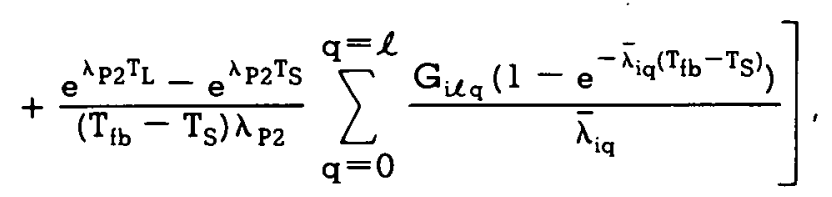


where $A_{i \ell}, B_{i \ell}, E_{i \ell q}$ and $G_{i \ell q}$ are defined by Equations (12), (13), (14), and (36), respectively.

In the previous discussion pertaining to the ingestion dose commitments accrued by people consuming food which has been subjected to the deposition of the released radionuclides and their daughters, the effect of deposition of radionuclides which had been deposited earlier and then resuspended is neglected. This is consistent with the procedures used previously in defining the immersion and surface deposit dose commitments. In general the AIRWAY program neglects the dose commitment contributions arising from the resuspension of previously deposited radionuclides with the exception of the inhalation dose commitments.

b. Food Supply Produced in Previously Contaminated Ground -In the discussion which follows of the development of the ingestion dose commitments resulting from the consumption of food in soil previously contaminated by the deposition of the released radionuclides and their daughters, the assumption is made that food crops and forage are grown continuously throughout the time interval that the dose commitments are being accrued. This approximation to the actual practice of growing food crops in discrete growing seasons does not alter the total a mount of tood produced in the contaminated soil nor does it change the quantities of food ingested by the population. It is considered that the additional complexities which are introduced into the AIRWAY program and its input by the incorporation of a discrete growing season for food crops are not warranted. In addition to the preceding approximation, the effect of the deposition of radionuclides which had been deposited previously and then resuspended will be neglected in the development of the dose commitments arising from the ingestion of food produced in soil contaminated by prior deposition of the released radionuclides and their daughters.

(1) Vicinity of Releases-The dose commitment from the ingestion of food produced in previously contaminated ground is given by the time integral of the product of the ingestion dose commitment conversion factor, the number of people at the position of interest, the ground concentration of the radionuclide of interest averaged over the food producing area, the activity taken up per unit food mass due to a unit activity concentration in the soil per unit thickness of the root bearing layer $\mathrm{K}_{t i}$ and the daily intake of the food type. Thus, the dose commitment to the $\mathrm{g}^{\text {th }}$ organ of the people in the $\mathrm{k}^{\text {th }}$ annulus of the $\mathrm{j}^{\text {th }}$ sector of the region immediately surrounding the release point due to the ingestion of food containing the $l^{\text {th }}$ daughter-of the released radionuclide $i$ which was produced in soil contaminated by earlier deposition is given by

$$
\begin{aligned}
& D_{\text {IIIl gik }}=\int_{T_{I}}^{T_{S}} 1.0 \times 10^{6} \mathrm{~K}_{\text {III } \ell g} P_{i k} e^{\lambda_{P} 1^{t}}\left(\bar{C}_{a i l}+C_{c i l}\right) \sum_{f} \frac{K_{u l} q_{f}}{24} d t \\
& +\int_{T_{S}}^{T_{i}^{L}} 1.0 \times 10^{6} K_{I I I l g} P_{j k} e^{\lambda_{P 1}}\left(\bar{C}_{b i l}+C_{d i l}\right) \sum_{f} \frac{K_{l \ell} q_{f}}{24} d t
\end{aligned}
$$

The $\overline{\mathrm{C}}_{\mathrm{ail}}$ and $\overline{\mathrm{C}}_{\mathrm{bil}}$ terms in the preceding equation are the ground concentrations of the $\ell^{\text {th }}$ daughter of the released radionuclide i during releases and subsequent to releases, respectively, in the region immediately surrounding the release point averaged over the area in this region in which the food is produced. These averages are obtained in the same manner as detined previously for the spatially averaged deposition rates in the region immediately surrounding the release point. Thus, if the people in a given annular segment of the immediate vicinity of the release point consume food produced in the same annular segment, then those average ground concentrations of the $\ell^{\text {th }}$ daughter of the released radionuclide i per unit area of the ground used to produce the food consumed by the people in the $\mathrm{k}^{\text {th }}$ annulus of the $\mathrm{j}^{\text {th }}$ sector of the immediate vicinity of the release point are given by

$$
\overline{\mathrm{C}}_{\mathrm{ail}}=\mathrm{C}_{\mathrm{ailjk}}
$$




$$
\overline{\mathrm{C}}_{\mathrm{bil}}=\mathrm{C}_{\mathrm{biljk}} \text {. }
$$

However, if the people in a given position consume food which is produced throughout the entire region surrounding the release point, then these average ground concentrations become

$$
\bar{C}_{\mathrm{ail}}=\frac{\sum_{\mathrm{k}=1}^{\mathrm{KMAX}} \sum_{\mathrm{j}=1}^{\text {JMAX }} \theta_{\mathrm{i}}\left[\left(\frac{\mathrm{r}_{\mathrm{k}+1}+\mathrm{r}_{\mathrm{k}}}{2}\right)^{2}-\left(\frac{\mathrm{r}_{\mathrm{k}}+\mathrm{r}_{\mathrm{k}-1}}{2}\right)^{2}\right] \mathrm{C}_{\mathrm{ai} / \mathrm{ik}}}{\sum_{\mathrm{k}=1}^{\text {KMAX JMAX }} \sum_{j=1}^{\text {JMAX }} \theta_{\mathrm{j}}\left[\left(\frac{\mathrm{r}_{\mathrm{k}+1}+\mathrm{r}_{\mathrm{k}}}{2}\right)^{2}-\left(\frac{\mathrm{r}_{\mathrm{k}}+\mathrm{r}_{\mathrm{k}-1}}{2}\right)^{2}\right]}
$$

and

$$
\bar{C}_{b i l}=\frac{\sum_{k=1}^{\text {KMAX JMAX }} \sum_{j=1}^{\text {KMAX JMAX }} \theta_{j}\left[\left(\frac{r_{k+1}+r_{k}}{2}\right)^{2}-\left(\frac{r_{k}+r_{k}-1}{2}\right)^{2}\right] C_{b i l j k}}{\sum_{k=1}^{\text {JMA }} \sum_{j=1} \theta_{i}\left[\left(\frac{r_{k+1}+r_{k}}{2}\right)^{2}-\left(\frac{r_{k}+r_{k}-1}{2}\right)^{2}\right]}
$$

Using the preceding definitions for $\overline{\mathrm{C}}_{\mathrm{a} i \ell}$ and $\overline{\mathrm{C}}_{\mathrm{bil}}$, substituting the expressions given in Equations (3), (6), (10), and (15) for $C_{a i \ell j k^{\prime}} C_{b i l j k^{\prime}} C_{c i l,}$ and $C_{d i l}$, respectively, and performing the indicated integrations yields the following expression for the dose commitment resulting from the ingestion nf fond produced in ground previously contaminated by deposition if the people in a given segment eat food produced in that segment

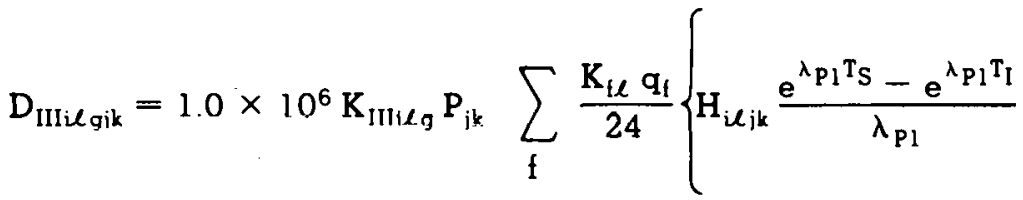

$$
\begin{aligned}
& +\sum_{q=0}^{q=\ell}\left[-I_{i \ell q i k} \frac{e^{-\left(\alpha_{i q}-\lambda_{P 1}\right) T_{1}}-e^{-\left(\alpha_{1 q}-\lambda_{P 1}\right) T_{S}}}{\alpha_{i q}-\lambda_{P 1}}+I_{1 \ell q} \frac{e^{\lambda_{P 1} T_{S}}-e^{\lambda P_{1} T_{1}}}{\lambda_{P 1}} .\right.
\end{aligned}
$$

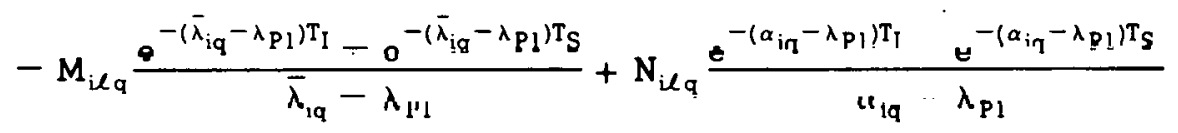

$$
\begin{aligned}
& +J_{i \ell q i k} e^{\alpha_{i q} T_{S}} \frac{e^{-\left(\alpha_{i q}-\lambda_{P 1}\right) T_{S}}-e^{-\left(\alpha_{i q}-\lambda_{p 1}\right) T_{L}}}{\alpha_{i q}-\lambda_{P 1}}+R_{i \ell q} e^{\alpha_{i q} T_{S}} \frac{e^{-\left(\alpha_{i q}-\lambda_{p 1}\right) T_{S}}-e^{-\left(u_{i q}-\lambda_{p 1}\right) T_{L}}}{\alpha_{i q}-\lambda_{P 1}} \\
& \left.\left.+S_{i \ell q} e^{\bar{\lambda}_{i q} T_{S}} \frac{e^{-\left(\bar{\lambda}_{i q}-\lambda_{P 1}\right) T_{S}}-e^{-\left(\dot{\lambda}_{i q}-\lambda_{P 1}\right) T_{L}}}{\bar{\lambda}_{i q}-\lambda_{P 1}}\right]\right\}
\end{aligned}
$$

where $J_{i \ell q i k^{\prime}} R_{i \ell q^{\prime}}$ and $S_{i \ell q}$ are given by Equations (7), (16), and (17), respectively, and $H_{i \ell j k^{\prime}} I_{i l q j k^{\prime}} L_{i \ell q^{\prime}}$ $\mathrm{M}_{\mathrm{i} \ell q^{\prime}}$ and $\mathrm{N}_{\mathrm{i} \ell q}$ are defined by the following expressions. 


$$
\begin{aligned}
& \mathrm{H}_{\mathrm{i} \ell \mathrm{jk}}=\frac{\sum_{\mathrm{q}=0}^{\mathrm{q}=\ell} \alpha_{\mathrm{i} 0} \alpha_{\mathrm{i} 1} \ldots \alpha_{\mathrm{iq}-1} \mathrm{Y}_{\mathrm{iqjk}} \lambda_{\mathrm{iq}+1} \lambda_{\mathrm{iq}+2 \ldots} \lambda_{\mathrm{il}}}{\alpha_{\mathrm{i} 0} \alpha_{\mathrm{i} 1} \ldots \alpha_{\mathrm{i} \ell}} . \\
& I_{i \ell q i k}=(-1)^{q} \sum_{h=0}^{h=q} \\
& \frac{(-1)^{\mathrm{h}} \mathrm{Y}_{\mathrm{ihik}} \lambda_{\mathrm{ih}+1} \lambda_{\mathrm{ih}+2 \ldots \lambda_{\mathrm{i} \ell}}}{\left(\alpha_{\mathrm{iq}}-\alpha_{\mathrm{ih}}\right)\left(\alpha_{\mathrm{iq}}-\alpha_{\mathrm{ih}+1}\right) \ldots\left(\alpha_{\mathrm{iq}}-\alpha_{\mathrm{iq}-1}\right) \alpha_{\mathrm{iq}}\left(\alpha_{\mathrm{iq}+1}-\alpha_{\mathrm{iq}}\right)\left(\alpha_{\mathrm{iq}+2}-\alpha_{\mathrm{iq}}\right) \ldots\left(\alpha_{\mathrm{i} \ell}-\alpha_{\mathrm{iq}}\right)} \\
& \mathrm{L}_{\mathrm{ilq}}=\frac{\gamma_{\mathrm{iq}} A_{\mathrm{iq}} \lambda_{\mathrm{iq}+1} \lambda_{\mathrm{iq}+2 \cdots \lambda_{i \ell}}}{\alpha_{\mathrm{iq}} \alpha_{\mathrm{iq}+1} \ldots \alpha_{\mathrm{i} \ell}} \\
& M_{i \ell q}=\sum_{h=q}^{h=\ell} \frac{\gamma_{i h} B_{i h} E_{i h q} \lambda_{i h+1} \lambda_{i h+2} \ldots \lambda_{i \ell}}{\left(\alpha_{i h}-\bar{\lambda}_{i q}\right)\left(\alpha_{i h+1}-\bar{\lambda}_{i q}\right) \ldots\left(\alpha_{i \ell}-\bar{\lambda}_{i q}\right)} \\
& \mathbf{N}_{\mathrm{ilq}}=\frac{(-1)^{q+1} \gamma_{\mathrm{ih}} \lambda_{\mathrm{ih}+1} \lambda_{\mathrm{ih}+2 \ldots \lambda_{\mathrm{iq}}}}{\left(\alpha_{\mathrm{iq}+1}-\alpha_{\mathrm{iq}}\right)\left(\alpha_{\mathrm{iq}+2}-\alpha_{\mathrm{iq}}\right) \ldots\left(\alpha_{\mathrm{il}}-\alpha_{\mathrm{iq}}\right)} \\
& \sum_{h=0}^{h=q} \frac{(-1)^{h} \gamma_{i h} \lambda_{i h+1} \lambda_{i h+2} \ldots \lambda_{i q}}{\left(\alpha_{i q}-\alpha_{i h}\right)\left(\alpha_{i q}-\alpha_{i h+1}\right) \ldots\left(\alpha_{i q}-\alpha_{i q}\right)}\left[\frac{\AA_{i h}}{\alpha_{i q}}-B_{i h} \sum_{p=0}^{p=h} \frac{E_{i h p}}{\alpha_{i q}-\bar{\lambda}_{i p}}\right]
\end{aligned}
$$

where $A_{i q^{\prime}} B_{i h^{\prime}}$ and $E_{i h q}$ are given by Equations (12), (13), and (14), respectively.

If the people in a given annular segment of the region in the immediate vicinity of the release point consume food produced throughout the entire region, the dose commitment to the $\mathrm{g}^{\text {th }}$ organ of the people in a specific annular segment of the region immediately surrounding the release point due to the consumption of food produced in soil contaminated by earlier deposition of the released radionuclide and its daughters is given by

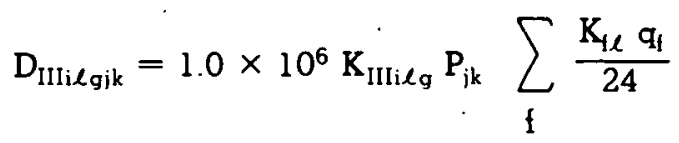

$$
\begin{aligned}
& \left\{\left[\left(\sum _ { k = 1 } ^ { K M A X } \sum _ { j = 1 } ^ { \text { JMAX } } \theta _ { j } \{ ( \frac { r _ { k + 1 } + r _ { k } } { 2 } ) ^ { 2 } - ( \frac { r _ { k } + r _ { k } - 1 } { 2 } ) ^ { 2 } \} \left\{H_{i \ell i k} \frac{e^{\lambda P_{1} T_{S}}-e^{\lambda_{P 1} T_{1}}}{\lambda_{p_{1}}}\right.\right.\right.\right.
\end{aligned}
$$

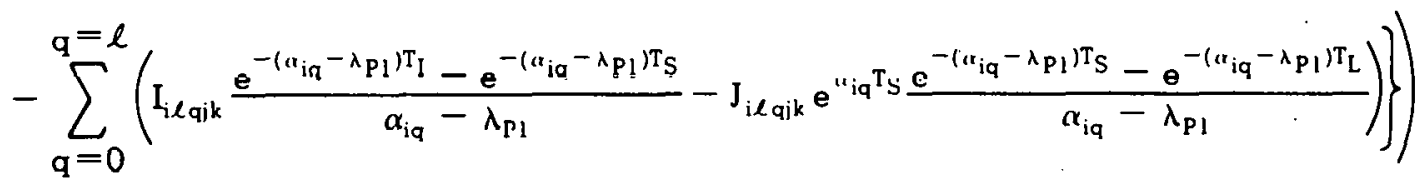




$$
\begin{aligned}
& \left.\int \sum_{k=1}^{\text {KMAX JMAX }} \sum_{j=1} \theta_{i}\left\{\left(\frac{r_{k+1}+r_{k}}{2}\right)^{2}-\left(\frac{r_{k}+r_{k}-1}{2}\right)^{2}\right\}\right] \\
& +\sum_{q=0}^{q=\ell}\left[L_{i \ell q} \frac{e^{\lambda_{P 1} T_{S}}-e^{\lambda_{P 1} T_{I}}}{\lambda_{P 1}}-M_{i \ell q} \frac{e^{-\left(\bar{\lambda}_{i q}-\lambda_{P 1}\right) T_{I}}-e^{-\left(\bar{\lambda}_{i q}-\lambda_{P 1}\right) T_{S}}}{\bar{\lambda}_{i q}-\lambda_{P 1}}\right. \\
& +N_{i \ell q} \frac{e^{-\left(\alpha_{i q}-\lambda_{p 1}\right) T_{I}}-e^{-\left(\alpha_{i q}-\lambda_{p 1}\right) T_{S}}}{\alpha_{i q 1}-\lambda_{P 1}}+R_{i \ell q} e^{\left(i_{i q} T_{S}\right.} \frac{e^{-\left(\mu_{i q}-\lambda_{p 1}\right) T_{S}}-e^{-\left(\alpha_{i q}-\lambda_{p 1}\right) T_{L}}}{\alpha_{i q}-\lambda_{P 1}} \\
& \left.\left.+\dot{S}_{i / q} e^{\bar{\lambda}_{i q} T_{S} e^{\left(\bar{\lambda}_{i q}-\lambda_{P 1}\right) T_{S}}-e^{-\left(\bar{\lambda}_{i q}-\Lambda_{P 1} T_{T}\right.}}\right]\right\}
\end{aligned}
$$

If the overall region affected by the release of the radionuclide $i$ is not large enough to include one passage around the earth, the soil in the vicinity of the release point on which food is produced can only be contaminated by the deposition of the released radionuclide and its daughters during their initial passage through the immediate neighborhood of the release point. Thus, the dose commitment due to ingestion of food produced in soil contaminated by prior deposition reduces to the following expression if the food is consumed by people residing within a few hundred meters of the area in which it is produced.

$$
\begin{aligned}
& D_{\text {IIIi } \ell \mathrm{gjk}}=1.0 \times 10^{6} \mathrm{~K}_{\mathrm{IIIi \ell g}} \mathrm{P}_{\mathrm{jk}} \sum_{\mathrm{f}} \frac{\mathrm{K}_{\mathrm{ll}} \mathrm{q}_{\mathrm{f}}}{24} \\
& \left\{H_{i \ell i k} \frac{e^{\lambda_{P 1} T_{S}}-e^{\lambda_{P 1} T_{I}}}{\lambda_{\bar{p} 1}}-\sum_{q=0}^{q=\ell} I_{i \ell \mathrm{q} l !} \frac{e^{-\left(\alpha_{i q}-\lambda_{P 1}\right) T_{I}}-e^{-\left(\alpha_{i q}-\lambda_{P 1}\right) T_{S}}}{\alpha_{i q}-\lambda_{P 1}}\right.
\end{aligned}
$$

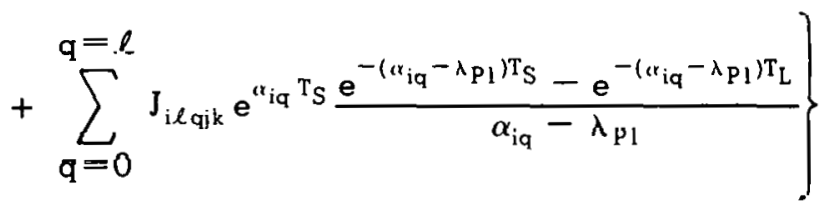

If the people in a qiven section of the region immediately surrnunding the noint of reloase consume food which is produced throughout the entire region in the vicinity of the release point, the dose commitment due to the ingestion of food produced in soil contaminated by prior deposition is qiven by

$D_{I I I i \ell g j k}=1.0 \times 10^{6} K_{\text {III } \ell g} P_{i k} \sum_{f} \frac{K_{l \ell} q_{f}}{24}$

$$
\left[\sum _ { k = 1 } ^ { \text { KMAX JMAX } } \sum _ { j = 1 } ^ { A = 1 } \theta _ { j } \{ ( \frac { r _ { k + 1 } + r _ { k } } { 2 } ) ^ { 2 } - ( \frac { r _ { k } + r _ { k } - 1 } { 2 } ) ^ { 2 } \} \left\{H_{i \ell j k} \frac{e^{\lambda_{P 1} T_{S}}-e^{\lambda_{P} 1^{1} I}}{\lambda_{P l}}\right.\right.
$$




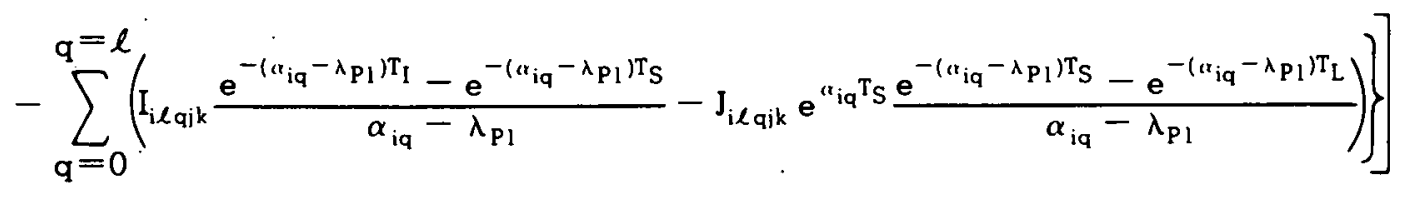

(2) Beyond Vicinity of Releases-The dose commitment to the $\mathrm{g}^{\text {th }}$ organ of the people in the overall region affected by the releases beyond the immediate neighborhood of the release point due to the ingestion of food which is produced in ground contaminated by the previous deposition of the released radionuclide $i$ and its daughters is given by

$$
\begin{aligned}
& D_{\text {III } \ell g}=1.0 \times 10^{6} \mathrm{~K}_{\text {III } \ell g} \mathrm{P}_{\mathrm{T}} \sum_{f} \frac{\mathrm{K}_{\ell \ell} \mathrm{q}_{\mathrm{i}}}{24} \\
& {\left[\int_{T_{I}}^{T_{S}} e^{\lambda P 2 t} C_{c i l} d t+\int_{T_{S}}^{T_{L}} e^{\lambda_{P 2} t} C_{d i l} d t\right]}
\end{aligned}
$$

Substituting the expressions for $\mathrm{C}_{\mathrm{cil}}$ and $\mathrm{C}_{\mathrm{dil}}$ given in Equations (10) and (15), respectively, into the preceding equation and performing the indicated integration yields the following expression for the ingestion dose commitment to the people in the overall region affected by the releases as a result of the consumption of food produced in soil contaminated by the earlier deposition of the released radionuclides and their daughters.

$$
\begin{aligned}
& D_{\text {IIIi g }}=1.0 \times 10^{6} \mathrm{~K}_{\text {IIIi g }} \mathrm{P}_{\mathrm{T}} \sum_{\mathrm{f}} \frac{\mathrm{K}_{\mathrm{i \ell}} \mathrm{q}_{\mathrm{f}}}{24} \\
& \sum_{q=0}^{q=\ell}\left[L_{i \ell q} \frac{e^{\lambda_{P 2} T_{S}}-e^{\lambda_{P 2} T_{I}}}{\lambda_{P 2}}-M_{i \ell q} \frac{e^{-\left(\bar{\lambda}_{i q}-\lambda_{P 2}\right) T_{1}}-e^{-\left(\bar{\lambda}_{i q}-\lambda_{P 2}\right) T_{S}}}{\bar{\lambda}_{i q}-\lambda_{P 2}}\right. \\
& +N_{i \ell q} \frac{e^{-\left(\alpha_{i q}-\lambda_{P 2}\right) T_{1}}-e^{-\left(\alpha_{i q}-\lambda_{p 2}\right) T_{S}}}{\alpha_{i q}-\lambda_{P 2}}+R_{i \ell q} e^{\alpha_{i q} T_{S}} \frac{e^{-\left(\alpha_{i q}-\lambda_{p 2}\right) T_{S}}-e^{-\left(\alpha_{i q}-\lambda_{P 2}\right) T_{L}}}{\alpha_{i q}-\lambda_{P 2}} \\
& \left.+S_{i \ell q} e^{\bar{\lambda}_{i q} T_{S}} \frac{e^{-\left(\bar{\lambda}_{i q}-\lambda_{P 2}\right) T_{S}}-e^{-\left(\bar{\lambda}_{i q}-\lambda_{P 2}\right) T_{L}}}{\bar{\lambda}_{i q}-\lambda_{P 2}}\right]
\end{aligned}
$$

\section{Inhalation Dose Commitments}

The AIRWAY program subdivides the inhalation dose commitments into two distinct pathways. The first of these pathways considers the inhalation of radionuclides which have remained in the atmosphere since their release or formation. This includes gaseous radionuclides (both released nuclides and daughter products) and all particulates which have not yet deposited. The dose commitments associated with this pathway are dependent upon the atmospheric concentration of the radionuclide of interest exclusive of the contributions to this concentration due to resuspension of previously deposited radionuclides. The second of these inhalation dose commitment pathways considers the inhalation of radionuclides which have been deposited and then resuspended. Thus, the dose commitments associated with the second inhalation pathway are dependent upon the ground concentrations of the released radionuclides and their daughters. 


\section{a. Radionuclides Not Previously Deposited}

(1) Vicinity of Releases-The dose commitment arising from the inhalation of radionuclides which have not been previously deposited is given by the time integral of the product of the inhalation dose commitment conversion factor $\mathrm{K}_{\mathrm{IV} \text { ilg }}$, the rate at which air is inhaled $\mathrm{B}_{\mathrm{a}^{\prime}}$ the number of people at the position of interest and the portion of the total ground level air concentration in the vicinity of the release point which can be attributed to radionuclides which have not been deposited previously. Thus, the dose commitment $D_{\text {IV } i l g}$ to the $g^{\text {th }}$ organ of the people in the $\mathrm{k}^{\text {th }}$ annulus of the $j^{\text {th }}$ sector of the region immediately surrounding the release point due to the inhalation of the $l^{\text {th }}$ daughter of the released radionuclide $i$ prior to its deposition is given by

$$
\begin{aligned}
& D_{I V i \ell g i k}=\int_{T_{I}}^{T_{3}} 1.0 \times 10^{f} K_{i V_{i} / y} B_{d} P_{j k} e^{\lambda P I^{\prime}}\left(x_{a i} / j k+x_{b i r}\right) d t \\
& +\int_{T_{S}}^{T_{L}} 1.0 \times 10^{6} K_{I V i l g} B_{a} P_{j k} e^{\lambda P 1^{t}} \chi_{c i l} d t
\end{aligned}
$$

Noting that $\chi_{\mathrm{ai} / \mathrm{jk}}$ is independent of time, using the expressions given in Equations (8) and (9) for $\chi_{\text {bil }}$ and $\chi_{\text {cil }}$, rearranging and then performing the indicated integrations as was done in References 2 and 3 yields the following equation for the inhalation dose commitment to the people in the immediate neighborhood of the release point due to breathing radionuclides which have not been deposited previously.

$$
\begin{aligned}
& D_{I V i l g j k}=1.0 \times 10^{6} K_{I V i l g} B_{a} P_{j k}\left[\left(X_{a i l j k}+A_{i \ell}\right) \frac{e^{\lambda_{P 1} T_{S}}-e^{\lambda_{P 1} T_{I}}}{\lambda_{P 1}}\right. \\
& -B_{i \ell} \sum_{q=0}^{q=\ell} E_{i \ell q} \frac{e^{-\left(\lambda_{i q}-\lambda_{P 1}\right) T_{1}}-e^{-\left(\bar{\lambda}_{i q}-\lambda_{P 1}\right) T_{S}}}{\bar{\lambda}_{i q}-\lambda_{P 1}}
\end{aligned}
$$

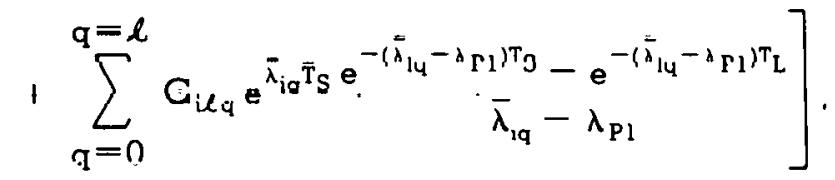

where $A_{i \ell}, B_{i \ell}, E_{i \ell q}$ and $G_{i \ell q}$ are defined by Equations (12), (13), (14), and (36), respectively.

If the release of the radionuclide i does not affect a region which encompasses at least one traverse around the earth, then the air concentration of the radionuclide of interest in the vicinity of the release point is composed solely of the air concentration resulting from the initial passage of the released radionuclide and its daughters through the region immediately surrounding the release point. Thus, there are no contributions from the $\chi_{\mathrm{bi} l}$ and $\chi_{\mathrm{ci}}$ terms in Equation (60) and the inhalation dose commitment equation reduces to

$$
D_{I V i l g j k}=1.0 \times 10^{6} K_{I V i l g} B_{a} P_{j k} \chi_{a i l j k}\left(\frac{e^{\lambda_{P 1} T_{S}}-e^{\lambda_{P 1} T_{I}}}{\lambda_{P 1}}\right)
$$


(2) Beyond the Vicinity of Release-The dose commitments to the $\mathrm{g}^{\text {th }}$ organ of the people in the overall region affected by the releases beyond the immediate vicinity of the release point due to the inhalation of the $l^{\text {th }}$ daughter of the released radionuclide $i$ before deposition of the released nuclide or its daughters can occur is given by

$$
\begin{aligned}
\mathrm{D}_{\mathrm{IV} i \ell g}= & \int_{\mathrm{T}_{\mathrm{I}}}^{\mathrm{T} \mathrm{S}} 1.0 \times 10^{6} \mathrm{~K}_{\mathrm{IV} i \ell g} \mathrm{~B}_{\mathrm{a}} \mathrm{P}_{\mathrm{T}} \mathrm{e}^{\lambda_{\mathrm{P} 2 t}} \chi_{\mathrm{bi} \ell} \mathrm{dt} \\
& +\int_{\mathrm{T}_{\mathrm{S}}}^{\mathrm{T}_{\mathrm{L}}} 1.0 \times 10^{6} \mathrm{~K}_{\mathrm{IVi \ell g}} \mathrm{B}_{\mathrm{a}} \mathrm{P}_{\mathrm{T}} \mathrm{e}^{\lambda \mathrm{P})^{\prime}} \chi_{\mathrm{c} i \ell} \mathrm{dt}
\end{aligned}
$$

Substituting the expressions for $\chi_{\mathrm{bi} \ell}$ and $\chi_{\mathrm{ci}}$ given in Equations (8) and (9), respectively, rearranging and then performing the indicated integrations as done in Reference 3 yields the following expression for the inhalation dose commitment to the people in the overall region affected by the releases resulting from breathing air containing the released radionuclide and its daughters before they can deposit on the ground.

$$
\begin{aligned}
\mathrm{D}_{\mathrm{IV} i \ell \mathrm{g}}= & 1.0 \times 10^{6} \mathrm{~K}_{\mathrm{IVi} \ell \mathrm{g}} \mathrm{B}_{\mathrm{a}} \mathrm{P}_{\mathrm{T}}\left[\mathrm{A}_{\mathrm{i} \ell} \frac{\mathrm{e}^{\lambda_{\mathrm{P} 2} \mathrm{~T}_{\mathrm{S}}}-\mathrm{e}^{\lambda_{\mathrm{P} 2} \mathrm{~T}_{\mathrm{I}}}}{\lambda_{\mathrm{P} 2}}\right. \\
& -\mathrm{B}_{\mathrm{i} \ell} \sum_{\mathrm{q}=0}^{\mathrm{q}=\ell} \mathrm{E}_{\mathrm{i} \ell \mathrm{q}} \frac{\mathrm{e}^{-\left(\bar{\lambda}_{\mathrm{iq}}-\lambda_{\mathrm{P} 2}\right) \mathrm{T}_{\mathrm{I}}}-\mathrm{e}^{-\left(\bar{\lambda}_{\mathrm{iq}}-\lambda_{\mathrm{P} 2}\right) \mathrm{T} \mathrm{S}}}{\bar{\lambda}_{\mathrm{iq}}-\lambda_{\mathrm{P} 2}} \\
& \left.+\sum_{\mathrm{q}=0}^{\mathrm{q}=\ell} \mathrm{G}_{\mathrm{i} \ell \mathrm{q}} \mathrm{e}^{\bar{\lambda}_{\mathrm{iq}} \mathrm{T}_{\mathrm{S}}} \frac{\mathrm{e}^{-\left(\bar{\lambda}_{\mathrm{iq}}-\lambda_{\mathrm{P} 2}\right) \mathrm{T} \mathrm{S}}-\mathrm{e}^{-\left(\bar{\lambda}_{\mathrm{iq}}-\lambda_{\mathrm{P} 2}\right) \mathrm{T}_{\mathrm{L}}}}{\bar{\lambda}_{\mathrm{iq}}-\lambda_{\mathrm{P} 2}}\right],
\end{aligned}
$$

where $A_{i \ell}, B_{i \ell}, E_{i \ell q}$ and $G_{i \ell q}$ are defined by Equations (12), (13), (14), and (36), respectively.

\section{(b) Resuspended Radionuclides.}

(1) Vicinity of Releases-The dose commitment resulting from the inhalation of resuspended radionnclides which had deposited earlier is given by the time integral of the product of the inhalation dose commitment conversion factor, the rate at which air is inhaled, the number of people at the position of interest and the portion of the total ground level air concentration in the vicinity of the release point which is comprised of resuspended radionuclides which had been deposited previously. The portion of the total ground level air concentration which is due to the resuspension of previously deposited radionuclides is given by the product of the resuspension factor $\beta$ and the ground concentration of the radionuclides at the position and time of interest. Thus, the dose commitment to the $\mathrm{g}^{\text {th }}$ organ of the people in the $\mathrm{k}^{\text {th }}$ annulus of the $j^{\text {th }}$ sector of the region in the immediate vicinity of the release point arising from the inhalation of resuspended particulates which contain the $\ell^{\text {th }}$ daughter of the released radionuclide $i$ is given by

$$
D_{I V i \ell g i k}=\int_{T_{I}}^{T_{S}} 1.0 \times 10^{6} K_{1 V i \ell g} B_{a} P_{j k} e^{\lambda} P^{t} \beta\left(C_{a l \ell j k}+C_{c i l}\right) d t
$$




$$
+\int_{T_{S}}^{T_{L}} 1.0 \times 10^{6} \mathrm{~K}_{I V_{i} \ell g} B_{a} P_{j k} e^{\lambda P^{t}} \beta\left(C_{b i l j k}+C_{d i l}\right) d t
$$

The resuspension factor $\beta$ in the preceding equation relates the amount of material which is resuspended to the amount of material which is on the ground and is in general a function of the time subsequent to deposition. However, the AIRWAY program uses a function of the time subsequent to the start of releases to approximate the resuspension factor and further it considers that this fartor is independent of the particular nuclide which is subject to resuspension. This factor is represented by the sum of a constant term and an exponentially decaying term in the manner defined in Reference 5 and given by the following expression.

$$
\beta=K_{0} e^{-\lambda} d^{t}+K_{1}
$$

Substituting the preceding expression for $\beta$ into Equation (65) and rearranging yields

$$
\begin{aligned}
& D_{I V i \ell g j k}=1.0 \times 10^{6} \mathrm{~K}_{\mathrm{IVi} \ell g} B_{a} P_{j k} \\
& {\left[K _ { 0 } \left\{\int_{T_{I}}^{T_{S}} e^{-\left(\lambda_{d}-\lambda_{P 1}\right) t}\left(C_{a i l j k}+C_{c i \ell}\right) d t\right.\right.} \\
& \left.+\int_{T_{S}}^{\mathrm{I}_{\mathrm{L}}^{\mathrm{L}}} \mathrm{e}^{-\left(\lambda_{\mathrm{d}}-\lambda_{\mathrm{PI}}\right) t}\left(\mathrm{C}_{\mathrm{bi} \ell \mathrm{jk}}+\mathrm{C}_{\mathrm{di} \ell}\right) \mathrm{dt}\right\} \\
& +K_{1}\left\{\int_{T_{I}}^{T_{S}} e^{\lambda P 1^{1}}\left(C_{a i l j k}+C_{c i l}\right) d t\right. \\
& \left.\left.+\int_{T_{S}}^{T_{L}} e^{\lambda{ }^{2} 1}\left(C_{b i l i k}+C_{d i \ell}\right) d t\right\}\right] \text {. }
\end{aligned}
$$

Using the expressions for $C_{a i \ell j k^{\prime}} C_{b i \ell j k}, C_{c i \ell}$ and $C_{d i \ell}$ given by Equations (3), (6), (10), and (15), respectively, and integrating in the manner performed in References 2 and 3 yields the following expression for the inhalation dose commitment in the virinity of the release point duo to the inhalation of resuspended radionuclides.

$$
\begin{aligned}
D_{I V i \ell g j k}= & 1.0 \times 10^{6} K_{I V i \ell g} B_{a} P_{j k}\left[K _ { 0 } \left\{H_{i \ell j k} \frac{e^{-\left(\lambda_{d}-\lambda_{P 1}\right) T_{I}}-e^{-\left(\lambda_{d}-\lambda_{p 1}\right) T_{S}}}{\lambda_{d}-\lambda_{P 1}}\right.\right. \\
& +\sum_{q=0}^{q=\ell}\left[\left(N_{i \ell q}+I_{i \ell q j k}\right) \frac{e^{-\left(\alpha_{i q}+\lambda_{d}-\lambda_{p 1}\right) T_{I}}-e^{-\left(\alpha_{i q}+\lambda_{d}-\lambda_{P 1}\right) T_{S}}}{\alpha_{i q}+\lambda_{d}-\lambda_{P 1}}\right.
\end{aligned}
$$




$$
\begin{aligned}
& +L_{i \ell q} \frac{e^{-\left(\lambda_{d}-\lambda_{P 1}\right) T_{I}}-e^{-\left(\lambda_{d}-\lambda_{P 1}\right) T_{S}}}{\lambda_{d}-\lambda_{P 1}}-M_{i \ell q} \frac{e^{-\left(\bar{\lambda}_{i q}+\lambda_{d}-\lambda_{P 1}\right) T_{I}}-e^{-\left(\bar{\lambda}_{i q}+\lambda_{d}-\lambda_{P 1}\right) T_{S}}}{\lambda_{i q}+\lambda_{d}-\lambda_{P 1}} \\
& +\left(J_{i \ell q j k}+R_{i \ell q}\right) e^{u_{i q} T_{S}} \frac{e^{-\left(u_{i q}+\lambda_{d}-\lambda_{P 1}\right) T_{S}}-e^{-\left(u_{i q}+\lambda_{d}-\lambda_{P 1}\right) T_{L}}}{\alpha_{i q}+\lambda_{d}-\lambda_{P J}} \\
& \left.\left.+S_{i \ell} e^{\bar{\lambda}_{i q} T_{S}} \frac{e^{-\left(\bar{\lambda}_{i q}+\lambda_{d}-\lambda_{P 1}\right) T_{S}}-e^{-\left(\bar{\lambda}_{i q}+\lambda_{d}-\lambda_{P 1}\right) T_{L}}}{\bar{\lambda}_{i q}+\lambda_{d}-\lambda_{P 1}}\right]\right\} \\
& +K_{1}\left\{H_{i \ell j k} \frac{e^{\lambda_{P 1} T_{S}}-e^{\lambda_{P 1} T_{I}}}{\lambda_{P 1}}+\sum_{q=0}^{q=\ell}\left[L_{i \ell q} \frac{e^{\lambda_{P 1} T_{S}}-e^{\lambda_{P 1} T_{1}}}{\lambda_{P 1}}\right.\right. \\
& +\left(N_{i \ell q}-I_{i \ell q i k}\right) \frac{e^{-\left(\mu_{i q}-\lambda_{P 1}\right) T_{1}}-e^{-\left(\mu_{i q}-\lambda_{P 1}\right) T_{S}}}{\alpha_{i q}-\lambda_{p 1}} \\
& -M_{i \ell, q} \frac{e^{-\left(\bar{\lambda}_{i q}-\lambda_{P 1}\right) T_{1}}-e^{-\left(\bar{\lambda}_{i q}-\lambda_{P 1}\right) T_{S}}}{\bar{\lambda}_{i q}-\lambda_{P I}} \\
& +\left(J_{i \ell q j k}+R_{i \ell q}\right) e^{\alpha_{i q} T_{S}} \frac{e^{-\left(u_{i q}-\lambda_{P 1}\right) T_{S}}-e^{-\left(\alpha_{i q}-\lambda_{P 1}\right) T_{L}}}{\alpha_{i q}-\lambda_{P l}} \\
& \left.\left.+S_{i \ell q} e^{\bar{\lambda}_{i q} T_{S}} \frac{e^{-\left(\bar{\lambda}_{i q}-\lambda_{P 1}\right) T_{S}}-e^{-\left(\bar{\lambda}_{i q}-\lambda_{P 1}\right) T_{L}}}{\bar{\lambda}_{1 q}-\lambda_{P 1}}\right]\right\} \text {. }
\end{aligned}
$$

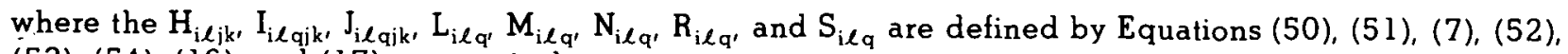
(53), (54), (16), and (17), respectively.

If the path followed by the released radionuclide and its daughters does not extend far enough to encircle the earth at least once, then only those radionuclides which result from deposition during the initial passage through the region immediately surrounding the release point contribute to the ground concentrations used to define the resuspended radionuclides. Thus, the $C_{c i l}$ and $C_{d i l}$ terms are not present in Equation (67) and the dose commitment due to the inhalation of resuspended radionuclides by the people in a specific annular segment of the region immediately surrounding the release point reduces to the following expression.

$$
\begin{aligned}
& D_{\text {IVilgjk }}=1.0 \times 10^{6} K_{I V i \ell g} B_{a} P_{j k}\left[K _ { 0 } \left\{H_{L j k} \frac{e^{-\left(\lambda_{d}-\lambda_{P 1}\right) T_{I}}-e^{-\left(\lambda_{d}-\lambda_{P 1}\right) T_{S}}}{\lambda_{d}-\lambda_{P 1}}\right.\right. \\
& -\sum_{q=0}^{q=\ell} I_{i \ell q i k} \frac{e^{-\left(\alpha_{i q}+\lambda_{d}-\lambda_{p 1}\right) T_{I}}-e^{-\left(\alpha_{i q}+\lambda_{d}-\lambda_{p i}\right) T_{S}}}{\alpha_{i q}+\lambda_{d}-\lambda_{p l}} \\
& \left.+\sum_{q=0}^{q=\ell} J_{i \ell q i k} e^{\alpha_{i q} T_{S}} \frac{e^{-\left(\alpha_{i q}+\lambda_{d}-\lambda_{p 1}\right) T_{S}}-e^{-\left(\mu_{i q}+\lambda_{d} \cdots \lambda_{p 1}\right) T_{L}}}{\alpha_{i q}+\lambda_{d}-\lambda_{P 1}}\right\}
\end{aligned}
$$




$$
\begin{aligned}
& +K_{1}\left\{H_{i \ell j k} \frac{e^{\lambda_{P 1} T_{S}}-e^{\lambda_{P 1} T_{I}}}{\lambda_{P 1}}-\sum_{q=0}^{q} I_{i \ell g i k} \frac{e^{-\left(\alpha_{i q}-\lambda_{P 1}\right) T_{I}}-e^{-\left(\alpha_{i q}-\lambda_{P 1}\right) T_{S}}}{\alpha_{i q}-\lambda_{P 1}}\right. \\
& \left.\left.+\sum_{q=0}^{q} J_{i \ell q j k} e^{\alpha_{i q} T_{S}} \frac{e^{-\left(\alpha_{i q}-\lambda_{P 1}\right) T_{S}}-e^{-\left(\alpha_{i q}-\lambda_{P 1}\right) T_{L}}}{\alpha_{i q}-\lambda_{P 1}}\right\}\right],
\end{aligned}
$$

where $H_{i \ell j k^{\prime}} I_{i \ell q j k^{\prime}}$ and $J_{i \ell q j k}$ are defined by Equations (50), (51), and (7), respectively.

(2) Beyond the Vicinity of Releases-The dose commitment to the $g_{t h}$ organ of the people in the overall region affected by the releases beyond the immediate vicinity of the release point from the inhalation of resuspended particulates containing the $\ell^{\text {th }}$ daughter of the released radionuclide $i$ is given by

$$
\begin{aligned}
& D_{I V i \ell g}=1.0 \times 10^{6} K_{I V i \ell g} B_{a} P_{T}\left[K _ { 0 } \left\{\int_{T_{1}}^{T_{S}} C_{c i l} e^{-\left(\lambda_{d}-\lambda_{P 2}\right) t} d t\right.\right. \\
& \left.+\int_{T_{S}}^{T_{L}} C_{d i l} e^{-\left(\lambda_{d}-\lambda_{P 2}\right) t} d t\right\} \\
& \left.+K_{1}\left\{\int_{T_{I}}^{T_{S}} C_{c i l .} e^{\lambda P 2^{t}} d t+\int_{T_{S}}^{T_{L}} C_{d i l} e^{\lambda P 2^{t}} d t\right\}\right]
\end{aligned}
$$

Substituting the expressions for $\mathrm{C}_{\mathrm{cil}}$ and $\mathrm{C}_{\mathrm{di} l}$ given in Equations (10) and (15), respectively, into the preceding equations and then performing the indicated integrations as was done in Reference 3 yields the following expression for the inhalation dose commitment accrued by the people in the overall region affected by the releases due to breathing resuspended particulates containing the released radinnnclide and its daughters.

$\mathrm{D}_{\mathrm{IVilg}}=1.0 \times 10^{6} \mathrm{~K}_{\mathrm{IVilg}} \mathrm{B}_{\mathrm{a}} \mathrm{P}_{\mathrm{T}}$

$$
\begin{aligned}
& {\left[\mathrm{K}_{0} \sum_{q=0}^{q_{\mathrm{q}}{ }^{m \ell}\left\{L_{i \ell q} \frac{e^{-\left(\lambda_{d}-\lambda_{P 2}\right) T_{I}}-e^{-\left(\lambda_{d}-\lambda_{P 2}\right) T_{S}}}{\lambda_{d}-\lambda_{P 2}}\right.}\right.} \\
& -M_{i \ell q} \frac{e^{-\left(\bar{\lambda}_{i q}+\lambda_{d}-\lambda_{P 2}\right) T_{I}}-e^{-\left(\bar{\lambda}_{i q}+\lambda_{d}-\lambda_{P 2}\right) T_{S}}}{\bar{\lambda}_{i q}+\lambda_{d}-\lambda_{P 2}} \\
& +N_{i \ell q} \frac{e^{-\left(\alpha_{i q}+\lambda_{d}-\lambda_{P 2}\right) T_{I}}-e^{-\left(\alpha_{i q}+\lambda_{d}-\lambda_{P 2}\right) T_{S}}}{\alpha_{i q}+\lambda_{d}-\lambda_{P 2}}
\end{aligned}
$$




$$
\begin{aligned}
& +R_{i \ell_{q}} e^{\alpha_{i q} T_{S}} \frac{e^{-\left(\alpha_{i q}+\lambda_{d}-\lambda_{P 2}\right) T_{S}}-e^{-\left(\alpha_{i q}+\lambda_{d}-\lambda_{P 2}\right) T_{L}}}{\alpha_{i q}+\lambda_{d}-\lambda_{P 2}} \\
& \left.+S_{i \ell q} e^{\bar{\lambda}_{i q} T_{S}} \frac{e^{-\left(\bar{\lambda}_{i q}+\lambda_{d}-\lambda_{P 2}\right) T_{S}}-e^{-\left(\bar{\lambda}_{i q}+\lambda_{d}-\lambda_{P 2}\right) T_{L}}}{\bar{\lambda}_{i q}+\lambda_{d}-\lambda_{P 2}}\right\} \\
& +K_{1} \sum_{q=0}^{q=\ell}\left\{L_{i \ell q} \frac{e^{\lambda_{P 2} T_{S}}-e^{\lambda_{P 2} T_{I}}}{\lambda_{P 2}}-M_{i \ell q} \frac{e^{-\left(\bar{\lambda}_{i q}-\lambda_{P 2}\right) T_{I}}-e^{-\left(\bar{\lambda}_{i q}-\lambda_{P 2}\right) T_{S}}}{\bar{\lambda}_{i q}-\lambda_{P 2}}\right. \\
& -N_{i \ell q} \frac{e^{-\left(\alpha_{i q}-\lambda_{P 2}\right) T_{1}}-e^{-\left(\alpha_{i q}-\lambda_{P 2}\right) T_{S}}}{\alpha_{i q}-\lambda_{P 2}} \\
& +R_{i \ell q} e^{\alpha_{i q} T_{S}} \frac{e^{-\left(\alpha_{i q}-\lambda_{P 2}\right) T_{S}}-e^{-\left(\alpha_{i q}-\lambda_{P 2}\right) T_{L}}}{\alpha_{i q}-\lambda_{P 2}} \\
& \left.\left.+S_{i \ell} e^{\bar{\lambda}_{i q} T_{S}} \frac{e^{-\left(\bar{\lambda}_{i q}-\lambda_{P 2}\right) T_{S}}-e^{-\left(\bar{\lambda}_{i q}-\lambda_{P 2}\right) T_{L}}}{\bar{\lambda}_{i q}-\lambda_{P 2}}\right\}\right]
\end{aligned}
$$

\section{Parameters}

In addition to the preceding set of equations which define the air and ground activity concentrations and the dose commitments, there are additional quantities which have to be defined to permit the $A$ IRWAY program to solve these equations. In general, these quantities are various coefficients, constants, etc. which are calculated from input data and/or intermediate calculational results. The equations necessary to define these additional quantities are presented in the remainder of this section.

\section{Precipitation Scavenging Constant}

The AIRWAY program uses the following expression which relates the average precipitation rate $R_{S}$ the precipitation scavenging ratio (by volume) $S_{s}$ and the height of the precipitation layer in the atmosphere $Z_{s}$ to define the precipitation scavenging constant $\lambda_{\mathrm{S}}$.

$$
\lambda_{\mathrm{S}}=\mathrm{R}_{\mathrm{S}} \mathrm{S}_{\mathrm{S}} / \mathrm{Z}_{\mathrm{S}}
$$

\section{Root Uptake Factor}

One of the parameters necessary to define the ingestion dose commitment arising from the eating uf food which is produced in soil which had been subjected to the deposition of the released radionuclides and their daughters prior to growing the food crop is the factor $\mathrm{K}_{\mathrm{fi}}$. This factor defines the amount of activity of the $\mathrm{i}^{\text {th }}$ radionuclide which is taken up per unit mass of food if there is a unit activity concentration of the $\mathrm{i}^{\text {th }}$ radionuclide in the soil per unit thickness of the root-bearing layer. The AIRWAY program uses the following relationship among the ratio of the concentration of an isotope in a plant $\mathrm{C}_{\mathrm{pi}}$ to the concentration of the same isotope in the soil producing the plant $C_{s i}$ the ratio of the activity intake rate via ingestion of the $\mathrm{i}^{\text {th }}$ radionuclide in the $\mathrm{f}^{\text {th }}$ food type $\mathrm{w}_{\mathrm{fi}}$ to the activity intake rate via ingestion of plants containing the $\mathrm{i}^{\text {th }}$ radionuclide $w_{1}$, the ratio of uormal ingestion rate of the $f^{\text {th }}$ food type $q_{0 \text { s }}$ to the average ingestion rate of 
plants $\mathrm{q}_{01}$, the density of the food producing soil $\rho_{\mathrm{g}}$ and the thickness $\mathrm{d}_{\mathrm{s}}$ of the root-bearing layer of the soil in which food is produced to define the activity uptake factor $\mathrm{K}_{\mathrm{fi}}$

$$
\mathrm{K}_{\mathrm{fi}}=\frac{1.0 \times 10^{-4}\left(\mathrm{C}_{\mathrm{pi}} / \mathrm{C}_{\mathrm{si}}\right)\left(\mathrm{w}_{\mathrm{fi}} / \mathrm{w}_{1 \mathrm{i}}\right)}{\rho_{\mathrm{s}} \mathrm{d}_{\mathrm{s}}\left(\mathrm{q}_{\mathrm{of}} / \mathrm{q}_{01}\right)}
$$

\section{Standard Deviation of Vertical Dispersion}

One of the quantities used to define the ground level air concentrations of the released radionuclides and their daughters in the region within 100 kilometers of the release point is $\sigma_{2 \mathrm{mk}}$ the standard deviation of the vertical dispersion. This parameter is a function of the distance from the release point and the meteorological stability category and defines the vertical dispersion of the released material as it moves away from the release point. In general, values of this parameter are available in graphical form in documents such as Reference 6 . However, the AIRWAY program requires that these vertical dispersion standard deviations be input in tabular form. 'T'hus, values of $\sigma_{z}$ are available in the AIRWAY program at various distances trom the release point for each of the meteorological stability categories considered in the problem. Since it is necessary to obtain $\sigma_{z}$ values at distances other than those specified via input, the AIRWAY program calculates the standard deviation of vertical dispersion at a given distance $r_{k}$ which lies between the distances $r_{v}$ and $r_{v+1}$ at which the standard deviation values $\sigma_{z v}$ and $\sigma_{z v+1}$ are provided by input in the following manner.

$$
\sigma_{z}\left(r_{k}\right)=\sigma_{z v}\left(r_{k} / r_{v}\right)^{\omega \nu}
$$

where the exponential term $\omega_{v}$ is defined as follows

$$
\omega_{\mathrm{v}}=\frac{\ln \left(\sigma_{z \mathrm{v}+1} / \sigma_{\mathrm{zv}}\right)}{\ln \left(\mathrm{r}_{\mathrm{v}+1} / \mathrm{r}_{\mathrm{v}}\right)}
$$

\section{Dry Deposition Integral Term}

The expression defining the ground level air concentration contains an integral in the term which defines the effect of dry deposition mechanisms. The integral is a function of the height of the release point above ground level $\mathrm{h}$ and the standard deviation of vertical dispersion $\sigma_{z}$. The integration is carried out over the radial distance between the point of release and the location at which the air concentration is being determined. The first step in solving this integral is to express the overall integral as the sum of a series of integrals each of which is integrated over the interval between the radial distance at which the standard deviation of vertical disposion are specified. Thus, the overall integral is defined as follows:

$$
\int_{0}^{r_{k}} \frac{e^{-\frac{1}{2}\left(\frac{h}{\sigma_{z}(r)}\right)^{2}}}{\sigma_{z}(r)} d r=\sum_{v=1}^{\text {VMAX }} \int_{r_{v}}^{r_{v y+1}} \frac{e^{-\frac{1}{2}\left(\frac{h}{\sigma_{z}(r)}\right)^{2}}}{\sigma_{z}(r)} d r+\int_{r_{v}=\operatorname{VMAX}}^{r_{k}} \frac{e^{-\frac{1}{2}\left(\frac{h}{\sigma_{z}(r)}\right)^{2}}}{\sigma_{v}(r)} d r
$$

where $r_{v=\operatorname{VMAX}} \leqslant r_{k}<r_{v}=\operatorname{VMAX}+1$

Substituting the expression for $\sigma_{z}$ given in Equation (74) into the preceding equation yields the following

$$
\int_{0}^{r_{k}} \frac{e^{-\frac{1}{2}\left(\frac{h}{r_{z}}\right)^{2}}}{\sigma_{z}} d r=\sum_{v=l}^{\text {VMAX }} \int_{r_{v}}^{r_{v}+1} \frac{e^{-\frac{h^{2}}{2 \sigma_{z v}^{2}\left(r / r_{v}\right)^{2\left(\omega_{v}\right.}}}}{\sigma_{z v}\left(r / r_{v}\right)^{\prime \prime \prime v}} d r
$$




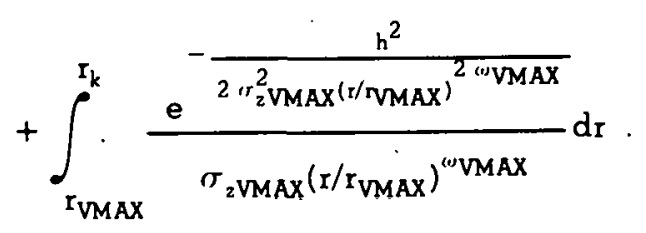

The integrals in the preceding equation are approximated in the AIRWAY program by subdividing the $v^{\text {th }}$ interval between $r_{v}$ and $r_{v+1}$ into smaller increments of equal width $\Delta r_{v}$ evaluating the value of the integrand at the centerline of each of these smaller increments, multiplying these integrand values at the centerline of the small increments by the width $\Delta \mathbf{r}_{\mathbf{v}}$ of the increments and then summing these products over all increments in the $v^{\text {th }}$ interval.

If the number of increments that the $v^{\text {th }}$ interval is subdivided into is denoted by $d_{v}$ then the width of each increment is given by

$$
\Delta \mathbf{r}_{v}=\left(r_{v+1}-r_{v}\right) / d_{v}
$$

The distance to the centerline of the $y^{\text {th }}$ increment is then given by

$$
\mathbf{r}=\mathbf{r}_{\mathrm{v}}+(\mathrm{y}-0.5) \Delta \mathbf{r}_{\mathrm{v}}
$$

Replacing the integrals in Equation (77) with the summation over $d_{v}$ increments and the term $r$ with the expression given in Equation (79) yields the following approximation to the integral used to define the effect of dry deposition mechanisms upon the ground level air concentrations of radionuclides.

$$
\begin{aligned}
& \int_{0}^{r_{k}} \frac{e^{-\frac{1}{2}\left(\frac{h}{\sigma_{z}}\right)^{2}}}{\sigma_{z}} d r=\sum_{v=1}^{\operatorname{VMAX}} \sum_{y=1}^{d_{v}} \frac{e^{2 \sigma_{z v}^{2}\left(1+\frac{(y-0.5) \Delta r_{v}}{r_{v}}\right)^{2(1) v}}}{\sigma_{z v}\left(1+\frac{(y-0.5) \Delta r_{v}}{r_{v}}\right)^{\left(\omega_{v}\right.}} \Delta r_{v}
\end{aligned}
$$

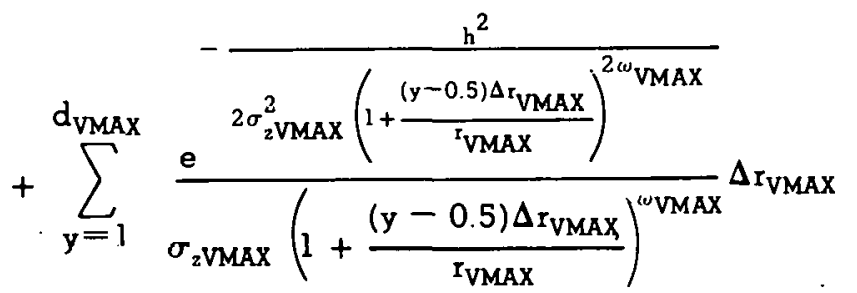

where

$$
\Delta_{\mathrm{VMAX}}=\left(\mathrm{r}_{\mathrm{k}}-\mathrm{r}_{\mathrm{VMAX}}\right) / \mathrm{d}_{\mathrm{VMAX}}
$$

\section{Modified Release Rates}

The calculation of the air and ground concentrations in the overall region affected by the releases beyond the immediate neighborhood of the release point is dependent upon the amount of the released radionuclide and its daughters which actually leaves the vicinity of the release point and enters the region beyond. An estimate of the number of the $i^{\text {th }}$ released radionuclides which are available to enter the region beyond the immediate vicinity of the release point is obtained by subtracting the total number of the $i^{\text {th }}$ released radionuclides and their daughters which deposit on the ground in the region surrounding the release point due to dry deposition mechanisms and precipitation scavenging effects from the total number 
of the $i^{\text {th }}$ radionuclides released into the atmosphere. Thus, an estimate of the activity of the radionuclide $\mathrm{i}$ released into the overall region affected by the releases beyond the immediate neighborhood of the release point is given by

$$
Q_{i}^{\prime}=\lambda_{i}\left\{\frac{Q_{i}}{\lambda_{i}}-\sum_{\ell} \frac{1}{\lambda_{i l}} \sum_{k=1}^{\text {KMAX JMAX }} \sum_{j=1}^{\operatorname{JMA}} \frac{Y_{i \ell j k} \theta_{j}}{2}\left[\left(\frac{r_{k+1}+r_{k}}{2}\right)^{2}-\left(\frac{r_{k}+r_{k}-1}{2}\right)^{2}\right]\right\}
$$

where $Y_{i \ell k}$ is the rate at which deposition of the $\ell^{\text {th }}$ daughter of the released radionuclide $i$ is occurring in the $\mathrm{k}^{\text {th }}$ annulus of the $\mathrm{j}^{\text {th }}$ sector of the region immediately surrounding the release point and is defined by Equation (5).

The preceding equation requires that the sum of the deposition of each daughter in the KMAX annuli and JMAX sectors of the region surrounding the release point be made to estimate the release rate of the radionuclide $i$ into the regions beyond the immediate vicinity of the release point. However, AIRWAY performs all calculations pertaining to a given daughter of a released radionuclide prior to moving on to con-. sidering the next daughter in the decay chain. As a result, the AIRWAY program uses an estimate of the rate of release of the radionuclide $i$ in the region beyond the vicinity of the release point which varies for each of the daughters of the radionuclide $i$. The expression used by the AIRWAY program to estimate the rate at which the radionuclide $i$ is introduced into the overall region beyond the immediate neighborhood of the release point is

$$
\left.\left.Q_{i l}^{\prime}=\lambda_{i 0}\left\{\frac{Q_{i l-1}^{\prime}}{\lambda_{i 0}}-\frac{1}{\lambda_{i l}} \sum_{k=1}^{\text {KMAX JMAX }} \sum_{j=1}^{Y_{i l j k} \theta_{j}} \frac{\left(\left(\frac{r_{k}+1}{2}+r_{k}\right.\right.}{2}\right)^{2}-\left(\frac{r_{k}+r_{k-1}}{2}\right)^{2}\right]\right\}
$$

where $Q_{i \ell}^{\prime}$ represents the release rate of the radionuclide $i$ in the region beyond the immediate vicinity of the release point for the precursor of the $\ell^{\text {th }}$ daughter of the $i^{\text {th }}$ radionuclide. The value of $Q_{i l-1}^{\prime}$ for the first daughters $(\ell=1)$ of the released radionuclide is the release rate $Q_{i}$ of the radionuclide $i$.

\section{Number Concentrations}

In general the AIRWAY program calculates air and ground activity concentrations in terms of the number of curies of a given radionuclide per unit volume of air or per unit area of ground. These activity concentrations are then used to define appropriate dose commitments. However, provisions were made in the AIRWAY program to provide the number concentrations (number of atoms of a given radionuclide per unit volume or per unit area) corresponding to the air and ground activity concentrations. This is accomplished by modifying each of the previously defined air and ground activity concentrations (i.e., $\chi_{\mathrm{a} i l j \mathrm{j},} \chi_{\mathrm{bil}}, \chi_{\mathrm{cil}}$ $\mathrm{C}_{\mathrm{ai} \ell \mathrm{j} k,} \mathrm{C}_{\mathrm{bi} \ell \mathrm{i},}, \mathrm{C}_{\mathrm{ci} \ell,}$ and $\left.\mathrm{C}_{\mathrm{di} \ell}\right)$ in the following manner.

$$
\mathrm{A}_{\mathrm{xi} \ell}=1.332 \times 10^{14} \mathrm{~N}_{\mathrm{xi} \ell} / \lambda_{\mathrm{i} \ell} .
$$

In the above expression the $\mathrm{N}_{\mathrm{xi}}$ term represents any of the previously defined air or ground activity

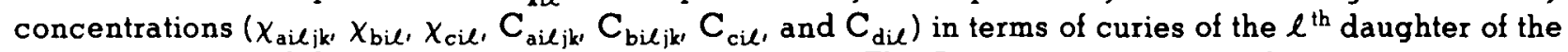
released radionuclide $i$ per unit volume or per unit area. The $A_{x i l}$ term in the preceding equation represents the air or ground number concentration in terms of number of atoms of the $\ell^{\text {th }}$ daughter of the released radionuclide $\mathrm{i}$ corresponding to the air or ground concentration used for $\mathrm{N}_{\mathrm{xil}}$.

\section{PROGRAM DESCRIPTION}

This section of the report presents a general description of the AIRWAY computer program. In addition to the information provided in this section, a detailed description of all the input necessary to run this program is presented in Appendix $C$ and a sample problem is supplied in Appendix D. The calculational checks which have been made to verify and qualify this program to date are described in Appendix E. 
The AIRWAY program was developed to estimate the effect upon people of releasing radioactive nuclides into the atmosphere. These effects are measured in terms of dose commitments which define the radiation dose a person may receive over a given period of time as the result of a specific exposure to radioactive material. Since the radioactive material released into the atmosphere may reach a person by means of various paths, the total dose commitment to a person or set of people resulting from the release of a specific radionuclide is given by the sum of the dose commitments which are accrued by that person or persons from each of the various paths by which the specific released radionuclide can reach man. The AIRWAY program considers six of these pathways:

1. Immersion in a cloud of radionuclides

2. Exposure to a surface deposit of radionuclides

3. Ingestion of food produced during the deposition of radionuclides

4. Ingestion of food produced in soil contaminated by the prior deposition of radionuclides

5. Inhalation of radionuclides prior to their initial deposition

6. Inhalation of radionuclides which have been resuspended subsequent to their depositions.

The dose commitments for each of these pathways are always calculated by the AIRWAY program and the results summed to define the total dose commitments associated with a given release. However, the results of each of these pathway dose commitment calculations can be modified by a multiplication factor prior to their summing if so desired. This feature may be used to examine the effect of any combination of these pathways, to reflect uncertainties in various pathway parameters, etc. by the proper choice of these individual pathway multiplication factors.

The actual dose commitments are dependent upon the exposure to the air and ground concentrations resulting from the release of radionuclides into the atmosphere. The AIRWAY program uses information delineating this exposure interval in conjunction with the time that releases stop to define the exposure limits to the various activity concentration behaviors in each of the dose commitment pathways considered. The program has the ability to examine exposure intervals which are entirely within the period that releases are occurring, which lie completely beyond the time that releases stop or which overlap the time at which releases stop.

The AIRWAY program requires that each radionuclide which is released into the atmosphere and its release rate be specified as input. The program then calculates the dose commitments arising from each of these specified released radionuclides and from each daughter of each released radionuclide. This latter function is performed automatically by the AIRWAY program by scanning decay chain information provided via input to define the daughters associated with a released radionuclide, their yields and their position in the decay chain. This information is then used to calculate the air and ground activity concentrations of each of these radionuclides and these in turn to estimate the dose commitments resulting from each released radionuclide and each of their daughters. Provisions are incorporated in the program to omit the consideration of daughters in a decay chain whose yield is below an arbitrary value specified via the input.

The dose commitment calculations are performed for two distinct physical regions. The first of these regions is the area which immediately surrounds the release point. This region may extend to approximately 100 kilometers from the release point. Beyond this radial distance, the equations which describe the detailed behavior of the released radionuclides and their daughters in the immediate vicinity of the release print can no longer be considered to be applicable and other procedures should be used to approximate the behavior of the nuclides. The actual distance used by the program to define the boundary of this region in the immediate vicinity of the release point is specified in the input data. This region which surrounds the release point is subdivided into annular segments and then calculations of the air and ground concentrations in each of these segments are made. The number and size of the annuli and the sectors into which this region is subdivided are defined by the user of the program to describe the problem being examined. The user also defines the number of people in each of these annular segments who will be exposed to the released radionuclides and for whom dose commitments will be obtained. The program permits the number of people in each of these segments to vary exponentially (gain or loss), however the population in all segments must vary in the same manner.

The dose commitments which are accrued by the people in the immediate vicinity of the release point are dependent upon the behavior of the released radionuclides and their daughters in this region subsequent to the releases. The behavior of the released radionuclides and their daughters is a function of the meteorological conditions which exist in this region during the period that exposure to these nuclides is occurring. The meteorological data which are necessary in the program are the fractions of the time during 
the exposure that the wind is blowing into each azimuthal sector at each of the wind speeds examined in the problem while each of the stability conditions considered in the job exists. Provisions are made in the AIRWAY program to supply this meteorological data in four different ways. These ways are:

1. Individual fractions for each possible combination of wind speed, wind direction and stability category

2. One set of data defining the fraction of the exposure time that the wind is blowing at certain speeds, another set of data defining the fraction of the exposure time that the wind is blowing in the various directions and a final set defining the fraction of the time that each of the stability categories exist

3. One set of data defining the fraction of the exposure time that each combination of the wind speed and stability category exist in conjunction with a set of data defining the fraction of the exposure time that the wind blows into each of the directions examined in the job

4. One set of data defining the fraction of the exposure time that each wind speed-wind direction combination exists and another set of data defining the fraction of the exposure time that each of the stability categories exist.

In addition to the above data the behavior of the released radionuclides and their daughters in the regions adjacent to the release point is dependent upon the dispersion associated with each stability category which is defined by the standard deviation of the vertical dispersion as provided in the input as a function of distance from the release point and upon the actual wind speed values. Further, the behavior of the radionuclides in the immediate vicinity of the release point is influenced by the height above the ground that the releases occur. It should be noted that the effect of plume rise is not incorporated directly in the program but its effect may be approximated by modifying the height above ground that the releases occur (Reference 7). Other items upon which the behavior of the radionuclides in the vicinity of the release point is dependent are the effects associated with precipitation scavenging, dry deposition mechanisms, radioactive decay and weathering or migration losses.

The second physical region in which dose commitments are estimated is the remainder of the world beyond the regions immediately adjacent to the release point which is affected by the releases. This second physical region is in itself divided into subregions-one which is associated with radionuclides which are in a gaseous form and one which is associated with radionuclides which are particulates. The extent of each of these two subregions which make up the remainder of the world affected by the releases which is beyond the immediate vicinity of the release point is defined by the atmospheric volume and the ground surface area of the respective subregions. In general, the regions affected by gaseous radionuclides are much larger than those affected by particulates.

The numerical values assigned by the user to the atmospheric volume and ground surface area parameters of the particulate and gaseous subregions beyond the immediate vicinity of the release point are dependent upon the specific radionuclides which are being examined in a job. For example, long-lived gaseous radionuclides would eventually affect the entire world while short-lived gaseous radionuclides may only affect a region within a few thousand kilometers of the release point before they decay. Conversely, the largest region which may be affected by the release of long-lived particulate radionuclides may also be within a few thousand kilometers of the release point since the atmosphere may essentually be depleted of these particulates due to the effect of precipitation scavenging and dry deposition during the time that the particulates travel through a region of this size. However, if the daughters of a long-lived gaseous radionuclide are particulates then the entire world may be affected by these particulates since the majority of them would not be formed until after the dispersion of their precursor throughout the world's atmosphere. A more general discussion of the factors which need to be considered in defining the extent of these subregions of the portion of the world which lies beyond that considered to be adjacent to the release point is presented in Reference 3.

The actual choice of whether a given radionuclide is to be treated as a gas or as a particulate (and thus the subregions into which the radionuclide is dispersed) is made by the value specified in the input for the dry deposition velocity of the radionuclide. It the dry deposition velocity of a given nuclide is greater than zero, that nuclide is assumed to be a particulate and it is subjected to precipitation scavenging and dry deposition mechanisms. It further is dispersed in the region beyond the immediate vicinity of the release point which is associated with particulates. If, however, the dry deposition velocity of a nuclide is equal to or less than zero, that nuclide is treated as a gas. Thus, it is not subjected to precipitation scavenging and dry deposition effects and it is assumed to be dispersed in the region beyond the immediate surroundings which is associated with gases. In the case of particulates the rate at which radionuclides enter the region 
beyond the immediate vicinity of the release point is obtained by reducing the number released into the atmosphere by the number which have been deposited in the region surrounding the release point via dry deposition and precipitation scavenging. This reduction is performed automatically by the AIRWAY program for particulate radionuclides. However, this is not done for gaseous radionuclides since the concentration of gases in the atmosphere is not considered to be affected by these mechanisms.

The AIRWAY program permits the population in each of the two subregions associated with the portion of the world affected by the releases which lies beyond the region in the immediate vicinity of the release point (one affected by gaseous radionuclides and the other affected by particulate radionuclides) to be specified separately. Further, each of these populations may change with time if so desired. These changes are assumed to be exponential and the population in either subregion may increase or decrease independently of the population in the other subregion.

One of the features which is incorporated in the AIRWAY program is the capability to include the effect of the released radionuclides and their daughters which have encircled the earth in the estimates of the dose commitments in the region which surrounds the release point. This effect is automatically available for any gaseous radionuclide when any exposure to the radioactive environment occurs more than 735 hours (between 30 and 31 days) after the releases start. Further, this effect can be forced for particulate radionuclides with the same exposure restriction by specitying that the population in the subregion affected by particulate radionuclide is equal to or greater than the population in the subregion affected by gaseous radionuclides. In general, the dose commitment contributions in the region adjacent to the release point from radionuclides which have circled the earth are negligible until the releases have stopped.

The AIRWAY program also permits the ingestion dose commitments in the vicinity of the release point to be obtained in two different ways at the discretion of the user. One of these procedures is to allow each person in the vicinity of the release point (i.e., all the people in all the azimuthal segments in the region which surrounds the release point) to consume food which is subjected to the average deposition in the region surrounding the release point. Thus, each person in the vicinity of the release point consumes food of the same level of contamination as every other person in this region. The second manner in which the ingestion dose commitments in the vicinity of the release point may be estimated is to require that all contaminated food consumed by an individual in the vicinity of the release point be produced in the specific annular segment in which the individual resides. This latter procedure corresponds to people eating the food which is grown in their own garden or produced in local farms. In either of these two procedures the individuals in the vicinity of the release point can consume food which is produced elsewhere but in both cases the food from other sources is not considered to be contaminated. Further, all people in the region adjacent to the release point are assumed to obtain the same fraction of their diet from these uncontaminated sources.

Another feature of the AIRWAY program is the capability of providing the basic nuclide data and some of the meteorological data in a general data file rather than as specific pieces of input. This feature permits a general data file containing information which does not vary significantly (if at all) from job to job to be generated and then all the data in this file made available to a specific job via the input of just one card to identify this file. Thus, a significant reduction in the a mount of input necessary to run a job can be obtained. The iniormation which can be provided in this file (or via input is so desired) includes the dose commitment conversion factors for each organ considered for the immersion, surface deposit, ingestion and inhalation pathways, the radioactive decay constants, the dry deposition velocities, the decay chain paths and the food uptake parameters for each nuclide. The file may also include meteorological data such as wind speeds, the standard deviations of vertical dispersion as a function of distance from the release point for various stability categories and some wind frequency distribution data. Since the information in this file does not in general chanqe from job to job, this basic data file is read only in the first case of a job and the data in it used for the remaining cases of that job unless specifically changed via the input for a case.

The AIRWAY program only includes the effect of resuspension in the calculation of the inhalation dose commitments. Thus, the direct effect of resuspended nuclides upon the immersion, surface deposit and ingestion dose commitments has not been included. However, the reduction in the ground activity concentrations to reflect resuspension effects was also not incorporated in the AIRWAY program. As a result, the neglect of resuspension yields an underestimate of the doses arising from the immersion pathway and overestimate of the doses due to exposure to surface deposits. Similarly the dose commitments due to the inges- 
tion of foods upon which radioactive material is depositing will be underestimated while those resulting from the ingestion of food produced in soil which had been contaminated previously by deposition will be overestimated.

The AIRWAY program provides dose commitments for a minimum of nine organs. These organs are the total body, bone, liver, kidneys, gonads, lungs, G.I. tract, thyroid and skin. Provisions are incorporated in the program to provide other organs or additional organs to be examined in a job. These dose commitments are provided for the total population in each of the annuli into which the region adjacent to the release point is subdivided and for all the people within this region for each released radionuclide and for each of their daughters. The dose commitments to the people in the remainder of the world (i.e., in the regions beyond the immediate vicinity of the release point) in the above organs for each released radionuclide and their daughters are also provided automatically as output of the program. $\AA$ sum of the above dose commitments over all the released radionuclides and their daughters is the final set of output information which is automatically provided by the program. However, provisions are available to print out other information which is generated in a job.

This auxiliary output includes the fractions of the total dose commitments from each released radionuclide and their daughters in each of the annuli in which the region adjacent to the release point is subdivided, the activity and number concentrations in the air and on the ground in each of the annular segments in which the region immediately surrounding the release point is subdivided and the activity and number concentrations in the atmosphere and on the ground of the regions beyond the immediate vicinity of the release point. The activity and number concentrations in this latter region and those concentrations on the ground in the region immediately adjacent to the release point are functions of time. These concentrations are calculated by the AIRWAY program starting at the time releases start (i.e., $t=0$ ) and continuing until the end of the exposure interval $\left(t=T_{L}\right)$. These calculations are made at intervals equal to the difference between the time releases stop and the beginning of the exposure interval or in 50 equal steps depending upon which procedure yields the minimum number of results. The maximum number of annular segments into which the region in the immediate vicinity of the release point may be subdivided should be limited to less than 5500 divided by the number of time steps plus four (i.e., $5500 /(\mathrm{NT}+4)$ ).

In addition to the output information identified above the AIRWAY program also provides a listing of the input cards and a printout of the information presented on these cards. The ability to print the contents of the basic data file is also provided in the program.

In summary, the AIRWAY computer program provides a means to estimate the radiation dnse cnmmit= ments which may be accrued by people who have been subjected to an environment whirh has heen contaminated by the atmospheric release of radionuclides. However, it should be emphasized that the AIRWAY program solves the equations associated with the models developed in References 2 and 3 . As such, its use is restricted by the limitations inherent in these equations due to the assumptions and approximations which are incorporated in the development of these models and which are discussed in detail in References 2 and 3. Thus, a thorough review of these references should be performed prior to the use of this program to ensure its applicability for the intended use, to define any pitfalls which may be encomntered in its use, and to identify any other paths or sources which should be considered in the solution beyond those performed by the AIRWAY program itself.

\section{ACKNOWLEDGEMENTS}

Over the period that the AIRWAY computer program was developed and this report prepared, many individuals on the Bettis Atomic Power Laboratory staff contributed to the overall effort to complete this task. However, specific acknowledgements of the contributions by the following individuals should be made.

Particular thanks must be given to M. M. Baker for the extensive effort performed in checking and verifying the proyram and its equations, in gathering and preparing much of the radionuclide data and sample problem information in the appendices and in reviewing the final report in detail. The author also wishes to express his gratitude to J. A. Milan for the aid provided in writing the output portion of the program, to J. G. Tirpak for the preparation and compilation of the basic file data presented in the appendices, to W. F. Grof for the work performed in checking derivations of the program equations, and to S. K. Beal for an extensive critical review of the program and this report and for the many constructive comments and con- 
cepts incorporated in this document. Finally, the author extends his thanks to L. R. Foulke and H. W. Ryals for their support and encouragement throughout the entire period associated with the development of the AIRWAY program and the preparation of this report.

\section{REFERENCES}

1. Light Water Breeder Reactor Program, Final Environmental Statement, ERDA-154 1, June 1976.

2. J. L. Rider and S. K. Beal, "A Model to Estimate the Local Radiation Doses to Man from the Atmo.spheric Release of Radionuclides," WAPD-TM-1273, April 1977.

3. J. L. Rider and S. K. Beal, "A Model to Estimate Radiation Dose Commitments to the World Population from the Atmospheric Release of Radionuclides," WAPD-TM-1274, February 1978.

4. L. Machta, et. al., "Regimal and Global Scale Dispersion of Krypton-85 for Population Dose Calculations," Proc. Symposium on the Physical Behavior of Radioactive Contaminants in the Atmosphere, pages 41 1-426, IAEA, Vienna, 1974.

5. L. R. Anspaugh, et. al., "Evaluation of the Resuspension Pathway Toward Protective Guidelines for Soil Contamination with Radioactivity," Lawrence Livermore Laboratory, STI/PUB-375, May 19, 1974; pp 513-524.

6. D. H. Slade, Ed., "Meteorology and Atomic Energy," TID-24190, July 1968 (latest printing March 1973) (NSA 22-45190).

7. R. E. Moore, "AIRDOS-A Computer Code for Estimating Population and Individual Doses Resulting from Atmospheric Releases of Radionuclides from Nuclear Facilities," ORNL-TM-4687, January 1975. 
APPENDIX A

\section{AIRWAY PROGRAM ABSTRACT}




\section{APPENDIX A}

\section{COMPUTER PROGRAM ABSTRACT}

\section{AIRWAY}

1. Program Name: AIRWAY-Environmental Dose Commitment Estimates to Man from the Atmospheric Release of Radionuclides.

2. Computer and Language (A): CDC-6600 and CDC-7600; FORTRAN-IV.

3. Problem Solved: The AIRWAY computer program (Reference c) performs the calculations to estimate the dose commitments to various organs of man arising from the transport and deposition of radionuclides released into the atmosphere. The program considers two major regions of interest: (a) the area immediately surrounding the point of release out to a radial distance of $80-100 \mathrm{~km}$ and (b) the remainder of the world which is affected by the releases. In general, the program considers the effects of release rates, meteorological conditions (i.e., direction and speed of wind, stability categories, etc.), radial and azimuthal locations relative to the release point, release times, exposure times, release heights, deposition velocities, population size and growth rates, radiological and physical removal constants and specific environmental pathways upon the transport and subsequent irradiation of man due to airborne radionuclides.

4. Method of Solution: The computer program solves explicit integral equations defining the dose commitments for the following six pathways: (a) immersion in a cloud of airborne radionuclides, (b) exposure to a surface deposit of radionuclides, (c) ingestion of food subjected to deposition of particulate radionuclides, (d) ingestion of food produced in soil which had been contaminated previously, $(e)$ inhalation of radionuclides prior to any deposition, and $(f)$ inhalation of radioactive material which had been resuspended subsequent to its deposition. The computer program also solves explicit integral equations to obtain the air and ground concentrations of the released radionuclides and their daughters for use in the dose commitment solutions.

5. Restrictions on Problem Complexity: The AIRWAY program calculates the air and ground concentrations of the released radionuclides and their daughters in each of the annular segments into which the region in the immediate vicinity of the release point is subdivided at each of the times the interval between the time releases start and the time at the end of the period of interest is divided into. This time interval may be divided into a maximum of $\mathbf{5 0}$ time steps. When a problem considers the maximum number of time steps (i.e., 50), a maximum of 100 annular segments may be examined in that problem. If the number of time steps is reduced below the maximum by a given factor then the maximum number of annular segments which can be examined in that job is increased by thal saime factor.

6. Related and Auxiliary Programs: The AIRWAY program uses the Bettis Programming Environment routines INTTAP, OUTTAP, and FINISH which are documented in Reference $d$. These routines can easily be replaced and do not require obtaining the entire programming environment. The program also uses the Bettis Programming Environment routine TRIO to move data to and from extended core storage locations and the CARDS and INP routines to read input data. These routines are documented in Reference d.

7. Typical Running Time: The running time for an AIRWAY problem varies primarily as a direct function of the number of released radionuclides, the number of their daughters, the complexity of the deoay chains of the released radionuclides, the number of annular segments in the vicinity of the release point and the number of times at which concentrations are obtained. The sample problem which examined 8 annular segments at 50 times for a total of 7 nuclides (four released radionuclides and three daughter nuclides arising from simple decay chains) required approximately 1 minute of CDC-6600 computer time.

8. Unusual Features: The AIRWAY program provides for the automatic growth of the daughters of the released radionuclides with time as the released material is transported from the regions in the immediate vicinity of the release point and is dispersed in the overall region affected by the releases.

9. Status: Production. 
10. Machine Requirements: The AIRWAY program requires $140 \mathrm{~K}$ octal words of CDC-6600 or CDC-7600 central memory and $200 \mathrm{~K}$ words of CDC-6600 Extended Core Storage or CDC-7600 Large Core Memory.

11. Operating System: The AIRWAY program operates with either the SCOPE 3.3 (CDC-6600) or the SCOPE 1.1 (CDC-7600) operating system.

12. Other Information: The program requires that a set of basic data for each radionuclide being examined be provided either directly by input or via a data file. The data for each radionuclide includes the decay constant, the deposition velocity, the food uptake factor, the dose commitment conversion factors for inhalation and ingestion, and the dose rate conversion factors for the immersion and surface deposit pathways. In addition, meteorological data such as wind speeds, frequency distributions of various wind speed, wind direction and stability category combinations, and standard deviations of vertical dispension as a function of radial distance from the point of release are required. Reference $c$ contains the basic radionuclide data for a total of 378 nuclides and the meteorological data averaged over 18 stations in the continental United States.

13. Availability: Copies of the computer proqram may be obtained by domestic users from Ärgonne Code Center

Attention: Mrs. Margaret Butler

Argonne National Laboratory

9700 South Cass Avenue

Argonne, Illinois 60440

14. References:

a. J. L. Rider and S. K. Beal, "A Model to Estimate the Local Radiation Doses to Man from the Atmospheric Release of Radionuclides," WAPD-TM-1273, April 1977.

b. J. L. Rider and S. K. Beal, "A Model to Estimate Radiation Dose Commitments to the World Population from the Atmospheric Release of Radionuclides," WAPD-TM-1274, February 1978.

c. J. L. Rider, "AIRWAY - A FORTRAN Computer Program Estimate Radiation Dose Commitments to Man From the Atmospheric Release of Radionuclides," WAPD-TM-1275, June 1979.

d. W. R. Cadwell, 2d., "Reference Manual-Bettis Programming Environment," WAPD-TM-1181, Bettis Atomic Power Laboratory (1974). 
APPENDIX B

AIRWAY PROGRAMM EQUATIONS 


\section{APPENDIX B}

\section{PROGRAM EQUATIONS}

\section{IMMERSION DOSE COMMITMENTS}

A. Vicinity of Releases-Gaseous Radionuclides and Particulate Radionuclides Affecting the Entire World

$$
D_{\mathrm{Ii} / \mathrm{gjk}}=\mathrm{F} \mathrm{K}_{\mathrm{I} / \mathrm{g}} \mathrm{P}_{\mathrm{jk}}\left[\left(\chi_{\mathrm{a} i / \mathrm{jk}} \operatorname{RINT} \AA\right)+\mathrm{RINT} 1+\mathrm{RINT} 2\right]
$$

B. Vicinity of Releases-Particulate Radionuclides Affecting Only Part of the World

$$
D_{\mathrm{I} i l g j k}=F K_{I i \ell g} P_{j k} X_{a i l j k} \text { RINT A }
$$

C. Remainder of World Affected by Releases-All Nuclides

$$
\mathrm{D}_{\mathrm{I} \ell_{g}}=\mathrm{F} \mathrm{K}_{\mathrm{I} \ell_{\mathrm{g}}} \mathrm{P}_{\mathrm{T}}[\text { RINT } 3+\text { RINT 4] }
$$

II. SURFACE DEPOSIT DOSE COMMITMENTS-Particulate Radionuclides Only

A. Vicinity of Releases-Entire World Affected

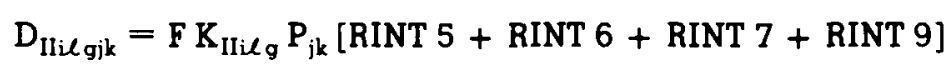

B. Vicinity of Releases-Only Part of World Affected

$$
D_{\text {IIi } g j k}=F_{\text {IIi } \text { Ig }} P_{i k}[\text { RINT } 5+\text { RINT 6] }
$$

C. Remainder of World Affected by Releases

$$
\mathrm{D}_{\mathrm{II} \ell \mathrm{g}}=\mathrm{F} \mathrm{K}_{\mathrm{II} \ell \mathrm{g}} \mathrm{P}_{\mathrm{T}} \text { [RINT } 16+\text { RINT 1 7] }
$$

\section{INGESTION DOSE COMMITMENTS-Particulate Radionuclides Only}

A. Vicinity of Releases

People consume food produced in immediate neighborhood (few hundred meters) of their residence.

1. Entire World Affected by Releases

a. Food subjected to direct deposition

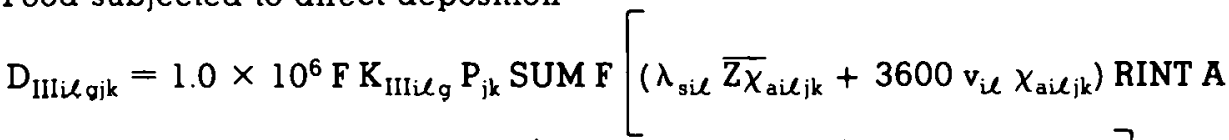

$$
\begin{aligned}
& \left.+\left(3600 v_{i l}+\lambda_{s i l} \mathrm{Z}\right)\left(\frac{\text { RINT 20 RINT A }}{\mathrm{T}_{\mathrm{S}}}\right)+\left(\frac{\text { RINT 2l RINT C }}{\mathrm{T}_{\mathrm{L}}-\mathrm{T}_{\mathrm{S}}}\right)\right]
\end{aligned}
$$


b. Food produced in previously contaminated ground

$$
\mathrm{D}_{\text {IIIi } \text { gik }}=1.0 \times 10^{6} \mathrm{~F} \mathrm{~K}_{\mathrm{III} \ell \text { g }} \mathrm{P}_{\mathrm{jk}} \text { SUM R [RINT } 5+\text { RINT } 6 \text { + RINT } 7+\text { RINT 9] }
$$

2. Only Part of World Affected by Releases

a. Food subjected to direct deposition

$$
D_{\text {III } \ell \text { gjk }}=1.0 \times 10^{6} \mathrm{FK}_{\text {III } \ell g} \mathrm{P}_{\mathrm{ik}} \operatorname{SUMF}\left(\lambda_{\mathrm{si} \ell} \overline{\mathrm{ZX}}_{\mathrm{ai} \ell \mathrm{jk}}+3600 \mathrm{v}_{\mathrm{i} \ell} \chi_{\mathrm{ai} \ell \mathrm{k} \mathrm{k}}\right) \operatorname{RINT} \AA
$$

b. Food produced in previously contaminated ground

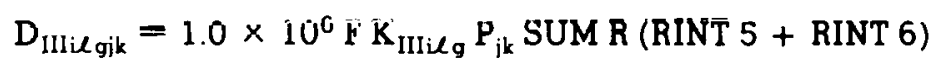

\section{B. Vicinity of Releases}

People consume food produced throughout region in vicinity of relcases.

1. Entire World Affected by Releases

a. Food subjected to direct deposition

$$
\begin{aligned}
& D_{\text {III } \ell \text { gjk }}=1.0 \times 10^{6} \mathrm{~F} \mathrm{~K}_{\text {III } \ell g} P_{j \mathrm{k}} \text { SUM F }\left[\frac{\text { SUM D RINT A }}{\text { SUM A }}\right. \\
& \left.+\left(3600 v_{i \ell}+\lambda_{3 i \ell} Z\right)\left(\frac{\text { RINT 20 RINT A }}{T_{S}}\right)+\left(\frac{\text { RINT 21 RINT C }}{T_{L}-T_{S}}\right)\right]
\end{aligned}
$$

b. Food produced in previously contaminated ground

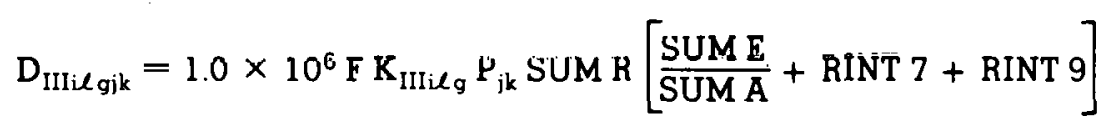

2. Only Part of World Affected by Releases

a. Food subjected to direct deposition

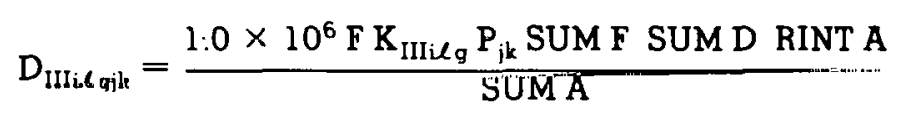

b. Food produced in previously contaminated ground

$$
D_{\text {IIIilgik }}=\frac{1.0 \times 10^{6} \mathrm{~F} \mathrm{~K}_{\text {III } \ell_{g}} P_{i \mathrm{k}} \text { SUM R SUM E }}{\text { SUM A }}
$$

\section{Remainder of World Affected by Releases}

1. Food Subjected to Direct Deposition

$$
\begin{gathered}
\mathrm{D}_{\text {IIILg }}=1.0 \times 10^{6} \mathrm{~F} \mathrm{~K}_{\text {III } \ell_{g}} \mathrm{P}_{\mathrm{T}} \operatorname{SUMF}\left(3600 \mathrm{v}_{\mathrm{i} \ell}+\lambda_{\mathrm{si}} \mathrm{Z}\right) \\
{\left[\left(\frac{\text { RINT 20 RINT B }}{\mathrm{T}_{\mathrm{S}}}\right)+\left(\frac{\text { RINT 21 RINT D }}{\mathrm{T}_{\mathrm{L}}-\mathrm{T}_{\mathrm{S}}}\right)\right]}
\end{gathered}
$$


2. Food Produced in Previously Contaminated Ground

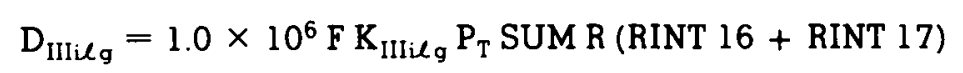

\section{INHALATION DOSE COMMITMENTS}

\section{A. Vicinity of Release-Entire World Affected by Release}

1. Gaseous and Particulate Radionuclides Not Previously Deposited

$$
D_{\text {IVi } l g j k}=1.0 \times 10^{6} \mathrm{~F} \mathrm{~B}_{\mathrm{a}} \mathrm{K}_{\text {IVilg }} \mathrm{P}_{\mathrm{jk}}\left[X_{\mathrm{a} i \ell_{\mathrm{jk}}} \text { RINT } \AA+\text { RINT } 1+\operatorname{RINT} 2\right]
$$

2. Resuspended Radionuclides

$$
\begin{aligned}
& \mathrm{D}_{\mathrm{IVi} \ell \mathrm{gik}}=1.0 \times 10^{6} \mathrm{~F} \mathrm{~B}_{\mathrm{a}} \mathrm{K}_{\mathrm{IVi \ell q}} \mathrm{P}_{\mathrm{jk}}\left[\mathrm{K}_{0}(\mathrm{RINT} 11+\mathrm{RINT} 12+\mathrm{RINT} 13+\mathrm{RINT} \text { 15) }\right. \\
& \left.+\mathrm{K}_{1}(\operatorname{RINT} 5+\operatorname{RINT} 6+\operatorname{RINT} 7+\operatorname{RINT} 9)\right]
\end{aligned}
$$

B. Vicinity of Release-Only Part of World Affected by Release

1. Gaseous and Particulate Radionuclides Not Previously Deposited

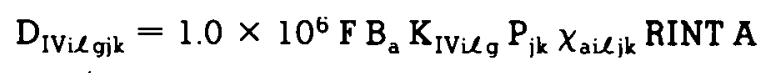

2. Resuspended Radionuclides

$$
\mathrm{D}_{\text {IVi } \text { g jk }}=1.0 \times 10^{6} \mathrm{~F} \mathrm{~B}_{\mathrm{a}} \mathrm{K}_{\mathrm{IVi \ell g}} \mathrm{P}_{\mathrm{jk}}\left[\mathrm{K}_{0}(\mathrm{RINT} 11+\mathrm{RINT} 12)+\mathrm{K}_{1}(\text { RINT } 5+\text { RINT 6) }]\right.
$$

C. Remainder of World Affected by Release

1. Gaseous and Particulate Radionuclides Not Previously Deposited

$$
\mathrm{D}_{\text {IVilg }}=1.0 \times 10^{6} \mathrm{~F} \mathrm{~B}_{\mathrm{a}} \mathrm{K}_{\mathrm{IVi \ell g}} \mathrm{P}_{\mathrm{T}}(\mathrm{RINT} 3+\mathrm{RINT} 4)
$$

2. Resuspended Radionuclides

$$
\mathrm{D}_{\mathrm{IVi} \ell g}=1.0 \times 10^{6} \mathrm{~F} \mathrm{~B}_{\mathrm{a}} \mathrm{K}_{\mathrm{IVi} \ell \mathrm{g}} \mathrm{P}_{\mathrm{T}}\left[\mathrm{K}_{0}(\text { RINT } 18+\mathrm{RINT} 19)+\mathrm{K}_{1}(\text { RINT } 16+\text { RINT 17) }]\right.
$$

v. SUMS

$$
\operatorname{SUM~} A=\sum_{k=1}^{\operatorname{KMAX}} \sum_{j=1}^{\text {JMAX }} \theta_{i}\left[\left(\frac{r_{k+1}+r_{k}}{2}\right)^{2}-\left(\frac{r_{k}+r_{k}-1}{2}\right)^{2}\right] / 2
$$

where

$$
\frac{\left(r_{k}+r_{k-1}\right)}{2}=0 \text { when } k=1
$$

and

$$
\frac{\left(r_{k+1}+r_{k}\right)}{2}=\text { RMAX when } k=\text { KMAX }
$$




$$
\operatorname{SUM~D}=\sum_{k=1}^{\operatorname{KMAX}} \sum_{j=1}^{\mathrm{JMAX}} \theta_{i}\left[\left(\frac{r_{k+1}+r_{k}}{2}\right)^{2}-\left(\frac{r_{k}+r_{k}-1}{2}\right)^{2}\right]\left[\lambda_{s i l}{\overline{Z_{\chi}}}_{a i l j k}+3600 v_{i l} \chi_{a i l j k}\right] / 2
$$

where

$$
\frac{\left(r_{k}+r_{k-1}\right)}{2}=0 \text { when } k=1
$$

and

$$
\begin{aligned}
& \frac{\left(r_{k+1}+r_{k}\right)}{2}=\operatorname{MMAX} \text { whin } k=\operatorname{IMMX} . \\
& \text { SUME }=\sum_{k=1}^{\operatorname{KMAX}} \sum_{j=1}^{\text {JMAX }} \theta_{i}\left[\left(\frac{r_{k+1}+r_{k}}{2}\right)^{2}-\left(\frac{r_{k}+r_{k}-1}{2}\right)^{2}\right][\text { RINT 5 + RINT 6] } / 2
\end{aligned}
$$

where

$$
\frac{\left(r_{k}+r_{k-1}\right)}{2}=0 \text { when } k=1
$$

and

$$
\frac{\left(x_{k+1}+r_{k}\right)}{2}=\operatorname{RMAX} \text { when } k=\operatorname{KMAX}
$$

$$
\operatorname{SUMF}=\sum_{\mathrm{f}=1}^{\mathrm{FMNX}} \mathrm{q}_{\mathrm{f}} \mathrm{w}_{\mathrm{f}} / \mathrm{q}_{\text {of }}
$$

$$
\text { SUM R }=\frac{4.16667 \times 10^{-6} C_{p i} q_{0 i}}{C_{s i} \rho_{s} d_{s} w_{1}} \sum_{f=1}^{\text {FMAX }} \frac{q_{f} w_{i}}{q_{0 f}}
$$

\section{INTEGRALS}

$$
\begin{aligned}
& \operatorname{RINTA}=\operatorname{XPINT}\left(\lambda_{\mathrm{P} 1}, \mathrm{~T}_{\mathrm{SI}}, \mathrm{T}_{\mathrm{I}}\right) \\
& \operatorname{RINTB}=\operatorname{XPINT}\left(\lambda_{\mathrm{P} 2}, \mathrm{~T}_{\mathrm{Sl}}, \mathrm{T}_{1}\right) \\
& \operatorname{RINTC}=\operatorname{XPINT}\left(\lambda_{\mathrm{P} 1}, \mathrm{~T}_{\mathrm{L}} \mathrm{T}_{\mathrm{SL}}\right) \\
& \operatorname{RINTD}=\operatorname{XPINT}\left(\lambda_{\mathrm{P} 2}, \mathrm{~T}_{\mathrm{L}} \mathrm{T}_{\mathrm{SL}}\right)
\end{aligned}
$$

$$
\operatorname{RINT1}=A_{i \ell} \operatorname{XPINT}\left(\lambda_{\mathrm{P} 1}, \mathrm{~T}_{\mathrm{SI}}, \mathrm{T}_{1}\right)-\mathrm{B}_{\mathrm{i} \ell} \sum_{\mathrm{q}=0}^{\mathrm{q}=\ell} \mathbf{E}_{\mathrm{i} \ell \mathrm{q}} \mathrm{XPINT}\left(-\bar{\lambda}_{\mathrm{iq}}+\lambda_{\mathrm{P} 1}, \mathrm{~T}_{\mathrm{SI}}, \mathrm{T}_{\mathrm{I}}\right)
$$




$$
\begin{aligned}
& \text { RINT2 }=\sum_{q=0}^{q=\ell} G_{i \ell q} X M P I N T\left(-\bar{\lambda}_{i q}+\lambda_{P 1}, T_{L} T_{S L} \bar{\lambda}_{i q^{\prime}} T_{S}\right) \\
& \operatorname{RINT3}=A_{i \ell} \operatorname{XPINT}\left(\lambda_{\mathrm{P} 2}, \mathrm{~T}_{\mathrm{Sl}}, \mathrm{T}_{\mathrm{I}}\right)-\mathrm{B}_{\mathrm{i} \ell} \sum_{\mathrm{q}=0}^{\mathrm{q}=\ell} \mathrm{E}_{\mathrm{i} \ell \mathrm{q}} \operatorname{XPINT}\left(-\bar{\lambda}_{\mathrm{iq}}+\lambda_{\mathrm{P} 1}, \mathrm{~T}_{\mathrm{SI}}, \mathrm{T}_{\mathrm{I}}\right) \\
& \operatorname{RINT4}=\sum_{q=0}^{q=\ell} G_{i \ell q} X M P I N T\left(-\bar{\lambda}_{i q}+\lambda_{P 2}, T_{L}, T_{S L}, \bar{\lambda}_{i q}, T_{S}\right) \\
& \operatorname{RINT5}=H_{i \ell, k} \operatorname{XPINT}\left(\lambda_{P_{1} 1} T_{S 1}, T_{1}\right)-\sum_{q=0}^{q=\ell} I_{i \ell q j k} X \operatorname{PINT}\left(-\alpha_{1 q}+\lambda_{P 1}, T_{S 1}, T_{1}\right) \\
& \operatorname{RINT6}=\sum_{\mathrm{q}=0}^{\mathrm{q}=\ell} \mathrm{J}_{\mathrm{i} \ell \mathrm{qik}} \mathrm{XMPINT}\left(-\alpha_{\mathrm{iq}}+\lambda_{\mathrm{P} 1}, \mathrm{~T}_{\mathrm{L}}, \mathrm{T}_{\mathrm{SL}}, \alpha_{\mathrm{iq}}, \mathrm{T}_{\mathrm{S}}\right) \\
& \operatorname{RINT7}=\sum_{\mathrm{q}=0}^{\mathrm{q}=\ell}\left[\mathrm{L}_{\mathrm{i} \ell \mathrm{q}} \operatorname{XPINT}\left(\lambda_{\mathrm{P} 1}, \mathrm{~T}_{\mathrm{SI}}, \mathrm{T}_{\mathrm{I}}\right)-\mathrm{M}_{\mathrm{i \ell q}} \mathrm{XPINT}\left(-\bar{\lambda}_{\mathrm{iq}}+\lambda_{\mathrm{Pl}}, \mathrm{T}_{\mathrm{SI}}, \mathrm{T}_{1}\right)\right. \\
& \left.+\mathrm{N}_{\mathrm{i} \ell q} \mathrm{XPINT}\left(-\alpha_{\mathrm{iq}}+\lambda_{\mathrm{Pl}}, \mathrm{T}_{\mathrm{SI}}, \mathrm{T}_{\mathrm{I}}\right)\right] \\
& \operatorname{RINT9}=\sum_{q=0}^{q=\ell}\left[R_{i \ell q} X M P I N T\left(-\alpha_{i q}+\lambda_{P 1}, T_{L}, T_{S L} \alpha_{i q}, T_{S}\right)\right. \\
& \left.+\mathrm{S}_{i \ell q} \mathrm{XMPINT}\left(-\bar{\lambda}_{\mathrm{iq}}+\lambda_{\mathrm{Pl}}, \mathrm{T}_{\mathrm{L}}, \mathrm{T}_{\mathrm{SL}}, \dot{\bar{\lambda}}_{\mathrm{iq}}, \mathrm{T}_{\dot{\mathrm{S}}}\right)\right]
\end{aligned}
$$

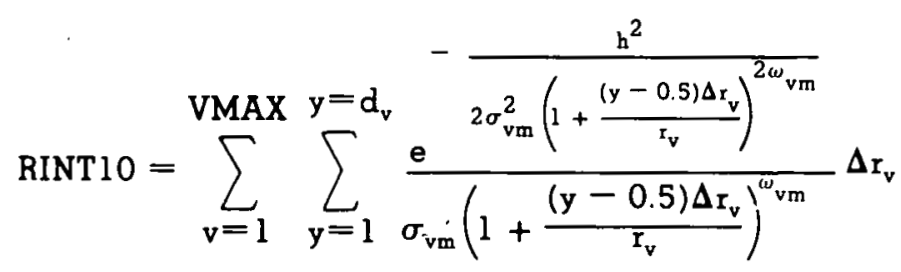

$$
\begin{aligned}
& \operatorname{RINT11}=\mathrm{H}_{\mathrm{i} \ell \mathrm{jk}} \mathrm{XPINT}\left(-\lambda_{\mathrm{d}}+\lambda_{\mathrm{P} 1}, \mathrm{~T}_{\mathrm{SI}}, \mathrm{T}_{\mathrm{I}}\right) \\
& -\sum_{\mathrm{q}=0}^{\mathrm{q}=\ell} \mathrm{I}_{\mathrm{i} \ell \mathrm{qik}} \mathrm{XPINT}\left(-\alpha_{\mathrm{iq}}-\lambda_{\mathrm{d}}+\lambda_{\mathrm{Pl}}, \mathrm{T}_{\mathrm{SI}}, \mathrm{T}_{\mathrm{l}}\right) \\
& \operatorname{RINT} 12=\sum_{\mathrm{q}=0}^{\mathrm{q}=\ell} \mathrm{J}_{\mathrm{i} \ell \mathrm{qik}} \mathrm{XMPINT}\left(-\alpha_{\mathrm{iq}}-\lambda_{\mathrm{d}}+\lambda_{\mathrm{Pl}}, \mathrm{T}_{\mathrm{SI}}, \mathrm{T}_{\mathrm{I}}, \alpha_{\mathrm{iq}}, \mathrm{T}_{\mathrm{S}}\right)
\end{aligned}
$$




$$
\begin{aligned}
& \operatorname{RINTI3}=\sum_{q=0}^{q=\ell}\left[L_{i \ell q} X \operatorname{PINT}\left(-\lambda_{d}+\lambda_{\mathrm{P} 1}, \mathrm{~T}_{\mathrm{SI}}, \mathrm{T}_{\mathrm{I}}\right)\right. \\
& -\mathrm{M}_{\mathrm{i} \ell \mathrm{q}} \operatorname{XPINT}\left(-\bar{\lambda}_{\mathrm{iq}}-\lambda_{\mathrm{d}}+\lambda_{\mathrm{Pl}}, \mathrm{T}_{\mathrm{SI}}, \mathrm{T}_{\mathrm{I}}\right) \\
& \left.+N_{i \ell q} \operatorname{XPINT}\left(-\alpha_{i q}-\lambda_{d}+\lambda_{P 1}, T_{S I}, T_{1}\right)\right] \\
& \operatorname{RINT15}=\sum_{q=0}^{q=\ell}\left[R_{i \ell q} \operatorname{XMPINT}\left(-\alpha_{i q}-\lambda_{d}+\lambda_{P 1}, T_{L}, T_{S L} \alpha_{i q}, T_{S}\right)\right.
\end{aligned}
$$

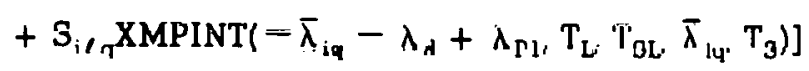

$$
\begin{aligned}
& \operatorname{RINT16}=\sum_{q=0}^{q=\ell}\left[L_{i \ell q} \operatorname{XPINT}\left(\lambda_{P 2}, T_{S 1}, T_{1}\right)-M_{i \ell q} \operatorname{XPINT}\left(-\bar{\lambda}_{i q}+\lambda_{P 2}, T_{S 1}, T_{I}\right)\right. \\
& \left.+\mathrm{N}_{\mathrm{i} \ell \mathrm{q}} \mathrm{XPINT}\left(-\alpha_{\mathrm{iq}}+\lambda_{\mathrm{P} 2}, \mathrm{~T}_{\mathrm{SI}}, \mathrm{T}_{1}\right)\right] \\
& \operatorname{RINTI7}=\sum_{q=0}^{q=\ell}\left[R_{i \ell q} \operatorname{XMPINT}\left(-\alpha_{\mathrm{iq}}+\lambda_{\mathrm{P}, 2} \mathrm{~T}_{\mathrm{L}} \mathrm{T}_{\mathrm{SL}} \alpha_{\mathrm{iq}} \mathrm{T}_{\mathrm{S}}\right)\right. \\
& \left.+S_{i \ell q} \text { XMPINT }\left(-\bar{\lambda}_{\text {iq }}+\lambda_{P 2}, T_{L}, T_{S L}, \bar{\lambda}_{i q}, T_{\varepsilon}\right)\right]
\end{aligned}
$$

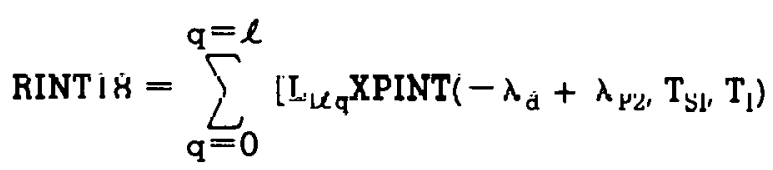

$$
\begin{aligned}
& -\mathrm{M}_{\mathrm{i} \mathrm{q}_{\mathrm{q}}} \operatorname{XPINT}\left(-\bar{\lambda}_{\mathrm{iq}}-\lambda_{\mathrm{d}}+\lambda_{\mathrm{P} 2}, \mathrm{~T}_{\mathrm{SI}}, \mathrm{T}_{\mathrm{I}}\right)
\end{aligned}
$$

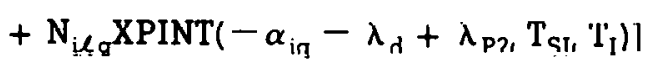

$$
\begin{aligned}
& \operatorname{RINT} I y=\sum_{q=0}^{q=\ell}\left[R_{i \ell q} X \operatorname{XMINT}\left(-\alpha_{i q}-\lambda_{d}+\lambda_{P 2}, T_{L}, T_{S L} \alpha_{i q^{\prime}} T_{S}\right)\right. \\
& \left.+\dot{s}_{\mathrm{i} \ell \mathrm{q}} \mathrm{XMPINT}\left(=\lambda_{\mathrm{iq}}-\lambda_{\mathrm{d}}+\lambda_{\mathrm{P} 2}, \mathrm{~T}_{\mathrm{L}} \mathrm{T}_{\mathrm{SL}} \overline{\bar{\lambda}}_{\mathrm{iq}} \mathrm{T}_{\mathrm{S}}\right)\right] \\
& \operatorname{RINT20}=\bar{A}_{i \ell} T_{S}-B_{i \ell} \sum_{q=0}^{q=\ell} E_{i \ell q} X P I N T\left(-\bar{\lambda}_{i q^{\prime}} T_{S}, 0\right) \\
& \operatorname{RINT21}=\sum_{\mathrm{q}=0}^{\mathrm{q}=\ell} \mathrm{G}_{\mathrm{iq} \mathrm{q}} \operatorname{XPINT}\left(-\bar{\lambda}_{\mathrm{iq}}, \mathrm{T}_{\mathrm{L}}-\mathrm{T}_{\mathrm{S}}, 0\right)
\end{aligned}
$$




$$
\begin{gathered}
\operatorname{XPINT}(a, b, c)=\left(e^{a b}-e^{a c}\right) / a \\
\operatorname{XMPINT}(a, b, c, d, e)=e^{d e}\left(e^{a b}-e^{a c}\right) / a
\end{gathered}
$$

\section{CONCENTRATIONS}

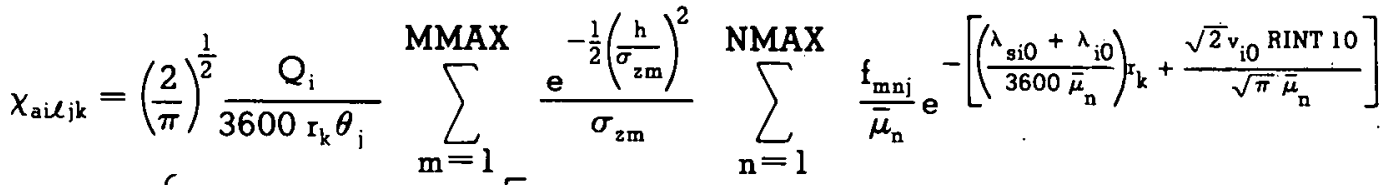

$$
\begin{aligned}
& \left\{\frac { \lambda _ { \mathrm { i } 1 } \lambda _ { \mathrm { i } 2 } \ldots \lambda _ { \mathrm { il } } } { 1 - \mathrm { e } ^ { - ( \overline { \lambda } _ { \mathrm { i } 0 \mathrm { r } _ { \mathrm { x } } / 3 6 0 0 \overline { \mu } _ { \mathrm { i } } ) } } } \left[\frac{1}{\overline{\bar{\lambda}}_{\mathrm{i} 1} \bar{\lambda}_{\mathrm{i} 2} \ldots \bar{\lambda}_{\mathrm{i} \ell}}-\bar{\lambda}_{\mathrm{i} 0}\right.\right. \\
& \left.\left.\sum_{q=0}^{q=\ell} \frac{(-1)^{q} e^{-\frac{\bar{\lambda}_{i q}{ }^{2} k}{3600 \bar{\mu}_{\mathrm{p}}}}}{\left(\bar{\lambda}_{\mathrm{iq}}-\bar{\lambda}_{\mathrm{iq}}\right)\left(\bar{\lambda}_{\mathrm{iq}}-\bar{\lambda}_{\mathrm{i} 1}\right) \ldots\left(\bar{\lambda}_{\mathrm{iq}}-\bar{\lambda}_{\mathrm{iq}-1}\right) \bar{\lambda}_{\mathrm{iq}}\left(\bar{\lambda}_{\mathrm{iq}+1}-\bar{\lambda}_{\mathrm{iq}}\right)\left(\bar{\lambda}_{\mathrm{iq}+2}-\bar{\lambda}_{\mathrm{iq}}\right) \ldots\left(\bar{\lambda}_{\mathrm{il}}-\bar{\lambda}_{\mathrm{iq}}\right)}\right]\right\} \\
& \chi_{\mathrm{bil}}=\bar{A}_{\mathrm{i} \ell}-\mathrm{B}_{\mathrm{i} \ell} \sum_{\mathrm{q}=0}^{\mathrm{q}=\ell} \mathbf{E}_{\mathrm{i} \ell \mathrm{q}} \mathrm{e}^{-\bar{\lambda}_{\mathrm{iq}}} \\
& x_{c i l}=\sum_{q=0}^{q=\ell} G_{i l q} e^{\left.-\bar{\lambda}_{i q}^{(i-T} S\right)}
\end{aligned}
$$

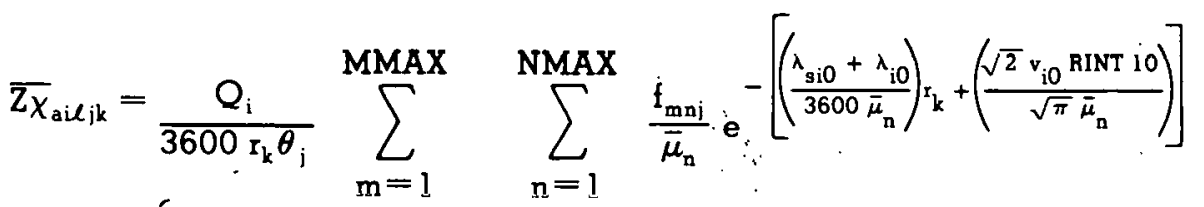

$$
\begin{aligned}
& \left\{\frac { \lambda _ { i 1 } \lambda _ { i 2 } \ldots \lambda _ { i l } } { 1 - e ^ { - ( \overline { \lambda } _ { i 0 } r _ { k } / 3 6 0 0 \overline { \mu } _ { n } ) } } \left[\frac{1}{\bar{\lambda}_{i 1} \bar{\lambda}_{i 2} \cdots \bar{\lambda}_{i l}}-\bar{\lambda}_{i 0}\right.\right. \\
& \left.\left.\sum_{q=0}^{q=\ell} \frac{(-1)^{q} e^{-\frac{\bar{\lambda}_{i q}{ }^{2}}{3600 \bar{\mu}_{n}}}}{\left(\bar{\lambda}_{i q}-\bar{\lambda}_{i \ell}\right)\left(\bar{\lambda}_{i q}-\bar{\lambda}_{i 1}\right) \ldots\left(\bar{\lambda}_{i q}-\bar{\lambda}_{i q-1}\right) \bar{\lambda}_{i q}\left(\bar{\lambda}_{i q+1}-\bar{\lambda}_{i q}\right)\left(\bar{\lambda}_{i q+2}-\bar{\lambda}_{i q}\right) \ldots\left(\bar{\lambda}_{i f}-\bar{\lambda}_{i q}\right)}\right]\right\}
\end{aligned}
$$$$
\mathrm{C}_{\mathrm{ai} / \mathrm{ik}}=\mathrm{H}_{\mathrm{i} / \mathrm{ik}}-\sum_{\mathrm{q}=0}^{\mathrm{q}=\ell} \mathrm{I}_{\mathrm{i} / \mathrm{qik}} \mathrm{e}^{-\alpha_{\mathrm{iq}}}
$$ 


$$
\begin{aligned}
& C_{b i l j k}=\sum_{q=0}^{q=\ell} J_{i \ell q i k} e^{\left.-\alpha_{i q}^{(t-T} S\right)} \\
& C_{c i l}=\sum_{q=0}^{q=\ell}\left[L_{i \ell q}-M_{i \ell q} e^{-\bar{\lambda}_{i q}^{t}}+N_{i \ell q} e^{-\alpha_{i q} t}\right] \\
& C_{d i \ell}=\sum_{q=0}^{q=\ell}\left[R_{i \ell q} e^{-\alpha_{i q}\left(t-T_{S}\right)}+S_{i \ell q} e^{\bar{\lambda}_{i q}^{\left(t-T_{S}\right)}}\right] \\
& A X_{a i l j k}=1.332 . \times 10^{14} \frac{\lambda_{a, l, k}}{\lambda_{j l}} \\
& \mathrm{~A}_{\chi_{\mathrm{bil}}}=1.332 \times 10^{14} \frac{\chi_{\mathrm{bil}}}{\lambda_{\mathrm{il}}} \\
& A \chi_{\mathrm{cil}}=1.332 \times 10^{14} \frac{\chi_{\mathrm{cil}}}{\lambda_{\mathrm{i} l}} \\
& \bar{A} \bar{Z}_{\chi_{a l i k}}=1.332 \times 10^{14} \frac{{\overline{Z_{X}}}_{a j \mathrm{jk}}}{\lambda_{\mathrm{i} \ell}} \\
& A C_{a i l j k}=1.332 \times 10^{14} \frac{C_{a i l j k}}{\lambda_{i \ell}} \\
& A C_{b i l j k}=1.332 \times 10^{14} \frac{C_{b i \ell j k}}{\lambda_{i \ell}} \\
& \mathrm{AC}_{\mathrm{ril}}=1.332 \times 10^{14} \frac{\mathrm{C}_{\mathrm{cil}}}{\lambda_{\mathrm{il}}} \\
& \mathrm{AC}_{\mathrm{di} l}=1.332 \times 10^{14} \frac{\mathrm{C}_{\mathrm{di} \ell}}{\lambda_{\mathrm{i} \ell}}
\end{aligned}
$$

\section{EXPRESSIONS}

$$
\begin{aligned}
& A_{i \ell}=\frac{\lambda_{i 1} \lambda_{i 2} \ldots \lambda_{i \ell}}{\bar{\lambda}_{10} \bar{\lambda}_{11} \ldots \bar{\lambda}_{\ell \ell}} \frac{Q_{i \ell}^{\prime}}{V_{a}} \\
& B_{i \ell}=\lambda_{i 1} \lambda_{i 2} \ldots \lambda_{i \ell} \frac{Q_{i \ell}^{\prime}}{V_{a}}
\end{aligned}
$$




$$
\begin{aligned}
& E_{i \ell q}=\frac{(-1)^{q}}{\left(\bar{\lambda}_{i q}-\bar{\lambda}_{i 0}\right)\left(\bar{\lambda}_{i q}-\bar{\lambda}_{i 1}\right) \ldots\left(\bar{\lambda}_{i q}-\bar{\lambda}_{i q-1}\right) \bar{\lambda}_{i q}\left(\bar{\lambda}_{i q+1}-\bar{\lambda}_{i q}\right)\left(\bar{\lambda}_{i q+2}-\bar{\lambda}_{i q}\right) \ldots\left(\bar{\lambda}_{i \ell}-\bar{\lambda}_{i q}\right)} \\
& G_{i \ell q}=\frac{\lambda_{i q+1} \lambda_{i q+2} \ldots \lambda_{i \ell}}{\left(\bar{\lambda}_{i q+1}-\bar{\lambda}_{i q}\right)\left(\bar{\lambda}_{i q+2}-\bar{\lambda}_{i q}\right) \ldots\left(\bar{\lambda}_{i \ell}-\bar{\lambda}_{i q}\right)} \\
& \left.\sum_{h=0}^{h=q} \frac{(-1)^{q+h} \lambda_{i h+1} \lambda_{i h+2 \ldots \lambda_{i q}}}{\left(\bar{\lambda}_{i q}-\bar{\lambda}_{i h}\right)\left(\bar{\lambda}_{i q}-\bar{\lambda}_{i h+1}\right) \ldots\left(\bar{\lambda}_{i q}-\bar{\lambda}_{i q-1}\right)} x_{b i h}\right|_{t=T_{S}}
\end{aligned}
$$

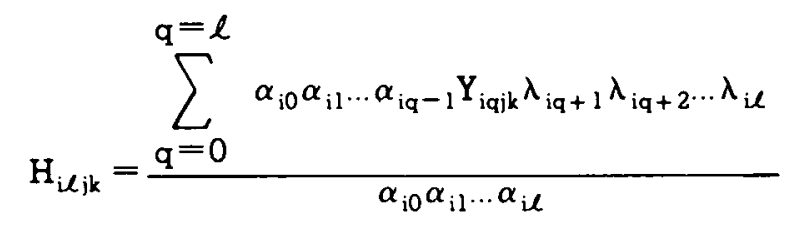

$$
\begin{aligned}
& I_{i \ell q j k}=(-1)^{\mathrm{q}} \sum_{h=0}^{h=q} \\
& \frac{(-1)^{\mathrm{h}} \mathbf{Y}_{\mathrm{ihjk}} \lambda_{\mathrm{ih}+1} \lambda_{\mathrm{ih}+2 \ldots \lambda_{\mathrm{il}}}}{\left(\alpha_{\mathrm{iq}}-\alpha_{\mathrm{ih}}\right)\left(\alpha_{\mathrm{iq}}-\alpha_{\mathrm{ih}+1}\right) \ldots\left(\alpha_{\mathrm{iq}}-\alpha_{\mathrm{iq}-1}\right) \alpha_{\mathrm{iq}}\left(\alpha_{\mathrm{iq}+1}-\alpha_{\mathrm{iq}}\right)\left(\alpha_{\mathrm{iq}+2}-\alpha_{\mathrm{iq}}\right) \ldots\left(\alpha_{\mathrm{il}}-\alpha_{\mathrm{iq}}\right)} \\
& \mathrm{J}_{\mathrm{i} \ell \mathrm{qjk}}=\frac{\lambda_{\mathrm{iq}+1} \lambda_{\mathrm{iq}+2 \cdots \lambda_{\mathrm{i} \ell}}}{\left(\alpha_{\mathrm{iq}+1}-\alpha_{\mathrm{iq}}\right)\left(\alpha_{\mathrm{iq}+2}-\alpha_{\mathrm{iq}}\right) \ldots\left(\alpha_{\mathrm{il}}-\alpha_{\mathrm{iq}}\right)} \\
& \left.\sum_{h=0}^{h=q} \frac{(-1)^{q+h} \lambda_{i h+1} \lambda_{i h+2} \ldots \lambda_{i q}}{\left(\alpha_{i q}-\alpha_{i h}\right)\left(\alpha_{i q}-\alpha_{i h+1}\right) \ldots\left(\alpha_{i q}-\alpha_{i q}-1\right)} C_{\text {aihjk }}\right|_{\mathrm{t}=\mathrm{T}_{S}} \\
& L_{i \ell q}-\frac{\gamma_{i q} A_{i q} \lambda_{i q+1} \lambda_{i q+2} \ldots \lambda_{i \ell}}{\alpha_{i q} \alpha_{i q+1} \ldots \alpha_{i \ell}} \\
& M_{i \ell q}=\sum_{h=q}^{h=\ell} \frac{\gamma_{i h} B_{i h} E_{i h q} \lambda_{i h+1} \lambda_{i h+2 \ldots \lambda_{i \ell}}}{\left(\alpha_{i h}-\bar{\lambda}_{i q}\right)\left(\alpha_{i h+1}-\bar{\lambda}_{i q}\right) \ldots\left(\alpha_{i \ell}-\bar{\lambda}_{i q}\right)} \\
& N_{i q_{q}}=\frac{(-1)^{q+1} \lambda_{i q+1} \lambda_{i q+2} \ldots \lambda_{i l}}{\left(\alpha_{i q+1}-\alpha_{i q}\right)\left(\alpha_{i q+2}-\alpha_{i q}\right) \ldots\left(\alpha_{i l}-\alpha_{i q}\right)} \\
& \sum_{h=0}^{h=q} \frac{(-1)^{h} \gamma_{i h} \lambda_{i h+1} \lambda_{i h+2} \ldots \lambda_{i q}}{\left(\alpha_{i q}-\alpha_{i h}\right)\left(\alpha_{i q}-\alpha_{i h+1}\right) \ldots\left(\alpha_{i q}-\alpha_{i h-1}\right)}
\end{aligned}
$$




$$
\left[\frac{A_{i h}}{\alpha_{i q}}-B_{i h} \sum_{p=0}^{p=h} \frac{E_{i h p}}{\alpha_{i q}-\bar{\lambda}_{i p}}\right]
$$

$$
\begin{aligned}
& R_{i \ell q}=\frac{\lambda_{i q+1} \lambda_{i q+2} \ldots \lambda_{i \ell}}{\left(\alpha_{i q+1}-\alpha_{i q}\right)\left(\alpha_{i q+2}-\alpha_{i q}\right) \ldots\left(\alpha_{i \ell}-\alpha_{i q}\right)} \\
& \sum_{h=0}^{h=q}(-1)^{q+h}\left[\left.\frac{\lambda_{i h+1} \lambda_{i h+2 \ldots \lambda_{i q}}}{\left(\alpha_{i q}-\alpha_{i q-1}\right)\left(\alpha_{i q}-\alpha_{i q}-2\right) \ldots\left(\alpha_{i q}-\alpha_{i h}\right)} C_{c i h}\right|_{t=T_{S}}\right. \\
& -\frac{1}{\alpha_{i q}-\bar{\lambda}_{i h}}\left\{\left.\sum_{p=0}^{p=h} \frac{(-1)^{h 1} \lambda_{i p \neq 1} \Lambda_{i p+2} \ldots \lambda_{i h}}{\left(\bar{\lambda}_{i h}-\bar{\lambda}_{i p}\right)\left(\bar{\lambda}_{i h}-\bar{\lambda}_{i p+1}\right) \ldots\left(\bar{\lambda}_{i h}-\bar{\lambda}_{i h-1}\right)} x_{b i p}\right|_{t=T_{S}}\right\} \\
& \left\{\sum_{p=h}^{p=q} \frac{(-1)^{h+p} \gamma_{i p} U_{i p h} \lambda_{i p+1} \lambda_{i p}+2 \ldots \lambda_{i q}}{\left(\alpha_{i q}-\alpha_{i q-1}\right)\left(\alpha_{i q}-\alpha_{i q}-2\right) \ldots\left(\alpha_{i q}-\alpha_{i p}\right)}\right\} \\
& S_{i \ell q}=\left[\sum_{h=q}^{h=\ell} \frac{\gamma_{i h} U_{i h q} \lambda_{i h+1} \lambda_{i h+2} \ldots \lambda_{i \ell}}{\left(\alpha_{i h}-\bar{\lambda}_{i q}\right)\left(\alpha_{i h+1}-\bar{\lambda}_{i q}\right) \ldots\left(\alpha_{i \ell}-\bar{\lambda}_{i q}\right)}\right] \\
& {\left[\left.\sum_{h=0}^{h=q} \frac{(-1)^{q+h} \lambda_{i h+1} \lambda_{i h+2 \cdots \lambda_{i q}}}{\left(\bar{\lambda}_{\text {iq }}-\bar{\lambda}_{\text {ih }}\right)\left(\bar{\lambda}_{\text {iq }} \cdots \bar{\lambda}_{\text {ih }+1}\right) \ldots\left(\bar{\lambda}_{\text {iq }}-\bar{\lambda}_{\text {iq }-1}\right)} x_{h i h}\right|_{t-T_{S}}\right]}
\end{aligned}
$$

$$
U_{i \ell q}=\frac{\lambda_{i q+1} \lambda_{i q+2 \ldots \lambda_{i \ell}}}{\left(\bar{\lambda}_{i q+1}-\bar{\lambda}_{i q}\right)\left(\bar{\lambda}_{i q+2}-\bar{\lambda}_{i q}\right) \ldots\left(\bar{\lambda}_{i \ell}-\bar{\lambda}_{i q}\right)}
$$

$Y_{\text {iqjk }}=3600 v_{\text {iq }} \chi_{\text {aiqjk }}+\lambda_{\text {siq }} \overline{Z \chi}_{\text {aiqjk }}$

\section{PARAMETERS}

$$
\begin{aligned}
& \Delta r_{v}=\frac{r_{v+1}-r_{v}}{d_{v}} \\
& \alpha_{i q}=\lambda_{i q}+\lambda_{w} \\
& \gamma_{\text {iq }}=3600 v_{i q}+\lambda_{\text {siq }} Z \\
& \bar{\lambda}_{\text {iq }}=\lambda_{\text {iq }}+\lambda_{\text {siq }}+3600 v_{\text {iq }} \frac{\AA_{a}}{V_{a}}
\end{aligned}
$$




$$
\begin{aligned}
& \lambda_{\mathrm{s}}=\frac{\mathrm{R}_{\mathrm{s}} \mathrm{S}_{\mathrm{s}}}{\mathrm{Z}_{\mathrm{s}}} \\
& \omega_{\mathrm{vm}}=\frac{\ln \left(\frac{\sigma_{2 \mathrm{v}+1 \mathrm{~m}}}{\left.-\frac{\sigma_{2 \mathrm{~mm}}}{\sigma}\right)}\right.}{\ln \left(\frac{\mathrm{r}_{\mathrm{v}+1}}{\mathrm{r}_{\mathrm{v}}}\right)} \\
& \sigma_{\mathrm{zkm}}=\sigma_{\mathrm{zvm}}\left(\frac{r_{\mathrm{k}}}{r_{\mathrm{v}}}\right)^{\omega_{\mathrm{vm}}} \\
& Q_{i l}^{\prime}=\lambda_{i 0}\left\{\frac{Q_{i \ell-1}^{\prime}}{\lambda_{i 0}}-\frac{1}{\lambda_{i \ell}} \sum_{k=1}^{\operatorname{KMAX}} \sum_{j=1}^{J \operatorname{MAX}} \frac{Y_{i \ell j k} \theta_{i}}{2}\left[\left(\frac{r_{k+1}+r_{k}}{2}\right)^{2}-\left(\frac{r_{k}+r_{k}-1}{2}\right)^{2}\right]\right\}
\end{aligned}
$$


APPENDIX C

AIRWAY INPUT DESCRIPTION 


\section{APPENDIX C}

\section{AIRWAY INPUT DESCRIPTION}

This appendix contains a detailed description of all the input necessary to run the AIRWAY computer program. Sufficient information is provided to define all the data which is required, the format in which it is to be presented and the units to be used. In addition, the default values assumed by the program if the user chooses to omit certain portions of the input data are specified.

\section{A. GENERAL INPUT DESCRIPTION}

The data necessary to run the AIRWAY program falls into two basic categories. The first of these is data which can be thought of as basic file information which does not vary from problem to problem. Data such as dose commitment conversion factors and decay chain information fall into this category. The second basic input data category contains data which are different from problem to problem or are only a few pieces of information which can be treated as default values. The AIRWAY program is written such that all information necessary to run a given problem can be read from the input stream (i.e., from input cards). However, an option is provided which permits certain portions of the input data to be read from a userdefined file. The AIRWAY program will first scan such a data file if provided by the user to obtain specific pieces of the input data and then obtain the remainder of the data from the input cards. If a given piece of input data is provided on both the data file and on the input cards, the information presented on the input cards will be used. Those portions of the input data which can be obtained from a data file in addition to the input cards will be identified in the detailed inpul description which follows. It should be noted that the format of the input information provided in a data file is identical to the format of the data on an input card.

Input data is provided on logical cards where a logical card consists of one or more physical cards. If more than one physical card is required then each continuation card is identified by a plus sign $(+)$ as the first non-blank character on a card. In this case all data on the card beyond the plus sign is treated as a continuation of the preceding card. The data on a physical card will extend to the end of the card (column 80 ) or until a dollar sign (\$) which is used to define the end of useful information on a physical card is encountered. Any information beyond the dollar sign will be ignored by the AIRWAY program. There are two other types of cards besides those containing input data. These are title cards which are identified by an equals $\operatorname{sign}(=)$ as the first non-blank character on a physical card and comment cards which are identified by an asterisk (") as the first non-blank character on a physical card. The information on title cards and comment cards is used for descriptive and definitive purposes only.

Input cards are subdivided into fields with each field containing one piece of numeric data which is stored in one computer word or a set of alphanumeric data which is stored in one or more computer words. Each field on an input card is delineated by commas (,) with the beginning and end of a logical card being treated as field delimiters. If the last field on a logical card is entirely blank it will be ignored.

A field is assumed to contain numeric information if the only characters it contains are the numerals $0-9$, blanks, plus signs $(+)$, minus signs $(-)$ or decimal points (.). If the field contains any other characters it is considered to be a Hollerith (alphanumeric) field. If the first non-blank character in a field is a left parenthesis and the last character in a field is a right parenthesis or the absolute delimiter $(\$)$, then all characters between the parentheses are treated as a set of one or more Hollerith words. This is the way in which a Hollerith word which contains only characters which are acceptable in numeric fields may be defined. Leading and trailing blanks in a Hollerith field are ignored unless the field is enclosed by parentheses in which case all characters within the parentheses are used as part of the Hollerith field. Hollerith fields are packed into computer words which contain 10 characters each. The characters in the last computer word used to store the data in a Hollerith field are left adjusted and the remainder of the word is filled with blanks.

A numeric field is considered to contain a real number if it contains a decimal point (.) and/or an embedded exponent sign ( + or - ). If a numeric field contains neither a decimal point nor an embedded exponent sign it is considered to contain an integer number. It a field containing a real number contains only an embedded exponent sign, the decimal point is assumed to be to the left of the first number character $(0-9)$ in the field. A numeric field which contains only a decimal point will be treated as a real zero and a numeric field which contains only blanks (except for the last field on a logical card) will be considered to 
be an integer zero. All blanks will be ignored in a numeric field and all leading zeros will be ignored except in a field containing a real number without a decimal point. A numeric field which contains more than one decimal point and/or more than one embedded exponent sign will be flagged as an error. In addition, a numeric field containing an embedded exponent sign must contain at least one number character (0-9) prior to and subsequent to the exponent sign.

Any field may be repeated if the first non-blank characters in the field are an integer number enclosed by slashes (/). The field to be repeated follows the second slash and the number of times it is to be repeated is defined by the integer number which is enclosed by the slashes.

The AIRWAY program can run more than one case in a job. The end of a case is indicated by a card which contains a slash $(/)$ as the only non-blank character on it and the end of the last case in a job is indicated by a card which contains a period (.) as the only non-blank character.

The first field on each input data card (i.e., all cards except title and comment cards) will contain an integer card number. All remaining fields on each data card will contain input data for the AIRWAY prngram If a set of cards defining the input data for a given case contains more than one input data card with the same card number, the information on the last card encountered with the same card number will be used as the input data for the AIRWAY program. If the last card encountered with the same card number contains only the card number, then no data from this card will be read from input for this case. The information on any physical card may start in column 1 and extend through column 80 .

\section{B. DETAILED INPUT DESCRIPTION}

Title Card-This card must contain an equals sign $(=)$ as the first non-blank character which is then followed by any desired descriptive information.

Comment Cards-These cards must contain an asterisk (") as the first non-blank character which may be followed by any desired descriptive information. Comment cards may be interspersed in any place among the input data cards.

Card \#0-This card is to provide the necessary information pertaining to the basic data file which contains data which does not vary from problem to problem. Since the basic data file is read only in the first case of a job, this card should only be provided in the first case. If this card is prnviden in any subsequent cases it will be ignored. The card contains the following two words.

Word 1-The name of the system file which conlains the basic data to be used in all the cases of this job. If this card is not provided as input, a default system file name of "BASDAT" will be used. This word is to be in Hollerith format and is to contain six alphanuviesic chataclers ur less.

Word 2-The contents of this word are used to define whether the information in the hasir data file is tn he printed or not. If thio word io oet to an integei l, all lle data cards in the basic data file will be printed. If this word is set to an integer 0 or is omitted the information in the basic data file will not be printcd.

Card \#1-The data defining the time at which the releases stop and the times at the beginning and end of the interval over which the dose commitments are to be obtained are provided on this card. The times on this card are to be measured from the time at which releases start which is considered to be time zero. This card contains 3 words each in real format.

Word $1-$ The time $T_{I}$ measured in hours from the time that releases start of the beginning of the exposure to the environment resulting from the releases being considered in this case is provided in this word. If the value of this word is less than zero, it will be changed to zero. If this card is omitted a default value of 254040.0 hours (approximately 29 years) will be used for this word 
Word 2-The time $\mathrm{T}_{\mathrm{S}}$ that the releases stop measured in hours from the time that releases start is supplied in this word. If this and the following word on this card are omitted a default value of 262800.0 hours (approximately 30 years) will be used for this word. If a negative or zero value is provided in this word, a message to that effect will be printed and the job aborted after checking the remainder of the input.

Word 3-This word contains the time $\mathrm{T}_{\mathrm{L}}$ of the end of the exposure to the environment resulting from the releases considered in this case. This time value is measured in hours from the time that releases start. If this word is omitted from the card a default value of 867240.0 hours (approximately 99 years) will be used. If this value is less than the value specified for word l (time of the beginning of the dose commitment interval) on this card, a message to that effect will be placed in the output and the case aborted after checking the remainder of the input.

Card \#2-This card contains the height of the releases, the radius of the immediate region surrounding the release point and the area and volume of the overall region affected by gaseous releases. Four pieces of information in real format are provided on this card. If any of these four words contain a negative number a message defining that fact will be written in the output and the case terminated after checking the remainder of the input.

Word 1 -The height $h$ in meters above the ground at which the releases occur is provided in this word. If this card is omitted, a default value of 100 meters will be used for this stack height.

Word 2-This word contains the radial distance RMAX measured in meters from the release point to the outer edge of the region which is considered to be the immediate neighborhood of the release point. It is within this region that dose commitments and air and ground concentrations are to be obtained at specific radial and azimuthal positions. If this word and all following words on the card are not provided as input, a default value of 80500.0 meters (approximately 50 miles) will be used for this word.

Word 3-The area of the overall region affected by the release of gaseous radionuclides is provided in square meters in this word. If this and the following word on the card are not supplied as input, a default value of $5.0 \times 10^{14}$ square meters (approximately the surface area of the earth) will be used for this word.

Word 4-This word contains the volume of the atmosphere of the overall region affected by the release of gaseous radionuclides in cubic meters. If this word is omitted from the input, a default value of $2.0 \times 10^{18}$ cubic meters (approximately the volume of the earth's atmosphere) will be used.

Card \#3-Tho information necessary tn define the resuspension factor is provided on this card. A total of three pieces of data expressed as real numbers are presented on this card. If any of these three words are negative, a message to that effect will be put into the output and the job aborted after checking the rest of the input data.

Word 1 -This word contains the constant term $\mathrm{K}_{\mathrm{o}}$ used in the expression which defines the resuspension factor. The units of this term are in inverse meters (i.e., $\mathrm{m}^{-1}$ ). If this card is omitted from the input a default value of $1.0 \times 10^{-9} \mathrm{~m}^{-1}$ will be used for this word. 
Word 2-The coefficient $\mathrm{K}_{1}$ of the time-dependent term used in the expression which defines the resuspension factor is provided in this word in units of inverse meters $\left(\mathrm{m}^{-1}\right)$. If this word and the following word are omitted from this card, a default value of $1.0 \times 10^{-5} \mathrm{~m}^{-1}$ will be used for this word.

Word 3-The decay constant $\lambda_{d}$ in inverse hours $\left(\mathrm{hrs}^{-1}\right)$ used in the time-dependent term of the expression for the resuspension factor is supplied in this word. If this word is not provided as input, a default valuo of $5.7775 \times 10^{-4} \mathrm{hrs}^{-1}$ (approximately a 50-day half life) will be used.

Card \#4-The data defining the ground loss constant and the breathing rate for this case are supplied on this card in the form of real numbers. If either of these two words is input as a negative number, a message to this effect will be printed and the case terminated after the remainder of the input data is checked.

Word 1 -The ground loss constant $\lambda_{\sigma}$ in inverse hours $\left(\mathrm{hrg}^{-1}\right)$ which deficues the rate at which deposited material is removed from the surface of the earth due to weathering, erosion, migration, etc. is provided in this word. If this card is not included as input, a default value of $8.78 \times 10^{-6}$ (approximately a nine-year half life) is used for this word.

Word 2-The rate at which a person inhales air $B_{a}$ in cubic meters per hour is supplied in this word. If this word is omitted from the card, a default value of $0.833 \mathrm{~m}^{3} / \mathrm{hr}$ will be used for this breathing rate.

Card \#5-The total population in the overall region affected by gaseous releases and the population growth constants for the region in the immediate vicinity of the release point and for the overall region affected by the gaseous releases are provided on this card. A total of three words each provided as a real number are supplied on this card.

Word 1-The total population in the overall regions affected by the release of gaseous radionuclides is proyided in this word If this card is not provided in the inpul dala, a delaull value of $4 \times 10^{3}$ (approximately the world's population) will be used for this word.

Word 2-The growth constant $\lambda_{\mathrm{Pl}}$ of the population in the immediate neighborhood of the release point (i.e., within RMAX of the release point) expressed in units of inverse houis $\left(\mathrm{lus}^{-1}\right)$. If this and the following word are not included on the card, a default value of 0.0 ( $\mathrm{nn}$ grnwth) will be used.

Word 3-This word contains the growth constant $\lambda_{P 2}$ of the population in the overall region affected by gaseous releases. This growth constant is provided in units of inverse hours $\left(\mathrm{hrs}^{-1}\right)$. If this word is not suppliod on this card, a defaull value of 0.0 will be used.

Card \#6-This card contains the information necessary to define the population and the size of the overall region affected by the release of particulates. $\mathbf{A}$ total of four words are presented in this card. Each of these words is to be expressed as a real number. If any of the first three words specified on this card is negative or if any of these first three words is greater than its default value, a message to this effert will be written in the output and all four words will be set equal to their default values.

Word 1-The total population in the overall region affected by the release of particulate radionuclides is supplied in this word. If this card is omitted a default value equal to the total population specified on Card \#5 for the overall region affected by the release of gaseous nuclides will be used for this word. 
Word 2-The second word on this card contains the area in square meters of the overall region affected by the release of particulate radionuclides. If this and all following words on the card are not provided as input, a default value equal to the area specified on card \#2 of the overall region affected by gaseous releases will be used.

Word 3-The atmospheric volume in cubic meters of the overall region affected by the release of particulate nuclides is supplied in this word. If this and the following word on the card are not included in the input, a default value equal to the atmospheric volume specified on card \#2 of the overall region affected by the gaseous releases will be used for this word.

Word 4-The final word on this card contains the population growth constant in inverse hours $\left(\mathrm{hrs}^{-1}\right)$ in the overall region affected by the release of particulates. If this word is omitted a default value equal to the population growth constant provided by word 3 on card \#5 for the overall region affected by the release of gaseous nuclides will be used for this word.

Card \#7-All of the information necessary to define the precipitation scavenging coefficient is provided on this card. A total of three words each expressed as a real number are to be supplied on this card. If any of the values provided on this card are negative, the negative value will be replaced with the default value and a message to this effect written.

Word 1-The first word on this card contains the rate $R_{s}$ at which precipitation in the form of rain occurs. If a portion of the precipitation is in the form of snow, sleet, etc., it should be changed into an equivalent a mount of rainfall and included in the precipitation rate. This value is to be provided in the units of meters per hour. If this card is not provided as part of the input data, a default value of $1.152 \times 10^{-4} \mathrm{~m} / \mathrm{hr}$ (equivalent to $40 \mathrm{in} / \mathrm{yr}$ of rain) will be used for this word.

Word 2-The height (depth) $Z_{s}$ of the rain-bearing layer of the atmosphere is provided on this card expressed in meters. If this word and the one following are not supplied on this card or if a value of zero is provided, a default value of 4000.0 meters will be used for this word.

Word 3-The scavenging ratio by volume $S_{s}$ (unitless) which is to be used to define the precipitation scavenging coefficient is provided as the last piece of data on the card. If this word is omitted from the card, a default value of $4.2 \times 10^{5}$ will be used.

Card \#8-The quantities of food (plants, milk and beef) consumed by each me mber of the food ingesting population and produced within the regions considered to be affected by the releases being examined in this case are input on this card. A total of three words each of which is expressed as a real number are provided. If any of these values is negative it will be replaced by its default value which is the average quantity of that food consumed per day.by an adult and a message to this effect will be written.

Word 1-The mass of food in plant form $q_{1}$ in grams per day that each member of the food ingesting population consumes is provided in this word. If this card is not provided as input, a default value of $250.0 \mathrm{gm} /$ day will be used for this word.

Word 2-The second word on this card contains the volume of milk $q_{2}$ in liters per day consumed by each member of the food-ingesting population. If this word and 
the following word on this card are not supplied as input, a default value of 1.0 liters/day will be used for this word.

Word 3-The quantity of meat $q_{3}$ in grams per day that each person of the foodingesting population eats is input as the last word on this card. A default value of $300.0 \mathrm{gm} / \mathrm{day}$ will be used for this word if it is not supplied as part of the input.

Card \#9-This card contains two words defining the density and the depth of the soil which has been sub jected to deposition of radionuclides and then used to produce food. These values are to be specified as real numbers. If either of these values is equal to or less than zero it will be replaced by its default value and a message stating that such a substitution was made printed in the output.

Word 1 -The density $\rho_{\mathrm{s}}$ in $\mathrm{gm} / \mathrm{cm}^{3}$ of the soil which had been subjected to radionurlide tapositinn prior to boing uned to produce food eropos is yiven as the first word on thio oard. If this eard is omitted fium the inpul this word will be defaulted to $1.5 \mathrm{gm} / \mathrm{cm}^{3}$.

Word 2-The depth $d_{s}$ in $\mathrm{cm}$ of the soil in which the deposited radioactive material is assumed to be distributed and available for uptake into the plants is provided in this word. A default value of $15.0 \mathrm{~cm}$ is used for this word if it is not supplied as input.

Card \# 10-'l'he radial distances in the immediate neighborhood of the release point at which dose commitments are to be calculated are provided on this card. Each word on this card will be checked to ensure that it lies between 1.0 and RMAX (the radial distance to the outer edge of the region immediately surrounding the release point specified in word 2 on card \#2). Any value which lies outside of this range will be ignored. If all values specified on this card lie outside the range between 1.0 and RMAX or if this card is not provided as input, the following set of 10 radial distances will be used- $805.0 \mathrm{~m}, 2415.0 \mathrm{~m}, 4025.0 \mathrm{~m}$, $5635.0 \mathrm{~m}, 7245.0 \mathrm{~m}, 12075.0 \mathrm{~m}, 24150.0 \mathrm{~m}, 40250.0 \mathrm{~m}, 50350 \mathrm{~m}$ and $72450.0 \mathrm{~m}$.

Words $1,2, \ldots$ KMAX-Each word on this card will contain a radial distance mcasured in meters from the release point at which dose commitment calculations are to be made. Each word on this card is to be expressed as a real number.

Card \# 11-This card contains the information necessary to define whether the food-ingesting population within the immediate vicinity of the release point consumes food which is produced throughout the region surrounding the release point or they consume food which is produced locally (i.e., within a few hundred meters of their residence). This card also contains the data defining the times at which deposition on food crops begins and ends.

Word $1-$ This word contains an integer number which is used to define the source of the food which is ingested by the people in the vicinity of the release point. If this word is a non-zero integer each member of the food ingesting population in the immediate vicinity of the release point will consume food which is produced through the region immediately around the release point. If this word is a zero each member of the food-ingesting population in the immediate neighborhood of the release point will consume fond which is produced within a few hundred meters of his residence. A default value of zero (i.e., people eat locally grown food) will be used for this word if this card is omitted from the input data.

Word 2-The second word on this card contains the time at which the deposition on the food crops begins. This value is measured in hours from the time at which releases begin and is expressed as a real number. A default value of 0.0 (the 
time at which releases start) will be used for this word if a negative value is provided in the input or if this word and the one following it are not provided as input.

Word 3-The time at which the deposition of radionuclides on the food crops ends is provided in the last word on this card. This value is measured in hours from the time at which releases start and is expressed as a real number. If this word is negative or if it is omitted from the input a default value equal to $\mathrm{T}_{\mathrm{L}}$ (the time at the end of the interval at which dose commitments are to be obtained and specified in word 3 on card \#1) will be used.

Card \#12-This card contains information which is used by the AIRWAY program to define when a number can essentially be considered zero and to define when a daughter in the decay chain of a parent nuclide can be ignored. This card contains two pieces of information each of which is to be sepcified as a real number.

Word 1-This word contains the number which the AIRWAY program uses to define air and ground concentrations which, if less than this number, may be considered to be zero. A default value of $1.0 \times 10^{-150}$ is used if this card is omitted from the input.

Word 2-The second word on this card contains the number which defines which of the daughters in the decay chain of a radionuclide are to be examined in this case. If the yield of a daughter per decay of its precursor in the decay chain is less than the value specified in this word, that daughter will not be considered in this case. If this word is not supplied as a piece of input data, a default value of 0.0001 will be used.

Card \#15-This card contains a set of multiplying factors which may be used to adjust the final dose commitments. One factor is supplied for each of the pathways by which radiation in the environment may reach man. If any of the values provided on the card are negative or if any of the words on the card are omitted, a default value of 1.0 will be used as the multiplying factor for that word. Each word on this card is expressed as a real number.

Word 1-The multiplying factor to be applied to the dose commitments from the immersion pathway is provided in this word. This factor is applied to the immersion dose commitment for each radionuclide released into the atmosphere and for each daughter of each released radionuclide.

Word 2-The multiplying factor to be applied to each dose commitment from the surface deposit pathway is supplied in this word.

Word 3-This word contains the multiplying factor which is to be applied to the ingestion dose commitments arising from the direct deposition of the released radionuclides and their daughters on the food crops.

Word 4-This word contains the multiplying factor which is to be applied to the ingestion dose commitments associated with consuming food which is produced in soil that has been subjected to previous deposition of the released radionuclides and their daughters.

Word 5-The multiplying fartor which is to be applied to the dose commitments due to the inhalation of the released radionuclides and their daughters prior to the initial deposition of these nuclides is provided in this word. 
Word 6-This word contains the multiplying factor which is to be applied to the dose commitments resulting from the inhalation of the radionuclides (both released parents and their daughters) which have been deposited and then resuspended into the air.

Card \#20-The widths of the azimuthal sectors into which the region immediately surrounding the release point is subdivided are provided on this card. The number of sectors into which the immediate neighborhood of the release point is defined by the number of words which are supplied on this card as input. Dose commitment calculations are made in each of these azimuthal sectors at each of the radial positions specified on card \# 10. The azimuthal sector widths on this card are to be specified in radians and each value is to be expressed as a real number. If this card is omitted entirely from the input data, the AIRWAY program will divide the region in the immediate vicinity of the release point into 16 sectors of equal width of 0.3927 radian as a default. If any of the specified input values on this card are less than or equal to zero, the program will use the default values and print a message to this effect in the output. A comparison of the sum of all the values supplied on this card with $2 \pi$ (the number of radians in a rircle) is also mado by the program. If the sum of the widths of the sectors provided on this card is not within $1 \%$ of $2 \pi$, the difference between the sum and $2 \pi$ is distributed (added if the sum is less than $2 \pi$ and removed if the sum is greater than $2 \pi$ ) uniformly among the sectors.

Words 1, 2,... JMAX-Each word on this card will contain the width in radians of one of the sectors into which the region immediately surrounding the release point is subdivided.

Card \#30-This card is used to define the fraction of the time during releases that the wind is blowing into the azimuthal sectors whose widths are specified on card \#20. This card is to contain the same number of words as card \#20 and there is a one to one correspondence between the data on this card and the sector widths on card \#20. Thus, the first word on this card is to be the fraction of the time that the wind is blowing into the sector whose width is given in the first word on card \#20, the second word on this card contains the fraction of the time that the wind is blowing into the sector whose width is specified on the second word on card \#20, etc. If this card contains more values than there are sectors defined via card \#20, the excess values on this card will be ignored. If there are fewer values sperified on this card than thcre are sectors defined by card \#20, the missinq values will be set equal in the last value supplied on this card. Eoch word un the card will be checked to ensure that it is not negative and any negative valun will be repleced by 0.0 . A message will be written in the output defining any input values which have been ignored or modified. Since this card contains the fraction of the time during releases that the wind is blowing into each of the sectors that the region immediately surrounding the release point is divided, the sum of all the values provided on this card should equal 1.0. To ensure that those fractions as used by the AIRWAY prngram do sum to 1.0 , each value obtained from this card will be divided hy the values of the sum of all valucs obtained from this card. A message will be placed in the output if the sum of the values supplied on this card is not within $1 \%$ of the expected value of 1.0 . The omission of this card from the input implies that the necessary information to define the fraction of the time that the wind is blowing into a given sector is supplied in combination with other fractional data in the card series which starts with card \#301.

Words 1, 2,... JMAX-Each word on this card contains the fraction of the time during releases that the wind is blowing into the sectors defined on card \#20. Each of these words is to be expressed as a real number.

Card \#35-This card is used to contain the fraction of the time during releases that a given meteorological stability category exists. The information on this card is to be given such that the first word on this card defines the fraction of the time that the meteorological stability category whose standard deviation of vertical dispersion data is supplied on card \#201 exists, the second word defines the fraction of the time that the stability category whose vertical dispersion standard deviation data is provided on card \#202 exists, etc. In general, there should be as many words on this card as there are cards in the series that starts with card \#201. If there are fewer values provided on this card than there are meteorological stability categories defined by the card \#201 series, only the stability categories defined by this card will be used in this case. 
However, if there are more values supplied on this card than there are stability categories idenfified via the card series starting with card \#201, the excess values provided on this card will be ignored. Each word on this card will be checked to ensure that it is not negative and any negative values will be set to 0.0 . A message will be written in the output defining any input values which have been so modified. Since this card contains the fraction of the time during releases that each of the meteorological stability categories considered exist, the sum of all the values provided on this card should equal 1.0. To ensure that this sum is equal to 1.0 , each value obtained from this card will be divided by the sum of all the values obtained from this card. A message will be written in the output if the sum of the values obtained from this card is not within $1 \%$ of the expected value of 1.0. The omission of this card from the input implies that the information necessary to define the fraction of the time that the various meteorological stability categories considered exist is given in combination with other information in the card series which starts with card \#301.

Words 1, 2,... MMAX-Each word on this card contains the fraction of the time during releases that a given meteorological stability class exists. Each of the words on this card is to be expressed as a real number.

Card \#40-Multiplication factors are provided on this card which permit the deposition velocity data supplied in the 1001 card series to be modified. There is a one to one correspondence between the words on this card and the cards in the series which begins with card \#1001. Thus, the first word on this card is the factor by which the deposition velocity provided on card \#1001 is to be multiplied, the second word on this card is the factor by which the deposition velocity provided on card \#1002 is to be multiplied, etc. If any of the words on this card are negative they will be replaced by a 1.0 and a message to this effect placed in the output. There should be as many words on this card as there are cards in the series that starts with card \#1001 (i.e., one for each nuclide for which basic data is provided and thus which may be considered in this problem). If there are fewer words on this card than there are cards in the 1001 card series, the last word specified on this card will be used as the deposition velocity multiplication factor for all the remaining isotopes described in the 1001 card series. If there are more values provided on this card than there are isotopes described via the card series starting with card \#1001, the excess values will be ignored. If this card is omitted from the input data, a default value of 1.0 will be provided as the multiplication factor to be applied to the deposition velocity of each of the nuclides described via the card series starting with card \#1001.

Words 1, 2, .. IMAX-Each word on this card will contain a multiplication factor which is to be applied to the deposition velocity of a given radionuclide. Each word on this card is to be expressed as a real number.

Card \#45-An alternate means of supplying the deposition velocity multiplication factors for each of the nuclides which can be considered in this proble $m$ is provided via this card. The information on this card is presented in doublets-one word identifying the nuclide whose deposition velocity is to be modified and the other to define the multiplication factor. The information provided on this card will override the data given on card \#40 or the default values of 1.0 if card \#40 is not provided as input. If an odd number of words is slipplied on this card, the last value will be ignored.

Words $1,3,5, \ldots$-The first word of each doublet on the card is the alphanumeric identifier of the nuclide whose deposition velocity is to be modified by the multiplying factor given in the second word of the doublet. If this word cannot be correlated with a nuclide identifier in the card series starting with card \#1001, the data in this doublet will be ignored and a message to this effect put into the output. The first word of each doublet on this card is to be in Hollerith format and is to contain a maximum of 10 characters.

Words $2,4,6, \ldots$-The second word of each doublet on this card contains the multiplying factor which is to be applied to the deposition velocity of the radionuclide identified by the first word of the doublet. If this word is negative, it will be replaced by a 
1.0 and a message to this effect will be written in the output. This second word of each doublet on this card is to be expressed as a real number.

Card \#50-This card contains the radionuclide release rate data $\mathrm{Q}$. The data on this card is presented such that there is a one to one correspondence between the release rates on this card and the radionuclides described by the card series which begins with card \#1001. Thus, the first word on this card is the release rate of the radionuclide which is described by the information on card \#1001, the second word on this card is the release rate of the radionuclide which is described by the data on card \#1002, etc. If this card contains fewer words than there are nuclides described by the 1001 card series, the remaining nuclide release rates which are unspecified will be defaulted to 0.0 . If this card supplies more release rate values than there are nuclides which can be examined in this problem, the excess release rates from this card will be ignored. Any negative release rate values specified on this card will be replaced by 0.0 . An output message will be written if any word on this card is ignored or modified. If neither this card nor card \#55 are provided as input, this case will be aborted after checking the remainder of the input data.

Words 1, 2, ...-Each word supplied on this rard is the release rate of a given radionuelid where the $\mathrm{i}^{\text {th }}$ word on this card is the release rate of the $\mathrm{i}^{\text {th }}$ radionuclide described via card $\# 1000+i$. Each release rate on this card is given in units of curies/hour and is expressed as a real number.

Card \#55-This card may be used to provide an alternate procedure to define the release rates of the radionuclides examined in a case. Since the data supplied on this card will override the data provided on card \#50, this card can be used to modify the release rate information provided on card \#50. The information on this card is presented in the form of doublets, one word to identify a radionuclide and the other to define its release rate. If an odd number of words is provided on this card the last value specified will be ignored. If neither this card nor card \#50 are provided as input or if none of the data provided results in defining at least one radionuclide with a positive (i.e., greater than zero) release rate, the case will abort after the remainder of the input has been checked and a message to this effect will be put into the output.

Words $1,3,5, \ldots-$ The first word of each doublet on this card contains the alphanumeric identio fier of the radionuclide whose release rate is sperified in the serond word of the doublet. If this word is not identical to one of the nurlide identifiers provided in the card series beginning with card \#1001, the data in the doublet will be ignored and a message to this effecl written in the outpt. The first word in each doublet is to be in Hollerith format and is to contain 10 or fewer characters.

Words $2,4,6, \ldots-$ The second word of each doublet on this card contains the release rate $Q$ in curies/hour of the radionuclide identified by the first word of the doublet. If this wnrd is negative it will he replaced by a valuo of 0.0 and a message will be put into the output to this effect. This second word of each dounlet on this card is to be exprssed as a real number

Card \#60-This card contains the number of annuli into which each interval specified on card \#200 by the radial distances at which the standard deviation of the vertical dispersion is supplied is to be subdivided. This data is used in evaluating the numerical integral used to define the effect of deposition upon the ground level air concentrations of the released radionuclides. There is a one to one correspondence between the words on this card and the intervals defined by the distances specified on card \#200. Thus, the first word on this card defines the number of annuli into which the interval between the first and second distances specified on card \#200 is to be subdivided, the second word on this card defines the number of annuli into which the interval between the second and third distances specified on card \#200 is to be subdivided, etc. If this card contains fewer words than there are intervals defined on card \#200, the last value specified on this card will be used as the number of annuli into which the remaining intervals defined on card \#200 are to be subdivided. If this card contains more words than there are intervals defined by the 
distances on card \#200, the excess values will be ignored. A default value of 10.0 will be used as the number of annuli into which each interval defined by card \#200 is divided if this card is omitted from the input data. Any values specified on this card which are less than 1.0 will be replaced by the default value of 10.0.

Words $1,2, \ldots$ VMAX-1-Each word on this card is the number of annuli $d_{\mathrm{v}}$ into which the $\mathrm{v}^{\text {th }}$ interval defined by the radial distances specified on Card \#200 (i.e., the interval between $r_{v}$ and $\left.r_{v+1}\right)$ is to be subdivided. Each word on this card is to be expressed as a real number.

Card \#65-This card is to define which of the various standard output quantities are to be printed. There is one integer word provided on this card for each of the six standard quantities which may be printed.. The default values on this card will be set such that only the total dose commitment values for each organ at the various radial positions (including the region beyond the immediate vicinity of the release) for each radionuclide released and its daughters will be printed if this card is omitted. The default values will be used for all unspecified words on this card.

Word 1 -This word is used to print the fractions of the total dose commitment to an organ at a given radial position which is due to each of the released radionuclides and its daughters. If this word is a non-zero integer, these fractions will be printed. If this word is zero (the default value) these fracions will not be included in the output.

Word 2-This word is used to define the printing of the ground level air concentrations in the immediate vicinity of the release point. If this word is an integer zero no ground level air concentrations will be printed. However, if this word is a positive (i.e., greater than zero) integer, both the activity and the number air concentrations at ground level for each released radionuclide and its daughters will be printed in each of the annular segments into which the region in the immediate vicinity of the release point is subdivided. If this word is a negative integer then only the activity air concentrations at ground level will be printed. This word will be defaulted to zero (i.e., no ground level air concentration output) if it is not supplied on the card.

Word 3-This word contains an integer to define the printing of the air concentrations in the region beyond the immediate neighborhood of the release point. If this word is a positive integer, the activity and number of air concentrations in the regions beyond the immediate vicinity of the release point (i.e., beyond the radial distance specified in word 2 on card \#2) will be printed as a function of time subsequent to release for each released radionuclide and its daughters. If this word is a negative integer then only the activity concentrations in the atmosphere of the region beyond the immediate neighborhood of the release point will be printed. If this word is an integer zero (default value) no printing of the air concentration data beyond the vicinity of the release will be performed.

Word 4-The fourth word on this card contains an integer which is used to define the printing of the ground concentrations within the region surrounding the release point. If this word is a positive integer, the activity and number concentrations on the ground in the immediate vicinity of the release point will be printed at each annular segment in the region as a function of time measured from the time releases begin for each released radionuclide and its daughters. If this word contains a negative integer only the activity concentration on the ground in the immediate vicinity of the release point will be 
printed. If an integer zero is supplied for this word no printing on ground concentration information within the region surrounding the release point will be done. If this word is not provided on the card, an integer zero (and thus no printing) will be used as the default value.

Word 5-The printing of the ground concentrations in the regions beyond that defined as the immediate vicinity of the release point is delineated by this word. If a positive integer is provided for this word, the activity and number of ground concentrations in the region beyond the immediate neighborhood of the release point (i.e., beyond the radial distance specified in word 2 on card \#2) will be printed for each released radionuclide and its daughters as a function of time subsequent to the start of releases. If a negative integer is supplied in this word only the astivity cnncentrations on the ground will be printed. If this word contains an integer zero no printing of ground concentration data beyond the immodiato noighborhood of the release puint will be perfurmed. A dofault value of eero will be used il this wurd is nut supplied on the card.

Word 6-The final word on this card defines the printing of the dose commitment data If this word contains a non-zero integer then no dose commitment data will be printed. However, if this word contains an integer zero or if this word is not supplied on the card, the dose commitment data for each organ from all the environmental paths considered will be printed for each released radionuclide and its daughters. The dose commitment data will be providen at each radial position specified via the card \#10 information and in the region beyond the immediate vicinity of the release point (i.e., beyond the radial distance defined by word 2 on card \#2). It should be noted that if the entire card is omitted only the dose commitment information will be printed.

Card \#99-This card is used to detine whether the input data provided in this case is to be saved for use in the next case. All input data for a given case will be saved for use as input to the next case unless this card is provided among the input cards. If the input data for a given case is not to be saved for use in the next case, this card should be provided as input to the case with at least one piece of data on it. This data word is to be an integer and it may assume any integer value.

Card \#100-The average wind speeds $\bar{\mu}_{n}$ which are used to define the meteorological conditions in the immediate vicinity of the release point (i.e., within the radial distance RMAX specified in word 2 of card \#2) are presented on this card. This card may either be incorporated in the basic data file whose name is given in word 1 of card \#0 or included in the input for a given case. If this card is provided in hoth the basic data file and the input stream, the data supplied as input to a given case will be used. The number of values provided on this card defines the number of wind speeds which will be considered in this job. If this data is not provided in either the input stream or the basic data file, no dose commitments can be obtained in the region immediately surrounding the release point. $A$ message to this effect will be put into the output if this data is not available.

Words $1,2, \ldots$ NMAX-Each word on this card is one of the average wind speeds used to define the meteorological conditions which will be encountered in the region immediately surrounding the release point during the period that releases are occurring. Each wind speed is given in the units of meters per second and is expressed as a real number.

Card \#200-The radial distances $r_{v}$ in the region immediately surrounding the release point (i.e., within the maximum radial distance RMAX defined by word 2 on card \#2) at which the standard deviations of the vertical dispersion for each meteorological stability category considered in this problem are specified on this card. This card may be included in the basic data file whose name is given by word 1 of card \#0 or as part of the input data. If it is provided in both the basic data file and among the input, the data provided via 
input will take precedence. The number of values provided on this card defines the number of standard deviations of vertical dispersion which are to be provided for each meteorological stability category which is to be considered in this job. If this card is omitted from both the input data and the basic data file or if there are fewer than two values specified, no calculations of the dose commitments in the immediate neighborhood of the release point can be made and a message to this effect will be written in the output.

Words $1,2, \ldots$ VMAX - Each word on this card is the radial distance measured in meters from the release point at which the standard deviation of vertical dispersion for each meteorological stability category considered in this job is specified. These radial distances are to be specified in increasing order. Thus, the smallest distance is to be provided first, the second smallest distance is to be supplied as the second word, etc., with largest radial distance being provided as the last word on the card. All values on this card are to be supplied as real numbers.

Cards \#201, 202, ... 200 + MMAX-The standard deviation of vertical dispersion data is supplied in this card series. Each card in this series is to contain the standard deviation of vertical dispersion at each of the radial distances defined on card \#200 for one meteorological stability category. One card in this series must be provided for each stability category which is to be considered in this job. These cards must have sequential card numbers starting with card \#201 since the first omission of a card in this series or a card which contains no data terminates the reading of data from the card series. The total number of meteorological stability categories which may be considered in the job is defined by the number of sequential cards in this card series. This card series may be included in the basic data file whose name is given in word 1 on card \#0 or it may be part of the input data. If this card series is provided in both the basic data file and the input data, the information supplied in this card series via the input stream will be used. If this entire card series is omitted from both the basic data file and the input stream, a message to this effect will be written in the output and the job will abort after checking the remainder of the input.

Words 1, 2,... VMAX-Each word on each card of the card series starting with card \#201 is the standard deviation of vertical dispersion at each of the radial distances provided on card \#200 for a given meteorological stability category. There is a one to one correspondence between the values presented on each of these cards and the distances presented on card \#200. Thus the first word on each of the cards in this series is the standard deviation of vertical dispersion for a given meteorological stability category at the first radial distance presented on card \#200, the second word on each card of this series is the standard deviation of vertical dispersion for a given stability category at the second radial distance specitied on card \#200, etc. If a given card in the series contains fewer words than there are on card \#200, the last value provided on the card will be used for the remaining distances specified on card \#200. If there are more words on any card in the series than there are words on card \#200, the excess will be ignored. Each word on these cards is to be expressed as a real number.

Cards \#301, 302 ...-The information necessary to define the fraction of the time during releases that the wind is blowing into a given azimuthal sector with a given speed while a specific meteorological stability category exists which is not provided on card \#30 and card \#35 is supplied in this card series. This card series may be provided as part of the basic data file whose name is specified in word I of card \#0 or it may be included as part of the input data. If this card series is present in both the basic data file and the input data, the information provided via the input stream will take precedence over the data supplied in the basic data file. The data in this card series is to be provided on cards with sequential numbers starting with card \#301. The information presented in this card series is terminated when a card with no data is encounlered or the sequential string of card numbers is broken. A maximum of 399 cards may be used in this card series. Each card in this card series is to contain one word for each wind speed that is specified on 
card \#100. Thus, there is to be a total of NMAX words on each card in this series where NMAX is the number of words on card \#100. The words on each card are to be arranged such that the first word on a card pertains to the first wind speed specified on card \#100, the second word on a card in this series pertains to the second wind speed specified on card \#100, etc. If a card in this series contains fewer than NMAX words, the last word specified on the card will be used for the remainder of the unspecified words. If there are more than NMAX words on a card in this series the excess words will be ignored. If any of the words on a card in this series are negative they will be set to 0.0 . The sum of all the data provided in the 301 card series is to add to 1.0. To ensure that this sum is 1.0 , all the values provided in the 301 card series will be summed and if the result is not within $1 \%$ of the desired value of 1.0 , each value obtained from the 301 series will be divided by the result of this summation. There are four distinct types of data which may be supplied in this card series depending upon the presence of cards \#30 and \#35 in the input stream. A description of the four different types of data which may be provided in this card series and the manner in which they are to be presented is delineated below.

1. Neither card \#30 nor card \#35 is provided as input. In this case the card seriea starting with card \#301 contains the fraction of the time during releases that the wind is blowing at given speeds irtu specific azimuthal sectors while a certain meteorological stability category is in existence. In this situation the cards in the 301 card series are subdivided into JMAX sets where each sct pertains to one of the JMAX azimuthal sectors being examined in this problem and JMAX is defined by the number of words on card \#20. There is to be a one to one correspondence between the JMAX sets of cards in the 301 card series and the sectors defined on card \#20. Thus, the first set of cards in the 301 series pertains to the azimuthal sector whose width is specified in the first word on card \#20, the second set of cards in this series pertains to the sector whose width is provided in the second word on card \#20, etc. Each of the JMAX sets of cards in the 301 card series is to contain one card for each of the meteorological stability categories which are to be considered in this problem. Thus, there may be a maximum of MMAX cards in each of the JMAX sets of cards in the 301 series where MMAX is the number of meteorological stability categories which can be considered in this job and is defined by the number of cards in the 201 card series. There is a one to one correspondence between the cards in a given set of the $30 \mathrm{l}$ card series and the meteorological stability categories defined by the 201 card series. Thus, the first card in any of the JMAX sets of cards in the 301 series pertains to the meteorological stability category whose standard deviation of vertical dispersion data is given on card \#201, the second card in any of the JMAX sets of cards in the 301 series pertains to the stability category whose vertical dispersion standard deviatinn data is provided on card \#202, etc. If there are more than MMAX cards provided in the JMAX sets of cards in the 301 forinn, the excess cards in each set will be ignored. However, if there are less than MMAX cards in each of the JMAX sets of cards in the 301 series, then only as many meteorological stability categories as there are cards in each of the JMAX sets will be examined in this problem. If the 301 card series is not provided in either the basic data file or in the input for this case, default values equal to l/(NMAX * JMAX * MMAX) will be used to define the fraction of the time that the wind is blowing at each of the wind spends given on card \#100 into each azimuthal sector defined by card \#20 for each meteorological stability categories presented in the 201 card series. In general, if neither rard \#30 nor oard \#36 io provided as iupul, llu 301 card series contains the fraction of the time during releases that the wind is blowing with the $\mathrm{n}^{\text {th }}$ wind speed into the $\mathrm{j}^{\text {th }}$ azimuthal sector while the $\mathrm{m}^{\text {th }}$ meteorological stahility category is in exictencc.

2. Only card \#30 is provided as input. In this situation the card series starting with card \#301 contains the fraction of the time during releases that the wind is blowing at given speeds while a specific meteorological stability condition exists. This data is applicable to all azimuthal sectors and the fraction of the time during releases that the wind is blowing into a given sector is supplied by the data on card \#30. Each card in the 301 card series will pertain to one meteorological stability category and there will be a one to one correspondence between the cards in this 301 series and the stability categories associated with the 201 card series. Thus, the first card in the 301 series pertains to the meteorological stability category whose standard deviation of vertical dispersion data is given on card \#201, the second card in the 301 series pertains to the stability category whose vertical dispersion standard deviation data is provided on card \#202, etc. A maximum of MMAX cards should be provided in the 301 card series in this case where the value of MMAX is given by the number of cards in the 201 card series. If the 301 card series contains more than MMAX cards then only the meteorological stability categories for which data is provided 
via the 201 card series will be examined in this job. If the 301 card series is not provided in either the basic data file or among the input cards for this case, then default values of $1 /($ NMAX * MMAX) will be used for each value of the fraction of the time during releases that the wind is blowing at the wind speeds specified on card \#100 while the meteorological stability categories defined by the 201 card series exist. In general, if only card \#30 is provided as input, the 301 card series contains the fraction of the time during releases that the wind is blowing at the $\mathrm{n}^{\text {th }}$ wind speed while the $\mathrm{m}^{\text {th }}$ meteorological stability category is in existence.

3. Only card \#35 is provided as input. In this case the card series starting with card \#301 contains the fraction of the time during releases that the wind is blowing at given wind speeds into the azimuthal sectors being considered in this problem. This data is applicable to all meteorological stability categories and the fraction of the time during releases that a specific meteorological stability category exists is supplied on card \#35. Each card in the 301 card series will pertain to the wind blowing into one azimuthal sector and there will be a one to one correspondence between the cards in the 301 series and the azimuthal sectors defined by card \#20. Thus, the first card in the $30 \mathrm{l}$ series pertains to the azimuthal sector whose width is supplied in the first word on card \#20, the second card in the 301 series pertains to the sector whose width is given in the second work on card \#20, etc. $A$ total of JMAX cards are to be supplied in the 301 card series for this case where JMAX is the total number of azimuthal sectors which are being considered in this problem and is equal to the number of words on card \#20. If the 301 card series contains more than JMAX cards the extra cards will be ignored. If the 301 card series contains less than JMAX cards the data specified on the last card supplied in the 301 series will be used for all unspecified cards. If the 301 card series is not provided in the basic data file or in the input data for a given problem, then default values of $1 /($ NMAX * JMAX) will be used for each value of the fraction of the time during releases that the wind is blowing at the speeds specified on card \#100 into the azimuthal sectors defined by the widths presented on card \#20. In general, if only card \#35 is provided as input in a given case, the 301 card series contains the fraction of the time during releases that the wind is blowing at the $\mathrm{n}^{\text {th }}$ wind speed into the $\mathrm{j}^{\text {th }}$ azimuthal sector.

4. Both card \#30 and card \#35 are provided as input. In this case the 301 card series contains only the one card which is to provide the fraction of the time during releases that the wind is blowing at the speeds given on card \#100. This data is then applicable to all azimuthal sectors and for all meteorological stability categories. The fractions of the time that the wind is blowing into the azimuthal sectors to be examined in this problem are given on card \#30 and fraction of the time that each of the meteorological stability categories exist is specified on card \#35 in this situation. The data on card \#301 will be arranged such that the first word on card \#301 is the fraction of the time during releases that the wind is blowing at the speed given in the first word on card \#100, the second word on card \#301 is the fraction of the time during releases that the wind is blowing at the speed specified by the second word on card \#100, etc. If more than card \#301 is provided when both card \#30 and card \#35 are included in the input, the extra cards in the 301 series will be ignored. If card \#30l is not provided in the basic data tile or among the input cards, then default values of $1 /$ NMAX will be used for each value of the fraction of the time during the releases that the wind is blowing with the speeds specified on card \#100

Card \#700-The information specified on this card is used in conjunction with the data provided in the card series starting with card \#701 to define the population distribution in the immediate vicinity of the release point (i.e., from the release point to the radial distance RMAX defined by word 2 on card \#2). This region in the immediate vicinity of the release point is considered to be subdivided into concentric annuli whose centers are defined by the radial distances specified on card \#10 and into azimuthal sectors whose widths are given on card \#20. This results in a subdivision of the region in the neighborhood of the release point into a set of annular segments and the population in each of these annular segments is defined by the information provided on card \#700 and in the 701 card series. This population distribution data may be supplied in one of the following two ways.

1. The population data in each of the annular segments is provided directly. 
2. The population data in each of the annular segments is obtained from the product of the total population in a given annulus and the fraction of the population in that annulus which is in the sector of interest.

If the first of these two procedures is to be used to define the population distribution in the vicinity of the release point, then card \#700 is omitted from the input and the actual population in each of the annular segments will be defined via the information specified in the 701 card series. If the second of the preceding ways to define the population distribution in the vicinity of the release point is to be used, then the total population in each of the concentric annuli is provided on card \#700. In this case there is a one to one correspondence between the data provided on card \#700 and the radial distances supplied on card \#10. Thus, the first word on card \#700 is the total population in the annulus whose center is given by the first radial distance specified on card \#10, the second word on card \#700 is the total population in the annulus whose center is defined by the second radial distance specified on card \#10, etc. There are to be a total of KMAX words on card \# 700 where KMAX is the number of radial distances which are specified in card \#10. If card \#700 contains fewer than KMAX words then the last value specified on card \#700 will be used for the remaining unspecified values. If there are more than KMAX words prnvided nn rard \#7nn then the excess words will be ignored. If any of the values on card \#700 are negative, they will be replaced by a 0.0 and a message to this effect written in the output. Each word on this card is to he expressed as a real number.

Cards \#701, 702, ... 700 + KMAX-The remainder of the information necessary to define the population distribution in the immediate vicinity of the release point is provided in this card series. The actual information which is supplied in this card series is dependent upon the presence or absence of card \#700 among the input data. If card \#700 is not provided as input to this job, then these cards contain the actual population in each of the annular segments into which the region immediately surrounding the release point is subdivided. However, if card \#700 is present in the input, then the 701 card series will contain data defining the fraction of the total population in a given annulus which is in each of the sectors into which each annulus is subdivided. There are to be a total of KMAX cards in this card series where KMAX is the number of radial positions which are being examined in this problem (defined by the number of radial distances specified via card \#10). There is to be a one to one correspondence between the cards in this series and the annuli whose centerline radii are defined by the data on card \#10. Thus, card \#70l is to contain the population distribution in each of the sectors examined in this problem for the concentric annulus whose centerline radius is given by the first value specified on card \#10, card \#702 contains the population distribution in each of the sectors evaluated in this job for the annulus whose centerline radius is given by the second value specified on card \#10, etc. Each of the cards in the 701 card series is to contain JMAX values where JMAX is the number of azimuthal sectors which are being examined in the job and is defined by the number of words on card \#20. If any of the cards in the 701 series (i.e. between card \#701 and card \#700 and KMAX inclusive) are omitted from the input data, a set of default values each equal to 1/JMAX will be used for the values on the card. If a card in the 101 series contains less than JMAX words, the last value provided on the card will be used for the remaining unspecified values. If a card in the 701 series contains more than JMAX words the extra words will be ignored. If any of the values in this card series is negative it will be replaced by a 0.0 and a message to this effect will be put into the output. There is to he a nne to one correspondence between the population distributions defined on each of the cards in the 701 series and the azimuthal sectors identified by the data on card \#20. Thus, the first word on card \#700+ $\mathrm{k}$ contains the population distribution in the sector of the $\mathrm{k}^{\text {th }}$ annulus whose width is given by the first word on card \#20, the second word on card \#700 + $\mathrm{k}$ contains the population distribution in the sector of the $\mathrm{k}^{\text {th }}$ annulus whose width is given in the second word on card $\# 20$, etc. In general, the $j^{\text {th }}$ word on card $\# 700+k$ contains the population distributions in the $\mathrm{j}^{\text {th }}$ sector of the $\mathrm{k}^{\text {th }}$ annulus of the region immediately surrounding the release point.

Cards \# 1001 thru 7000-The remainder of the input for the AIRWAY program is associated with providing basic data for the various radionuclides which may be examined in a job. $A$ total of six categories of basic information is to be supplied for each of the radionuclides which may be examined. The data for each of these six categories is supplied in an input card series starting with cards \#1001, 2001, 3001, 4001, 5001 and 6001 . The information which is to be supplied in each of these card series is delineated in detail 
below. Each of these card series may be provided in the basic data file whose name is given in word l of card \#0 or it may be included as part of the input data. If cards in any of these series are provided in both the basic data file and the input data, the information provided via the input data will take precedence. Each radionuclide which may be examined in a job is assigned a unique integer number between $l$ and 1000 inclusive and this number along with a unique alphanumeric identifier is included on all cards in these series which contain data pertaining to that radionuclide. In addition, the card number of each card containing data associated with a given radionuclide is to include the assigned integer number for that isotope. Thus, the number of the card which contains data in category 1 for the radionuclide identified by the integer $i$ is given by $1000+i$, the number of the card which contains the category 2 type data for the radionuclide identified by the number $i$ is given by $2000+i$, etc. If data is provided in one or more categories for a specific radionuclide but not in all six data categories, then a set of values equal to 0.0 will be provided for the unspecified data categories for that radionuclide.

Cards \#1001, 1002, ... 2000-This card series provides the decay constant, the deposition velocities, the uptake factors for the various food paths considered and the ground and plant concentrations of each of the radionuclides for which data is provided in a job. If all the cards in this series do not contain the same number of words, the card with the maximum number of words will be used to define the number of food paths considered in this problem (equal to the number of input words on this card less six): However, a minimum of three food paths (surface crops, milk and beef) must be considered in all jobs. One uptake factor is to be provided on each card for each food path considered. If any of the cards in this series contains less than $\mathrm{NF}+6$ words where NF is the number of food paths considered in problem, the last value provided on the card will be used for all unspecified values

Word 1 -Integer identification number $i$ assigned to the radionuclide whose data is defined on this card. This value is used to define the card number (i.e., $1000+i)$.

Word 2-Hollerith identifier of the radionuclide whose data is provided on this card. This word is in Hollerith format and has 10 characters or less in it.

Word 3-Radioactive decay constant $\lambda_{d}$ in hours ${ }^{-1}$.

Word 4-Deposition velocity $\mathrm{v}$ in meters per second.

Word 5-Uptake factor $w_{1}$ for above surface food crops in square meters:

Word 6-Uptake factor $w_{2}$ for milk in square meters.

Word 7-Uptake factor $w_{3}$ for beef in square meters.

Word $N F+4-U$ iptake factor $\mathrm{w}_{\mathrm{NF}}$ for the $\mathrm{NF}^{\text {th }}$ food type- $\mathrm{m}^{2}$

Word NF + 5-Concentration of the elemental form of the radionuclide in the soil-gm element/gm soil.

Word NF + 6-Concentration of the elemental form of the radionuclide in plants-gm element/gm plant.

It should be noted that for a few materials the concentration in the soil and plants are isotopic-dependent rather than just element-dependent. Values for the parameters supplied in this card series are given in Appendix F. The 
first word on each card in this series is an integer, the second word is presented in Hollerith format and the remaining words are expressed as real numbers.

Cards \#2001, 2002, ... 3000-This card series provides the ingestion dose commitment conversion factors for each of the organs examined in this job for each radionuclide which may be considered. If all the cards in this series or in the series starting with cards \#3001,4001 and 5001 do not contain the same number of words, then the card in these series which has the maximum number of words on it will be used to define the number of organs for which dose commitments are to be obtained. This number is equal to two less than the maximum number of words on any card between card \#2001 and card \#6000, inclusive. However, a minimum of nine organs (total body, bone, liver, kidney, gonads, lung, GI tract, thyroid and skin) must be considered in each job. One ingestion dose commitment conversion factor must be provided on each card for each organ considered. If any card in this series has less than NG +2 words where NG is the number of organs examined in this job, the last value supplied on that card will be used for the remaining unspecified values. The first word on each card in this series is given as an integer, the second word on each card is in Hollerith format and the remaining words on each card in this series are expressed as real numbers and are given in the units of rem per microcurie. Values of the 70-year dose commitment factors for ingestion of various radionuclides are presented in Appendix F.

Word 1-Integer identification number $i$ assigned to the radionuclide whose dose commitment conversion factors for ingestion are provided on this card. This integer is used to define the card number (i.e., $2000+i$ ).

Word 2-Alphanumeric identifier of the radionuclide whose ingestion dose commitment conversion factor data is provided on this card. This word is to contain 10 or fewer characters.

Word 3-Ingestion dose commitment conversion factor for the total body.

Word 4-Ingestion dose commitment conversion factor for bone.

Word 5-Ingestion dose commitment conversion factor for the liver.

Word 6-Ingestion dose commitment conversion factor for the kidneys.

Word 7-Ingestion dose commitment conversion factor for the gonads.

Word 8-Ingestion dose commitment conversion factor for the lung.

Word 9-Ingestion dose commitment conversion factor for the GI tract.

Word 10-Ingestion dose commitment conversion factor for the thyroid.

Word 11-Ingestion dose commitment conversion faclor for the skin.

Word NG + 2-Ingestion dose commitment conversion factor the NG ${ }^{\text {th }}$ organ.

Cards \#3001, 3002, ... 400-This card series is used to define the inhalation dose commitment conversion factors. These cards are identical to the 2001 card series except that inhalation dose commitment conversion factors in rem per microcurie are provided on each card rather than ingestion dose commitment factors. 
Cards \#4001, 4002, ...5000-The immersion dose rate conversion factors are provided in this card series. The cards in this series are identical to those supplied on the 2001 card series except that the immersion dose rate conversion factors in $\mathrm{re} / \mathrm{hr}$ per microcuries $/ \mathrm{m}^{3}$ are provided on each card rather than the ingestion dose commitment conversion factors.

Cards \#5001, 5002, ... 6000-These cards contain the conversion factors which define the dose rates to the various organs due to surface deposits of the various radionuclides which may be examined in a job. The cards in this series are identical to the cards in the $200 \mathrm{l}$ series with the exception that surface deposit dose rate conversion factors in re $\mathrm{m} / \mathrm{hr}$ per microcurie $/ \mathrm{m}^{2}$ are provided on each card instead of the ingestion dose commitment conversion factors.

Cards \#6001,6002, ... 7000-This card series is used to define the decay paths for each of the radionuclides which may be examined in a job. Each card in the series contains the decay path for one radionuclide. The decay path information in a chain is presented in the form of doublets where each doublet defines the name of a daughter nuclide and the fraction of its precursor which decays to this daughter. The doublets are arranged on the card such that the first daughter in the decay chain of a given radionuclide is defined by the first doublet on the card, the second daughter is defined by the second doublet, etc. Samples of the decay chain complexities which are handled in the AIRWAY program (including repetitions and combinations as noted) are presented below (Figure $\mathrm{C}-1$ ).

Word 1 -Integer identification number $i$ assigned to the radionuclide whose decay chain data is provided on this card. This value is used to define the card number (i.e., $6000+i$ ) and is the same value as was used for this radionuclide in the 1000 thru the 5000 card series.

Word 2-Alphanumeric identifier of the radionuclide whose decay path information is supplied on this card. This word is in Hollerith format and contains 10 or fewer characters.

Words $3,5,7, \ldots$-These are the first words of the doublets which define the decay chain of the radionuclides described by the words 1 and 2 . The fraction of the decays of the precursor which yield the daughter product defined by this doublet is presented in this word. Thus, the maximum value this word can assume is 1.0 and the minimum value should be greater than 0.0 . If a value greater than 1.0 is provided it will be reduced to 1.0 and if a negative value is supplied, its absolute value will be used. Each of these words is to be expressed as a real number.

Words $4,6,8, \ldots-$ These are the second words of the doublets which define the decay paths associated with the radionuclide identified by words 1 and 2 . This word of the doublet is to contain the alphanumeric identifier of the daughter nuclide which is defined by this doublet and whose yield fraction from its precursor is provided in the first word of the doublet. Each of these words is to be expressed in Hollerith format. If this word does not correspond to one of the alphanumeric identifiers of the radionuclide for which information is presented in the 1000-5000 card series, then the decay product defined by this doublet will be ignored and a message to this effect written. 


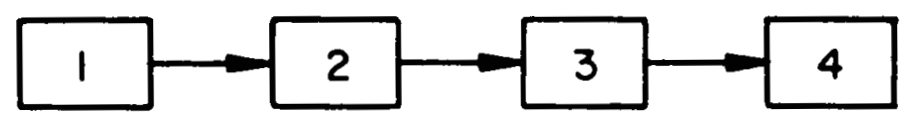

(a)

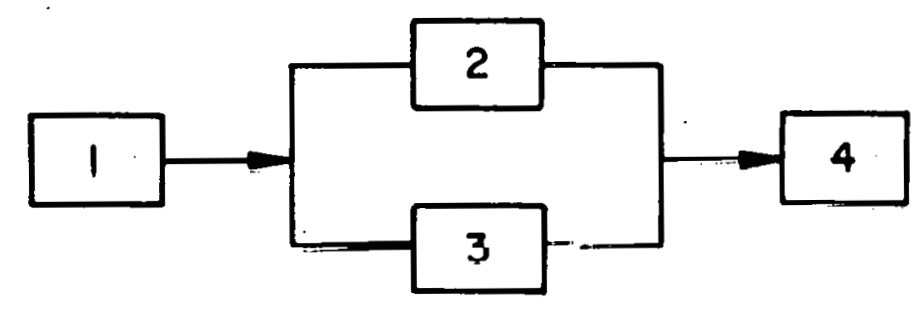

(b)
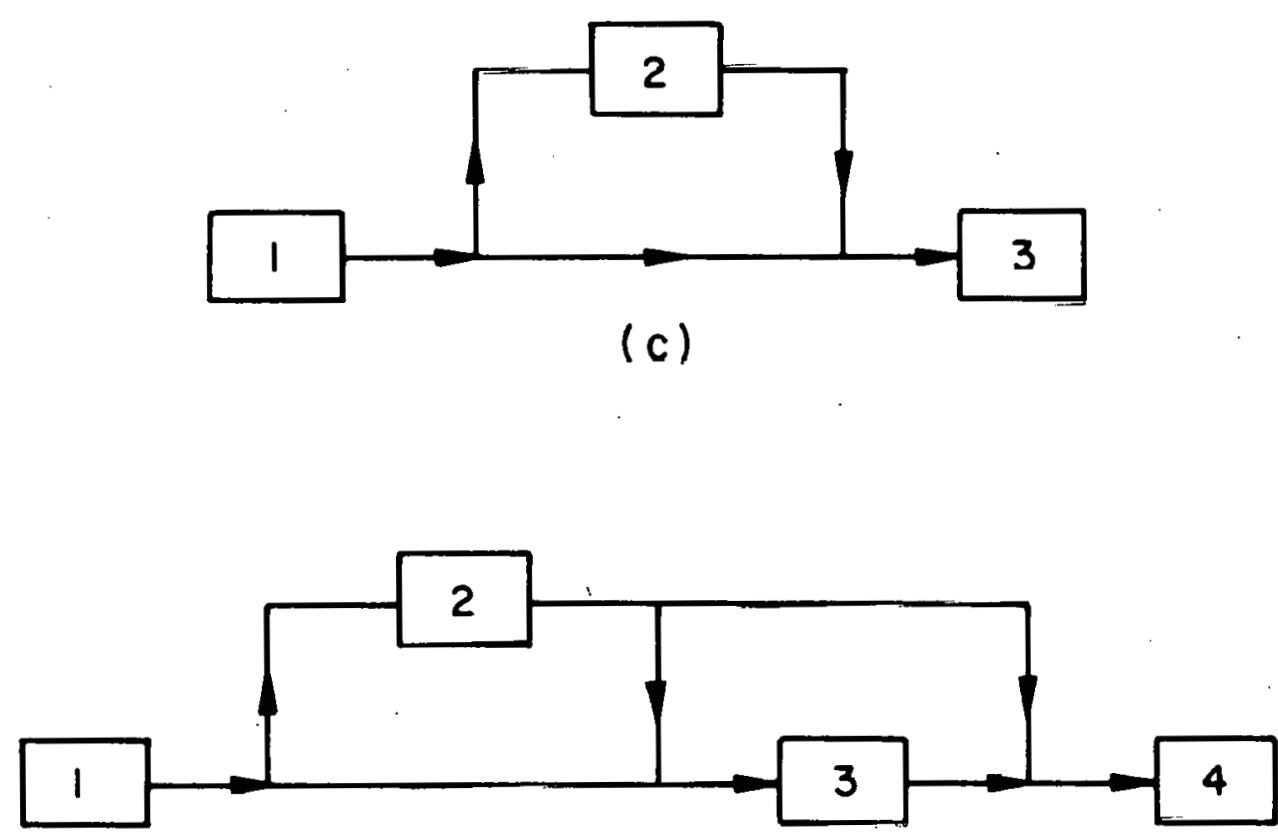

(d)

Figure C-1. Sample Decay Chain Segments Handled by Airway Program 


\section{APPENDIX D}

SAMPLE PROBLEM 


\section{APPENDIX D}

\section{SAMPLE PROBLEM}

A sample problem depicting the necessary input and output for a simple AIRWAY computer problem is presented in this appendix. This problem is representative of those which can be solved using the AIRWAY computer program and uses information which is provided via input cards, default values and a file of input data. This appendix contains a brief description of the problem to be solved, a listing of the cards provided to the AIRWAY program via the input stream, a listing of the cards provided via a file of input data, a copy of the day file which contains the control cards used to run the job and the program output providing a summary of the input data and the results of the dose commitment calculations.

The sample problem is to provide an estimate of the 70 -year dose commitment which results from the continuous release of four radionuclides at a rate of $1.0 \mathrm{Ci} / \mathrm{hr}$ for period of a year. The four released radionuclides are ${ }^{85} \mathrm{Kr},{ }^{127 m} \mathrm{Te}^{133} \mathrm{I}$, and ${ }^{133} \mathrm{Xe}$ where the krypton and xenon nuclides behave as gases and the iodine and tellurium isotopes are treated as particulates. These radionuclides are released from a $10 \mathrm{~m}$ high stack. The wind patterns are such that the wind blows into one $22.5^{\circ}$ sector $25^{\prime \prime}$. of the time during a year and into each of the remaining 15 equal size sections $5^{\prime}$ of the time during a year. Since a person at a given radial position in any one of these 15 sectors into which the wind blows $5^{\prime \prime}$.. of the time receives the same dose commitment as a person at the same radial position in any of the other sectors, these 15 sectors may be replaced by one sector into which the wind blows $75^{\cdots}$ of the time during a year. The immediate vicinity of the release point will be subdivided into four annuli centered at 500,4000, 20000 and 80500 meters from the release point with each annulus divided into the two sectors defined by the directions in which the wind blows. The people in the immediate vicinity of the release point are distributed such that 240 are in the annulus centered at 500 meters with 30 of these people in the $22.5^{\circ}$ sector and 210 people in the $337.5^{\circ}$ sector, 3520 people are in the annulus centered at 4000 meters with 440 of the $m$ in the $22.5^{\circ}$ sector, 164800 people are in the annulus centered at 20000 meters with 20000 of the $m$ in the smaller $22.5^{\circ}$ sector, and 832000 people in the annular centered at 80500 meters from the release point with 104000 of these people located in the $22.5^{\circ}$ sector.

The gaseous radionuclides which are released are to affect the entire world using a total world population of $4 \times 10^{9}$. people and the particulate radionuclides are to affect a portion of the world which encompasses an atmospheric volume of $4 \times 10^{17}$ cubic meters, a surface of $5 \times 10^{13}$ square meters and contains $1 \times 10^{8}$ people. The particulates are to be removed from the surface via weathering, erosion and migration with a removal constant of $1 \times 10^{-10} \mathrm{hrs}^{-1}$ (note that smaller values of this parameter should not be used for radionuclides with long half lives, i.e., decay constants less than $1 \times 10^{-12} \mathrm{hrs}^{-1}$ ). The wind speeds and the fraction of the time that the wind blows with a given speed which a specific stability category exists will be taken from the 18 station average meteorological data (presented in Table F-1 of Appendix F) and the standard deviation of vertical dispersion data will be that associated with the 18 station average data (presented in Table F-3 of Appendix F). The dose commitment conversion faclors associated with the ingestion and inhalation pathways for the released radionuclide and their daughters will be taken from Tables F-8 and F-9 of Appendix F and the dose rate conversion factors associated with the immersions and surface deposit pathways for these nuclides will be taken from Tables F-6 and F-7 of Appendix F. The decay chain information for each of the released radionuclides is given in Table F-10 of Appendix F while the general nuclide data for each released radionuclide and their daughters is obtained from Table F-5 of Appendix F. The standard AIRWAY default values will be used for all remaining parameters such as the precipitation scavenging, resuspension, population growth, food consumption, food producing locations, breathing rates and soil parameters.

The data for this sample problem which was obtained from the BASDAT data file is presented below. The card input data necessary to run the AIRWAY sample problem and the results of this problem are provided on the following pages.

$100,0.8,2.5,4.5,6.9,9.5,12.5,15.6$

$200,1.0,100.0,150.0,200.0,300.0,400.0,500.0,700.0,1000.0,2000.0$ $+3000.0,4000.0,5000.0,7000.0,10000.0,20000.0,40000.0$, $+60000.0,80000.0,100000.0$ 
$201,2.9,10.2,15.0,20.0,31.0,43.0,55.0,85.0,140.0,400.0,900.0$ $+1000.0,1000.0,1000.0,1000.0,1000.0,1000.0,1000.0,1000.0,1000.0$

$202,2.0,7.6,11.4,14.5,21.0,28.0,34.0,47.0,64.0,116.0,165.0,210,0$, $+249.0,340.0,440.0,760.0,1000.0,1000.0,1000.0,1000.0$

$203,2.1,4.9,6.8,8.7,12.1,15.8,19.0,24.7,32.0,53.5,70.0,84.0,96.0$, $+118.0,145.0,204.0,290.0,348.0,400.0,440.0$

$204,0.49,1.5,2.3,3.0,4.7,6.2,7.7,10.2,13.5,21.7,27.6,32.0,35.5,41.2$, $+48.0,60.8,75.0,83.0,90.0,93.0$

$1072,72, \mathrm{KR}-85,7.369-5,0.0,0.0$

$1147,147, \mathrm{TE}-127 \mathrm{M}, 2.65-4,0.01,1.07,0.467,57.9,1.0-3,1.3-3$

$1148,148, \mathrm{TE}-127,7.413-2,0.01,0.034,0.008,0.013,1.0-3,1.3-3$

$1164,164,1-133,3.301-2,0.01,0.072,0.167,0.007,5.0,1.0-1$

$1170,170, \mathrm{XE}-133 \mathrm{M}, 1.256-2,0.0,0.0$

$1171,171, \mathrm{XE}-133,5.459-3,0.0,0.0$

$2072,72, \mathrm{KR}-85,4.6-6,4.6-6,2.3-5,7.7-6,4.6-6,4.6-6,4.6-6,4.6-6,0.0$

$2147,147, \mathrm{TE}-127 \mathrm{M}, 1.09-3,8.31-3,3.17-3,3.6-2,9.8-3,1.09-3,3.88-2,2.28-3,0.0$ $2148,148,1 ' \mathrm{E}-127,2.39-5,1.13-4,4.03-5,4.57-4,1.29-4,2.39-5,9.7-3,8.13-5,0.0$ $2164,164,1-133,7.48-4,7.48-4,7.48-4,7.48-4,7.48-4,7.48-4,3.49-2,0.497,0.0$ 2170,170, XE-133M, 1.4-5, 1.4-5, 7.0-5, 2.3-5, 1.4-5, 1.4-5, 1.4-3, 1.4-5, 0.0 $2171,171, \mathrm{XE}-133,9.5-6,9.5-6,4.7-5,1.6-5,9.5-6,9.5-6,9.5-4,9.5-6,0.0$ $3072,72, \mathrm{KR}-85,4.4-8,4.4-8,4.4-8,2.8-8,3.6-8,3.2-6,4.4-8,4.4-8,0.0$ 3147, 147, TE-127M, 1.65-3, 1.23-2, 6.35-3, 5.39-2, 1.44-2, 1.64-1, 2.37-2, 3.47-3, 0.0 $3148,148, \mathrm{TE}-127,3.63-5,1.67-4,8.07-5,6.86-4,1.89-4,8.55-4,7.11-3,1.24-4,0.0$ $3164,164,1-133,3.12-4,3.12-4,3.12-4,3.12-4,3.12-4,1.46 .2,7.78-3,0.209,0.0$ $3170,170, \mathrm{XE}-133 \mathrm{M}, 2.4-7,2.4-7,2.4-7,2.4-7,2.4-7,9.7-6,2.4-7,2.4-7,0.0$ $3171,171, \mathrm{XE}-133,1.7-7,1.7-7,1.7-7,1.7-7,1.7-7,6.8-6,1.7-7,1.7-7,0.0$ $4072,72, \mathrm{KR}-85,1.71,2.09,1.43,1.52,1.89,1.61,1.71,1.8,248.0$ $4147,147, \mathrm{TE}-127 \mathrm{M}, 3.95,6.02,1.51,2.24,5.4,2.02,1.58,4.17,467.0$ $4148,148, \mathrm{TE}-127,3.37,4.18,2.79,2.96,3.78,3.16,3.29,3.53,243.0$ $4164,164, \mathrm{I}-133,558.0,660.0,466.0,486.0,580.0,527.0,541.0,562.0,1120.0$

D-2 
$4170,170, \mathrm{XE}-133 \mathrm{M}, 26.9,40.9,17.7,18.3,35.7,21.0,15.9,26.5,235.0$

$4171,171, \mathrm{XE}-133,32.6,56.5,21.8,22.6,33.4,25.1,19.4,38.7,196.0$

$5072,72, \mathrm{KR}-85,0.304-1,0.371-1,0.253-1,0.269-1,0.338-1,0.287-1,0.304-1,0.32-1,0.371-1$

$5147,147, \mathrm{TE}-127 \mathrm{M}, 0.301,0.459,0.115,0.17,0.411,0.154,0.12,0.317,0.703$

$5148,148, T E-127,0.0605,0.0751,0.0501,0.0531,0.0679,0.0568,0.0591,0.0635,0.0741$

$5164,164, \mathrm{I}-133,9.77,11.5,8.16,8.52,10.1,9.23,9.49,9.85,11.9$

$5170,170, \mathrm{XE}-133 \mathrm{M}, 0.985,1.5,0.646,0.67,1.3,0.768,0.581,0.97,1.51$

$5171,171, \mathrm{XE}-133,0.957,1.66,0.637,0.663,0.978,0.735,0.567,1.13,1.32$

6147, 147, TE-127M, 0.98, TE-127

$6164,164, \mathrm{I}-133,0.024, \mathrm{XE}-133 \mathrm{M}, 1.0, \mathrm{XE}-133$

$6170,170, \mathrm{XE}-133 \mathrm{M}, 1.0, \mathrm{XE}-133$ 
$05 / 29 / 79$ SAPL-A VERSION 44 SCUPE $3.302 / 28 / 79$-FULL ECS-

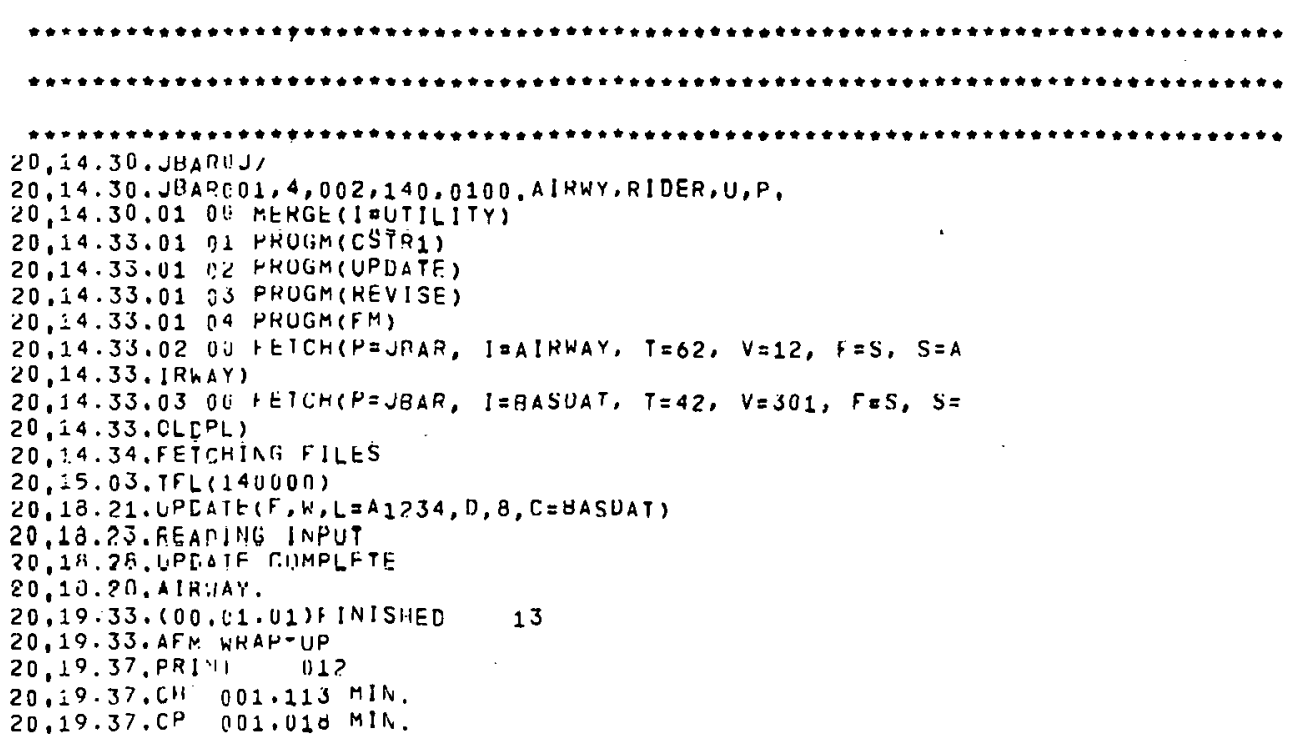




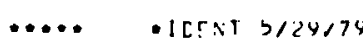

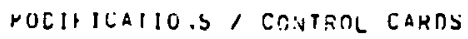

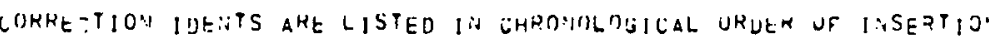

\begin{tabular}{|c|c|c|c|c|c|c|c|}
\hline $\begin{array}{l}\text { EASUAT } \\
\text { EXRFM }\end{array}$ & $\begin{array}{l}F: \forall 5 \\
A 1: G 7\end{array}$ & $\begin{array}{l}\text { SEEIO } \\
\because \text { RRLE }\end{array}$ & $\begin{array}{l}\text { INREM } \\
\text { DAUTER }\end{array}$ & $\begin{array}{l}\text { MAYSO } \\
\text { HCEDHF }\end{array}$ & $\begin{array}{l}\text { Rours } \\
\text { CHEHCl }\end{array}$ & $\begin{array}{l}\text { NCV6 } \\
\text { AF R? }\end{array}$ & $\begin{array}{l}E 2 U 1 L, \\
2,9,72,\end{array}$ \\
\hline $7 / 26 / 77$ & $3 / 29 / 79$ & & & & & & \\
\hline
\end{tabular}

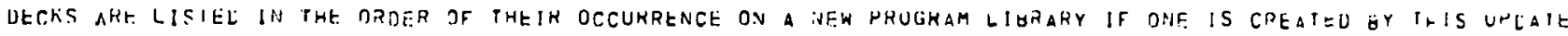

YANGSO HASIIAT

LECKS HRITTH.N TO COMPILE HILE

EASDAT

THIS IFUATE HEGUIREI 33ZONH HOQRUS OH CORE, 
PROGRAY AIRWAY - DE/DO/7B

LISTIN. JF CAMTIS FROM THF INPUT STHEAM FOR LASE 1 .
= AJKHAY SAMOLE PROHLEM
$0002 \quad 2,0.0010$
$4,1,0 \cdot 10$
$0004 \quad 5,4,0$
$0005 \quad 6,1,7+4,3,0,13,4,0+17$
$10,500,0,4053,0,20000,0,50500.0$
$20,5,3421,5,390$
$30,0.23,0,75$

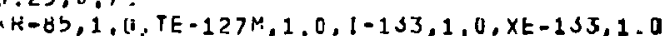
$0010 \quad 301, .0 \$ 36, .0072,0462,0192, .00496, .00091,+00048$
$0011 \quad 302, .0142,0448,0496,0256,36576,00015,0002$

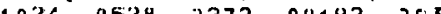

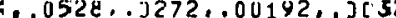
$0014 \quad 701.30 .0, ? 16,0$
$702,440,0,3080.0$
$703,20600,0,144200,0$ 


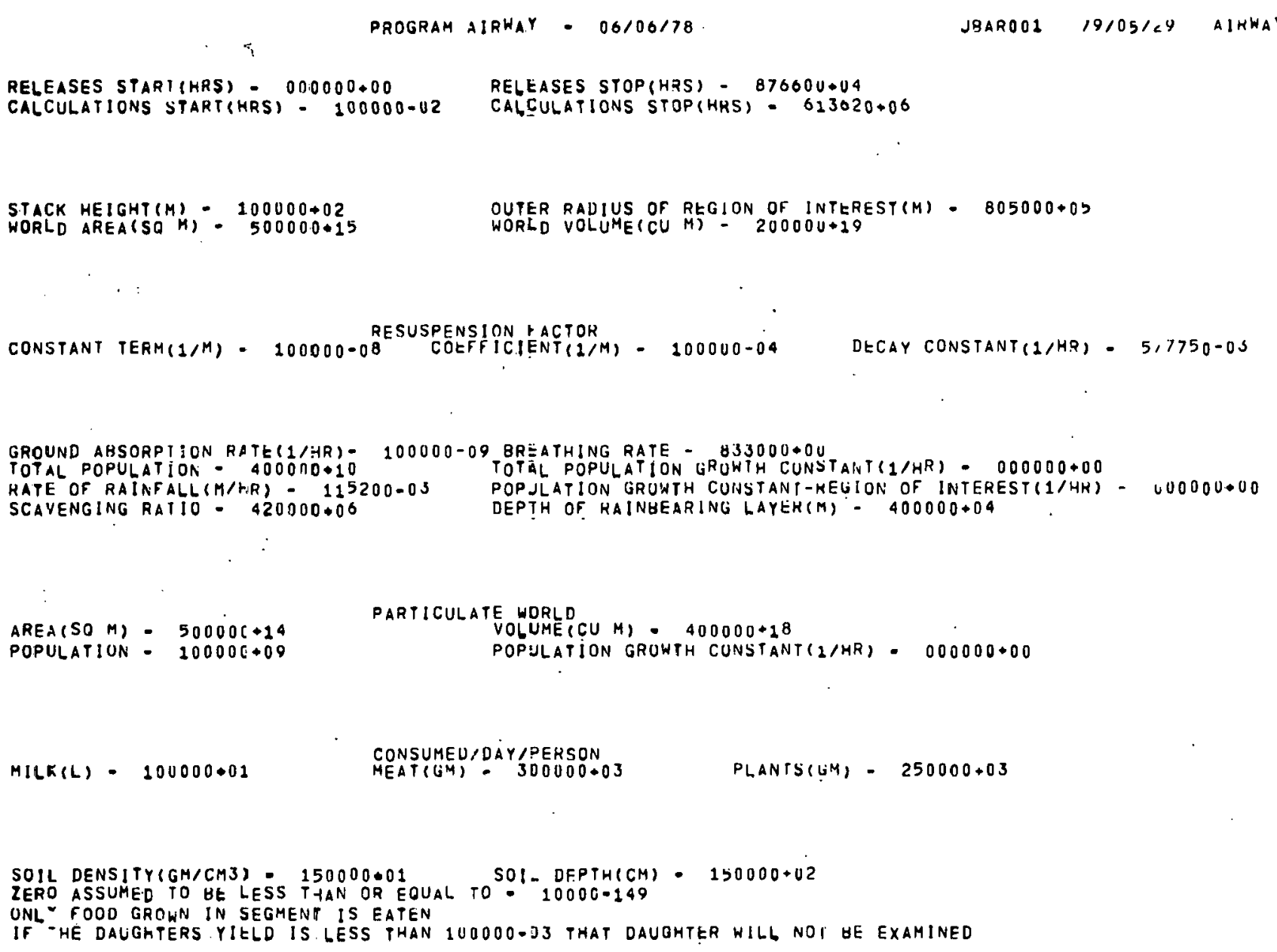

RELEASES STARI(HRS) - 000000+00 RELEASES STOP(HRS) - 876000004

CALCULATIONS START(HRS) - $100000-02$ CALCULATIONS STOP(HRS) $=613020+06$

PLUME SUGMERSION - 100000001 DOSE MULTIPLISTS GROUND FOOD INGESTICN. 100000+01 INHALATION. 100000001 
FOPULATION AT TIME OOOOOO-LO HHS

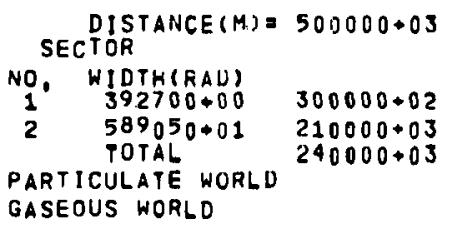

$\begin{array}{cccc}400000-04 & 200000+05 & 805[00+05 & \text { TOTAL } \\ & & & \\ 940000-03 & 200000+05 & 104 C 00+06 & 125070+06 \\ 308000-04 & 144200+06 & 728 C 00+06 & 815490+06 \\ 352000-04 & 106800+06 & 832600+06 & 100056+07 \\ & & & 100000+09 \\ & & & 400000+10\end{array}$

POPULATION AP TIME 100000-02 HKS

\begin{tabular}{|c|c|c|c|}
\hline $400000-04$ & $200000+05$ & $805000-05$ & IOTAL \\
\hline $\begin{array}{l}440000-03 \\
308000-04 \\
352000-04\end{array}$ & $\begin{array}{l}206000+05 \\
144200+05 \\
109800+06\end{array}$ & $\begin{array}{l}10<00 j+06 \\
72 \varepsilon .00 j+06 \\
83<00 j+06\end{array}$ & $\begin{array}{l}125070+08 \\
8 / 5<90+08 \\
1 \cup 0 \cup 56+07 \\
1 \cup 0 \cup 00+09 \\
4 \cup 0 \cup 00+10\end{array}$ \\
\hline
\end{tabular}

POPULAIIOV AT TIME $876600+04$ HKS

$\begin{array}{llll}400000+04 & 200000+05 & 805003+05 & 10 T A L \\ & & & \\ 440000+03 & 206000+05 & 104001+06 & 125070+06 \\ 308000+04 & 144200+06 & 728003+06 & 815490+06 \\ 352000+04 & 164800+06 & 832000+06 & 100056+07 \\ & & & 100000+0.9 \\ & & & 400000+10\end{array}$

PJPULATION AT TIME 613620+06 HRS

$400000+04 \quad 200000+05 \quad 805000+05$

IOTAL 
NO, WIDTH(F\&L)

$10.392700+\therefore 0 \quad 300000+02$ $569050+: 11$

PARTICULATE :ORL GASEDUS KORLT $\begin{array}{lllll}400000+02 & 440000+13 & 206000+05 & 104000+06 & 125070+06\end{array}$

$\begin{array}{lllll}210020+13 & 308000+114 & 144200+06 & 728000+06 & 8 / 5490+06 \\ 240000+03 & 352000+14 & 164900+06 & 832000+06 & 140056+07\end{array}$

$100050+07$ $100000+09$
$4 \cup 0 \cup 00+10$ 
HOSES FUH ISIIUUE 7ä(Kn-3E, - FEM

\begin{tabular}{|c|c|c|c|c|c|c|c|c|c|}
\hline DISTANCr. & TOT & BO NE. & LIVER & KIISNE! & GONAUS & LUNS: & SI IRACT & TMYROID & Sixing \\
\hline $\begin{array}{l}500000+03 \\
400000+114\end{array}$ & $\begin{array}{l}506314-02 \\
304235=02\end{array}$ & $\begin{array}{l}6397 \delta 4-J 2 \\
4 R(0)=J 9=\end{array}$ & $\begin{array}{l}475094-02 \\
331040-02\end{array}$ & $\begin{array}{l}500565-172 \\
348345-102\end{array}$ & $\begin{array}{l}6<2734-02 \\
453563-02\end{array}$ & $\begin{array}{l}138670-01 \\
965051=02\end{array}$ & $\begin{array}{l}=66>144-0 \geq \\
=94<39-00 \geq\end{array}$ & $\begin{array}{l}345704-n 2 \\
+14555-02\end{array}$ & $\begin{array}{l}304569+00 \\
554164+00\end{array}$ \\
\hline $\begin{array}{l}200000+05 \\
805000+055\end{array}$ & $\begin{array}{l}151 \cup 0 a-n 1 \\
10.5476-01\end{array}$ & $\begin{array}{l}232501-111 \\
2.914 ? 7-111\end{array}$ & $\begin{array}{l}1603935=01 \\
138447-01\end{array}$ & $\begin{array}{l}168771-01 \\
146213-01\end{array}$ & $\begin{array}{l}203461-01 \\
101090-01\end{array}$ & $\begin{array}{l}467560-01 \\
405066-01\end{array}$ & $\begin{array}{l}191006-0 ! \\
165476-00=\end{array}$ & $\begin{array}{l}200848-01 \\
: 14005-01\end{array}$ & $\begin{array}{l}271<92+01 \\
234 y 53-01\end{array}$ \\
\hline TOTAL & $4 \longleftarrow 2357.01$ & $5=10: 5-01$ & $380000-01$ & $59997.7-n_{1}$ & $4 y 746 y-01$ & $=10>81+00$ & $45 \geq 557-0:$ & $475676-01$ & $542263+111$ \\
\hline WOKLL & $\triangle 1: 890>01$ & $513282+41$ & $345021 \div 01$ & 363053401 & $401060+01$ & $100581+02$ & $4.13090+c=$ & $=32062+91$ & $583400+03$ \\
\hline
\end{tabular}


DUSES FOH ISTIOPE $147(T F-127 \mathrm{H})$ - QEM

\begin{tabular}{|c|c|c|c|c|c|c|c|c|c|}
\hline DISTA:CL & TUT BO!IY & BONE. & $L I V \equiv R$ & KIDNEY & GONAUS & LUNG & $311 K_{A}=T$ & IHYKOL!) & $\operatorname{saln}$ \\
\hline $500000+03$ & $3>817 \mathrm{~s}+04$ & $546792+05$ & $246>31.05$ & $279402+116$ & $702231+05$ & $89564 n+n 4$ & $301600+0 s$ & $1 / 82 S=02$ & $286073+03$ \\
\hline $\begin{array}{l}40 C 000+04 \\
20 \therefore 00 n+05\end{array}$ & $\begin{array}{l}328037+[4 \\
744041+[44\end{array}$ & $\begin{array}{l}247237+05 \\
501 ? 25+05\end{array}$ & $\begin{array}{l}942562+04 \\
213414+05\end{array}$ & $\begin{array}{l}100324+166 \\
242490+06\end{array}$ & $\begin{array}{l}241363+05 \\
601393+05\end{array}$ & $\begin{array}{l}342030 \bullet 04 \\
774589 \cdot 04\end{array}$ & $\begin{array}{l}1150.09+05 \\
261<54+00\end{array}$ & $\begin{array}{l}081315+04 \\
15465 B+05\end{array}$ & $\begin{array}{l}109086+03 \\
240 y 3 u+03\end{array}$ \\
\hline $805000+05$ & $2>1 / 16+c 4$ & $189715+45$ & $? 23090.04$ & $819706+02$ & $2\langle 3>7>+05$ & $250790 \circ 04$ & $863,56+95$ & $522801+04$ & $941 \mathrm{i} 41.02$ \\
\hline -OTAL & $218257+C 5$ & $104497+06$ & $626490+05$ & $710747+06$ & $1 \times 3056+06$ & $22.7305+0.5$ & $765,38+03$ & $4 b 330=+15$ & $729 ! 00+03$ \\
\hline WORELL & $000000+C .0$ & $9 \pi 0000+00$ & $000000+00$ & $000000+00$ & $0000110+00$ & $000000 \rightarrow 00$ & $600<00+01)$ & $00000 E+n 0$ & $000000 \cdot 08$ \\
\hline
\end{tabular}

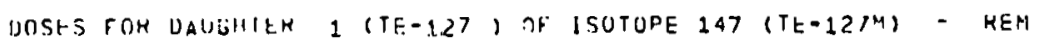

\begin{tabular}{|c|c|c|c|c|c|c|c|c|c|}
\hline DISTAIICE & TLT HUQ̣T & $E \geqslant$ & LIVER & KIONEY & GONAUS & LUNG & $3 I \mid R_{A} C T$ & IHYROID & $\sin 1 \mathrm{in}$ \\
\hline $\begin{array}{l}50(0,0 n+113 \\
40 \times 030+104\end{array}$ & $\begin{array}{l}2 b \cup 214+02 \\
953031+01\end{array}$ & $\begin{array}{l}343175 \cdot 02 \\
131263+02\end{array}$ & $\begin{array}{l}215264+02 \\
323211+01\end{array}$ & $\begin{array}{l}510104+1) 2 \\
149428+02\end{array}$ & $\begin{array}{l}3<n<61+02 \\
1<? 105+02\end{array}$ & $\begin{array}{l}237224+02 \\
908367+01\end{array}$ & $\begin{array}{l}401<37+05 \\
153697+05\end{array}$ & $\begin{array}{l}284606+02 \\
108053002\end{array}$ & $\begin{array}{l}295040 \cdot 02 \\
112030 * 02\end{array}$ \\
\hline $\begin{array}{l}200030+05 \\
801.330+05\end{array}$ & $\begin{array}{l}? 17311+0 ? \\
i S 546 C+01\end{array}$ & $\begin{array}{l}298431+02 \\
101353+02\end{array}$ & $\begin{array}{l}187090.02 \\
534379.01\end{array}$ & $\begin{array}{l}341048+02 \\
116383+112\end{array}$ & $\begin{array}{l}2 / 9112+02 \\
944359+01\end{array}$ & $\begin{array}{l}207107+02 \\
7 \cap 755.4+01\end{array}$ & $\begin{array}{l}352 \times 90+0.5 \\
122+15+0.3\end{array}$ & $\begin{array}{l}241474+02 \\
034934+01\end{array}$ & $\begin{array}{l}250452 \cdot 02 \\
869012001\end{array}$ \\
\hline IDTAL & $658 / 40+02$ & $87427 ?+02$ & 548113.02 & $997612+112$ & $H_{1}>43 b+02$ & $61: 5923+02$ & $102>d 4+04$ & $124931+02$ & $751<99+42$ \\
\hline AnFiLL & $12522 \%+01$ & $219189+111$ & $135807+01$ & $264468+111$ & $206291+01$ & $15>796+01$ & $3016 \% 4+02$ & $101053+n 1$ & $184 y 25+01$ \\
\hline
\end{tabular}


DOSES FOR ISOTOFE $164(1-133$, - REM

\begin{tabular}{|c|c|c|c|c|c|c|c|c|c|}
\hline DISTANCE & TCT BOOY & BONE & LIVER & KIDYEY & LONADS & LUNG & GI $\mid R_{A} C T$ & THYROID & SKIn \\
\hline $\begin{array}{l}500000+03 \\
400000+04 \\
200000+05 \\
805000+05\end{array}$ & $\begin{array}{l}539218+02 \\
202530+02 \\
436445+02 \\
124442+02\end{array}$ & $\begin{array}{l}558459+02 \\
254778+02 \\
4 \pm 4373+02 \\
1 \geqslant 8101+02\end{array}$ & $\begin{array}{l}4 E 4172+02 \\
191857002 \\
391905 * 02 \\
11174 \% 02\end{array}$ & $\begin{array}{l}496457+02 \\
186474+02 \\
401852+02 \\
114582+02\end{array}$ & $\begin{array}{l}500393+02 \\
206802+02 \\
+45044+02 \\
127062+02\end{array}$ & $\begin{array}{l}872737+02 \\
325102 * 02 \\
686827 * 02 \\
189391 * 02\end{array}$ & $\begin{array}{l}573,27+05 \\
355<91+0 \\
738585+0 \\
235=99+04\end{array}$ & $\begin{array}{l}136716 * 05 \\
113584 * 04 \\
210715 * 05 \\
315862 * 04\end{array}$ & $\begin{array}{l}119043 \bullet 02 \\
151579 \cdot 02 \\
338490+02 \\
563011+01\end{array}$ \\
\hline TOTAL & $130263+13$ & $194571+03$ & 11.6468 .03 & $11993 ?+03$ & $=33$ is $10+03$ & $207406+03$ & $2.35020+04$ & $53037 b+05$ & 101279003 \\
\hline WORLD & $000000 \div 00$ & $00,0000+00$ & $09000 E \div 00$ & $00000 c+00$ & $000000+00$ & $000000+00$ & $000000+00$ & $000000 * 00$ & $000000 \div 00$ \\
\hline
\end{tabular}

DOSES FOR DAUGHTER I (XE-1331) JF ISCTOPE :64 (1.133) - REM

\begin{tabular}{|c|c|c|c|c|c|c|c|c|c|}
\hline DISTANCE & TO' $B O D Y$ & BONE' & LIVER & KIDNEY & UONADS & LUNG & GI $\| H_{A} C^{-}$ & - HYROID & SKIN \\
\hline $\begin{array}{l}500000+03 \\
400000+04 \\
200000+05 \\
805000+05\end{array}$ & $\begin{array}{l}768393-01 \\
286908-01 \\
62<132-01 \\
1 / 8762-01\end{array}$ & $\begin{array}{l}11 ? 014 \div 00 \\
43996:-01 \\
951453001 \\
272226-01\end{array}$ & $\begin{array}{l}503941-01 \\
189477-01 \\
409330-01 \\
11 ; 240-01\end{array}$ & $\begin{array}{l}522664-01 \\
196516-01 \\
424537-01 \\
121595-c 1\end{array}$ & $\begin{array}{l}101412+00 \\
351300-01 \\
823728-01 \\
235931-01\end{array}$ & $\begin{array}{l}599128.02 \\
22527201 \\
486696-01 \\
139429.01\end{array}$ & $\begin{array}{l}453=35-01 \\
170.412-01 \\
368+43-11 \\
105+43-61\end{array}$ & $\begin{array}{l}756692=01 \\
264508=01 \\
614621=01 \\
176040-01\end{array}$ & $\begin{array}{l}1=1803+00 \\
4+3086-01 \\
9581180-01 \\
255162-01\end{array}$ \\
\hline TOTAL & $18 \in 020+00$ & $28 \div 27 \varepsilon+00$ & $121999+00$ & $126531 \cdot c 0$ & $275008+00$ & $145053 \times 00$ & $109: 23+[0$ & $1 E 318 / 000$ & $25.5440+00$ \\
\hline WORLD & $214 \$ 44-01$ & $32 \leqslant 854.01$ & $146782=01$ & $146007-01$ & $233297-01$ & $168033=01$ & $125017-01$ & 211376.01 & $3<b 919-01$ \\
\hline
\end{tabular}

DOSES FOH DAUGHTER 2 (XE-133), OF ISO-OPE 134 (1-133, - REM

\begin{tabular}{|c|c|c|c|c|c|c|c|c|c|}
\hline DISTANCE & TOT BODY & BONE & LIVEF & KIONEY & $\angle O N A D S$ & LUNG & $G_{I} \mid H_{A C T}$ & $i \dashv Y R O_{10}$ & $5 \times ! N$ \\
\hline $\begin{array}{l}500000+03 \\
400000+04 \\
200000+05 \\
805000+05\end{array}$ & $\begin{array}{l}311117+01 \\
116973+01 \\
252673+01 \\
723511+00\end{array}$ & $\begin{array}{l}539359.01 \\
202399.01 \\
43 E 383+01 \\
125499+01\end{array}$ & $\begin{array}{l}207386+01 \\
77899500 \\
16 E .185+01 \\
483580+00\end{array}$ & $\begin{array}{l}215535+0.1 \\
8103 / 4+01 \\
175049+01 \\
501242+00\end{array}$ & $\begin{array}{l}3174<4+01 \\
119339+01 \\
228218+01 \\
759389+00\end{array}$ & $\begin{array}{l}238949+01 \\
8984010+00 \\
194069+02 \\
555742+00\end{array}$ & $\begin{array}{l}18 \leq 030+02 \\
692035+03 \\
145,03+01 \\
428035+00\end{array}$ & $\begin{array}{l}367359+01 \\
12.0118+01 \\
290350+01 \\
85+305+00\end{array}$ & $\begin{array}{l}423339+01 \\
161358+01 \\
34 \times 999+01 \\
09.3540+00\end{array}$ \\
\hline TOTAL & $753114+01$ & $13 a 634.02$ & 501289.01 & $521750+0:$ & $7007040+01$ & $578432 \bullet 01$ & $440<\mathrm{CJ} J+0 I$ & $885: 25 /+01$ & $103 y 16+02$ \\
\hline WORLD & $871180+00$ & $151: 09.01$ & $579933 \cdot 00$ & $6035 \% 0+06$ & $8 y j 33 z+00$ & $671082+40$ & $5162118+06$ & $10 \leq 874+01$ & $12=482+01$ \\
\hline
\end{tabular}


PROGRAM a LRWA - 06/00/7H

DOSES FOH ISOIOPE $172(\times E-153)$ - REM

DISTANCE TOT BODY

BONE.

$-83598+100$

$127133+40$

$268038+00$

$\$ 63694+00$

WORLE $105152+00$

$181909+00$
LIVE?

RIDNEY

$737147-91$

49243.01

$232318+00 \quad 24074$

$240788+00$
$1361 ; 3+0 i$

$532311.00 \quad 551720000$

$551720+00$

730364-01
GONAUS

$148 / 22+00$

72584.01

$2 / 4587+00$

$813 \% 32+00$
J8AROOL

$19105 / 29$

$70407 \geq-01$
LUNG

$907197-01$

$690512=01$
$325733+00$

$251851+30$

$746356+00$
$107721+00 \quad 988022.01$

$938022-01$
SI $I R_{A} C$ T

$533422-01$.

$438<14-01$

$206906+00$

$159476+00$

$474187+00$

$627=94-01$
I.HYROIL

$125901+00$

4) $1600-11$

$317975+00$

$942312+10$

$124745+100$
SKIN

$63514+00$

$439 y 25+00$
$207525+01$

160454002

$475303+01$

$629469+00$ 
TOTAL DOSES TUR ALL ISOTOPES - FEM

\begin{tabular}{|c|c|c|c|c|c|c|c|c|c|}
\hline DISTANCE & Tבי & BaNE & GIVER & KI [INE : & GONADS & $L J_{N G}$ & 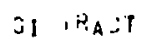 & THYROIO & Sxin \\
\hline $\begin{array}{l}500000+03 \\
400000+04 \\
200000+05 \\
805000+05\end{array}$ & $\begin{array}{l}8.56397+04 \\
3.91247+04 \\
731474+0.4 \\
253799+0.4\end{array}$ & $\begin{array}{l}647791+05 \\
247615+05 \\
552059+05 \\
139972+05\end{array}$ & $\begin{array}{l}247252+05 \\
945089+04 \\
214312+05 \\
724916+04\end{array}$ & $\begin{array}{l}279553+06 \\
100859+10 \\
242507+1.6 \\
819945+1.6\end{array}$ & $\begin{array}{l}703236+05 \\
241706+05 \\
602248+05 \\
223008+05\end{array}$ & $\begin{array}{l}706996+04 \\
34629 C+04 \\
783764+04 \\
263478+04\end{array}$ & $\begin{array}{l}502456 \cdot n= \\
115009 \cdot 00 \\
262097 \times 0= \\
\varepsilon \varepsilon 6=38 \cdot 0=\end{array}$ & $\begin{array}{l}315278+05 \\
119614+05 \\
265655+05 \\
839623+04\end{array}$ & $\begin{array}{l}364 \$ 12 * 03 \\
134385 * 03 \\
316545 * 03 \\
107930 * 03\end{array}$ \\
\hline TOTAL & $220282+05$ & $154744+116$ & $628 ? 65.05$ & $7109 / 3-10$ & $1 \times 4 \cup 80+06$ & $23005 \Omega+05$ & $769126-0 \equiv$ & $? 8450^{9}+05$ & $\partial_{2} / \underline{y} 73+03$ \\
\hline WORLD & $000897+01$ & $E 02041+V 1$ & $547276+01$ & $701646-c 1$ & $700001+01$ & $124027+112$ & $349,99-013$ & $: 30577+01$ & $58 / 174+03$ \\
\hline
\end{tabular}


APPENDIX E

PROGRAM VERIFICATION AND QUALIFICATION 


\section{APPENDIX E}

\section{PROGRAM VERIFICATION AND QUALIFICATION}

Representative results of the effort expended to verify and qualify the AIRWAY computer program are presented in this appendix. In general, the verification and qualification efforts consisted of comparisons with previously reported results, hand calculations and check problems with another computer program. Specifically, comparisons were made with LMFBR air concentration results (Reference E-1). relative deposition rates from Reference E-2, check problem results from the AIRDOS computer program of ORNL (Reference E-3), and the results of hand calculations of check problems. With the exception of the comparisons made with the LMFBR air concentrations, only a representative fraction of the total number of comparisons actually made are presented in this appendix.

The first effort performed to qualify and verify the results obtained from the AIRWAY program was to compare the $X / Q$ 's (air concentrations per unit release rate) reported in Reference E-l for the LMFBR plant with those predicted by the AIRWAY computer program for the same meteorological conditions as a function of distance from the release point for three release heights. The LMFBR results depicted the effect of dispersion upon a long-lived radionuclide using the 18 station average meteorological conditions (see Table F-1 in Appendix F for these wind speed-stability category frequency distributions) with the wind assumed to be blowing in one direction throughout the release. The air concentrations per unit release rates were obtained with and without considering the effect of deposition. The AIRWAY computer program was run using ${ }^{85} \mathrm{Kr}$ to represent a long-lived gaseous radionuclide which is not affected by deposition and ${ }^{129} \mathrm{I}$ to represent a long-lived párticulate radionuclide which is subject to deposition mechanisms. The deposition processes considered in the AIRWAY run were the atmospheric scavenging associated with an annual precipitation rate equivalent to $40^{\prime \prime}$ of rain and the effect of a dry deposition velocity of $1 \mathrm{~cm} / \mathrm{sec}$. The results of these comparisons at 15 radial distances ranging from 400 meters to 80000 meters from the release point are presented in Tables E-1, E-2 and E-3 for release heights of 0,10 and 100 meters, respectively. Examination of these results indicate that the average difference between the reported LMFBR air concentrations and those obtained from the AIRWAY program is $4 \%$ with a maximum discrepancy between the two of $21.3 \%$

A second set of calculations performed to qualify and verify the AIRWAY program was a comparison between the dose commitment estimates provided by the AIRDOS computer program of the Oak Ridge $\mathrm{Na}$ tional Laboratories and those predicted by the AIRWAY program. This effort was primarily performed to ensure that the dose commitment results obtained from the AIRWAY program would not be an order of magnitude different from those obtained from other predictive tools designed to perform similar functions when no special efforts are taken to ensure that equivalent parameters are used among the various programs. Thus, two check problems were defined requesting that dose commitment estimates be obtained for eight specific organe due to the release of given radionuclides at a constant rate over a fixed length of time. The two problems were considered to be representative of a reactor plant placed in an area with a relatively large population and of another facility in the overall fuel cycle such as a fuel reprocessing plant set in a sparsely populated region. Each of these check problems calculated the dose commitments for a "maximum individual" located on the site boundary of the plant and the dose commitments to the general population within a 50 -mile radius of the facility. The specific information provided to ORNL to run this computer program (and thus the only data known to be exactly the same in comparing the ORNL results and the AIRW $\Lambda$ Y results) was

1. Released radionuclides, their release rates and the length of releases

2. Release heights

3. Site boundaries

4. Population distribution within a 50-mile radius of the release point

5. Wind speed-stability category frequency distribution

6. Wind direction.

The above set of parameters were those which were considered would be provided to any organization requested to provide dose commitment estimates. All other parameters were considered to be part of the model used to estimate the dose commitments and thus were not specified. Selected results of these dose commitment comparisons for the "maximum individual" at the reactor plant, the population in the vicinity of the reactor plant, the "maximum individual" at a fuel facility and the population in the vicinity of the fuel 
TABLE E-1. LMFBR X/Q COMPARISONS

$$
\mathrm{h}=0
$$

\begin{tabular}{ccccccc}
$\begin{array}{c}\text { Distance } \\
\text { (meters) }\end{array}$ & \multicolumn{3}{c}{ No Deposition } & & & Deposition \\
\hline & LMFBR & AIRWAY & A/L & LMFBR & AIRWAY & A/L \\
\hline 400 & $2.05-4$ & $2.0-4$ & 0976 & $1.38-4$ & 1.0861 & 0.707 \\
600 & $9.47-5$ & $9.25-5$ & 0.977 & $5.77-5$ & $4.63-5$ & 0.802 \\
800 & $5.57-5$ & $5.47-5$ & 0.982 & $3.16-4$ & $2.575-5$ & 0.815 \\
1000 & $3.69-5$ & $3.66-5$ & 0.992 & $1.98-5$ & $1.642-5$ & 0.829 \\
1500 & $1.82-5$ & 1.8165 & 0.998 & $8.79-6$ & $7.38-6$ & 0.840 \\
2000 & $1.08-5$ & $1.107-5$ & 1025 & $4.83-6$ & $4.18-6$ & 0.865 \\
3000 & $5.75-6$ & $5.69-6$ & 0.990 & $2.25-6$ & $1.913-6$ & 0.850 \\
4000 & $3.68-6$ & $3.645-6$ & 0.990 & $1.31-6$ & $1.216-6$ & 0.860 \\
5000 & $2.62-6$ & $2.61-6$ & 0.996 & $8.54-7$ & $7.53-7$ & 0.882 \\
10000 & $9.47-7$ & $9.42-7$ & 0.995 & $2.33-7$ & $2.123-7$ & 0.911 \\
15000 & $5.38-7$ & $5.4-7$ & 1.004 & $1.09-7$ & $1.023-7$ & 0.939 \\
20000 & $3.6-7$ & $3.642-7$ & 1.012 & $6.23-8$ & $6.0-8$ & 0.963 \\
40000 & $1.47-7$ & $1.45-7$ & 0.986 & 1.618 & $1.617-8$ & 1.004 \\
60000 & $8.72-8$ & $8.69-8$ & 0.997 & $6.93-9$ & $7.45-9$ & 1.075 \\
80000 & $6.01-8$ & $5.99-8$ & 0.997 & $3.74-9$ & $4.21-9$ & 1.126
\end{tabular}


TABLE E-2. LMFBR X/Q COMPARISONS

$$
h=10 \text { meters }
$$

\begin{tabular}{rrrrrrr}
$\begin{array}{r}\text { Distance } \\
\text { (meters) }\end{array}$ & \multicolumn{3}{c}{ No Deposition } & & & Deposition \\
\hline & LMFBR & AIRWAY & A/L & LMFBR & AIRWAY & A/L \\
\hline 400 & $8.85-5$ & $8.92-5$ & 1.008 & $8.28-5$ & $8.28-5$ & 1.0 \\
600 & $6.03-5$ & $6.03-5$ & 1.0 & $5.39-5$ & $5.35-5$ & 0.993 \\
800 & $4.16-5$ & $4.14-5$ & 0.995 & $3.52-5$ & $3.47-5$ & 0.986 \\
1000 & $3.01-5$ & $3.0-5$ & 0.997 & $2.41-5$ & $2.38-5$ & 0.988 \\
1500 & $1.62-5$ & $1.615-5$ & 0.997 & $1.16-5$ & $1.14-5$ & 0.983 \\
2000 & $1.0-5$ & $1.02-5$ & 1.02 & $6.51-6$ & $6.55-6$ & 1.006 \\
3000 & $5.46-6$ & $5.411-6$ & 0.991 & $3.04-6$ & $2.98-6$ & 0.980 \\
4000 & $3.54-6$ & $3.509-6$ & 0.991 & $1.75-6$ & $1.73-6$ & 0.989 \\
5000 & $2.53-6$ & $2.531-6$ & 1.0004 & $1.13-6$ & $1.13-6$ & 1.0 \\
10000 & $9.3-7$ & $9.259-7$ & 0.996 & $2.96-7$ & $2.98-7$ & 1.007 \\
15000 & $5.3-7$ & $5.329-7$ & 1.005 & $1.34-7$ & $1.38-7$ & 1.030 \\
20000 & $3.56-7$ & $3.606-7$ & 1.013 & $7.59-8$ & $7.91-7$ & 1.042 \\
40000 & $1.46-7$ & $1.44-7$ & 0.986 & $1.89-8$ & $2.0-8$ & 1.058 \\
60000 & $8.66-8$ & $8.634-8$ & 0.997 & $7.91-9$ & $8.85-9$ & 1.119 \\
80000 & $5.97-8$ & $5.959-8$ & 0.998 & $4.18-9$ & $4.86-9$ & 1.163
\end{tabular}


TABLE E-3. LMFBR X/Q COMPARISONS

$$
\mathrm{h}=100 \text { meters }
$$

\begin{tabular}{rrrrrrr}
$\begin{array}{r}\text { Distance } \\
\text { (meters) }\end{array}$ & \multicolumn{3}{c}{ No Deposition } & & & Deposition \\
\cline { 2 - 6 } 400 & $6.64-7$ & $0.671-7$ & 1011 & $6.63-7$ & 6.77 & 1.011 \\
600 & $1.63-6$ & $1.66-6$ & 1.018 & $1.63-6$ & $1.65-6$ & 1.012 \\
800 & $1.72-6$ & $1.74-6$ & 1.012 & $1.7-6$ & $1.72-6$ & 1.012 \\
1000 & $1.54-6$ & $1.52-6$ & 0.987 & $1.52-6$ & $1.5-6$ & 0.987 \\
1500 & $1.12-6$ & $1.12-6$ & 1.0 & $1.1-6$ & $1.1-6$ & 1.0 \\
2000 & $9.2-7$ & $9.44-7$ & 1.026 & $8.95-7$ & $9.14-7$ & 1.021 \\
3000 & $6.66-7$ & $6.79-7$ & 1.020 & $6.36-7$ & $6.44-7$ & 1.013 \\
4000 & $5.19-7$ & $5.41-7$ & 1.042 & $4.84-7$ & $4.99-7$ & 1.031 \\
5000 & $4.26-7$ & $4.51-7$ & 1.059 & $3.87-7$ & $4.05-7$ & 1.047 \\
10000 & $2.4-7$ & $2.58-7$ & 1.075 & $2.02-7$ & $2.12-7$ & 1.050 \\
15000 & $1.72-7$ & $1.83-7$ & 1.064 & $1.34-7$ & $1.39-7$ & 1.037 \\
20000 & $1.35-7$ & $1.43-7$ & 1.059 & $9.62-8$ & $9.83-8$ & 1.022 \\
40000 & $6.93-8$ & $749-8$ & 1.081 & 3.368 & $3.4-8$ & 1.012 \\
60000 & $4.61-8$ & $5.0-8$ & 1.085 & $1.58-8$ & $1.6-8$ & 1.013 \\
80000 & $3.42-8$ & $3.72-8$ & 1.088 & $8.78-9$ & $9.02-9$ & 1.027
\end{tabular}


facility are presented in Tables E-4, E-5, E-6 and E-7, respectively. Each of these tables presents a comparison of the Oak Ridge dose commitments and those obtained from the AIRWAY program arising from immersion in the release plume, surface deposits, ingestion and inhalation for the gaseous radionuclide ${ }^{85} \mathrm{Kr}$ and the particulate radionuclide ${ }^{133} \mathrm{I}$. Results are presented for eight organs. The results of these comparisons with the Oak Ridge results indicate that dose commitment estimates obtained from the AIRWAY program will not be grossly different from those provided by other models for the same basic problem. Further examination of the model used by Oak Ridge identified differences in the dose commitment conversion factors and in the standard deviation of vertical dispersion data as a function of distance from the release point and of the stability category from those used in the AIRWAY program which could reduce the differences between the results obtained from the two models.

Throughout the development of the AIRWAY computer program a continuing comparison of the program results were made with hand calculations of various quantities to verify that the computer program was performing the desired calculations correctly. To accomplish this check, hand calculation problems were set up to examine the air concentrations, the ground concentrations and the 70-year dose commitments arising from the continuous release at a constant rate of six radionuclides ( 3 gaseous nuclides and 3 particulate nuclides). These problems considered four radial positions, two azimuthal sectors and two release heights. The releases were assumed to take place over a 30-year period and the dose commitment estimates started at the end of the $29^{\text {th }}$ year of releases and continued for a 70 -year period. These dose commitment estimates were made assuming a constant population over a 70-year dose commitment period and assuming an increasing population over that period. The following specific comparisons between the AIRWAY program results and the hand calculations were made:

1. Air concentrations at four radial positions and two azimuthal positions in the vicinity of the release point for each of two release heights for each of the six released radionuclides and for each of the five daughters of the released radionuclides during the period that releases were occurring.

2. The time-dependent air concentrations in the overall region affected by the releases at 49 times for each of the two release heights and for each of the six released radionuclides. The 49 times covered both the period during releases and the period subsequent to releases.

3. The time-dependent ground concentrations of the three released particulate radionuclides at each of four radial positions and two azimuthal positions in the vicinity of the release point for each of the two release heights. These 16 comparisons for each released particulate were made at 49 times covering the period during releases and the period subsequent to releases.

4 The time-dependent ground concentrations of the three released particulate radionuclides in the overall region affected by the releases for each of the two release heights. These comparisons were made at 49 times extending over the period that releases were occurring and after they had stopped.

5. The 70-year dose commitments for each of nine organs in the vicinity of the release point and in the overall region affected by the releases for each of the six released radionuclides and their five daughters assuming a stable population throughout. These comparisons wère made for each of six pathways (immersion, surface deposits, ingestion of food contaminated during the releases, ingestion of food produced in ground conta minated by prior releases, inhalation of the released radionuclides and their daughters prior to any deposition and the inhalation of resuspended radionuclides) and for each of the two release heights.

6. The 70-year dose commitments for each of nine organs in the vicinity of the release point and in the overall region affected by the releases assuming the population is increasing in both regions for two of the released radionuclides (one gaseous nuclide and one particulate nuclide) for each of the two release heights from earh of the six pathways.

A representative sampling of these comparisons are presented in Tables E-8 through E-17. All hand calculation values a re within $1 "$ : of the AIRWAY program results.

To further qualify the AIRWAY computer program a series of comparisons were made with Figures 7 through 10 of Reference E-2. These figures present relative deposition rates as a function of distance from the release point, the height of the releases and the meteorological stability categories. These relative deposition rates depicted in Reference E-2 may be used to estimate the deposition which occurs due to dry deposition mechanisms.

The AIRWAY computer program was run using the 1974-1975 Allegheny County meteorological data (presented in Table F-2 in Appendix F) and the standard deviation of vertical disposion data presented in 
TABLE E-4. ORNL COMPARISONS-DOSE COMMITMENTS-REACTOR FACILITY

Maximum Individual

\begin{tabular}{|c|c|c|c|c|c|c|c|}
\hline \multirow[b]{2}{*}{ Pathway } & \multirow[b]{2}{*}{ Organ } & \multicolumn{3}{|c|}{$K_{r}-85$} & \multicolumn{3}{|c|}{ I-133 } \\
\hline & & Oak Ridge & ĀIRWAY & OR/A & Oak Ridge & AIRWAY & $\overline{O R / A}$ \\
\hline \multirow{8}{*}{$\begin{array}{l}\text { Air Im- } \\
\text { mersion }\end{array}$} & Total Body & $0.138-4$ & $0.155-4$ & 0.89 & $0.431-2$ & $0.480-2$ & 0.90 \\
\hline & Bone & $0.168-4$ & $0.190-4$ & 0.88 & $0.513-2$ & $0.568-2$ & 0.90 \\
\hline & Liver & $0.115-4$ & $0.130-4$ & 0.88 & $0.361-2$ & $0.401-2$ & 0.90 \\
\hline & Kidney & $0.122-4$ & $0.138-4$ & 0.88 & $0.386-2$ & $0.418-2$ & 0.92 \\
\hline & Gonad & $0.152-4$ & $0.171-4$ & 0.89 & $0.460-2$ & $0.499-2$ & 0.92 \\
\hline & Lung & $0.120-4$ & $0.146-4$ & 0.88 & $0.412-2$ & $0.453-2$ & 0.91 \\
\hline & GI Tract & $0.990-5$ & $0.155-4$ & 064 & $0.322-2$ & 0.1662 & 0.69 \\
\hline & Thyroid & $0.145-4$ & $0.163-4$ & 0.89 & & & \\
\hline \multirow{8}{*}{$\begin{array}{l}\text { Surface } \\
\text { Deposit }\end{array}$} & Total Body & & & & 0.0898 & 00944 & 0.95 \\
\hline & Bone & & & & 0.107 & 0.111 & 0.96 \\
\hline & Liver & & & & 0.0752 . & 0.0788 & 0.95 \\
\hline & Kidney & & & & 0.0782 & 0.0523 & 0.95 \\
\hline & Gonad & & & & 0.0934 & 0.0975 & 0.96 \\
\hline & Lung & & & & 0.0850 & 0.0891 & 0.95 \\
\hline & GI Tract & & & & 0.0654 & 0.0317 & 0.71 \\
\hline & Thyroid & & & & 0.0744 & 0.0951 & 0.78 \\
\hline \multirow{8}{*}{ Ingestion } & Total Body & & & & 0.107 & 0.0585 & 1.83 \\
\hline & Bone & & & & 0.107 & 0.0585 & 1.83 \\
\hline & Liver & & & & 0.107 & 00.585 & 1.83 \\
\hline & Kidney & & & & 0.107 & 0.0585 & 1.83 \\
\hline & Gonad & & & & 0.107 & 0.0585 & 1.83 \\
\hline & Lung & & & & 0.107 & 0.0585 & 1. 83 \\
\hline & GI Tract & & & & 0.453 & 2.13 & 0.17 \\
\hline & Thyroid & & & & 71.7 & 38.8 & 1.85 \\
\hline \multirow{8}{*}{ Inhalation } & Total Body & & & & $0.369-2$ & $0.224-2$ & 1.65 \\
\hline & Bone & & & & $0.379-2$ & $0.224-2$ & 1.69 \\
\hline & Liver & & 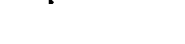 & & 0.3803 & $0.224-2$ & 1.70 \\
\hline & Kidney & & & & 0.367 .2 & $0.224-2$ & 1.64 \\
\hline & Gonad & & & & $0.376-2$ & $0.224-2$ & 1.68 \\
\hline & Lung & $0.153-4$ & $0.172-4$ & 0.89 & 0.033 & 0.105 & 0.31 \\
\hline & GI Tract & & & & 0.0139 & 0.0558 & 0.25 \\
\hline & Thyroid & . & & & 2.53 & 1.5 & 1.69 \\
\hline
\end{tabular}


TABLE E-5. ORNL COMPARISONS-DOSE COMMITMENTS-REACTOR FACILITY

Population

\begin{tabular}{|c|c|c|c|c|c|c|c|}
\hline \multirow[b]{2}{*}{ Pathway } & \multirow[b]{2}{*}{ Organ } & \multicolumn{3}{|c|}{$\mathrm{K}_{\mathrm{r}-85}$} & \multicolumn{3}{|c|}{ I-133 } \\
\hline & & Oak-Ridge & AIRWAY & OR/A & Oak Ridge & AIRWAY & $\mathrm{OR} / \mathrm{A}$ \\
\hline \multirow{8}{*}{$\begin{array}{l}\text { Air Im- } \\
\text { mersion }\end{array}$} & Total Body & 0.26 & 0.193 & 1.35 & 15.0 & 10.1 & 1.49 \\
\hline & Bone & 0.32 & 0.236 & 1.36 & 18.0 & 11.9 & 1.51 \\
\hline & Liver & 0.22 & 0.162 & 1.36 & 12.0 & 8.42 & 1.43 \\
\hline & Kidney. & 0.23 & 0.172 & 1.34 & 13.0 & 8.78 & 1.48. \\
\hline & Gonad & 0.29 & 0.214 & 1.36 & 15.0 & 10.5 & 1.43 \\
\hline & Lung & 0.25 & 0.182 & 1.37 & 14.0 & 9.52 & 1.47 \\
\hline & GI Tract & $0: 19$ & 0.193 & 0.98 & 11.0 & 9.77 & 1.13 \\
\hline & Thyroid & 0.28 & 0.203 & 1.38 & & & \\
\hline \multirow{8}{*}{$\begin{array}{l}\text { Surface } \\
\text { Deposit }\end{array}$} & Total Body & & & & 330.0 & 209.0 & 1.58 \\
\hline & Bone & & & & 390.0 & 246.0 & 1.59 \\
\hline & Liver & & & & 270.0 & 174.0 & 1.55 \\
\hline & Kidney & . & & & 290.0 & 182.3 & 1.59 \\
\hline & Gonad & & & & 340.0 & 216.0 & 1.57 \\
\hline & Lung & & & & 310.0 & 197.0 & 1.57 \\
\hline & GI Tract & & & & 240.0 & 203.0 & 1.18 \\
\hline & Thyroid & & & & 330.0 & 211.0 & 1.56 \\
\hline \multirow{3}{*}{ 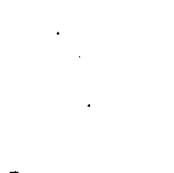 } & Total Body & & & & 390.0 & 195.0 & 2.0 \\
\hline & Bone & & & & 390.0 & 195.0 & 2.0 \\
\hline & Liver & & & & 390.0 & 195.0 & 2.0 \\
\hline \multirow[t]{5}{*}{ Ingestion } & Kidney & & & & 390.0 & 195.0 & 2.0 \\
\hline & Gonad & & & . & 390.0 & 195.0 & 2.0 \\
\hline & Lung & & & & 390.0 & 195.0 & 2.0 \\
\hline & GI Tract & & & & 1700 & 9110 & 0.19 \\
\hline & Thyroid & & & & $0.26+6$ & $0.13+6$ & 2.0 \\
\hline \multirow{8}{*}{ Inhalation } & Total Body & & & & 13.0 & 4.69 & 2.77 \\
\hline & Bone & & & & 13.0 & 4.69 & 2.77 \\
\hline & Liver & & & & 13.0 & 1.68 & 2.77 \\
\hline & Kidney & & & & 13.0 & 4.69 & 2.77 \\
\hline & Gonad & & & & 13.0 & 4.69 & 2.77 \\
\hline & Lung & 0.29 & 0.215 & 1.35 & 110.0 & 220.0 & 0.50 \\
\hline & GI Tract & & & & 47.0 & 117.0 & 0.40 \\
\hline & Thyroid & & & & 8700 & 3140. & 2.77 \\
\hline
\end{tabular}


TABLE E-6. ORNL COMPARISONS-DOSE COMMITMENTS-FUEL FACILITY

Maximum Individual

\begin{tabular}{|c|c|c|c|c|c|c|c|}
\hline \multirow[b]{2}{*}{ Pathway } & \multicolumn{4}{|c|}{$\mathrm{K}_{\mathrm{r}-85}$} & \multicolumn{3}{|c|}{ I-133 } \\
\hline & Organ & Oak Ridge & AIRWĀY & OR/A & Oak Ridge & AIRWAY & OR/A \\
\hline \multirow{8}{*}{$\begin{array}{l}\text { Air Im- } \\
\text { mersion }\end{array}$} & Total Body & $0.034=\mathrm{C}$ & $0.820-0$ & 1.02 & $0.277-3$ & $0.257-3$ & 1.08 \\
\hline & Bone & $0.102-5$ & $0.1-5$ & 1.02 & $0.316-3$ & $0.304-3$ & 1.04 \\
\hline & Liver & $0.694-6$ & $0.686-6$ & 1.01 & $0.23-3$ & $0.215-3$ & 1.07 \\
\hline & Kidney & $0.739-6$ & $0.729-6$ & 1.01 & $0.233-3$ & $0.224-3$ & 1.04 \\
\hline & Gonad & $0.922-6$ & $0.907-6$ & 1.02 & $0.281-3$ & $0.267-3$ & 1.05 \\
\hline & Lung & $0.785=6$ & $0.772-6$ & 1.02 & $0.256-3$ & $0.243-3$ & 1.05 \\
\hline & GI Tract & $0.6-6$ & $0.82-6$ & $\cap 73$ & $0191=3$ & $0.249-3$ & 0.77 \\
\hline & Thyrold & $0.877-8$ & 0.06.1-6 & $14 \%$ & & & \\
\hline \multirow{8}{*}{$\begin{array}{l}\text { Surface } \\
\text { Deposit }\end{array}$} & Total Body & & & & $0.642-2$ & $0.613-2$ & 10.5 \\
\hline & Bune & & & & $0.758-2$ & $0.721-2$ & 1.05 \\
\hline & Liver & & & & $0.536-2$ & $0.512-2$ & 1.05 \\
\hline & Kidney & & & & $0.558-2$ & $0.534-2$ & 1.04 \\
\hline & Gonad & & & & $0.665-2$ & $0.634-2$ & 1.05 \\
\hline & Lung & & & & $0.605-2$ & $0.579-2$ & 1.04 \\
\hline & GI Tract & & & & $0.469-2$ & $0.595-2$ & 0.79 \\
\hline & Thyroid & & & & 0.528 .2 & $0.618-2$ & 0.85 \\
\hline \multirow{8}{*}{ Ingestion } & Total Body & & & & $0.767-2$ & $0.38-2$ & 2.02 \\
\hline & Bone & & & & $0.765-2$ & $0.38-2$ & 2.01 \\
\hline & Liver & & & & $0.767-2$ & $0.38-?$ & 2. ก). \\
\hline & Kidney & & & & $0.764-2$ & $0.38-2$ & 2.01 \\
\hline & Gonad & & & & $0.764-2$ & $0.38-2$ & 2.01 \\
\hline & Lung & & & & $0.763-2$ & $0.38-2$ & 2.01 \\
\hline & GI Tracl & & & & $0.323-1$ & 0.177 & 0.18 \\
\hline & Thyroid & & & & 5.12 & 2.52 & 2.03 \\
\hline \multirow{8}{*}{ Inhalation } & Total Body & & & & $0.234-3$ & $0.12-3$ & 1.95 \\
\hline & Bone & & & & $0.237-3$ & $0.12-3$ & 1.98 \\
\hline & T.iver & & & & $0.23-3$ & $0.13: 3$ & 1.92 \\
\hline & Kidney & & & & $0.233-3$ & $0.12-3$ & 1.94 \\
\hline & Gonad & & & & $0.237-3$ & $0.12-3$ & 1.98 \\
\hline & Lung & $0.925-6$ & $0.911-6$ & 1.02 & $0.205-2$ & $0.56-2$ & 0.37 \\
\hline & GI Tract & & & & $0.876-3$ & $0.298-2$ & 0.29 \\
\hline & Thiyruid & & & & 0.158 & 0.0801 & 1.97 \\
\hline
\end{tabular}


TABLE E-7. ORNL COMPARISONS-DOSE COMMITMENTS-FUEL FACILITY

Population

\begin{tabular}{|c|c|c|c|c|c|c|c|}
\hline \multirow[b]{2}{*}{ Pathway } & \multirow{2}{*}{ Organ } & \multicolumn{3}{|c|}{$K_{r}-85$} & \multicolumn{3}{|c|}{ I-133 } \\
\hline & & Oak Ridge & AIRWAY & OR/A & Oak Ridge & AIRWAY & OR/A \\
\hline \multirow{8}{*}{$\begin{array}{l}\text { Air Im- } \\
\text { mersion }\end{array}$} & Total Body & 0.016 & 0.0323 & 0.50 & 3.2 & 2.57 & 1.25 \\
\hline & Bone & 0.019 & 0.0394 & 0.48 & 3.7 & 3.04 & 1.22 \\
\hline & Liver & 0.013 & 0.027 & 0.48 & 2.6 & 2.15 & 1.21 \\
\hline & Kidney & 0.014 & 0.0287 & 0.49 & 2.8 & 2.24 & 1.25 \\
\hline & Gonad & 0.017 & 0.0357 & 0.48 & 3.3 & 2.67 & 1.24 \\
\hline & Lung & 0.015 & 0.0304 & 0.49 & 3.0 & 2.43 & 1.23 \\
\hline & GI Tract & 0.011 & 0.0323 & 0.34 & 2.3 & 2.49 & 0.92 \\
\hline & Thyroid & 0.017 & 0.034 & 0.50 & 3.2 & 2.59 & 1.24 \\
\hline \multirow{8}{*}{$\begin{array}{l}\text { Surface } \\
\text { Deposit }\end{array}$} & Total Body & & & & 80.0 & 56.4 & 1.42 \\
\hline & Bone & & & & 95.0 & 66.4 & $1.43^{\circ}$ \\
\hline & Liver & & & & 67.0 & 47.1 & 1.42 \\
\hline & Kidney & & & & 70.0 & 49.2 & 1.42 \\
\hline & Gonad & & & & 83.0 & 58.3 & 1.42 \\
\hline & Lung & & & & 76.0 & 53.3 & 1.43 \\
\hline & GI Tract & & & & 59.0 & 54.8 & 1.08 \\
\hline & Thyroid & & & & 81.0 & 56.9 & 1.42 \\
\hline \multirow{8}{*}{ Ingestion } & Total Body & & & & 96.0 & 36.6 & 2.62 \\
\hline & Bone & & & & 96.0 & 36.6 & 2.62 \\
\hline & Liver & & & & 96.0 & 36.6 & 2.62 \\
\hline & Kidney & & & & 96.0 & 36.6 & 2.62 \\
\hline & Gonad & & & & 96.0 & 36.6 & 2.62 \\
\hline & Lung & & & & 96.0 & 36.6 & 2.62 \\
\hline & GI Tract & & & - & 400.0 & 1710 & 0.23 \\
\hline & Thyroid & & & & $0.64+5$ & $0.24+5^{-}$ & 2.63 \\
\hline \multirow{8}{*}{ Inhalation } & Total Body & & . & & 2.7 & 1.2 & 2.25 \\
\hline & Bone & & & & 2.7 & 1.2 & 2.25 \\
\hline & Liverer & & & & 2.7 & 1.2 & 2.25 \\
\hline & Kidney & & & & 2.7 & 1.2 & 2.25 \\
\hline & Gonad & & & & 2.7 & 1.2 & 2.25 \\
\hline & Lung & 0.017 & 0.0358 & 0.47 & 24.0 & 56.0 & 0.43 \\
\hline & GI Tract & & & & 10.0 & 29.9 & 0.33 \\
\hline & Thyroid & & & & 1900 & 802 & 2.37 \\
\hline
\end{tabular}


TABLE E-8. HAND CALCULATION COMPARISONS-AIR CONCENTRATIONSVICINITY OF RELEASE POINT DURING RELEASES

\begin{tabular}{|c|c|c|c|c|c|c|c|c|}
\hline \multirow[b]{2}{*}{ Nuclide } & \multirow{2}{*}{$\begin{array}{c}\mathbf{h} \\
(\mathbf{m})\end{array}$} & \multirow{2}{*}{$\begin{array}{c}\mathbf{r} \\
\text { meters } \\
\end{array}$} & \multicolumn{3}{|c|}{$\theta=0.3927$ radian } & \multicolumn{3}{|c|}{$\theta=5.8905$ radian } \\
\hline & & & AIRWAY & Verification- & $\mathbf{V} / \mathbf{A}$ & AIRWAY & Verification & $\mathrm{V} / \mathrm{A}$ \\
\hline & 100 & $\begin{array}{r}500 \\
4000 \\
20000 \\
80500\end{array}$ & $\begin{array}{l}8.74475-11 \\
3.75923-11 \\
9.95032-12 \\
2.56503-12\end{array}$ & $\begin{array}{l}8.742-11 \\
3.752-11 \\
9.948-12 \\
2.565-12\end{array}$ & $\begin{array}{l}0.9997 \\
0.9981 \\
0.9998 \\
1.0\end{array}$ & $\begin{array}{l}1.74895-11 \\
7.51845-12 \\
1.99006-12 \\
5.13005-13\end{array}$ & $\begin{array}{l}1.748-11 \\
7.521-12 \\
1.99-12 \\
5.129-13\end{array}$ & $\begin{array}{l}0.9994 \\
1.0003 \\
1.0 \\
0.9998\end{array}$ \\
\hline & 0 & $\begin{array}{r}500 \\
4000 \\
20000 \\
80500\end{array}$ & $\begin{array}{l}9.00514-9 \\
2.53148-10 \\
2.52939-11 \\
4.12893-12\end{array}$ & $\begin{array}{l}9.005-9 \\
2.526-10 \\
2.53-11 \\
4.125-12\end{array}$ & $\begin{array}{l}1.9 \\
0.9978 \\
1.0002 \\
0.999\end{array}$ & $\begin{array}{l}1.80103-9 \\
5.06296-11 \\
5.05878-12 \\
8.25786-13\end{array}$ & $\begin{array}{l}1.8-9 \\
5.062-11 \\
5.06-12 \\
8.25-13\end{array}$ & $\begin{array}{l}0.9994 \\
0.9998 \\
1.0002 \\
0.999\end{array}$ \\
\hline & 100 & $\begin{array}{r}500 \\
4000 \\
20000 \\
80500\end{array}$ & $\begin{array}{l}8.71605-11 \\
3.46614-11 \\
6.81799-12 \\
6.16573-13\end{array}$ & $\begin{array}{l}8.63202-11 \\
3.45873-11 \\
6.81827-12 \\
6.16808-13\end{array}$ & $\begin{array}{l}0.9904 \\
0.9979 \\
1.0 \\
1.0004\end{array}$ & $\begin{array}{l}1.74321-11 \\
6.93227-12 \\
1.3636-12 \\
1.23315-13\end{array}$ & $\begin{array}{l}1.72759-11 \\
6.9322-12 \\
1.36365-12 \\
1.23362-13\end{array}$ & $\begin{array}{l}0.99 \\
1.0 \\
1.0 \\
1.0004\end{array}$ \\
\hline 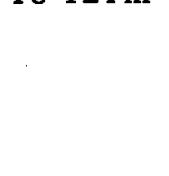 & 0 & $\begin{array}{r}500 \\
4000 \\
20000 \\
80500\end{array}$ & $\begin{array}{l}4.69966-9 \\
7.87262-11 \\
4.19339-12 \\
2.89478-13\end{array}$ & $\begin{array}{l}4.67342-9 \\
7.85527-11 \\
4.23049-12 \\
2.89421-13\end{array}$ & $\begin{array}{l}0.9944 \\
0.9978 \\
1.0088 \\
0.9998\end{array}$ & $\begin{array}{l}9.39932-10 \\
1.57452-11 \\
8.38679-13 \\
5.78955-14\end{array}$ & $\begin{array}{l}9.34353-10 \\
1.5744-11 \\
8.46099-13 \\
5.78843-14\end{array}$ & $\begin{array}{l}0.9941 \\
0.9999 \\
1.0088 \\
0.9998\end{array}$ \\
\hline & 100 & $\begin{array}{r}500 \\
4000 \\
20000 \\
80500\end{array}$ & $\begin{array}{l}3.53603-13 \\
9.96455-13 \\
7.96737-13 \\
1.40119-13\end{array}$ & $\begin{array}{l}3.51181-13 \\
9.94322-13 \\
7.96763-13 \\
1.40168-13\end{array}$ & $\begin{array}{l}0.9932 \\
0.9979 \\
1.0 \\
1.0003\end{array}$ & $\begin{array}{l}1.01205-14 \\
1.99291-13 \\
1.59 .347-1.3 \\
2.802 .38-14\end{array}$ & $\begin{array}{l}1.02112-14 \\
1.99288-13 \\
159353-13 \\
2.80336-14\end{array}$ & $\begin{array}{l}0.9928 \\
1.0 \\
1.0 \\
1.0003\end{array}$ \\
\hline $\begin{array}{l}\text { (daughter } \\
\text { of } \\
\text { Te- } 127 \mathrm{~m} \text { ) }\end{array}$ & 0 & $\begin{array}{r}500 \\
4000 \\
20000 \\
80500\end{array}$ & $\begin{array}{l}1.39136-11 \\
1.39674-12 \\
2.88261-13 \\
5.98733-14\end{array}$ & $\begin{array}{l}1.37474-11 \\
1.39359-12 \\
2.91125-13 \\
5.98676-14\end{array}$ & $\begin{array}{l}0.9981 \\
0.9977 \\
1.0099 \\
0.9999\end{array}$ & $\begin{array}{l}2.78272-12 \\
2.79349-13 \\
5.76523-14 \\
1.19747-14\end{array}$ & $\begin{array}{l}2.74851-12 \\
2.79312-13 \\
5.8225-14 \\
1.19735-14\end{array}$ & $\begin{array}{l}0.9877 \\
0.9999 \\
1.0099 \\
0.9999\end{array}$ \\
\hline
\end{tabular}


TABLE E-9. HAND CALCULATION COMPARISONS-AIR CONCENTRATIOONSWORLD-DURING RELEASES

\begin{tabular}{|c|c|c|c|c|c|}
\hline Nuclide & $h(m)$ & Time-Yrs & AIRWAY & Verification & V/A \\
\hline & & 2.0 & $8.2966-15$ & $8.2966-15$ & 1.0 \\
\hline \multirow[t]{5}{*}{$\mathrm{Kr}-85$} & any & 10.1 & $3.25048-14$ & $3.25043-14$ & 1.0 \\
\hline & & 20.2 & $4.9438-14$ & $4.9438-14$ & 1.0 \\
\hline & & 28.3 & $5.69229-14$ & $5.69229-14$ & 1.0 \\
\hline & 100 & 2.0 & $3.58002-17$ & $3.58068-17$ & 1.0002 \\
\hline & & 28.3 & $3.58002-17$ & $3.58068-17$ & 1.0002 \\
\hline \multicolumn{6}{|l|}{$\mathrm{Te}-127 \mathrm{~m}$} \\
\hline & 0 & 2.0 & $1.04418-17$ & $1.04525-17$ & 1.001 \\
\hline & & 28.3 & $1.04418-17$ & $1.04525-17$ & 1.001 \\
\hline
\end{tabular}


TABLE E-10. HAND CALCULATION COMPARISONS-AIR CONCENTRATIONS-

WORLD-AFTER RELEASE

\begin{tabular}{|c|c|c|c|c|c|}
\hline Nuclide & $\mathbf{h}(\mathbf{m})$ & Time-Yrs & AIRWAY & Verification & $\mathbf{V} / \mathbf{A}$ \\
\hline \multirow{8}{*}{ Kr-85 } & \multirow{7}{*}{ any } & 30.3 & $5.69316-14$ & $5.69315-14$ & 1.0 \\
\hline & & 40.4 & $2.96582-14$ & $296582-14$ & 1.0 \\
\hline & & 50.5 & $1.54503-14$ & $1.54503-14$ & 1.0 \\
\hline & & 60.6 & $8.04874-15$ & $8.04874-15$ & 1.0 \\
\hline & & 70.7 & 4.19295-15 & 4.19295-15 & 1.0 \\
\hline & & 80.8 & $2.18429-1.5$ & . $2.18429-15$ & 1.0 \\
\hline & & 90.9 & $1.1379-15$ & $1.1379-15$ & 1.0 \\
\hline & & 98.9 & $6.75361-16$ & $6.75361-16$ & 1.0 \\
\hline \multirow{4}{*}{$\mathrm{Te}-127 \mathrm{~m}$} & 100 & 30.3 & $8.26526-37$ & $8.26679-37$ & 1.0002 \\
\hline & & 32.3 & 0 & 0 & 1.0 \\
\hline & - & & & . & \\
\hline & \multirow[t]{2}{*}{0} & 30.3 & $2.41073-37$ & $2.41318-31$ & 1.001 \\
\hline . & & 32.3 & 0 & 0 & 1.0 \\
\hline
\end{tabular}


TABLE E-11. HAND CALCULATION COMPARISONS-GROUND CONCENTRATIONSVICINITY OF RELEASES-RELEASES STOP AT 30 YEARS

\begin{tabular}{|c|c|c|c|c|c|c|c|}
\hline \multirow{3}{*}{$\begin{array}{l}\text { Time } \\
\text { yrs }\end{array}$} & & & $\begin{array}{r}\mathrm{Te} \\
\theta=0.3\end{array}$ & $7 \mathrm{~m}$ & & & \\
\hline & \multirow{2}{*}{$\begin{array}{c}\mathbf{r} \\
\text { meters }\end{array}$} & \multicolumn{3}{|c|}{$\mathbf{h}=100$} & \multicolumn{3}{|c|}{$\mathrm{h}=0$} \\
\hline & & AIRWAY & Verification & $\mathbf{V} / \mathbf{A}$ & AIRWAY & Verification & V/A \\
\hline \multirow{4}{*}{2.0} & 500 & $1.863-5$ & $1.84553-5$ & 0.9906 & $6.3701-4$ & $6.27631-4$ & 0.9853 \\
\hline & 4000 & $5.48467-6$ & $5.47472-6$ & 0.9982 & $1.10088-5$ & $1.09855-5$ & 0.9979 \\
\hline & 20000 & $1.04012-6$ & $1.04016-6$ & 1.0 & $6.23737-7$ & $6.28856-7$ & 1.0082 \\
\hline & 80500 & $9.66348-8$ & $9.66679-8$ & 1.0003 & $4.76499-8$ & $4.76432-8$ & 0.9998 \\
\hline \multirow{4}{*}{4.0} & 500 & $1.88011-5$ & $1.86248-5$ & 0.9906 & $6.42861-4$ & $6.39268-4$ & 0.9944 \\
\hline & 4000 & $5.53505-6$ & $5.52501-2$ & 0.9982 & $1.11099-5$ & $1.10864-5$ & 0.9979 \\
\hline & 20000 & $1.04968-6$ & $1.04972-6$ & 1.0 & $6.29466-7$ & $6.34632-7$ & 1.0082 \\
\hline & 80500 & $9.75224-8$ & $9.75558-8$ & 1.0003 & $4.80876-8$ & $4.80808-8$ & 0.9998 \\
\hline \multirow{4}{*}{28.3} & 500 & $1.88027-5$ & $1.86264-5$ & 0.9906 & $6.42916-4$ & $6.39322-4$ & 0.9944 \\
\hline & 4000 & $5.53552-6$ & $5.52547-6$ & 0.9982 & $1.11109-5$ & $1.10873-5$ & 0.9979 \\
\hline & 20000 & $1.04977-6$ & $1.04981-6$ & 1.0 & $6.29519-7$ & $6.34686-7$ & 1.0082 \\
\hline & 80500 & $9.75307-8$ & $9.75641-8$ & 1.0003 & $4.80916-8$ & $4.80848-8$ & 0.9998 \\
\hline \multirow{4}{*}{30.3} & 500 & $9.23841-2$ & $9.15176-6$ & 0.9906 & $3.15886-4$ & $3.1412-4$ & 0.9944 \\
\hline & 4000 & $2.71978-2$ & $2.71485-6$ & 0.9982 & $5.45914-6$ & $5.44756-6$ & 0.9979 \\
\hline & 20000 & $5.15785-7$ & $5.15805-7$ & 1.0 & $3.09304-7$ & $3.11842-7$ & 1.0082 \\
\hline & 80500 & $4.792-8$ & $4.79365-8$ & 1.0003 & $2.3629-8$ & $2.36257-8$ & 0.9998 \\
\hline \multirow{4}{*}{50.5} & 500 & $3.94835-26$ & $3.91132-26$ & 0.9906 & $1.35005-24$ & $1.3425-24$ & 0.9944 \\
\hline & 4000 & $1.16239-26$ & $1.16028-26$ & 0.9982 & $2.33315-26$ & $2.3282-26$ & 0.9979 \\
\hline & 20000 & $2.20439-27$ & $2.20447-27$ & 1.0 & $1.32192-27$ & $1.33277-27$ & 1.0082 \\
\hline & 80500 & $2.04803-28$ & $2.04873-27$ & 1.0003 & $1.00987-28$ & $1.00972-28$ & 0.9998 \\
\hline \multirow{4}{*}{08.9} & 500 & $5.13312-75$ & $5.08498-75$ & 0.9906 & $1.75515-73$ & $1.74534-73$ & 0.9944 \\
\hline & 4000 & $1.51118-75$ & $1.50845-75$ & ก 998?. & $3.03325-75$ & $3.02682-75$ & 0.9979 \\
\hline & 20000 & $2.86585-76$ & $2.86595-76$ & 1.0 & $1.71858-76$ & $1.73268-76$ & 1.0082 \\
\hline & 80500 & $2.66257-77$ & $2.66348-77$ & 1.0003 & $1.31289-77$ & $1.31271-77$ & 0.9999 \\
\hline
\end{tabular}


TABLE E-12. HAND CALCULATION COMPARISONS-GROUND CONCENTRATIONSWORLD-RELEASES STOP AT 30 YRS

$$
\text { Te-127m }
$$

\begin{tabular}{rllllll}
\multirow{2}{*}{$\begin{array}{c}\text { Time } \\
\text { yrs }\end{array}$} & \multicolumn{2}{c}{$\mathbf{h}=100$} & & \multicolumn{3}{c}{$\mathbf{h}=0$} \\
\hline 2.0 & $1.12951-11$ & $1.12972-11$ & 1.0002 & $3.29446-12$ & $3.29781-12$ & 1.001 \\
4.0 & $1.13989-11$ & $1.1401-11$ & 1.0002 & $3.32472-12$ & $3.3281-12$ & 1.001 \\
6.1 & $1.13998-11$ & $1.1402-11$ & 1.0002 & $3.325-12$ & $3.32837-12$ & 1.001 \\
28.3 & $1.13999-11$ & $11411 \%-11$ & 1.0002 & $3.335-13$ & $3.32038-12$ & 1.001 \\
30.3 & $5.69056-12$ & $5.6916-12$ & 1.0002 & $1.65977-12$ & $1.66145-12$ & 1.001 \\
40.4 & $3.72019-22$ & $3.72086-22$ & 1.0002 & $1.08507-22$ & $1.08617-22$ & 1.001 \\
50.5 & $2.43206-32$ & $2.4325-32$ & 1.0002 & $7.09359-33$ & $7.10078-33$ & 1.001 \\
60.6 & $1.58995-42$ & $1.59024-42$ & 1.0002 & $4.63741-43$ & $4.64211-4.3$ & 1001 \\
80.8 & $6.7952-63$ & $6.79644-63$ & 1.0002 & $1.98196-63$ & $1.98397-63$ & 1.001 \\
98.9 & $3.16184-81$ & $3.16241-81$ & 1.0002 & $9.22214-82$ & $9.23148-82$ & 1.001
\end{tabular}


TABLE E-13. HAND CALCULATION COMPARISONS-70 YR DOSE COMMITMENTSAIR IMMERSION-VICINITY OF RELEASES

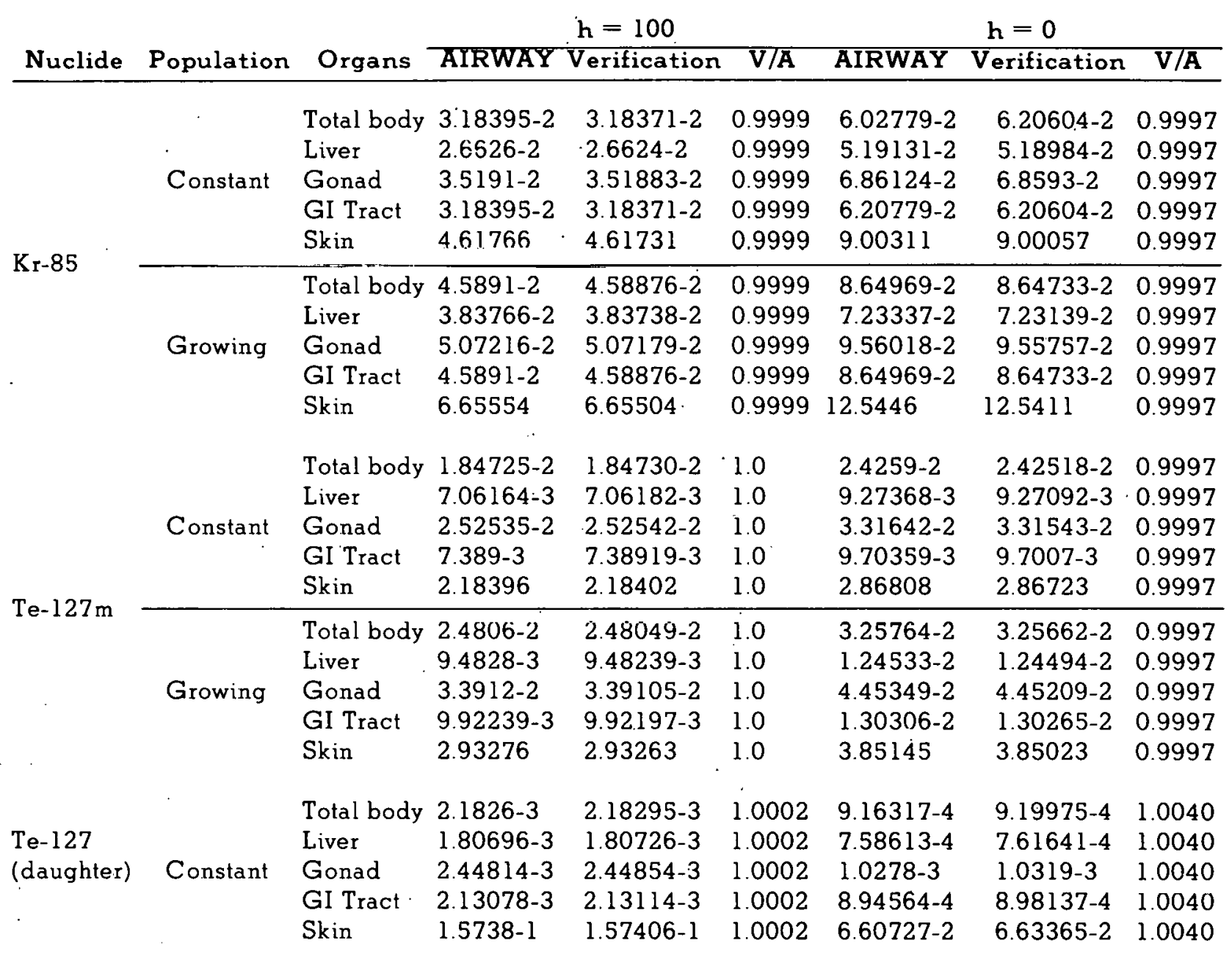


TABLE E-14. HAND CALCULATION COMPARISONS-70 YR DOSE COMMITMENTSAIR IMMERSION-WORLD

\begin{tabular}{|c|c|c|c|c|c|c|c|c|}
\hline \multirow[b]{2}{*}{ Nuclide } & \multirow[b]{2}{*}{ Population } & \multirow[b]{2}{*}{ Organ } & \multicolumn{3}{|c|}{$h=100$} & \multicolumn{3}{|c|}{$\mathrm{h}=0$} \\
\hline & & & AIRWAY & Verification & $\mathbf{V} / \mathbf{A}$ & AIRWAY & Verification & V/A \\
\hline \multirow{18}{*}{$\mathbf{K}_{\mathbf{r}-85}$} & \multirow{9}{*}{ Constant } & Total body & 56.7182 & 56.7182 & 1.0 & 56.7182 & 56.7182 & 1.0 \\
\hline & & Bone & 69.3222 & 69.3222 & 1.0 & 69.3222 & 6.9.32.2.? & 1.0 \\
\hline & & Liver & 47.431 & 47.431 & 1.0 & 47.431 & 47.431 & 1.0 \\
\hline & & Kidney & 50.4161 & 50.4161 & 1.0 & 50.4161 & 50.4161 & 1.0 \\
\hline & & Gonad & 62.6885 & 62.6885 & 1.0 & 62.6885 & 62.6855 & 1.0 \\
\hline & & Lung & 53.4013 & 53.4013 & 1.0 & 53.4013 & 53.4013 & 1.0 \\
\hline & & GI Tract & 56.7182 & 56.7182 & 1.0 & 56.7182 & 56.7182 & 10 \\
\hline & & Thyroid & 59.7033 & 59.7033 & 1.0 & 59.7033 & 59.7033 & 1.0 \\
\hline & & Skin & 8225.79 & 8225.79 & 1.0 & 8225.79 & 82.2 .579 & 10 \\
\hline & \multirow{9}{*}{ Growing } & Total body & 141.868 & 141.868 & 1.0 & 141.868 & 141.868 & 1.0 \\
\hline & & Bone & 173.394 & 173.394 & 1.0 & 17.3 .394 & 173.394 & 1.0 \\
\hline & & Liver & 118.638 & 118.638 & 1.0 & 118.638 & 118.638 & 1.0 \\
\hline & & Kidney & 126.106 & 126.106 & 1.0 & 126.106 & 126.106 & 1.0 \\
\hline & & Gonad & 156.802 & 156.802 & 1.0 & 156.802 & 156.802 & 1.0 \\
\hline & & Lung & 133.572 & 133.572 & 1.0 & 133.572 & 133.572 & 1.0 \\
\hline & & GI Tracl & 141.868 & 141.868 & 1.0 & 141.868 & 141.868 & 1.0 \\
\hline & & Thyroid & 149.335 & 149.335 & 1.0 & 149.335 & 149.335 & 1.0 \\
\hline & & Skin & 20575 & 20575 & 1.0 & 20575 & 20575 & 1.0 \\
\hline \multirow{9}{*}{$\mathrm{Te}-127 \mathrm{~m}$} & \multirow{9}{*}{ Constant } & Total body & $1.24714-4$ & $1.23489-4$ & 0.9902 & $3.63755-5$ & $3.60481-5$ & 0.9910 \\
\hline & & Bone & $1.90071-4$ & $1.88204-4$ & 0.9902 & $5.54381-5$ & $5.49392-5$ & 0.9910 \\
\hline & & Livẹr & $4.76757-5$ & $4.72074-5$ & 0.9902 & $1.39056=5$ & $1.37804-5$ & 0.9910 \\
\hline & & Kidney & $7.07242-5$ & $7.00294-5$ & 0.9902 & $2.06281-5$ & $2.04425-5$ & 0.9910 \\
\hline & & Gonad & $1.70496-4$ & $1.68821-4$ & 0.9902 & $4.97285-5$ & $4.9281-5$ & 0.9910 \\
\hline & & Lung & $6.3778-5$ & $6.31516-5$ & 0.9902 & $1.86022-5$ & $1.84347-5$ & 0.9910 \\
\hline & & GI Tract & $4.98858-5$ & $4.93958-5$ & 0.9902 & $1.45502-5$ & $1.44193-5$ & 0.9910 \\
\hline & & Thyroid & $1.31661-5$ & $1.30367-5$ & 0.9902 & $3.84015-5$ & $3.80559-5$ & 0.9910 \\
\hline & & Skin & $1.47447-2$ & $1.45999-2$ & 0.9902 & $4.3006-3$ & $4.26189-3$ & 0.9910 \\
\hline
\end{tabular}


TABLE E-15. HAND CALCULATION COMPARISONS-70 YR DOSE COMMITMENTSSURFACE DEPOSITS

\begin{tabular}{|c|c|c|c|c|c|c|c|c|}
\hline \multirow[b]{2}{*}{ Nuclide } & \multirow[b]{2}{*}{ Location } & \multicolumn{6}{|c|}{ Population-Constant } & \multirow[b]{2}{*}{ V/A } \\
\hline & & Organs & AIRWAY & Verification & V/A & AIRWAY & Verification & \\
\hline \multirow{2}{*}{$\mathrm{Te}-127 \mathrm{~m}$} & $\begin{array}{l}\text { Vicinity } \\
\text { of } \\
\text { Release }\end{array}$ & $\begin{array}{l}\text { Total body } \\
\text { Liver } \\
\text { Gonad } \\
\text { GI Tract } \\
\text { Skin }\end{array}$ & $\begin{array}{l}314.799 \\
120.272 \\
429.842 \\
161.06 \\
735.229\end{array}$ & $\begin{array}{l}314.8 \\
120.272 \\
429.843 \\
161.061 \\
735.231\end{array}$ & $\begin{array}{l}1.0 \\
1.0 \\
1.0 \\
1.0 \\
1.0\end{array}$ & $\begin{array}{l}381.6 \\
145.794 \\
521.055 \\
195.237 \\
891.245\end{array}$ & $\begin{array}{l}381.512 \\
145.76 \\
520.934 \\
195.192 \\
891.039\end{array}$ & $\begin{array}{l}0.9998 \\
0.9998 \\
0.9998 \\
0.9998 \\
0.9998\end{array}$ \\
\hline & World & $\begin{array}{l}\text { Total body } \\
\text { Liver } \\
\text { Gonad } \\
\text { GI Tract } \\
\text { Skin } \\
\text {. }\end{array}$ & $\begin{array}{c}4.32107 \\
1.65091 \\
5.9002 \\
2.21078 \\
10.0921\end{array}$ & $\begin{array}{l}4.27862 \\
1.63469 \\
5.84224 \\
2.18906 \\
9.99293\end{array}$ & $\begin{array}{l}0.9902 \\
0.9902 \\
0.9902 \\
0.9902 \\
0.9902\end{array}$ & $\begin{array}{l}1.26033 \\
0.48152 \\
1.72091 \\
0.644819 \\
2.94356\end{array}$ & $\begin{array}{l}1.24898 \\
0.477187 \\
1.70542 \\
0.639016 \\
2.91706\end{array}$ & $\begin{array}{l}0.9910 \\
0.9910 \\
0.9910 \\
0.9910 \\
0.9910\end{array}$ \\
\hline $\begin{array}{l}\text { Te-127 } \\
\text { (daughter) }\end{array}$ & $\begin{array}{l}\text { Vicinity } \\
\text { of } \\
\text { Releases }\end{array}$ & $\begin{array}{l}\text { Total body } \\
\text { Liver } \\
\text { Gonad } \\
\text { GI Tract } \\
\text { Skin }\end{array}$ & $\begin{array}{l}62.0969 \\
51.4224 \\
69.6922 \\
60.6601 \\
76.056\end{array}$ & $\begin{array}{l}62.0905 \\
51.4171 \\
69.6851 \\
60.6537 \\
76.0481\end{array}$ & $\begin{array}{l}0.9999 \\
0.9999 \\
0.9999 \\
0.9999 \\
0.9999\end{array}$ & $\begin{array}{l}75.2569 \\
62.3202 \\
84.4617 \\
73.5154 \\
92.174\end{array}$ & $\begin{array}{l}75.2378 \\
62.3044 \\
84.4404 \\
73.4968 \\
92.1508\end{array}$ & $\begin{array}{l}0.9997 \\
0.9997 \\
0.9997 \\
0.9997 \\
0.9997\end{array}$ \\
\hline
\end{tabular}

TABLE E-16. HAND CALCULATION COMPARISONS70 YR DOSE COMMITMENTS-INGESTION
Population-Constant
$h=100$ meters

\begin{tabular}{|c|c|c|c|c|c|c|c|c|}
\hline \multirow[b]{2}{*}{ Nuclide } & \multirow[b]{2}{*}{ Location } & \multirow[b]{2}{*}{ Organs } & \multicolumn{3}{|c|}{ During Deposition } & \multicolumn{3}{|c|}{ Root Uptake } \\
\hline & & & AIRWAY & Verification & V/A. & AIRWAY & Verification & V/A \\
\hline \multirow{2}{*}{$\mathrm{Te}-127 \mathrm{~m}$} & $\begin{array}{c}\text { Vicinity } \\
\text { of } \\
\text { Releases }\end{array}$ & $\begin{array}{l}\text { Total body } \\
\text { Liver } \\
\text { Gonad } \\
\text { GI Tract } \\
\text { Thyroid }\end{array}$ & $\begin{array}{l}1.25495+4 \\
3.64971+4 \\
1.1283+5 \\
4.46715+5 \\
2.62503+4\end{array}$ & $\begin{array}{l}1.255+4 \\
3.64987+4 \\
1.12835+5 \\
4.46735+5 \\
2.62514+4\end{array}$ & $\begin{array}{l}1.0 \\
1.0 \\
1.0 \\
1.0 \\
1.0\end{array}$ & $\begin{array}{r}3811.16 \\
.1 .10838+4 \\
3.42654+4 \\
1.35663+5 \\
7.97106+3\end{array}$ & $\begin{aligned} 3811.33 \\
1.10843+4 \\
3.4267+4 \\
1.35669+5 \\
7.97331+3\end{aligned}$ & $\begin{array}{l}1.0 \\
1.0 \\
1.0 \\
1.0 \\
1.0\end{array}$ \\
\hline & World & $\begin{array}{l}\text { Total body } \\
\text { Liver } \\
\text { Gonad } \\
\text { GI Tract } \\
\text { Thyroid }\end{array}$ & $\begin{array}{c}172.57 \\
501.879 \\
155.155 \\
6142.87 \\
360.973\end{array}$ & $\begin{array}{c}170.875 \\
496.948 \\
153.631 \\
6082.52 \\
357.426\end{array}$ & $\begin{array}{l}0.9902 \\
0.9902 \\
0.9902 \\
0.9902 \\
0.9902\end{array}$ & $\begin{array}{c}52.3136 \\
152.141 \\
470.342 \\
1862.17 \\
109.427\end{array}$ & $\begin{array}{c}51.7996 \\
150.646 \\
465.722 \\
1843.88 \\
108.352\end{array}$ & $\begin{array}{l}0.9902 \\
0.9902 \\
0.9902 \\
0.9902 \\
0.9902\end{array}$ \\
\hline $\begin{array}{l}\text { Te-127 } \\
\text { (daughter) }\end{array}$ & $\begin{array}{l}\text { Vicinity } \\
\text { of } \\
\text { Releases }\end{array}$ & $\begin{array}{l}\text { Total hody } \\
\text { Liver } \\
\text { Gonad } \\
\text { GI Traet } \\
\text { Thyroid }\end{array}$ & $\begin{array}{l}3.5524-2 \\
5.99003-2 \\
0.19174 \\
11.4177 \\
0.120841\end{array}$ & $\begin{array}{c}3.5529-2 \\
5.99088-2 \\
0.191767 \\
14.4137 \\
0.120858\end{array}$ & $\begin{array}{l}1.0001 \\
1.0001 \\
1.0001 \\
1.0001 \\
1.0001\end{array}$ & $\begin{array}{c}2.38829 \\
4.02711 \\
12.8908 \\
969.306 \\
8.12418\end{array}$ & $\begin{array}{c}2.38805 \\
4.02672 \\
12.8895 \\
969.21 \\
8.12337\end{array}$ & $\begin{array}{l}0.9999 \\
0.9999 \\
0.9999 \\
0.9999 \\
0.9999\end{array}$ \\
\hline
\end{tabular}


TABLE E-17. HAND CALCULATION COMPARISONS70 YR DOSE COMMITMENTS-INHALATION

Population-Constant

$$
h=100 \text { meters }
$$

\begin{tabular}{|c|c|c|c|c|c|c|c|c|}
\hline \multirow[b]{2}{*}{ Nuclide } & \multirow[b]{2}{*}{ Location } & \multirow[b]{2}{*}{ Organs } & \multicolumn{3}{|c|}{ Prior to Deposition } & \multicolumn{3}{|c|}{ Resuspended } \\
\hline & & & AIRWAY & Verification & V/A & AIRWAY & Verification & $\mathbf{V} / \mathbf{A}$ \\
\hline \multirow{2}{*}{$\mathrm{Te}-127 \mathrm{~m}$} & $\begin{array}{c}\text { Vicinity } \\
\text { of } \\
\text { Releases }\end{array}$ & $\begin{array}{l}\text { Total body } \\
\text { Boñe } \\
\text { Kidney } \\
\text { Lung } \\
\text { Thyroid }\end{array}$ & $\begin{array}{c}6.42773 \\
47.9158 \\
209.972 \\
638.877 \\
13.5177\end{array}$ & $\begin{array}{c}6.42784 \\
47.9158 \\
209.972 \\
638.893 \\
13.518\end{array}$ & $\begin{array}{l}1.0 \\
1.0 \\
1.0 \\
1.0 \\
1.0\end{array}$ & $\begin{array}{l}1.43726-3 \\
1.07156-2 \\
4.69571-2 \\
0.142875 \\
3.02302-3\end{array}$ & $\begin{array}{l}1.43753-3 \\
1.07161-2 \\
4.69592-2 \\
0.142881 \\
3.02316-3\end{array}$ & $\begin{array}{l}1.0 \\
1.0 \\
1.0 \\
1.0 \\
1.0\end{array}$ \\
\hline & World & $\begin{array}{l}\text { Total body } \\
\text { Bone } \\
\text { Kidney } \\
\text { J.tung } \\
\text { Thyroid }\end{array}$ & $\begin{array}{l}4.33959-2 \\
0.323497 \\
1.4176 \\
4.31339 \\
9.13639-2\end{array}$ & $\begin{array}{c}4.29696-2 \\
0.320319 \\
1.40367 \\
4.27092 \\
9.03604-2\end{array}$ & $\begin{array}{l}0.9902 \\
0.9902 \\
0.9902 \\
0.9902 \\
0.9902\end{array}$ & $\begin{array}{l}1.97312-5 \\
1.47087-4 \\
6.44553-4 \\
1.96116=3 \\
4.14953-5\end{array}$ & $\begin{array}{l}1.95374-5 \\
1.45642-4 \\
6.38222-4 \\
1.9419-3 \\
1.10877-5\end{array}$ & $\begin{array}{l}0.9902 \\
0.9902 \\
0.9902 \\
0.9902 \\
0.9902\end{array}$ \\
\hline $\begin{array}{l}\text { Tc.137 } \\
\text { (daughter) }\end{array}$ & $\begin{array}{l}\text { Vicinily } \\
\text { of } \\
\text { Releases }\end{array}$ & $\begin{array}{l}\text { Total body } \\
\text { Bu!ly } \\
\text { Kidney } \\
\text { Lung } \\
\text { Thyroid }\end{array}$ & $\begin{array}{l}1.958 .37-2 \\
9.00959-2 \\
0.370094 \\
0.461269 \\
6.68976-2\end{array}$ & $\begin{array}{l}1.95869-2 \\
y . U 11 \cup 8-2 \\
0.370156 \\
0.461346 \\
6.69086-2\end{array}$ & $\begin{array}{l}1.0002 \\
1.0002 \\
1.0002 \\
1.0002 \\
1.0002\end{array}$ & $\begin{array}{l}3.10361-5 \\
1.42783-4 \\
5.86522-4 \\
7.31015-4 \\
1.06018-4\end{array}$ & $\begin{array}{l}3.1033-5 \\
1.42769-4 \\
5.86464-4 \\
7.30943-4 \\
1.06008-4\end{array}$ & $\begin{array}{l}0.9999 \\
0.9999 \\
0.9999 \\
0.9999 \\
0.9999\end{array}$ \\
\hline \multirow{2}{*}{$K_{I}-85$} & $\begin{array}{l}\text { Vicinity } \\
\text { of } \\
\text { Releases }\end{array}$ & $\begin{array}{l}\text { Total body } \\
\text { Bone } \\
\text { Kidney } \\
\text { Lung } \\
\text { Thyroid }\end{array}$ & $\begin{array}{l}4.86243-4 \\
4.86243-4 \\
3.09427-4 \\
3.53631-2 \\
4.86243-4\end{array}$ & $\begin{array}{l}4.86204-4 \\
4.86204-4 \\
3.09403-4 \\
3.53603-2 \\
4.86204-4\end{array}$ & $\begin{array}{l}0.9999 \\
0.9999 \\
0.9999 \\
0.9999 \\
0.9999\end{array}$ & . & & \\
\hline & World : & $\begin{array}{l}\text { Total body } \\
\text { Bone } \\
\text { Kidney } \\
\text { Lung } \\
\text { Thyroid }\end{array}$ & $\begin{array}{c}0.866181 \\
0.866181 \\
0.551206 \\
62.995 \\
0.866181\end{array}$ & $\begin{array}{l}0.866181 \\
0.866181 \\
0.551206 \\
62.995 \\
0.866181\end{array}$ & $\begin{array}{l}1.0 \\
1.0 \\
1.0 \\
1.0 \\
1.0\end{array}$ & . & & \\
\hline
\end{tabular}


Figure 1 of Reference E-l to obtain the relative deposition rates used for comparison purposes. Values were obtained for various radial distances from the release point and at release heights of 30,60 and 100 meters above the ground as well as at ground level. In general, a deposition velocity of $0.01 \mathrm{~m} / \mathrm{sec}(1 \mathrm{~cm} / \mathrm{sec})$ was used for stability categories A, B, C and D comparisons and a deposition velocity of $0.001 \mathrm{~m} / \mathrm{sec}$ was used for the $\mathrm{E}, \mathrm{F}$ and $\mathrm{G}$ stability category comparisons.

Figures E-1, E-2 and E-3 show the comparisons between the relative deposition rates obtained from the AIRWAY program and the curves presented in Reference E-2 for stability categories $A, B$ and $C$ at release heights of 30,60 and 100 meters above the ground. Figures E-4 through E-6 depict the same results using only the maximum values of the relative deposition rates predicted by the AIRWAY program for each of these unstable stability classes (i.e., $A, B$, and $C$ ). The relative deposition rate comparisons for the neutral stability category (D) are presented in Figures E-7, E-8 and E-9 for the 30-, 60- and 100-meter release heights, respectively. The comparison results for the stable stability categories ( $E, F$ and $G$ ) are shown on Figures E-10 and E-1I for release heights of 30 and 60 meters above the ground. It should be noted that while Reference $\mathrm{E}-2$ does not provide estimates of the relative deposition rates for 100 -meter-high releases for the stable meteorological stability categories, the AIRWAY program does define these deposition rates although they are not presented here. The final Figure E-12 depicts the relative deposition rates for ground level releases for the four stability categories (B, C, D and F) considered in the 18 station average wind speed-frequency data provided in Table F-l of Appendix F.

These comparisons (presented in Figures E-1 through E-12) of the relative deposition rates obtained using the AIRWAY program with those presented in Reference E-2 for general use show that the AIRWAY program will provide deposition estimates which agree quite well with those obtained using the procedures presented in Reference E-2 for the meteorological stability categories A, B, C and D. The comparisons for stability categories E, F, and G indicate that the AIRWAY program will provide more conservative estimates of the deposition than would be obtained using the procedures defined in Reference E- 2 .

\section{REFERENCES}

E-1. Proposed Final Environmental Statement, Liquid Metal Fast Breeder Reactor Program, USAEC, WASH-1535, December 1974.

E-2. NRC Regulatory Guide 1.111, "Methods for Estimating Atmospheric Transport and Dispersion of Gaseous Effluents in Routine Releases from Light-Water-Cooled Reactors," USNRC Office of Standards Development, Revision 1, July 1977.

E-3. R. E. Moore, "AIRDOS-A Computer Code for Estimating Population and Individual Doses Resulting from Atmospheric Releases of Radionuclides from Nuclear Facilities," ORNL-TM-4687, January 1975. 


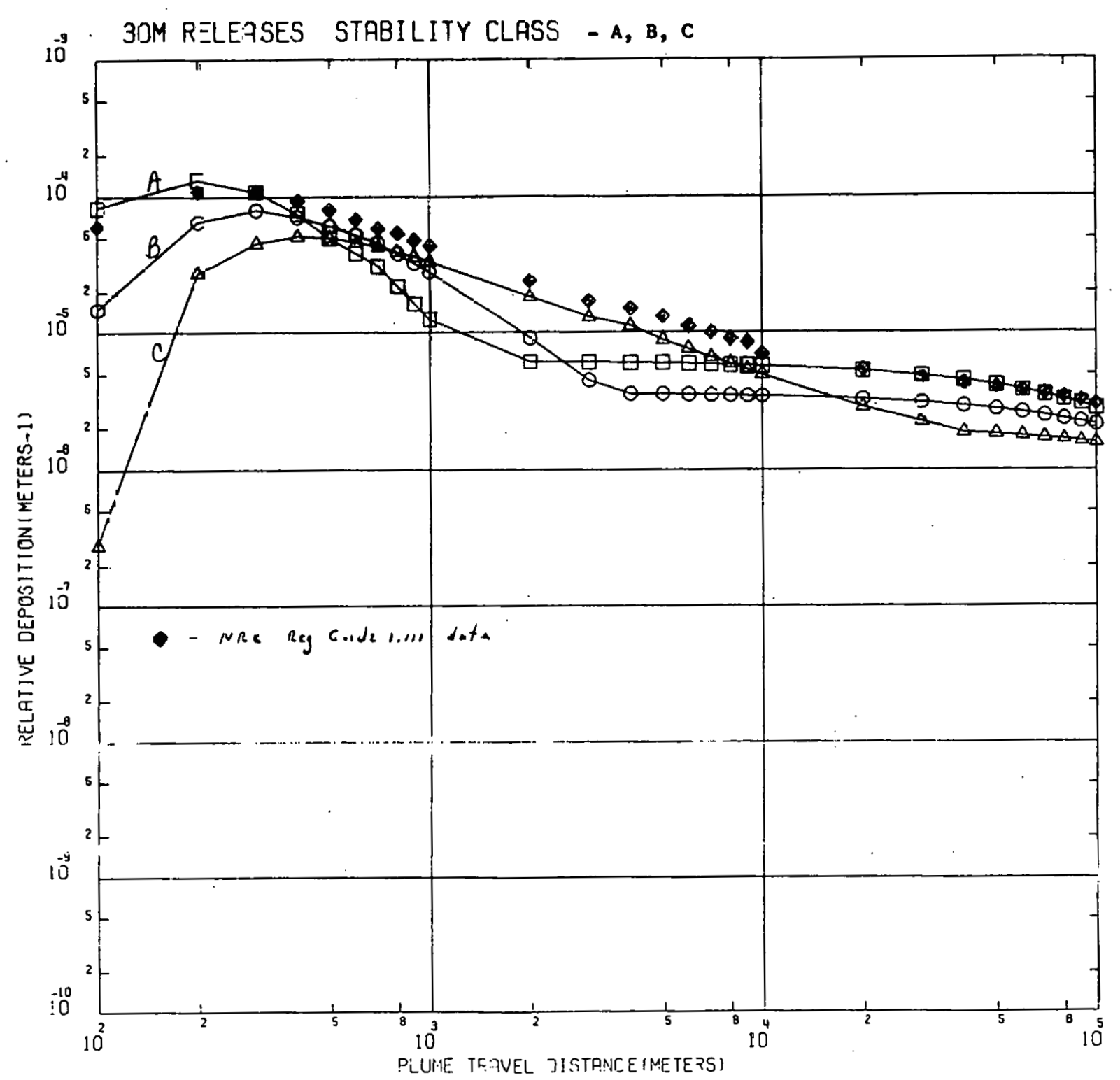

Figure E-1 


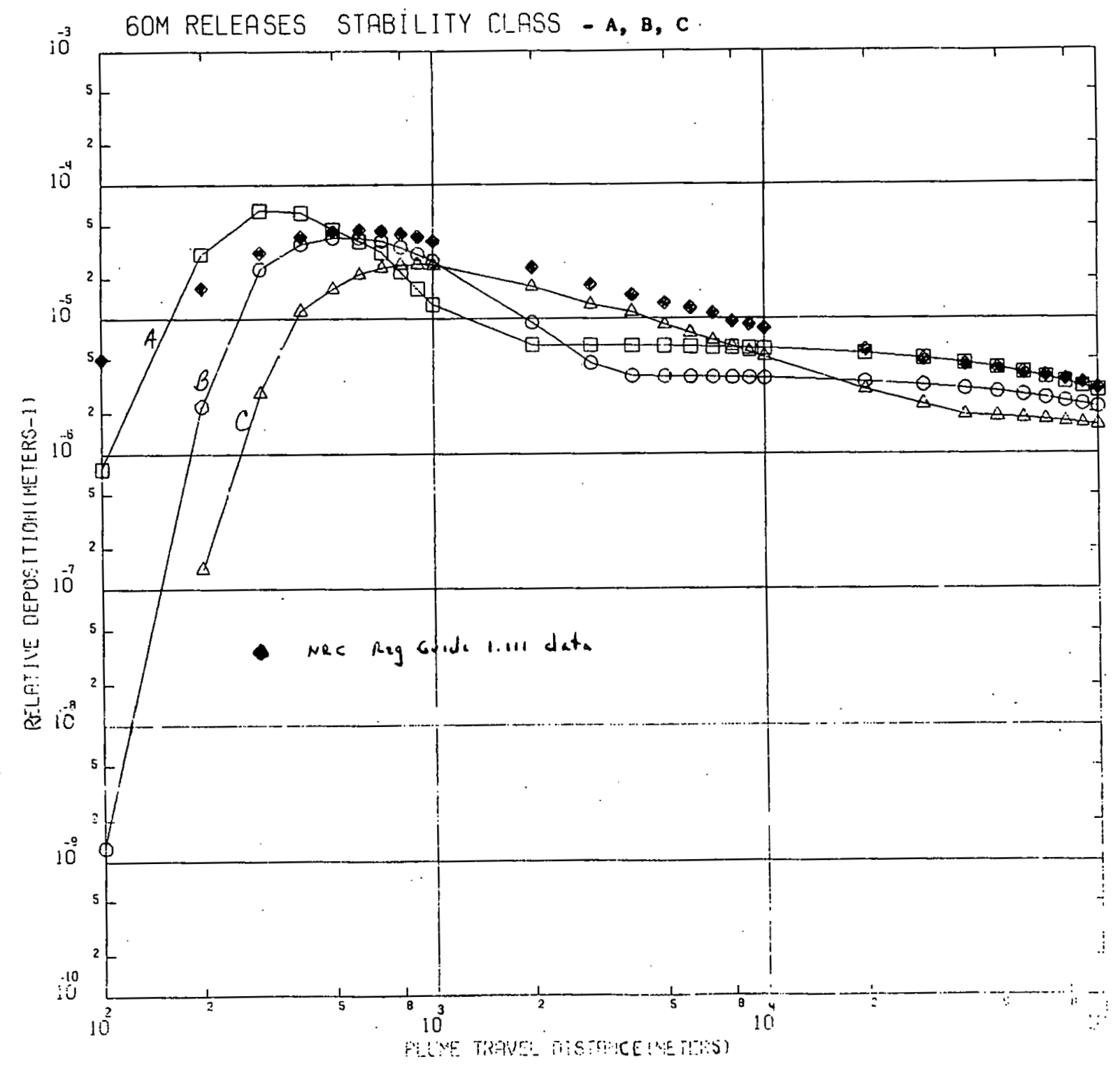




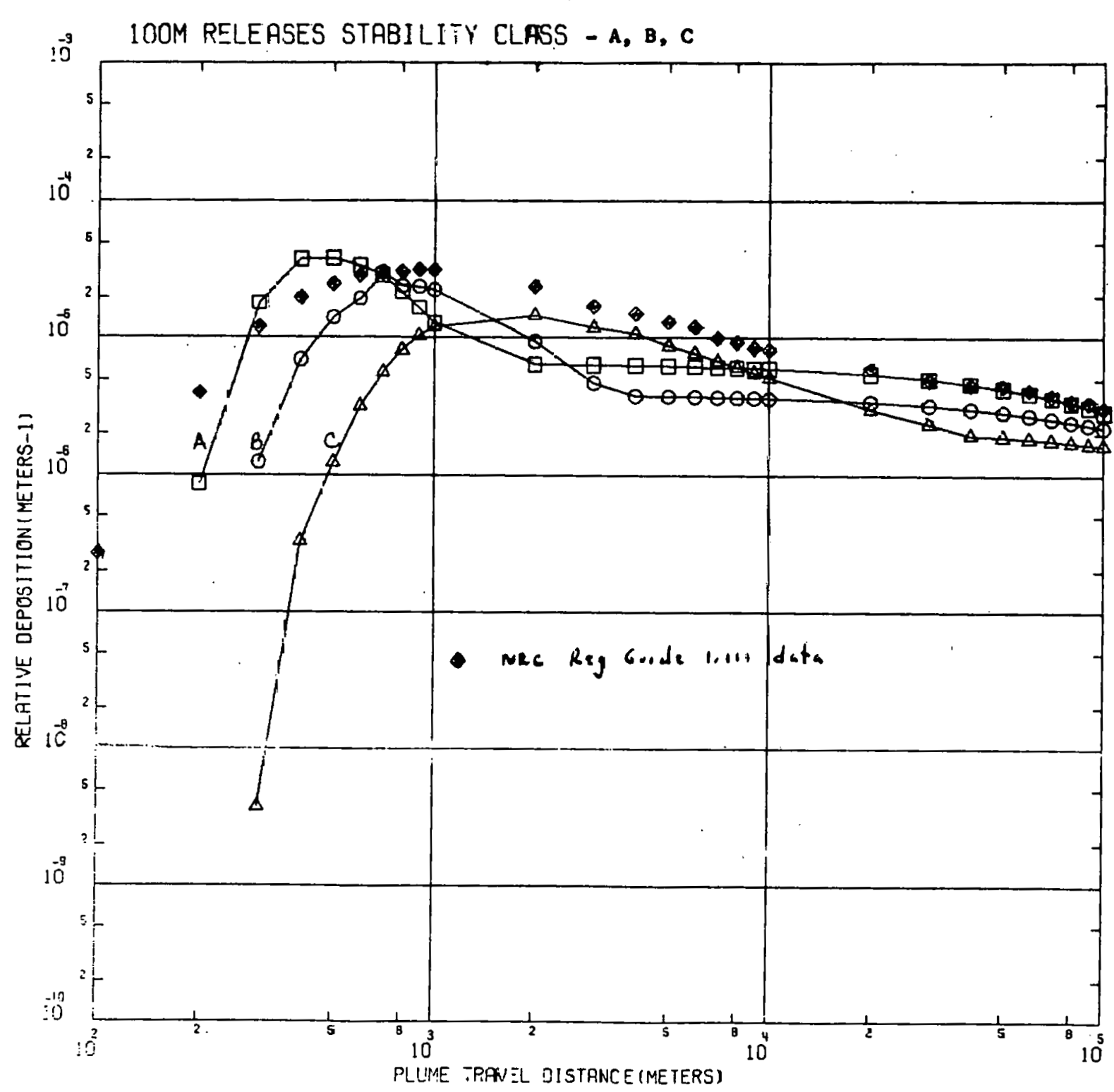

Figure E-3 


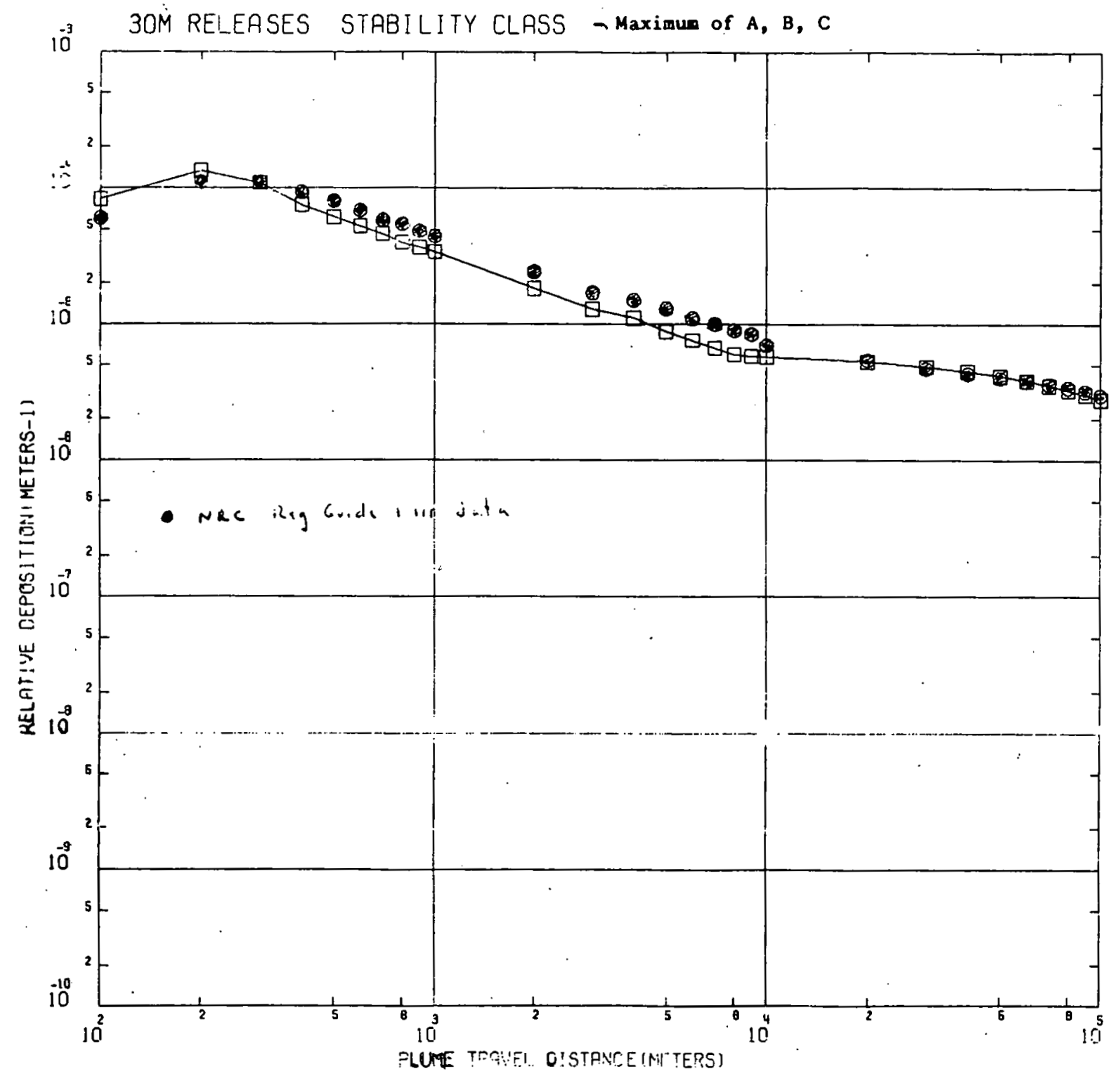




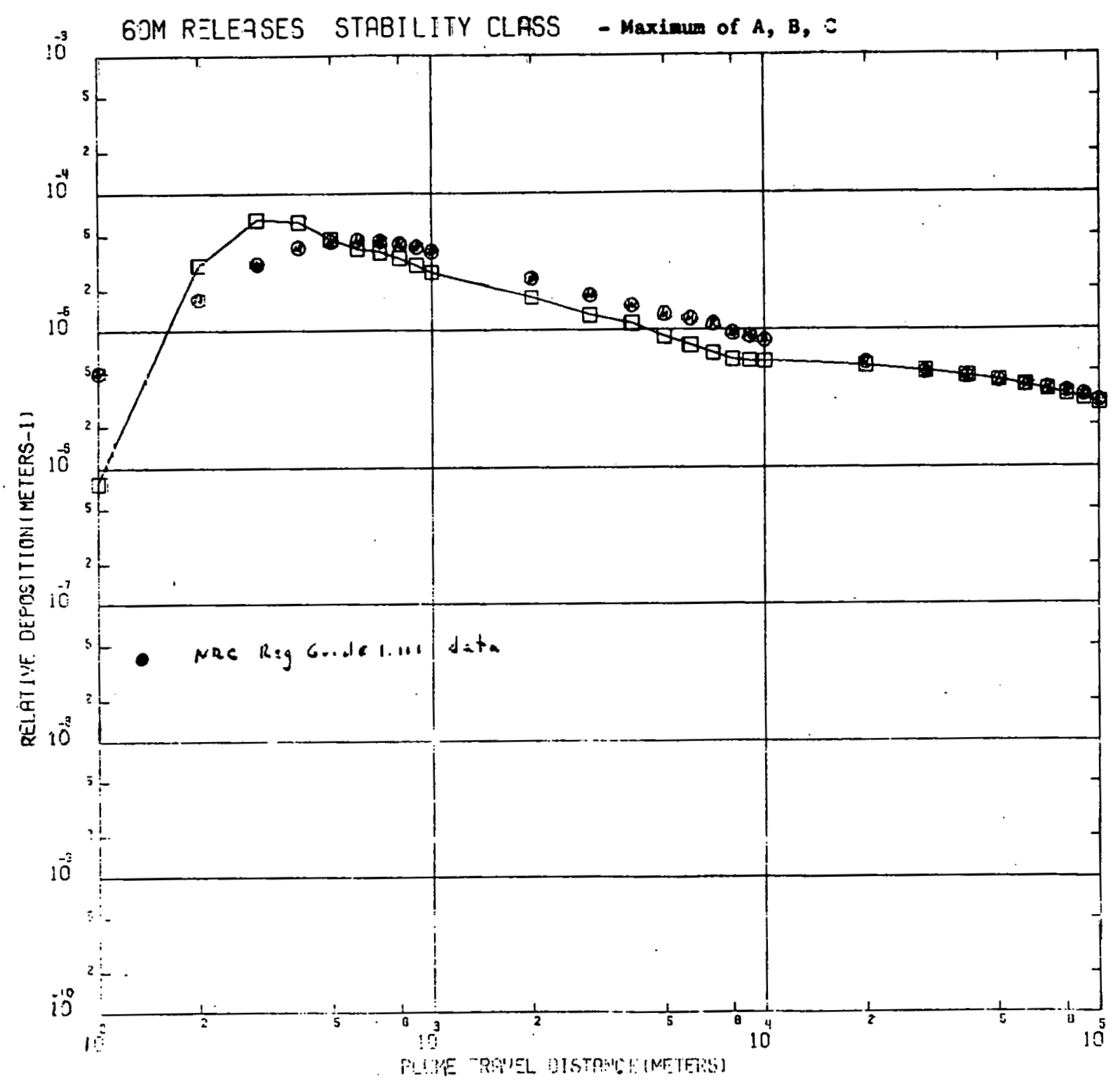

Figuze E-5 


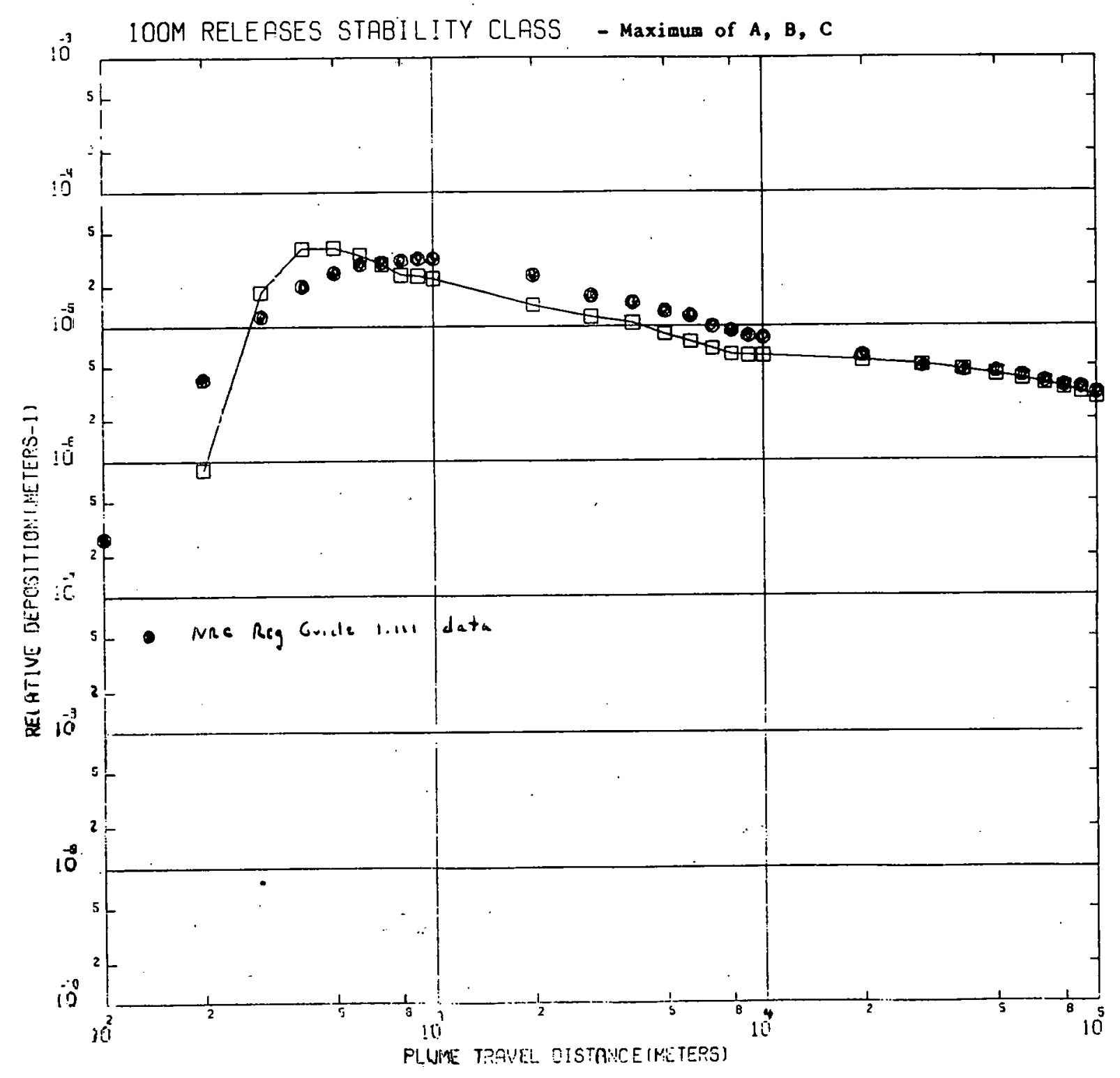

Figure E-6 


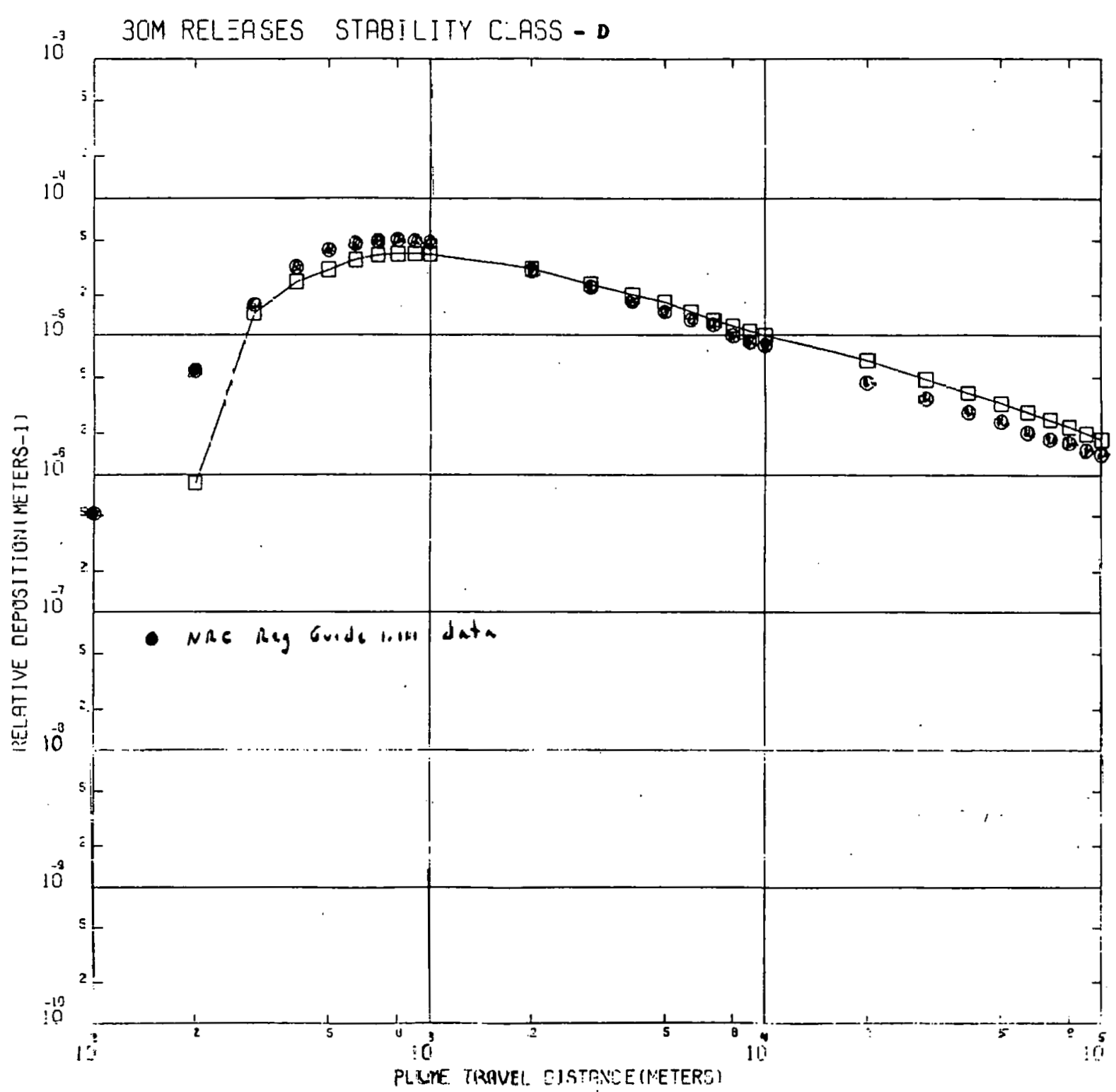

Figure E-7 


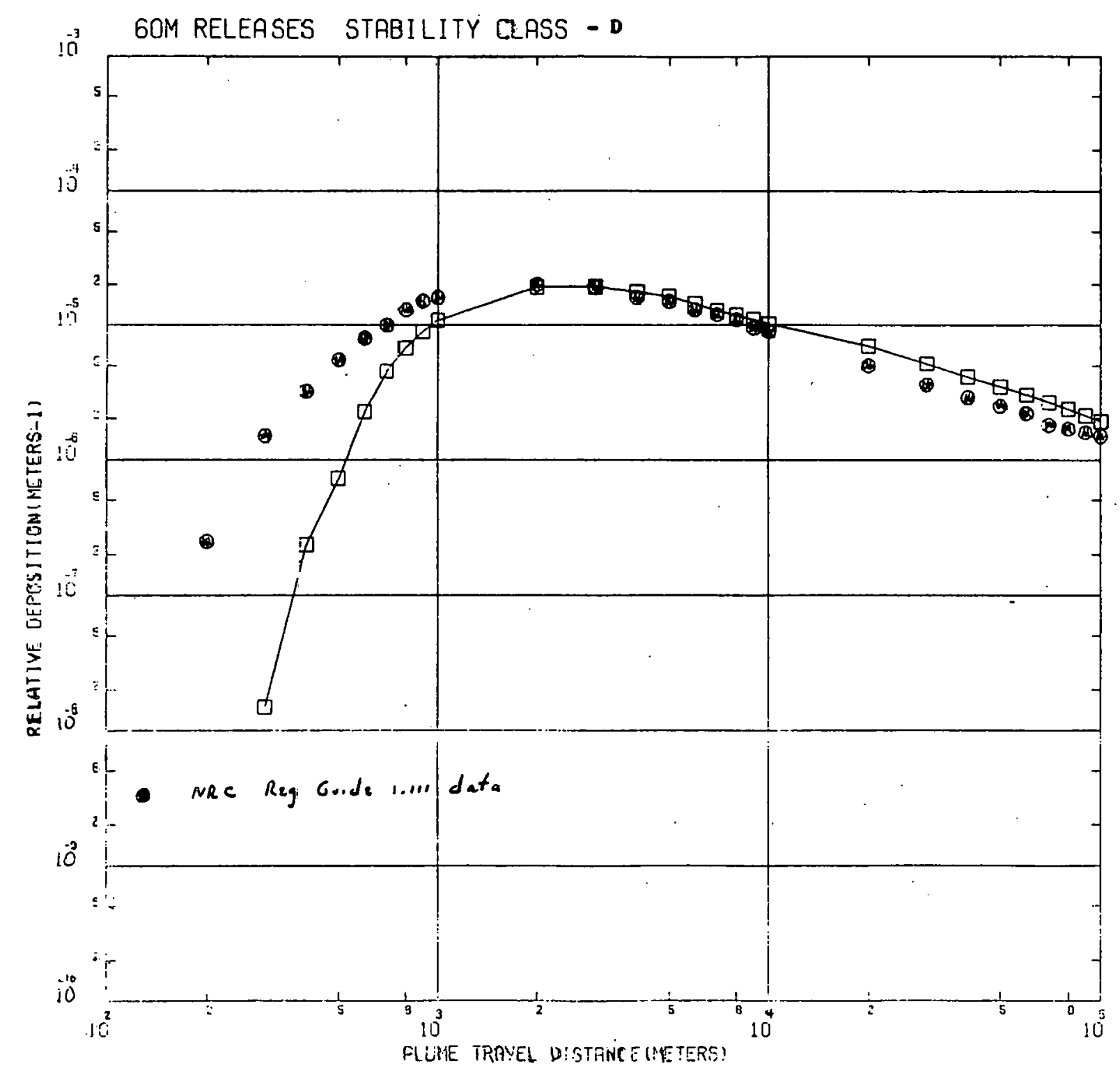




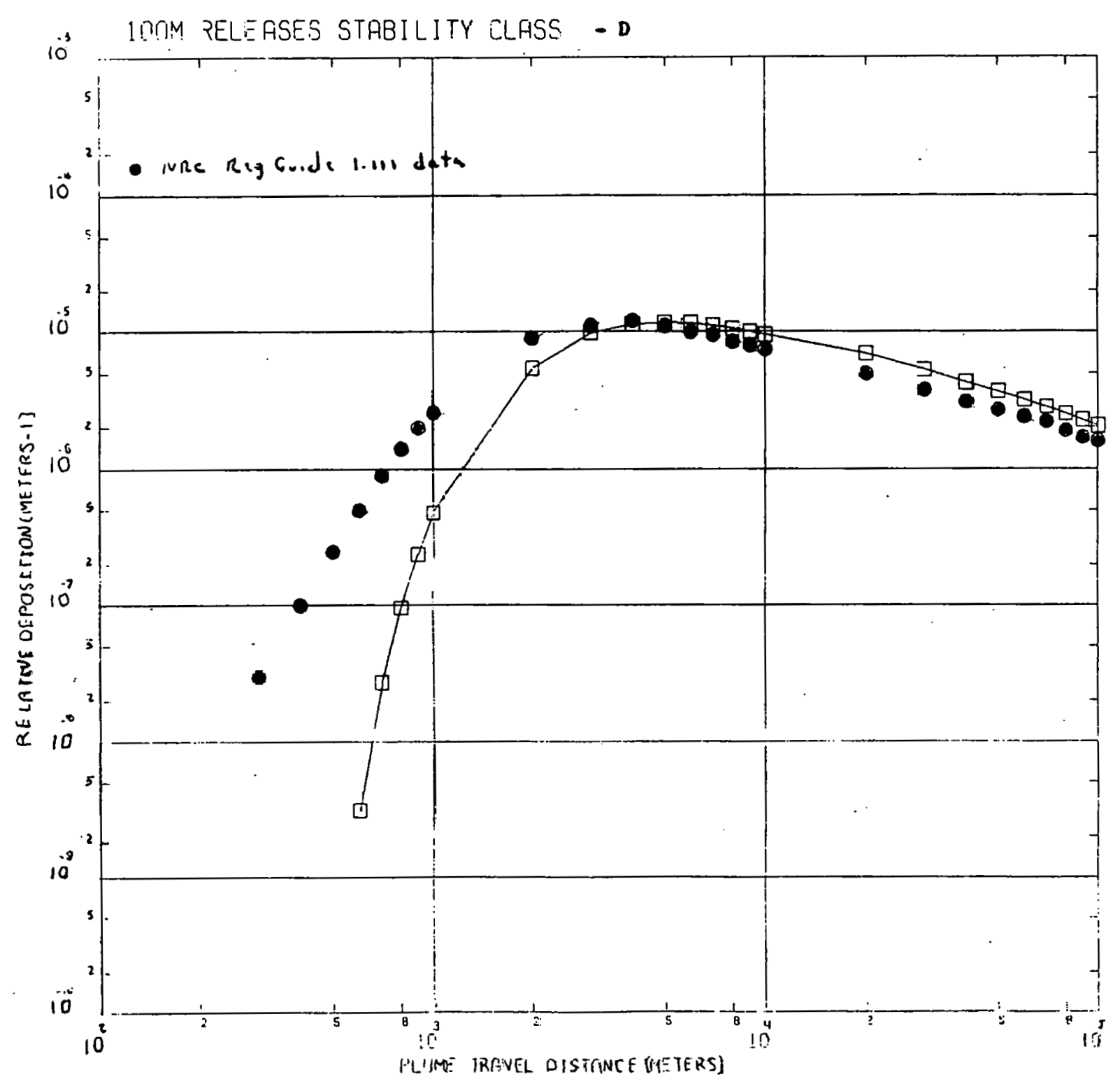

Figure E-9 


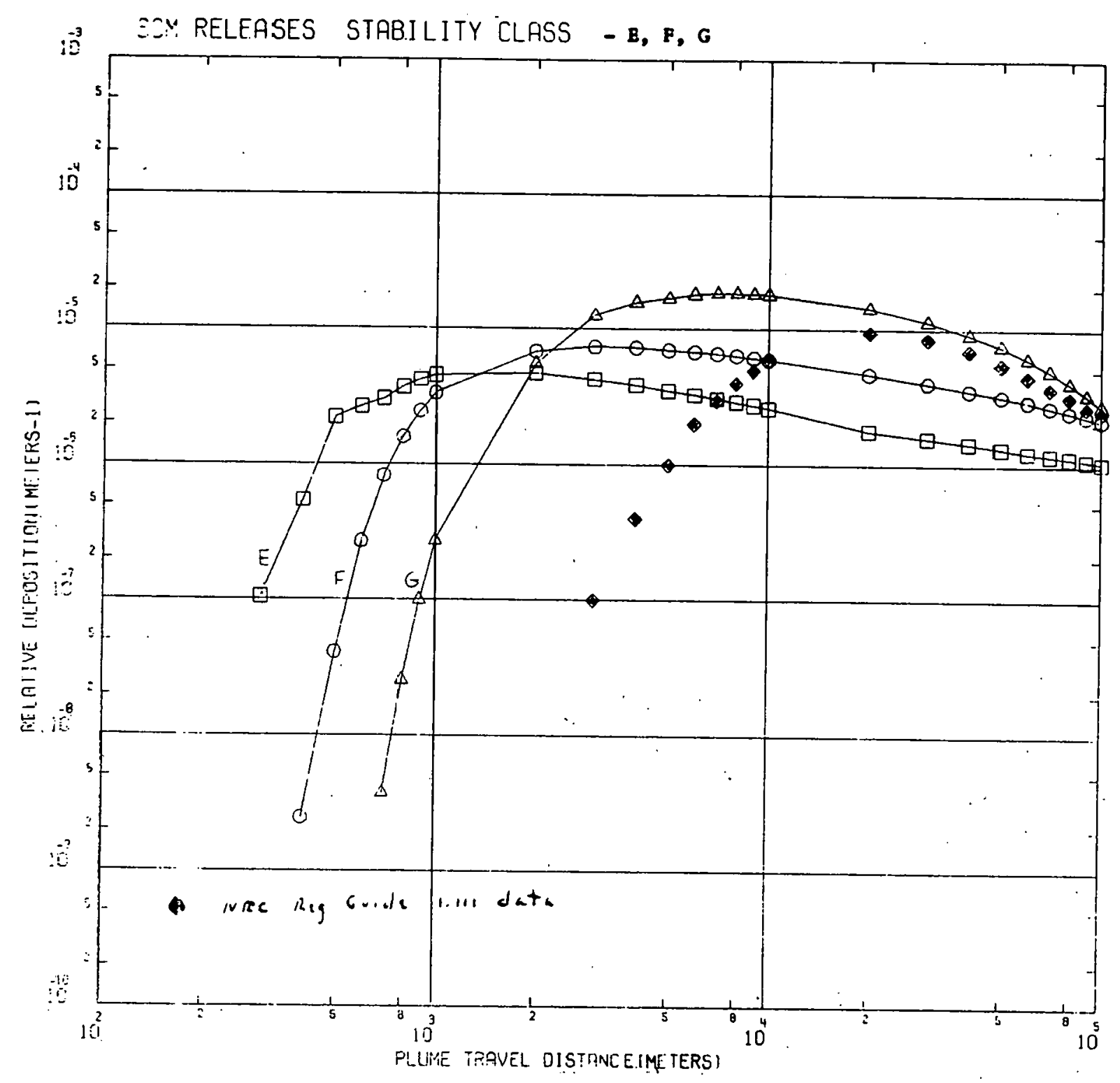




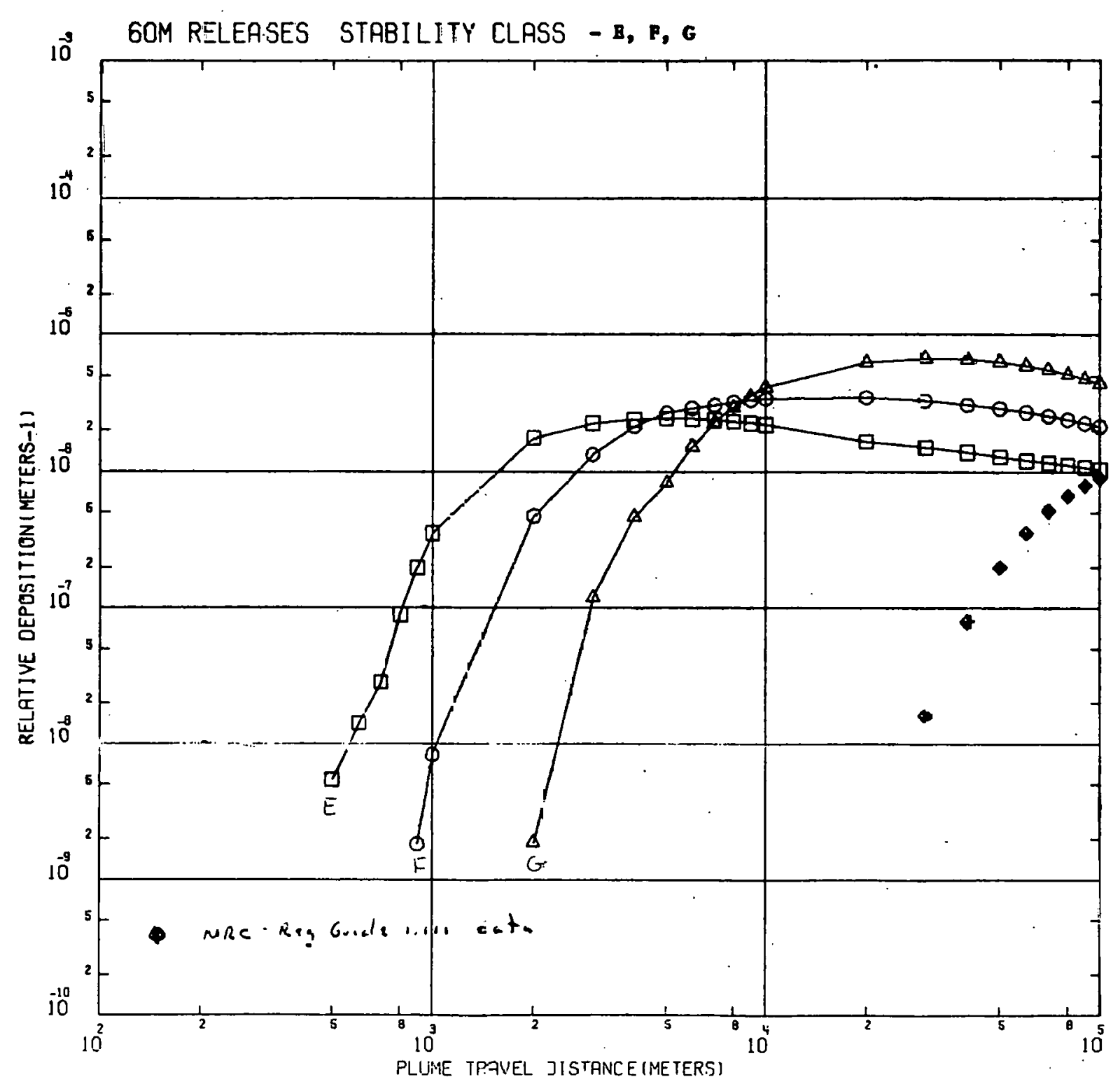

Figlire E-11 


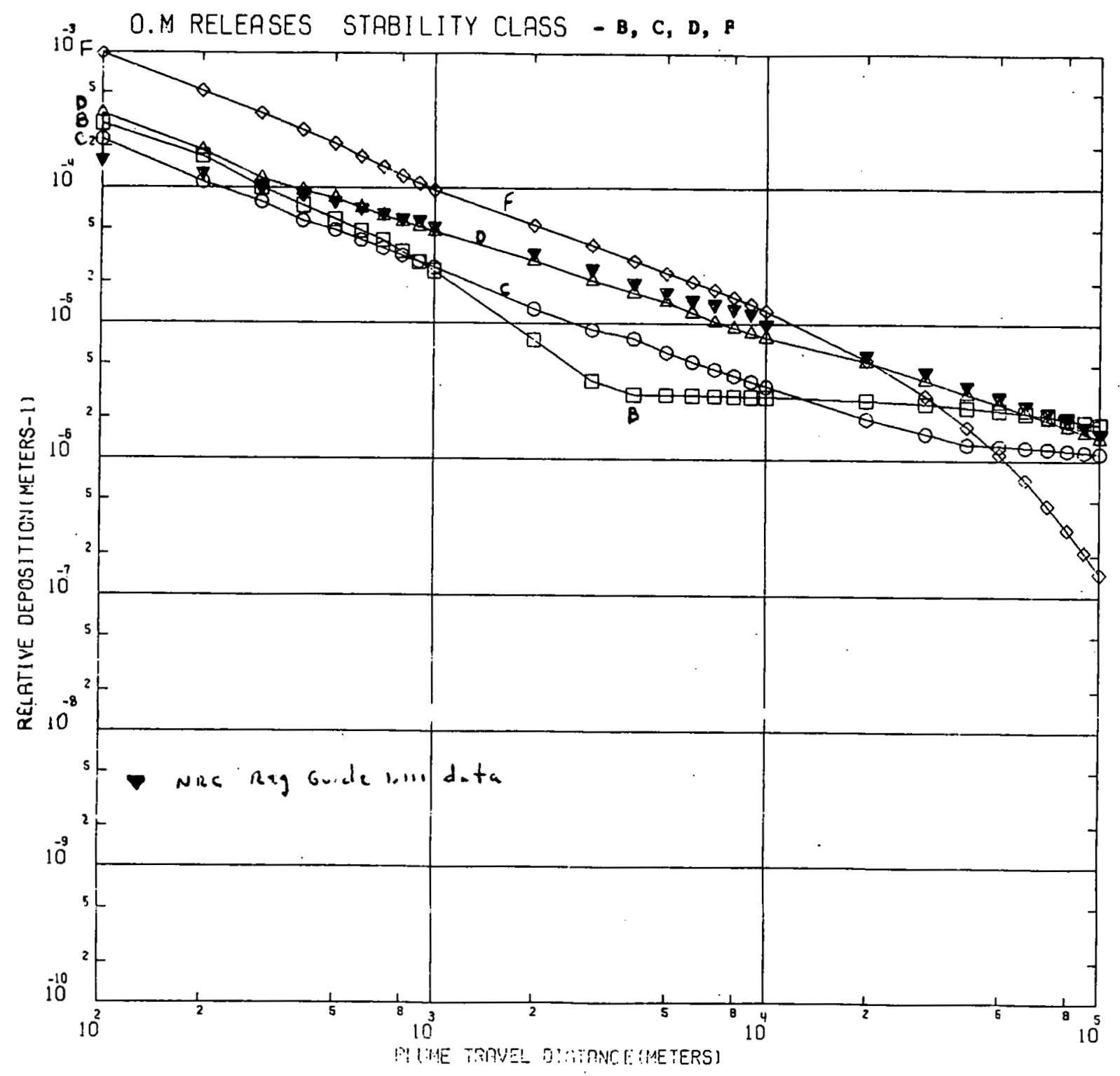

m

Figure E-12 
APPENDIX F

GENERAL INPUT DATA 


\section{APPENDIX F}

\section{GENERAL INPUT DATA}

This appendix provides general input data which is required to run a problem using the AIRWAY computer program. This information is provided in ten tables and covers meteorological data, standard deviations of vertical dispersion, dose commitment conversion factors, decay chains and general data for various radionuclides. A specific description of the data in each of the tables in this appendix is presented below.

The first table in this appendix defines the meteorological data (i.e., the wind speed-stability category frequency distribution) obtained by averaging meteorological data taken from 18 stations in the continental United States (with the exception of Alaska). The data obtained from these 18 stations consisted of wind speed-stability category frequencies independent of wind direction and were averaged to yield wind speed-stability category frequencies in a typical $22.5^{\circ}$ sector. The averaged data were further simplified by combining (adding) the $\mathbf{A}$ and $B$ stability category data and the $\mathbf{E}$ and $\mathbf{F}$ stability category data. The data in the first table in this appendix was obtained from Appendix IX-B of Reference F-l of this appendix with the exception of the frequency for an average wind speed-stability category combination of $15.6 \mathrm{~m} / \mathrm{sec}$ and an F Pasquill category which was modified to ensure that the sum of all frequencies in the table was 1.0.

The second table in this appendix presents the wind speed-stability category frequency data at the $\mathrm{Al}$ legheny County Airport in Pennsylvania during 1974 and 1975. This table is presented as an example of the meteorological data which, combined with wind direction frequency information, is necessary to run a problem using the AIRWAY computer program.

Table F-3 in this appendix provides the standard deviation of vertical dispersion data used with the 18 station average meteorological data. This information is based upon the curves supplied on Page 409 of Reference F-2. The values specified for a distance of 1.0 meters were obtained by extrapolation of these curves.

Table F-4 in this appendix presents the standard deviation of vertical dispersion data which are given in Reference F-3. These data were modified to estimate the values at a distance of 1.0 meter via extrapolation back towards zero.

The fifth table of this appendix provides general information for a total of 378 radionuclides. The data provided for each radionuclide in this table are

1. $\lambda$-decay constant in $\mathrm{hrs}^{-1}$

2. $v$-dry deposition velocity in $\mathrm{m} / \mathrm{sec}$. The values specified in this table for this parameter have been arbitrarily chosen to be $0.01 \mathrm{~m} / \mathrm{sec}(1 \mathrm{~cm} / \mathrm{sec})$ for radionuclides which are considered to behave as particulates (i.e., are subject to dry deposition effects and to precipitation scavenging) and to be 0.0 for all radionuclides which are considered to behave as gases.

3. $\mathrm{w}_{1}$-amount of radioactivity in $\mu \mathrm{Ci}$ taken into the body during a day due to the daily ingestion of 250 grams of above-surface plant foods which had been subjected to a deposition rate of the radionuclide of $1 \mu \mathrm{Ci} / \mathrm{m}^{2}$ on the land per day.

4. $\mathrm{w}_{2}$-amount of radioactivity in $\mu \mathrm{Ci}$ taken into the body during a day due to the daily ingestion of 1 liter of milk which is obtained from cows pastured on land which hac been subjected to a $1.0 \mu \mathrm{Ci} / \mathrm{m}^{2}$ deposition rate per day.

5. $w_{3}$-amount of radioactivity in $\mu \mathrm{Ci}$ taken into the body in a day due to the daily ingestion of 300 grams of beef obtained from cattle pastured on land which had been subjected to a $1 \mu \mathrm{Ci} / \mathrm{m}^{2}$ deposition rate per day.

6. $\mathrm{C}_{\mathrm{s}}$-concentration of the element (in some cases of the specific radionuclide) in the soil-gms element/gm soil.

7. $\mathrm{C}_{\mathrm{p}}$-concentration of the element (or of specific radionuclides) in plants-gms element/gm plant.

In general, the food uptake and concentration data presented in this table were obtained from Appendix IX-C of Reference F-l or as described in this reference from the TERMOD computer program (Reference $F-4$ ).

Table F-6 through Table F-9 provide conversion factors relating the radiation doses accrued by man via various nathways due to the radioactivity in the environment. These conversion factors are provided for nine organs (total body, bone, liver, kidneys, gonads, lung, GI tract, thyroid and the skin) for each of the radionuclides. Table $\mathrm{F}-6$ provides the dose rate conversion factors in $\mathrm{mrem} / \mathrm{hr}$ per $\mathrm{Ci} / \mathrm{m}^{3}$ due to immersion in 
a cloud of radionuclides and Table F-7 supplies the dose rate conversion factors in $\mathrm{mrem} / \mathrm{hr}$ per $\mathrm{Ci} / \mathrm{m}^{2}$ associated with exposure to a surface deposit of radionuclides. Table F-8 presents dose commitment conversion factors in rem $/ \mu \mathrm{Ci}$ defining the dose commitments arising from the ingestion of radionuclides and Table F-9 provides dose commitment conversion factors due to the inhalation of radionuclides. The data in these tables were obtained in the manner defined in Reference F-1.

The final table in this appendix defines the radioactive decay chain data for various radionuclides. It should be noted that the stable isotopes which are final product of these decay chains are omitted from these chains and thus all radionuclides which decay immediately to a stable nuclide are not incorporated in this table.

\section{REFERENCES}

F-1. Light Water Breeder Reactor Program, Final Environmental Statement, ERDA-1541, June 1976.

F-2. D. H. Slade, Ed., "Meteorology and Atomic Energy," TID-24190, July 1968 (latest printing March 1973) (NSA 22-45190).

F-3. NRC Regulatory Guide 1.111, "Methods for Estimating Atmospheric Transport and Dispersion of Gaseous Effluents in Routine Releases from Light-Water-Cooled Reactors," USNRC Office of Standards Development, March 1976.

F-4: R. S. Booth, et. al., "A Systems Analysis Methodology for Predicting Dose to Man from a Radioactivity Contaminated Terrestrial Environment," Proceedings of Third National Symposium on Radioecology, Vol. 2, Oak Ridge, Tenn., May 10-12, 1972. 
TABLE F1. WINDSPEED-STABILITY CATEGORY FREQUENCY DISTRIBUTIONEIGHTEEN STATION AVERAGE

\begin{tabular}{|c|c|c|c|c|}
\hline \multirow{2}{*}{$\begin{array}{c}\text { Windspeed } \\
\begin{array}{c}(\mathrm{m} / \mathrm{sec}) \\
\overline{\mathrm{u}}\end{array}\end{array}$} & \multicolumn{4}{|c|}{ Pasquill Stability Class } \\
\hline & $\mathbf{B}$ & C & D & $\boldsymbol{F}$ \\
\hline $\begin{array}{r}0.8 \\
2.5 \\
4.5 \\
6.9 \\
9.5 \\
12.5 \\
15.6\end{array}$ & $\begin{array}{l}0.0336 \\
0.0672 \\
0.0464 \\
0.0192 \\
0.00496 \\
0.00091 \\
0.00048\end{array}$ & $\begin{array}{l}0.0192 \\
0.0448 \\
0.0496 \\
0.0256 \\
0.00576 \\
0.00015 \\
0.00022\end{array}$ & $\begin{array}{l}0.0448 \\
0.0912 \\
0.072 \\
0.0512 \\
0.0288 \\
0.00224 \\
0.00256\end{array}$ & $\begin{array}{l}0.0688 \\
0.1328 \\
0.1024 \\
0.0528 \\
0.0272 \\
0.00192 \\
0.0032\end{array}$ \\
\hline
\end{tabular}

TABLE F-2. WINDSPEED-STABILITY CATEGORY FREQUENCY DISTRIBUTIONALLEGHENY COUNTY AIRPORT (1974-1975)

\begin{tabular}{|c|c|c|c|c|c|c|c|}
\hline \multirow{2}{*}{$\begin{array}{c}\text { Windspeed } \\
\text { (m/sec) } \\
\frac{\bar{u}}{}\end{array}$} & \multicolumn{7}{|c|}{ Pasquill Stability Class } \\
\hline & $\mathbf{A}$ & $\mathbf{B}$ & $\mathbf{C}$ & $\mathbf{D}$ & $\mathbf{E}$ & $\boldsymbol{F}$ & $\mathbf{G}$ \\
\hline 0.7717 & 0.002742 & 0.009424 & 0.003256 & 0.009767 & 0.0 & 0.009767 & 0.018341 \\
\hline 2.5722 & 0.002742 & 0.025017 & 0.033071 & 0.120459 & 0.071967 & 0.103324 & 0.0 \\
\hline 4.3728 & 0.0 & 0.015936 & 0.048663 & 0.27381 & 0.076251 & 0.0 & 0.0 \\
\hline 6.945 & 0.0 & 0.0 & 0.005312 & 0.157642 & 0.0 & 0.0 & 0.0 \\
\hline 9.7744 & 0.0 & 0.0 & 0.0 & 0.010624 & 0.0 & 0.0 & 0.0 \\
\hline 16.1306 & 0.0 & 0.0 & 0.0 & 0.001885 & 0.0 & 0.0 & 0.0 \\
\hline
\end{tabular}


TABLE F-3. STANDARD DEVIATION OF VERTICAL DISPERSION (USED WITH EIGHTEEN STATION AVERAGE DATA)

\begin{tabular}{r} 
Distance \\
$r$ \\
\hline 1.0 \\
100.0 \\
150.0 \\
200.0 \\
300.0 \\
400.0 \\
500.0 \\
700.0 \\
1000.0 \\
2000.0 \\
3000.0 \\
4000.0 \\
5000.0 \\
7000.0 \\
10000.0 \\
20000.0 \\
40000.0 \\
60000.0 \\
80000.0 \\
100000.0
\end{tabular}

Pasquill Stability Class

\begin{tabular}{|c|c|c|c|}
\hline $\mathbf{B}$ & C & D & $\boldsymbol{F}$ \\
\hline 29 & 2.0 & 2.1 & 0.49 \\
\hline 10.2 & 7.6 & 4.9 & .1 .5 \\
\hline 15.0 & 11.4 & 6.8 & 2.3 \\
\hline 20.0 & 14.3 & 8.7 & 3.0 \\
\hline 31.0 & . 21.0 & 12.1 & 4.7 \\
\hline 430 & 28.0 & 15.8 & 6.2 \\
\hline 55.0 & 34.0 & 19.0 & 7.7 \\
\hline 85.0 & 47.0 & 24.7 & 10.2 \\
\hline 140.0 & 64.0 & 32.0 & 13.5 \\
\hline 400.0 & 116.0 & 53.5 & 21.7 \\
\hline 900.0 & 165.0 & 70.0 & 27.6 \\
\hline 1000.0 & 210.0 & 84.0 & 32.0 \\
\hline 1000.0 & 249.0 & 96.0 & 35.5 \\
\hline 1000.0 & 340.0 & 118.0 & 41.2 \\
\hline 1000.0 & 440.0 & 145.0 & 48.0 \\
\hline 1000.0 & 760.0 & 204.0 & 60.8 \\
\hline 1000.0 & 1000.0 & 290.0 & 75.0 \\
\hline 1000.0 & 1000.0 & 348.0 & 83.0 \\
\hline 1000.0 & 1000.0 & 400.0 & 90.0 \\
\hline 1000.0 & 1000.0 & 440.0 & 93.0 \\
\hline
\end{tabular}

TABLE F-4. STANDARD DEVIATION OF VERTICAL DISPERSION

\begin{tabular}{|c|c|c|c|c|c|c|c|}
\hline \multirow{2}{*}{$\begin{array}{c}\text { Distance } \\
\mathrm{r}\end{array}$} & \multicolumn{7}{|c|}{ Pasquill Stability Class } \\
\hline & $\mathbf{A}$ & $\mathbf{B}$ & $\mathbf{C}$ & $\mathbf{D}$ & $\mathbf{E}$ & $\mathbf{F}$ & $\mathbf{G}$ \\
\hline 1.0 & 0.4 & 0.34 & 0.13 & 0.12 & 0.11 & 0.072 & 0.038 \\
\hline 100.0 & 17.0 & 12.0 & 80 & 5.0 & 3.6 & 2.4 & 1.5 \\
\hline 150.0 & 22.5 & 15.0 & 11.0 & 7.0 & 4.6 & 3.0 & 1.8 \\
\hline 200.0 & 30.0 & 20.0 & 16.0 & 9.0 & 6.3 & 4.0 & 2.4 \\
\hline 300.0 & 50.0 & 33.0 & 22.0 & 14.0 & 9.0 & 5.4 & 3.3 \\
\hline 400.0 & 80.0 & $44 . \dot{U}$ & 30.0 & 17.0 & i i. U & 6.7 & $4 . \bar{I}$ \\
\hline 500.0 & 125.0 & 55.0 & 35.0 & 19.0 & 15.0 & 8.0 & 5.0 \\
\hline 700.0 & 200.0 & 77.5 & 47.0 & 25.0 & 17.0 & 11.0 & 6.5 \\
\hline 1000.0 & 500.0 & 130.0 & 65.0 & 32.0 & 23.0 & 15.0 & 8.6 \\
\hline 2000.0 & 1000.0 & 400.0 & 125.0 & 50.0 & 37.0 & 22.5 & 13.0 \\
\hline 3000.0 & 1000.0 & 800.0 & 175.0 & 67.0 & 46.0 & 28.0 & 17.0 \\
\hline 4000.0 & 1000.0 & 1000.0 & 200.0 & 80.0 & .53 .0 & 33.0 & 19.5 \\
\hline 5000.0 & 1000.0 & 1000.0 & 250.0 & 90.0 & 60.0 & 37.0 & 21.0 \\
\hline 7000.0 & 1000.0 & 1000.0 & 325.0 & 120.0 & 70.0 & 41.0 & 25.0 \\
\hline 10000.0 & 1000.0 & 1000.0 & 425.0 & 150.0 & 84.0 & 47.0 & 29.0 \\
\hline 20000.0 & 1000.0 & 1000.0 & 700.0 & 200.0 & 125.0 & 60.0 & 35.0 \\
\hline 40000.0 & 1000.0 & 1000.0 & 1000.0 & 290.0 & 150.0 & 73.0 & 40.0 \\
\hline 60000.0 & 1000.0 & 1000.0 & 1000.0 & 350.0 & 170.0 & 80.0 & 42.0 \\
\hline 80000.0 & 1000.0 & 1000.0 & 1000.0 & 400.0 & 180.0 & 88.0 & 44.0 \\
\hline 100000.0 & 1000.0 & 1000.0 & 1000.0 & 450.0 & 190.0 & 94.0 & 46.0 \\
\hline
\end{tabular}


TABLE F-5. GENERAL ISOTOPIC DATA

\begin{tabular}{|c|c|c|c|c|c|c|c|}
\hline Isotope & $\begin{array}{c}\lambda \\
\left(h \mathbf{r}^{-1}\right) \\
\end{array}$ & $\begin{array}{c}\mathbf{v} \\
(\mathrm{m} / \mathrm{sec})\end{array}$ & $\begin{array}{c}w_{1} \\
\left(m^{2}\right)\end{array}$ & $\begin{array}{c}\mathbf{w}_{2} \\
\left(\mathbf{m}^{2}\right)\end{array}$ & $\begin{array}{c}\mathbf{w}_{3} \\
\left(\mathbf{m}^{2}\right)\end{array}$ & $\begin{array}{c}\mathrm{C}_{\mathrm{s}} \\
\text { (gm/gm } \\
\text { soil) }\end{array}$ & $\begin{array}{c}C_{p} \\
\text { (gm/gm } \\
\text { plant) }\end{array}$ \\
\hline $\mathrm{H}-3$ & $6.402-6^{*}$ & 0.0 & 0.0 & 0.0 & 0.0 & 0.0 & 0.0 \\
\hline $\mathrm{Be}-7$ & $5.418-4$ & 0.01 & & & & & \\
\hline C-11 & $2.039+0$ & 0.01 & & & & & \\
\hline C-14 & $1.379-8$ & 0.0 & & & . & & \\
\hline $\mathrm{N}-13$ & $4.171+0$ & 0.01 & & & - & & \\
\hline O-15 & $2.029+1$ & 0.01 & & & & & \\
\hline$F-18$ & $3.787-1$ & 0.01 & & & & & \\
\hline $\mathrm{Na}-22$. & $3.041-5$ & 0.01 & & & & & \\
\hline $\mathrm{Na}-24$ & $4.621-2$ & 0.01 & & & & & \\
\hline $\mathrm{Mg}-28$ & $3.285-2$ & 0.01 & & & & & \\
\hline Al-28 & $1.86+1$ & 0.01 & & & & $7.14+4$ & $1.3+1$ \\
\hline $\mathrm{Si}-31$ & $2.65-1$ & 0.01 & & & & & \\
\hline P-32 & $2.021-3$ & 0.01 & & & & & \\
\hline S-35 & $3.282-4$ & 0.01 & & $:$ & & & \\
\hline Cl-36 & $2.627-10$ & 0.01 & & & & & \\
\hline Cl-38 & $1.12+0$ & 0.01 & & & & & \\
\hline K-40 & $6.177-14$ & 0.01 & & & & & \\
\hline $\mathrm{K}-42$ & $5.608-2$ & 0.01 & & & & & \\
\hline $\mathrm{K}-43$ & $3.094-2$ & 0.01 & & & & & \\
\hline $\mathrm{Ca}-45$ & $1.761-4$ & 0.01 & & & & & \\
\hline $\mathrm{Ca}-47$ & $6.375-3$ & 0.01 & & & & & \\
\hline Ca-49 & $4.726+0$ & 0.01 & & & & $\cdot$ & \\
\hline Sc-46 & $3.446-4$ & 0.01 & & & & $7.0+0$ & $7.5-3$ \\
\hline Sc-47 & $8.494-3$ & 0.01 & & & & $7.0+0$ & $7.5-3$ \\
\hline Sc-48 & $1.59-2$ & 0.01 & $1.45-1$ & $1.0-3$ & $5.0-3$ & $7.0+0$ & $7.5-3$ \\
\hline Sc-49 & $7.232-1$ & 0.01 & & & & $7.0+0$ & $7.5-3$ \\
\hline$V-48$ & $1.81-3$ & 0.01 & & & & $1.0+2$ & $1.3-1$ \\
\hline Cr-51 & $1.042-3$ & 0.01 & $8.13-1$ & $4.37-1$ & $1.29-2$ & $2.0+2$ & $5.0-2$ \\
\hline$M n-52 m$ & $1.98+0$ & 0.01 & & & & $8.5+2$ & $2.5+1$ \\
\hline$M n-52$ & $5.093-3$ & 0.01 & & & & $8.5+2$ & $2.5+1$ \\
\hline Mn-54 & $9.24-5$ & 0.01 & $1.15+0$ & $1.3-1$ & $1.1-1$ & $8.5+2$ & $2.3+1$ \\
\hline Mn-56 & $2.68-1$ & 0.01 & $9.63-3$ & $1.54-4$ & $2.38-7$ & $8.5+2$ & $2.5+1$ \\
\hline $\mathrm{Fe}-52$ & $8.452-2$ & 0.01 & & & & $3.8+4$ & $2.5+1$ \\
\hline Fe-55 & $2.929-5$ & 0.01 & $1.19+0$ & $6.4-1$ & $8.19+0$ & $3.8+4$ & $2.5+1$ \\
\hline Fe-59 & $6.475-4$ & 0.01 & $9.31-1$ & $2.78-1$ & $3.69-1$ & $3.8+4$ & $2.5+1$ \\
\hline Co-56 & $3.736-4$ & 0.01 & & & & $8.0+0$ & $7.5-2$ \\
\hline Co-57 & $1.07-4$ & 0.01 & & & & $8.0+0$ & $7.5-2$ \\
\hline Co-58m & $2.49+8$ & 0.01 & & & & $8.0+0$ & $7.5-2$ \\
\hline Co-58 & $4.05-4$ & 0.01 & $1.01+0$ & $4.5-1$ & $7.6-1$ & $8.0+0$ & $7.5-2$ \\
\hline Co- 60 & $1.503-5$ & 0.01 & $1,19+0$ & $5.5-1$ & $2.97+0$ & $8.0+0$ & $7.5-2$ \\
\hline $\mathrm{Ni}-56$ & $4.734-3$ & 0.01 & & & & & \\
\hline $\mathrm{Ni}-59$ & $9.88-10$ & 0.01 & & & & . & \\
\hline $\mathrm{Ni}-63$ & $8.594-7$ & 0.01 & & & & & $\cdot$ \\
\hline $\mathrm{Ni}-65$ & $2.75-1$ & 0.01 & & & & & \\
\hline $\mathrm{Cu}-64$ & $5.415-2$ & 0.01 & & & & & \\
\hline $\mathrm{Zn}-65$ & $1.185-4$ & 0.01 & $1.14+0$ & $1.95+1$ & $3.87+0$ & $5.0-3$ & $2.0+1$ \\
\hline$Z_{n-69}$ & $4.99-2$ & 0.01 & & & & $5.0-3$ & $2.0+1$ \\
\hline
\end{tabular}

$* 6.402-6=0.000006402$ 
TABLE F-5 (Cont'd)

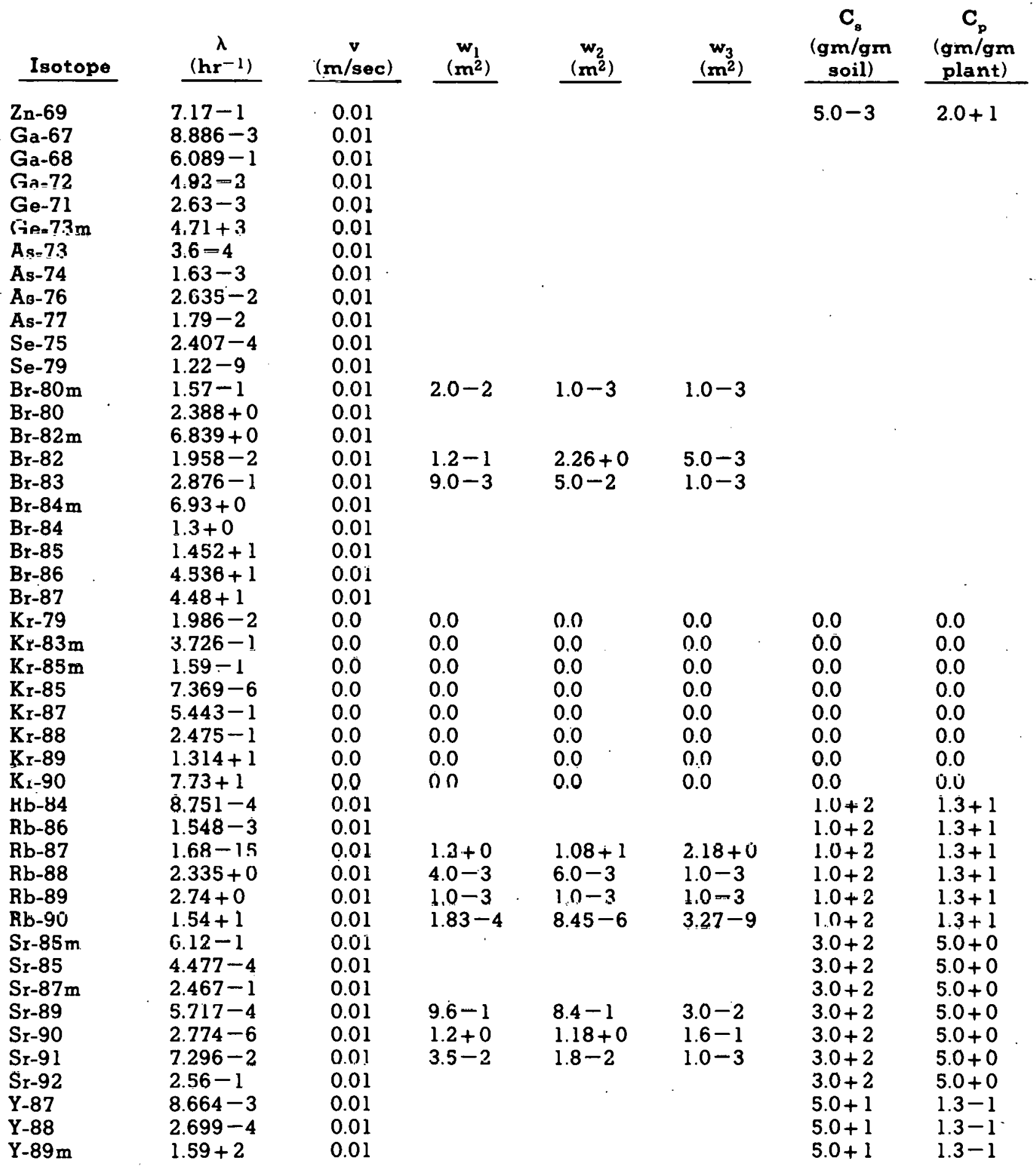


TABLE F-5 (Cont'd)

\begin{tabular}{|c|c|c|c|c|c|c|c|}
\hline Isotope & $\begin{array}{c}\lambda \\
\left(h r^{-1}\right) \\
\end{array}$ & $\begin{array}{c}\mathbf{v} \\
(\mathrm{m} / \mathrm{sec})\end{array}$ & $\begin{array}{c}w_{1} \\
\left(m^{2}\right)\end{array}$ & $\begin{array}{c}w_{2} \\
\left(\mathrm{~m}^{2}\right)\end{array}$ & $\begin{array}{r}w_{3} \\
\left(\mathrm{~m}^{2}\right) \\
\end{array}$ & $\begin{array}{c}\mathrm{C}_{\mathrm{s}} \\
(\mathrm{gm} / \mathrm{gm} \\
\text { soil) }\end{array}$ & $\begin{array}{c}C_{p} \\
\text { (gm/gm } \\
\text { plant) } \\
\end{array}$ \\
\hline Y-90 & $1.083-2$ & 0.01 & $2.0-1$ & $1.0-3$ & $1.0-3$ & $5.0+1$ & $1.3-1$ \\
\hline$Y-91 \mathrm{~m}$ & $8.285-1$ & 0.01 & $3.15-3$ & $4.89-7$ & $1.04-3$ & $5.0+1$ & $1.3-1$ \\
\hline$Y-91$ & $4.903-4$ & 0.01 & $9.7-1$ & $1.0-3$ & $2.2-1$ & $5.0+1$ & $1.3-1$ \\
\hline Y-92 & $1.96-1$ & 0.01 & & & & $5.0+1$ & $1.3-1$ \\
\hline Y-93 & $6.762-2$ & 0.01 & & & & $5.0+1$ & $1.3-1$ \\
\hline Zr-93 & $8.33-11$ & 0.01 & & & & $3.0+2$ & $5.0-2$ \\
\hline$Z_{\text {I-95 }}$ & $4.409-4$ & 0.01 & $9.94-1$ & $1.0-3$ & $1.42+0$ & $3.0+2$ & $5.0-2$ \\
\hline $\mathrm{Zr}-97$ & $4.077-2$ & 0.01 & & & & $3.0+2$ & $5.0-2$ \\
\hline $\mathrm{Nb}-93 \mathrm{~m}$ & $6.59-6$ & 0.01 & & & & $5.3+0$ & $5.0-2$ \\
\hline $\mathrm{Nb}-95 \mathrm{~m}$ & $8.0-3$ & 0.01 & $2.64-1$ & $1.58-1$ & $7.68-2$ & $5.3+0$ & $5.0-2$ \\
\hline Nb-95 & $8.228-4$ & 0.01 & $8.7-1$ & $9.6-1$ & $5.25+0$ & $5.3+0$ & $5.0-2$ \\
\hline $\mathrm{Nb}-97 \mathrm{~m}$ & $4.151+1$ & 0.01 & & & & $5.3+0$ & $5.0-2$ \\
\hline $\mathrm{Nb}-97$ & $5.776-1$ & 0.01 & & & & $5.3+0$ & $5.0-2$ \\
\hline Mo-99 & $1.047-2$ & 0.01 & $2.1-1$ & $6.5-1$ & $5.0-3$ & $2.0+0$ & $2.5-1$ \\
\hline Tc-96m & $7.98-1$ & 0.01 & & & & $1.0-13$ & $2.5-14$ \\
\hline Tc-96 & $6.71-3$ & 0.01 & & & & $1.0-13$ & $2.5-14$ \\
\hline Tc-97m & $3.21-4$ & 0.01 & & & & $1.0-13$ & $2.5-14$ \\
\hline Tc-97 & $3.05-11$ & 0.01 & & & & $1.0-13$ & $2.5-14$ \\
\hline Tc-99m & $1.151-1$ & 0.01 & $2.23-2$ & $6.25-2$ & $1.62-6$ & $1.0-13$ & $2.5-14$ \\
\hline Tc-99 & $3.73-10$ & 0.01 & $1.2+0$ & $3.6+1$ & $2.84-1$ & $1.0-13$ & $2.5-14$ \\
\hline Ru-103m & $1.6+6$ & 0.01 & & & & $1.0-3$ & $5.0 \div 5$ \\
\hline Ru-103 & $7.293-4$ & 0.01 & $9.0-1$ & $1.0-3$ & $8.97+0$ & $1.0-3$ & $5.0-5$ \\
\hline Ru-105 & $1.56-1$ & 0.01 & $1.64-2$ & $1.52-6$ & $4.41-4$ & $1.0-3$ & $5.0-5$ \\
\hline Ru-106 & $7.826-5$ & 0.01 & $1.16+0$ & $1.0-3$ & $5.97+1$ & $1.0-3$ & $5.0-5$ \\
\hline Rh-103m & $7.296-1$ & 0.01 & $3.58-3$ & $9.84-4$ & $6.33-8$ & $1.0-3$ & $1.3-2$ \\
\hline Rh- $105 \mathrm{~m}$ & $6.56+1$ & 0.01 & & & & $1.0-3$ & $1.3-2$ \\
\hline Rh-105 & $1.952-2$ & 0.01 & $1.21-1$ & $2.6-1$ & $7.89-5$ & $1.0-3$ & $1.3-2$ \\
\hline Rh-106 & $8.208+1$ & 0.01 & $3.13-5$ & $8.32-8$ & $4.8-12$ & $1.0-3$ & $1.3-2$ \\
\hline Pd-103 & $1.699-3$ & 0.01 & & & & & \\
\hline Pd-107 & $1.32=11$ & 0.01 & & & & & \\
\hline $\mathrm{Pd}-109$ & $5.15-2$ & 0.01 & & & & & \\
\hline Ag-105 & $7.04-4$ & 0.01 & & & & $10-1$ & $1.5-2$ \\
\hline $\mathrm{Ag}-110 \mathrm{~m}$ & $1.153-4$ & 0.01 & $1.14+0$ & $2.52+1$ & $5.8-1$ & $1.0-1$ & $1.5-2$ \\
\hline Ag-111 & $3.876-3$ & 0.01 & & & & $1.0-1$ & $1.5-2$ \\
\hline Cd-109 & $6.38-5$ & 0.01 & & & & & \\
\hline Cd-113 & $8.78-21$ & 0.01 & & & & & \\
\hline $\mathrm{Cd}-115 \mathrm{~m}$ & $6.48-4$ & 0.01 & & & & & . \\
\hline$C_{d-115}$ & $1.3-2$ & 0.01 & & & & & \\
\hline $\ln -113 m$ & $4.18-1$ & 0.01 & & & & & \\
\hline $\mathrm{In}=114 \mathrm{~m}$ & $5.67+4$ & 0.01 & & & & & \\
\hline In-114 & $3.47+1$ & 0.01 & & & & & \\
\hline In-1 15m & $1.54-1$ & 0.01 & & & & & \\
\hline In-115 & $1.59-19$ & 0.01 & & & & & \\
\hline$S_{n-113}$ & $2.511-4$ & 0.01 & & & & & \\
\hline Sn-119m & $1.155-4$ & 0.01 & & & & & \\
\hline $\mathrm{Sn}-121$ & $2.59-2$ & 0.01 & & & & & \\
\hline
\end{tabular}


TABLE F-5 (Cont'd)

\begin{tabular}{|c|c|c|c|c|c|c|c|}
\hline Igotopo & $\begin{array}{c}\lambda \\
\left(\mathbf{h} \mathbf{r}^{-1}\right) \\
\end{array}$ & $\stackrel{v}{(m / s e c})$ & $\begin{array}{c}w_{1} \\
\left(\mathrm{sin}^{2}\right)\end{array}$ & $\begin{array}{c}w_{2} \\
\left(\mu u^{2}\right)\end{array}$ & $\begin{array}{r}w_{3} \\
\left(m^{0}\right)\end{array}$ & $\begin{array}{c}\mathrm{C}_{\mathrm{s}} \\
(\mathrm{gm} / \mathrm{gm} \\
\mathrm{so1l}) \\
\end{array}$ & $\begin{array}{c}C_{p} \\
(g m / g m \\
\text { plant })\end{array}$ \\
\hline $\begin{array}{l}S n-123 \\
S n-125\end{array}$ & $\begin{array}{l}2.31-4 \\
2.99-3\end{array}$ & $\begin{array}{l}0.01 \\
0.01\end{array}$ & & & & & \\
\hline Sn-126 & $7.91-10$ & 0.01 & & & & & \\
\hline $\begin{array}{l}\text { Sb-122 } \\
\text { Sb-124 }\end{array}$ & $\begin{array}{l}1.07-2 \\
4.797-4\end{array}$ & $\begin{array}{l}0.01 \\
0.01\end{array}$ & & & & $\begin{array}{l}2.3+0 \\
2.3+0\end{array}$ & $\begin{array}{l}2.5-2 \\
2.5-2\end{array}$ \\
\hline $8 b-123$ & $2.855-5$ & 0.01 & $1.10+0$ & $7.9-1$ & $7.8-1$ & $2.3+0$ & $2.5=2$ \\
\hline $5 b-126 \mathrm{~m}$ & $\dot{z} . \dot{y}+\dot{U}$ & U.01 & & & & $2.3+0$ & $2 . \overline{5}-2$ \\
\hline Sb-126 & $2.33-3$ & 0.01 & & & & $2.3+0$ & $2.5-2$ \\
\hline Sb-127 & $-7.502-3$ & 0.01 & & & & $2.3+0$ & $2.5-2$ \\
\hline$S b-128$ & $7.7 \cdots 2$ & 0.01 & & & & $2.3+0$ & $2.5-2$ \\
\hline Sb-129 & $1.6-1$ & 0.01 & & & & $2.3+0$ & $2.5-2$ \\
\hline $\mathrm{T}_{\theta}-123 \mathrm{~m}$ & $2.41-4$ & 0.01 & & & & $1.0-3$ & $1.3-3$ \\
\hline $\mathrm{Te}-125 \mathrm{~m}$ & $4.979-4$ & 0.01 & $9.75-1$ & $4.29-1$ & $4.05+0$ & $1.0-3$ & $1.3-3$ \\
\hline $\mathrm{Te}-127 \mathrm{~m}$ & $2.65-4$ & 0.01 & $1.07+0$ & $4.67-1$ & $5.79+1$ & $1.0-3$ & $1.3-3$ \\
\hline Te-127 & $7.413-2$ & 0.01 & $3.4-2$ & $8.0-3$ & $1.3-2$ & $1.0-3$ & $1.3-3$ \\
\hline $\mathrm{Te}-129 \mathrm{~m}$ & $8.596-4$ & 0.01 & $8.6-1$ & $3.8-1$ & $6.2-1$ & $1.0-3$ & $1.3-3$ \\
\hline Te-129 & $5.97-1$ & 0.01 & $4.0-3$ & $1.0-3$ & $1.0-3$ & $1.0-3$ & $1.3-3$ \\
\hline $\mathrm{Te}-13 \mathrm{~lm}$ & $2.31-2$ & 0.01 & $1.03-1$ & $2.14-2$ & $2.83-3$ & $1.0-3$ & $1.3-3$ \\
\hline $\mathrm{Te}-131$ & $1.663+0$ & 0.01 & $1.55-3$ & $1.96-5$ & $5.94-7$ & $1.0-3$ & $1.3-3$ \\
\hline Te-132 & $8.886-3$ & 0.01 & $2.4-1$ & $1.0-1$ & $7.0-2$ & $1.0-3$ & $1.3-3$ \\
\hline Te-134 & $9.9-1$ & 0.01 & & & & $1.0-3$ & $1.3-3$ \\
\hline I-123 & $5.251-2$ & 0.01 & & & & $5.0+0$ & $1.0-1$ \\
\hline I- 124 & $6.925-3$ & 0.01 & & . & & $5.0+0$ & $1.0-1$ \\
\hline I-125 & $4.813-4$ & 0.01 & & & & $5.0+0$ & $1.0-1$ \\
\hline T- 126 & $2.221-3$ & 0.01 & & & & $5.0+0$ & $1.0-1$ \\
\hline I-128 & $1.666+0$ & 0.01 & $1.56-3$ & $1.99-4$ & $3.15-7$ & $5.0+0$ & $1.0-1$ \\
\hline I- 129 & $4.65-12$ & 0.01 & $1.2+0$ & $1.27+1$ & $3.81+0$ & $5.0+0$ & $1.0-1$ \\
\hline I-130 & $5.59-2$ & 0.01 & $4.5-2$ & $8.8-2$ & $3.0-3$ & $5.0+0$ & $1.0-1$ \\
\hline I-131 & $3.583-3$ & 0.01 & $4.54-1$ & $1.39+0$ & $4.11-1$ & $5.0+0$ & $1.0-1$ \\
\hline I- 1.32 & $3.04-1$ & 0.01 & $2.35-1$ & $6.87-1$ & $8.8-2$ & 5.0 .0 & $1.0-1$ \\
\hline I-133 & $3.301-2$ & 0.01 & $7.2-2$ & $1.67-1$ & $7.0-3$ & $5.0+0$ & $1.0-1$ \\
\hline I-134 & $7.91 \div 1$ & 0.01 & $3.0-3$ & $1.0-3$ & $1.0-3$ & $5.0+0$ & $1.0-1$ \\
\hline I- 135 & $1.035-1$ & 0.01 & $2.5-2$ & $3.6-2$ & $1.0-3$ & $5.0+0$ & $1.0-1$ \\
\hline $1-136$ & $2.934+1$ & 0.01 & $8.69-5$ & $6.39-7$ & $9.63-10$ & $5.0+0$ & $1.0-1$ \\
\hline I-137 & $1.015+2$ & 0.01 & & & & $5.0+0$ & $1.0-1$ \\
\hline Xe-13lm & $2.427-3$ & 0.0 & 0.0 & 0.0 & 0.0 & 0.0 & 0.0 \\
\hline Xe-133m & $1.256-2$ & 0.0 & 0.0 & 0.0 & 0.0 & 0.0 & 0.0 \\
\hline $\mathrm{Xe-133}$ & $5.459-3$ & 0.0 & 0.0 & 0.0 & 0.0 & 0.0 & 0.0 \\
\hline $\mathrm{Xe-135m}$ & $2.666+0$ & 0.0 & 0.0 & 0.0 & 0.0 & 0.0 & 0.0 \\
\hline$X_{\theta-135}$ & $7.591-2$ & 0.0 & 0.0 & 0.0 & 0.0 & 0.0 & 0.0 \\
\hline Xe-137 & $1.083+1$ & 0.0 & 0.0 & 0.0 & 0.0 & 0.0 & 0.0 \\
\hline Xe-138 & $2.927+0$ & 0.0 & 0.0 & 0.0 & 0.0 & 0.0 & 0.0 \\
\hline Xe-139 & $6.299+1$ & 0.0 & 0.0 & 0.0 & 0.0 & 0.0 & 0.0 \\
\hline Xe-140 & $1.836+2$ & 0.0 & 0.0 & 0.0 & 0.0 & 0.0 & 0.0 \\
\hline Cs-134 & $3.857-5$ & 0.01 & $1.18+0$ & $7.39+0$ & $7.8-1$ & $5.0+0$ & $5.0-2$ \\
\hline Cs-135 & $2.63-11$ & 0.01 & $1.2+0$ & $5.04+0$ & $2.84-1$ & $5.0+0$ & $5.0-2$ \\
\hline Cs-136 & $2.239-3$ & 0.01 & $6.09-1$ & $2.13+0$ & $8.61-3$ & $5.0+0$ & $5.0-2$ \\
\hline
\end{tabular}


TABLE F-5 (Cont'd)

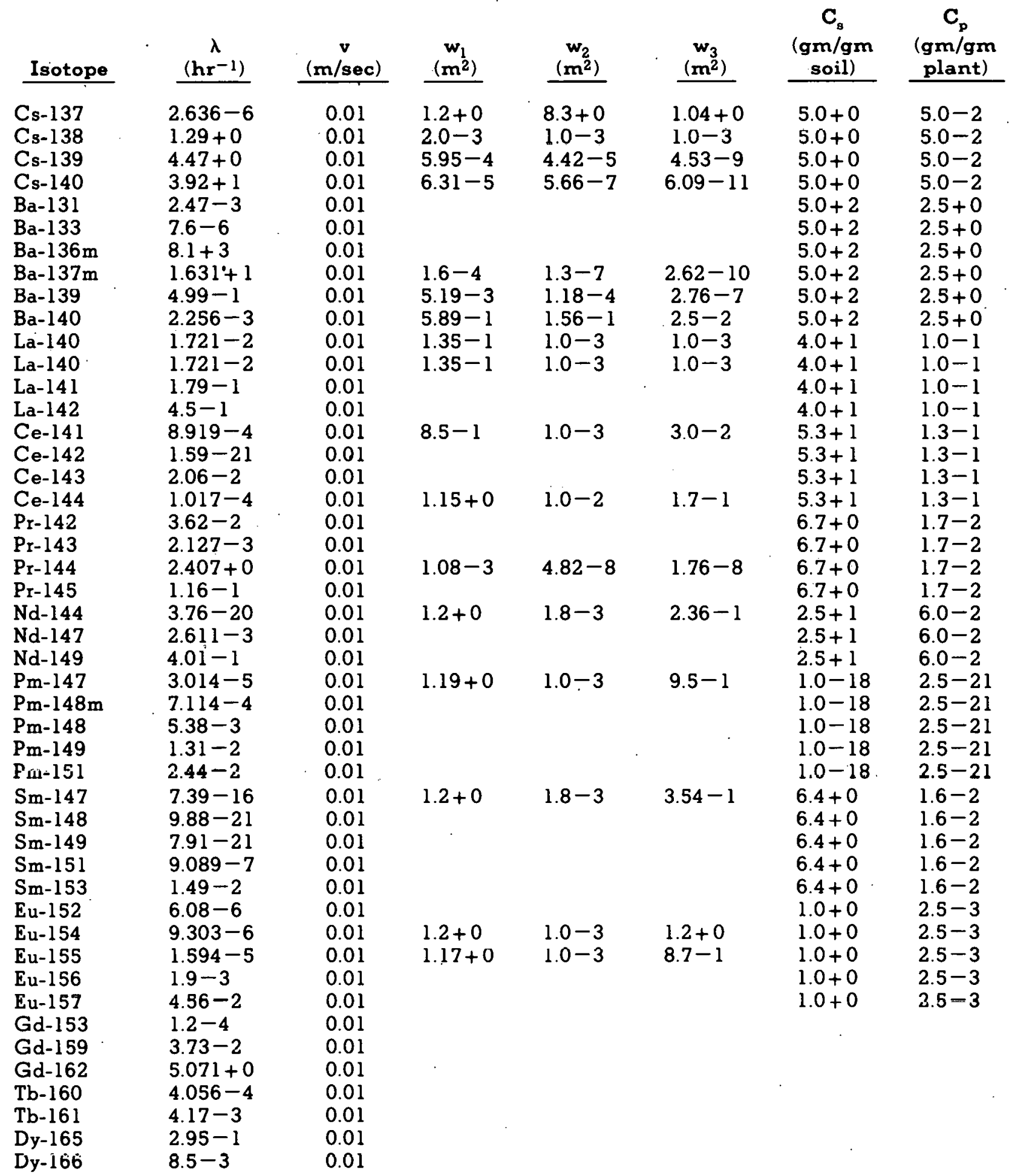


TABLE F-5 (Cont'd)

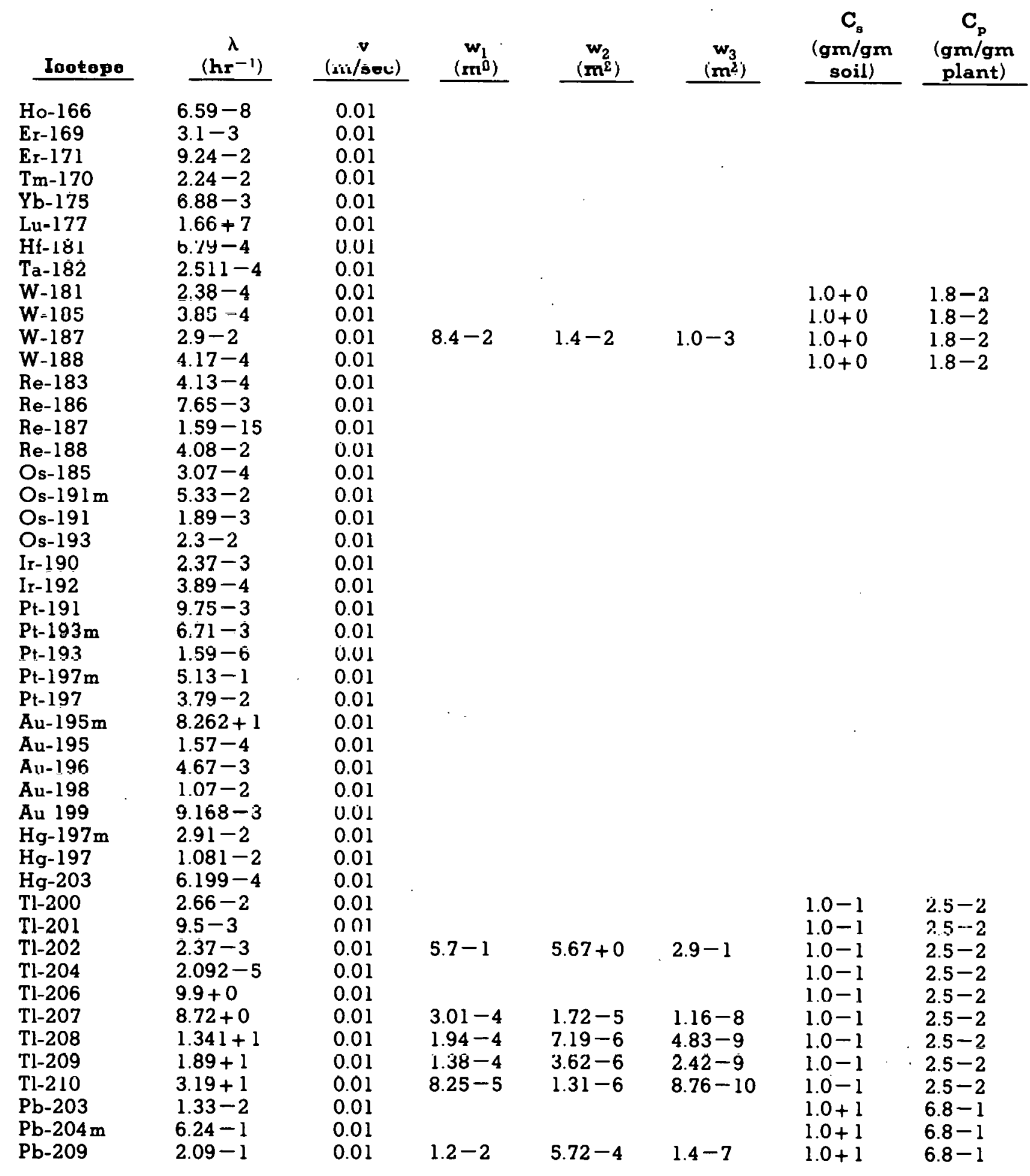


TABLE F-5 (Cont'd)

\begin{tabular}{|c|c|c|c|c|c|c|c|}
\hline Isotope & $\begin{array}{c}\lambda \\
\left(h r^{-1}\right) \\
\end{array}$ & $\begin{array}{c}\mathbf{v} \\
(\mathrm{m} / \mathrm{sec})\end{array}$ & $\begin{array}{c}w_{1} \\
\left(\mathrm{~m}^{2}\right)\end{array}$ & $\begin{array}{c}\mathbf{w}_{2} \\
\left(\mathbf{m}^{2}\right)\end{array}$ & $\begin{array}{c}w_{3} \\
\left(\mathbf{m}^{2}\right) \\
\end{array}$ & $\begin{array}{c}\mathrm{C}_{\mathrm{s}} \\
\text { (gm/gm } \\
\text { soil) }\end{array}$ & $\begin{array}{c}\mathrm{C}_{\mathrm{p}} \\
\text { (gm/gm } \\
\text { plant) }\end{array}$ \\
\hline $\mathrm{Pb}-210$ & $3.765-6$ & 0.01 & $1.2+0$ & $3.6-1$ & $7.0-2$ & $1.0+1$ & $6.8-1$ \\
\hline $\mathrm{Pb}-21 \mathrm{l}$ & $1.15+0$ & 0.01 & $2.26-3$ & $2.52 \div 5$ & $4.83-9$ & $1.0+1$ & $6.8-1$ \\
\hline $\mathrm{Pb}-212$ & $6.51-2$ & 0.01 & $3.88-2$ & $3.59-3$ & $1.46-6$ & $1.0+1$ & $6.8-1$ \\
\hline $\mathrm{Pb}-214$ & $1.55+0$ & 0.01 & $1.68-3$ & $1.42-5$ & $2.65-9$ & $1.0+1$ & $6.8-1$ \\
\hline $\mathrm{Bi}-206$ & $4.63-3$ & 0.01 & & & & $1.0-1$ & $1.5-2$ \\
\hline $\mathrm{Bi}-207$ & $2.08-6$ & 0.01 & & & & $1.0-1$ & $1.5-2$ \\
\hline $\mathrm{Bi}-210$ & $5.767-3$ & 0.01 & $3.28-1$ & $7.0-2$ & $1.0-3$ & $1.0-1$ & $1.5-2$ \\
\hline $\mathrm{Bi}-21 \mathrm{l}$ & $1.94+1$ & 0.01 & $1.35-4$ & $7.77-8$ & $7.8-10$ & $1.0-1$ & $1.5-2$ \\
\hline $\mathrm{Bi}-212$ & $6.863-1$ & 0.01 & $3.78-3$ & $5.44-5$ & $6.09-7$ & $1.0-1$ & $1.5-2$ \\
\hline $\mathrm{Bi}-213$ & $9.04-1$ & 0.01 & $2.94-3$ & $3.38-5$ & $3.69-7$ & $1.0-1$ & $1.5-2$ \\
\hline $\mathrm{Bi}-214$ & $2.1+0$ & 0.01 & $1.23-3$ & $6.23-6$ & $6.48-8$ & $1.0-1$ & $1.5-2$ \\
\hline Po-210 & $2.09-4$ & 0.01 & $1.09+0$ & $1.44+0$ & $1.01+1$ & $2.0-11$ & $1.0-11$ \\
\hline Po-211. & $4.71+3$ & 0.01 & & & & $2.0-11$ & $1.0-11$ \\
\hline Po-212 & $8.32+9$ & 0.01 & & & & $2.0-11$ & $1.0-11$ \\
\hline Po-213 & $6.24+8$ & 0.01 & & & & $2.0-11$ & $1.0-11$ \\
\hline Po-214 & $1.52+7$ & 0.01 & & & & $2.0-11$ & $1.0-11$ \\
\hline Po-215 & $1.4+6$ & 0.01 & & & & $2.0-11$ & $1.0-11$ \\
\hline Po-216 & $1.66+4$ & 0.01 & & & & $2.0-11$ & $1.0-11$ \\
\hline Po-218 & $1.36+1$ & 0.01 & $1.91-4$ & $9.29-7$ & $1.4-8$ & $2.0-11$ & $1.0-11$ \\
\hline At-211 & $9.61-2$ & 0.01 & & & & & \\
\hline At-215 & $2.49+7$ & 0.01 & & & & & \\
\hline At-217 & $7.8+4$ & 0.01 & & & & & \\
\hline At-218 & $1.25+3$ & 0.01 & & & & & \\
\hline $\mathrm{Rn}-219$ & $6.3+2$ & 0.0 & 0.0 & 0.0 & 0.0 & 0.0 & 0.0 \\
\hline $\mathrm{Rn}-220$ & $4.5+1$ & 0.0 & 0.0 & 0.0 & 0.0 & $1.0-13$ & $3.5-13$ \\
\hline $\mathrm{Rn}-222$ & $7.54-3$ & 0.0 & $2.67-1$ & $2.26+0$ & $2.3-2$ & $1.0-13$ & $3.5-13$ \\
\hline $\mathrm{Ra}-223$ & $2.53-3$ & 0.01 & $5.51-1$ & $2.08+0$ & $5.73-2$ & $8.0-7$ & $2.5-10$ \\
\hline $\mathrm{Ra}-224$ & $7.934-3$ & 0.01 & $2.58-1$ & $9.27-1$ & $9.0-3$ & $8.0-7$ & $2.5-10$ \\
\hline Ra-225 & $1.95-3$ & 0.01 & $6.3-1$ & $2.37+0$ & $8.3-2$ & $8.0-7$ & $2.5-10$ \\
\hline Ra-22G & $4.93-8$ & 0.01 & $1.2+0$ & $9.49+0$ & $9.75+0$ & $8.0-7$ & $2.5-10$ \\
\hline Ra-228 & $1.37-5$ & 0.01 & 1. $19+0$ & $4.7+0$ & $1.97+0$ & $8.0-7$ & $2.5-10$ \\
\hline$F_{1}-221$ & $8.64+0$ & 0.01 & $3.01-4$ & $3.81-5$ & $5.79-8$ & $1.0-18$ & $1.0-20$ \\
\hline $\mathrm{Fr}-223$ & $1.89+0$ & 0.01 & $1.38-3$ & $7.74-4$ & $1.21-6$ & $1.0-18$ & $1.0-20$ \\
\hline Ac-225 & $2.89-3$ & 0.01 & $5.2-1$ & $6.42-4$ & $2.73-4$ & $1.0-10$ & $2.5-13$ \\
\hline Ac-227 & $3.63-6$ & 0.01 & $1.2+0$ & $1.66-3$ & $5.1+0$ & $1.0-10$ & $2.5-13$ \\
\hline Ac-228 & $1.13-1$ & 0.01 & $2.26-2$ & $1.28-5$ & $1.33-4$ & $1.0-10$ & $2.5-13$ \\
\hline Th-227 & $1.54-3$ & 0.01 & $6.94-1$ & $8.68-4$ & $6.33-4$ & $6.0+0$ & $2.5-2$ \\
\hline Th-228 & $4.133-5$ & 0.01 & $1.18+0$ & $3.0-3$ & $3.7-2$ & $6.0+0$ & $2.5-2$ \\
\hline Th-229 & $1.08-8$ & 0.01 & $1.2+0$ & $3.17-3$ & $5.67-2$ & $6.0+0$ & $2.5-2$ \\
\hline Th-230 & $9.881-10$ & 0.01 & $1.2+0$ & $3.0-3$ & $5.7-2$ & $6.0+0$ & $2.5-2$ \\
\hline Th-231 & $2.718-2$ & 0.01 & $8.9-2$ & $1.0-3$ & $1.0-3$ & $6.0+0$ & $2.5-2$ \\
\hline Th-232 & $5.608-15$ & 0.01 & $1.2+0$ & $7.0-3$ & $1.18-1$ & $6.0+0$ & $2.5-2$ \\
\hline Th-234 & $1.198-3$ & 0.01 & $7.75-1$ & $2.0-3$ & $3.0-3$ & $6.0+0$ & $2.5-2$ \\
\hline $\mathrm{Pa}-230$ & $1.66-3$ & 0.01 & & & & $1.0-7$ & $2.5-10$ \\
\hline $\mathrm{Pa}-231$ & $2.43-9$ & 0.01 & $1.19+0$ & $1.8-3$ & $6.12+0$ & $1.0-7$ & $2.5-10$ \\
\hline $\mathrm{Pa}-233$ & $1.07-3$ & 0.01 & $8.06-1$ & $1.01-3$ & $4.47-1$ & $1.0-7$ & $2.5-10$ \\
\hline $\mathrm{Pa}-234 \mathrm{~m}$ & $3.55+1$ & 0.01 & $7.38-5$ & $2.32-5$ & $1.51-9$ & $1.0-7$ & $2.5-10$ \\
\hline
\end{tabular}


TABLE F-5 (Cont'd)

\begin{tabular}{|c|c|c|c|c|c|c|c|}
\hline Isotope & $\begin{array}{c}\lambda \\
\left(h r^{-1}\right)\end{array}$ & $\begin{array}{c}\mathbf{v} \\
(\mathrm{m} / \mathrm{sec})\end{array}$ & $\begin{array}{c}w_{1} \\
\left(m^{2}\right)\end{array}$ & $\begin{array}{c}\mathbf{w}_{2} \\
\left(\mathbf{m}^{2}\right)\end{array}$ & $\begin{array}{c}w_{3} \\
\left(m^{2}\right) \\
\end{array}$ & $\begin{array}{c}(\mathrm{gm} / \mathrm{gm} \\
\text { soil) }\end{array}$ & $\begin{array}{l}\text { (gm/gm } \\
\text { plant) }\end{array}$ \\
\hline $\mathrm{Pa}-234$ & $1.04-1$ & 0.01 & & & & $1.0-7$ & $2.5-10$ \\
\hline $\mathrm{Pa}-235$ & $1.73+0$ & 0.01 & $1.79-3$ & $8.96-8$ & $6.09-7$ & $1.0-7$ & $2.5-10$ \\
\hline U-230 & $1.39-3$ & 0.01 & & & & $1.0+0$ & $2.5-3$ \\
\hline U-232 & $1.098-6$ & 0.01 & $1.2+0$ & $3.1-1$ & $9.0=2$ & $1.0+0$ & $2.5-3$ \\
\hline $\mathrm{U}-233$ & $4.88-10$ & 0.01 & $1.2+0$ & $3.2-1$ & $9.0-2$ & $1.0+0$ & 2.53 \\
\hline U-234 & $3.2-10$ & 0.01 & $1.2+0$ & $3.2-1$ & $0.0-2$ & $1.0+0$ & $2.5-3$ \\
\hline U-235 & $1.114-13$ & 0.01 & $1.2+0$ & $3.2-1$ & $9.0=2$ & $1.0+0$ & $2.5-3$ \\
\hline U-2̇36 & $3.309-12$ & 0.01 & $1.2+0$ & $3.2-1$ & $9.0-2$ & $1.0+0$ & $2.5-3$ \\
\hline U-23? & $4.2 .9-3$ & 0.01 & $4.2 \cdots 1$ & $9.0-2$ & $1.0-3$ & $1.0+0$ & $2.5-3$ \\
\hline $\mathrm{U}=238$ & $1.754-14$ & 0.01 & $1.2+0$ & $3.2-1$ & $9.0-2$ & $1.0+0$ & $2.5-3$ \\
\hline U-239 & $1.77+0$ & 0.01 & $1.48-3$ & $8.84-6$ & $2.33-9$ & $1.0+0$ & $2.5-3$ \\
\hline $\mathrm{Np}_{\mathrm{p}-237}$ & $3.695-11$ & 0.01 & $1.2+0$ & $3.0-3$ & $6.0-2$ & $1.0-12$ & $2.5-15$ \\
\hline Np-238 & $1.36-2$ & 0.01 & $1.63-1$ & $1.0-3$ & $2.0-3$ & $1.0-12$ & $2.5-15$ \\
\hline Np-239 & $1.226-2$ & 0.01 & $1.8-1$ & $2.07-4$ & $2.38-5$ & $1.0-12$ & $2.5-15$ \\
\hline $\mathrm{Np}-240 \mathrm{~m}$ & $5.55+0$ & 0.01 & $4.56-4$ & $8.75-9$ & $2.6-9$ & $1.0-12$ & $2.5-15$ \\
\hline Np-240 & $6.419-1$ & 0.01 & $4.26-3$ & $5.91-7$ & $9.99-9$ & $1.0-12$ & $2.5-15$ \\
\hline Pu-238 & $9.194-7$ & 0.01 & $1.2+0$ & $1.0-3$ & $1.6-2$ & $1.0-13$ & $2.5-17$ \\
\hline $\mathrm{Pu}-239$ & $3.301-9$ & 0.01 & $1.2+0$ & $1.0-3$ & $1.7-2$ & $1.0-13$ & $2.5-17$ \\
\hline $\mathrm{Pu}-240$ & $1.202-8$ & 0.01 & $1.2+0$ & $1.0-3$ & $1.7-2$ & $1.0-13$ & $2.5-17$ \\
\hline Pu-241 & $5.991-6$ & 0.01 & $1.2+0$ & $1.0-3$ & $1.5-2$ & $1.0-13$ & $2.5-17$ \\
\hline $\mathrm{Pu}-242$ & 2. $04-10$ & 0.01 & $1.2+0$ & $1.0-3$ & $1.7-2$ & $1.0-13$ & $2.5-17$ \\
\hline $\mathrm{Pu}-243$ & $1.4-1$ & 0.01 & & & & $1.0-13$ & $2.5-17$ \\
\hline $\mathrm{Pu}-244$ & $9.53-13$ & $0 \cap 1$ & & & & (.) 1$)-13$ & $3.6-17$ \\
\hline Am-211 & $1.826-7$ & 0.01 & $1.2+0$ & $3.0-3$ & $6.0-2$ & $1.0-30$ & $2.5-31$ \\
\hline$A m=242 \mathrm{~m}$ & $1.78+5$ & 0.01 & & & & $1.0-30$ & $2.5-34$ \\
\hline Am-242 & $4.332-2$ & 0.01 & $1.2+0$ & $3.0-3$ & $5.0-2$ & $1.0-30$ & $2.5-34$ \\
\hline Ám-243 & $9.946-9$ & 0.01 & $1.2+0$ & $3.0-3$ & $6.0-2$ & $1.0-30$ & $2.5-34$ \\
\hline$A-2-244$ & $6.86-2$ & 0.01 & & & & $1.0-30$ & $2.5-34$ \\
\hline $\mathrm{Cm}_{\mathrm{m}-242}$ & $1.772-4$ & 0.01 & $1.11+0$ & $2.0-3$ & $2.0-2$ & $1.0-30$ & $2.5-33$ \\
\hline $\mathrm{Cm}-2.43$ & $2.636-6$ & 0.01 & $1.2+0$ & $3.0-3$ & $5.0-2$ & $1.0-30$ & $2.5-33$ \\
\hline $\mathrm{Cm}-244$ & $4.43-6$ & 0.01 & $1.2+0$ & $3.0-3$ & $5.0-2$ & $1.0-30$ & $2.5-33$ \\
\hline Cm.245 & $9.3-9$ & 0.01 & $1.2+0$ & $1.8-3$ & $1.42-2$ & $1.0-30$ & $2.5-33$ \\
\hline $\mathrm{Cm}-246$ & $1.67-8$ & 0.01 & $1.2+0$ & $1.8-3$ & 1.422 & $1.0-30$ & $2.5-33$ \\
\hline$e_{\text {m- }}-24 \%$ & $5.13-12$ & 0.01 & $1.2+0$ & $1.8-3$ & $1.42-2$ & $1.0-30$ & $2.5-33$ \\
\hline $\mathrm{Cm}-248$ & $2.26-10$ & 0.01 & $1.2+0$ & $1.8-3$ & $1.42-2$ & $1.0-30$ & $2.5 \cdots 33$ \\
\hline $\mathrm{Cm}-249$ & $6.48-1$ & 0.01 & & & & $1.0-30$ & $2.5-3.3$ \\
\hline Bk-249 & $929-5$ & 0.01 & $1.15+0$ & $4.32-4$ & $2.87+0$ & $1.0-30$ & $2.5-30$ \\
\hline $\mathrm{Bk}-250$ & $2.15-1$ & 0.01 & & & & & \\
\hline Cf- -249 & $2.25-7$ & 0.01 & & & & & \\
\hline Cf-250 & $6.03-6$ & 0.01 . & $1.2+0$ & $4.86-4$ & $4.92+0$ & $1.0-30$ & $2.5-30$ \\
\hline $\mathrm{Ct}-25 \mathrm{l}$ & $8.78-8$ & 0.01 & & & & & \\
\hline Cf-252 & $3.01-5$ & 0.01 & $1.19+0$ & $4.52-4$ & $3.99+0$ & $1.0-30$ & $2.5-30$ \\
\hline Cf-253 & $1.62-3$ & 0.01 & & & & & \\
\hline Cf-254 & $4.83-4$ & 0.01 & & & & & \\
\hline Es-253 & $1.41-3$ & 0.01 & & & & & \\
\hline Es-254 m & $1.76-2$ & 0.01 & & & & & \\
\hline
\end{tabular}


TABLE F-5 (Cont'd) .

\begin{tabular}{|c|c|c|c|c|c|c|c|}
\hline Isotope & $\begin{array}{c}\lambda \\
\left(\mathbf{h} \mathbf{r}^{-1}\right) \\
\end{array}$ & $\begin{array}{c}\mathbf{v} \\
(\mathrm{m} / \mathrm{sec})\end{array}$ & $\begin{array}{c}w_{1} \\
\left(\mathrm{~m}^{2}\right)\end{array}$ & $\begin{array}{c}w_{2} \\
\left(\mathbf{m}^{2}\right)\end{array}$ & $\begin{array}{c}\mathbf{w}_{3} \\
\left(\mathbf{m}^{2}\right) \\
\end{array}$ & $\begin{array}{c}\mathrm{C}_{\mathrm{s}} \\
\begin{array}{c}\mathrm{gm} / \mathrm{gm} \\
\text { soil) }\end{array} \\
\end{array}$ & $\begin{array}{c}\mathrm{C}_{\mathrm{p}} \\
\text { (gm/gm } \\
\text { plant) }\end{array}$ \\
\hline $\begin{array}{l}\text { Es-254 } \\
\text { Es-255 } \\
\text { Fm-254 } \\
\text { Fm-255 } \\
\text { Fm-256 }\end{array}$ & $\begin{array}{l}1.05-4 \\
7.42-4 \\
2.14-1 \\
3.45-2 \\
2.64-1\end{array}$ & $\begin{array}{l}0.01 \\
0.01 \\
0.01 \\
0.01 \\
0.01\end{array}$ & & & & & \\
\hline
\end{tabular}


TABLE F-6. IMMERSION DOSE RATE CONVERSION FACTORS

(rem/hr per ci/m $\mathrm{m}^{3}$ )

\begin{tabular}{|c|c|c|c|c|c|c|c|c|c|}
\hline Isotope & $\begin{array}{l}\text { Total } \\
\text { Body }\end{array}$ & Bone & Liver & Kidney & Gonad & Lung & G.I. Tract & Thyroid & Skin \\
\hline-3 & 0.0 & 0.0 & 0.0 & 0.0 & 0.0 & 0.0 & 0.0 & 0.0 & $6.34+0$ \\
\hline & $4.09+1$ & $.99+1$ & & $3.63+1$ & $4.54+1$ & $3.86+1$ & & & $5.0+1$ \\
\hline & $47+2$ & $03+3$ & $05+2$ & $52+2$ & $9.41+2$ & $0+2$ & $*$ & $8.92+2$ & $1.44+3$ \\
\hline & & 0.0 & 0.0 & 0.0 & 0.0 & 0.0 & 0.0 & 0.0 & $5.51+1$ \\
\hline-13 & $8.47+2$ & $.03+3$ & $7.07+2$ & $7.52+2$ & $9.41+2$ & $8.0+2$ & $*$ & $8.93+2$ & $1.56+3$ \\
\hline-15 & & $04+3$ & $n 7+?$ & $3+2$ & 8.1313 & $8.01+2$ & $*$ & $4+2$ & $1.81+3$ \\
\hline-18 & & & $85+2$ & & & & * & & \\
\hline & $95+3$ & $2.18+3$ & $1.63+3$ & $1.52+3$ & $1.74+3$ & & $1.87+3$ & & $2.43+3$ \\
\hline$a-24$ & $.56+3$ & $3.7+3$ & $3.13+3$ & $2.68+3$ & & $3.42+3$ & & & $4.75+3$ \\
\hline & $1.19+3$ & $1.28+3$ & $9.87+2$ & $8.98+2$ & & & & $1.13+3$ & $1.53+3$ \\
\hline & $5+3$ & $1.74+3$ & $39+3$ & & & & & $*$ & $8+3$ \\
\hline & & & & & & & & & \\
\hline & & & & & & & & & \\
\hline & & & & & & & 0. & & \\
\hline & & & & & & & & & +2 \\
\hline & & & & & & & & & $2+3$ \\
\hline & & +2 & $22+2$ & & & & & & $6.24+2$ \\
\hline & $6+2$ & +2 & $.15+2$ & $1.8+2$ & & & & • & $1.74+3$ \\
\hline$K=4$ & $15+2$ & $9.95+2$ & $6.8+2$ & & & & & $8.54+2$ & $1+3$ \\
\hline & & & & & & & & & $8.92+1$ \\
\hline & & +3 & & & $79+2$ & & & & \\
\hline & & & & & & & & & \\
\hline & & $.74+3$ & +3 & & & & & & $7+3$ \\
\hline & +3 & & +1 & +1 & & $29+1$ & & & $2.9+2$ \\
\hline & & & & & & & & & 0.0 \\
\hline & & & & & & & & & $8.34+2$ \\
\hline V -48 & & & & & & & & & \\
\hline Cr-51 & $2.66+1$ & & $2.03+1$ & $.93+1$ & & & & & $3.7+1$ \\
\hline$M n-52 m$ & & & & & & & & & $3+3$ \\
\hline$M n-52$ & & $3.24+3$ & $5+3$ & $7+3$ & & & & & +3 \\
\hline & $56+2$ & $7.19+2$ & $5.5+2$ & +2 & $5.92+2$ & $6.14+2$ & $5.92+2$ & $4.86+2$ & $8.52+2$ \\
\hline & & & & & & $00^{\circ}$ & & & 0.0 \\
\hline Fe- & $6.1+2$ & $2+2$ & $5.0+2$ & +2 & $7.03+2$ & & 5.65 & $63+2$ & $9.43+2$ \\
\hline & $\cdots 2$ & $4-2$ & $8-5$ & & & & 1 & $5-3$ & +0 \\
\hline & $2+3$ & $1.09+3$ & $5+2$ & & & & & +2 & $5+3$ \\
\hline & $3+3$ & $3.32+3$ & $2.69+3$ & $1+3$ & & & & $1+3$ & $3.82+3$ \\
\hline Co-57 & $1.06+2$ & $1.88+2$ & $7.85+1$ & $7.72+1$ & $9.91+1$ & $8.87+1$ & $6.87+1$ & $1.29+2$ & $1.45+2$ \\
\hline Co-58m & 0.0 & & 0.0 & & ?0 & 0.0 & 0.0 & 0.0 & 0.0 \\
\hline Co-58 & $1.73+2$ & $8.63+2$ & $6.47+2$ & $7.16+2$ & $7.24+2$ & $7.25+2$ & $6.83+2$ & $6.15+2$ & $1.02+3$ \\
\hline Co-60 & $2.16+3$ & & $1.81+3$ & $1.74+3$ & $1.76+3$ & $2.04+3$ & $2.1+3$ & $1.92+3$ & $2.64+3$ \\
\hline $\mathrm{Ni}-56$ & $1.43+3$ & $1.67+3$ & $1.18+3$ & $1.23+3$ & $1.37+3$ & $1.32+3$ & $1.26+3$ & $1.21+3$ & $1.78+3$ \\
\hline $\mathrm{Ni}-59$ & 0.0 & 0.0 & 0.0 & 0.0 & 0.0 & 0.0 & 0.0 & 0.0 & 0.0 \\
\hline
\end{tabular}

- Data not available. Use of Total Body Factor is recommended. 
TABLE F-6 (Cont)

\begin{tabular}{|c|c|c|c|c|c|c|c|c|c|}
\hline Isotope & $\begin{array}{l}\text { Iotal } \\
\text { Body }\end{array}$ & Bone & Liver & Kidney & Gonad & Lung & G.I. Tract & Thyroid & Skin \\
\hline $\mathrm{Ni}-63$ & 0.0 & 0.0 & 0.0 & 0.0 & 0.0 & 0.0 & 0.0 & 0.0 & $12+$ \\
\hline $\mathrm{Ni}-65$ & 0.0 & 0.0 & 0.0 & 0.0 & 0.0 & 0.0 & 0 & 0.0 & 0.0 \\
\hline $\mathrm{Cu}-64$ & $1.64+2$ & $.99+2$ & $1.37+2$ & $1.45+2$ & $1.79+2$ & $1.55+2$ & $1.63+2$ & $1.72+2$ & $3.3+2$ \\
\hline$n-65$ & $5+2$ & $01+2$ & $82+2$ & $26+2$ & $4.14+2$ & $4.26+2$ & $1+2$ & $3.41+2$ & $5.96+2$ \\
\hline$n-69 m$ & $46+2$ & $22+2$ & $2.88+2$ & $3.07+2$ & $3.84+2$ & $3.26+2$ & $*$ & $3.64+2$ & $4.45+2$ \\
\hline$n-69$ & 0.0 & 0.0 & 0.0 & 0.0 & 0.0 & 0.0 & 0.0 & 0.0 & $3.36+2$ \\
\hline Ga-67 & $53+2$ & $31+2$ & $1.18+2$ & $1.15+2$ & $1.8+2$ & $1.35+2$ & $1.09+2$ & $1.58+2$ & +2 \\
\hline Ga-68 & $4+2$ & $.52+2$ & $6.54+2$ & $6.97+2$ & $8.62+2$ & $.41+2$ & $7.79+2$ & $8.15+2$ & $1.71+3$ \\
\hline Ga-72 & 0.0 & 0.0 & 0.0 & 0.0 & 0.0 & 0.0 & 0.0 & 0.0 & 0.0 \\
\hline Ge-71 & 0.0 & 0.0 & 0.0 & 0.0 & 0.0 & 0.0 & 0. & 0.0 & 0.0 \\
\hline s-73 & 0.0 & 0.0 & 0.0 & 0.0 & 0.0 & 0. & 0. & 0.1 & 0.0 \\
\hline Âs-74 & 0.0 & 0.0 & 0.0 & 0.0 & 0.0 & 0.0 & 0. & 0. & 0.0 \\
\hline As-76 & $.71+2$ & $.33+2$ & $3.1-1-1$ & $3.1+2$ & 3.68 & $3.52+2$ & & 3.82 & $1.52+3$ \\
\hline s-77 & 0.0 & 0.0 & 0.0 & 0.0 & 0. & 0.0 & 0. & 0. & 0.0 \\
\hline$e-2$ & $89+2$ & $62+2$ & $3.89+2$ & $3.57+2$ & -2 & 4.44 & & 4.98 & $5.87+2$ \\
\hline$e-7$ & 0.0 & & & & & & & & 0.0 \\
\hline $3 r-8$ & & 1 & & & & & & & $2+1$ \\
\hline $3 r-8 c$ & $7.0+$ & 1 & $5.83+1$ & $6.2+1$ & $7.74+1$ & $6.61+1$ & 6.99 & $7.36+1$ & $8.28+2$ \\
\hline $3-8$ & 0.0 & 0. & 0.0 & 0. & 0. & 0. & 0. & 0. & 0.0 \\
\hline$r-82$ & $2.24+3$ & $.51+3$ & $.87+3$ & $1.92+3$ & $2.08+3$ & $2.11+3$ & $2.04+3$ & $2.03+3$ & $2.88+3$ \\
\hline$r-83$ & $5+0$ & $68+0$ & $3.1+0$ & $5.44+0$ & $7.33+0$ & $5.86+0$ & $6.23+0$ & $6.47+0$ & $1.91+2$ \\
\hline r-84 & $4+3$ & $*$ & $*$ & * & $*$ & * & $*$ & $\bullet$ & $2.82+3$ \\
\hline $\mathrm{r}-84$ & $9+3$ & $1.67+3$ & $4+3$ & $3+3$ & $1.43+3$ & $1.5+3$ & $1.7+3$ & $1.86+3$ & $13+3$ \\
\hline$r-2$ & +3 & & $*$ & & & * & * & & $2.03+3$ \\
\hline$r-\varepsilon$ & & * & * & * & * & & * & & $6+3$ \\
\hline & & * & $*$ & $*$ & $*$ & * & * & $*$ & $3+3$ \\
\hline$r-79$ & $4+2$ & $45+2$ & $59+2$ & $66+2$ & $2.18+2$ & $8+2$ & $.78+$ & $1.96+2$ & $2.65+2$ \\
\hline$I-8$ & -1 & & & & & & & -2 & $3+1$ \\
\hline$r-85 m$ & $1+2$ & $9+2$ & $1.0+2$ & $9.51+1$ & & +2 & $9.51+1$ & $26+2$ & $38+2$ \\
\hline$r-8$ & +0 & +0 & +0 & +0 & +0 & & * : & 0 & $8+2$ \\
\hline$r-8$ & $8+2$ & $.07+2$ & $5.65+2$ & $5.31+2$ & $4+2$ & $9+2$ & $6.81+2$ & $1+2$ & $5+3$ \\
\hline$r-\varepsilon$ & $8+3$ & $78+3$ & $1.48+3$ & $1.31+3$ & $1.67+3$ & $1.61+3$ & $1.93+3$ & $1.55+3$ & $9+3$ \\
\hline & $8+3$ & $*$ & $*$ & * & * & • & * & * & $2.25+3$ \\
\hline$r-$ & & * & * & * & * & * & * & * & $1.73+3$ \\
\hline lb-84 & $2+2$ & $7.91+2$ & $5.88+2$ & $6.46+2$ & $6.67+2$ & $.59+2$ & $19+$ & 5.79 & $02+3$ \\
\hline lb- & $7.44+1$ & $8.17+1$ & $24+1$ & $6.96+1$ & $6.72+1$ & $6.96+1$ & $6.72+1$ & $5.52+1$ & $7.56+2$ \\
\hline$b-8 ?$ & 0. & & & & & 0.0 & 0.0 & 0.0 & 0.0 \\
\hline $\mathrm{Rb}-88$ & $5.65+2$ & $5.9+2$ & $5.01+2$ & $4.55+2$ & $5.52+2$ & $5.41+2$ & $6.5+2$ & $5.3+2$ & $2.82+3$ \\
\hline Rb-89 & $02+3$ & $16+3$ & $1.74+3$ & $1.63+3$ & $1.74+3$ & $1.92+3$ & $2.1+3$ & $1.87+3$ & $3.02+3$ \\
\hline Rb-90 & $4.01+3$ & $*$ & * & $*$ & $*$ & $m$ & * & $*$ & $5.77+3$ \\
\hline Sr-85m & 0.0 & 0.0 & 0.0 & 0.0 & 0.0 & 0.0 & 0.0 & 0.0 & 0.0 \\
\hline Sr-85 & $4.25+2$ & $5.18+2$ & $3.54+2$ & $3.77+2$ & $4.71+2$ & $4.01+2$ & $4.23+2$ & $4.46+2$ & $5.34+2$ \\
\hline Sr-87m & $2.74+2$ & $3.34+2$ & $2.28+2$ & $2.43+2$ & $3.04+2$ & $2.58+2$ & " & $2.88+2$ & $4.03+2$ \\
\hline Sir-09 & 0.0 & 0.0 & 0.0 & 0.0 & 0.0 & 0.0 & 0.0 & 0.0 & $5.65+2$ \\
\hline
\end{tabular}

\footnotetext{
*Data not available. Use of Total Body Factor is recommended.
} 
TABLE F-6 (Cont)

Total

Isotope Body

Sr-90

Sr-92

Y-87

Y-88

$\mathrm{Y}-90$

Y-9lm

Y-91

Y-92

$Y-93$

Zr-93

$\mathrm{Z}_{\mathrm{r}-95}$

Zr-97

Nb-93m

$\mathrm{Nb}-95 \mathrm{~m}$

$\mathrm{Nb}-95$

Nb-97m

$\mathrm{Nb}-97$

Mo-99

Tc-96m

Tc-96

Tc-97m

Tc-97

Tc- $99 \mathrm{~m}$

Tc-99

Ru-103m

Ru-103

Ru-105

Ru-106

Rh-103m

Rh-105

Rh-106

Pd-103

Pd 107

Pd-109

Ag-105

Ag-110m

Ag-111

Cd-109

Cd-113

Cd-115m

Cd-115

0.0

$6.11+2$

Bone

Liver Kidney Gonad

Lung

$$
0.0
$$

0.0

0.0

0.0

0.0

0.0

0.0

$3.87+2$

0.0

$5.11+2$

$5.38+2$

$5.9+2$

$5.75+2$

$5.62+2$

$3.10+3$

$\begin{array}{ll}4.71+2 & 3.22+2 \\ 3.4\end{array}$

0.0

$2.31+3$

$1.93+3$

$3.42+2 \quad 4.29+2 \quad 3.64+2$

0.0

$3.86+2$

0.0

0.0

$1.81+3$

$2.04+32.1+3$

$4.34+2$

$2.25+0$

$5.29+2$

$3.62+2$

0.0

0.0

$2.1+3$

$2.47+3$

0.0

0.0

0.0

$1.89+0$

$3.85+24.82+2$

$4.1+2$

$6.22+1$

0.0

0.0

0.0

$2.15+0$

$2.29+0$

0.0
$6.11+$

0.0

$$
0.0
$$

$7.41+1$
0.0

$5.25+1$

$4.98+1$

$5.35+1 \quad 5.82+1$

0.0

$5.84+2$

$6.73+2$

0.0

0.0

0.0

0.0

$5.32+2$

$1.52+2$

$1.71+2$

$4.89+2$
$1.28+2$

$5.34+2$

$5.82+2 \quad 5.48+2$

$1.39+2$

$4.76+1$

0.0

0.0

0.0

$\begin{array}{ll}1.38+2 & 1.44+2\end{array}$

0.0

$6.23+1 \quad 4.23+1$

$3.47+1$

$6.0+2 \quad 6.59+2$

$5.03+2$

$3.47+1$

$5.42+25.62+2$

$6.06+2$

$5.55+2$

$7.4+2$

$5.06+2$

$5.62+2$

$5.39+2$

$6.73+25.73+2$

$1.31+2$

$6.71+2$

$4.6+2$

$4.9+2$

$6.1+2$

$5.22+2$

$5.42+2$

$1.6+2$

$1.09+2$

$1.16+2$

$1.42+2 \quad 1.23+2$

0.0

0.0

0.0

0.0

0.0

0.0

0.0

0.0

0.0

0.0

0.0

0.0

0.0

0.0

0.0

0.0

0.0

0.0

0.0

$5.5+2$

$1.22+2$

0.0

0.0

0.0

0.0

0.0

$1.95+2$

$8.09+1$

$7.94+1$

$1.02+2$

$9.13+1$

$7.03+1$

0.0

0.0

0.0

0.0

0.0

0.0

0.0

$4.01+2 \quad 4.9+2$

$3.33+2$

0.0

0.0

0.0

0.0

0.0

0.0

$3.56+$
0.0

$4.45+2 \quad 3.78+2$

$$
0.0
$$

0.0

0.0

0.0

0.0

0.0

0.0

0.0

$3.36-1 \quad 3.33-1$

$6.52+19.86+1$

$3.58-2$

$1.01-1$

$4.75-1 \quad 5.55-2$

$*$

0.0

0.0

4.55-2

$4.74+1$

$1.54+2 \quad 1.88+2$

$5.0+1$

$4.74+1$

$8.53+15.79+1$

$1.58+0 \quad 2.82+0$

$1.29+2$

$1.37+2$

$1.69+2 \quad 1.46+2$

$1.53+2$

$1.09+0$

0.0

0.0

U.U

$1.07+0$

$1.45+0$

$1.2+0$

0.0
0.0

0.0

0.0

0.0

0.0

0.0

0.0

0.0

0.0

0.0

0.0

0.0

0.0

0.0

$2.09+3$

$1.85+1$

$\begin{array}{lll}2.56+1 & 3.87+1 & 1.96+1\end{array}$

$1.86+1$

$\begin{array}{ll}0.12+3 & 2.15+3\end{array}$

0.0

0.0

$2.27+1$

0.0

0.0

0.0

0.0

0.0

0.0

0.0

0.0

$2.6+1$

0.0

0.0

$2.34+1$

$2.59+1$

0.0

$2.09+2 \quad 2.56+2$

0.0

$\begin{array}{lll}1.86+2 & 2.33+2 & 1.97+2\end{array}$

*

$1.35+2$

Thyroid

$\underline{\text { Ekint }}$

In-1 $14 \mathrm{~m}$

$\begin{array}{lll}1.84+2 & 2.67+2 & 1.43+2\end{array}$

$1.39+2 \quad 2.34+2 \quad 1.64+2$

$0.0 \quad 1.92+2$

$5.56+2 \quad 1.39+3$

$0.0 \quad 0.0$

$4.06+2 \quad 49.3+2$

$1.92+3 \quad 2.74+3$

$0.0 \quad 9.26+2$

$1.67+2 . \quad 5.6+2$

* $6.0+2$

$0.0 \quad 0.0$

$5.65+1 \quad 1.26+3$

$0.0 \quad 0.0$

$5.15+2 \quad 8.71+2$

$1.43+2 \quad 8.92+2$

$\begin{array}{ll}0.0 & 0.0\end{array}$

$4.57+1 \quad 2.2+2$

$4.45+2 \quad 8.29+2$

$6.38+2 \quad 7.53+2$ 
TABLE F-6 (Cont)

\begin{tabular}{|c|c|c|c|c|c|c|c|c|c|}
\hline Isotope & $\begin{array}{l}\text { Total } \\
\text { Body }\end{array}$ & Bone & Liver & Kidney & Gonad & Lung & G.I. Tract & Thyroid & Skin \\
\hline $\begin{array}{l}\text { In-115m } \\
\text { In-115 }\end{array}$ & $\begin{array}{c}2.57+2 \\
0.0\end{array}$ & $\begin{array}{c}3.89+2 \\
0.0\end{array}$ & $\begin{array}{c}1.96+2 \\
0.0\end{array}$ & $\begin{array}{c}1.86+2 \\
0.0\end{array}$ & $\begin{array}{c}3.37+2 \\
0.0\end{array}$ & $\begin{array}{c}2.27+2 \\
0.0\end{array}$ & $\begin{array}{c}1.86+2 \\
0.0\end{array}$ & $\begin{array}{c}2.45+2 \\
0.0\end{array}$ & $\begin{array}{c}3.26+2 \\
0.0\end{array}$ \\
\hline $\mathrm{Sn}-113$ & $9.03+0$ & $1.1+1$ & $3.6+0$ & $4.39+0$ & $1.23+1$ & $4.36+0$ & $3.29+0$ & $5.7+0$ & $3.65+1$ \\
\hline Sn-119m & $33+0$ & $4.43+0$ & $9.33-1$ & $1.56+0$ & $4.6+0$ & $1.28+0$ & & $2.71+0$ & $9.01+1$ \\
\hline $\mathrm{Sn}-12$ & 0.0 & 0 & 0 & 0.0 & 0.0 & 0.0 & & & 0.0 \\
\hline Sn-1 & 0 & & 0 & & $0 . c$ & 0.0 & & & $34+2$ \\
\hline & 0.0 & 0 & & & 0.0 & 0.0 & & & 0.0 \\
\hline Sn-1 & 0.0 & 0.0 & 0.0 & 0.0 & 0.0 & 0.0 & 0.0 & 0.0 & 0.0 \\
\hline b-122 & $.42+2$ & $.17+2$ & $85+2$ & $3.05+2$ & $3.78+2$ & $3.23+2$ & $3.4+2$ & $3.57+2$ & $9.95+2$ \\
\hline & $1+3$ & & $.36+3$ & $7+3$ & $1.41+3$ & $1.53+3$ & & & \\
\hline & $2+2$ & $49+2$ & $.96+2$ & $3.16+2$ & $4.06+2$ & $3.37+2$ & & $8+2$ & $.86+2$ \\
\hline & 0.0 & & 0.0 & 0.0 & 0.0 & 0.0 & & 0.0 & 0.0 \\
\hline & $.46+2$ & $61+2$ & $1.54+2$ & 4.8 & $5.88+2$ & $5.14+2$ & & $.41+2$ & $9.99+2$ \\
\hline & 0.0 & & 0.0 & & 0.0 & 0.0 & & & 0.0 \\
\hline & 0.0 & & & & & 0.0 & & & 0.0 \\
\hline & $1.18+2$ & $1.78+2$ & $j+1$ & & $1.54+2$ & $1.02+2$ & & & $.49+2$ \\
\hline & $3+1$ & +1 & +0 & & $2.15+1$ & +0 & & & $1+2$ \\
\hline & +0 & +0 & & & & & & & +2 \\
\hline Te- & & & $1+0$ & & $3.78+0$ & $3.16+0$ & +0 & +0 & $.43+2$ \\
\hline & $32+1$ & $4+1$ & $2.19+1$ & $2.4+$ & $3.18+1$ & $2.51+1$ & $9+1$ & $3+1$ & $8+2$ \\
\hline & $9+1$ & $48+1$ & $.29+1$ & $4.61+1$ & $5.79+1$ & $4.87+1$ & & $5+1$ & $3.86+2$ \\
\hline & $3+3$ & $29+3$ & $45+2$ & $9+2$ & $1.02+3$ & $6+3$ & & +2 & $1.68+3$ \\
\hline & $1+2$ & +2 & +2 & $2+2$ & $6+2$ & $3.12+2$ & +2 & $6+2$ & $3+3$ \\
\hline & $1+2$ & +2 & +2 & +2 & +2 & +2 & & $6+2$ & $2.77+2$ \\
\hline & & & & & & & & & \\
\hline & $1+2$ & +2 & $1+1$ & & +2 & $3+2$ & & & +2 \\
\hline & & & & & & & & & +3 \\
\hline & +1 & +1 & & & & & & +1 & $1+1$ \\
\hline & $5+2$ & $34+2$ & $.93+2$ & $3.13+2$ & $3.93+2$ & $3.33+2$ & $3.5+2$ & $3.72+2$ & $1+2$ \\
\hline & $1+1$ & $*$ & * & * & ${ }^{*}$ & * & * & * & $5.59+2$ \\
\hline & $9+1$ & $66+1$ & $11+0$ & $14+0$ & $5+1$ & $.54+0$ & & $5+1$ & $7+1$ \\
\hline & $9+3$ & $07+3$ & & +3 & $1.84+3$ & $1.59+3$ & $0+3$ & $3+3$ & $2.37+3$ \\
\hline & $4+2$ & $89+2$ & $2.6+2$ & $6+2$ & $3.52+2$ & $2.96+2$ & $8+2$ & +2 & $6+2$ \\
\hline & $5+3$ & $12+3$ & $1.54+3$ & $1.63+3$ & $1.8+3$ & $1.74+3$ & 1.7 & $1.7+3$ & $5+3$ \\
\hline & $8+2$ & $6+2$ & $.66+2$ & $4.86+2$ & $5.8+2$ & $5.27+2$ & $1+2$ & $2+2$ & $1.12+3$ \\
\hline & +3 & $12+3$ & $1+3$ & $1.74+3$ & $1.75+3$ & $1.8+3$ & $4+3$ & $.55+3$ & $19+3$ \\
\hline & +3 & $62+$ & $28+3$ & $1.19+3$ & $24+3$ & $1.44+$ & $1.48+3$ & $.39+3$ & +3 \\
\hline & & & & & & & & * & \\
\hline I-137 & & * & * & * & * & * & * & * & $6+3$ \\
\hline & $93+0$ & $51+1$ & $4.76+0$ & & & & & & $4+2$ \\
\hline & & & & & & & & & $35+2$ \\
\hline & $26+1$ & $65+1$ & $2.18+1$ & $2.26+1$ & $3.34+1$ & & & $3.87+1$ & $1.96+2$ \\
\hline & & & & & & & & $3.73+2$ & \\
\hline Xe-135 & $2.04+2$ & $3.05+2$ & $1.58+2$ & $1.51+2$ & $2.65+2$ & $1.81+2$ & $1.5+2$ & $1.97+2$ & $5.83+2$ \\
\hline
\end{tabular}

"Data not available. Use of Total Body Factor is recommended. 
TABLE F-6 (Cont)

Total

Isotope Bndy Bnne T.iver Kidney Gonad Lung G.I.Tract Thyruid Dhist

Xe-137

Xe-138

Xe-139

$\mathrm{Xe}-140$

Cs-134

Cs-135

Cs-136

Cs-137

Cs-138

Cs-139

Ba-131

Ba-133

$\mathrm{Ba}-137 \mathrm{~m}$

Ba-139

Ba-140

La-140

La-141

La-142

$\mathrm{Ce}-141$

Ce-142

$\mathrm{Ce}-143$

$\mathrm{Ce}-144$

Pr 142

Pr-143

Pr-144

Pr-145

Nd-144

Nd-147

Nd-149

Pm-147

Pm-148m

Pm-148

Pm-149

Pm-151

Sm-147

$\mathrm{Sm}-148$

Sm-149

Sm-151

Sm-153

Eu-152

Eu-154

Eu-155

Eu-156
$151+2$

$6.81+2$

$6.86+2$

$4.58+3$

$1.26+3$

0.0

$1.71+3$

0.0

$+3 \quad 2.0+3$

$1.89+2$

0.0

$2.89+2 \quad 3.89+$

$4.66+25.68+2$

$4.25+1$

$1.6+2$

$2.04+3$

0.0

$6.55+1$

0.0

$1.16+2$
0.0

$2.02+2$

$2.21+3$

0.0

0.0

$1.16+2$

$1.54+1$

0.0

$3.34+2$

$2.72+$
0.0

0.0

$2.56+1$

$2.81+1$

0.0

0.0

0.0

0.0

$1.13+2 \quad 1.56+2$

0.0

0.0

0.0

().)

$\begin{array}{cc}1.63+3 & 1.97+3 \\ 5.51+2 & 6.05+2\end{array}$

0.0

0.0

0.0

0.0

0.0

0.0

0.0

$3.5-3$

0.0

0.0

0.0

0.0

0.0

$2.79-3$

0.0

0.0

$1.09+3$

$1.23+3$

$5.42+1$

0.0
$9.79+1$

0.0

\section{$1.27+2$}

$5.78+2 \quad 5.17+2$

$1.53+2 \quad 1.43+2$

$6.44+2$

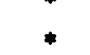

*

$73+$

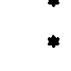

$1.47+2$

$6.88+2$

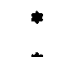

$1.14+3 \quad 1.26+3 \quad 1.19+3$

(III)

$1111 \quad 0.0$

0.0

$1.42+3$

$\begin{array}{lll}1.51+3 & 1.6+3 & 1.6+3\end{array}$

0.0

0.0

0.0

0.0

$1.61+3$

$1.44+3 \quad 1.67+3$

1.79

0.0

0.0 .

0.0

0.0

$3.3+2 \quad 2.6+2$

$3.88+2$

$4.13+2$

$5.18+2 \quad 4.39+2$

*

*

$1.48+2$

$1.31+2$

$1.38+$

$\begin{array}{rr}1.81+2 & 1.48+2 \\ 1.69+3 & 1.94+3\end{array}$

0.0

0.0

0.0

0.0

0.0

0.0

0.0

0.0

$\begin{array}{llll}4.75+1.4 .71+1 & 6.22+1 & 5.39+1\end{array}$

$$
0.0
$$

0.0

0.0

0.0

$\begin{array}{llll}1.91+2 & 1.96+2 & 2.84+2 & 2.19+2\end{array}$

$1.09+1$

$1.09+1$

$1.51+1$

$1.23+1$

0.0

0.0

0.0

0.0

0.0

0.0

0.0

0.0

$2.21+1$

$2.05+1$

$2.07+1$

$2.44+1$

0.0

0.0

0.0

0.0

$8.77+1$

0.0

0.0

0.0

0.0

$1.23+2$

$1.0+2$

0.0

$\begin{array}{ll}0.0 & 0.0\end{array}$

0.0

$\begin{array}{llll}1.36+3 & 1.46+3 & 1.77+3 & 1,54+3\end{array}$

$4.63+2$

$4.35+2$

$\begin{array}{ll}4.74+2 & 5.24+2\end{array}$

0.0

0.0

0.0

0.0

0.0

00

0.0

0.0

0.0

0.0

0.0

0.0

0.0

0.0

0.0

0.0
$4.43-3$

0.0

$8.01-4$

$2.68-4$
0.0
0.0

0.0

$0: 0$

$4.84-$

$\begin{array}{ll}0.0 & 0.0\end{array}$

0.0

0.0

0.0

$9.06+2$

$8.89+2$

\section{0}

$1.02+3$

0.0

$3.85+1$

0.0

$5.0+1$

$4.43+1$

0.0
$1.15+3$

0.0

$1.53+3$

0.0

$1.46+3$

0.0

$2.51+2$

$4.65+2$

$1.52+2$

$2.01+3$

0.0

0.0

$4.14+1$

0.0

$1.88+2$

$9.55+0$

0.0

0.0

$2.65+1$

0.0

0.0

$9.69+1$

0.0

0.0

$1.58+3$

$5.22+2$

0.0

0.0

0.0

0.0

0.0

$5.06-4$

0.0

0.0

$1.0+3$

$3.47+1$

0.0

$1.54+2 \quad 2.04+3$

$6.65+2 \quad 1.53+3$

* $1.77+3$

* $\quad 5.47+3$

$1.13+3 \quad 1.76+3$

$0.0 \quad 6.73+1$

$\begin{array}{ll}1.37+3 & 2.31+3\end{array}$ 
TABLE F-6 (Cont)

\begin{tabular}{|c|c|c|c|c|c|c|c|c|c|}
\hline Isotope & $\begin{array}{l}\text { Total } \\
\text { Body }\end{array}$ & Bone & Liver & Kidney & Gonad & Lung & G.I. Tract & Thyroid & Skin \\
\hline Eu-157 & 0.0 & 0.0 & 0.0 & 0.0 & 0.0 & 0.0 & 0.0 & 0.0 & 0.0 \\
\hline Gd-153 & 0.0 & 0.0 & 0.0 & 0.0 & 0.0 & 0.0 & 0.0 & 0.0 & 0.0 \\
\hline Gd-159 & 0.0 & 0.0 & 0.0 & 0.0 & 0.0 & 0.0 & 0.0 & 0.0 & 0.0 \\
\hline Gd-162 & $3.5+2$ & $428+2$ & $2.92+2$ & $3.12+2$ & $3.89+2$ & $3.31+2$ & $3.49+2$ & $3.7+2$ & $7.86+2$ \\
\hline Tb-160 & $8.8+2$ & $1.01+3$ & $7.29+2$ & $7.73+2$ & $8.12+2$ & $8.2+2$ & $7.89+2$ & $7.11+2$ & $1.39+3$ \\
\hline Tb-16l & 0.0 & 0.0 & 0.0 & 0.0 & 0.0 & 0.0 & 0.0 & 0.0 & 0.0 \\
\hline Dy-165 & 0.0 & 0.0 & 0.0 & 0.0 & 0.0 & 0.0 & 0.0 & 0.0 & 0.0 \\
\hline Dy-166 & 0.0 & 0.0 & 0.0 & 0.0 & 0.0 & 0.0 & 0.0 & 0.0 & 0.0 \\
\hline Ho-166 & 0.0 & 0.0 & 0.0 & 0.0 & 0.0 & 0.0 & 0.0 & 0.0 & 0.0 \\
\hline$E_{r-169}$ & 0.0 & 0.0 & 0.0 & 0.0 & 0.0 & 0.0 & 0.0 & 0.0 & 0.0 \\
\hline$E_{r-171}$ & 0.0 & 0.0 & 0.0 & 0.0 & 0.0 & 0.0 & 0.0 & 0.0 & 0.0 \\
\hline Tm-170 & 0.0 & 0.0 & 0.0 & 0.0 & 0.0 & 0.0 & 0.0 & 0.0 & 0.0 \\
\hline Yb-175 & 0.0 & 0.0 & 0.0 & 0.0 & 0.0 & 0.0 & 0.0 & 0.0 & 0.0 \\
\hline Lu-177 & 0.0 & 0.0 & 0.0 & 0.0 & 0.0 & 0.0 & 0.0 & 0.0 & 0.0 \\
\hline Hf-181 & 0.0 & 0.0 & 0.0 & 0.0 & 0.0 & 0.0 & 0.0 & 0.0 & 0.0 \\
\hline Ta-182 & $1.1+3$ & $1.24+3$ & $9.13+2$ & $8.92+2$ & $9.38+2$ & $1.03+3$ & $1.03+3$ & $9.78+2$ & $1.54+3$ \\
\hline W-181 & 0.0 & 0.0 & 0.0 & 0.0 & 0.0 & 0.0 & 0.0 & 0.0 & 0.0 \\
\hline W-185 & 0.0 & 0.0 & 0.0 & 0.0 & 0.0 & 0.0 & 0.0 & 0.0 & 0.0 \\
\hline W-187 & $1.42+2$ & $1.83+2$ & $1.15+2$ & $1.22+2$ & $1.54+2$ & $1.31+2$ & $1.35+2$ & $1.5+2$ & $4.39+2$ \\
\hline W-188 & 0.0 & 0.0 & 0.0 & 0.0 & 0.0 & 0.0 & 0.0 & 0.0 & 0.0 \\
\hline $\operatorname{Re}-183$ & 0.0 & 0.0 & 0.0 & 0.0 & 0.0 & 0.0 & 0.0 & 0.0 & 0.0 \\
\hline Re-186 & 0.0 & 0.0 & 0.0 & 0.0 & 0.0 & 0.0 & 0.0 & 0.0 & 0.0 \\
\hline Re- 187 & 0.0 & 0.0 & 0.0 & 0.0 & 0.0 & 0.0 & 0.0 & 0.0 & 0.0 \\
\hline $\operatorname{Re}-188$ & 0.0 & 0.0 & 0.0 & 0.0 & 0.0 & 0.0 & 0.0 & 0.0 & 0.0 \\
\hline Os-185 & 0.0 & 0.0 & 0.0 & 0.0 & 0.0 & 0.0 & 0.0 & 0.0 & 0.0 \\
\hline Os-19lm & 0.0 & 0.0 & 0.0 & 0.0 & 0.0 & 0.0 & 0.0 & 0.0 & 0.0 \\
\hline Os-191 & 0.0 & 0.0 & 0.0 & 0.0 & 0.0 & 0.0 & 0.0 & 0.0 & 0.0 \\
\hline Os-193 & 0.0 & 0.0 & 0.0 & 0.0 & 0.0 & 0.0 & 0.0 & 0.0 & 0.0 \\
\hline $\mathrm{I}_{\mathrm{r}-1}-190$ & 0.0 & 0.0 & 0.0 & 0.0 & 0.0 & 0.0 & 0.0 & 0.0 & 0.0 \\
\hline$I_{Y}=192$ & 0.0 & 0.0 & 0.0 & 0.0 & 0.0 & 0.0 & 0.0 & 0.0 & 0.0 \\
\hline Pt-19l & 0.0 & 0.0 & 0.0 & 0.0 & 0.0 & 0.0 & 0.0 & 0.0 & 0.0 \\
\hline Pt-193m & 0.0 & 0.0 & 0.0 & 0.0 & 0.0 & 0.0 & 0.0 & 0.0 & 0.0 \\
\hline Pt-193 & 0.0 & 0.0 & 0.0 & 0.0 & 0.0 & 0.0 & 0.0 & 0.0 & 0.0 \\
\hline$P t-197 m$ & 0.0 & 0.0 & 0.0 & 0.0 & 0.0 & 0.0 & 0.0 & 0.0 & 0.0 \\
\hline Pt-197 & 0.0 & 0.0 & 0.0 & 0.0 & 0.0 & 0.0 & 0.0 & 0.0 & 0.0 \\
\hline Au-195m & $1.63+2$ & $2.52+2$ & $1.23+2$ & $1.18+2$ & $2.08+2$ & $1.43+2$ & $1.15+2$ & $1.56+2$ & $3.12+2$ \\
\hline Au-195 & $6.69+1$ & $1.27+2$ & $4.13+1$ & $4.3+1$ & $5.89+1$ & $4.97+1$ & $4.21+1$ & $6.83+1$ & $1.55+2$ \\
\hline Аu-196 & 0.0 & 0.0 & 0.0 & 0.0 & 0.0 & 0.0 & 0.0 & 0.0 & 0.0 \\
\hline Au-198 & $3.36+2$ & $4.1+2$ & $2.8+2$ & $2.98+2$ & $3.72+2$ & $3.16+2$ & $3.34+2$ & $3.53+2$ & $7.55+2$ \\
\hline Au-199 & $7.91+1$ & $1.23+2$ & $5.94+1$ & $5.68+1$ & $9.95+1$. & $6.89+1$ & $5.56+1$ & $7.66+1$ & $2.57+2$ \\
\hline $\mathrm{Hg}-19^{\prime} \mathrm{m}$ & 0.0 & 0.0 & 0.0 & 0.0 & 0.0 & 0.0 & 0.0 & 0.0 & 0.0 \\
\hline $\mathrm{Hg}-197$ & $5.0+1$ & $9.57+1$ & $2.99+1$ & $3.14+1$ & $4.41+1$ & $3.63+1$ & $3.14+1$ & $4.89+1$ & $1.36+2$ \\
\hline $\mathrm{Hg}-203$ & $1.97+2$ & $3.02+2$ & $1.51+2$ & $1.43+2$ & $2.56+2$ & $1.75+2$ & $1.42+2$ & $1.89+2$ & $3.47+2$ \\
\hline Pl-200 & 0.0 & 0.0 & 0.0 & 0.0 & 0.0 & 0.0 & 0.0 & 0.0 & 0.0 \\
\hline
\end{tabular}

* Data not available. Use of Total Body Factor is recommended. 
TABLE F-6 (Cont)

\begin{tabular}{|c|c|c|c|c|c|c|c|c|c|}
\hline Isotope & $\begin{array}{l}\text { Total } \\
\text { Body }\end{array}$ & Bone & Liver & Kidney & Gonad & Lung & G.I. Tract & Thyroid & Skin \\
\hline Tl-20l & 0.0 & 0.0 & 0.0 & 0.0 & 0.0 & 0.0 & 0.0 & 0.0 & 0.0 \\
\hline Tl-202 & 0.0 & 0.0 & 0.0 & 0.0 & 0.0 & 0.0 & 0.0 & 0.0 & 0.0 \\
\hline Tl-204 & $8.0-1$ & $1.55+0$ & $4.79-1$ & $5.06-1$ & $7.03-1$ & $5.83-1$ & $5.06-1$ & $7.92-1$ & $2.52+2$ \\
\hline Tl-207 & $1.77+0$ & $*$ & $*$ & * & $*$ & * & * & $*$ & $3.52+?$ \\
\hline Tl-208 & $2.0+3$ & $3.01+3$ & $2.48+3$ & $2.25+3$ & $2.58+3$ & $2.68+3$ & $3.15+3$ & $2.66+3$ & $4.03+3$ \\
\hline Tl-209 & $1.77+3$ & $*$ & $*$ & * & $*$ & • & $*$ & * & $328+3$ \\
\hline $\mathrm{Pb}-303$ & $\tilde{0} . \dot{U}$ & 0.0 & 0.00 & U.U & 0.0 & 0.0 & 0.0 & 0.0 & 0.0 \\
\hline $\mathrm{Pb}-204 \mathrm{~m}$ & 0.0 & 0.0 & 0.0 & 0.0 & 0.0 & 0.0 & 0.0 & 0.0 & 0.0 \\
\hline $\mathrm{Pb}-209$ & 0.0 & 0.0 & 0.0 & 0.0 & 0.0 & 0.0 & 0.0 & 0.0 & $1.22+2$ \\
\hline $\mathrm{Pb}-210$ & $1.63+0$ & $2.8+0$ & $8.3-1$ & $8.76-1$ & $1.38+0$ & $1.03+0$ & $8.97+1$ & $1.37+0$ & $4.5+1$ \\
\hline $\mathrm{Pb}-211$ & $.48+1$ & $*$ & $*$ & $*$ & *• & * & • & $*$ & $3.24+2$ \\
\hline $\mathrm{Pb}-212$ & $.2+2$ & $1.89+2$ & $8.94+1$ & $8.57+1$ & $1.48+2$ & $1.04+2$ & $8.31+1$ & $1.16+2$ & $3.33+2$ \\
\hline $\mathrm{Pb}-214$ & $18+2$ & $*$ & • & * & * & * & * & * & $3.5+2$ \\
\hline $\mathrm{Bi}-206$ & 0.0 & 0.0 & 0.0 & 0.0 & 0.0 & 0.0 & 0.0 & 0.0 & 0.0 \\
\hline $\mathrm{Bi}-207$ & 0.0 & 0.0 & 0.0 & 0.0 & 0.0 & 0.0 & 0. & 0.0 & 0.0 \\
\hline $\mathrm{Bi}-210$ & 0.0 & 0.0 & 0.0 & 0.0 & 0.0 & 0.0 & 0.0 & 0.0 & $4.06+2$ \\
\hline $\mathrm{Bi}-21 \mathrm{l}$ & $4.63+1$ & $\star$ & $*$ & * & * & $*$ & * & * & $4.66+1$ \\
\hline $\mathrm{Bi}-212$ & $9.26+1$ & $1.06+2$ & $7.72+1$ & $7.69+1$ & $8.45+1$ & $8.73+1$ & $8.73+1$ & $9.07+1$ & $6.29+2$ \\
\hline $\mathrm{Bi}-213$ & $1.43+2$ & $*$ & * & $*$ & * & * & * & * & $4.56+2$ \\
\hline $\mathrm{Bi}-214$ & $17+3$ & * & * & * & * & * & * & * & $1.54+3$ \\
\hline Po-210 & $97-2$ & $*$ & $*$ & $*$ & $*$ & $*$ & $*$ & $*$ & $*$ \\
\hline Po-212 & 0.0 & 0.0 & 0.0 & 0.0 & 0.0 & 0.0 & 0.0 & 0.0 & 0.0 \\
\hline $\mathrm{Po}=213$ & 0.0 & 0.0 & 00 & 0.0 & 0.0 & 0.0 & 0.0 & 0.0 & 0.0 \\
\hline $0-215$ & $.03-1$ & * & $*$ & $*$ & * & * & * & $*$ & * \\
\hline Po-216 & $39-2$ & * & * & * & * & * & * & * & * \\
\hline Po-218 & $.59-3$ & $*$ & $*$ & $*$ & $*$ & * & $*$ & * & $1.44-2$ \\
\hline At-211 & 0.0 & 0.0 & 0.0 & 0.0 & 0.0 & 0.0 & 0.0 & 0.0 & 0.0 \\
\hline & $2.61-1$ & $\bullet$ & $*$ & $*$ & $*$ & 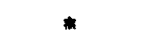 & $\cdot$ & + & $\$$ \\
\hline Rn-219 & $1+1$ & $*$ & $*$ & * & * & * & * & * & * \\
\hline $\mathrm{R}_{\mathrm{u}}=220$ & $2.97-1$ & $3.63-1$ & $2.48-1$ & $2.64-1$ & $3.3-1$ & $2.81-1$ & * & $3.13-1$ & $3.63-1$ \\
\hline Rn-222 & $2.94-1$ & * & * & * & * & * & * & + & • \\
\hline Ra-224 & $8.37+0$ & $1.28+1$ & $6.42+0$ & $6.08+0$ & $1.08+1$ & $7.42+0$ & $6.05+0$ & $8.1+0$ & $1.29+1$ \\
\hline Ra-225 & $2.08+1$ & * & * & $*$ & $*$ & * & * & * & $6.51+1$ \\
\hline Ra-226 & $5.59+0$ & $8.58+0$ & $4.28+0$ & $4.06+0$ & $7.16+0$ & $4.94+0$ & $4.02+0$ & $5.47+0$ & $2 n 4+1$ \\
\hline $\mathrm{Ra}-228$ & $142+1$ & $2.17+1$ & $5.35+0$ & $8.01+0$ & $1.95+1$ & $7.21+U$ & $5.6+0$ & $1.5+1$ & $8.18+1$ \\
\hline$F_{T-221}$ & $3.29+1$ & * & * & * & $*$ & * & • & * & * \\
\hline Ac-225 & $2.7+1$ & $*$ & * & * & * & * & * & * & $*$ \\
\hline Ac-227 & 0.0 & 0.0 & 0.0 & 0.0 & 0.0 & 0.0 & 0.0 & 0.0 & 0.0 \\
\hline Ac-228 & $6.13+2$ & $7.09+2$ & $5.08+2$ & $5.21+2$ & $5.59+2$ & $5.72+2$ & $5.49+2$ & $5.31+2$ & $8.8+2$ \\
\hline & $1.09+2$ & • & * & $*$ & * & * & * & $*$ & * \\
\hline Th-228 & $2.1+0$ & $3.39+0$ & $1.47+0$ & $1.43+0$ & $2.17+0$ & $1.69+0$ & $1.3+0$ & $2.21+0$ & $2.52+1$ \\
\hline & $1.72+2$ & $*$ & $*$ & $*$ & $\star$ & * & * & $*$ & * \\
\hline Th-230 & $4.1-1$ & $6.06-1$ & $1.92-1$ & $1.95-1$ & $3.62-1$ & $2.4-1$ & $1.93-1$ & $2.98-1$ & $1.67+1$ \\
\hline
\end{tabular}

- Data not available. Use of Total Body Factor is recommended. 
TABLE F-6 (Cont)

\begin{tabular}{|c|c|c|c|c|c|c|c|c|c|}
\hline Isotope & $\begin{array}{l}\text { Total } \\
\text { Body }\end{array}$ & Bone & Liver & Kidney & Gonad & Lung & G.I. Tract & Thyroid & Skin \\
\hline Th-231 & $1.4+1$ & $2.28+1$ & $8.65+0$ & $8.87+0$ & $1.38+1$ & $1.01+1$ & $7.73+0$ & $1.48+1$ & $2.02+2$ \\
\hline Th-232 & $2.01-1$ & $2.36-1$ & $5.33-2$ & & $1.54-1$ & $7.67-2$ & & $8.64-2$ & $1.38+1$ \\
\hline Th-234 & $5.98+0$ & $1.07+1$ & $4.3+0$ & $4.5+0$ & $5.54+0$ & $4.9+0$ & $3.8+0$ & $7.1+0$ & $2.1+1$ \\
\hline $\mathrm{Pa}-230$ & 0.0 & 0.0 & 0.0 & 0.0 & 0.0 & 0.0 & 0.0 & 0.0 & 0.0 \\
\hline $\mathrm{Pa}-231$ & $7.36+1$ & * & $*$ & $*$ & * & * & $*$ & $*$ & 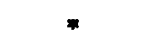 \\
\hline $\mathrm{Pa}-233$ & $1.64+2$ & $2.55+2$ & $1.26+2$ & $1.21+2$ & $2.01+2$ & $1.45+2$ & $1.16+2$ & $1.67+2$ & $4.27+2$ \\
\hline$a-234 m$ & $2.25-1$ & $04-1$ & $1.8-1$ & & $2.69-1$ & $2.07-1$ & & $2.27-1$ & $8.62+2$ \\
\hline & $1.27+1$ & $*$ & $\cdot$ & $*$ & * & * & $*$ & * & $1.87+1$ \\
\hline 0 & 0.0 & 0.0 & 0.0 & 0.0 & 0.0 & 0.0 & & 0.0 & 0.0 \\
\hline & $3.78-1$ & $82-1$ & $1.39-1$ & -1 & $3.2-1$ & 1.79 & & & $1.91+1$ \\
\hline 33 & $36-1$ & $09+0$ & $4.75-1$ & & $8.36-1$ & -1 & & & $8.74+0$ \\
\hline-234 & $2.84-1$ & $36-1$ & $8.72-2$ & $9.28-2$ & $2.39-1$ & $1.18-1$ & -2 & $6-1$ & $1.55+1$ \\
\hline-235 & $1.24+2$ & $93+2$ & $9.34+1$ & $8.94+1$ & $1.53+2$ & $1.08+2$ & $8.68+1$ & $1.24+2$ & $1.96+2$ \\
\hline 6 & $78-1$ & $53-1$ & $2.1-2$ & $2.6-2$ & $1.43-1$ & $3.97-2$ & $4.92-2$ & $3.72-2$ & $1.31+1$ \\
\hline 7 & $5+2$ & $1.92+2$ & $8.25+1$ & +1 & $1.24+2$ & $9.53+1$ & 7.3 & $1.21+2$ & $3.55+2$ \\
\hline $\mathrm{U}-238$ & $1.79-1$ & $1.75-1$ & $3.6-2$ & $3.98-2$ & $1.47-1$ & $5.44-2$ & & $6.15-2$ & $1.14+1$ \\
\hline $\mathrm{U}-23$ & 0.0 & 0.0 & 0.0 & 0.0 & 0.0 & 0.0 & & & 0.0 \\
\hline $\mathrm{Np}-2$ & $7.01+1$ & $1.19+2$ & $5.19+1$ & $5.06+1$ & $7.43+1$ & $5.94+1$ & $4.6+1$ & $7.9+1$ & $1.06+2$ \\
\hline$p-23$ & 0.0 & 0.0 & 0.0 & 0.0 & 0.0 & 0.0 & & & 0.0 \\
\hline Np-239 & $1.11+2$ & $1.78+2$ & $8.3+1$ & $7.98+1$ & $1.29+2$ & $9.54+1$ & $7.57+1$ & $1.16+2$ & $4.17+2$ \\
\hline p-240m & $2.56+2$ & $*$ & $*$ & $*$ & $*$ & * & $*$ & * & $7.06+2$ \\
\hline$p-240$ & $1.01+3$ & * & * & * & * & $*$ & $*$ & * & $1.2+3$ \\
\hline $1-238$ & $1.89-1$ & $1.44-1$ & $1.52-2$ & $2.02-2$ & $1.51-1$ & $3.45-2$ & $5.08-2$ & $2.81-2$ & $1.24+1$ \\
\hline$-23+2+3$ & $8.24-2$ & $7.32-2$ & $1.07-2$ & & 6.97 & & & & $8.38+0$ \\
\hline 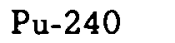 & $.67-1$ & $1.32-1$ & $1.6-2$ & $3-2$ & $1.34-1$ & $3.3-$ & & -2 & $1.11+1$ \\
\hline & 0.0 & & & & & & & & $5.87+0$ \\
\hline & $.59-1$ & $1.19-1$ & $1.07-2$ & $1.51-2$ & $1.26-1$ & 2.69 & & -2 & $1.08+1$ \\
\hline & & & & & & & & & 0.0 \\
\hline $1-$ & 0.0 & 0 & 0.0 & & 0.0 & & & & 0.0 \\
\hline$m-241$ & $.64+1$ & $3.1+1$ & $9.47+0$ & $1.0+1$ & $1.44+1$ & $1.15+1$ & $1.0+$ & $1.58+1$ & $5.95+1$ \\
\hline$A \mathrm{~m}-2$ & 0.0 & & & & & & & & 0.0 \\
\hline Åm-242 & $.13+1$ & $1.96+1$ & $7.94+0$ & $7.86+0$ & $1.07+1$ & & & & $4.11+1$ \\
\hline$\AA \mathrm{m}-243$ & $3.61+1$ & $6.94+1$ & $2.13+1$ & $2.26+1$ & $3.14+1$ & $2.6+1$ & $2.26+1$ & $3.53+1$ & $6.63+1$ \\
\hline$m-244$ & 0.0 & 0.0 & 0.0 & 0.0 & 0.0 & 0.0 & 0.0 & 0.0 & 0.0 \\
\hline$C m-242$ & $1.8-1$ & $1.32-1$ & $1.15-2$ & $1.64-2$ & $1.44-1$ & $2.99-2$ & $4.74-2$ & $2.19-2$ & $1.16+1$ \\
\hline $\mathrm{C}_{\mathrm{m}-243}$ & $.06+2$ & $1.71+2$ & $7.93+1$ & $7.63+1$ & $1.22+2$ & $9.11+1$ & $7.21+1$ & $1.12+2$ & $2.68+2$ \\
\hline $\mathrm{Cm}-244$ & $5.14+0$ & $9.57+0$ & $3.26+0$ & $3.36+0$ & $4.58+0$ & $3.87+0$ & $3.21+0$ & $5.4+0$ & $1.21+1$ \\
\hline & 0.0 & 0 & 00 & & 0.0 & & & & 0.0 \\
\hline$m-246$ & 0.0 & & 0.0 & & 0.0 & & & & 0.0 \\
\hline & 0.0 & 0.0 & 0.0 & & & & & & 0.0 \\
\hline & 0.0 & 0.0 & 0.0 & 0 & & & & & 0.0 \\
\hline & 0. & 0.0 & 0.0 & 0 & & 0. & 0.0 & & 0.0 \\
\hline & & & & & & 0.0 & 0.0 & & 0.0 \\
\hline Bk-250 & 0.0 & 0.0 & 0.0 & 0.0 & 0.0 & 0.0 & 0.0 & 0.0 & 0.0 \\
\hline
\end{tabular}

* Data not available. Use of Total Body Factor is recommended. 
TABLE F-6 (Cont)

Total

\begin{tabular}{|c|c|c|c|c|c|c|c|c|c|}
\hline Isntnpe & Rndy & Bone & Livor & Kidney & Gonad & Luxisy & G.I. Traul & Thyroid & skin \\
\hline Cf-249 & 0.0 & 0.0 & 0.0 & 0.0 & 0.0 & 0.0 & 0.0 & 0.0 & 0.0 \\
\hline $\mathrm{Cf}-250$ & 0.0 & 0.0 & 0.0 & 0.0 & 0.0 & 0.0 & 0.0 & 0.0 & 0.0 \\
\hline Cf-25l & 0.0 & 0.0 & 0.0 & 0.0 & 0.0 & 0.0 & 0.0 & 0.0 & 0.0 \\
\hline Cf-252 & 0.0 & 0.0 & 0.0 & 00 & 0.0 & 0.0 & 0.0 & 0.0 & 0.0 \\
\hline Cf-253 & 0.0 & 0.0 & 0.0 & 0.0 & 0.0 & 0.0 & 0.0 & 0.0 & 0.0 \\
\hline$r: t=2.54$ & $n$ & 0.0 & 0.0 & 0.0 & 0.0 & 0.0 & 0.0 & 0.0 & 0.0 \\
\hline his-253 & 0.0 & 0.0 & 0.0 & 0.0 & 0.0 & 0.0 & 0.0 & 0.0 & U.u \\
\hline Es-254m & 0.0 & 0.0 & 0.0 & 0.0 & 0.0 & 0.0 & 0.0 & 0.0 & 0.0 \\
\hline Es-254 & 0.0 & 0.0 & 00 & 0.0 & 0.0 & 0.0 & 0.0 & 0.0 & 0.0 \\
\hline Es-255 & 0.0 & 0.0 & 0.0 & 0.0 & 0.0 & 0.0 & 0.0 & 0.0 & 0.0 \\
\hline$F m-254$ & 0.0 & 0.0 & 0.0 & 0.0 & 0.0 & 0.0 & 0.0 & 0.0 & 0.0 \\
\hline$F_{m-255}$ & 0.0 & 0.0 & 0.0 & 0.0 & 0.0 & 0.0 & 0.0 & 0.0 & 0.0 \\
\hline$F_{m-256}$ & 0.0 & 0.0 & 0.0 & 0.0 & 0.0 & 0.0 & 0.0 & 0.0 & 0.0 \\
\hline
\end{tabular}


TABLE F-7. SURFACE DEPOSIT DOSE RATE CONVERSION FACTORS

(rem/hr per ci $/ \mathrm{m}^{2}$ )

\begin{tabular}{|c|c|c|c|c|c|c|c|c|c|}
\hline Isotope & $\begin{array}{l}\text { Total } \\
\text { Body }\end{array}$ & Bone & Liver & Kidney & Gonad & Lung & G.I. Tract & Thyroid & Skin \\
\hline H-3 & 0.0 & 0.0 & 0.0 & 0.0 & 0.0 & 0.0 & 0.0 & 0.0 & 0.0 \\
\hline-7 & $26-1$ & $87-1$ & $.05-1$ & $6.45-1$ & $8.07-1$ & $6.86-1$ & 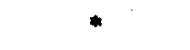 & $7.65-1$ & $8.87-$ \\
\hline$C-11$ & $51+1$ & $84+1$ & $1.26+1$ & $.34+1$ & $1.68+1$ & $1.43+1$ & $*$ & $1.59+1$ & $1.84+1$ \\
\hline 4 & 0.0 & 0.0 & 0.0 & 0.0 & 0.0 & 0.0 & 0.0 & 0.0 & 0.0 \\
\hline-13 & $51+1$ & $.84+1$ & $1.26+1$ & $.34+1$ & $1.68+1$ & $1.43+1$ & * & $1.59+1$ & $1.84+1$ \\
\hline $0-15$ & $1+1$ & $34+1$ & $1.26+1$ & $4+1$ & $8+1$ & $43+1$ & * & $.59+1$ & $84+1$ \\
\hline$F-18$ & $46+1$ & $.78+1$ & $1.22+1$ & $3+1$ & $1.62+1$ & $1.38+1$ & * & $1.54+1$ & $1.78+1$ \\
\hline $\mathrm{Na}-22$ & $3+1$ & $68+1$ & $2.76+1$ & $.56+1$ & $2.95+1$ & $3.14+1$ & $3.16+1$ & $3.37+1$ & $3.77+1$ \\
\hline $\mathrm{Na}-24$ & $17+1$ & $.38+1$ & $4.54+1$ & $.89+1$ & $5.27+1$ & $4.98+1$ & $5+1$ & $4.95+1$ & $6.07+1$ \\
\hline $\mathrm{Mg}-28$ & $05+1$ & $2+1$ & $1.71+1$ & $.55+1$ & $1.68+1$ & $1.94+1$ & $.0+1$ & $1.96+1$ & $2.36+1$ \\
\hline Al-28 & $5+1$ & $.63+1$ & $2.1+1$ & $6+1$ & $3+1$ & $2.39+1$ & $6+1$ & & $2.73+1$ \\
\hline & 0.0 & 0.0 & 0.0 & 0.0 & 0.0 & 0.0 & 0. & 0.0 & 0.0 \\
\hline & 0 & & 0.0 & & & 0.0 & & & 0.0 \\
\hline & 0 & & 0.0 & & & & & & 0.0 \\
\hline & $37-5$ & & & & & & & & $1.19-3$ \\
\hline & & & & & & & & +1 & $5+1$ \\
\hline & $1+0$ & & & & & & 0 & * & $2.52+0$ \\
\hline & $2+0$ & +0 & $3.38+0$ & 0 & 3.74 & 3.84 & $11+0$ & * & $39+0$ \\
\hline $\mathrm{K}-\mathrm{C}$ & $4+1$ & $.76+1$ & $1.2+1$ & $8+1$ & 1.6 & $1.36+1$ & $*$ & $1.51+$ & $1.76+1$ \\
\hline $\mathrm{Ca}-$ & 0.0 & 0.0 & 0.0 & & 0. & 0.0 & $0 .($ & 0 & 0.0 \\
\hline $\mathrm{Ca}-47$ & $59+1$ & $.68+1$ & $1.34+1$ & $4+1$ & $1.44+1$ & 1.52 & $61+1$ & +1 & $1.76+1$ \\
\hline $\mathrm{Ca}-4$ & $4+1$ & * & $3.61+1$ & & $*$ & 3.61 & 4 & & $4.27+1$ \\
\hline Sc- & $6+1$ & $.92+1$ & $.24+1$ & $3+1$ & 2.41 & 2.49 & 2 & 1.97 & $4+1$ \\
\hline & $17+0$ & $.23+0$ & $1.13+0$ & $7+0$ & & $1.3+0$ & 1 & & $1.78+0$ \\
\hline & & & & & & & & & \\
\hline & & & & & & & & & 0.0 \\
\hline & 0 & & & & & 0.0 & & & 0.0 \\
\hline$C_{I}-51$ & $7-1$ & 0 & $5.13-1$ & $4.86-1$ & $8.77-1$ & $.94-1$ & & -1 & $8.38-1$ \\
\hline $\mathbf{M n}$ & $2+1$ & & $3.04+1$ & & $3.2+$ & 3.45 & $3.47+1$ & -1 & $4.12+1$ \\
\hline$M_{n}-5$ & $92+1$ & $5.43+1$ & $4.12+1$ & $3.97+1$ & $4.34+1$ & 4.66 & $4.57+1$ & $4.68+1$ & $5.78+1$ \\
\hline & $1.16+1$ & $1.27+1$ & $9.73+0$ & $1.08+1$ & $1.05+1$ & $1.08+1$ & $1.05+1$ & $8.61+0$ & $1.5+1$ \\
\hline & 0.0 & 0.0 & 0.0 & & 0.0 & 0.0 & 0.0 & 0. & 0.0 \\
\hline & $07+1$ & $38+1$ & $8.8+1$ & -0 & $1.24+1$ & $1.0+$ & $95+$ & -1 & $1.31+1$ \\
\hline & $11-2$ & $17-3$ & $1.83-5$ & $5-6$ & $1.54-2$ & $1.74-3$ & $34-3$ & $8.76-4$ & $3.94-1$ \\
\hline $\mathrm{Fe}-$ & $68+1$ & $1: 8+1$ & $1.4+1$ & $1.37+1$ & $1.38+1$ & $1.59+1$ & $1.62+1$ & $1.47+1$ & $1.99+1$ \\
\hline Co-5 & $97+1$ & $5.29+1$ & $4.28+1$ & $3.98+1$ & $4.21+1$ & $4.7+1$ & $5.07+1$ & $5.1+1$ & $5.83+1$ \\
\hline Co-5 & $.56+0$ & $4.53+0$ & $1.88+0$ & $1.85+0$ & $2.39+0$ & $2.13+0$ & $1.66+0$ & $3.1+0$ & $3.05+0$ \\
\hline Co-5 & 0. & & 0.0 & & & 0.0 & & 0.0 & 0.0 \\
\hline Co-58 & $1.38+1$ & $1.54+1$ & $1.15+1$ & $1.28+1$ & $1.29+1$ & $1.29+1$ & $1.22+1$ & $1.1+1$ & $1.76+1$ \\
\hline $\mathrm{C}_{0}-60$ & $3.52+1$ & $3.76+1$ & $2.96+1$ & $2.82+1$ & $2.87+1$ & $3.33+1$ & $3.41+1$ & $3.13+1$ & $4.13+1$ \\
\hline $\mathrm{Ni}-56$ & $2.48+1$ & $2.89+1$ & $2.04+1$ & $2.13+1$ & $2.39+1$ & $2.31+1$ & $2.18+1$ & $2.1+1$ & $3.07+1$ \\
\hline $\mathrm{Ni}-59$ & 0.0 & 0.0 & 0.0 & 0.0 & & & & & 0.0 \\
\hline $\mathrm{Ni}-63$ & 0.0 & 0.0 & 0.0 & 0.0 & 0.0 & 0.0 & 0.0 & 0.0 & 0.0 \\
\hline
\end{tabular}

* Data not available. Use of Total Body Factor is recommended. 
TABLE F-7 (Cont)

Total

\begin{tabular}{|c|c|c|c|c|c|c|c|c|c|}
\hline Isotope & Body & Bone & Liver & Kidney & Gonad & Lung & G.I. Tract & Thyroid & Skin \\
\hline Ni-65 & 0.0 & 0.0 & 0.0 & 0.0 & 0.0 & 0.0 & 0.0 & 0.0 & 0.0 \\
\hline $\mathrm{Cu}-64$ & $3.12+0$ & $3.78+0$ & $2.6+0$ & $2.74+0$ & $3.41+0$ & $2.95+0$ & $3.1+0$ & $3.28+0$ & $3.81+0$ \\
\hline $\mathrm{Zn}-65$ & $8.12+0$ & $8.93+0$ & $6.8+0$ & $7.58+0$ & $7.37+0$ & $7.59+0$ & $7.31+0$ & $6.08+0$ & $1.05+1$ \\
\hline $\mathrm{Zn}-69 \mathrm{~m}$ & $6.19+0$ & $.54+0$ & $5.16+0$ & $5.49+0$ & $6.87+0$ & $5.84+0$ & $*$ & $6.52+0$ & $7.56+0$ \\
\hline $\mathrm{Zn}-69$ & 0.0 & 0.0 & 0.0 & 0.0 & 0.0 & 0.0 & 0.0 & $n \cap n$ & $n \cap$ \\
\hline Ga-67 & $3.36+0$ & $5.09+0$ & $2.59+0$ & $2.55+0$ & $3.97+0$ & $2.97+0$ & $2.4+0$ & $346+0$ & $4.12+0$ \\
\hline Ga-68 & $39+1$ & $1.7+1$ & $1.16+1$ & $1.24+1$ & $1.54+1$ & $1.32+1$ & 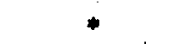 & $1.45+1$ & $1.71+1$ \\
\hline $\mathrm{G}_{\mathrm{a}-72}$ & 0.0 & 0.0 & 0.0 & 0.0 & 0.0 & 0.0 & 0.0 & 0.0 & 0.0 \\
\hline $\mathrm{Ge}_{\theta-71}$ & 0.0 & 0.0 & 0.0 & 0.0 & 0.0 & 0.0 & 0.0 & 0.0 & 0.0 \\
\hline$\overline{\mathbf{A}} \mathbf{E} \cdot 73$ & 0.0 & 0.0 & 0.0 & 0.0 & 0.0 & 0.0 & 0.0 & 0.0 & 0.0 \\
\hline Ass-74 & 0.0 & 0.0 & 0.0 & 0.0 & 0.0 & 0.0 & 0.0 & 0.0 & 0.0 \\
\hline As-76 & $6.39+0$ & $7.47+0$ & $5.37+0$ & $5.35+0$ & $6.34+0$ & $6.06+0$ & $6.26+0$ & $6.6+0$ & $7.6+0$ \\
\hline As-77 & 0.0 & 0.0 & 0.0 & 0.0 & 0.0 & 0.0 & 0.0 & 0.0 & 0.0 \\
\hline Se-75 & $9.37+0$ & $1.27+1$ & $7.45+0$ & $6.86+0$ & $9.65+0$ & $8.53+0$ & $7.75+0$ & 9.54 & $1.05+1$ \\
\hline-79 & 0.0 & 0.0 & 0.0 & 0.0 & 0.0 & 0.0 & 0. & 0. & 0.0 \\
\hline $\mathrm{Br}-80 \mathrm{~m}$ & $8.66-1$ & $1.24+0$ & $3.05-1$ & $4.52-1$ & $1.14+0$ & $4.14-1$ & $3.3-1$ & $8.44-1$ & $2.91+0$ \\
\hline $\mathrm{Br}-80$ & $1.31+0$ & $1.6+0$ & $1.1+0$ & $1.16+0$ & $1.45+0$ & $1.24+0$ & * & $1.38+0$ & $1.61+0$ \\
\hline $\mathrm{Br}-82 \mathrm{~m}$ & 0.0 & 0.0 & 0.0 & 0.0 & 0.0 & 0.0 & 0.0 & 0.0 & 0.0 \\
\hline $\mathrm{Br}-82$ & $3.82+1$ & $4.3+1$ & $3.21+1$ & $3.29+1$ & $3.55+1$ & $3.6 l+1$ & $3.49+1$ & $3.48+1$ & $4.65+1$ \\
\hline $8 r-83$ & $.1+0$ & $1.23+0$ & $8.18-1$ & $8.72-1$ & $1.18+0$ & $9.38-1$ & $1.0+0$ & $1.04+0$ & $3.05+0$ \\
\hline$-84 \mathrm{~m}$ & 0.0 & 0.0 & 0.0 & 0.0 & 0.0 & 0.0 & & 0 & 0.0 \\
\hline-84 & $37+1$ & $2.49+1$ & $2.1+1$ & $1.95+1$ & 2.13 & $2.24+1$ & 2.53 & & $2.81+1$ \\
\hline 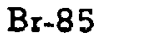 & 0.0 & 0.0 & 0.0 & & & 0.0 & 0 & & 0.0 \\
\hline-86 & 0.0 & 0.0 & 0.0 & 0.0 & 0.0 & 0.0 & 0 & 0. & 0.0 \\
\hline Br-87 & 0.0 & 0.0 & 0.0 & 0.0 & 0.0 & 0.0 & 0. & 0.0 & 0.0 \\
\hline $\mathrm{Kr}-79$ & $61+0$ & $5.83+0$ & $3.77+0$ & $3.93+0$ & $5.19+0$ & $4.29+0$ & $4.22+0$ & $4.66+0$ & $5.75+0$ \\
\hline $\mathrm{Kr}-83 \mathrm{~m}$ & $67-2$ & $3.9-2$ & $1.94-3$ & $2.56-3$ & $4.85-2$ & $8.98-3$ & $1.56-2$ & $4.31-3$ & $6.77-1$ \\
\hline $\mathrm{Kr}-85 \mathrm{~m}$ & $2.26+0$ & $3.42+0$ & $1.74+0$ & $1.64+0$ & $2.96+0$ & $2.01+0$ & $1.64+0$ & $2.17+0$ & $2.75+0$ \\
\hline Kr-85 & $3.04-2$ & $3.71-2$ & $2.53-2$ & $2.69-2$ & $3.38-2$ & $2.87-2$ & $3.04-2$ & $3.2-2$ & $3.71-2$ \\
\hline $\mathrm{K}_{\mathrm{r}-87}$ & $1.0+1$ & $1.09+1$ & $8.74+0$ & $8.21+0$ & $7.34+0$ & $9.59+0$ & $1.05+1$ & $9.77+0$ & $1.22 \cdots .1$ \\
\hline $\mathrm{Kr}-88$ & $2.49+1$ & $2.65+1$ & $2.19+1$ & $1.94+1$ & $2.47+1$ & $2.39+1$ & $2.85+1$ & $2.31+1$ & $3.01+1$ \\
\hline$K_{1}=09$ & 0.0 & 0.0 & 0.0 & 0.0 & 0.0 & 0.0 & 0.1 & 0.1 & 0.0 \\
\hline $\mathrm{K}_{\mathrm{r}-90}$ & 0.0 & 0.0 & 0.0 & 0.0 & 0.0 & 0.0 & 0.0 & 0.0 & 0.0 \\
\hline$R^{R} \mathbf{b} \cdot \mathbf{8}$ & $3+1$ & $1.48+1$ & $1.11+1$ & $1.21+i$ & $1.26+1$ & $1.23+1$ & 1.16 & 1.09 & $1.69+1$ \\
\hline Rh-86 & $1.2 .4+0$ & $1.37+0$ & $1.05+0$ & $1.16+0$ & $1.13+0$ & $1.16+0$ & $1.13+0$ & $9.25 \cdots 1$ & $1.61+0$ \\
\hline 07 & 0.0 & 0.0 & 0.0 & & & 0.0 & & & 0.0 \\
\hline $\mathrm{Rb}-88$ & $62+0$ & $9.01+0$ & $7.65+0$ & $6.95+0$ & $8.42+0$ & $8.25+0$ & $9.91+0$ & $8.08+0$ & $1.05+0$ \\
\hline $\mathrm{Rb}-\varepsilon$ & $3.18+1$ & $3.39+1$ & $2.74+1$ & $2.57+1$ & $2.73+1$ & $3.04+1$ & $3.3+1$ & $2.96+1$ & $3.81+1$ \\
\hline & 0.0 & 0.0 & 0.0 & 0.0 & 0.0 & 0.0 & 0.0 & 0.0 & 0.0 \\
\hline$S_{r}-85 m$ & 0.0 & 0.0 & 0.0 & 0.0 & 0.0 & 0.0 & 0.0 & 0.0 & 0.0 \\
\hline Sr-85 & $9.01+0$ & $1.1+1$ & $7.5+0$ & $7.99+0$ & $1.0+1$ & $8.49+0$ & $8.98+0$ & $9.47+0$ & $1.12+1$ \\
\hline $5 r-87 m$ & $4.84+0$ & $5.91+0$ & $4.04+0$ & $4.3+0$ & $5.38+0$ & $4.58+0$ & $4.84+0$ & $5.1+0$ & $5.91+0$ \\
\hline $5-89$ & 0.0 & 0.0 & 0.0 & 0.0 & 0.0 & 0.0 & 0.0 & 0.0 & 0.0 \\
\hline$r-9$ & 0.0 & 0.0 & 0.0 & 0.0 & 0.0 & 0.0 & 0.0 & 0.0 & 0.0 \\
\hline
\end{tabular}

"Data not available. Use of Total Body Factor is.recommended. 
TABLE F-7 (Cont)

\begin{tabular}{|c|c|c|c|c|c|c|c|c|c|}
\hline Isotope & $\begin{array}{l}\text { Total } \\
\text { Body }\end{array}$ & Bone & Liver & Kidney & Gonad & Lung & G.I. Tract & Thyroid & Skin \\
\hline Sr-9l & $1.04+1$ & $1.19+1$ & $8.71+0$ & $9.17+0$ & $1.01+1$ & $9.81+0$ & $9.57+0$ & $9.47+0$ & $1.28+1$ \\
\hline Sr-92 & 0.0 & 0.0 & 0.0 & 0.0 & 0.0 & 0.0 & 0.0 & 0.0 & 0.0 \\
\hline Y-87 & $8.6+0$ & $1.05+1$ & $7.15+0$ & $7.63+0$ & $9.54+0$ & $8.1+0$ & $8.57+0$ & $9.03+0$ & $1.07+1$ \\
\hline Y-88 & $3.6+1$ & $3.78+1$ & $3.16+1$ & $2.97+1$ & $3.34+1$ & $3.44+1$ & $4.03+1$ & $3.14+1$ & $4.46+1$ \\
\hline Y-90 & 0.0 & 0.0 & 0.0 & 0.0 & 0.0 & 0.0 & 0.0 & 0.0 & 0.0 \\
\hline Y-9lm & $7.79+0$ & $9.5+0$ & $6.48+0$ & $6.92+0$ & $8.64+0$ & $7.35+0$ & $7.77+0$ & $8.2+0$ & $9.51+0$ \\
\hline$Y-91$ & $3.68-2$ & $3.86-2$ & $3.09-2$ & $2.59-2$ & $3.44-2$ & $3.52-2$ & $3.77-2$ & $*$ & $4.02-2$ \\
\hline Y-92 & 0.0 & 0.0 & 0.0 & 0.0 & 0.0 & 0.0 & 0.0 & 0.0 & 0.0 \\
\hline$Y-93$ & $1.01+0$ & $1.2+0$ & $8.48-1$ & $8.05-1$ & $8.64-1$ & $9.42-1$ & $9.87-1$ & $9.14-1$ & $1.23+0$ \\
\hline$Z_{r-93}$ & 0.0 & 0.0 & 0.0 & 0.0 & 0.0 & 0.0 & 0.0 & 0.0 & 0.0 \\
\hline Zr-95 & $1.03+1$ & $1.18+1$ & $8.57+0$ & $9.37+0$ & $1.02+1$ & $9.63+0$ & $9.34+0$ & $9.03+0$ & $1.29+1$ \\
\hline Zr-97 & $2.57+0$ & $2.89+0$ & $2.15+0$ & $2.15+0$ & $2.33+0$ & $2.42+0$ & $2.35+0$ & $2.41+0$ & $3.07+0$ \\
\hline $\mathrm{Nb}-93 \mathrm{~m}$ & 0.0 & 0.0 & 0.0 & 0.0 & 0.0 & 0.0 & 0.0 & 0.0 & 0.0 \\
\hline $\mathrm{Nb}-95 \mathrm{~m}$ & $8.0-1$ & $1.21+0$ & $6.14-1$ & $5.82-1$ & $1.05+0$ & $7.11-1$ & $5.82-1$ & $7.67-1$ & $9.7-1$ \\
\hline Nb-95 & $05+1$ & $1.15+1$ & $8.79+0$ & $9.81+0$ & $9.47+0$ & $9.81+0$ & $9.47+0$ & $7.77+0$ & $1.36+1$ \\
\hline $\mathrm{Nb}-97 \mathrm{~m}$ & $1.06+1$ & $1.3+1$ & $8.86+0$ & $9.44+0$ & $1.18+1$ & $1.0+1$ & $1.06+1$ & $1.12+1$ & $1.3+1$ \\
\hline Nb-97 & $9.73+0$ & $1.19+1$ & $8.1+0$ & $8.64+0$ & $1.07+1$ & $9.19+0$ & $9.69+0$ & $1.02+1$ & $1.19+1$ \\
\hline Mo-99 & $2.53+0$ & $3.08+0$ & $2.09+0$ & $2.24+0$ & $2.73+0$ & $2.36+0$ & $2.36+0$ & $2.45+0$ & $3.16+0$ \\
\hline Tc-96m & 0.0 & 0.0 & 0.0 & & 0.0 & 0.0 & 0.0 & 0.0 & 0.0 \\
\hline$=-96$ & 0.0 & 0.0 & 0.0 & 0.0 & 0. & $0 . \mathrm{C}$ & & & 0.0 \\
\hline$-97 \mathrm{~m}$ & 0.0 & 0.0 & 0.0 & 0.0 & 0. & & & & 0.0 \\
\hline Tc-97 & 0.0 & 0.0 & 0.0 & 0.0 & 0.0 & 0.0 & 0.0 & 0.0 & 0.0 \\
\hline Tc-99m & $1.86+0$ & $3.32+0$ & $1.37+0$ & $1.35+0$ & $1.74+0$ & $1.55+0$ & $1.2+0$ & $2.27+0$ & $2.17+0$ \\
\hline Tc-9s & 0.0 & 0.0 & & 0.0 & 0 . & 0.0 & ) & 0.0 & 0.0 \\
\hline Ru-103m & 0.0 & 0.0 & 0.0 & 0.0 & 0.0 & 0.0 & 0.0 & 0.0 & 0.0 \\
\hline Ru-103 & $7.32+0$ & $8.94+0$ & $6.1+0$ & $6.5+0$ & $8.14+0$ & $6.91+0$ & $7.31+0$ & $7.71+0$ & $8.96+0$ \\
\hline Ru-105 & 0.0 & 0.0 & 0.0 & 0.0 & 0.0 & 0.0 & 0.0 & 0.0 & 0.0 \\
\hline Ru-106 & 0.0 & 0.0 & 0.0 & 0.0 & 0.0 & 0.0 & 0.0 & 0.0 & 0.0 \\
\hline $\mathrm{Rh}-103 \mathrm{~m}$ & $4.26-2$ & $4.22-2$ & $4.53-3$ & $1.28-2$ & $6.02-2$ & $7.02-3$ & $5.75-3$ & $1.66-2$ & $2.04-1$ \\
\hline Rh-105 & $1.14+0$ & $1.74+0$ & $8.78-1$ & $8.32-1$ & $1.5+0$ & $1.02+0$ & $8.32-1$ & $1.1+0$ & $1.39+0$ \\
\hline Rh-106 & $2.73+0$ & $3.32+0$ & $2.27+0$ & $2.42+0$ & $2.98+0$ & $2.58+0$ & $2.69+0$ & $2.84+0$ & $3.33+0$ \\
\hline Pd-103 & $3.13-2$ & $5.62-2$ & $2.0-2$ & $2.12-2$ & $2.89-2$ & $2.39-2$ & $2.18-2$ & $3.13-2$ & $4.23-2$ \\
\hline Pd-107 & 0.0 & 0.0 & 0.0 & 0.0 & 0.0 & 0.0 & 0.0 & 0.0 & 0.0 \\
\hline Pd-109 & 0.0 & 0.0 & 0.0 & 0.0 & 0.0 & 0.0 & $0: 0$ & 0.0 & 0.0 \\
\hline Ag-105 & 0.0 & 0.0 & 0.0 & 0.0 & 0.0 & 0.0 & 0.0 & 0.0 & 0.0 \\
\hline Ag-110m & $3.88+1$ & $4.37+1$ & $3.25+1$ & $3.33+1$ & $3.62+1$ & $3.66+1$ & $3.56+1$ & $3.56+1$ & $4.71+1$ \\
\hline Ag-111 & $4.51-1$ & $6.84-1$ & $3.46-1$ & $3.28-1$ & $5.89-1$ & $4.01-1$ & $3.28-1$ & $4.33-1$ & $5.47-1$ \\
\hline $\mathrm{Cd}-109$ & 0.0 & 0.0 & 0.0 & 0.0 & $0 . \tilde{0}$ & 0.0 & 0.0 & 0.0 & 0.0 \\
\hline $\mathrm{Cd}-113$ & 0.0 & 0.0 & 0.0 & 0.0 & 0.0 & 0.0 & 0.0 & 0.0 & 0.0 \\
\hline$C d-115 m$ & $4.61-1$ & $5.0-1$ & $3.87-1$ & $3.87-1$ & $3.93-1$ & $4.35-1$ & $4.36-1$ & $3.97-1$ & $5.57-1$ \\
\hline Cd-115 & 0.0 & 0.0 & 0.0 & 0.0 & 0.0 & 0.0 & 0.0 & 0.0 & 0.0 \\
\hline In-113m & $3.71+0$ & $4.53+0$ & $3.09+0$ & $3.3+0$ & $4.12+0$ & $3.5+0$ & $3.71+0$ & $3.9+0$ & $4.53+0$ \\
\hline In- $114 \mathrm{~m}$ & $3.6+0$ & $5.22+0$ & $2.77+0$ & $2.72+0$ & $4.57+0$ & $3.21+0$ & $2.63+0$ & $3.49+0$ & $4.49+0$ \\
\hline In-115m & $5.07+0$ & $7.67+0$ & $3.87+0$ & $3.68+0$ & $6.64+0$ & $4.47+0$ & $3.66+0$ & $4.85+0$ & $6.28+0$ \\
\hline
\end{tabular}

*Data not available. Use of Total Body Factor is recommended. 
TABLE F-7 (Cont)

\begin{tabular}{|c|c|c|c|c|c|c|c|c|c|}
\hline Isotope & Body & Bone & Liver. & Kidney & Gonad & Lung & G.I. Tract & Thyroid & Skin \\
\hline & 0.0 & 0.0 & 0.0 & 0.0 & 0.0 & 0.0 & 0.0 & 0.0 & 0.0 \\
\hline $5 n-113$ & $7.28-1$ & $8.85-1$ & $2.9-1$ & $3.54-1$ & $9.94-1$ & $3.52-1$ & $2.65-1$ & $4.59-1$ & $2.39+1$ \\
\hline $\mathrm{Sn}-119 \mathrm{~m}$ & $3.21-1$ & $4.27-1$ & $9.0-2$ & $1.51-1$ & $4.4-1$ & $1.23-1$ & $9.76-2$ & $2.61-1$ & $1.06+0$ \\
\hline Sn-121 & 0.0 & 0.0 & 0.0 & 0.0 & 0.0 & 0.0 & 0.0 & 0.0 & 0.0 \\
\hline Sn-123 & 0.0 & 0.0 & 0.0 & 0.0 & 0.0 & 0.0 & 0.0 & 0.0 & 0.0 \\
\hline $5 n-125$ & 0.0 & 0.0 & 0.0 & 0.0 & 0.0 & 0.0 & 0.0 & 0.0 & 00 \\
\hline Sn-126 & 0.0 & 0.0 & 0.0 & 0.0 & 0.0 & 0.0 & 0.0 & 0.0 & 0.0 \\
\hline Sb-122 & $6.11+0$ & $7.42+0$ & $5.09+0$ & $5.43+0$ & $6.73+0$ & $5.76+0$ & $6.06+0$ & $6.36+0$ & $7.47+0$ \\
\hline Sb-124 & $2.64+1$ & $2.95+1$ & $2.21+1$ & $2.07+1$ & $2.32+1$ & $2.51+1$ & $2.52+1$ & $2.67+1$ & $3.05+1$ \\
\hline $5 b-125$ & $36+0$ & $.13+0$ & $6.03+0$ & $6.44+0$ & $8.28+0$ & $6.85+0$ & $\overline{5}+0$ & $7.74+0$ & $9.21+0$ \\
\hline Sb-126 & 0.0 & 0.0 & 0.0 & 0.0 & 0.0 & 0.0 & 0.0 & 0.0 & 0.0 \\
\hline Sb-127 & $9.65+0$ & $1.16+1$ & $8.01+0$ & $8.57+0$ & $1.04+1$ & $9.06+0$ & $9.2+0$ & $9.57+0$ & $1.19+1$ \\
\hline Sb- 128 & 0.0 & 0.0 & 0.0 & 0.0 & 0.0 & & 0.0 & & 0.0 \\
\hline Sb-129 & 0.0 & 0.0 & 0.0 & 0.0 & 0. & 0.0 & 0.0 & & 0.0 \\
\hline$\Gamma \mathrm{e}-123 \mathrm{~m}$ & $2.52+0$ & $3.81+0$ & $1.88+0$ & $1.81+0$ & $3.31+0$ & $2.19+0$ & $1.77+0$ & $2.43+0$ & $3.21+0$ \\
\hline e-12 & $11+0$ & $1.7+0$ & $4.26-1$ & & $1.52+0$ & $71-1$ & $4.44-1$ & $18+0$ & $2.55+0$ \\
\hline Te-l & $1-1$ & $4.59-1$ & $1.15-1$ & $1.7-1$ & $4.11-1$ & $54-1$ & $1.2-1$ & $3.17-1$ & $7.03-1$ \\
\hline Te-12 & $05-2$ & $7.51-2$ & $5.01-2$ & $5.31-2$ & 6.79 & $68-2$ & $91-2$ & -2 & $7.41-2$ \\
\hline $\mathrm{Te}-129 \mathrm{~m}$ & -1 & $1.04+0$ & $6.46-1$ & 7.05 & 9.41 & & $7.63-1$ & $8.64-1$ & $1.13+0$ \\
\hline Te-129 & $8+0$ & & $9.58-1$ & 1.0 & 1.29 & $9+0$ & 1. & $*$ & $1.51+0$ \\
\hline $\mathrm{Te}-131 \mathrm{~m}$ & $4+1$ & $2.2+1$ & $1.62+1$ & 1.6 & 1.7 & $1.81+1$ & $1.77+$ & $1.6+1$ & $2.43+1$ \\
\hline $\mathrm{Te}-131$ & & $7.8+0$ & $4.77+0$ & $5.03+0$ & $5.79+$ & $5.37+0$ & 4. & & $7.25+0$ \\
\hline $\mathrm{Te}-132$ & $2.88+0$ & $4.42+0$ & $2.19+0$ & $2.09+0$ & $3.71+0$ & $2.55+0$ & $2.07+0$ & $2.79+0$ & $3.5+0$ \\
\hline Te-134 & 0.0 & 0.0 & 0.0 & 0.0 & 0.0 & 0.0 & 0.0 & 0.0 & 0.0 \\
\hline I-123 & $3.18+0$ & $4.75+0$ & $2.35+0$ & $2.31+0$ & $4.13+0$ & $2.74+0$ & $2.2+0$ & $3.09+0$ & $4.14+0$ \\
\hline$I-124$ & $1.63+1$ & $1.89+1$ & $1.37+1$ & $1.35+1$ & $1.58+1$ & $1.54+1$ & $59+1$ & $1.68+1$ & $1.95+1$ \\
\hline $\mathrm{I}-125$ & $1.29+0$ & $1.96+0$ & $4.85-1$ & $7.27-1$ & $1.77+0$ & $6.54-1$ & $9-1$ & $1.36+0$ & $2.97+0$ \\
\hline$I-126$ & $6.8+0$ & $8.3+0$ & $5.63+0$ & $5.99+0$ & $7.52+0$ & $6.38+0$ & $6.71+0$ & $7.12+0$ & $8.4+0$ \\
\hline I- 128 & 0.0 & 0.0 & 0.0 & & 0.0 & 0.0 & 0.0 & 0.0 & 0.0 \\
\hline I-129 & $6.54-1$ & $9.96-1$ & $2.47-1$ & $3.69-1$ & $9.0-1$ & $3.32-1$ & $2.59-1$ & $6.89-1$ & $1.51+0$ \\
\hline$I-130$ & $4+1$ & $3.62+1$ & $2.45+1$ & $2.61+1$ & 3.22 & $77+1$ & $9+1$ & $3.06+1$ & $3.61+1$ \\
\hline I-131 & $5.58+0$ & $6.92+0$ & $4.63+0$ & $4.92+0$ & 6.26 & $5.26+0$ & $5.48+0$ & $5.87+0$ & $6.83+0$ \\
\hline I-132 & $6+1$ & $3.77+1$ & $2.73+1$ & & $3.2+1$ & $3.07+1$ & $3.02+1$ & $3.01+1$ & $4.05+1$ \\
\hline I-133 & $9.77+0$ & $1.15+1$ & $8.16+0$ & & $1.01+1$ & $9.23+0$ & $9.49+0$ & $9.85+0$ & $1.19+1$ \\
\hline $1-134$ & $3.26+1$ & $3.62+1$ & $2.74+1$ & $2.96+1$ & $2.9 \%+1$ & $3.07+1$ & $2.95+1$ & $2.64+1$ & $4.12+1$ \\
\hline I-135 & $2.45+1$ & $2.62+1$ & $2.07+1$ & $1.92+1$ & $2.02+1$ & $2.33+1$ & $2.41+1$ & $2.26+1$ & $2.84+$ \\
\hline I-136 & & & 0.0 & & 0.0 & 0.0 & 0.0 & 0.0 & 0.0 \\
\hline I- 137 & 0.0 & & 0.0 & 0. & 0. & 0.0 & 0.0 & 0.0 & 0.0 \\
\hline $\mathrm{Xe}-131 \mathrm{~m}$ & $5.56-1$ & $8.45-1$ & $2.66-1$ & 3.3 ? & 7.5 & $3.38-1$ & $2.58-1$ & $5.71-1$ & $1.13+0$ \\
\hline $\mathrm{Xe}-13$ & $9.85-1$ & 1.5 & $6.46-1$ & $6.7-1$ & & $7.68-1$ & $5.81-1$ & $9.7-1$ & $1.51+0$ \\
\hline $\mathrm{Xe}-13$ & $9.57-1$ & $1.66+0$ & $6.37-1$ & $6.63-1$ & $9.78-1$ & $7.35-1$ & $5.67-1$ & $1.13+0$ & $1.32+0$ \\
\hline $\mathrm{Xe}-135 \mathrm{~m}$ & $6.46+0$ & $7.9+0$ & $5.37+0$ & $5.73+0$ & $7.18+0$ & $6.08+0$ & $6.44+0$ & $6.8+0$ & $7.92+0$ \\
\hline $\mathrm{Xe}-135$ & $3.53+0$ & $5.27+0$ & $2.72+0$ & $2.6+0$ & $4.57+0$ & $3.14+0$ & $2.57+0$ & $3.4+0$ & $4.29+0$ \\
\hline Xe-137 & $2.59+0$ & $3.07+0$ & $2.18+0$ & $2.24+0$ & $2.64+0$ & $2.45+0$ & $2.55+0$ & $2.66+0$ & $3.14+0$ \\
\hline
\end{tabular}

* Data not available. Use of Total Body Factor is recommended. 
TABLE F-7 (Cont)

Total

\begin{tabular}{|c|c|c|c|c|c|c|c|c|c|}
\hline Isotope & Body & Bone & Liver & Kidney & Gonad & Lung & G.I. Tract & Thyroid & Skin \\
\hline Xe-138 & $1.19+1$ & $1.36+1$ & $1.0+1$ & $8.98+0$ & $9.94+0$ & $1.12+1$ & $1.2+1$ & $1.15+1$ & $1.4+1$ \\
\hline Xe-139 & 0.0 & 0.0 & 0.0 & 0.0 & 0.0 & 0.0 & 0.0 & 0.0 & 0.0 \\
\hline Xe-140 & 0.0 & 0.0 & 0.0 & 0.0 & 0.0 & 0.0 & 0.0 & 0.0 & 0.0 \\
\hline Cs-134 & $2.2+1$ & $2.55+1$ & $1.85+1$ & $2.0+1$ & $2.2+1$ & $2.08+1$ & $2.02+1$ & $1.97+1$ & $2.75+1$ \\
\hline Cs-135 & 0.0 & 0.0 & 0.0 & 0.0 & 0.0 & 0.0 & 0.0 & 0.0 & 0.0 \\
\hline$C s-136$ & $2.92+1$ & $3.34+1$ & $2.43+1$ & $2.58+1$ & $2.73+1$ & $2.73+1$ & $2.61+1$ & $2.43+1$ & $3.68+1$ \\
\hline Cs-137 & 0.0 & 0.0 & 0.0 & 0.0 & 0.0 & 0.0 & $0: 0$ & 0.0 & 0.0 \\
\hline Cs-138 & $2.95+1$ & $3.13+1$ & $2.52+1$ & $2.26+1$ & $2.61+1$ & $2.81+1$ & $3.07+1$ & $2.83+1$ & $3.41+1$ \\
\hline Cs-139 & 0.0 & 0.0 & 0.0 & 0.0 & 0.0 & 0.0 & 0.0 & 0.0 & 0.0 \\
\hline $\mathrm{Ba}-13 \mathrm{l}$ & 0.0 & 0.0 & 0.0 & 0.0 & 0.0 & 0.0 & 0.0 & 0.0 & 0.0 \\
\hline $\mathrm{Ba}-133$ & $6.11+0$ & $8.23+0$ & $4.81+0$ & $5.03+0$ & $6.97+0$ & $5.49+0$ & $5.31+0$ & $6: 4+0$ & $7.76+0$ \\
\hline $\mathrm{Ba}-137 \mathrm{~m}$ & $8.29+0$ & $1.01+1$ & $6.89+0$ & $7.36+0$ & $9.21+0$ & $7.82+0$ & $8.28+0$ & $8.73+0$ & $1.01+1$ \\
\hline Ba-139 & 0.0 & 0.0 & 0.0 & 0.0 & 0.0 & 0.0 & 0.0 & 0.0 & 0.0 \\
\hline $\mathrm{Ba}-140$ & $3.17+0$ & $4.0+0$ & $2.59+0$ & $2.73+0$ & $3.61+0$ & $2.95+0$ & $3.0+0$ & $3.3+0$ & $3.92+0$ \\
\hline La-140 & $3.28+1$ & $3.56+1$ & $2.75+1$ & $2.49+0$ & $2.72+1$ & $3.12+1$ & $3.22+1$ & $3.16+1$ & $3.74+1$ \\
\hline La-14l & 0.0 & 0.0 & 0.0 & 0.0 & 0.0 & 0.0 & 0.0 & 0.0 & 0.0 \\
\hline La- 142 & 0.0 & 0.0 & 0.0 & 0.0 & 0.0 & 0.0 & 0.0 & 0.0 & 0.0 \\
\hline Ce-14l & $1.16+0$ & $2.07+0$ & $8.46-1$ & $8.39-1$ & $1.11+0$ & $9.59-1$ & $7.39-1$ & $1.42+0$ & $1.4+0$ \\
\hline $\mathrm{Ce}-142$ & 0.0 & 0.0 & 0.0 & 0.0 & 0.0 & 0.0 & 0.0 & 0.0 & 0.0 \\
\hline $\mathrm{Ce}-143$ & $4.76+0$ & $6.51+0$ & $3.71+0$ & $3.81+0$ & $5.51+0$ & $4.26+0$ & $3.65+0$ & $4.46+0$ & $6.06+0$ \\
\hline $\mathrm{Ce}-144$ & $3.21-1$ & $5.65-1$ & $2.26-1$ & $2.27-1$ & $3.13-1$ & $2.58-1$ & $1.99-1$ & $3.86-1$ & $4.08-1$ \\
\hline Pr-142 & 0.0 & 0.0 & 0.0 & 0.0 & 0.0 & 0.0 & 0.0 & 0.0 & 0.0 \\
\hline Pr-143 & 0.0 & 0.0 & 0.0 & 0.0 & 0.0 & 0 & 0.0 & 0.0 & 0.0 \\
\hline Pr-144 & $4.02-1$ & $4.42-1$ & $3.48-1$ & $3.23-1$ & $3.24-1$ & $3.85-1$ & $4.17-1$ & $3.96-1$ & $4.82-1$ \\
\hline Pr-145 & 0.0 & 0.0 & 0.0 & 0.0 & 0.0 & 0.0 & 0.0 & 0.0 & 0.0 \\
\hline Nd-144 & 0.0 & 0.0 & 0.0 & 0.0 & 0.0 & 0.0 & 0.0 & 0.0 & 0.0 \\
\hline Nd-147 & $2.24+0$ & $3.1+0$ & $1.75+0$ & $1.84+0$ & $2.45+0$ & $1.99+0$ & $1.93+0$ & $2.41+0$ & $2.84+0$ \\
\hline $\mathrm{Nd}-149$ & 0.0 & 0.0 & 0.0 & 0.0 & 0.0 & 0.0 & 0.0 & 0.0 & 0.0 \\
\hline$P \mathrm{~m}-147$ & 0.0 & 0.0 & $0 \cap$ & 0.0 & 0.0 & 0.0 & 0.0 & 0.0 & 0.0 \\
\hline Pm-148m & $2.88+1$ & $3.48+1$ & $2.4+1$ & $2.57+1$ & $3.1+1$ & $2.72+1$ & $2.76+1$ & $2.87+1$ & $3.55+1$ \\
\hline Pm-148 & $9.14+0$ & $1.0+1$ & $7.67+0$ & $7.21+0$ & $7.86+0$ & $8.69+0$ & $8.65+0$ & $8.82+0$ & $1.06+1$ \\
\hline Pm-149 & 0.0 & 0.0 & 0.0 & 0.0 & 0.0 & 0.0 & 0.0 & 0.0 & 0.0 \\
\hline Pm-15l & 0.0 & 0.0 & 0.0 & 0.0 & 0.0 & 0.0 & 0.0 & 0.0 & 0.0 \\
\hline Sm-147 & 0.0 & 0.0 & 0.0 & 0.0 & 0.0 & 0.0 & 0.0 & 0.0 & 0.0 \\
\hline Sm-148 & 0.0 & 0.0 & 0.0 & 0.0 & 0.0 & 0.0 & 0.0 & 0.0 & 0.0 \\
\hline$S_{m-1} 49$ & 0.0 & 0.0 & 0.0 & 0.0 & 0.0 & 0.0 & 0.0 & 0.0 & 0.0 \\
\hline Sm-151 & $6.6-4$ & $5.23-4$ & $5.03-5$ & $1.51-4$ & $8.33-4$ & $9.09-5$ & $9.49-5$ & $1.94-4$ & $5.21-5$ \\
\hline Sm-153 & 0.0 & 0.0 & 0.0 & 0.0 & 0.0 & 0.0 & 0.0 & 0.0 & 0.0 \\
\hline Eu-152 & 0.0 & 0.0 & 0.0 & 0.0 & 0.0 & 0.0 & 0.0 & 0.0 & 0.0 \\
\hline Eu-154 & $1.84+1$ & $2.09+1$ & $1.53+1$ & $1.51+1$ & $1.61+1$ & $1.72+1$ & $1.69+1$ & $1.7+1$ & $2.19+1$ \\
\hline Eu-155 & $1.03+0$ & $1.86+0$ & $7.4-1$ & $7.33-1$ & $9.51-1$ & $8.44-1$ & $6.61-1$ & $1.22+0$ & $1.23+0$ \\
\hline Eu-156 & 0.0 & 0.0 & 0.0 & 0.0 & 0.0 & 0.0 & 0.0 & 0.0 & 0.0 \\
\hline Eu-157 & 0.0 & 0.0 & 0.0 & 0.0 & 0.0 & 0.0 & 0.0 & 0.0 & 0.0 \\
\hline
\end{tabular}


TABLE F-7 (Cont)

Total

\begin{tabular}{|c|c|c|c|c|c|c|c|c|c|}
\hline Isotope & Body & Bone & $\underline{\text { Liver }}$ & $\underline{\text { Kidney }}$ & Gonad & Lung & G.I. Tract & Thyroid & Skin \\
\hline Gd-153 & 0.0 & 0.0 & 0.0 & 0.0 & 0.0 & 0.0 & 0.0 & 0.0 & 0.0 \\
\hline Gd-159 & 0.0 & 0.0 & 0.0 & 0.0 & 0.0 & 0.0 & 0.0 & 0.0 & 0.0 \\
\hline Gd-162 & $6.34+0$ & $7.75+0$ & $5.27+0$ & $5.63+0$ & $7.04+0$ & $5.98+0$ & $6.32+0$ & $6.68+0$ & $7.76+0$ \\
\hline Tb-160 & $1.51+1$ & $1.72+1$ & $1.24+1$ & $1.32+1$ & $1.38+1$ & $1.4+1$ & $1.35+1$ & $1.22+1$ & $1.88+1$ \\
\hline Tb-161 & 0.0 & 0.0 & $n n$ & 0.0 & 0.0 & 0.0 & 0.0 & 0.0 & 0.0 \\
\hline Dy-165 & 0.0 & 0.0 & 0.0 & 0.0 & 0.0 & 0.0 & 0.0 & 0.0 & 0.0 \\
\hline Dy-166 & 0.0 & 0.0 & 00 & 0.0 & 0.0 & 0.0 & 0.0 & 0.0 & 0.0 \\
\hline $\mathrm{H} \cap-166$ & 0.0 & 0.0 & 0.0 & 0.0 & 0.0 & 0.0 & 0.0 & 0.0 & 0.0 \\
\hline$E_{r-169}$ & 0.0 & 0.0 & 0.0 & 0.0 & 0.0 & 0.0 & 00 & 0.0 & 0.0 \\
\hline Er-1 1]1 & 0.0 & 0.0 & 0.0 & 0.0 & 0.0 & 0.0 & 0.0 & 0.0 & 0.0 \\
\hline $\mathrm{Tm}-170$ & 0.0 & 0.0 & 0.0 & 0.0 & 0.0 & 0.0 & 0.0 & 0.0 & 0.0 \\
\hline Yb-175 & 0.0 & 0.0 & 0.0 & 0.0 & 0.0 & 0.0 & 0.0 & 0.0 & 0.0 \\
\hline Lu-177 & 0.0 & 0.0 & 0.0 & 0.0 & 0.0 & 0.0 & 0.0 & 0.0 & 0.0 \\
\hline $\mathrm{Ht}-181$ & 0.0 & 0.0 & 0.0 & 0.0 & 0.0 & 0.0 & 0.0 & 0.0 & 0.0 \\
\hline Ta-182 & $1.86+1$ & $2.11+1$ & $1.54+1$ & $1.51+1$ & $1.59+1$ & $1.74+1$ & $1.74+1$ & $1.66+1$ & $2.24+1$ \\
\hline W-181 & 0.0 & 0.0 & 0.0 & 0.0 & 0.0 & 0.0 & 0.0 & 0.0 & 0.0 \\
\hline W-185 & 0.0 & 0.0 & 0.0 & 0.0 & 0.0 & 0.0 & 0.0 & 0.0 & 0.0 \\
\hline W-187 & $2.47+0$ & $3.2+0$ & $2.02+0$ & $2.13+0$ & $2.68+0$ & $2.28+0$ & $2.35+0$ & $2.63+0$ & $3.01+0$ \\
\hline$W-188$ & 0.0 & 0.0 & 0.0 & 0.0 & 0.0 & 0.0 & 0.0 & 0.0 & 0.0 \\
\hline $\operatorname{Re}-183$ & 0.0 & 0.0 & 0.0 & 0.0 & 0.0 & 0.0 & 0.0 & 0.0 & 0.0 \\
\hline $\operatorname{Re}-186$ & 0.0 & 0.0 & 0.0 & 0.0 & 0.0 & 0.0 & 0.0 & 0.0 & 0.0 \\
\hline $\operatorname{Re}-187$ & 0.0 & 0.0 & 0.0 & 0.0 & 0.0 & 0.0 & 0.0 & 0.0 & 0.0 \\
\hline Re-188 & 0.0 & 0.0 & 0.0 & 0.0 & 0.0 & 0.0 & 0.0 & 0.0 & 0.0 \\
\hline Os-185 & 0.0 & 0.0 & 0.0 & 0.0 & 0.0 & 0.0 & 0.0 & 0.0 & 0.0 \\
\hline Os-19lm & 0.0 & 0.0 & 0.0 & 0.0 & 0.0 & 0.0 & 0.0 & 0.0 & 0.0 \\
\hline Os-191 & 0.0 & 0.0 & 0.0 & 0.0 & 0.0 & 0.0 & 0.0 & 0.0 & 0.0 \\
\hline Os-193 & 0.0 & 0.0 & 0.0 & 0.0 & 0.0 & 0.0 & 0.0 & 0.0 & 0.0 \\
\hline Ir-190 & 0.0 & 0.0 & 0.0 & 0.0 & 0.0 & 0.0 & 0.0 & 0.0 & 0.0 \\
\hline$I_{r-192}$ & 0.0 & 0.0 & 0.0 & 0.0 & 0.0 & 0.0 & 0.0 & 0.0 & 0.0 \\
\hline Pt-191 & 0.0 & 0.0 & 0.0 & 0.0 & 0.0 & 0.0 & 0.0 & 0.0 & 0.0 \\
\hline Pt-193m & 0.0 & 0.0 & 0.0 & 0.0 & 0.0 & 0.0 & 0.0 & 0.0 & 0.0 \\
\hline Pl-193 & 0.0 & 0.0 & 0.0 & 0.0 & 0.0 & 0.0 & 0.0 & 0.0 & 0.0 \\
\hline Pt-197m & 0.0 & 0.0 & 0.0 & 0.0 & 0.0 & 0.0 & 0.0 & 0.0 & $n \cap$ \\
\hline Pt 197 & 0.0 & 0.0 & 0.0 & 0.0 & 0.0 & 0.0 & 0.0 & 0.0 & 0.0 \\
\hline Au-195m & $3.53+0$ & $5.44+0$ & $2.66+0$ & $2.53+0$ & $4.5+0$ & $3.09+0$ & $2.5+0$ & $3.39+0$ & $4.39+0$ \\
\hline Áu-195 & $2.13+0$ & $4.06+0$ & $1.32+0$ & $1.37+0$ & $1.88+0$ & $1.59+0$ & $1.35+0$ & $2.18+0$ & $3.04+0$ \\
\hline Au-196 & 0.0 & 0.0 & 0.0 & 0.0 & 0.0 & 0.0 & 0.0 & 0.0 & 0.0 \\
\hline Au-198 & $5.97+0$ & $7.31+0$ & $4.97+0$ & $5.3+0$ & $6.62+0$ & $5.64+0$ & $5.96+0$ & $6.28+0$ & $7.29+0$ \\
\hline Au-199 & $1.55+0$ & $2.42+0$ & $1.16+0$ & $1.11+0$ & $1.95+0$ & $1.36+0$ & $1.09+0$ & $1.51+0$ & $1.93+0$ \\
\hline $\mathrm{Hg}-197 \mathrm{~m}$ & 0.0 & 0.0 & 0.0 & 0.0 & 0.0 & 0.0 & 0.0 & 0.0 & 0.0 \\
\hline $\mathrm{Hg}-197$ & $1.61+0$ & $3.08+0$ & $9.61-1$ & $1.01+0$ & $1.42+0$ & $1.16+0$ & $1.01+0$ & $1.58+0$ & $2.35+0$ \\
\hline $\mathrm{Hg}-203$ & $3.49+0$ & $5.35+0$ & $2.66+0$ & $2.53+0$ & $4.52+0$ & $3.08+0$ & $2.51+0$ & $3.36+0$ & $4.27+0$ \\
\hline Tl-200 & 0.0 & 0.0 & 0.0 & 0.0 & 0.0 & 0.0 & 0.0 & 0.0 & 0.0 \\
\hline Tl-201 & 0.0 & 0.0 & 0.0 & 0.0 & 0.0 & 0.0 & 0.0 & 0.0 & 0.0 \\
\hline
\end{tabular}


TABLE F-7 (Cont)

\begin{tabular}{|c|c|c|c|c|c|c|c|c|c|}
\hline Isotope & $\begin{array}{l}\text { Total } \\
\text { Body }\end{array}$ & Bone & Liver & Kidney & Gonad & Lung & G.I. Tract & Thyroid & Skin \\
\hline Tl-202 & 0.0 & 0.0 & 0.0 & 0.0 & 0.0 & 0.0 & 0.0 & 0.0 & 0.0 \\
\hline Tl-204 & $2.57-2$ & $4.94-2$ & $1.52-2$ & $1.61-2$ & $2.24-2$ & $1.85-2$ & $1.61-2$ & $2.51-2$ & $3.76-2$ \\
\hline $\mathrm{T} l-207$ & $3.37-2$ & * & * & $*$ & $\star$ & $*$ & * & * & $*$ \\
\hline Tl-208 & $4.14+1$ & $4.45+1$ & $3.65+1$ & $3.33+1$ & $3.81+1$ & $3.97+1$ & $4.65+1$ & $3.93+1$ & $5.06+1$ \\
\hline Tl-209 & $3.02+1$ & * & * & * & * & $*$ & $*$ & $*$ & * \\
\hline $\mathrm{Pb}-203$ & 0.0 & 0.0 & 0.0 & 0.0 & 0.0 & 0.0 & 0.0 & 0.0 & 0.0 \\
\hline $\mathrm{Pb}-204 \mathrm{~m}$ & 0.0 & 0.0 & 0.0 & 0.0 & 0.0 & 0.0 & 0.0 & 0.0 & 0.0 \\
\hline $\mathrm{Pb}-209$ & 0.0 & 0.0 & 0.0 & 0.0 & 0.0 & 0.0 & 0.0 & 0.0 & 0.0 \\
\hline $\mathrm{Pb}-210$ & $2.59-\mathrm{J}$ & $4.45-1$ & $1.32-1$ & $1.39-1$ & $2.19-1$ & $1.64-1$ & $1.43-1$ & $2.18-1$ & $7.59-1$ \\
\hline $\mathrm{Pb}-21 \mathrm{l}$ & $9.4-1$ & $*$ & $*$ & * & $*$ & $*$ & $*$ & * & $*$ \\
\hline $\mathrm{Pb}-2 \mathrm{l} 2$ & $2.29+0$ & $3.62+0$ & $1.7+0$ & $1.63+0$ & $2.83+0$ & $1.99+0$ & $1.59+0$ & $2.24+0$ & $2.84+0$ \\
\hline $\mathrm{Pb}-214$ & $3.75+0$ & $*$ & $*$ & $*$ & $*$ & * & $*$ & $*$ & * \\
\hline $\mathrm{Bi}-206$ & 0.0 & 0.0 & 0.0 & 0.0 & 0.0 & 0.0 & 0.0 & 0.0 & 0.0 \\
\hline $\mathrm{Bi}-207$ & 0.0 & 0.0 & 0.0 & 0.0 & 0.0 & 0.0 & 0.0 & 0.0 & 0.0 \\
\hline $\mathrm{Bi}-210$ & 0.0 & 0.0 & 0.0 & 0.0 & 0.0 & 0.0 & 0.0 & 0.0 & 0.0 \\
\hline $\mathrm{Bi}-21 \mathrm{l}$ & $8.23-1$ & * & $*$ & $*$ & $*$ & $*$ & $*$ & * & $*$ \\
\hline $\mathrm{Bi}-212$ & $1.74+0$ & $2.0+0$ & $1.45+0$ & $1.44+0$ & $1.66+0$ & $1.64+0$ & $1.63+0$ & $1.7+0$ & $2.09+0$ \\
\hline $\mathrm{Bi}-213$ & $2.61+0$ & * & $*$ & $*$ & $*$ & $*$ & $*$ & * & $*$ \\
\hline $\mathrm{Bi}-214$ & $2.05+1$ & * & * & * & * & $*$ & * & * & * \\
\hline Po-210 & $7.55-4$ & $*$ & $*$ & $*$ & $*$ & $*$ & $*$ & $*$ & * \\
\hline Po-212 & 0.0 & 0.0 & 0.0 & 0.0 & 0.0 & 0.0 & 0.0 & 0.0 & 0.0 \\
\hline Po-213 & 0.0 & 0.0 & 0.0 & 0.0 & 0.0 & 0.0 & 0.0 & 0.0 & 0.0 \\
\hline Po-215 & $3.73-3$ & $*$ & $*$ & $*$ & $*$ & $*$ & $*$ & $*$ & $*$ \\
\hline Po-216 & $2.64-4$ & * & * & * & * & * & $*$ & * & $*$ \\
\hline Po-218 & $1.42-4$ & $*$ & $*$ & $*$ & $*$ & $*$ & $*$ & $*$ & $*$ \\
\hline At-21l & 0.0 & 0.0 & 0.0 & 0.0 & 0.0 & 0.0 & 0.0 & 0.0 & 0.0 \\
\hline At -217 & $4.44-3$ & $*$ & $*$ & $*$ & $*$ & $*$ & $*$ & $*$ & * \\
\hline $\mathrm{Rn}-219$ & $8.38-1$ & $*$ & * & $*$ & * & $*$ & $*$ & * & * \\
\hline $\mathrm{Rn}-220$ & $5.27-3$ & $6.44-3$ & $4.39-3$ & $4.6 y-3$ & $5.87-3$ & $4.99-3$ & $3.27-3$ & $3.56-3$ & 6.44 \\
\hline $\mathrm{Rn}-222$ & $5.62-3$ & * & $*$ & $*$ & $*$ & $*$ & $*$ & * & * \\
\hline Ra-224 & $1.5-1$ & $2.28-1$ & $1.15-1$ & $1.09-1$ & $1.94-1$ & $.1 .32-1$ & $1.09-1$ & $1.45-1$ & $1.83-1$ \\
\hline Ra-225 & $5.55-1$ & * & * & $*$ & * & $*$ & $*$ & $*$ & * \\
\hline $\mathrm{Ra}-226$ & $1.08-1$ & $1.66-1$ & $8.25-2$ & $7.84-2$ & $1.38-1$ & $9.54-2$ & $7.76-2$ & $1.06-1$ & $1.32-1$ \\
\hline Ra-228 & $7.57-1$ & $1.15+0$ & $2.85-1$ & $4.28-1$ & $1.04+0$ & $3.85-1$ & $2.99-1$ & $7.99-1$ & $1.71+0$ \\
\hline Fr-221 & $3.81-2$ & * & $*$ & $*$ & $*$ & * & $*$ & $*$ & * \\
\hline Ac-225 & $5.01-1$ & $*$ & $*$ & $*$ & $*$ & * & $*$ & * & * \\
\hline Ac-227 & 0.0 & 0.0 & 0.0 & 0.0 & 0.0 & 0.0 & 0.0 & 0.0 & 0.0 \\
\hline Ac-228 & $1.03+1$ & $1.19+1$ & $8.54+0$ & $8.76+0$ & $9.41+0$ & $9.61+0$ & $9.23+0$ & $8.94+0$ & $1.27+1$ \\
\hline Th-227 & $2.6+0$ & $*$ & $*$ & * & $*$ & $*$ & $*$ & $*$ & $*$ \\
\hline Th-228 & $1.89-1$ & $3.07-1$ & $1.32-1$ & $1.29-1$ & $1.96-1$ & $1.53-1$ & $1.18-1$ & $2.0-1$ & $3.23-1$ \\
\hline Th-229 & $2.7+0$ & $*$ & $*$ & * & $*$ & $*$ & $*$ & * & $*$ \\
\hline Th-230 & $6.99-2$ & $1.03-1$ & $3.26-2$ & $3.33-2$ & $6.16-2$ & $4.1-2$ & $3.29-2$ & $5.09-2$ & $2.51-1$ \\
\hline Th-231 & $1.47+0$ & $2.39+0$ & $9.05-1$ & $9.29-1$ & $1.44+0$ & $1.05+0$ & $8.09-1$ & $1.55+0$ & $3.1+0$ \\
\hline
\end{tabular}

* Data not available. Use of Total Body Factor is recommended. 
TABLE F-7 (Cont)

Total

Isotope

Body

Th-232

Th-234

$\mathrm{Pa}-230$

$\mathrm{Pa}-231$

$\mathrm{Pa} \cdot 233$

$\mathrm{Pa}-234 \mathrm{~m}$

Pa 335

$\mathrm{U}-230$

U-232

Uิ-233

U-234

$\mathrm{U}-235$

U-236

$\mathrm{U}-237$

$\mathrm{U}-238$

U-239

$\mathrm{Np}-237$

$\mathrm{Np}-238$

$\mathrm{Np}-239$

$\mathrm{Np}-240 \mathrm{~m}$

$\mathrm{Np}-240$

$\mathrm{Pu}-238$

Pu-239

$\mathrm{Pu}-240$

Pu-24 1

$\mathrm{Pu}-242$

Pu-243

$\mathrm{Pu}-244$

$A \mathrm{~m}=241$

Am. $242 \mathrm{~m}$

Am-242

A $\mathrm{m}-243$

Am-244

Cm-242

Cm 243

$\mathrm{C} m-244$

$\mathrm{Cm}-245$

$\mathrm{C} \mathrm{m}-246$

$\mathrm{Cm}-247$

Cm-248

Cm-249

Bk-249

Bk-250

Bone Liver Kidney Gonad

$1.89-1$

$4.79-2$

$1.08-2$

$1.14-2$

$3.13-2$

Lung

0.0
$7.18-1$

$3.38-1$
0.0

. $36-1$

$1.35-1$

$1.75-1$

$1.56-2$

G.I. Tract

Thyroid

Skin

$3.7+0$

$5.71+0 \quad 2.82+0$

0.0

$1.55-1$
0.0

1.35-2

1.76-2 2.4-1

$5.03-3$

$6.79-3 \quad 4$ ก.3-3

$2.72+0$

$4.5+0 \quad 3.24+0$

$1.2-1$.

$2.25-1 \cdot 2.4-1$

$0.0 \quad 0.0$

0.0

$0.0 \quad 0.0$

$4.1-3$

$6.01-3$

$2.61+0$

$3.73+0 \quad 4.55+0$

0.0

0.0

0.0

0.0

0.0

U.U

$4.25 \quad 3$

$3.07-3 \quad 6.28-3$

6.99-2 8.92-2

2.57-2

0.0

0.0

0.0

0.0

$0.0 \quad 0.0$

$8.92-2 \quad 1.31-1$

$5.75-2$

2.67-2. $5.91-2 \quad 3.31-2$

0.0

0.0

0.0

$5.46-2 \quad 6.43-2$

$3.25+0 \quad 5.07+0$

$1.67-2$

$5.57-2 \quad 1.01-1$

$6.77-2$

2.57-2

$5.26-2$

$42.8-2 \quad 3.36-1$

$3.66-2 \quad 3.14-2$

$2.45+0$
$4.31-3$

$1.78-2 \quad 4.57-2$

$2.25-2$

$1.87-2$

$\begin{array}{ll}7.86-2 & 2.01-1\end{array}$

$4.21+0 \quad 7.03+0$

$3.62-2$

$3.52-2$

$3.02+0$

$2.34+0$

$4.01+0 \quad 2.82+0$

$2.27+0$

$2.81-2 \quad 2.97-1$

$$
0.0
$$

0.0

$7.25-3$

$\begin{array}{lll}5.34-3 & 2.93-2 & 8.16-3\end{array}$

$1.01-2$

$3.25+0$

$7.65-3$

$4.11+0$

$\begin{array}{ll}1.52+0 & 2.57+0\end{array}$

$0.0 \quad 0.0$

$4.52+0 \quad 3.49+0$

$2.69+0$

$4.45+0$

$2.74-1$

0.0

0.0

$1.13+0$

0.0

0.0

0.0

$1.12-2$

0.0

$2.84+0$

$4.58+0$

$0.0 \quad 0.0$

$1.61+0$

$1.29+0$

9.99-1

0.0

0.0

0.0

$2.13+0$

$0.0 \quad 0.0$

0.0

$1.94+0$

0.0

0.0

$3.71-2 \quad 2.82-2$

0.0

0.0

$3.31+$
0.0

0.0

0.0

0.0

$9.94-3$
$4.11-3$

$1.56-2 \quad 1.39-2$

$2.97-3$

$3.95-3$

$2.96-2$

$6.76-3$

$3.25-2 \quad 2.57-2$

$3.1-3$

$\begin{array}{lll}2.66-3 & 1.32-2 & 3.7-3\end{array}$

$8.89-3$

0.0

0.0

0.0

$3.96-3$

2.6-2

$6.44-3$

$8.89-3$
0.0

$3.1-2 \quad 2.32-2$

$2.1-3$

0.0

$0.0 \quad 0.0$

0.0

0.0

0.0

$2.96-3$

$2.48-2$

$5.29-3$

$8.17-3$

0.0

0.0

0.0

0.0

0.0

$8.56-1 \quad 1.62+0$

$4.94-1$

$5.04-1$

2.5-1

0.0

0.0

0.0

0.0

0.0

0.0

0.0

$5.23-1$

0.0

0.0

0.0

6.5-1 $\quad 1.12+0 \quad 4.54-1$.

$4.5-1$

$6.11-15.16-1$

$3.97-1$

0.0

$2.98+0$

$1.85+0$

$7.58-1 \quad 1.46+0$

4.5-1

4.75-1

$6.6-1 \quad 5.47-1$

4.75-1

0.0

$\begin{array}{cc}0.0 & 0.0 \\ 3.3-2 & 2.4 \overline{2}-2\end{array}$

0.0

0.0

0.0

0.0

$2.69+0 \quad 4.36+0$

$2.1-3$
$2.02+0$

$3.0-3$

$2.63-2 \quad 5.47-3$

$8.65-3$

$8.65-3$
$1.84+0$

$1.29-1$

0.0

$3.84-1$

$1.31-1$

$1.94+0$

$3.12+0$

$2.33+0$

0.0

0.0

0.0

0.0

0.0

0.0

0.0

0.0

0.0

0.0

0.0

0.0

0.0

0.0

0.0

0.0

0.0

0.0

0.0

0.0

0.0

0.0

0.0

0.0

0.0

0.0
0.0

0.0

0.0

0.0

0.0

0.0

0.0

0.0

0.0

0.0

0.0

0.0

$3.56+0$

$5.49-3 \quad 2.98-1$

$4.1-3 \quad 1.13-1$

$5.63-3 \quad 2.56-1$

$0.0 \quad 0.0$

$3.96-3 \quad 2.53-1$

$0.0 \quad 0.0$

$0.0 \quad 0.0$ 
TABLE F-7 (Cont)

Total

Isotope Body Bone Liver Kidney Gonad Lung G.I.Tract Thyroid Skin

$\begin{array}{llllllllll}\text { Cf-249 } & 0.0 & 0.0 & 0.0 & 0.0 & 0.0 & 0.0 & 0.0 & 0.0 & 0.0 \\ \text { Cf-250 } & 0.0 & 0.0 & 0.0 & 0.0 & 0.0 & 0.0 & 0.0 & 0.0 & 0.0 \\ \text { Cf-251 } & 0.0 & 0.0 & 0.0 & 0.0 & 0.0 & 0.0 & 0.0 & 0.0 & 0.0 \\ \text { Cf-252 } & 0.0 & 0.0 & 0.0 & 0.0 & 0.0 & 0.0 & 0.0 & 0.0 & 0.0 \\ \text { Cf-253 } & 0.0 & 0.0 & 0.0 & 0.0 & 0.0 & 0.0 & 0.0 & 0.0 & 0.0 \\ \text { Cf-254 } & 0.0 & 0.0 & 0.0 & 0.0 & 0.0 & 0.0 & 0.0 & 0.0 & 0.0 \\ \text { Es-253 } & 0.0 & 0.0 & 0.0 & 0.0 & 0.0 & 0.0 & 0.0 & 0.0 & 0.0 \\ \text { Es-254m } & 0.0 & 0.0 & 0.0 & 0.0 & 0.0 & 0.0 & 0.0 & 0.0 & 0.0 \\ \text { Es-254 } & 0.0 & 0.0 & 0.0 & 0.0 & 0.0 & 0.0 & 0.0 & 0.0 & 0.0 \\ \text { Es-255 } & 0.0 & 0.0 & 0.0 & 0.0 & 0.0 & 0.0 & 0.0 & 0.0 & 0.0 \\ \text { Fm-254 } & 0.0 & 0.0 & 0.0 & 0.0 & 0.0 & 0.0 & 0.0 & 0.0 & 0.0 \\ \text { Fm-255 } & 0.0 & 0.0 & 0.0 & 0.0 & 0.0 & 0.0 & 0.0 & 0.0 & 0.0 \\ \text { Fm-256 } & 0.0 & 0.0 & 0.0 & 0.0 & 0.0 & 0.0 & 0.0 & 0.0 & 0.0\end{array}$


TABLE F-8. INGESTION DOSE COMMITMENT CONVERSION FACTORS $\left(\mathrm{rem} / \mu \mathrm{Ci}_{\mathrm{i}}\right)$

\begin{tabular}{|c|c|c|c|c|c|c|c|c|c|}
\hline Isotope & $\begin{array}{l}\text { Total } \\
\text { Body }\end{array}$ & Bone & Liver & Kidney & Gonad & Lung & $\begin{array}{c}\text { GI } \\
\text { Tract } \\
\end{array}$ & Thyroid & Skin \\
\hline$B c=7$ & $3.01=0$ & $2.73-8$ & $\dot{b} .21-6$ & $6.51-6$ & * & * & $9.7-4$ & * & 0.0 \\
\hline C-11 & 0.0 & 0.0 & 0.0 & 0.0 & 0.0 & 0.0 & 0.0 & 0.0 & 0.0 \\
\hline $\mathrm{N}-13$ & 0.0 & 0.0 & 0.0 & 0.0 & 0.0 & 0.0 & 0.0 & 0.0 & 0.0 \\
\hline $0-15$ & 0.0 & 0.0 & 0.0 & 0.0 & 0.0 & 0.0 & 0.0 & 0.0 & 0.0 \\
\hline$F-18$ & $7.27-5$ & $4.57-4$ & $*$ & * & $*$ & $*$ & $3.88-3$ & $*$ & 0.0 \\
\hline $\mathrm{Na}-22$ & $1.84-2$ & $*$ & * & $*$ & $*$ & $n$ & $6.47-2$ & * & 0.0 \\
\hline $\mathrm{Na}-24$ & $1.7-3$ & $*$ & $*$ & $*$ & * & * & $6.47-2$ & $*$ & 0.0 \\
\hline$M y-28$ & 0.0 & 0.0 & 0.0 & $\cap \cap$ & 0.0 & 0.0 & 0.0 & 0.0 & U.U \\
\hline Al-28 & $0.0^{\circ}$ & n & 0.0 & 0.0 & 0.0 & 0.0 & 0.0 & 0.0 & 0.0 \\
\hline Si-3l & $5.78-5$ & $*$ & $*$ & $*$ & $5.11-5$ & $4.28-4$ & $9.7-3$ & * & 0.0 \\
\hline P-32 & $7.31-3$ & $1.94-1$ & 1.19 & * & 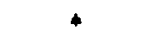 & * & $9.7-?$ & * & 0.0 \\
\hline 3-35 & $2.6-3$ & $6.69-3$ & * & * & $1.02-2$ & * & $6.47-3$ & * & 0.0 \\
\hline Cl-36 & $7.89-3$ & $*$ & * & * & $*$ & * & $3.23-2$ & * & 0.0 \\
\hline Cl-38 & $6.26-5$ & * & * & * & * & * & $4.85-3$ & * & 0.0 \\
\hline K -40 & 0.0 & 0.0 & 0.0 & 0.0 & 0.0 & 0.0 & 0.0 & 0.0 & 0.0 \\
\hline $\mathrm{K}-42$ & $8.71-4$ & * & $6.73-4$ & $*$ & $*$ & * & $9.7-2$ & * & 0.0 \\
\hline $\mathrm{K}-43$ & 0.0 & 0.0 & 0.0 & 0.0 & 0.0 & 0.0 & 0.0 & 0.0 & 0.0 \\
\hline $\mathrm{Ca}-45$ & $8.75-3$ & $3.94-1$ & * & * & * & * & $9.7-3$ & * & 0.0 \\
\hline $\mathrm{Ca}-47$ & $4.31-3$ & $7.2-2$ & $*$ & $*$ & $*$ & $*$ & $6.47-2$ & $*$ & 0.0 \\
\hline $\mathrm{Ca}-49$ & 0.0 & 0.0 & 0.0 & 0.0 & 0.0 & 0.0 & 0.0 & 0.0 & 0.0 \\
\hline Sc-46 & $2.99-6$ & $4.52-6$ & $1.03-5$ & $9.77-6$ & * & $*$ & $4.85-2$ & * & 0.0 \\
\hline $\mathrm{Sc}-47$ & $8.44-8$ & $5.78-7$ & $4.21-7$ & $3.23-7$ & * & * & $2.16-2$ & * & 0 \\
\hline Sc -48 & $3.93-7$ & 5.77 & $1.21=6$ & $7.48-1$ & $*$ & * & $6.47-2$ & $*$ & 0.0 \\
\hline Sc-49 & 0.0 & 0.0 & 0.0 & 0.0 & 0.0 & 0.0 & 0.0 & 0.0 & 0.0 \\
\hline$V-48$ & $4.6 l-4$ & $5.07-4$ & $6.1-4$ & $1.81-3$ & * & $*$ & $6.47-2$ & $*$ & 0.0 \\
\hline Cr-3l & $.3 .48-6$ & * & * & $1.01-6$ & * & $5.46-6$ & $9.7-4$ & $3.69-6$ & 0.0 \\
\hline $\mathrm{Mn} \cdot 52 \mathrm{~m}$ & 0.0 & 0.0 & 0.0 & 0.0 & 0.0 & 0.0 & 0.0 & 0.0 & 0.0 \\
\hline $\mathrm{Mn}-52$ & $9.23-4$ & * & $3.73-3$ & * & $*$ & $*$ & $6.47-2$ & $*$ & 0.0 \\
\hline $\mathrm{Mn}-54$ & $8.65-4$ & * & $4.56-3$ & * & * & * & $1.94-2$ & * & 0.0 \\
\hline$M n-56$ & $2.19-5$ & $*$ & $1.23-4$ & * & $*$ & $*$ & $1.94-2$ & * & 0.0 \\
\hline $\mathrm{Fe}-52$ & 0.0 & 0.0 & 0.0 & 0.0 & 0.0 & 0.0 & 0.0 & 0.0 & 0.0 \\
\hline $\mathrm{Fe}-55$ & $3.15-4$ & $4.53-4$ & $1.34-3$ & $*$ & $*$ & $7.8-4$ & $9.7-4$ & $*$ & 0.0 \\
\hline $\mathrm{Fe}-59$ & $3.63-3$ & $*$ & $9.83-3$ & $*$ & * & * & $5.17-2$ & • & 0.0 \\
\hline Co-56 & 0.0 & 0.0 & 0.0 & 0.0 & 0.0 & 0.0 & 0.0 & 0.0 & 0.0 \\
\hline Co-57 & $2.6-4$ & * & $1.47-4$ & $6.07-5$ & • & 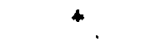 & $4.85-3$ & * & 0.0 \\
\hline Co-58m & $1.15-5$ & * & $6.59-6$ & $2.6-6$ & $*$ & * & $9.7-4$ & * & 0.0 \\
\hline Co-58 & $1.6-3$ & * & $7.38-4$ & $1.11-3$ & * & * & $3.06 \cdots 2$ & - & 0.0 \\
\hline Co-60 & $4.49-3$ & * & $2.07-3$ & $7.8-4$ & $*$ & $*$ & $8.02-2$ & $*$ & 0.0 \\
\hline $\mathrm{Ni}-56$ & 0.0 & 0.0 & 0.0 & 0.0 & 0.0 & 0.0 & 0.0 & 0.0 & 0.0 \\
\hline $\mathrm{Ni}-59$ & $1.61-3$ & $9.67-3$ & $3.32-3$ & $*$ & $*$ & $*$ & $9.7-4$ & $*$ & 0.0 \\
\hline $\mathrm{Ni}-63$ & $4.3-3$ & $1.35-1$ & $8.91-3$ & * & * & * & $2.77-3$ & * & 0.0 \\
\hline $\mathrm{Ni}-65$ & $4.84-5$ & $9.16-4$ & $1.14-4$ & $*$ & * & * & $1.94-2$ & * & 0.0 \\
\hline $\mathrm{Cu}-64$ & $3.88-5$ & 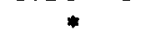 & $8.68-5$ & $2.12-4$ & $*$ & * & $9.7-3$ & * & 0.0 \\
\hline Zn-65 & $6.5-3$ & $3.04-3$ & $1.49-2$ & $1.0-2$ & $1.52-3$ & - & $9.7-3$ & * & 0.0 \\
\hline $\mathrm{Zn}-69 \mathrm{~m}$ & $3.89-5$ & $1.91-4$ & $4.38-4$ & $2.66-4$ & $9.14-5$ & * & $3.23-2$ & * & 0.0 \\
\hline $\mathrm{Zn-69}$ & $1.39-6$ & $1.07-5$ & $2.01-5$ & $1.35-5$ & $4.88-6$ & $*$ & $9.7-4$ & $*$ & 0.0 \\
\hline Ga-67 & 0.0 & 0.0 & 0.0 & 0.0 & 0.0 & 0.0 & 0.0 & 0.0 & 0.0 \\
\hline Ga-68 & 0.0 & 0.0 & 0.0 & 0.0 & 0.0 & 0.0 & 0.0 & 0.0 & 0.0 \\
\hline
\end{tabular}

- Data nol available. Use of Total Body Factor is recommended. 
TABLE $F-8$ (Cont)

Total

Isotope

Ga-72

Ge-71

As-73

As-74

As-76

As-77

Se-75

Se-79

$\mathrm{Br}-80 \mathrm{~m}$

$\mathrm{Br}-80$

$\mathrm{Br}-82 \mathrm{~m}$

$\mathrm{Br}-82$

$\mathrm{Br}-83$

$\mathrm{Br}-84 \mathrm{~m}$

$\mathrm{Br}-84$

$\mathrm{Br}-85$

$\mathrm{Br}-86$

Br-87

Kr-79

$\mathrm{Kr}-83 \mathrm{~m}$

$\mathrm{Kr}-85 \mathrm{~m}$

$\mathrm{Kr}-85$

$\mathrm{Kr}-87$

$\mathrm{Kr}-88$

Kr-89

$\mathrm{Kr}-90$

$\mathrm{Rb}-84$

$\mathrm{Rb}-86$

$\mathrm{Rb}-87$

$\mathrm{Rb}-88$

$\mathrm{Rb}-89$

$\mathrm{Rb}-90$

$\mathrm{Sr}-85 \mathrm{~m}$

$\mathrm{S}-85$

Sr $-87 \mathrm{~m}$

$\mathrm{S}$ - 89

$\mathrm{Sr}-90$

Sr-91

Sr-92

Y -87

Y-88

Y -90

Y $-91 \mathrm{~m}$

Y-91

Y -92

Y-93

Z $\mathrm{r}-93$

$\mathrm{Zr}-95$

\section{Body}

$1.02-6$

$9.63-8$

1.15-4

$2.9-4$

4.49-5

$1.21-5$

$1.9-3$

0.0

1.38-4

$2.4-6$

0.0

2.46-3

2.95-5

6.8-7

$1.42-5$

$1.1-7$

0.0

0.0

0.0

6.7-7

$5.8-6$

4. $6-6$

2.4-5

$5.6-5$

$1.0-5$

0.0

0.0

9.67-3

4. $24-3$

$3.57-5$

$4.56-6$

$2.8-7$

$6.46-6$

$6.71-3$

0.0

$8.69-3$

$1.69-1$
$2.64-4$
$8.98-5$

$8.98-5$

0.0

0.0

$2.48-1$

$3.41-9$

$3.6-6$

$2.51-8$

$7.48-8$

$1.04-6$

6. $39-6$

Bone

Liver Kidney Gonad Lung

$4.57-6$

$6.28-6$

$1.07-4$
1.51

$2.39-6$

$2.51-4$

4.69-5

$1.49-5$

$4.86-3$

$2.39-6$
$4.4-6$
$.77-4$

4. $24-4$

8.87-5

$2.81-5$

0.0

0.0

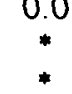

0.0

$*$
$*$
$*$
$*$
$*$
$*$
0.0
$*$
0.0

0.0

0.0

0.0

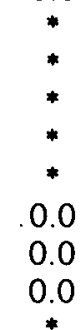

*

$*$

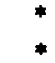

* 0.0

*

G.I.

Tract Thyroid Skin

$4.85-2$

$9.7-4$

$3.88-3$

$3.88-2$

$9.7-2$

2.43-2

6.47-3

0.0

$8.4-4$

0.0

$3.84-2$

$2.09-3$

4.5-4

$4.47-3$

$2.4-4$

0.0

0.0

0.0

0.0

0.0

$0.0 \cdot \quad 0.0$

$0.0 \quad 0.0$

0.0

$6.7-5$

$5.8-4$

4.6-4

$2.4-3$

$5.6-3$

$*$
$* \quad 1.2-5$
$* \quad 2.8-4$

$7.7-6$

$4.0-5$

* $\quad 5.0-5$

9.3-5

0.0

0.0

1.7-5

$1.0-3$

0.0

0.0

0.0

0.0

0.0

0.0

0.0

0.0

9.7-2

$9.7-3$

4.85-3

$1.94-3$

$5.0-4$

$6.9-7$

$2.77-4$

$9.7-3$

0.0

9.9-2

$3.84-2$

$3.88-2$

$3.23-2$

$\begin{array}{llll}0.0 & 0.0 & 0.0 & 0.0\end{array}$

0.0

0.0

1.17-1

6.47-4

$1.06-1$

3.23-2

6.47-2

2.43-3

$3.23-2$

.

0.0

0.0

0.0

0.0

0.0

0.0

0.0

0.0

0.0

0.0

$\begin{array}{ll}0.0 & 0.0\end{array}$

* $\quad 0.0$

* $\quad 0.0$

* $\quad 0.0$

* $\quad 0.0$

$-\quad 0.0$
$-\quad 0.0$

$\begin{array}{ll}0.0 & 0.0\end{array}$

$0.0 \quad 0.0$

$\begin{array}{ll}0.0 & 0.0\end{array}$

$*$
$*$

* $\quad 0.0$

* $\quad 0.0$

* $\quad 0.0$

* 0.0

$*$
$*$

$\begin{array}{ll}0.0 & 0.0\end{array}$

$0.0 \quad 0.0$

* $\quad 0.0$

* $\quad 0.0$

* $\quad 0.0$

* $\quad 0.0$

* $\quad 0.0$

* $\quad 0.0$

* $\quad 0.0$

$\begin{array}{ll}0.0 & 0.0\end{array}$

* $\quad 0.0$

$*$
$*$

* 0.0

* 0.0

$\begin{array}{ll}0.0 & 0.0\end{array}$

$\begin{array}{ll}0.0 & 0.0\end{array}$

* $\quad 0.0$

* $\quad 0.0$

*

* $\quad 0.0$

* $\quad 0.0$

* $\quad 0.0$

*Data not available. Use of Total Body Factor is recommended. 
TABLE F-8 (Cont)

\begin{tabular}{|c|c|c|c|c|c|c|c|c|c|}
\hline Isotope & $\begin{array}{l}\text { Total } \\
\text { Body }\end{array}$ & Bone & Liver & Kidney & Gonad & Lung & $\begin{array}{c}\text { G.I. } \\
\text { Tract }\end{array}$ & Thyroid & Skin \\
\hline Zr-97 & $1.56-7$ & $1.66-6$ & $3.43-7$ & $5.2-7$ & * & * & $9.7-2$ & * & 0.0 \\
\hline $\mathrm{Nb}-93 \mathrm{~m}$ & $2.51-6$ & $3.76-5$ & $1.01-5$ & $1.19-5$ & * & $*$ & $4.85-3$ & * & 0.0 \\
\hline $\mathrm{Nb}-95 \mathrm{~m}$ & $2.54-7$ & $5.95-7$ & $4.63-7$ & $4.56-7$ & * & * & $2.43-2$ & * & 0.0 \\
\hline Nb-95 & $1.79-6$ & $4.98-6$ & $3.39-6$ & $3.27-6$ & * & * & $1.94-2$ & * & 0.0 \\
\hline $\mathrm{Nb}-97 \mathrm{~m}$ & 0.0 & 0.0 & 0.0 & 0.0 & 0.0 & 0.0 & 0.0 & 0.0 & 0.0 \\
\hline $\mathrm{Nb}-97$ & $4.65-9$ & $4.87-8$ & $1.27-8$ & $1.49-8$ & $*$ & $*$ & $2.16-3$ & $*$ & 0.0 \\
\hline Mo-99 & $8.14-4$ & * & $4.31-3$ & $1.01-2$ & * & $*$ & $4.85-2$ & * & 0.0 \\
\hline l'c-96m & $5.65-6$ & $1.47-1$ & $1.4-b$ & $1.85-5$ & * & $4.51-1$ & $1.94-4$ & * & 0.0 \\
\hline Tc-96 & $5.86-4$ & $1.36-5$ & $1.57-4$ & $2.01-3$ & * & $4.85-5$ & $3.88-2$ & * & 0.0 \\
\hline Tc-97m & $4.66-5$ & $7: 75-5$ & $1.34-4$ & $1.76-3$ & $*$ & $1.39-5$ & $9.7-3$ & $*$ & 0.0 \\
\hline Tc-97 & $1.05-5$ & $4.97-6$ & $3.88-5$ & $4.89-4$ & * & $3.3-6$ & $2.43-3$ & + & 0.0 \\
\hline Tc-99m & $8.38-6$ & $5.23-8$ & $5.66-7$ & $7.94-6$ & $*$ & $2.77-7$ & $6.47-4$ & $*$ & 0.0 \\
\hline Tc-99 & 0.0 & 0.0 & 0.0 & 0.0 & 0.0 & 0.0 & 0.0 & 0.0 & 0.0 \\
\hline$R_{u}-103 m$ & 0.0 & 0.0 & 0.0 & 0.0 & 0.0 & 0.0 & 0.0 & 0.0 & 0.0 \\
\hline$R u-103$ & $8.57-5$ & $1.87-4$ & $*$ & $7.74-4$ & $*$ & $*$ & $2.43-2$ & $*$ & 0.0 \\
\hline Ru-105 & $7.16-6$ & $1.67-5$ & * & $2.22-4$ & * & * & $1.94-2$ & * & 0.0 \\
\hline Ru-106 & $3.17-4$ & $2.46-3$ & $*$ & $4.73-3$ & * & * & $2.37-1$ & * & 0.0 \\
\hline Rh-103m & $4.38-7$ & $7.56-7$ & $7.21-7$ & $3.01-6$ & * & * & $1.94-4$ & * & 0.0 \\
\hline Rh-105 & $5.57-5$ & $1.38-4$ & $9.17-5$ & $4.01-4$ & * & * & $1.94-2$ & * & 0.0 \\
\hline Rh-106 & 0.0 & 0.0 & 0.0 & 0.0 & 0.0 & 0.0 & 0.0 & 0.0 & 0.0 \\
\hline Pd-103 & $5.23-5$ & $*$ & $4.89-4$ & $3.28-3$ & * & $*$ & $6.47-3$ & * & 0.0 \\
\hline Pतू-107 & 0.0 & 00 & 0.0 & 0.0 & 0.0 & 0.0 & 0.0 & 0.0 & 0.0 \\
\hline Pd-109 & $4.49-5$ & * & $1.99-4$ & $1.15-3$ & $*$ & $*$ & $2.77-2$ & * & 0.0 \\
\hline Ag-105 & $2.9-5$ & $1.42-5$ & $4.13-5$ & $8.6-5$ & * & * & $1.94-2$ & * & 0.0 \\
\hline $\mathrm{Ag}-110 \mathrm{~m}$ & $8.72-5$ & $1.55-4$ & $1.54-4$ & $3.18-4$ & $8.72-5$ & * & $6.47-2$ & * & 0.0 \\
\hline$\Lambda g-111$ & $1.26-5$ & $5.65=5$ & $2.46-5$ & $7.23=5$ & * & * & $4.86-2$ & * & 0.0 \\
\hline Cd-109 & $4.03-5$ & * & $1.15-3$ & $1.1-3$ & * & $*$ & $9.7-3$ & $*$ & 0.0 \\
\hline $\mathrm{Cd}-113$ & 0.0 & 0.0 & 0.0 & 0.0 & 0.0 & 0.0 & 0.0 & 0.0 & 0.0 \\
\hline $\mathrm{Cd}-115 \mathrm{~m}$ & $5.59-5$ & $*$ & $1.75-3$ & $1.42-3$ & $*$ & * & $6.47-2$ & $*$ & 0.0 \\
\hline Cd-115 & $4.09-6$ & * & $1.05-4$ & $7.52-5$ & * & * & $4.85-2$ & * & 0.0 \\
\hline In-113m & $4.43-8$ & $1.77-7$ & $1.85-7$ & $2.71-7$ & * & * & $1.94-3$ & $3.24-8$ & 0.0 \\
\hline $\mathrm{In}-114 \mathrm{~m}$ & $4.87-5$ & $4.16-4$ & $2.95-4$ & $4.91-4$ & $*$ & * & $9.7-2$ & $1.94-5$ & 0.0 \\
\hline In-115m & $1.03-7$ & $5.01-7$ & $4.59-7$ & $7.05-7$ & * & $*$ & $4.85-3$ & $8.91-8$ & 0.0 \\
\hline In-1 15 & $1.71-5$ & $1.73-4$ & $1.19-4$ & $1.99-4$ & * & * & $2.16-2$ & $4.19-6$ & 0.0 \\
\hline Sn-113 & $4.52-4$ & $7.77-3$ & $2.13-4$ & $*$ & $t$ & * & $3.35-3$ & $1.26-4$ & 0.0 \\
\hline Sn-119m & $9.8-5$ & $3.89-4$ & $4.47-5$ & $*$ & $*$ & $*$ & $6.69-2$ & $1.7-5$ & 0.0 \\
\hline Sn-121 & 0.0 & 0.0 & 0.0 & 0.0 & 0.0 & 0.0 & 0.0 & 0.0 & 0.0 \\
\hline Sn-123 & $7.13-1$ & $3.02-2$ & $5.03-1$ & * & $*$ & $*$ & $3.13=2$ & $4.38=4$ & 0.0 \\
\hline $\mathrm{Sn}-125$ & $3.69-4$ & $8.75-3$ & $1.7-4$ & $*$ & $*$ & $*$ & $9.7-2$ & $1.43-4$ & 0.0 \\
\hline Sn-126 & $0: 0$ & 0.0 & 0.0 & 0.0 & 0.0 & 0.0 & 0.0 & 0.0 & 0.0 \\
\hline Sb-122 & $6.7-5$ & $2.29-4$ & $4.51-6$ & $*$ & $*$ & $1.19-4$ & $6.47-2$ & $3.11-6$ & 0.0 \\
\hline Sb-124 & $1.16-3$ & $2.75-3$ & $5.47-7$ & * & * & $2.31-3$ & $9.7-2$ & $7.14-6$ & 0.0 \\
\hline Sb-125 & $4.07-4$ & $1.75-3$ & $2.14-5$ & * & $*$ & $1.37-3$ & $1.94-2$ & $1.85-6$ & 0.0 \\
\hline Sb-126 & 0.0 & 0.0 & 0.0 & 0.0 & 0.0 & 0.0 & 0.0 & 0.0 & 0.0 \\
\hline Sb-127 & 0.0 & 0.0 & 0.0 & 0.0 & 0.0 & 0.0 & 0.0 & 0.0 & 0.0 \\
\hline Sb-128 & 0.0 & 0.0 & 0.0 & 0.0 & 0.0 & 0.0 & 0.0 & 0.0 & 0.0 \\
\hline Sb-129 & 0.0 & 0.0 & 0.0 & 0.0 & 0.0 & 0.0 & 0.0 & 0.0 & 0.0 \\
\hline $\mathrm{Te}-123 \mathrm{~m}$ & 0.0 & 0.0 & 0.0 & 0.0 & 0.0 & 0.0 & 0.0 & 0.0 & 0.0 \\
\hline
\end{tabular}

* Data not available. Use of Total Body Factor is recommended. 
TABLE $F-8$ (Cont)

\begin{tabular}{|c|c|c|c|c|c|c|c|c|c|}
\hline Isotope & $\begin{array}{l}\text { Total } \\
\text { Body }\end{array}$ & Bone & Liver & Kidney & Gonad & Lung & $\begin{array}{c}\text { G.I. } \\
\text { Tract } \\
\end{array}$ & Thyroid & Skin \\
\hline $\mathrm{Te}-125 \mathrm{~m}$ & $4.71-4$ & $2.46-3$ & $1.21-3$ & $1.37-2$ & $3.02-3$ & * & $1.94-2$ & $7.86-4$ & 0.0 \\
\hline $\mathrm{Te}-127 \mathrm{~m}$ & $1.09-3$ & $8.31-3$ & $3.17-3$ & $3.6-2$ & $9.8-3$ & * & $3.38-2$ & $2.28-3$ & 0.0 \\
\hline $\mathrm{Te}-127$ & $2.39-5$ & $1.13-4$ & $4.03-5$ & $4.57-4$ & $1.29-4$ & $*$ & $9.7-3$ & $8.13-5$ & 0.0 \\
\hline $\mathrm{Te}-129 \mathrm{~m}$ & $89-3$ & $1.23-2$ & $5.73-3$ & $6.11-2$ & $1.52-2$ & $*$ & $1.65-2$ & $4.43-3$ & 0.0 \\
\hline Te-129 & $1.31-5$ & $3.44-5$ & $1.61-5$ & $1.69-4$ & $4.21-5$ & * & $2.43-3$ & $2.8-5$ & 0.0 \\
\hline $\mathrm{Te}-131 \mathrm{~m}$ & $4.82-4$ & $7.51-4$ & $5.02-4$ & $4.75-3$ & 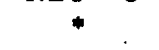 & * & $4.85-2$ & $6.95-4$ & 0.0 \\
\hline Te-131 & $1.3-6$ & $7.9-6$ & $2.3-6$ & $3.2-5$ & * & $*$ & $1.1-3$ & 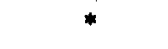 & 0.0 \\
\hline $\mathrm{Te}-1$ & $1.3-3$ & $2.17-3$ & $1.38-3$ & $1.36-2$ & $2.92-3$ & $*$ & $1.38-3$ & $1.63-3$ & 0.0 \\
\hline $\mathrm{Te}-1$ & 0.0 & 0.0 & 0.0 & 0.0 & 0.0 & 0.0 & 0.0 & 0.0 & 0.0 \\
\hline I- 123 & 0.0 & 0.0 & 0.0 & 0. & 0.0 & 0.0 & 0.0 & 0. & 0.0 \\
\hline I. & 0.0 & 0.0 & 0.0 & 0.0 & 0.0 & 0.0 & 0.0 & 0 . & 0.0 \\
\hline $\mathrm{I}-12$ & 0.0 & 0.0 & 0.0 & 0.0 & 0.0 & 0.0 & 0.0 & 0.0 & 0.0 \\
\hline I-126 & $2.91-3$ & $*$ & $*$ & * & $*$ & $*$ & $2.16-2$ & $2.06+0$ & 0.0 \\
\hline I- & $3-6$ & $2.1-5$ & $2.1-5$ & $3.8-5$ & * & * & $1.1-3$ & $*$ & 0.0 \\
\hline I. & $22-3$ & * & $*$ & 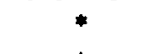 & * & * & $1.34-2$ & $5.17+0$ & 0.0 \\
\hline I- & $3-4$ & $*$ & $*$ & $*$ & $*$ & * & $7.52-2$ & & 0.0 \\
\hline I. & $51-3$ & * & * & $*$ & * & * & $4.09-2$ & +0 & 0.0 \\
\hline $\mathrm{I}-13$ & $1.28-4$ & * & $*$ & * & $*$ & * & $9.15-3$ & -2 & 0.0 \\
\hline I- & $48-4$ & * & * & * & * & * & $3.49-2$ & -1 & 0.0 \\
\hline 4 & $.52-5$ & $*$ & $*$ & $*$ & * & $*$ & $2.75-3$ & -2 & 0.0 \\
\hline $\mathrm{I}-13$ & $3.43-4$ & * & * & * & * & * & $3.33-2$ & $1.44-1$ & 0.0 \\
\hline & $7.4-8$ & $3.6-7$ & $3.6-7$ & $6.4-7$ & $*$ & $*$ & $3.2-4$ & $*$ & 0.0 \\
\hline I- & 0.0 & 0.0 & 0.0 & 0.0 & 0.0 & 0.0 & 0.0 & 0.0 & 0.0 \\
\hline $\mathrm{Xe}$ - & $3.0-6$ & $*$ & $1.5-5$ & $5.0-$ & $*$ & * & $3.0-4$ & $*$ & 0.0 \\
\hline $3 \mathrm{~m}$ & $4-5$ & * & $7.0-5$ & $2.3-5$ & * & * & $1.4-3$ & * & 0.0 \\
\hline $\mathrm{Xe}-$ & $5-6$ & * & $4.7-5$ & $1.6-5$ & $*$ & * & $9.5-4$ & * & 0.0 \\
\hline $35 \mathrm{~m}$ & $2.5-6$ & * & $1.3-5$ & $4.2-6$ & $*$ & * & $2.5-4$ & * & 0.0 \\
\hline & $7.6-6$ & * & $3.8-5$ & $1.3 \cdot-5$ & * & * & $7.6-4$ & * & 0.0 \\
\hline $\mathrm{X}$ & $7.6-6$ & $*$ & $3.8-5$ & $1.3-5$ & * & * & $7.6-4$ & $*$ & 0.0 \\
\hline$x$ & $1.0-5$ & $*$ & $5.0-5$ & $1.7-5$ & * & $*$ & $1.0-3$ & $*$ & 0.0 \\
\hline $\mathrm{Xe}-$ & 0.0 & 0.0 & 0.0 & 0.0 & 0.0 & 0.0 & 0.0 & 0.0 & 0.0 \\
\hline & 0.0 & 0.0 & 0.0 & & 0.0 & 0.0 & 0.0 & 0.0 & 0.0 \\
\hline $\mathrm{Cs}-134$ & $7.5-2$ & $4.98-2$ & $1.39-1$ & $4.5-2$ & 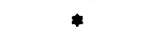 & * & $6.94-2$ & $*$ & 0.0 \\
\hline$s-135$ & $4.84-3$ & $1.93-2$ & $1.79-2$ & $6.77-3$ & $*$ & $2.03-3$ & $9.7-3$ & $*$ & 0.0 \\
\hline & $7.5-3$ & $3.6-3$ & $1.21-2$ & $7.1-3$ & * & $*$ & $4.11-2$ & * & 0.0 \\
\hline 37 & $4.33-2$ & $8.1-2$ & $1.1-1$ & $3.81-2$ & * & * & $6.23-2$ & * & 0.0 \\
\hline & $6.21-5$ & & 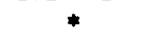 & & * & * & $4.85-3$ & $*$ & 0.0 \\
\hline 139 & $2.5-6$ & $7.1-6$ & $7.4-6$ & $5.7-6$ & $*$ & * & $1.3-3$ & * & 0.0 \\
\hline & $7.5-8$ & $1.0-7$ & $1.7-7$ & $1.2-7$ & $*$ & $*$ & $2.1-4$ & $*$ & 0.0 \\
\hline 31 & $1.95-4$ & $3.95-4$ & $2.83-6$ & $8.38-7$ & $*$ & $1.61-6$ & $9.7-3$ & $*$ & 0.0 \\
\hline & 0.0 & 0.0 & 0.0 & 0.0 & 0.0 & 0.0 & 0.0 & 0.0 & 0.0 \\
\hline $37 \mathrm{~m}$ & $1.2-9$ & $2.4-13$ & $3.1-11$ & $1.3-11$ & $*$ & $*$ & $2.5-5$ & * & 0.0 \\
\hline & $1.6-6$ & $7.7-5$ & $4.3-8$ & $1.8-8$ & * & * & $2.2-3$ & * & 0.0 \\
\hline & $1.29-3$ & $1.65-2$ & $2.29-5$ & $7.5-6$ & $*$ & $*$ & $5.49-2$ & $*$ & 0.0 \\
\hline & $3.34-7$ & $1.9-6$ & $1.19-6$ & $*$ & * & * & $9.7-2$ & $*$ & 0.0 \\
\hline & 0.0 & 0.0 & 0.0 & 0.0 & 0.0 & 0.0 & 0.0 & 0.0 & 0.0 \\
\hline $1-144$ & 0.0 & 0.0 & 0.0 & 0.0 & 0.0 & 0.0 & 0.0 & 0.0 & 0.0 \\
\hline Ce-14l & $6.6-7$ & $7.89-6$ & $5.63-6$ & $2.64-6$ & * & $*$ & $2.16-2$ & * & 0.0 \\
\hline
\end{tabular}

*Data not available. Use of Total Body Factor is recommended. 
TABLE F-8 (Cont)

Isotope

Ce-142

Ce-143

Ce-144

Pr-142

Pr-143

Pr-144

Pr-145

Nd-144

Nd-147

Nd-149

Pm-147

Pm-148m

Pm-148

Pm-149

Pm-151

Sm-147

Sm-148

Sm-149

$\mathrm{Sm}-151$

Sm-153

Eu-152

Eu-154

Eu-155

Eu-156

Eu-157

Gd-153

Gd-159

Gd-162

Tb-160

Tb-161

Dy-165

Dy-166

Ho-166

Er-169

Er-171

Tm-170

Yb-175

Lı-177

Hf-181

Ta-182

W-181

W-185

W-187

W-188

Re-183

Re-186

Re-187

Re-188
Total Body$$
3.0-7
$$

Bone

$\underline{\text { Liver Kidney }}$

Gonad

$4.59-5$

$7.12-8$

4.52-7

$3.5-10$

0.0

$1.31-3$

4.65-7

$9.56-9$

0.0

$4.83-4$

$31-6$

$9.9-9$

0.0

$5.5-2$

$5.75-6 \quad 6.9-6$

$2.77-6$

$1.43-7$

$6.08-6$

5.99-7

$7.31-5 \quad 6.84-6$

$1.24-7$

0.0

$7.1-6$

$1.58-3$

0.0

0.0

$6.32-$

0.0

0.0

0.0

$2.84-6$

$6.87-5 \quad 1.18-5$

$6.13-8$
$3.86-5$

$7.9-7 \quad 7.61-7$

$1.93-4 \quad 4.41-5$

$7.79-5$

$1.21-3 \quad 1.15-4$

$5.26-6$

$4.63-5$

0.0

0.0

0.0

0.0

$2.94-6$

$\begin{array}{llll}2.83-8 & 2.65-7 & 1.28-7\end{array}$

$$
0.0
$$

$5.87-6$

00

0.0

4.7

$4.7-5$

0.0

5. $18-9$

$2.81-7$

$9.14-8$

$\begin{array}{lll}8.06-8 & 2.51-6 & 1.96-7\end{array}$

$\begin{array}{llll}3.6-7 & 3.39-6 & 2.62-7\end{array}$

$2.11-8 \quad 3.89-5$

$3.81-6 \quad 1.31-4$

$5.87-8 \quad 1 . \overline{7} \overline{7}-6$

1. $19-7 \quad 36.5-6$

$2.25-6,5.0-6$

$8.75-6{ }^{*} 1.72-5$

$2.09-5 \quad 2.93-5$

$1.47-5$

$$
3.99-4
$$

$9.23-5$

$3.56-5$

$2.23-$

$3.35-4$

$4.97-4$

$1.71-4$

$4.4-5$

$1.14-5 \quad 3.38-4$

$3.15-4 \quad 1.21-4 \quad 1.25-4$
0.0

0.0

$2.05-4$

$5.33-7$

$21-4$

$5.59-7$

$3.17-7$

$3.64-6$

$2.11-6$

$7-9$

$1.6-9$

0.0

0.0

1.6-2

$1.77-7$

$4.2-6$

$9.83-8$

$1.29-5$

$7.77-6$

$1.18-5$

$1.19-6$

$2.14-6$

$4.51-7$

0.0

0.0

0.0

0.0

$7.37-3$

0.0

0.0

0.0

$1.32-5$

$2.38-7$

$1.07-5$

$2.06-4$

$6.57-4$

0.0

$2.66-5$

0.0

$2.0-6$

0.0

0.0

0.0

0.0

$*$

$\begin{array}{ll}0.0 & 0 \\ * & \\ * & \\ * & \\ 0.0 & \end{array}$

*

$0.0 \quad 0.0$

$0.0 \quad 0.0$

$0.0 \quad 0.0$

*

$0.0 \quad 0.0$

$0.0 \quad 0.0$

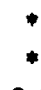

$\begin{array}{lll}0.0 & 0.0 & 0.0\end{array}$

$1.93-5$

0.0

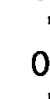

$3.71-\%$

$8.63-7$

$6.97-8$

$1.53-5$

$1.51-1$

$2.62 \cdot 7$

$5.25-6$

$2.42-6$

$2.9-5$

$6.37-5$
$8.78-5$

$1.38-4$

$9.11-5$

$8.75-4$

*

0.0

G.I.

Tract Thyroid Skin

$\begin{array}{lll}0.0 & 0.0 & 0.0\end{array}$

4.85-2 * 0.0

$2.37-1 \quad * \quad 0.0$

6.47-2 * 0.0

$3.88-2 * 0.0$

$1.3-3 \quad * \quad 0.0$

$\begin{array}{lll}0.0 & 0.0 & 0.0\end{array}$

$2.43-2 \quad * \quad 0.0$

3.23-2 * 0.0

$6.47-3 * 0.0$

$\begin{array}{lll}9.7-3 & * & 0.0\end{array}$

6.93-2 *

$1.02-1 \quad * \quad 0.0$

$4.85-2 * \quad * \quad 0.0$

$\begin{array}{lll}0.0 & 0.0 & 0.0\end{array}$

$2.77-2 \quad * \quad 0.0$

0.0

0.0

$4.85-3$

$2.43-2$

$2.43-2$

$9.7-2$

$9.7-3$

0.0

0.0

$2.43-2$

0.0

$4.85-2$

0.0

$4.85-3$

$4.85-2$

$6.47-2$

2. $16-2$.

$1.94-2$

$3.88-2$

$1.94-2$

$1.94-2$

$2.77-2$

4.85-2

$6.47-3$

$1.94-2$

3.23-2

$6.47-2$

$6.47-3$

$3.88-2$

$\begin{array}{lll}9.7-4 & 4.62-4 & 0.0 \\ 6.47-2 & 5.85-3 & 0.0\end{array}$

0.0

$0.0 \quad 0.0$

* $\quad 0.0$

* $\quad 0.0$

* $\quad 0.0$

* $\quad 0.0$

* $\quad 0.0$

$0.0 \quad 0.0$

$0.0 \quad 0.0$

* $\quad 0.0$

$0.0 \quad 0.0$

$\begin{array}{ll}* & 0.0 \\ 0 & 0.0\end{array}$

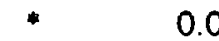

- $\quad 0.0$

* 0.0

* 0 n

* 0.0

* $\quad 0.0$

$+\quad 00$

- 0.0

* 0.0

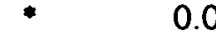

* 0.0

* $\quad 0.0$

- $\quad 0.0$

* 0.0

* $\quad 0.0$

"Data not available. Use of Total Body Factor is recommended. 
TABLE F-8.(Cont)

\begin{tabular}{|c|c|c|c|c|c|c|c|c|c|}
\hline Isotope & $\begin{array}{l}\text { Total } \\
\text { Body }\end{array}$ & Bone & Liver & Kidney & Gonad & Lung & $\begin{array}{c}\text { G.I. } \\
\text { Tract }\end{array}$ & Thyroid & Skin \\
\hline Os-185 & $1.07-4$ & * & $2.6-4$ & $1.47-3$ & * & * & $2.77-2$ & * & 0.0 \\
\hline Os $-191 \mathrm{~m}$ & $2.83-6$ & * & $4.39-6$ & $2.48-5$ & * & * & $9.7-4$ & * & 0.0 \\
\hline Os-191 & $3.01-5$ & $*$ & $8.48-5$ & $5.11-4$ & * & * & $9.7-3$ & $*$ & 0.0 \\
\hline Os-193 & $3.18-5$ & * & $6.55-5$ & $4.64-4$ & * & * & $3.88-2$ & $*$ & 0.0 \\
\hline Ir-190 & $2.91-4$ & * & $1.32-3$ & $1.28-3$ & * & * & $9.7-3$ & * & 0.0 \\
\hline Ir-192 & $1.82-3$ & * & $1.19-2$ & $1.65-2$ & $*$ & $*$ & $4.85-2$ & * & 0.0 \\
\hline $\mathrm{Pt}-191$ & $1.98-4$ & * & $2.78-5$ & $1.56-3$ & * & $*$ & $1.94-2$ & $*$ & 0.0 \\
\hline Pt-1.93m & $2.36-5$ & * & $3.53-6$ & $1.8-4$ & * & * & $1.94-3$ & $*$ & 0.0 \\
\hline Pt-193 & $1.08-4$ & * & $1.31-5$ & $2.05-3$ & * & $*$ & $9.7-4$ & $*$ & 0.0 \\
\hline Pt-197m & $3.23-6$ & $*$ & $9.85-7$ & $6.84-5$ & $*$ & $*$ & $2.16-3$ & $*$ & 0.0 \\
\hline Pt- 197 & $1.99-5$ & $*$ & $5.96-6$ & $4.16-4$ & $*$ & $*$ & $1.94-2$ & * & 0.0 \\
\hline Au-195m & 0.0 & 0.0 & 0.0 & 0.0 & 0.0 & 0.0 & 0.0 & 0.0 & 0.0 \\
\hline$A \dot{u}-195$ & 0.0 & 0.0 & 0.0 & 0.0 & 0.0 & 0.0 & 0.0 & 0.0 & 0.0 \\
\hline Au-196 & $2.6-4$ & * & $1.99-4$ & $6.05-4$ & $*$ & $*$ & $1.94-2$ & $*$ & 0.0 \\
\hline$A u-198$ & I. $58-4$ & $*$ & $2.05-4$ & $8.11-4$ & * & $*$ & $3.88-2$ & * & 0.0 \\
\hline Au-199 & $5.84-5$ & $*$ & $6.95-5$ & $2.73-4$ & $*$ & * & $9.7-3$ & * & 0.0 \\
\hline $\mathrm{Hg}-197 \mathrm{~m}$ & $2.14-4$ & * & $8.38-4$ & $1.07-2$ & $*$ & $*$ & $9.7-2$ & * & 0.0 \\
\hline $\mathrm{Hg}-197$ & $1.6-4$ & * & $5.67-4$ & $6.28-3$ & $*$ & * & $3.88-3$ & * & 0.0 \\
\hline $\mathrm{Hg}-203$ & $1.61-3$ & $*$ & $8.38-3$ & $1.05-1$ & * & * & $1.94-2$ & * & 0.0 \\
\hline Tl-200 & $1.73-4$ & $2.41-5$ & $1.43-4$ & $7.09-4$ & * & $3.38-5$ & $9.7-3$ & * & 0.0 \\
\hline Tl-201 & $1.52-4$ & $2.42-4$ & $1.97-4$ & $1.3-3$ & * & $4.75-5$ & $9.7-3$ & $*$ & 0.0 \\
\hline Tl-202 & $6.27-4$ & $1.08-3$ & $8.15-4$ & $5.93-3$ & * & $2.14-4$ & $2.77-2$ & * & 0.0 \\
\hline $\mathrm{Tl}-204$ & $5.89-4$ & $2.38-3$ & $1.08-3$ & $9.83-3$ & * & $2.97-4$ & $2.23-4$ & $*$ & 0.0 \\
\hline Tl-207 & $7.2-8$ & $3.0-7$ & $1.1-7$ & $8.1-7$ & $*$ & $*$ & $2.1-4$ & * & 0.0 \\
\hline Tl-208 & $3.5-8$ & $1.5-7$ & $5.4-8$ & $3.9-7$ & * & * & $1.6-4$ & * & 0.0 \\
\hline Tl-209 & $3.0-8$ & $1.3-7$ & $4.7-8$ & $3.4-7$ & $*$ & $*$ & $1.5-4$ & $*$ & 0.0 \\
\hline Tl-2 10 & $2.0-8$ & $2.9-7$ & $5.2-8$ & $1.8-7$ & * & * & $8: 0-5$ & $*$ & 0.0 \\
\hline $\mathrm{Pb}-203$ & $4.0-5$ & $2.32-5$ & $5.63-5$ & $3.64-4$ & * & * & $4.85-3$ & * & 0.0 \\
\hline $\mathrm{Pb}-204 \mathrm{~m}$ & $5.27-6$ & $3.05-6$ & $7.65-6$ & $5.05-5$ & $*$ & * & $4.85-2$ & * & 0.0 \\
\hline $\mathrm{Pb}-209$ & $2.4-6$ & $4.7-5$ & $7.6-6$ & $7.6-6$ & * & * & $8.8-3$ & * & 0.0 \\
\hline $\mathrm{Pb}-210$ & $5.1-1$ & $5.0+1$ & $4.7+0$ & $5.6 \cdot 1 \cdot 0$ & * & * & $3.5-3$ & $*$ & 0.0 \\
\hline $\mathrm{Pb}-21 \mathrm{l}$ & $6.3-5$ & $1.2-3$ & $2.0-4$ & $2.0-3$ & $*$ & * & $1.3-3$ & * & 0.0 \\
\hline $\mathrm{Pb}-212$ & $2.7-3$ & $5.4-2$ & $8.5-3$ & $8.6-2$ & * & * & $9.1-3$ & * & 0.0 \\
\hline $\mathrm{Pb}-214$ & $2.6-5$ & $5.1-4$ & $8.4-5$ & $8.3-4$ & * & * & $1.8-3$ & $*$ & 0.0 \\
\hline $\mathrm{Bi}-206$ & $5.28-5$ & $5.81-6$ & $2.33-4$ & $1.32-3$ & $*$ & * & $4.85-2$ & * & 0.0 \\
\hline $\mathrm{Bi}-207$ & $5.23-5$ & $9.95-6$ & $4.34-4$ & $1.45-3$ & * & * & $3.23-2$ & * & 0.0 \\
\hline $\mathrm{Bi}-210$ & $2.2-4$ & $8.7-4$ & $4.2-3$ & $3.9-2$ & *. & * & $6.1-2$ & * & 0.0 \\
\hline $\mathrm{Bi}-21 \mathrm{l}$ & $4.7-8$ & $9.9-8$ & $2.7-7$ & $3.2-6$ & $*$ & * & $1.4-4$ & * & 0.0 \\
\hline $\mathrm{Bi}-212$ & $1.7-5$ & $3.6-5$ & $1.0-4$ & $1.2-3$ & * & 中 & $3.4-3$ & * & 0.0 \\
\hline $\mathrm{Bi}-213$ & $1.4-5$ & $3.1-5$ & $8.4-5$ & $9.7-4$ & $*$ & $*$ & $2.0-3$ & * & 0.0 \\
\hline $\mathrm{Bi}-214$ & $1.4-6$ & $2.9-6$ & $8.0-6$ & $9.1-5$ & * & * & $1.8-3$ & $1.4-6$ & 0.0 \\
\hline Po-210 & $8.4-2$ & $3.8-1$ & $9.0-1$ & $4.6+0$ & $*$ & $*$ & $9.7-2$ & $*$ & 0.0 \\
\hline Po-2 12 & 0.0 & 0.0 & 0.0 & 0.0 & 0.0 & 0.0 & 0.0 & 0.0 & 0.0 \\
\hline Po-2.13 & 0.0 & 0.0 & 0.0 & 0.0 & 0.0 & 0.0 & 0.0 & 0.0 & 0.0 \\
\hline Po-2 16 & 0.0 & 0.0 & 0.0 & 0.0 & 0.0 & 0.0 & 0.0 & 0.0 & 0.0 \\
\hline Po-218 & $1.9-11$ & $1.1-10$ & $1.7-10$ & $5.7-10$ & $*$ & $*$ & $3.8-4$ & * & 0.0 \\
\hline At-21l & $1.92-2$ & $*$ & $*$ & $*$ & $3.35-1$ & $*$ & $2.77-2$ & $2.01+0$ & 0.0 \\
\hline At-2 17 & 0.0 & 0.0 & 0.0 & 0.0 & 0.0 & 0.0 & 0.0 & 0.0 & 0.0 \\
\hline
\end{tabular}

"Data not available. Use of Total Body Factor is recommended. 
TABLE F-8 (Cont)

\begin{tabular}{|c|c|c|c|c|c|c|c|c|c|}
\hline Isotope & $\begin{array}{l}\text { Total } \\
\text { Body }\end{array}$ & Bone & Liver & Kidney & Gonad & Lung & $\begin{array}{c}\text { G.I. } \\
\text { Tract } \\
\end{array}$ & Thyroid & Skin \\
\hline $\begin{array}{l}R n-220 \\
R n-222\end{array}$ & $\begin{array}{l}1.5-4 \\
2.0-3\end{array}$ & * & $\begin{array}{l}7.5-4 \\
10-2\end{array}$ & $\begin{array}{l}2.5-4 \\
33-3\end{array}$ & * & * & $\begin{array}{l}1.5-2 \\
2.0-1\end{array}$ & 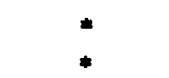 & $\begin{array}{l}0.0 \\
0.0\end{array}$ \\
\hline $\begin{array}{l}\text { Rn-22z } \\
\text { Ra-223 }\end{array}$ & $4.9-1$ & $1.3+1$ & $\begin{array}{l}1.0-2 \\
8.8-3\end{array}$ & $\begin{array}{l}3.5-3 \\
2.0-1\end{array}$ & * & * & $\begin{array}{l}2.0-1 \\
6.2-1\end{array}$ & * & $\begin{array}{l}0.0 \\
0.0\end{array}$ \\
\hline Ra-224 & $2.03-1$ & $1.6+0$ & $3.92-3$ & $1.11-1$ & * & * & $5.44-1$ & * & 0.0 \\
\hline Ra-225 & $13+0$ & $9.0+0$ & $8.1-3$ & $2.4-1$ & $*$ & * & $6.9-2$ & $*$ & 0.0 \\
\hline Ra-226 & $3.11+1$ & $3.7+2$ & 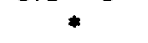 & 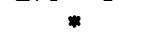 & * & * & $6.47-2$ & * & 0.0 \\
\hline Ra-228 & $1.66+1$ & $1.25+2$ & + & " & + & * & h $4 \%$ & * & 0.0 \\
\hline$\overline{\mathbf{F}}_{\mathrm{r}-2} 221$ & $1.3-5$ & $2.0-4$ & $2.0-4$ & $1.6-4$ & * & * & $9.7-4$ & $m^{\circ}$ & 0.0 \\
\hline$\overline{\mathrm{F}}_{\mathrm{r}}-223$ & $7.3-4$ & $2.0-3$ & $2.0-3$ & $1.6-3$ & * & * & $1.7-3$ & * & 0.0 \\
\hline A $\mathrm{c}-225$ & $2.9-4$ & $6.1-3$ & $5.6-3$ & $6.5-4$ & * & $*$ & $5.7-1$ & * & 0.0 \\
\hline$A c-227$ & $1.19-1$ & $2.07+0$ & $2.54-1$ & $8.61-2$ & - & * & $6.47-3$ & - & 0.0 \\
\hline$\AA_{c-228}$ & $6.26-6$ & $7.92-5$ & $3.14-5$ & $3.49-6$ & * & * & $2.16-2$ & * & 0.0 \\
\hline Th-227 & $3.85-4$ & $1.33-2$ & $2.42-4$ & $1.37-3$ & * & * & $9.7-2$ & * & 0.0 \\
\hline Th-228 & $1.7-2$ & $1.1+0$ & $8.0-3$ & $4.52-2$ & * & * & $9.8-2$ & * & 0.0 \\
\hline Th-229 & $3.5-1$ & $2.3+1$ & $1.5-1$ & $7.0-1$ & * & * & $9.1-2$ & $*$ & 0.0 \\
\hline Th-230 & $7.65-2$ & $2.77+0$ & $1.57-1$ & $7.13-1$ & * & * & $6.47-2$ & * & 0.0 \\
\hline Th-231 & $2.02-8$ & $4.39-7$ & $3.69-8$ & $1.83-7$ & * & * & $9.7-3$ & * & 0.0 \\
\hline Th-232 & $9.92-2$ & $2.72+1$ & $1.28-1$ & $5.92-1$ & * & * & $8.8-2$ & * & 0.0 \\
\hline Th-234 & $2.31-6$ & $1.12-4$ & $4.43-6$ & $2.57-5$ & * & * & $1.7-1$ & * & 0.0 \\
\hline $\mathrm{Pa}-230$ & $1.07-4$ & $2.67-3$ & 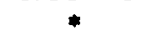 & $5.19-4$ & * & * & $9.7-3$ & * & 0.0 \\
\hline $\mathrm{Pa}-231$ & $2.11-1$ & $7.81+0$ & $1.97-1$ & $1.12+0$ & * & * & $1.0-1$ & * & 0.0 \\
\hline $\mathrm{Pa}-233$ & $9.22-7$ & $7.44-6$ & $1.01-6$ & $3.78-6$ & * & * & $2.5-1$ & * & 0.0 \\
\hline $\mathrm{Pa}-234 \mathrm{~m}$ & $1.7-12$ & $5.6-11$ & $3.5-12$ & $1.5-11$ & * & * & $9.3-5$ & * & 0.0 \\
\hline $\mathrm{Pa}-235$ & 0.0 & 0.0 & 0.0 & 0.0 & 0.0 & 0.0 & 0.0 & 0.0 & 0.0 \\
\hline $\mathrm{U}-230$ & $6.3-2$ & $4.04-1$ & $*$ & $8.18-1$ & $*$ & $*$ & $3.88-1$ & $*$ & 0.0 \\
\hline U-232 & $2.94+0$ & $5.77+i$ & 4 & $4.3 i+0$ & * & * & $1.03-1$ & - & 0.0 \\
\hline U-233 & $5.26-1$ & $1.21+1$ & * & $1.96+0$ & * & $*$ & $9.28-2$ & * & 0.0 \\
\hline $\mathrm{U}-234$ & $5.15-1$ & $1.17+1$ & * & $1.92+0$ & * & * & $9.28-2$ & * & 0.0 \\
\hline U-235 & $4.84-1$ & $1.12+1$ & * & $1.8+0$ & * & * & $8.85-2$ & $*$ & 0.0 \\
\hline$U=236$ & $4.94-1$ & $1.12+1$ & * & $1.84+0$ & * & * & $8.47-2$ & * & 0.0 \\
\hline U-237 & $9.29-5$ & $1.07-3$ & * & $2.43-3$ & * & * & $6.28-3$ & * & 0.0 \\
\hline U-238 & $4.52-1$ & $1.07+1$ & * & $1.68+0$ & * & * & $8.12-2$ & $*$ & 0.0 \\
\hline U-239 & $2.6-\%$ & $1.5-5$ & $*$ & $3.7-6$ & $*$ & * & $7.0-4$ & * & 0.0 \\
\hline Np-237 & $6.34-1$ & $2.32+1$ & $9.3+0$ & $1.19+0$ & $3.4-1$ & * & $321-2$ & * & 0.0 \\
\hline $\mathrm{Np}-238$ & $9.6-7$ & $2.9-5$ & $2.0-6$ & $6.5-6$ & * & * & $5.3-2$ & * & 0.0 \\
\hline $\mathrm{Np}-239$ & $7.07-8$ & $1.08-6$ & $1.11-7$ & $3.59-7$ & $*$ & * & $1.94-2$ & * & 0.0 \\
\hline $\mathrm{Np}-240 \mathrm{~m}$ & 0.0 & 0.0 & 0.0 & 0.0 & 0.0 & 0.0 & 0.0 & 0.0 & 0.0 \\
\hline $\mathrm{N}_{00}=240$ & $1.9-8$ & $6.1-7$ & $4.0=8$ & $1.3-7$ & 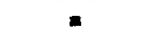 & $\because$ & $9.7-4$ & 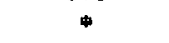 & 0.0 \\
\hline $\mathrm{Pu}-238$ & $1.75-2$ & $6.1-1$ & $2.61-1$ & $3.36-2$ & $1.06-2$ & * & $1.05-1$ & $*$ & 0.0 \\
\hline $\mathrm{Pu}-239$ & $2.06-2$ & $7.5-1$ & $3.0-1$ & $3.87-2$ & $1.11-2$ & * & $1.0-1$ & * & 0.0 \\
\hline $\mathrm{Pu}-240$ & $2.05-2$ & $7.5-1$ & $3.0-1$ & $3.86-2$ & $1.11-2$ & * & $1.0-1$ & * & 0.0 \\
\hline $\mathrm{Pu}-24 \mathrm{l}$ & $2.87-4$ & $1.2-2$ & $1.98-3$ & $6.41-4$ & $2.73-4$ & * & $1.95-3$ & * & 0.0 \\
\hline $\mathrm{Pu}-242$ & $1.98-2$ & $7.0-1$ & $2.89-1$ & $373-2$ & $1.07-2$ & * & $9.37-3$ & * & 0.0 \\
\hline $\mathrm{Pu}_{\mathrm{u}} 243$ & $2.42-9$ & $1.04-7$ & $1.01-8$ & $1.13-8$ & 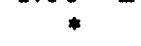 & * & $6.47-3$ & * & 0.0 \\
\hline $\mathrm{Pu}-244$ & $2.84-2$ & $1.15+0$ & * & $1.16-1$ & $*$ & * & $1.94-1$ & * & 0.0 \\
\hline Ám-24l & $7.04-1$ & $2.47+1$ & $1.03+1$ & $1.33+0$ & $3.9-1$ & * & $3.68-2$ & * & 0.0 \\
\hline$\AA \mathrm{m}-242 \mathrm{~m}$ & $6.86-1$ & $2.44+1$ & $9.5+0$ & $1.23+0$ & $3.7-1$ & * & $1.44-2$ & * & 0.0 \\
\hline
\end{tabular}

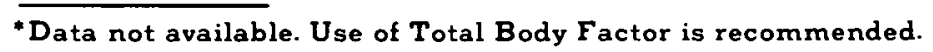


TABLE F-8 (Cont)

Total

Iscotope

Am-242

A $m-243$

Am-244

$\mathrm{C} \mathrm{m}-242$

Cm-243

Cm-244

$\mathrm{Cm}-245$

$\mathrm{Cm}-246$

Cm-247

$\mathrm{C} \mathrm{m}-248$

Cm-249

Bk-249

Bk-250

Cf-249

Cf-250

Cf-251

Cf-252

Cf-253

Cf-254

Es-253

Es-254 m

Es-254

Es-255

Fm-254

$\mathrm{Fm}-255$

Fm-256

Body
G.I.

Tract Thyroid Skin

$$
1.94-2
$$

$$
3.74-2
$$

$3.88-4$

4.0-2

3.94-2

$3.94-2$

$3.68-2$

$3.54-2$

$3.6 \mathrm{l}-2$

$3.35-2$

$9.7-4$

$1.71-3$

$9.7-3$

9.7-2

3.92-2

$6.47-2$

$1.38-1$

$1.94-2$

$1.94+1$

9.7-2

9.7-2

$1.94-1$

6.47-2

1.94-2

$6.47-2$

$2.16+0$
0.0

0.0

0.0

0.0

0.0

0.0

0.0

0.0

0.0

0.0

0.0

0.0

0.0

0.0

0.0

0.0

0.0

0.0

0.0

0.0

0.0

0.0

0.0

0.0

0.0

0.0

\footnotetext{
"Data not available. Use of Total Body Factor is recommended.
} 
TABLE F-9 Inhalation Dose Commitment Conversion Factors (rem $/ \mu \mathrm{Ci}$ )

\begin{tabular}{|c|c|c|c|c|c|c|c|c|c|}
\hline Isotope & $\begin{array}{l}\text { Total } \\
\text { Body }\end{array}$ & Bone & Liver & Kidney & Gonad & Lung & $\begin{array}{c}\text { G.I. } \\
\text { Tract } \\
\end{array}$ & Thyroid & Skin \\
\hline $\mathrm{Be}-7$ & $3.76-4$ & $3.42-4$ & $7.76-4$ & $8.13-4$ & $*$ & $5.43-3$ & $7.11-4$ & $*$ & 0.0 \\
\hline C-11 & 0.0 & 0.0 & 0.0 & 0.0 & 0.0 & 0.0 & 0.0 & 0.0 & 0.0 \\
\hline $\mathrm{N}-13$ & 0.0 & 0.0 & 0.0 & 0.0 & 0.0 & 0.0 & 0.0 & 0.0 & 0.0 \\
\hline $0-15$ & 0.0 & 0.0 & 0.0 & 0.0 & 0.0 & 0.0 & 0.0 & 0.0 & 0.0 \\
\hline F-18 & $5.45-5$ & $4.57-4$ & $*$ & * & * & $3.86-4$ & $2.37-3$ & $*$ & 0.0 \\
\hline $\mathrm{Na}-22$ & $1.38-2$ & $*$ & $\phi=$ & * & * & $7.81-1$ & $4.27-2$ & * & 0.0 \\
\hline $\mathrm{Na}-24$ & $1.27-3$ & $*$ & * & * & $*$ & $8.61-3$ & $4.27-2$ & $*$ & 0.0 \\
\hline $\mathrm{Mg} \cdot 38$ & 0.0 & 0.0 & 0.0 & 0.0 & 0.0 & 0.0 & 0.0 & 0.0 & 0.0 \\
\hline Al-28 & 0.0 & 0.0 & 0.0 & 0.0 & 0.0 & 0.0 & 0.0 & 0.0 & 0.0 \\
\hline $\mathrm{Si}-31$ & $4.62-5$ & $*$ & * & * & $4.04-5$ & $5.94-4$ & $7.11-3$ & • & 0.0 \\
\hline P-32 & $6.14-3$ & $1.65-1$ & $9.52-3$ & * & * & $8.08-2$ & $5.34-2$ & * & 0.0 \\
\hline S-35 & $1.95-3$ & $4.46-3$ & $*$ & * & $7.68-3$ & $2.59-2$ & $4.27-3$ & * & 0.0 \\
\hline Cl-36 & $5.92-3$ & $*$ & * & * & * & $2.86-1$ & $2.13-2$ & * & 0.0 \\
\hline $\mathrm{Cl}-38$ & $4.7-5$ & * & $*$ & $*$ & $*$ & $4.53-4$ & $2.13-2$ & $*$ & 0.0 \\
\hline $\mathrm{K}-40$ & 0.0 & 0.0 & 0.0 & 0.0 & 0.0 & 0.0 & 0.0 & 0.0 & 0.0 \\
\hline $\mathrm{K}-42$ & $6.53-4$ & $*$ & $5.04-4$ & $*$ & $*$ & $7.11-3$ & $5.34-2$ & $*$ & 0.0 \\
\hline $\mathrm{K}-43$ & 0.0 & 0.0 & 0.0 & 0.0 & 0.0 & 0.0 & 0.0 & 0.0 & 0.0 \\
\hline $\mathrm{Ca}-45$ & $8.02-3$ & $3.65-1$ & $*$ & $*$ & $*$ & $5.46-2$ & $7.11-3$ & $*$ & 0.0 \\
\hline $\mathrm{Ca}-47$ & $3.95-3$ & $6.67-2$ & • & $*$ & * & $3.49-2$ & $3.56-2$ & $*$ & 0.0 \\
\hline $\mathrm{Ca}-49$ & 0.0 & 0.0 & 0.0 & 0.0 & 0.0 & 0.0 & 0.0 & 0.0 & 0.0 \\
\hline $\mathrm{Sc}-46$ & $7.49-3$ & $1.13-2$ & $2.76-2$ & $2.44-2$ & $*$ & $2.89-1$ & $3.05-2$ & $*$ & 0.0 \\
\hline Sc-47 & $2.11-4$ & $1.44-3$ & $1.12-3$ & $8.06-4$ & * & $6.41-3$ & $1.07-2$. & * & 0.0 \\
\hline Sc-48 & $9.79-4$ & $1.42-3$ & $3.23-3$ & $1.87-3$ & $*$ & $1.82-2$ & $4.27-2$ & $*$ & 0.0 \\
\hline Sc -49 & 0.0 & 0.0 & 0.0 & 0.0 & 0.0 & 0.0 & 0.0 & 0.0 & 0.0 \\
\hline$V-48$ & $6.0-3$ & $7.24-3$ & $1.02-2$ & $2.26-2$ & * & $1.17-1$ & $4.27-2$ & + & 0.0 \\
\hline$C_{5}-51$ & $1.74-4$ & + & * & $5.3-5$ & * & $2.89-3$ & $2.11-4$ & $1.88-4$ & 0.0 \\
\hline$M n-52 m$ & 0.0 & 0.0 & 0.0 & 0.0 & 0.0 & 0.0 & 0.0 & 0.0 & 0.0 \\
\hline$M n-52$ & $2.77-3$ & * & $1.3-2$ & $*$ & $*$ & $1.02-1$ & $4.27-2$ & $*$ & 0.0 \\
\hline $\mathrm{Mn}-54$ & $2.59-3$ & * & $1.6-2$ & * & * & $1.81-1$ & $1.07 \div 2$ & $*$ & 0.0 \\
\hline Mn-56 & $6.57-5$ & * & $4.31-4$ & $*$ & $*$ & $1.31-3$ & $1.07-2$ & $*$ & 0.0 \\
\hline $\mathrm{Fe}-52$ & 0.0 & 0.0 & 0.0 & 0.0 & 0.0 & 0.0 & 0.0 & 0.0 & 0.0 \\
\hline $\mathrm{Fe}-55$ & $9.45-4$ & $1.36-3$ & $4.13-3$ & $*$ & $*$ & $6.44-3$ & $5.34-4$ & * & 0.0 \\
\hline$F_{0-59}$ & $1.96-2$ & 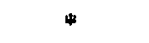 & $5.31-2$ & $*$ & $=$ & $3.41-1$ & $1.75-2$ & * & 0.0 \\
\hline Co-56 & 0.0 & 0.0 & 0.0 & 0.0 & 0.0 & 0.0 & 0.0 & 0.0 & 0.0 \\
\hline Co-57 & $3.47=4$ & * & $4.2-4$ & $8.09-5$ & $*$ & $4.03-2$ & $3.05-3$ & $*$ & 0.0 \\
\hline Co-58m & $1.53-5$ & * & $1.88-5$ & $3.47-6$ & * & $6.94-4$ & $5.34-4$ & * & 0.0 \\
\hline Co..58 & $2.95-3$ & * & $2.32-3$ & $2.03 \cdots 3$ & * & $7.18-1$ & $1.06-2$ & * & 0.0 \\
\hline Co-60 & $8.21-3$ & $*$ & $6.5-3$ & $1.4 .3-3$ & $*$ & $5.29+0$ & $3: 7-2$ & $*$ & 0.0 \\
\hline $\mathrm{Ni}-56$ & 0.0 & 0.0 & 0.0 & 0.0 & 0.0 & 0.0 & 0.0 & 0.0 & 0.0 \\
\hline $\mathrm{Ni}-59$ & $2.15-3$ & $1.29-2$ & $4.98-3$ & * & * & $8.47-3$ & $7.11-4$ & * & 0.0 \\
\hline $\mathrm{Ni}-63$ & $5.73-3$ & $1.79-1$ & $1.34-2$ & * & * & $2.3-2$ & $2.13-3$ & * & 0.0 \\
\hline $\mathrm{Ni}-65$ & $6.45-5$ & $1.22-3$ & $1.71-4$ & $*$ & $*$ & $1.21-3$ & $1.07-2$ & $*$ & 0.0 \\
\hline $\mathrm{Cu}-64$ & $5.41-5$ & $*$ & $1.3-4$ & $4.24-4$ & $*$ & $9.19-4$ & $5.34-3$ & * & 0.0 \\
\hline $\mathrm{Zn}-65$ & $1.95-2$ & $8.11-4$ & $4.7-2$ & $3.0-2$ & $4.56-3$ & $1.11-1$ & $7.11-3$ & $*$ & 0.0 \\
\hline $\mathrm{Zn}-69 \mathrm{~m}$ & $1.17-4$ & $5.74-4$ & $1.37-3$ & $7.99-4$ & $2.74-4$ & $2.62-3$ & $2.13-2$ & * & 0.0 \\
\hline Zn-69 & $4.18-6$ & $3.22-5$ & $6.32-5$ & $3.91-5$ & $1.46-5$ & $1.34-4$ & $7.11-4$ & * & 0.0 \\
\hline
\end{tabular}

*Data not available. Use of Total Body Factor is recommended. 
TABLE F-9 (Cont)

\begin{tabular}{|c|c|c|c|c|c|c|c|c|c|}
\hline Isotope & $\begin{array}{l}\text { Total } \\
\text { Body }\end{array}$ & Bone & Liver & Kidney & Gonad & Lung & $\begin{array}{c}\text { G.I. } \\
\text { Tract } \\
\end{array}$ & Thyroid & Skin \\
\hline Ga-67 & 0.0 & 0.0 & 0.0 & 0.0 & 0.0 & 0.0 & 0.0 & 0.0 & 0.0 \\
\hline Ga-68 & 0.0 & 0.0 & 0.0 & 0.0 & 0.0 & 0.0 & 0.0 & 0.0 & 0.0 \\
\hline Ga-72 & $2.54-4$ & $1.14-3$ & $1.58-3$ & $5.98-4$ & $*$ & $5.92-3$ & $3.56-2$ & * & 0.0 \\
\hline Ge-7l & $2.5-6$ & $*$ & $9.92-6$ & $1.17-4$ & * & $9.99-4$ & $7.11-4$ & $*$ & 0.0 \\
\hline As-73 & $1.03-3$ & * & $9.47-4$ & $1.59-3$ & * & $1.75-2$ & $2.67-3$ & * & 0.0 \\
\hline As-74 & $2.61-3$ & $*$ & $2.23-3$ & $3.81-3$ & * & $5.32-2$ & $2.37-2$ & $*$ & 0.0 \\
\hline As-76 & $4.04-4$ & * & $4.17-4$ & $7.98-4$ & * & $1.1-2$ & $7.11-2$ & * & 0.0 \\
\hline As-77 & $1.09-4$ & $*$ & $1.32-2$ & $2.53-4$ & * & $3.51-3$ & $2.13-2$ & * & 0.0 \\
\hline Se-75 & 0.0 & 0.0 & 0.0 & 0.0 & 0.0 & 0.0 & 0.0 & 0.0 & 0.0 \\
\hline Se-79 & 0.0 & 0.0 & 0.0 & 0.0 & 0.0 & 0.0 & 0.0 & 0.0 & 0.0 \\
\hline Br- $80 m$ & 0.0 & 0.0 & 0.0 & 0.0 & 0.0 & 0.0 & 0.0 & 0.0 & 0.0 \\
\hline $\mathrm{Br}-80$ & $6.0-7$ & $*$ & $*$ & $*$ & $*$ & $5.0-4$ & $4.3-5$ & $*$ & 0.0 \\
\hline $\mathrm{Br}-82 \mathrm{~m}$ & 0.0 & 0.0 & 0.0 & 0.0 & 0.0 & 0.0 & 0.0 & 0.0 & 0.0 \\
\hline $\mathrm{Br}-82$ & $1.13-3$ & $*$ & $*$ & $*$ & $*$ & $3.02-2$ & $9.56-3$ & $*$ & 0.0 \\
\hline $\mathrm{Br}-83$ & $7.5-6$ & * & * & * & * & $1.25-3$ & $2.38-4$ & * & 0.0 \\
\hline $\mathrm{Br}-84 \mathrm{~m}$ & $2.1-7$ & * & * & * & * & $3.5-4$ & $1.2-5$ & * & 0.0 \\
\hline $\mathrm{Br}-84$ & $5.36-6$ & * & * & * & * & $3.59-5$ & $2.95-4$ & * & 0.0 \\
\hline Br-85 & $3.8-8$ & * & $*$ & $*$ & $*$ & $1.1-4$ & $3.5-6$ & * & 0.0 \\
\hline $\mathrm{Br}-86$ & 0.0 & 0.0 & 0.0 & 0.0 & 0.0 & 0.0 & 0.0 & 0.0 & 0.0 \\
\hline $\mathrm{Br}-87$ & 0.0 & 0.0 & 0.0 & 0.0 & 0.0 & 0.0 & 0.0 & 0.0 & 0.0 \\
\hline Kr-79 & 0.0 & 0.0 & 0.0 & 0.0 & 0.0 & 0.0 & 0.0 & 0.0 & 0.0 \\
\hline $\mathrm{K}_{\mathrm{r}-83 \mathrm{~m}}$ & $7.1-9$ & $*$ & $*$ & $4.6-9$ & $5.8-9$ & $5.3-7$ & $*$ & $*$ & 0.0 \\
\hline $\mathrm{Kr}-85 \mathrm{~m}$ & $5.9-8$ & * & * & $3.7-8$ & $4.7-8$ & $4.3-6$ & * & $*$ & 0.0 \\
\hline $\mathrm{K}_{\mathrm{I}-85}$ & $4.4-8$ & * & * & $2.8-8$ & $3.6-8$ & $3.2-6$ & * & * & 0.0 \\
\hline $\mathrm{K}_{\mathrm{r}-87}$ & $2.8-7$ & * & * & $1.8-7$ & $2.3-7$ & $2.0-5$ & * & * & 0.0 \\
\hline $\mathrm{Kr}-88$ & $5.8-7$ & * & * & $3.7-7$ & $4.7-7$ & $4.3-5$ & * & * & 0.0 \\
\hline $\mathrm{Kr}-89$ & $6.5-7$ & * & * & $4.1-7$ & $5.3-7$ & $4.8-5$ & * & $*$ & 0.0 \\
\hline $\mathrm{K}_{\mathrm{r}-90}$ & 0.0 & 0.0 & 0.0 & 0.0 & 0.0 & 0.0 & 0.0 & 0.0 & 0.0 \\
\hline Rb-84 & 0.0 & 0.0 & 0.0 & 0.0 & 0.0 & 0.0 & 0.0 & 0.0 & 0.0 \\
\hline $\mathrm{Rb}-86$ & $7.26-3$ & * & $1.64-2$ & $*$ & $*$ & $9.78-2$ & $5.34-2$ & $*$ & 0.0 \\
\hline $\mathrm{Rb}-87$ & $3.18-3$ & * & $9.78-3$ & * & * & $9.89-2$ & $7.11-3$ & * & 0.0 \\
\hline $\mathrm{Rb}-88$ & $2.68-5$ & * & * & * & * & $2.91-4$ & $2.67-3$ & * & 0.0 \\
\hline $\mathrm{Rb}-89$ & $3.42-6$ & * & * & * & * & $2.43-5$ & $1.07-3$ & * & 0.0 \\
\hline $\mathrm{Rb}-90$ & $1.0-7$ & $*$ & $2.4-7$ & * & * & $2.3-4$ & $6.9-6$ & * & 0.0 \\
\hline $\mathrm{Sr}-85 \mathrm{~m}$ & $8.62-6$ & $1.72-5$ & $*$ & $*$ & $*$ & $7.18-5$ & $2.13-4$ & $*$ & 0.0 \\
\hline$S_{I}-85$ & $8.94-3$ & $1.73-2$ & ${ }^{*}$ & $*$ & $*$ & $6.18-2$ & $7.11-3$ & $*$ & 0.0 \\
\hline $\mathrm{S}_{\mathrm{r}}-87 \mathrm{~m}$ & 0.0 & 0.0 & 0.0 & 0.0 & 0.0 & 0.0 & 0.0 & 0.0 & 0.0 \\
\hline Sr-89 & $1.59-2$ & $5.69-1$ & * & $*$ & $*$ & $4.74-1$ & $4.36-2$ & * & 0.0 \\
\hline Sr-90 & $1.78+0$ & $2.67+1$ & * & * & * & $9.71+0$ & $1.88-2$ & * & 0.0 \\
\hline Sr-91 & $3.52-4$ & $7.27-3$ & " & * & * & $5.48-3$ & $2.37-2$ & * & 0.0 \\
\hline Sr-92 & $1.2-4$ & $2.58-3$ & $*$ & $*$ & $*$ & $2.01-3$ & $2.13-2$ & $*$ & 0.0 \\
\hline$Y-87$ & 0.0 & 0.0 & 0.0 & 0.0 & 0.0 & 0.0 & 0.0 & 0.0 & 0.0 \\
\hline Y-88 & 0.0 & 0.0 & 0.0 & 0.0 & 0.0 & 0.0 & 0.0 & 0.0 & 0.0 \\
\hline Y-90 & $1.15-3$ & $4.26-2$ & $*$ & $*$ & * & $3.51-2$ & $3.24-2$ & * & 0.0 \\
\hline Y-9lm & $8.52-6$ & $2.09-4$ & * & * & * & $1.76-4$ & $3.56-4$ & * & 0.0 \\
\hline Y-9l & $1.93-2$ & $7.08-1$ & * & * & * & $5.75-1$ & $3.6-2$ & * & 0.0 \\
\hline Y-92 & $6.28-5$ & $2.06-3$ & * & * & * & $2.06-3$ & $2.13-2$ & * & 0.0 \\
\hline Y-93 & $1.87-4$ & $5.43-3$ & $*$ & * & * & $5.75-3$ & $4.27-2$ & $*$ & 0.0 \\
\hline
\end{tabular}

*Data not available. Use of Total Body Factor is recommended. 
TABLE F-9 (Cont)

Total

Isotope

ZI-93

2r-95

Zr-97

$\mathrm{Nb}-93 \mathrm{~m}$

$\mathrm{Nb}-95 \mathrm{~m}$

$\mathrm{Nb}-95$

$\mathrm{Nb}-97 \mathrm{~m}$

Nb-97

Mo-99

Tc-96m

Tc-96

Tc-97 m

Tc-97

Tc-99m

Tc-99

Ru-103m

Ru-103

Ru-105

Ru-106

Rh-103m

Rh-105

Rh-106

Pd-103

Pd-10\%

Pd-109

Ag-105

Aq-1 10m

Ag-1 11

Cd-109

Cd-1 13

Cd-115m

Cd-115

In-113m

In-114m

In-115m

In-113

Sn-113

Sn-119m

Sn-12.1

$\mathrm{Sn}-123$

Sn-125

$\mathrm{Sn}-126$

Sb-122

$\mathrm{Sb}-124$

Sb-125

Sb-126

Sb-127

Sb-128

$6.45-5$

4.03-3
Body

$2.59-$

$1.5-$

$3.9-4$

$6.27-3$

$1.32-3$

$4.47-3$

0.0

1. $16-$

6.61-4

$5.65-$

$5.86-$

$4.66-5$

$1.05-5$

8. $38-6$

0.0

0.0

$7.71-4$

$5.68-3$

$7.66-7$

$$
0.0
$$

9.15-5

0.0

7.85-5

7.55-4

$2.27-3$

$3.27-$

$$
0.0
$$

5.59-

4.09-4

$5.54-6$

$6.09-3$

$1.29-5$

$2.14-3$

$2.53-$

5.49

$$
0.0
$$

4.16-3

$2.07-3$

0.0

6.03-4

$1.04-2$
$3.66-3$

$3.66-3$

0.0

0.0

0.0
Bone

$9.42-2$

$4.15-3$

$9.89-2$

$1.57-3$

0.0

$1.28-4$

$1.47-7$

1. $36-5$

$7.75-5$

$4.97-6$

$23-8$
0.0

0.0

$1.56-3$

$1.39-4$

4.4-2

$1.51-6$
$2.77-4$

0.0

0.0

0.0
$*$

3. $7-4$

$4.04-3$

$1.47-3$

0.0

$\begin{array}{cc}0.0 & 0.0 \\ * & 1.75-1\end{array}$

* $1.05-2$

$2.08-5 \quad 2.64-5$

$4.9-2$

$5.89-5 \quad 4.21-2$

$2.03-2$

$6.55-5$

$1.7-2$

1.19-3

3.11-2

$2.51-4$

0.0

00

$1.21-1 \quad 2.82-3$

$1.21-$

0.0

9.53-4

0.0

$2.29-3$

$3.75-5$

$2.75-2$

$1.75-$

$4.56-6$
$1.78-4$

0.0

0.0

0.0
0.0
Kidney Gonad Lung

\section{$2.42-2$}

$3.31-2$

$1.3-3$

$2.97-2$

1. $14-3$

$8.18-3$

$n \cap$

$3.74-5$

$8.43-3$

$1.85-5$

$2.01-3$

$1.76-3$

$4.89-4$

$7.94-6$

0.0

0.0

$6.45-3$

$1.85-3$

8.44-2

$5.01-6$

$6.68-4$

0.0

$4.92-3$

0.0

$1.72-3$

$2.15-3$

$7.94-3$

1.8] -3

1. $1-1$

0.0

$$
1.42-1
$$

$7.52 \cdots 3$

$3.39-5$

$6.13-2$

$8.82-5$

$2.48-2$

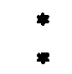

0.0

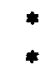

0.0
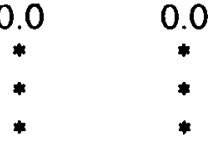

0.0

0.0

$*$
$*$
$*$
$*$
$*$
0.0
$*$
$*$
$*$
$*$
$*$
$*$
0.0
0.0

2.2-1

$1.03-2$

4.05-2

$1.07-2$

6.45-2

U. 0

$2.99-4$

$1.2-2$

$2.18-4$

$2.43-2$

$4.29 \cdots 2$

$2.2-2$

$8.06-5$

0.0

0.0

$7.56-2$

$1.58-3$

$5.48+0$

$1.91-5$

$2.61-3$

0.0

8. $59-3$

0.0

$2.18-3$

$7.97-2$

6.28-1

2.16-2

$8.78-2$

0.0

1.77-1

1.15-2

$1.4-4$

3. $0-1$

$3.48-4$

1.87-1

$1.22-1$

$2.82-2$

0.0

$2.92-1$

7.74-2

0.0

$1.68-2$

$3.39-1$

$2.42-1$

0.0

0.0
G.I.

Tract Thyroid Skin

0.0

2. $1.3-3$

$2.13-2$

7.11-2

$3.05-3$

$1.33-2$

$1.07-2$

0.0

$1.07-3$

$3.05-2$

$1.07-4$

$2.67-2$

$7.11-3$

$2.13-3$

$4.27-4$

0.0

0.0

2.13-2

$1.07-2$

9.48-2

$1.07-4$

$1.07-2$

0.0

4. $27-3$

0.0

2.13-2

$1.07-2$

4.27-2

2.67-2

7. $11-3$

$$
0.0
$$

5.34-2

$3.56-2$ 
TABLE F-9 (Cont)

\begin{tabular}{|c|c|c|c|c|c|c|c|c|c|}
\hline Isotope & $\begin{array}{l}\text { Total } \\
\text { Body }\end{array}$ & Bone & Liver & Kidney & Gonad & Lung & $\begin{array}{c}\text { G.I. } \\
\text { Tract }\end{array}$ & Thyroid & Skin \\
\hline Sb-129 & 0.0 & 0.0 & 0.0 & 0.0 & 0.0 & 0.0 & 0.0 & 0.0 & 0.0 \\
\hline $\mathrm{Te}-123 \mathrm{~m}$ & 0.0 & 0.0 & 0.0 & 0.0 & 0.0 & 0.0 & 0.0 & 0.0 & 0.0 \\
\hline $\mathrm{Te}-125 \mathrm{~m}$ & $7.16-4$ & $3.63-3$ & $2.41-3$ & $2.05-2$ & $4.43-3$ & $5.01-2$ & $2.13-2$ & $1.19-3$ & 0.0 \\
\hline $\mathrm{Te}-127 \mathrm{~m}$ & $1.65-3$ & $1.23-2$ & $6.35-3$ & $5.39-2$ & $1.44-2$ & $1.64-1$ & 2. $37-2$ & $3.47-3$ & 0.0 \\
\hline Te-127 & $3.63-5$ & $1.67-4$ & $8.07-5$ & $6.86-4$ & $1.89-4$ & $8.55-4$ & $7.11-3$ & $1.24-4$ & 0.0 \\
\hline Te-129m & $6.32-3$ & $2.64-2$ & $1.57-2$ & $1.17-1$ & $3.32-2$ & $4.87-1$ & $5.43-3$ & $9.7-3$ & 0.0 \\
\hline Te-129 & $1.99-5$ & $5.08-5$ & $3.21-5$ & $2.54-4$ & $6.17-5$ & $3.41-4$ & $2.13-3$ & $4.26-5$ & 0.0 \\
\hline $\mathrm{Te}-131 \mathrm{~m}$ & $7.32-4$ & $1.11-3$ & $1.0-3$ & $7.12-3$ & $*$ & $1.13-2$ & $3.56-2$ & $1.06-3$ & 0.0 \\
\hline $\mathrm{Te}-131$ & $1.3-6$ & $7.5-6$ & $2.2-6$ & $3.0-5$ & * & $8.7-4$ & $6.4-5$ & $*$ & 0.0 \\
\hline Te-132 & $2.49-3$ & $4.07-3$ & $3.31-3$ & $2.29-2$ & $5.6-3$ & $7.63-2$ & $3.86-3$ & $3.13-3$ & 0.0 \\
\hline Te-134 & 0.0 & 0.0 & 0.0 & 0.0 & 0.0 & 0.0 & 0.0 & 0.0 & 0.0 \\
\hline I -123 & 0.0 & 0.0 & 0.0 & 0.0 & 0.0 & 0.0 & 0.0 & 0.0 & 0.0 \\
\hline I- 124 & 0.0 & 0.0 & 0.0 & 0.0 & 0.0 & 0.0 & 0.0 & 0.0 & 0.0 \\
\hline I-125. & 0.0 & 0.0 & 0.0 & 0.0 & 0.0 & 0.0 & 0.0 & 0.0 & 0.0 \\
\hline I-l & $2.19-3$ & $*$ & * & $*$ & $*$ & $1.97-2$ & $1.07-2$ & $1.58+0$ & 0.0 \\
\hline I- 128 & $1.0-6$ & $5.1-6$ & $5.0-6$ & $9.3-6$ & * & $7.3-4$ & $6.5-5$ & $*$ & 0.0 \\
\hline I- 129 & $4.19-3$ & * & * & $*$ & * & $4.45-1$ & $7.1-3$ & $3.0+0$ & 0.0 \\
\hline I- 130 & $3.05-4$ & * & * & * & * & $1.28-2$ & $1.45-2$ & $9.64-2$ & 0.0 \\
\hline I-I & $1.93-3$ & * & * & * & * & $3.23-2$ & $1.35-2$ & $1.04+0$ & 0.0 \\
\hline I-132 & $3.44-5$ & * & * & * & * & $3.27-3$ & $1.05-3$ & $1.38-2$ & 0.0 \\
\hline I-133 & $3.12-4$ & * & * & * & * & $1.46-2$ & $7.78-3$ & $2.09-1$ & 0.0 \\
\hline & $7.19-6$ & * & * & * & * & $1.4-3$ & $2.2-4$ & $4.13-3$ & 0.0 \\
\hline I-I & $1.07-4$ & * & * & * & * & $6.59-3$ & $5.03-3$ & $4.49-2$ & 0.0 \\
\hline$I-136$ & $2.6-8$ & $1.3-7$ & $1.3-7$ & $2.4-8$ & * & $1.5-4$ & $2.5-6$ & * & 0.0 \\
\hline I-137 & 0.0 & 0.0 & 0.0 & 0.0 & 0.0 & 0.0 & 0.0 & 0.0 & 0.0 \\
\hline $\mathrm{Xe}-$ & $5.3-8$ & $*$ & $*$ & $*$ & $*$ & $2.2-6$ & $*$ & $*$ & 0.0 \\
\hline $\mathrm{Xe}-$ & $2.4-7$ & * & * & * & * & $9.7-6$ & * & * & 0.0 \\
\hline & $1.7-7$ & * & * & * & * & $3-6$ & * & * & 0.0 \\
\hline $\mathrm{Xe}-135 \mathrm{~m}$ & $2.2-7$ & * & * & * & * & $9.0-6$ & * & * & 0.0 \\
\hline & $1.3-7$ & * & - & * & * & $5.5-6$ & $*$ & * & 0.0 \\
\hline & $6.2-7$ & * & * & * & * & $2.5-5$ & * & * & 0.0 \\
\hline 38 & $3.1-7$ & 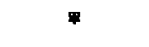 & $*$ & $*$ & $*$ & $1.3-5$ & + & $*$ & 0.0 \\
\hline $\mathrm{Xe}-139$ & 0.0 & 0.0 & 0.0 & 0.0 & 0.0 & 0.0 & 0.0 & 0.0 & 0.0 \\
\hline Xe-140 & 0.0 & 0.0 & 0.0 & 0.0 & 0.0 & 0.0 & 0.0 & 0.0 & 0.0 \\
\hline Cs-134 & $4.35-2$ & $2.89-2$ & $8.1-2$ & $2.61-2$ & $*$ & $3.31+0$ & $2.98-2$ & $*$ & 0.0 \\
\hline Cs-135 & $3.63-3$ & $1.45-2$ & $1.28-2$ & $5.08-3$ & * & $7.26-2$ & $5.34-3$ & * & 0.0 \\
\hline Cs-136 & $4.35-3$ & $2.09-3$ & $7.0-3$ & $4.12-3$ & * & $8.66-2$ & $1.31-2$ & * & 0.0 \\
\hline Cs-137 & $2.51-2$ & $4.7-2$ & $6.4-2$ & $2.21-2$ & * & $3.65+0$ & $3.11-2$ & * & 0.0 \\
\hline $\mathrm{C}_{\text {E- }} 138$ & $4.66-5$ & * & * & 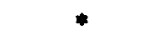 & * & $3.94-4$ & $2.67-3$ & * & 0.0 \\
\hline Cs-139 & $7.5-7$ & $2.1-6$ & $2.2-6$ & $1.7-6$ & * & $9.2-4$ & $4.5-5$ & * & 0.0 \\
\hline Cs- 140 & $2.8-8$ & $3.9-8$ & $6.3-8$ & $4: 6-8$ & $*$ & $1.6-4$ & $1.2-6$ & $*$ & 0.0 \\
\hline $\mathrm{Ba}-131$ & $1.09-3$ & $2.14-3$ & $1.6-5$ & $4.69-6$ & * & $1.84-2$ & $7.11-3$ & * & 0.0 \\
\hline $\mathrm{Ba}-133$ & 0.0 & 0.0 & 0.0 & 0.0 & 0.0 & 0.0 & 0.0 & 0.0 & 0.0 \\
\hline Ba-137m & $8.0-9$ & $8.0-8$ & $2.2-10$ & $9.0-11$ & $*$ & $2.6-5$ & $3.2-7$ & $*$ & 0.0 \\
\hline Ba-139 & $6.4-6$ & $3.2-4$ & $1.8-7$ & $7.3-8$ & * & $2.8-3$ & $2.1-4$ & * & 0.0 \\
\hline Ba-140 & $1.34-2$ & $1.71-1$ & $2.37-4$ & $7.78-5$ & * & $3.41-1$ & $1.87-2$ & * & 0.0 \\
\hline & $8.35-4$ & $4.75-3$ & $3.19-3$ & $*$ & $*$ & $1.67-2$ & $5.34-2$ & $*$ & 0.0 \\
\hline La-14] & 0.0 & 0.0 & 0.0 & 0.0 & 0.0 & 0.0 & 0.0 & 0.0 & 0.0 \\
\hline
\end{tabular}

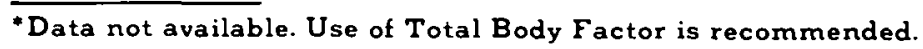


TABLE F-9 (Cont)

\begin{tabular}{|c|c|c|c|c|c|c|c|c|c|}
\hline Isotope & $\begin{array}{l}\text { Total } \\
\text { Body }\end{array}$ & Bone & Liver & Kidney & Gonad & Lung & $\begin{array}{c}\text { G.I. } \\
\text { Tract }\end{array}$ & Thyroid & Skin \\
\hline La-143 & 0.0 & 0.0 & 0.0 & 0.0 & 0.0 & 0.0 & 0.0 & 0.0 & 0.0 \\
\hline $\mathrm{Ce}_{\theta-141}$ & $1.65-3$ & $1.97-2$ & $1.35-2$ & $6.6-3$ & - & $4.17-2$ & $1.07-2$ & $*$ & 0.0 \\
\hline $\mathrm{Ce}-142$ & 0.0 & 0.0 & 0.0 & 0.0 & 0.0 & 0.0 & 0.0 & 0.0 & 0.0 \\
\hline $\mathrm{Ce}-143$ & $3.38-4$ & $3.97-3$ & $2.9-3$ & $1.33-3$ & $*$ & $1.0-2$ & $3.05-2$ & $*$ & 0.0 \\
\hline Ce-144 & $1.39-1$ & $2.59+0$ & $1.1+0$ & $6.5-1$ & * & $4.43+0$ & $9.24-2$ & * & 0.0 \\
\hline $\mathrm{Pr}-142$ & $1.78-4$ & $3.27-4$ & $1: 4-3$ & $7.91-4$ & * & $5.9-3$ & $4.27-2$ & * & 0.0 \\
\hline Pr-143 & $1.13-3$ & $2.28-2$ & $9.11-3$ & $5.28-3$ & * & $3.61-2$ & $2.37-2$ & * & 0.0 \\
\hline Pr-144 & $9.0-7$ & $2.5-5$ & $1.0-6$ & $4.0-6$ & * & $7.6-4$ & $6.4-5$ & * & 0.0 \\
\hline Pr-145 & 0.0 & $n \cap$ & nก & 0.0 & 0.0 & $0.0^{\circ}$ & 0.0 & 0.0 & 0.0 \\
\hline Nd-144 & $3.43+0$ & $1.41+2$ & $1.47+1$ & $3.21+1$ & $*$ & $2.2+1$ & $2.13-2$ & $*$ & 0.0 \\
\hline Nd. 147 & $1.16-3$ & $1.48-2$ & $1.78-2$ & $8.41-3$ & * & $2.84-2$ & $2.13-2$ & क & 0.0 \\
\hline Nd-149 & $2.39-5$ & $3.68-4$ & $4.61-4$ & $1.97-4$ & * & $7.52-4$ & $4.27-3$ & * & 0.0 \\
\hline Pm-147 & $6.92-3$ & $1.88-1$ & $2.28-2$ & $3.23-2$ & * & $6.71-2$ & $5.34-3$ & * & 0.0 \\
\hline Pm-148m & $1.52-2$ & $5.0-2$ & $2.59-2$ & $2.94-2$ & * & $2.17-1$ & $4.27-2$ & * & 0.0 \\
\hline$P m-148$ & $1.5-3$ & $1.83-2$ & $3.97-3$ & $5.36-3$ & * & $4.07-2$ & $6.28-2$ & * & 0.0 \\
\hline Pm-l 49 & $3.11-4$ & $3.94-3$ & $8.35-4$ & $1.13-3$ & $*$ & $8.71-3$ & $2.67-2$ & * & 0.0 \\
\hline Pm-15l & 0.0 & 0.0 & 0.0 & 0.0 & 0.0 & 0.0 & 0.0 & 0.0 & 0.0 \\
\hline Sm-147 & $3.95+0$ & $1.63+2$ & $1.67+1$ & $1.84+1$ & * & $2.53+1$ & $2.13-2$ & * & 0.0 \\
\hline Sm-148 & 0.0 & 0.0 & 0.0 & 0.0 & 0.0 & 0.0 & 0.0 & 0.0 & 0.0 \\
\hline $\mathrm{Sm}-149$ & 0.0 & 0.0 & 0.0 & 0.0 & 0.0 & 0.0 & 0.0 & 0.0 & 0.0 \\
\hline $\mathrm{Sm}-151$ & $7.09-3$ & $1.77-1$ & $3.03-2$ & $3.31-2$ & * & $4.6-2$ & $3.05-3$ & * & 0.0 \\
\hline $\mathrm{Sm}-153$ & $1.53-4$ & $2.03-3$ & $1.96-3$ & $5.95-4$ & * & $4.59-3$ & $2.13-2$ & * & 0.0 \\
\hline Eu-152 & $9.66-2$ & $8.82-1$ & $1.06-1$ & $5.15-1$ & * & $3.54-1$ & $2.13-2$ & * & 0.0 \\
\hline Eu-154 & $1.95-1$ & $3.03+0$ & $2.76-1$ & $1.64+0$ & * & $9.26-1$ & $5.34-2$ & * & 0.0 \\
\hline Eu-155 & $1.31-2$ & $1.16-1$ & $2.58-2$ & $6.66-2$ & * & $8.75-2$ & $5.34-3$ & $*$ & $\cap \cap$ \\
\hline$\overline{\mathbf{E}} \mathbf{u}-156$ & 0.0 & 0.0 & 0.0 & 0.0 & 0.0 & 0.0 & 0.0 & 0.0 & 0.0 \\
\hline Eu-157 & 0.0 & 0.0 & 0.0 & 0.0 & 0.0 & 0.0 & 0.0 & 0.0 & 0.0 \\
\hline Gd-153 & $7.34-3$ & $6.9-2$ & $4.99-3$ & $*$ & $*$ & $7.28-2$ & $5.34-3$ & $*$ & 0.0 \\
\hline Gd-159 & $7.07-5$ & $6.48-4$ & $3.2-4$ & $*$ & * & $2.25-3$ & $2.13-2$ & * & 0.0 \\
\hline Gd-162 & 0.0 & 0.0 & 0.0 & 0.0 & 0.0 & 0.0 & 0.0 & 0.0 & 0.0 \\
\hline Tb-160 & $1.47-2$ & $1.18-1$ & $*$ & $4.84-2$ & $*$ & $1.98-1$ & $2.67-2$ & * & 00 \\
\hline Tb-161 & 0.0 & 0.0 & 0.0 & 0.0 & 0.0 & 0.0 & 0.0 & 0.0 & 0.0 \\
\hline Dy-165 & $1.29-5$ & $2.29-4$ & .7265 & * & • & $3.46-4$ & $3.05-3$ & • & 0.0 \\
\hline Dy-166 & 2.03-4 & $2.08-2$ & $2.29-3$ & $*$ & * & $2.27-2$ & $3.05-2$ & * & 0.0 \\
\hline Ho- 166 & $2.01-4$ & $6.27-3$ & $6.54-4$ & $9.27-4$ & " & $6.89-3$ & $3.56-2$ & * & 0.0 \\
\hline Er-169 & $9.01-4$ & $8.47-3$ & $6.54-4$ & $2.16-3$ & * & $1.76-2$ & $1.07-2$ & - & 0.0 \\
\hline E. $r=171$ & $52.7-5$ & $9.74-2$ & & $1.74-4$ & * & $1.39-3$ & $1.07-2$ & * & 0.0 \\
\hline Tm-1 70 & $9.52-3$ & $3.22-1$ & * & $3.82-2$ & * & $1.92-1$ & $2.67-2$ & * & 0.0 \\
\hline Yb-175 & $1.72-4$ & $4.57-3$ & * & $1.95-3$ & $*$ & $5.45-3$ & $1.07-2$ & * & 0.0 \\
\hline Lu-177 & $2.98-4$ & $9.13-3$ & * & $6.55-4$ & * & $9.43-3$ & $1.07-2$ & - & 0.0 \\
\hline Hf-181 & $5.63-3$ & $1.33-2$ & $5.91-2$ & $1.31-2$ & * & $8.83-2$ & $2.13-2$ & * & 0.0 \\
\hline Тa-182 & $2.19-2$ & $4.29-2$ & $1.7-1$ & $7.26-2$ & * & $2.97-1$ & $3.05-2$ & * & 0.0 \\
\hline$W-181$ & $6.28-5$ & $8.37-5$ & $2.93-4$ & 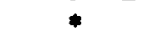 & * & $5.15-2$ & $3.56-3$ & * & 0.0 \\
\hline W-185 & $4.4-5$ & $1.14-3$ & $4.59-4$ & * & * & $5.87-2$ & $1.07-2$ & * & 0.0 \\
\hline W-187 & $1.07-4$ & $2.64-4$ & $3.03-4$ & * & * & $4.0-3$ & $2.13-2$ & * & 0.0 \\
\hline W-188 & $2.67-4$ & $6.37-3$ & $2.92-3$ & * & * & $3.59-1$ & $4.27-2$ & * & 0.0 \\
\hline $\operatorname{Re}-183$ & $3.35-4$ & $*$ & * & * & * & $9.98-2$ & $4.27-3$ & * & 0.0 \\
\hline $\operatorname{Re}-186$ & $4.97-4$ & $1.71-4$ & $2.38-4$ & * & * & $1.25-2$ & $2.67-2$ & $7.85-3$ & 0.0 \\
\hline
\end{tabular}

* Data not available. Use of Total Body Factor is recommended. 
TABLE F-9 (Cont)

Total

\begin{tabular}{|c|c|c|c|c|c|c|c|c|c|}
\hline Isotope & Body & Bone & Liver & Kidney & Gonad & Lung & Tract & Thyroid & $\underline{\text { Skin }}$ \\
\hline Re-187 & $4.4-5$ & $1.14-5$ & $3.62-5$ & * & * & $1.32-2$ & $1.07-3$ & $4.62-4$ & 0.0 \\
\hline & $3.15-4$ & $1.21-4$ & $1.25-4$ & $*$ & * & $5.5-3$ & $3.56-2$ & $5.85-3$ & 0.0 \\
\hline Os-185 & $3.2-4$ & $*$ & $6.5-4$ & $5.86-3$ & $*$ & $1.41-1$ & $2.13-2$ & 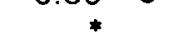 & 0.0 \\
\hline Os-19lm & $8.48-6$ & * & $1.1-5$ & $9.91-5$ & * & $6.87-4$ & $5.34-4$ & * & 0.0 \\
\hline Os-19l & $9.05-5$ & * & $2.12-2$ & $2.04-3$ & * & $1.55-2$ & $7.11-3$ & $*$ & 0.0 \\
\hline Os-193 & $9.55-5$ & * & $1.64-4$ & $1.86-3$ & * & $4.48-3$ & $2.37-2$ & * & 0.0 \\
\hline Ir -190 & $8.72-4$ & * & $4.01-3$ & $3.98-3$ & * & $1.6-2$ & $7.11-3$ & * & 0.0 \\
\hline $\mathrm{Ir}-192$ & $5.46-3$ & * & $3.62-2$ & $5.13-2$ & * & $2.53-1$ & $3.56-2$ & * & 0.0 \\
\hline Pt- 191 & $5.94-4$ & * & $8.34-5$ & $4.68-3$ & * & $8.31-3$ & $1.07-2$ & * & 0.0 \\
\hline Pt-193m & $7.07-5$ & * & $1.06-5$ & $5.39-4$ & * & $9.69-4$ & $1.07-3$ & * & 0.0 \\
\hline Pt-193 & $3.24-4$ & * & $3.93-5$ & $6.16-3$ & * & $2.09-2$ & $7.11-4$ & * & 0.0 \\
\hline Pt- $197 \mathrm{~m}$ & $9.67-6$ & * & $2.95-6$ & $2.05-4$ & * & $2.38-4$ & $1.07-3$ & * & 0.0 \\
\hline Pt-197 & $5.96-5$ & * & $1.79-5$ & $1.25-3$ & $*$ & $1.64-3$ & $1.07-2$ & * & 0.0 \\
\hline $\mathrm{Au}-195 \mathrm{~m}$ & 0.0 & 0.0 & 0.0 & 0.0 & 0.0 & 0.0 & 0.0 & 0.0 & 0.0 \\
\hline Au- 195 & 0.0 & 0.0 & 0.0 & 0.0 & 0.0 & 0.0 & 0.0 & 0.0 & 0.0 \\
\hline Au-196 & $7.8-4$ & $*$ & $4.98-4$ & $1.81-3$ & * & $1.03-2$ & $7.11-3$ & $*$ & 0.0 \\
\hline Au-198 & $4.74-4$ & * & 5. $12-4$ & $2.43-3$ & * & $1.06-2$ & $2.67-2$ & * & 0.0 \\
\hline Au-199 & $1.75-4$ & * & $1.74-4$ & $8.18-4$ & * & $3.66-3$ & $7.11-3$ & * & 0.0 \\
\hline $\mathrm{Hg}-197 \mathrm{~m}$ & $1.8-4$ & * & $6.86-4$ & $9.09-3$ & * & $1.82-3$ & $7.11-2$ & * & 0.0 \\
\hline $\mathrm{Hq}-197$ & 1. $34-4$ & * & $4.64-4$ & $5.31-3$ & * & $1.26-3$ & $2.37-3$ & * & 0.0 \\
\hline $\mathrm{Hg}-203$ & $1.35-3$ & $*$ & $6.86-3$ & $8.87-2$ & * & $5.16-2$ & $1.07-2$ & * & 0.0 \\
\hline Tl-200 & $1.85-4$ & $2.51-5$ & $1.43-4$ & $7.39-4$ & * & $1.85-3$ & $5.34-3$ & * & 0.0 \\
\hline Tl-2C & $1.62-4$ & $2.51-4$ & $1.97-4$ & $1.35-3$ & * & $3.22-3$ & $7.11-3$ & * & 0.0 \\
\hline Tl-2 & $6.68-4$ & $1.13-3$ & $8.15-4$ & $6.19-3$ & * & $2.7-2$ & $2.13-2$ & * & 0.0 \\
\hline TI-204 & $6.28-4$ & $2.48-3$ & $1.08-3$ & $1.03-2$ & * & $2.48-1$ & $2.13-2$ & * & 0.0 \\
\hline $\mathrm{Tl}-2 \mathrm{C}$ & $5.2-8$ & $2.2-7$ & $8.1-8$ & $5.8-7$ & * & $1.1-4$ & $4.6-6$ & * & 0.0 \\
\hline Tl-2 & $2.6-8$ & $1.1-7$ & $4.1-8$ & $3.0-7$ & * & $7.4-5$ & $2.4-6$ & * & 0.0 \\
\hline Tl-2 & $2.3-8$ & $9.8-8$ & $3.7-8$ & $2.7-7$ & * & $8.8-5$ & $1.7-6$ & * & 0.0 \\
\hline $\mathrm{Tl}-210$ & $1.6-8$ & $2.4-7$ & $4.2-8$ & $1.5-7$ & * & $7.6-5$ & $5.7-7$ & * & 0.0 \\
\hline $\mathrm{Pb}-203$ & $1.45-4$ & $9.27-5$ & $2.02-4$ & $1.46-3$ & * & $1.84-3$ & $3.56-3$ & $*$ & 0.0 \\
\hline $\mathrm{Pb}-204 \mathrm{~m}$ & $1.91-5$ & $1.22-5$ & $2.75-5$ & $2.02-4$ & * & $2.54-4$ & $2.67-2$ & * & 0.0 \\
\hline $\mathrm{Pb}-209$ & $1.4-6$ & $2.8-5$ & $4.3-6$ & $4.3-5$ & * & $5.3-4$ & $1.1-3$ & * & 0.0 \\
\hline $\mathrm{Pb}-210$ & $3.4+0$ & $3.3+2$ & $3.1+1$ & $3.7+1$ & * & $6.4+2$ & $1.6-3$ & * & 0.0 \\
\hline $\mathrm{Pb}-2$ & $18-4$ & $3.4-3$ & $5.5-4$ & $5.6-3$ & * & $1.1-1$ & $9.3-5$ & * & 0.0 \\
\hline $\mathrm{Pb}-212$ & $1.1-2$ & $2.0-1$ & $3.3-2$ & $3.4-1$ & * & $1.7+0$ & $1.6-3$ & * & 0.0 \\
\hline $\mathrm{Pb}-214$ & $13-4$ & $2.6-3$ & $4.2-4$ & $4.2-3$ & * & $9.3-2$ & $1.1-4$ & * & 0.0 \\
\hline $\mathrm{Bi}-206$ & $1.37-3$ & $1.49-4$ & $6.21-3$ & $3.51-2$ & $*$ & $4.45-2$ & $3.05-2$ & * & 0.0 \\
\hline & $1.36-3$ & $2.55-4$ & $1.16-2$ & $3.87-2$ & * & $4.75-1$ & $2.13-2$ & * & 0.0 \\
\hline $\mathrm{Bi}-210$ & $1.1-2$ & $4.3-2$ & $8.2-1$ & $1.9+0$ & * & $7.5+0$ & $1.8-2$ & * & 0.0 \\
\hline $\mathrm{Bi}-21 \mathrm{l}$ & $1.7-6$ & $3.5-6$ & $9.7-6$ & $1.1-4$ & * & $6.4-3$ & $1.6-6$ & $*$ & 0.0 \\
\hline Bi-212 & $4.1-4$ & $8.7-4$ & $2.4-3$ & $2.8-2$ & $*$ & $2.1-1$ & $2.9-4$ & $*$ & 0.0 \\
\hline $\mathrm{Bi}-2$ & $3.1-4$ & $6.5-4$ & $1.8-3$ & $2.1-2$ & * & $1.7-1$ & $1.5-4$ & * & 0.0 \\
\hline $\mathrm{Bi}-214$ & $8.7-5$ & $1.8-4$ & $5.0-4$ & $5.8-3$ & $*$ & $6.8-2$ & $9.3-5$ & * & 0.0 \\
\hline Po-210 & $7.5-1$ & $3.5+0$ & $8.3+0$ & $4.1+1$ & * & $1.9+2$ & $3.5-2$ & * & 0.0 \\
\hline Po-212 & 0.0 & 0.0 & 0.0 & 0.0 & 0.0 & 0.0 & 0.0 & 0.0 & 0.0 \\
\hline Po-213 & 0.0 & 0.0 & 0.0 & 0.0 & 0.0 & 0.0 & 0.0 & 0.0 & 0.0 \\
\hline Po-216 & 0.0 & 0.0 & 0.0 & 0.0 & 0.0 & 0.0 & 0.0 & 0.0 & 0.0 \\
\hline Po-218 & $6.4-6$ & $3.6-5$ & $5.5-5$ & $1.9-4$ & $*$ & $1.9-2$ & $5.6-6$ & * & 0.0 \\
\hline
\end{tabular}

"Data not available. Use of Total Body Factor is recommended. 
TABLE F-9 (Cont)

Total

Isotope

At-211

At-217

Rn-220

$\mathrm{Rn}-222$

$\mathrm{Ra}-223$

Ra-224

$\operatorname{Ra}=22.5$

Ra-226

$\mathrm{Ra}-228$

Fr-221

Fr-223

Ac-225

Ac- 227

Ac-228

Th-227

Th-228

Th-229

Th-230

Th-231

Th-232

Th-234

$\mathrm{Pa}-230$

$\mathrm{Pa}-231$

$\mathrm{Pa}-233$

$\mathrm{Pa}-234 \mathrm{~m}$

$\mathrm{Pa}-235$

U-230

U-232

U-233

$\mathrm{U}-234$

U-235

U-236

U-237

IJ.238

U-239

$\mathrm{Np}-237$

$\mathrm{Np}-238$

$\mathrm{Np}-239$

$\mathrm{Np}-240 \mathrm{~m}$

$\mathrm{Np}-240$

Pu-238

Pu-239

$\mathrm{Pu}-240$

$\mathrm{Pu}-241$

Pu-242

$\mathrm{Pu}-243$

$\mathrm{Pu}-244$

A m-24 1

Am-242m
Body

$1.44-$

0.0

$5.28-4$
$4.68-4$

$8.1-1$

$3.04-$

2. $2 .-1$

$4.15+1$

$2 \% 1+1$

$2.4-5$

$1.8-4$

$1.7-1$

$2.98+2$

$1.57-2$

9.63-1

$2.2+1$

$4.7+2$

$1.91+27.11+3$

$5.04-5 \quad 1.13-3$

$1.31+2 \quad 3.7+4$

$\begin{array}{ll}1.73-3 & 8.62-2\end{array}$

$\begin{array}{ll}2.69-1 & 6.53+0\end{array}$

$2.79+2 \quad 1.01+4$

$7.19-4 \quad 5.62-3$

$6.2-9$

0.0

$1.9-7$

0.0

$1.58+0 \quad 1.03+1$

$5.21+0 \quad 1.02+2$

9.3-1 $2.15+1$

$9.12-12.07+1$

$\begin{array}{ll}8.56-1 & 1.98+1\end{array}$

$8.75-1 \quad 1.98+1$

$\begin{array}{ll}1.63-4 & 1.9-3\end{array}$

กก. $1.9+1$

6.3-7 $3.7-5$

$8.37+1 \quad 3.07+3$

$4.2-4 \quad 1.3-2$

$1.77-4 \quad 2.63-3$

0.0

0.0

$4.0-6 \quad 1.2-4$

$7.71+12.69+3$

$9.04+1 \quad 3.31+3$

$9.03+1 \quad 3.3+3$

$1.26+0 \quad 5.13+1$

$8.71+1 \quad 3.07+3$

$2.01-5 \quad 8.71-4$

$2.37+2 \quad 9.61+3$

$9.29+13.26+3$

$9.06+1 \quad 3.22+3$
Liver

$0.0 \quad 0.0$

$1.68-4$

$1.56-4 \quad 1.44-4$

$1.5-2 \quad 3.4-1$

$5.88-3 \quad 1.67-1$

$1.4-3 \quad 3.9-2$

*

ค 4 - $5 \quad 54-5$

$4.8-4 \quad 4.0-4$

$3.4+0 \quad 3.9-1$

$6.6+2 \quad 2.58+2$

$8.16-2 \quad 1.05-2$

$4.84-1 \quad 2.74+0$

$8.5+0 \quad 4.79+1$

$2.01+2 \quad 9.27+2$

$3.15+2 \quad 1.43+3$

$7.38-5 \quad 3.66-4$

$1.35+2 \quad 6.27+2$

$2.66-3 \quad 1.54-2$

$1.3+0$

$2.71+2 \quad 1.49+3$

$8.27-4 \quad 2.93-3$

$1.3-8$

0.0

$5.7-8$

0.0

* $\quad 2.08+1$

* $7.64+0$

* $\quad 3.47+0$

* $3.04+0$

* $\quad 3.2+0$

* $3.26+0$

* $4.32-3$

* $\quad 2.99+0$

* $\quad 9.0-6$

$1.21+3$

$8.7-4$

$1.58+2$

$2.8-3$

$2.87-4 \quad 8.97-4$

0.0

0.0

$8.2-7$

$2.1-5$

$1.15+3$

$1.48+2$

$1.32+3$

$1.32+3$

$8.7+0$

$1.7+2$

$1.7+2$

$2.82+0$

$1.27+3$

$1.64+2$

$8.52-5$

$9.4-5$

$9.7+2$

$1.36+3$

$1.26+3$

$1.75+2$

$1.62+2$
Gonad Lung

$$
2.51-1 \quad 1.67-1
$$

0.0

0.0

4.08-3

$8.4-5 \quad 4.56-3$

* $1.0+2$

* $1.94+1$

* $\quad 1.3+2$

$1.21+2$

$1.67+2$

4.7-2.

$2.6-1$

$8.7+1$

$2.49+2$

$3.8-1$

$3.83+1$

$2.36+3$

$4.21+3$

$6.42+2$

$1.08-3$

$3.77+3$

$7.06-1$

$8.48+0$

$8.78+2$

$1.6-1$

$$
4.1-5
$$

0.0

$5.68+1$

$3.35+3$

$8.13+2$

$7.97+2$

$7.48+2$

$7.64+2$

2.5-2

6.9912

4.5-4

$4.51+1$

$7.98+2$

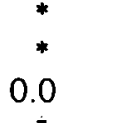

3. $35-3$

$67+1$

$4.88+1$

$4.88+1$

$1.2+0$

$4.71+1$

0.0

$1.9-3$

$9.12+2$

$8.62+2$

$8.62+2$

$4.45-1$

$8.29+2$

$3.62-4$

* $1.95+2$

$5.13+1 \quad 9.27+2$

$4.91+13.66+2$
G.I.

Tract Thyroid Skin

2.13-2 $1.54+0 \quad 0.0$

$\begin{array}{lll}0.0 & 0.0 & 0.0\end{array}$

$* \begin{array}{lll}* & * & 0.0 \\ * & * & 0.0\end{array}$

$1.9-1$

$1.56-1$

$2.2-2$

$3.56-2$

$5.34-2$

$2.1-5$

$9.6-5$

$1.8-1$

$4.27-3$

$1.07-2$

$7.11-2$

$4.3-2$

$4.55-2$

$3.56-2$

$5.34-3$

$4.4-2$

$5.8-2$

$5.34-3$

$5.2-2$

$8.4-3$

$6.0-7$

0.0

2.67-1

$5.1-2$

4.66-2

4.66-2

4. $46-2$

4.2-2

2.14-3

$1.01-2$

4.0-5

$1.61-2$

$1.4-2$

1.07-2

0.0

$8.6-5$

$5.24-2$

$5.0-2$

$5.0-2$

$1.07-3$

4.68-2

$3.56-3$

1.07-1

$1.83-2$

$7.22-3$

0.0

0.0

0.0

0.0

0.0

0.0

0.0

0.0

0.0

0.0 
TABLE F-9 (Cont)

Total

Isotope

Am-242

Am-243

A $\mathrm{m}-244$

$\mathrm{C} \mathrm{m}-242$

Cm-243

Cm-244

Cm-245

$\mathrm{C} \mathrm{m}-246$

$\mathrm{Cm}-247$

$\mathrm{Cm}-248$

$\mathrm{Cm}-249$

$\mathrm{Bk}-249$

$\mathrm{Bk}-250$

Cl-249

Cf -250

$\mathrm{Cf}-25]$

Ct-252

C $1-253$

Cf-254

Es-253

Es-254m

Es-254

Es-255

Fm-254

$\mathrm{F}_{\mathrm{m}}-255$

$\mathrm{Fm}_{\mathrm{m}-256}$

\section{Body}

\begin{abstract}
$1.17-$
\end{abstract}
$9.31+1 \quad 3.3+3$

$2.09-4$

$1.52+0$

$5.8+1$

$4.06+1 \quad 1.38+3.6$

$9.54+13.43+3$

$9.54+1 \quad 3.42+3$

$9.4+1 \quad 3.31+3$

$7.74+2 \quad 2.75+4$

$5.99-5 \quad 9.33-4$

$7.11+0 \quad 5.4+0$

$2.0-3$

$2.32+$

$8.14-2$

3.

$3.36+$
$2.33+$

$1.14+3$

$2.58+1 \quad 8.85+2$

$3.44-1 \quad 1.39+1$

$5.57+1 \quad 2.22+3$

$3.82-1 \cdot 1.55+1$

$5.44-2 \quad 2.21+0$

$1.49+1 \quad 6.19+2$

$\begin{array}{ll}5.89-1 & 2.39+1\end{array}$

$4.66-3 \quad 1.89-1$

$1.76-2 \quad 7.13-1$

$1.05-14.18+0$
G.I.

Tract Thyroid Skin

$1.59-1 \quad 8.01-2$

$1.34+3 \quad 1.73+2$

$1.37-3 \quad 1.45-1$

$3.03+1 \quad 3.91+0$

$8.85+2$

$1.14+2$

$8.23+1$

$1.8+2$

$1.4+3$

$1.79+2$

$1.77+2$

$1.39+3$

$1.13+4$

$1.46+3$

$*$
$3.0+0$

$3.86-1$
$*$
$*$

$5.42+2 \quad 7.0+1$

$7.0+$

$4.83+2 \quad 6.23+1$

83
$*$
$*$
$*$
$*$
$*$
$*$
$*$
$*$
$*$
Gonad Lung

* $\quad 1.38-1$

$4.97+1 \quad 8.78+2$

* $2.65-4$

$1.54+0 \quad 2.56+2$

$4.07+1 \quad 9.37+2$

$3.24+1 \quad 9.06+2$

$5.15+18.95+2$

$5.15+1 \quad 9.11+2$

$5.07+18.95+2$

$4.17+2 \quad 7.37+3$

* $\quad 1.25-4$

$1.52-1 \quad 4.33-1$

$4.33-1$
$2.82-3$

$6.59+1$

$2.88+1 \quad 9.14+2$

* $\quad 6.48+1$

* $\quad 2.25+3$

* $\quad 8.46+0$

* $\quad 1.33+3$

* $\quad 1.07+1$

* $\quad 1.1+0$

* $\quad 6.07+1$

* $\quad 1.61+1$

$9.64-2$

$5.95-1$

$3.66+0$

$1.07-2$
$1.86-2$
$2.67-4$
$1.64-2$
$1.97-2$
$1.97-2$
$1.83-2$
$1.77-2$
$1.8-2$
$1.67-2$
$5.34-4$
$7.17-4$
$5.34-3$
$5.34-2$
$1.97-2$
$4.27-2$
$6.09-2$
$7.11-3$
$1.07+1$
$5.34-2$
$7.11-2$
$7.11-2$
$4.27-2$
$1.07-2$
$3.56-2$
$1.07+0$

0.0

0.0

0.0

0.0

0.0

0.0

0.0

0.0

0.0

0.0

0.0

0.0

0.0

0.0

0.0

0.0

0.0

0.0

0.0

0.0

0.0

0.0

0.0

0.0

- 0.0

*Data not available. Use of Total Body Factor is recommended. 
TABLE F-10 DECAY CHAINS

\begin{tabular}{|c|c|c|c|c|c|c|c|c|}
\hline Parent & Fraction & Daughter & Fraction & Daughter & Fraction & Daughter & Fraction & Daughter \\
\hline $\mathrm{Mg}-28$ & 1.0 & Al-28 & & & & & & \\
\hline $\mathrm{Ca}-47$ & 1.0 & $\mathrm{Sc}-47$ & & & & & & \\
\hline $\mathrm{Ca}-49$ & 1.0 & Sc-49 & & & & & & \\
\hline$M n-\zeta \angle m$ & U.UL & $M n-5<$ & & & & & & \\
\hline$F_{c-52}$ & 1.0 & $\mathrm{Mn}-52 \mathrm{~m}$ & 0.02 & $\mathrm{Mn}_{\mathrm{n}-52}$ & & & & \\
\hline Co- $58 \mathrm{~m}$ & 1.0 & Co-58 & & & & & & \\
\hline $\mathrm{Ni}-56$ & 1.0 & Co-56 & & & . & & & \\
\hline $\mathrm{Zn}-69 \mathrm{~m}$ & 1.0 & $\mathrm{Zn}-69$ & & & & & & \\
\hline As-73 & 1.0 & $\mathrm{Ge}-73 \mathrm{~m}$ & & & & & & \\
\hline $\mathrm{Rr}_{\mathrm{r}=} 8 \cap \mathrm{m}$ & 10 & Br-80 & & & . & & & \\
\hline $\mathrm{Br}-82 \mathrm{~m}$ & 1.0 & $\mathrm{Br}-82$ & & & & & & \\
\hline$D_{1}-83$ & 1.0 & $\mathrm{Kr}-83 \mathrm{~m}$ & & & & & & \\
\hline Br-83 & 1.0 & $\mathrm{~K}_{\mathrm{r}-85 \mathrm{~m}}$ & 0.225 & $K_{1}=05$ & & & & \\
\hline Br-87 & 0.97 & $K_{\tau}-87$ & 0.97 & RL-87 & & & & \\
\hline $\mathrm{K}_{\mathrm{I}-85 \mathrm{~m}}$ & 0.225 & $\mathrm{~K}_{\mathrm{r}-85}$ & & & & & & \\
\hline $\mathrm{Kr}-87$ & 1.0 & $\mathrm{Rb}-87$ & & & & & & \\
\hline K T-88 & 1.0 & $\mathrm{Rb}-8 \mathrm{8}$ & & & & & & \\
\hline $\mathrm{K}_{\mathrm{r}-89}$ & 1.0 & $\mathrm{Rb}-89$ & 1.0 & $\mathrm{Sr}_{\mathrm{r}} 89$ & 0.0002 & $Y-89 m$ & & \\
\hline $\mathrm{K}_{\mathrm{T}-90}$ & 1.0 & Rb-90 & 1.0 & Sr-90 & 1.0 & $Y-90$ & & \\
\hline $\mathrm{Rb}-89$ & 1.0 & $\mathrm{~S}_{\mathrm{I}-89}$ & 0.0002 & Y $-89 \mathrm{~m}$ & & & & \\
\hline Si-85tn & 0.87 & Sr-85 & & & & & & \\
\hline Sr-89 & 0.0002 & $\mathrm{Y}-89 \mathrm{~m}$ & & & & & & \\
\hline$S_{\text {I }-90}$ & 1.0 & $Y-90$ & & & & & & \\
\hline$S_{r-91}$ & 0.6 & Y $-91 \mathrm{~m}$ & 1.0 & Y-91 & & & & \\
\hline SI -92 & 1.0 & Y-92 & & & & & & \\
\hline Y.87 & 1.0 & $\mathrm{Sr}-87 \mathrm{~m}$ & & & & & & \\
\hline $\mathrm{Y}-9 \mathrm{~lm}$ & 1.0 & Y-91 & & & & & & \\
\hline Y.93 & 1.0 & $Z_{r-93}$ & 1.0 & $\mathrm{Nb}-93 \mathrm{~m}$ & & & & \\
\hline$Z_{r-93}$ & 1.0 & $\mathrm{Nb}-93 \mathrm{~m}$ & & & & & & \\
\hline$Z_{1}-95$ & 0.02 & $\mathrm{Nb}-95 \mathrm{~m}$ & 1.0 & $\mathrm{Nb}-95$ & & & & \\
\hline Zr-97 & 0.96 & $\mathrm{Nb}-97 \mathrm{~m}$ & 1.0 & $\mathrm{Nb}-97$ & & ' & & \\
\hline $\mathrm{Nb}-95 \mathrm{~m}$ & 1.0 & Nb-95 & & & & & & \\
\hline $\mathrm{Nb}-97 \mathrm{~m}$ & 1.0 & Nb-97 & & & & & & \\
\hline Mo-99 & 0.87 & l'c-99m & 1.0 & $T c-99$ & & & & \\
\hline $\mathrm{Te}-96 \mathrm{~m}$ & 0.98 & $\mathrm{Tc}-96$ & & & & & & \\
\hline Tc-97m & 1.0 & $T_{c}-97$ & & & & & & \\
\hline Tc-99m & 1.0 & Tc-99 & & & & & & \\
\hline$R u-103$ & 0.995 & Rh. $103 \mathrm{~m}$ & & & & & & \\
\hline Ru-105 & 1.0 & $\mathrm{Rh}-105 \mathrm{~m}$ & 1.0 & Rh-105 & & & & \\
\hline $\mathrm{Ru}-106$ & 1.0 & Rh-106 & & & & & & \\
\hline $\mathrm{Rh}-105 \mathrm{~m}$ & 1.0 & $\mathrm{Rh} 105$ & & & & & & \\
\hline Ful-100 & 1.0 & $n_{1} \cdot 10 J_{111}$ & & & & & & \\
\hline$P, j-1 \cap 9$ & 10 & $A_{1,-10} A_{1 ! 1}$ & & & & & & \\
\hline $\mathrm{Cd}-109$ & 1.0 & $A y-109 m$ & & & & & & \\
\hline ritsligm & $\ln$ & In-115 & & & & & & \\
\hline Cd-115 & 1.0 & In-1 $15 \mathrm{~m}$ & 0.95 & In-115 & & & & \\
\hline $\mathrm{In}-1 \mathrm{l} 4 \mathrm{~m}$ & 0.965 & In-114 & & & & & & \\
\hline$I_{H}=115 \mathrm{~m}$ & 0.95 & $\ln -115$ & & & & & & \\
\hline $\mathrm{Sn}-125$ & 1.0 & Sb-125 & 0.3 & $\mathrm{Te}-125 \mathrm{~m}$ & & & & \\
\hline Sn- 126 & 1.0 & $3 \mathrm{~b}-126 \mathrm{~m}$ & 0.99 & SL-12G & & & & \\
\hline Sb-125 & 0.3 & $\mathrm{Te}-125 \mathrm{~m}$ & & & & & & \\
\hline $\mathrm{Sb} \cdot 126 \mathrm{~m}$ & 0.99 & Sb-126 & & & & & & \\
\hline Sb-127 & 0.22 & $\mathrm{Te}-127 \mathrm{~m}$ & 1.0 & Té-127 & & & & \\
\hline Sb-129 & 0.36 & $\mathrm{Te}-129 \mathrm{~m}$ & 1.0 & Te-129 & 1.0 & I-129 & & \\
\hline $\mathrm{Te}-127 \mathrm{~m}$ & 0.98 & $\mathrm{Te}-127$ & & & & & & \\
\hline Tc $129 \mathrm{~m}$ & 1.0 & $T_{\theta-129}$ & 1.0 & I-129 & & & & \\
\hline $\mathrm{Te}-129$ & 1.0 & I-129 & & & & & & \\
\hline $\mathrm{Te}-131 \mathrm{~m}$ & 0.2 & $\mathrm{Te}-131$ & 1.0 & $1-131$ & 0.008 & $\mathrm{Xe}-131 \mathrm{~m}$ & & \\
\hline $\mathrm{Te}-131$ & 1.0 & I-131 & 0.008 & $\mathrm{Xe}-131 \mathrm{~m}$ & & & & \\
\hline $\mathrm{Te}-132$ & 1.0 & I-132 & & & & & & \\
\hline $\mathrm{Te}-134$ & 1.0 & $\mathrm{I}-134$ & & & & & & \\
\hline
\end{tabular}


TABLE F-10 (Cont)

\begin{tabular}{|c|c|c|c|c|c|c|c|c|}
\hline Parent & Fraction & Daughter & Fraction & Daughter & Fraction & Daughter & Fraction & Daughter \\
\hline I-13I & 0.008 & $\mathrm{Xe}-13 \mathrm{~lm}$ & & & & & & \\
\hline I- 133 & 0.024 & $\mathrm{Xe}-133 \mathrm{~m}$ & 1.0 & Xe-1 33 & & & & \\
\hline $1-135$ & 0.3 & $\mathrm{Xe}-135 \mathrm{~m}$ & 1.0 & $X e-135$ & 1.0 & $\mathrm{Cs}-135$ & & \\
\hline I-137 & 0.96 & $X e-137$ & 0.96 & Cs-137 & 0.883 & $\mathrm{Ba}-137 \mathrm{~m}$ & & \\
\hline $\mathrm{Xe}-133 \mathrm{~m}$ & 1.0 & $\mathrm{Xe}-133$ & & & & & & \\
\hline $\mathrm{Xe}-135 \mathrm{~m}$ & 1.0 & $\mathrm{Xe}-135$ & 1.0 & Cs-135 & & & & \\
\hline $\mathrm{Xe}-135$ & 1.0 & C.s-135 & & & & & . & \\
\hline $\mathrm{Xe-1} 37$ & 1.0 & Cs-137 & 0.92 & $\mathrm{Ba}-137 \mathrm{~m}$ & & & & \\
\hline Xe-138 & 1.0 & Cs- 138 & & & & & & \\
\hline $\mathrm{Xe}-139$ & 1.0 & Cs-139 & 1.0 & Ba-1 39 & & & & \\
\hline $\mathrm{Xe}-140$ & 1.0 & Cs- 140 & 1.0 & $\mathrm{Ba}-140$ & 1.0 & La-140 & & \\
\hline Cs-136 & 1.0 & $\mathrm{Ba}-136 \mathrm{~m}$ & & & & & & \\
\hline Cs-137 & 0.92 & $\mathrm{Ba}-137 \mathrm{~m}$ & & & & & & \\
\hline Cs-139 & 1.0 & Ba-139 & & & & & & \\
\hline Cs. 140 & 1.0 & $\mathrm{Ba}-140$ & 1.0 & L.a- 140 & & & & \\
\hline $\mathrm{Ba}-140$ & 1.0 & La-140 & & & & & & \\
\hline $\mathrm{Ce}-144$ & 1.0 & $\operatorname{Pr}=144$ & 1.0 & Nd- 144 & & & & \\
\hline La-14 l & 1.0 & $\mathrm{Ce}-14 \mathrm{l}$ & & & & 一 & & \\
\hline $\begin{array}{l}\text { Ce-143 } \\
\text { Pr-144 }\end{array}$ & $\begin{array}{l}1.0 \\
1.0\end{array}$ & $\begin{array}{l}\text { Pr-143 } \\
\text { Nd-144 }\end{array}$ & & & & & & \\
\hline Nd-147 & 1.0 & $\mathrm{Pm}-147$ & 1.0 & $\mathrm{Sm}-147$ & & & & \\
\hline$P_{m-147}$ & 1.0 & $\mathrm{Sm}-147$ & & & & & & \\
\hline$P m-151$ & 1.0 & $S_{n-151}$ & & & & & & \\
\hline TI-209 & 1.0 & $\mathrm{~Pb}-209$ & & & & & & \\
\hline TI-210 & 1.0 & $\mathrm{~Pb}-210$ & 1.0 & $\mathrm{Bi}-210$ & $\begin{array}{l}0.0000013 \\
0.9999987\end{array}$ & $\begin{array}{l}\text { Tl-206 } \\
\text { Po-210 }\end{array}$ & & \\
\hline \multirow[t]{2}{*}{$\mathrm{Pb}-210$} & 1.0 & $\mathrm{Bi}-210$ & 0.0000013 & Tl-206 & & & & \\
\hline & & & 0.9999987 & Po-210 & & & & . \\
\hline $\mathrm{Pb}-21 \mathrm{l}$ & 1.0 & $\mathrm{Bi}-21 \mathrm{l}$ & $\begin{array}{l}0.0028 \\
0.9972\end{array}$ & $\begin{array}{l}\text { Po-211 } \\
\text { Tl-207 }\end{array}$ & & & & \\
\hline \multirow[t]{2}{*}{$\mathrm{Pb}-212$} & 1.0 & $\mathrm{Bi}-212$ & 0.36 & Tl-208 & & & & \\
\hline & & & 0.64 & Po-212 & & & 10 & $\mathrm{Bi}-210$ \\
\hline \multirow{3}{*}{$\mathrm{Pb}-214$} & 1.0 & $B \mathrm{i}-214$ & $\begin{array}{l}0.0002 \\
0.9998\end{array}$ & $\begin{array}{l}\text { Tl-210 } \\
\text { Po-214 }\end{array}$ & 1.0 & $\mathrm{~Pb}-210$ & & Di-c10 \\
\hline & 0.0000013 & Tl-206 & & & & & & \\
\hline & 0.9999987 & Po-210 & & & & & & \\
\hline \multirow[t]{2}{*}{$\mathrm{Bi}-210$} & 0.0000013 & Tl-206 & & & & & & \\
\hline & 0.9999987 & Po-210 & & & & & & \\
\hline \multirow[t]{2}{*}{$\mathrm{Bi}-2 \mathrm{ll}$} & 0.0028 & Po-211 & & & & & & \\
\hline & $0.9 y \% 2$ & T'1-20\% & & & & & & \\
\hline \multirow[t]{2}{*}{$\mathrm{Bi}-212$} & 0.36 & Tl-208 & & & & & & \\
\hline & 0.61 & Po-212 & & & & & & \\
\hline \multirow[t]{2}{*}{$\mathrm{Bi}-213$} & 0.022 & Tl-209 & 1.0 & $\mathrm{~Pb}-209$ & & & & \\
\hline & 0.978 & Po-213 & & & & & & \\
\hline \multirow[t]{2}{*}{$\mathrm{Bi}-214$} & 0.0002 & $\mathrm{Tl}-210$ & 1.0 & $\mathrm{~Pb}-210$ & 1.0 & $\mathrm{Bi}-210$ & 0.0000013 & $\mathrm{Tl}-206$ \\
\hline & 0.9998 & Po-214 & & & & & 0.9999987 & Po-2 10 \\
\hline Po-213 & 1.0 & $\mathrm{~Pb}-209$ & & & & & & \\
\hline Po-214 & 1.0 & Pb-210 & 1.0 & $\mathrm{Bi}-210$ & $\begin{array}{l}0.0000013 \\
0.9999987\end{array}$ & $\begin{array}{l}\text { Tl-206 } \\
\text { Po-210 }\end{array}$ & & \\
\hline \multirow[t]{2}{*}{ Po-215 } & 0.0000023 & $\begin{array}{l}\text { At-215 } \\
P b-211\end{array}$ & 1.0 & $\mathrm{Bi}-211$ & $\begin{array}{l}0.0028 \\
0.9972\end{array}$ & Po-211 & & \\
\hline & 0.9999977 & $\mathrm{~Pb}-211$ & 1.0 & $B_{1}-212$ & $\begin{array}{l}0.9972 \\
0.36\end{array}$ & $\begin{array}{l}\text { Tl-207 } \\
\text { Tl-208 }\end{array}$ & & \\
\hline Po-216 & 1.0 & $\mathrm{~Pb}-212$ & 1.0 & & 0.64 & Po-212 & & \\
\hline \multirow[t]{3}{*}{ Po-218 } & 0.0002 & At-218 & 1.0 & $\mathrm{Bi}-214$ & 0.0002 & Tl-210 & 1.0 & $\mathrm{~Pb}-210$ \\
\hline & 0.9998 & $\mathrm{~Pb}-214$ & & & 0.9998 & Po-214 & & \\
\hline & 1.0 & $\mathrm{Bi}-210$ & $\begin{array}{l}0.0000013 \\
0.9999987\end{array}$ & $\begin{array}{l}\text { Tl-206 } \\
\text { Po-210 }\end{array}$ & & & . & \\
\hline At-215 & 0.9999977 & Pb-2 Il & 1.0 & $\mathrm{Bi}-21 \mathrm{l}$ & $\begin{array}{l}0.0028 \\
0.8972\end{array}$ & $\begin{array}{l}\text { Po-21 1 } \\
\text { Tl-207 }\end{array}$ & & \\
\hline
\end{tabular}


TABLE F-10 (Cont)

\begin{tabular}{|c|c|c|c|c|c|c|c|c|}
\hline Paxent & Fraction & Daughter & Fraction & Daughter & Fraction & Daughter & Fraction & Daughter \\
\hline At-217 & 1.0 & $\mathrm{Bi}-213$ & $\begin{array}{l}0.022 \\
0.978\end{array}$ & $\begin{array}{l}\text { Tl-209 } \\
\text { Po-213 }\end{array}$ & 1.0 & $\mathrm{~Pb} \cdot 209$ & & \\
\hline \multirow[t]{2}{*}{ At-2 18} & 1.0 & $\mathrm{Bi}-214$ & $\begin{array}{l}0.0002 \\
0.9998\end{array}$ & $\begin{array}{l}\mathrm{Tl}-210 \\
\text { Po-214 }\end{array}$ & 1.0 & $\mathrm{~Pb}-210$ & 1.0 & $\mathrm{Bi}-210$ \\
\hline & $\begin{array}{l}0.0000013 \\
0.9999987\end{array}$ & $\begin{array}{l}\text { Tl-206 } \\
\text { Po-210 }\end{array}$ & & & & & & \\
\hline$R n-219$ & 1.0 & Po-215 & $\begin{array}{l}0.0000023 \\
0.9999977\end{array}$ & $\begin{array}{l}\mathrm{At}-215 \\
\mathrm{~Pb}-211\end{array}$ & 1.0 & $B i-21 l$ & $\begin{array}{l}0.0028 \\
0.9972\end{array}$ & $\begin{array}{l}\text { Po-211 } \\
\text { Tl-207 }\end{array}$ \\
\hline $\operatorname{Rn} 330$ & 1.0 & $\Gamma \cdot-210$ & 10 & $\mathrm{~Pb}-212$ & 1.0 & $b_{1}-212$ & $\begin{array}{l}0.36 \\
0.64\end{array}$ & $\begin{array}{l}\text { Tl-208 } \\
\text { Pn-212 }\end{array}$ \\
\hline \multirow[t]{2}{*}{$\mathrm{Rn}_{\mathrm{n}}-222$} & 1.0 & $\mu_{0-218}$ & $\begin{array}{l}0.0002 \\
0.9998\end{array}$ & $\begin{array}{l}A_{t-2} 18 \\
\mathrm{~Pb}-214\end{array}$ & 1.0 & $\mathrm{Bi}-2.14$ & $\begin{array}{l}n \text { กnก? } \\
\text { ก } 4498\end{array}$ & $\begin{array}{l}\text { TL210 } \\
\text { Po-214 }\end{array}$ \\
\hline & 1.0 & $\mathrm{~Pb}-210$ & 1.0 & $\mathrm{Bi}-210$ & $\begin{array}{l}0.0000013 \\
09999987\end{array}$ & $\begin{array}{l}\text { T1-206 } \\
\mathrm{P}_{0-2} 10\end{array}$ & & \\
\hline \multirow[t]{2}{*}{$\mathrm{Ra}-223$} & 1.0 & $\mathrm{Rn}-219$ & 1.0 & Po-215 & $\begin{array}{l}0.0000023 \\
0.9999977\end{array}$ & $\begin{array}{l}\text { At-215 } \\
\text { Pb-211 }\end{array}$ & 1.0 & $\mathrm{Bi}-21 \mathrm{l}$ \\
\hline & $\begin{array}{l}0.0028 \\
0.99 \% 2\end{array}$ & $\begin{array}{l}\text { Po-211 } \\
111-207\end{array}$ & & & & & & \\
\hline \multirow[t]{2}{*}{$\mathrm{Ra}-224$} & 1.0 & $\mathrm{kn}-220$ & 1.0 & Po-216 & 1.0 & Pb-212 & 1.0 & $\mathrm{Bi}-212$ \\
\hline & $\begin{array}{l}0.36 \\
0.64\end{array}$ & $\begin{array}{l}\text { Tl-208 } \\
\text { Po-212 }\end{array}$ & & & & & & \\
\hline \multirow[t]{2}{*}{ Ra-225 } & 1.0 & Ac-225 & 1.0 & $4 x-221$ & 1.0 & At-217 & 1.0 & $\mathrm{Bi}-213$ \\
\hline & $\begin{array}{l}0.022 \\
0.978\end{array}$ & $\begin{array}{l}\text { Tl-209 } \\
\text { Po-213 }\end{array}$ & 1.0 & $\mathrm{~Pb}-209$ & & & & \\
\hline \multirow[t]{2}{*}{ Ra-226 } & 1.0 & $R n 233$ & 1.0 & Pu.210 & $\begin{array}{l}0.0002 \\
0.9998\end{array}$ & $\begin{array}{l}\text { At-218 } \\
\mathrm{Pb}-214\end{array}$ & 1.0 & $\mathrm{Bi}-214$ \\
\hline & $\begin{array}{l}0.0002 \\
0.9998 \\
1.0\end{array}$ & $\begin{array}{l}\text { Tl-210 } \\
\text { Po } 214 \\
\text { Ac-228 }\end{array}$ & $\begin{array}{l}1.0 \\
1.0\end{array}$ & $\begin{array}{l}\text { Ph-210 } \\
\text { Th-228 }\end{array}$ & $\begin{array}{l}1.0 \\
1.0\end{array}$ & $\begin{array}{l}\text { Bi } 210 \\
\text { Ra-224 }\end{array}$ & $\begin{array}{l}0.0000013 \\
0.99999811 \\
1.0\end{array}$ & $\begin{array}{l}\text { Tl-206 } \\
\text { Yn-210 } \\
\text { Rn-220 }\end{array}$ \\
\hline $\mathrm{Ra}-228$ & 1.0 & Po-216 & 1.0 & $\mathrm{~Pb}-212$ & 1.0 & $\mathrm{Bi}-212$ & $\begin{array}{l}0.36 \\
0.64\end{array}$ & $\begin{array}{l}\text { Tl-208 } \\
\text { Po-212 }\end{array}$ \\
\hline$F_{r}-221$ & 1.0 & At-217 & 1.0 & $\mathrm{Bi}-213$ & $\begin{array}{l}0.022 \\
0.978\end{array}$ & $\begin{array}{l}\text { Tl-209 } \\
\text { Po-213 }\end{array}$ & 1.0 & Pb-209 \\
\hline \multirow[t]{2}{*}{$F_{T}-223$} & 1.0 & $\mathrm{Ra}-223$ & 1.0 & $\mathrm{Rn}-219$ & 1.0 & Po-215 & $\begin{array}{l}000 \cap 0 \cap 2.3 \\
0.4999477\end{array}$ & $\begin{array}{l}\text { At_215 } \\
\text { Ph_?11 }\end{array}$ \\
\hline & 1.0 & $\mathrm{Bi}-211$ & $\begin{array}{l}0.0028 \\
0.9972\end{array}$ & $\begin{array}{l}\text { Po-211 } \\
\text { 'll-20'? }\end{array}$ & & & & \\
\hline Ác-225 & 1.0 & $F_{r}-221$ & 1.0 & At- 217 & 1.0 & $\mathrm{Bi}-213$ & $\begin{array}{l}0.022 \\
0.978\end{array}$ & $\begin{array}{l}\text { Tl-209 } \\
\text { Po-2l3 }\end{array}$ \\
\hline \multirow[t]{2}{*}{ Ac- $2 Z Z$} & $\begin{array}{l}1.0 \\
0.014 \\
0.986\end{array}$ & $\begin{array}{l}\text { Ph-2099 } \\
F_{r-223} \\
\text { Th-227 }\end{array}$ & 1.0 & Ra-223 & 1.0 & $R n-219$ & 1.0 & Po-215 \\
\hline & $\begin{array}{l}0.0000023 \\
0.9999977\end{array}$ & $\begin{array}{l}\text { At-2 } 15 \\
\mathrm{~Pb}-211\end{array}$ & 1.0 & $\mathrm{Bi}-211$ & $\begin{array}{l}0.0028 \\
0.9972\end{array}$ & $\begin{array}{l}\text { Po-211 } \\
\text { T1-207 }\end{array}$ & & \\
\hline \multirow[t]{2}{*}{ Ac-228 } & 1.0 & Th-228 & 1.0 & $\mathrm{Ra}-224$ & 1.0 & $\mathrm{Rn}-220$ & 1.0 & Po-216 \\
\hline & 1.0 & $\mathrm{~Pb}-212$ & 1.0 & $\mathrm{Bi}-212$ & $\begin{array}{l}0.36 \\
0.64\end{array}$ & $\begin{array}{l}\text { T1-208 } \\
\text { Po-212 }\end{array}$ & & \\
\hline \multirow[t]{2}{*}{ Th-227 } & 1.0 & Ra-223 & 1.0 & $\mathrm{Rn}-219$ & $1.0^{\circ}$ & Po-2.15 & $\begin{array}{l}0.0000023 \\
0.9999977\end{array}$ & $\begin{array}{l}A t-215 \\
P b-211\end{array}$ \\
\hline & 1.0 & $\mathrm{Bi}-211$ & $\begin{array}{l}0.0028 \\
0.9972\end{array}$ & $\begin{array}{l}\text { Po-211 } \\
\text { Tl-207 }\end{array}$ & & & & \\
\hline
\end{tabular}


TABLE F-10 (Cont)

\begin{tabular}{|c|c|c|c|c|c|c|c|c|}
\hline Parent & Fraction & Daughter & Fraction & Daughter & Fraction & Daughter & Fraction & Daughter \\
\hline \multirow[t]{2}{*}{ Th-228 } & 1.0 & $\mathrm{Ra}-224$ & 1.0 & $\mathrm{Rn}-220$ & 1.0 & Po-216 & 1.0 & $\mathrm{~Pb}-212$ \\
\hline & 1.0 & $\mathrm{Bi}-212$ & $\begin{array}{l}0.36 \\
0.64\end{array}$ & $\begin{array}{l}\text { Tl-208 } \\
\text { Po-212 }\end{array}$ & & & & \\
\hline \multirow[t]{2}{*}{ Th-229 } & 1.0 & Ra-225 & 1.0 & Ac-22S & 1.0 & Fr.221 & 1.0 & At-217 \\
\hline & 1.0 & $\mathrm{Bi}-213$ & $\begin{array}{l}0.022 \\
0.978\end{array}$ & $\begin{array}{l}\text { Tl-209 } \\
\text { Po-213 }\end{array}$ & 1.0 & $\mathrm{~Pb}-209$ & & \\
\hline \multirow[t]{3}{*}{ Th-230 } & 1.0 & $\mathrm{Ra}-226$ & 1.0 & $\mathrm{Rn}-222$ & 1.0 & Po.218 & $\begin{array}{l}0.0002 \\
0.9998\end{array}$ & $\begin{array}{l}\text { At-218 } \\
\mathrm{Pb}-214\end{array}$ \\
\hline & 1.0 & $\mathrm{Bi}-214$ & $\begin{array}{l}0.0002 \\
0.9998\end{array}$ & $\begin{array}{l}\text { Tl-210 } \\
\text { Po-214 }\end{array}$ & 1.0 & $\mathrm{~Pb}-210$ & 1.0 & $\mathrm{Bi}-210$ \\
\hline & $\begin{array}{l}0.0000013 \\
0.9999987\end{array}$ & $\begin{array}{l}\text { Tl-206 } \\
\text { Po-210 }\end{array}$ & & & & & & \\
\hline \multirow[t]{3}{*}{ Th-231 } & 1.0 & Po-231 & 1.0 & Ac-227 & $\begin{array}{l}0.014 \\
0.986\end{array}$ & $\begin{array}{l}\text { Fr-223 } \\
\text { Th-227 }\end{array}$ & 1.0 & Ra-223 \\
\hline & 1.0 & $R n-219$ & 1.0 & Po-215 & $\begin{array}{l}0.0000023 \\
0.9999977\end{array}$ & $\begin{array}{l}\text { At-215 } \\
\mathrm{Pb}-211\end{array}$ & 1.0 & $\mathrm{Bi}-211$ \\
\hline & $\begin{array}{l}0.0028 \\
0.9972 \\
1.0\end{array}$ & $\begin{array}{l}\text { Po-211 } \\
\text { Tl-207 } \\
\text { Ra-228 }\end{array}$ & 1.0 & Ac-228 & 1.0 & Th-228 & 1.0 & Ra-224 \\
\hline \multirow{2}{*}{ Th-232 } & 1.0 & $R n-220$ & 1.0 & Po-216 & 1.0 & $\mathrm{~Pb}-212$ & 1.0 & $\mathrm{Bi}-212$ \\
\hline & $\begin{array}{l}0.36 \\
0.64\end{array}$ & $\begin{array}{l}\text { Tl-208 } \\
\text { Po-212 }\end{array}$ & & & & & & \\
\hline \multirow[t]{4}{*}{ Th-234 } & 1.0 & $\mathrm{~Pa}-234 \mathrm{~m}$ & 0.0013 & $\mathrm{~Pa}-234$ & 1.0 & U-234 & $1: 0$ & Th-230 \\
\hline & 1.0 & Ra-226 & 10 & $\mathrm{Rn}-222$ & 1.0 & Po-218 & $\begin{array}{l}0.0002 \\
0.9998\end{array}$ & $\begin{array}{l}\text { At-218 } \\
\mathrm{Pb}-214\end{array}$ \\
\hline & 1.0 & $\mathrm{Bi}-214$ & $\begin{array}{l}0.0002 \\
0.9998\end{array}$ & $\begin{array}{l}\mathrm{Tl}-210 \\
\text { Po-214 }\end{array}$ & 1.0 & $\mathrm{~Pb}-210$ & 1.0 & $\mathrm{Bi}-210$ \\
\hline & $\begin{array}{l}0.0000013 \\
0.9999987\end{array}$ & $\begin{array}{l}\text { Tl-206 } \\
\text { Po-210 }\end{array}$ & & & & & & \\
\hline \multirow[t]{2}{*}{$\mathrm{Pa}-231$} & 1.0 & Ac-227 & $\begin{array}{l}0.014 \\
0.986\end{array}$ & $\begin{array}{l}\text { Fr }_{\mathrm{r}-223} \\
\text { Th-227 }\end{array}$ & 1.0 & Ra-223 & 1.0 & $\mathrm{Rn}-219$ \\
\hline & 1.0 & Po-215 & $\begin{array}{l}0.0000023 \\
0.9999977\end{array}$ & $\begin{array}{l}\mathrm{At}-215 \\
\mathrm{~Pb}-211\end{array}$ & 1.0 & Bi-21l & $\begin{array}{l}0.0028 \\
0.9972\end{array}$ & $\begin{array}{l}\text { Po-211 } \\
\text { Tl-207 }\end{array}$ \\
\hline \multirow[t]{3}{*}{$\mathrm{Pa}-233$} & 1.0 & U-233 & 1.0 & Th-229 & 1.0 & Ra-225 & 1.0 & Ac-225 \\
\hline & 1.0 . & $F_{\mathrm{r}}-221$ & 1.0 & At-2 17 & 1.0 & $\mathrm{Bi}-213$ & $\begin{array}{l}0.022 \\
0.978\end{array}$ & $\begin{array}{l}\text { Tl-209 } \\
\text { Po-213 }\end{array}$ \\
\hline & $\begin{array}{l}1.0 \\
0.0013\end{array}$ & $\begin{array}{l}\mathrm{Pb}-209 \\
\mathrm{~Pa}-234\end{array}$ & 1.0 & U-234 & 1.0 & Th.230 & 1.0 & $\mathrm{Ra}-226$ \\
\hline \multirow{2}{*}{$\mathrm{Pa}-234 \mathrm{~m}$} & 1.0 & $\mathrm{Rn}-222$ & 1.0 & Po-218 & $\begin{array}{l}0.0002 \\
0.9998\end{array}$ & $\begin{array}{l}\text { At-218 } \\
\mathrm{Pb}-214\end{array}$ & 1.0 & Bi-214 \\
\hline & $\begin{array}{l}0.0002 \\
0.9998\end{array}$ & $\begin{array}{l}\text { Tl-210 } \\
\text { Po-214 }\end{array}$ & 1.0 & $\mathrm{~Pb}-210$ & 1.0 & $\mathrm{Bi}-210$ & $\begin{array}{l}0.0000013 \\
0.9999987\end{array}$ & $\begin{array}{l}\text { Tl-206 } \\
\text { Po-210 }\end{array}$ \\
\hline
\end{tabular}


TABLE F-10 (Cont)

\begin{tabular}{|c|c|c|c|c|c|c|c|c|}
\hline Parent & Fraction & Daughter & Fraction & Daughter & Fraction & Daughter & Fraction & Daughter \\
\hline \multirow[t]{3}{*}{$\mathrm{Pa}-234$} & 1.0 & U-234 & 1.0 & Th-230 & 1.0 & Ra-226 & 1.0 & $\mathrm{Rn}-222$ \\
\hline & 1.0 & Po-218 & $\begin{array}{l}0.0002 \\
0.9998\end{array}$ & $\begin{array}{l}\text { At-218 } \\
\text { Pb-214 }\end{array}$ & 1.0 & $\mathrm{Bi}-214$ & $\begin{array}{l}0.0002 \\
\dot{0} .99988\end{array}$ & $\begin{array}{l}\text { Tl-210 } \\
\text { Po- } 214\end{array}$ \\
\hline & 1.0 & $\mathrm{~Pb}-210$ & 1.0 & $\mathrm{Bi}-210$ & $\begin{array}{l}0.0000013 \\
0.9999987\end{array}$ & $\begin{array}{l}\text { Tl-206 } \\
\text { Po-210 }\end{array}$ & & \\
\hline \multirow[t]{2}{*}{$\mathrm{U}-232$} & 1.0 & Th-228 & 1.0 & Ra-224 & 1.0 & $\mathrm{Rn}-220$ & 1.0 & Po-216 \\
\hline & 1.0 & $\mathrm{~Pb}-212$ & 1.0 & B $\mathrm{i}-\underline{2} 12$ & $\begin{array}{l}0.36 \\
0.64\end{array}$ & $\begin{array}{l}\text { Tl-208 } \\
\text { Po- } 2 \perp 2\end{array}$ & & \\
\hline \multirow[t]{2}{*}{ U 233} & 1.0 & Th-228 & 1.0 & Ra 225 & 1.0 & Ao 335 & 1.0 & Fr 331 \\
\hline & 1.0 & $\Lambda \mathrm{t}-217$ & 1.0 & $B_{i}=213$ & $\begin{array}{l}0.022 \\
0.978\end{array}$ & $\begin{array}{l}\text { Tl-209 } \\
\text { Po-213 }\end{array}$ & 1.0 & PL-209 \\
\hline \multirow[t]{3}{*}{ U-234 } & 1.0 & Th-230 & 1.0 & $\mathrm{Ra}-226$ & 1.0 & $R n-222$ & 1.0 & Po 218 \\
\hline & $\begin{array}{l}0.0002 \\
0.9998\end{array}$ & $\begin{array}{l}\text { At-218 } \\
\text { Pb-214 }\end{array}$ & 1.0 & $\mathrm{Bi}-214$ & $\begin{array}{l}0.0002 \\
0.9998\end{array}$ & $\begin{array}{l}\text { Tl-210 } \\
\text { Po-214 }\end{array}$ & 1.0 & $\mathrm{~Pb}-210$ \\
\hline & 1.0 & $\mathrm{Bi}-210$ & $\begin{array}{l}0.0000013 \\
0.9999987\end{array}$ & $\begin{array}{l}\text { T1-20G } \\
\text { Po-210 }\end{array}$ & & & & \\
\hline \multirow[t]{3}{*}{ U-235 } & 1.0 & Th-231 & 1.0 & $\mathrm{~Pa}-231$ & 1.0 & Ac-227 & $\begin{array}{l}0.014 \\
U .986\end{array}$ & $\begin{array}{l}F_{\mathrm{r}-223} \\
\text { l'h-2'2l }\end{array}$ \\
\hline & 1.0 & $R \rightarrow-2.23$ & 1.0 & $R n-219$ & 1.0 & Po-215 & $\begin{array}{l}0.0000023 \\
0.9999977\end{array}$ & $\begin{array}{l}\text { At-215 } \\
\text { Pb-211 }\end{array}$ \\
\hline & 1.0 & $\mathrm{Bi}-211$ & $\begin{array}{l}0.0028 \\
0.9972\end{array}$ & $\begin{array}{l}\text { Po-21 1 } \\
\text { Tl-207 }\end{array}$ & & & . & \\
\hline \multirow[t]{2}{*}{$\overline{\mathrm{U}}-236$} & 1.0 & $\mathrm{~T} h-232$ & 1.0 & $\mathrm{Ra}-228$ & 1.0 & Ac-228 & 1.0 & Th-228 \\
\hline & $\begin{array}{l}1.0 \\
1.0\end{array}$ & $\begin{array}{l}\mathrm{Ra}-224 \\
\mathrm{Bi}-212\end{array}$ & $\begin{array}{l}1.0 \\
0.36 \\
0.64\end{array}$ & $\begin{array}{l}\mathrm{Rn}-220 \\
\mathrm{Tl}-208 \\
\mathrm{P} 0-212\end{array}$ & $1.0^{\circ}$ & Po-216 & 1.0 & $\mathrm{~Pb}-212$ \\
\hline \multirow[t]{3}{*}{$\mathrm{U}-237$} & 1.0 & Np-237 & 1.0 & $\mathrm{~Pa}-233$ & 1.0 & U-233 & 1.0 & Th-229 \\
\hline & 1.0 & $\mathrm{Ra}-225$ & 1.0 & Ac-225 & 1.0 & Fr-22l & 1.0 & $A t-217$ \\
\hline & 1.0 & $\mathrm{Bi}-213$ & $\begin{array}{l}0.022 \\
0.978\end{array}$ & $\begin{array}{l}\text { Tl-209 } \\
\text { Po-2 } 13\end{array}$ & 1.0 & $\mathrm{~Pb}-209$ & & \\
\hline \multirow[t]{2}{*}{$\mathrm{U}-238$} & 1.0 & Th-234 & 1.0 & $P_{a}-234 \mathrm{~m}$ & 0.0013 & $\mathrm{~Pa}-234$ & 1.0 & $\mathrm{U}-234$ \\
\hline & 1.0 & Th-230 & 1.0 & $\mathrm{Ra}-226$ & 1.0 & $\mathrm{Rn}-222$ & 1.0 & $\mathrm{Po}_{0} 218$ \\
\hline & $\begin{array}{l}0.0002 \\
0.9998\end{array}$ & $\begin{array}{l}\text { At-218 } \\
\text { Pb-2 } 14\end{array}$ & 1.0 & $B i-214$ & $\begin{array}{l}0.0002 \\
0.9998\end{array}$ & $\begin{array}{l}\text { Tl-210 } \\
\text { Po-214 }\end{array}$ & 1.0 & $\mathrm{~Pb}-210$ \\
\hline & 1.0 & $B i-210$ & $\begin{array}{l}0.0000013 \\
0.9999987\end{array}$ & $\begin{array}{l}\text { Tl-2UE } \\
\text { Po-2 } 10\end{array}$ & & & & \\
\hline \multirow[t]{4}{*}{ U-239 } & 1.0 & Np-239 & 1.0 & $\mathrm{Pu}-239$ & 1.0 & U-235 & 1.0 & Th-231. \\
\hline & 1.0 & $\mathrm{~Pa}-231$ & 1.0 & Ac-227 & $\begin{array}{l}0.014 \\
0.986\end{array}$ & $\begin{array}{l}\text { Fr-223 } \\
\text { Th-227 }\end{array}$ & 1.0 & $\mathrm{Ra}-223$ \\
\hline & 1.0 & $R n-219$ & 1.0 & $P_{n-215}$ & $\begin{array}{l}0.0000023 \\
0.9999977\end{array}$ & $\begin{array}{l}\text { At-2.15 } \\
\mathrm{Pb}-211\end{array}$ & 1.0 & $\mathrm{Bi}-2.11$ \\
\hline & $\begin{array}{l}0.0028 \\
0.9972\end{array}$ & $\begin{array}{l}\text { Po-211 } \\
\text { Tl-207 }\end{array}$ & & & & & & \\
\hline
\end{tabular}


TABLE F-10 (Cont)

\begin{tabular}{|c|c|c|c|c|c|c|c|c|}
\hline Parent & Fraction & Daughter & Fraction & Daughter & Fraction & Daughter & Fraction & Daughter \\
\hline \multirow[t]{3}{*}{$\mathrm{Np}-237$} & 1.0 & $\mathrm{~Pa}-233$ & 1.0 & U-233 & 1.0 & Th-229 & 1.0 & $\mathrm{Ra}-225$ \\
\hline & 1.0 & Ac-225 & 1.0 & $F_{r-221}$ & 1.0 & At-217 & 1.0 & $\mathrm{Bi}-213$ \\
\hline & $\begin{array}{l}0.022 \\
0.978\end{array}$ & $\begin{array}{l}\text { Tl-209 } \\
P_{0-2.13}\end{array}$ & 1.0 & $\mathrm{~Pb}-209$ & & & & \\
\hline \multirow[t]{2}{*}{$\mathrm{N}_{\mathrm{p}-238}$} & 1.0 & $\mathrm{Pu}-238$ & 1.0 & $\mathrm{U}-234$ & 1.0 & Th-230 & 1.0 & Ra-226 \\
\hline & 1.0 & $\mathrm{Rn}-222$ & 1.0 & $P_{n}-218$ & $\begin{array}{l}0.0002 \\
0.9998\end{array}$ & $\begin{array}{l}\text { At-218 } \\
\text { Pb-214 }\end{array}$ & 1.0 & Bi-214 \\
\hline \multirow{3}{*}{ Np-239 } & $\begin{array}{l}0.0002 \\
0.9998 \\
10\end{array}$ & $\begin{array}{l}\text { Tl-2.10 } \\
\text { Po-214 } \\
\text { Pu-239 }\end{array}$ & 1.0 & $\mathrm{~Pb}-210$ & 1.0 & $\begin{array}{l}\mathrm{Bi}-210 \\
\mathrm{Th}-231\end{array}$ & $\begin{array}{l}0.0000013 \\
0.9999987 \\
1.0\end{array}$ & $\begin{array}{l}\text { Tl-206 } \\
\text { Po-210 } \\
\text { Pa-231 }\end{array}$ \\
\hline & 1.0 & Ac-227 & $\begin{array}{l}0.014 \\
0.986\end{array}$ & $\begin{array}{l}F_{r}-223 \\
\text { Th-227 }\end{array}$ & 1.0 & Ra-223 & 1.0 & $R n-219$ \\
\hline & 1.0 & Po-215 & $\begin{array}{l}0.0000023 \\
0.9999977\end{array}$ & $\begin{array}{l}\mathrm{At}-215 \\
\mathrm{~Pb}-211\end{array}$ & 1.0 & $\mathrm{Bi}-21 !$ & $\begin{array}{l}0.0028 \\
0.9972\end{array}$ & $\begin{array}{l}\text { Po-211 } \\
\text { Tl-207 }\end{array}$ \\
\hline \multirow[t]{3}{*}{ Np-240 } & 1.0 & $\mathrm{Pu}-240$ & $1.0, \cdots$ & $\mathrm{U}-236$ & 1.0 & Th-232 & 1.0 & $\mathrm{Ra}-228$ \\
\hline & 1.0 & Ac- 228 & $1: 0$ & Th-228 & 1.0 & Ra-224 & 1.0 & $\mathrm{Rn}-220$ \\
\hline & 1.0 & Po-216 & 1.0 & $\mathrm{~Pb}-212$ & 1.0 & $\mathrm{Bi}-212$ & $\begin{array}{l}0.36 \\
0.64\end{array}$ & $\begin{array}{l}\text { Tl-208 } \\
\text { Po-212 }\end{array}$ \\
\hline \multirow[t]{3}{*}{$\mathrm{Pu}-238$} & 1.0 & U-234 & 1.0 & Th-230 & 1.0 & Ra-226 & 1.0 & Rn-222 \\
\hline & 1.0 & Po-218 & $\begin{array}{l}0.0002 \\
0.9998\end{array}$ & $\begin{array}{l}\text { At-218 } \\
\text { Pb-214 }\end{array}$ & 1.0 & $B i-214$ & $\begin{array}{l}0.0002 \\
0.9998\end{array}$ & $\begin{array}{l}\text { Tl-210 } \\
\text { Po-214 }\end{array}$ \\
\hline & 1.0 & $\mathrm{~Pb}-210$ & 1.0 & $\mathrm{Bi}-210$ & $\begin{array}{l}0.0000013 \\
0.9999987\end{array}$ & $\begin{array}{l}\text { Tl-206 } \\
\text { Po-210 }\end{array}$ & & \\
\hline \multirow[t]{3}{*}{ Pu-239 } & 1.0 & U-235 & 1.0 & Th-231 & 1.0 & $\mathrm{~Pa}-231$ & 1.0 & Ac-227 \\
\hline & $\begin{array}{l}0.014 \\
0.986\end{array}$ & $\begin{array}{l}\text { Fr-223 } \\
\text { Th-227 }\end{array}$ & 1.0 & Ra-223 & 1.0 & $R n-219$ & 1.0 & Po-215 \\
\hline & $\begin{array}{l}0.0000023 \\
0.9999977\end{array}$ & $\begin{array}{l}\text { At-215 } \\
\mathrm{Pb}-211\end{array}$ & 1.0 & $\mathrm{Bi}-21 \mathrm{l}$ & $\begin{array}{l}0.0028 \\
0.9972\end{array}$ & $\begin{array}{l}\text { Po-211 } \\
\text { Tl-207 }\end{array}$ & & \\
\hline \multirow{3}{*}{$\overline{\mathrm{P}} \mathrm{u}-240$} & 1.0 & $0-236$ & 1.0 & Th-2̌32 & 1.0 & Ra-228 & 1.0 & $A=-228$ \\
\hline & 1.0 & Th-228 & 1.0 & $R a-224$ & 1.0 & $R n-220$ & 1.0 & Po-216 \\
\hline & 1.0 & $\mathrm{~Pb}-212$ & 1.0 & $B i-212$ & $\begin{array}{l}0.36 \\
0.64\end{array}$ & $\begin{array}{l}\text { Tl-208 } \\
\text { Po-212 }\end{array}$ & 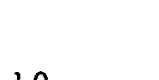 & 202 \\
\hline \multirow{2}{*}{$\mathrm{Pu}-241$} & 10 & Th-229 & 10 & $\mathrm{R}$ - 225 & 10 & $A c-225$ & 10 & $F_{r-2} 21$ \\
\hline & 1.0 & At-217 & 1.0 & $B i-213$ & $\begin{array}{l}0.022 \\
0.978\end{array}$ & $\begin{array}{l}\text { Tl-209 } \\
\text { Po-213 }\end{array}$ & 1.0 & $\mathrm{~Pb}-209$ \\
\hline \multirow[t]{4}{*}{$\mathrm{Pu}-242$} & 1.0 & $\mathrm{U}-238$ & 1.0 & Th-234 & 1.0 & $\mathrm{Pd}-234 \mathrm{d1}$ & 0.0013 & $\mathrm{~Pa}-234$ \\
\hline & 1.0 & U-234 & 1.0 & Th-230 & 1.0 & $\mathrm{Ra}-226$ & 1.0 & $\mathrm{Rn}-222$ \\
\hline & 1.0 & Po-218 & $\begin{array}{l}0.0002 \\
0.9998\end{array}$ & $\begin{array}{l}\text { At-2 } 18 \\
\mathrm{~Pb}-214\end{array}$ & 1.0 & $\mathrm{Bi}-214$ & $\begin{array}{l}0.0002 \\
0.9998\end{array}$ & $\begin{array}{l}\text { Tl-210 } \\
\text { Po-214 }\end{array}$ \\
\hline & 1.0 & $\mathrm{~Pb}-210$ & 1.0 & $\mathrm{Bi}-210$ & $\begin{array}{l}0.0000013 \\
\cap 9999987\end{array}$ & $\begin{array}{l}\text { Tl-206 } \\
P_{n}-210\end{array}$ & & \\
\hline
\end{tabular}


TABLE F-10 (Cont)

\begin{tabular}{|c|c|c|c|c|c|c|c|c|}
\hline Parent & Fraction & Daughter & Fraction & Daughter & Fraction & Daughter & Fraction & Daughter \\
\hline \multirow[t]{3}{*}{ Am-24l } & 1.0 & $\mathrm{~N}_{\mathrm{p}-237}$ & 1.0 & $\mathrm{~Pa}-233$ & 1.0 & U-233 & 1.0 & $T h-229$ \\
\hline & 1.0 & Ra-225 & 1.0 & $F_{I-221}$ & 1.0 & A1-217 & 1.0 & $\mathrm{Bi}-213$ \\
\hline & $\begin{array}{l}0.022 \\
0.978\end{array}$ & $\begin{array}{l}\text { Tl-209 } \\
\text { Po-213 }\end{array}$ & 1.0 & $\mathrm{~Pb}-209$ & & & & \\
\hline \multirow[t]{3}{*}{$A m-242 m$} & 1.0 & Np-238 & 1.0 & Pu-238 & 1.0 & U-234 & 1.0 & Th-230 \\
\hline & 1.0 & Ra-226 & 1.0 & $R n-222$ & 1.0 & Po-218 & $\begin{array}{l}0.0002 \\
0.9998\end{array}$ & $\begin{array}{l}\mathrm{At}-218 \\
\overline{\mathrm{P} b}-214\end{array}$ \\
\hline & 1.0 & Ri-214 & $\begin{array}{l}0.0002 \\
0.9998\end{array}$ & $\begin{array}{l}\text { Tl-210 } \\
\text { Po-214 }\end{array}$ & 1.0 & $\mathrm{~Pb}-210$ & 1.0 & $\mathrm{Bi}-210$ \\
\hline \multirow{4}{*}{ Am-243 } & $\begin{array}{l}0.0 n 00013 \\
0.9999987\end{array}$ & $\begin{array}{l}\text { T1 } 206 \\
\text { Po-210 }\end{array}$ & & & & & & \\
\hline & 1.0 & Np-239 & 1.0 & Pu-239 & 1.0 & U-235 & 1.0 & Th-231 \\
\hline & 1.0 & $\mathrm{~Pa}-231$ & 1.0 & Ac-227 & $\begin{array}{l}0.14 \\
0.986\end{array}$ & $\begin{array}{l}\text { Fy-223 } \\
\text { Th-227 }\end{array}$ & 1.0 & Ra-223 \\
\hline & 1.0 & $R n-219$ & 1.0 & Po-215 & $\begin{array}{l}0.0000023 \\
0.9999977\end{array}$ & $\begin{array}{l}\text { At-215 } \\
\mathrm{Pb}-211\end{array}$ & 1.0 & $\mathrm{Bi}-211$ \\
\hline \multirow{3}{*}{$\mathrm{Cm} \cdot 242$} & $\begin{array}{l}0.0028 \\
0.9972\end{array}$ & $\begin{array}{l}\text { Po-211 } \\
\text { Tl-207 }\end{array}$ & & & & & & \\
\hline & 1.0 & Pu-238 & 1.0 & U-234 & 1.0 & Th-230 & 1.0 & Ra-226 \\
\hline & 1.0 & Rn-222 & 1.0 & Po-218 & $\begin{array}{l}0.0002 \\
0.9998\end{array}$ & $\begin{array}{l}\text { At-218 } \\
\mathrm{Pb}-214\end{array}$ & 1.0 & $B i-214$ \\
\hline \multirow{3}{*}{$C_{\mathrm{m}-244}$} & $\begin{array}{l}0.0002 \\
0.9938 \\
0\end{array}$ & $\begin{array}{l}\text { T1-2.10 } \\
P_{u}=214 \\
P_{u}-240\end{array}$ & 1.0 & $\mathrm{~Pb}_{\mathrm{b}} 210$ & 1.0 & $\mathrm{Bi}-210$ & $\begin{array}{l}0.0000 n 13 \\
0.9999987 \\
10\end{array}$ & $\begin{array}{l}\text { Tl-206 } \\
\text { Po-210 } \\
\text { Ra-228 }\end{array}$ \\
\hline & 1.0 & Ac-228 & 1.0 & $T_{h}-228$ & 1.0 & Ra-224 & 1.0 & $R n-220$ \\
\hline & 1.0 & Po-216 & 1.0 & $\mathrm{~Pb}-212$ & 1.0 & $\mathrm{Bi}-212$ & $\begin{array}{l}0.36 \\
0.64\end{array}$ & $\begin{array}{l}\text { Tl-208 } \\
\text { Po-212 }\end{array}$ \\
\hline
\end{tabular}

